Supporting Information for

\title{
Mechanistic Studies and Expansion of the Substrate Scope of Direct Enantioselective Alkynylation of $\alpha$-Ketiminoesters Catalyzed by Adaptable (Phebox)Rh(III) Complexes
}

\author{
Kazuhiro Morisaki, ${ }^{\dagger \dagger}$ Masanao Sawa ${ }^{\dagger,}{ }^{\dagger}$ Ryohei Yonesaki, ${ }^{\dagger}$ Hiroyuki Morimoto,,,$\dagger$ \\ Kazushi Mashima, ${ }^{\S}$ Takashi Ohshima*,† \\ ${ }^{\dagger}$ Graduate School of Pharmaceutical Sciences, Kyushu University, Fukuoka 812-8582, \\ Japan. ${ }^{\S}$ Department of Chemistry, Graduate School of Engineering Science, Osaka \\ University, Toyonaka, Osaka 560-8531, Japan. \\ e-mail address: ohshima@phar.kyushu-u.ac.jp,hmorimot@phar.kyushu-u.ac.jp
}

\section{Table of Contents}

1. General Experimental Details $\quad$ S2

2. Isolation of (Alkynyl)Rh(III) Complexes $\quad$ S3

3. Identification of the Proton Source $\quad \mathrm{S} 10$

4. Kinetic Experiments (1): Comparison of Reactivity of Rh(III) Complexes $\mathrm{S} 14$

5. Kinetic Experiments (2): Initial Rate Kinetic Studies of the Catalytic Cycle S16

6. Kinetic Experiments (3): Reaction Progress Kinetic Analysis $\quad$ S21

7. Kinetic Experiments (4): Kinetic Isotope Effects $\quad$ S24

8. Kinetic Experiments (5): Eyring Plots $\quad$ S25

$\begin{array}{ll}\text { 9. Reversibility of Reaction Steps } & \text { S27 }\end{array}$

$\begin{array}{ll}\text { 10. Non-Linear Effects } & \text { S31 }\end{array}$

11. Computational Analysis of the Transition States $\quad$ S32

12. Improvement of the Catalytic Activity (1): Development of Precatalyst S47

13. Improvement of the Catalytic Activity (2): Substrate Scope S50

14. Expansion of the Substrate Scope (1): $\alpha$-Ketiminophosphonate $\quad$ S60

15. Expansion of the Substrate Scope (2): Cyclic $N$-Sulfonyl $\alpha$-Ketiminoesters S65

16. Determination of Absolute Configuration of Products S71

$\begin{array}{ll}\text { 17. Spectral Data } & \text { S76 }\end{array}$ 


\section{General Experimental Details}

All reactions were performed in flame-dried or oven-dried glassware under an argon atmosphere unless otherwise noted. Reagents and solvents were obtained from commercial sources and used as received unless otherwise stated. Flash silica gel column chromatography was performed with Kanto Chemical silica gel 60N (spherical neutral, particle size $40-50 \mu \mathrm{m})$.

Nuclear magnetic resonance (NMR) spectra were acquired on $400 \mathrm{MHz}$ Varian Unity and $500 \mathrm{MHz}$ Bruker Avance III spectrometers. ${ }^{1} \mathrm{H}$ and ${ }^{13} \mathrm{C}\left\{{ }^{1} \mathrm{H}\right\}$ NMR chemical shifts are reported in ppm and referenced to tetramethylsilane or residual solvent peaks as internal standards (for $\mathrm{CDCl}_{3}$, tetramethylsilane $0 \mathrm{ppm}$ for ${ }^{1} \mathrm{H}$ and $\mathrm{CDCl}_{3} 77.0 \mathrm{ppm}$ for ${ }^{13} \mathrm{C}\left\{{ }^{1} \mathrm{H}\right\}$; for DMSO- $d_{6}, 2.50 \mathrm{ppm}$ for ${ }^{1} \mathrm{H}$ and $39.5 \mathrm{ppm}$ for ${ }^{13} \mathrm{C}\left\{{ }^{1} \mathrm{H}\right\}$; for $\mathrm{C}_{6} \mathrm{D}_{6}, 7.16 \mathrm{ppm}$ for ${ }^{1} \mathrm{H}$ and 128.06 ppm for ${ }^{13} \mathrm{C}\left\{{ }^{1} \mathrm{H}\right\}$; for toluene- $d_{8}, 2.08$ ppm for ${ }^{1} \mathrm{H}$ and $20.4 \mathrm{ppm}$ for $\left.{ }^{13} \mathrm{C}\left\{{ }^{1} \mathrm{H}\right\}\right) .{ }^{19} \mathrm{~F}$ NMR chemical shifts are reported in ppm relative to $\alpha, \alpha, \alpha$-trifluorotoluene at $-63.72 \mathrm{ppm}$ as external reference. ${ }^{31} \mathrm{P}$ NMR chemical shifts are reported in ppm relative to phosphoric acid at $0 \mathrm{ppm}$ as external reference. Coupling constants are reported in hertz. The following abbreviations are used: $\mathrm{s}=$ singlet, $\mathrm{d}=$ doublet, $\mathrm{t}=$ triplet, $\mathrm{q}=$ quartet, $\mathrm{m}=$ multiplet, $\mathrm{br}=$ broad. Infrared (IR) spectra were recorded with Shimadzu FTIR-8400. High-resolution mass spectroscopy (HRMS) was obtained with Waters ACQUITY UPLC ${ }^{\circledR}$-LCT-Premier ${ }^{\text {TM }}$ XE system. High performance liquid chromatography (HPLC) was performed with JASCO PU-2089plus pump and UV-2075plus detector. Chiral HPLC analysis was performed with DAICEL CHIRALCEL OD-3, CHIRALPAK AD-3 and CHIRALPAK AS-3 column series. Optical rotation was measured with JASCO P2200 polarimeter. Melting points were measured by Yanaco Micro Melting Point System MP-J3 and are uncorrected. Elemental analysis was performed by Yanaco CHN coder MT-5 or MT-6 at the Service Center of the Elementary Analysis of Organic Compounds in Kyushu University.

(Aqua)(diacetato)(phebox)Rh(III) complexes 1 were prepared according to the literature procedure. ${ }^{1} \alpha$-Ketiminoesters $\mathbf{2}, \mathbf{9}$ and $\mathbf{1 1}$ were prepared according to the literature procedure $^{2}$ with some modification for 9 (see Chapter 14 of this SI for details). Alkynes 3 were either purchased from commercial sources or prepared according to the literature procedure. $^{3}$ 


\section{Isolation of (Alkynyl)Rh(II) Complexes}

$\left(\right.$ Acetato-k $\left.\kappa^{2} O, O^{\prime}\right)($ alkynyl)(phebox)Rh(III) complexes 5, 7, 13 were prepared according to literature procedure. ${ }^{4}$

\section{(Acetato-k $\left.{ }^{2} O, O^{\prime}\right)(2,6-b i s((3 a S, 8 a R)-8,8 a-d i h y d r o-3 a H$-indeno[1,2-d]} oxazol-2-yl)-4-nitrophenyl- $\left.\kappa^{3} N, C^{1}, N^{\prime}\right)($ phenylethynyl)rhodium(III) (5a)

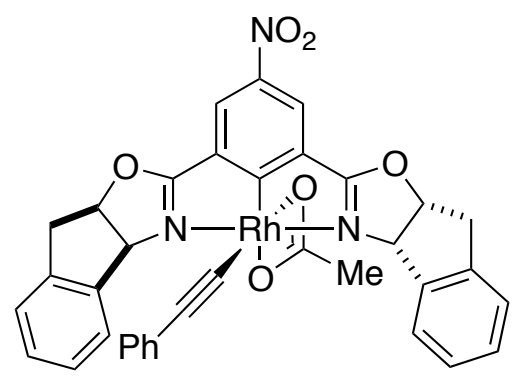

Reaction was performed according to the literature procedure on a $0.20 \mathrm{mmol}$ scale, and the mixture was purified using hexane/EtOAc $=1 / 1$ to EtOAc as eluent to give $\mathbf{5 a}$ as a yellow solid ( $74.0 \mathrm{mg}, 53 \%$ yield).

${ }^{1} \mathrm{H}$ NMR $\left(500 \mathrm{MHz}, \mathrm{CDCl}_{3}\right) \delta 8.38(\mathrm{~d}, J=2.0 \mathrm{~Hz}, 1 \mathrm{H})$, $8.34(\mathrm{~d}, J=2.0 \mathrm{~Hz}, 1 \mathrm{H}), 8.14(\mathrm{~d}, J=8.0 \mathrm{~Hz}, 1 \mathrm{H}), 7.89(\mathrm{~d}, J$ $=8.0 \mathrm{~Hz}, 1 \mathrm{H}) 7.34-7.278 .14(\mathrm{~m}, 6 \mathrm{H}), 6.95-6.91(\mathrm{~m}, 3 \mathrm{H}), 6.58(\mathrm{dd}, J=8.0,2.0 \mathrm{~Hz}, 2 \mathrm{H})$, 6.06 (ddd, $J=8.0,8.0,2.5 \mathrm{~Hz}, 1 \mathrm{H}), 5.98(\mathrm{dd}, J=7.0,7.0 \mathrm{~Hz}, 1 \mathrm{H}), 5.84(\mathrm{~d}, J=7.0 \mathrm{~Hz}, 1 \mathrm{H})$, $5.66(\mathrm{~d}, J=8.0 \mathrm{~Hz}, 1 \mathrm{H}), 3.71-3.50(\mathrm{~m}, 4 \mathrm{H}), 2.01(\mathrm{~s}, 3 \mathrm{H}) ;{ }^{13} \mathrm{C}\left\{{ }^{1} \mathrm{H}\right\} \mathrm{NMR}(125 \mathrm{MHz}$, $\left.\mathrm{CDCl}_{3}\right) \delta 197.72\left(J_{C-R h}=25.0 \mathrm{~Hz}\right), 187.15,171.24\left(J_{C-R h}=4.0 \mathrm{~Hz}\right), 170.87\left(J_{C-R h}=4.4 \mathrm{~Hz}\right)$, 144.17, 139.43, 138.39, 138.16, 137.74, 131.52, 131.21, 131.16, 129.63, 129.38, 127.97, $127.81,127.72,127.16,126.81,126.57,125.31,125.10,124.34,122.00,121.85,102.59$, $89.09,88.37,80.73\left(\mathrm{~d}, J_{C-R h}=47.0 \mathrm{~Hz}\right), 73.39,71.79,39.61,39.33$, 24.57. IR (KBr) 3595, 3387, 3071, 2924, 2855, 2122, 1528, 1466, 1142, 1335, 1296, 1011, 980, 826, 756, 679 $\mathrm{cm}^{-1}$. HRMS (ESI-TOF) $\mathrm{m} / z$ calcd. for $\mathrm{C}_{38} \mathrm{H}_{29} \mathrm{~N}_{5} \mathrm{O}_{4} \mathrm{Rh}\left[\mathrm{M}^{+}-{ }^{-} \mathrm{OAc}+2 \mathrm{MeCN}\right] 722.1275$, found 722.1263. Anal. calcd. for $\mathrm{C}_{36} \mathrm{H}_{26} \mathrm{~N}_{3} \mathrm{O}_{6} \mathrm{Rh}: \mathrm{C}, 61.81 \%$; H, 3.75\%; N, 6.01; found: $\mathrm{C}$, $61.79 \%$; H, 3.80\%; N, 6.00\%.

\section{(Acetato-k $\left.{ }^{2} O, O^{\prime}\right)\left(2,6-b i s((R)-4-p h e n y l o x a z o l i n-2-y l) p h e n y l-\kappa^{3} N, C^{1}, N^{\prime}\right)($ phenylethynyl)} rhodium(III) (7a)

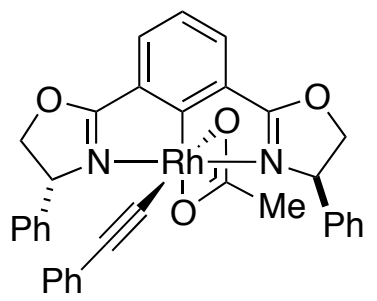

Reaction was performed according to the literature procedure (0.20 mmol scale), and the mixture was purified using hexane/EtOAc $=1 / 1$ to EtOAc as eluent to give $7 \mathbf{a}$ as a yellow solid (61.1 mg, 48\% yield). 
${ }^{1} \mathrm{H}$ NMR $\left(500 \mathrm{MHz}, \mathrm{CDCl}_{3}\right) \delta 7.66(\mathrm{dd}, J=8.5,1.0 \mathrm{~Hz}, 1 \mathrm{H}), 7.63(\mathrm{dd}, J=8.0,1.0 \mathrm{~Hz}$, $1 \mathrm{H}), 7.52-7.50(\mathrm{~m}, 2 \mathrm{H}), 7.31-7.25(\mathrm{~m}, 9 \mathrm{H}), 7.12-7.00(\mathrm{~m}, 5 \mathrm{H}), 5.28-5.12(\mathrm{~m}, 4 \mathrm{H})$, 4.67-4.60 (m, 2H) $1.46(\mathrm{~s}, 3 \mathrm{H}) ;{ }^{13} \mathrm{C}\left\{{ }^{1} \mathrm{H}\right\} \mathrm{NMR}\left(125 \mathrm{MHz}, \mathrm{CDCl}_{3}\right) \delta 186.61\left(\mathrm{~d}, J_{C-R h}=25.3\right.$ $\mathrm{Hz}), 183.43,173.02\left(\mathrm{~d}, J_{C-R h}=4.8 \mathrm{~Hz}\right), 172.79\left(\mathrm{~d}, J_{C-R h}=4.9 \mathrm{~Hz}\right), 138.87,137.92,131.38$, $130.50,128.56$, 128.51, 128.35, 128.24, 128.12, 127.79, 127.68, 127.45, 124.77, 122.36, $102.02\left(\mathrm{~d}, J_{C-R h}=8.8 \mathrm{~Hz}\right), 86.41\left(\mathrm{~d}, J_{C-R h}=51.4 \mathrm{~Hz}\right), 78.60,78.30,66.47,66.40,23.47 . \mathrm{IR}$ (KBr) 3421, 3051, 3030, 3009, 2976, 2947, 2110, 1614, 1589, 1533, 1483, 1454, 1398, 1327, 1242, 1215, 1148, 1059, 1013, 962, $931 \mathrm{~cm}^{-1}$. HRMS (ESI-TOF) $\mathrm{m} / z$ calcd. for $\mathrm{C}_{36} \mathrm{H}_{30} \mathrm{~N}_{4} \mathrm{O}_{2} \mathrm{Rh}\left[\mathrm{M}^{+}-{ }^{-} \mathrm{OAc}+2 \mathrm{MeCN}\right]$ 653.1424, found 653.1425. Anal. calcd. for $\mathrm{C}_{34} \mathrm{H}_{27} \mathrm{~N}_{2} \mathrm{O}_{4} \mathrm{Rh}$ : C, 64.77\%; H, 4.32\%; N, 4.44\%; found: C, 64.78\%; H, 4.33\%; N, 4.46\%.

\section{(Acetato-k $\left.{ }^{2} O, O^{\prime}\right)(2,6-b i s((3 a S, 8 a R)-8,8 a-d i h y d r o-3 a H$-indeno[1,2-d]} oxazol-2-yl)-4-nitrophenyl- $\left.\kappa^{3} N, C^{1}, N^{\prime}\right)(4-t$ rifluoromethylphenylethynyl)rhodium(III) (5b)

Reaction was performed according to the literature procedure on a $0.10 \mathrm{mmol}$ scale, and the mixture was purified using hexane/EtOAc $=2 / 1$ to EtOAc as eluent to give $\mathbf{5 b}$ as a yellow solid (46.5 mg, 61\% yield).

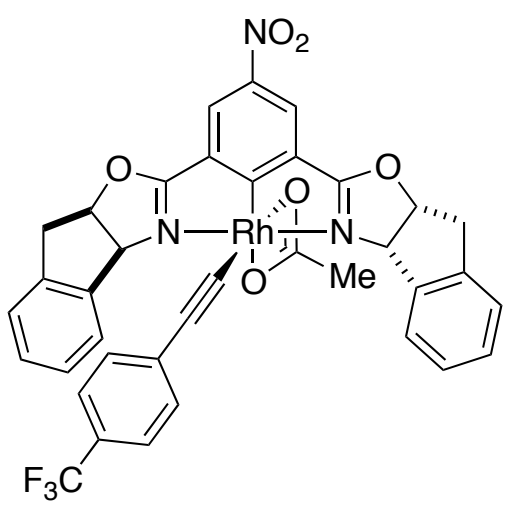

${ }^{1} \mathrm{H}$ NMR $\left(500 \mathrm{MHz}, \mathrm{CDCl}_{3}\right) \delta 8.42(\mathrm{~s}, 1 \mathrm{H}), 8.38$ (s, $1 \mathrm{H}), 8.12(\mathrm{~d}, J=5.0 \mathrm{~Hz}, 1 \mathrm{H}), 7.89(\mathrm{~d}, J=6.5 \mathrm{~Hz}, 1 \mathrm{H})$, 7.35-7.29 (m, 6H), $7.18(\mathrm{~d}, J=8.0 \mathrm{~Hz}, 2 \mathrm{H}), 6.62(\mathrm{~d}, J=$ $8.0 \mathrm{~Hz}, 2 \mathrm{H}), 6.09$ (ddd, $J=6.0,6.0,2.0 \mathrm{~Hz}, 1 \mathrm{H}), 6.03$ (dd, $J=7.5,7.5 \mathrm{~Hz}, 1 \mathrm{H}), 3.73-3.51(\mathrm{~m}, 4 \mathrm{H}), 2.04(\mathrm{~s}, 3 \mathrm{H})$; ${ }^{13} \mathrm{C}\left\{{ }^{1} \mathrm{H}\right\}$ NMR $\left(125 \mathrm{MHz}, \mathrm{CDCl}_{3}\right) \delta 197.16\left(\mathrm{~d}, J_{C-R h}=25.0\right.$ $\mathrm{Hz}), 187.65,171.27,170.93\left(\mathrm{~d}, J_{C-R h}=4.5 \mathrm{~Hz}\right), 144.36$, $139.46,138.46,137.71,131.57,131.15,130.51,129.73$, 129.46, 127.92, 127.81, 127.75, $126.65\left(\mathrm{q}, J_{C-F}=32.1 \mathrm{~Hz}\right), 126.58,125.37,124.83,124.29$ $\left(\mathrm{q}, J_{C-F}=135 \mathrm{~Hz}\right), 124.10,124.08\left(\mathrm{q}, J_{C-F}=3.8 \mathrm{~Hz}\right), 124.07,122.17,122.02,102.11,89.09$, $88.37,86.42\left(\mathrm{q}, J_{C-F}=46.5 \mathrm{~Hz}\right), 73.54,71.72,39.69,39.45,24.49 ;{ }^{19} \mathrm{~F}$ NMR $(360 \mathrm{MHz}$, $\left.\mathrm{CDCl}_{3}\right) \delta$-75.97. IR (KBr) 3431, 2926, 2123, 1611, 1528, 1470, 1373, 1321, 1242, 1161, 1121, 1065, 1047, 1022, 1009, $843 \mathrm{~cm}^{-1}$; HRMS (ESI-TOF) $\mathrm{m} / z$ calcd. for 
$\mathrm{C}_{39} \mathrm{H}_{28} \mathrm{~F}_{3} \mathrm{~N}_{5} \mathrm{O}_{4} \mathrm{Rh}\left[\mathrm{M}^{+}-{ }^{-} \mathrm{OAc}+2 \mathrm{MeCN}\right]$ 790.1148, found 790.1146. Anal. calcd for $\mathrm{C}_{37} \mathrm{H}_{25} \mathrm{~F}_{3} \mathrm{~N}_{3} \mathrm{O}_{6} \mathrm{Rh}: \mathrm{C}, 57.90 \%$; H, 3.28\%; N, 5.47; found: C, 58.11\%; H, 3.44\%; N, 5.46\%.

(Acetato- $\left.{ }^{2} O, O^{\prime}\right)(2,6-b i s((3 a S, 8 a R)-8,8 a-d i h y d r o-3 a H-i n d e n o[1,2-d]$ oxazol-2-yl)-4-nitrophenyl-k $\left.{ }^{3} N, C^{1}, N^{\prime}\right)$ (trimethylsilylethynyl)rhodium(III) (5d)

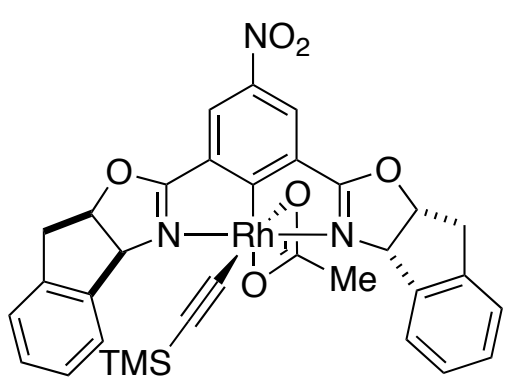

To a $20 \mathrm{~mL}$ screw vial were added activated molecular sieves 4A (200 mg), (aqua)(diacetato)(phebox)Rh(III) 1a (67.5 mg, $0.10 \mathrm{mmol}$ ), trimethylsilylacetylene 3d (138 $\mu \mathrm{L}$, $1.00 \mathrm{mmol}, 10.0$ equiv) and toluene $(4.0 \mathrm{~mL})$ in a glove box and the mixture was heated on a hot plate at $60{ }^{\circ} \mathrm{C}$ for

$24 \mathrm{~h}$. The crude mixture was directly purified by column chromatography using hexane/EtOAc $=2 / 1$ to EtOAc as eluent to give $\mathbf{5 d}$ as a yellow solid (46.6 mg, 67\% yield).

${ }^{1} \mathrm{H}$ NMR (500 MHz, $\left.\mathrm{CDCl}_{3}\right) \delta 8.38(\mathrm{~s}, 1 \mathrm{H}), 8.33(\mathrm{~s}, 1 \mathrm{H}), 8.05(\mathrm{~d}, J=8.5 \mathrm{~Hz}, 1 \mathrm{H}), 7.87$ (d, $J=8.5 \mathrm{~Hz}, 1 \mathrm{H}), 7.39-7.26(\mathrm{~m}, 8 \mathrm{H}), 6.04$ (ddd, $J=8.5,8.5,2.5 \mathrm{~Hz}, 1 \mathrm{H}), 5.98$ (ddd, $J=$ $8.5,8.5,2.0 \mathrm{~Hz}, 1 \mathrm{H}), 5.78(\mathrm{~d}, J=8.5 \mathrm{~Hz}, 1 \mathrm{H}), 5.60(\mathrm{~d}, J=8.5 \mathrm{~Hz}, 1 \mathrm{H}), 3.71-3.49(\mathrm{~m}, 4 \mathrm{H})$, $2.00(\mathrm{~s}, 3 \mathrm{H}),-0.36(\mathrm{~s}, 9 \mathrm{H}) ;{ }^{13} \mathrm{C}\left\{{ }^{1} \mathrm{H}\right\} \mathrm{NMR}\left(125 \mathrm{MHz}, \mathrm{CDCl}_{3}\right) \delta 197.96\left(\mathrm{~d}, J_{C-R h}=25.2 \mathrm{~Hz}\right)$, 187.41, 171,32 (d, $\left.J_{C-R h}=4.8 \mathrm{~Hz}\right), 170.85\left(\mathrm{~d}, J_{C-R h}=4.8 \mathrm{~Hz}\right), 144.15,139.49,138.32$, $138.15,137.61,131.24,131.23,129.65,129.52$, 128.09, 128.00, 127.71, 126.61, 125.31, 124.70, 121.93, 121.68, $108.18\left(\mathrm{~d}, J_{C-R h}=7.1 \mathrm{~Hz}\right), 102.56\left(\mathrm{~d}, J_{C-R h}=51.4 \mathrm{~Hz}\right), 89.05,88.35$, 73.40, 71.67, 39.67, 39.41, 24.53, 0.93. IR (KBr) 3437, 2353, 2052, 1613, 1595, 1526, 1460, 1427, 1410, 1373, 1333, 1298, 1244, 1203, 1047, 1024, 1006, 978, 860, $837 \mathrm{~cm}^{-1}$. HRMS (ESI-TOF) $\mathrm{m} / z$ calcd. for $\mathrm{C}_{35} \mathrm{H}_{33} \mathrm{~N}_{5} \mathrm{O}_{4} \mathrm{RhSi}\left[\mathrm{M}^{+}{ }^{-}{ }^{-} \mathrm{OAc}+2 \mathrm{MeCN}\right]$ 718.1357, found 718.1351. Anal. calcd. for $\mathrm{C}_{33} \mathrm{H}_{30} \mathrm{~N}_{3} \mathrm{O}_{6} \mathrm{RhSi}$ : C, 56.98\%; H, 4.35\%; N, 6.04\%; found: C, $56.09 \%$; H, 4.35\%; N, 5.87\%.

(Acetato- $\left.{ }^{2} O, O^{\prime}\right)\left(2,6-b i s((R)-4-p h e n y l o x a z o l i n-2-y l) p h e n y l-\kappa^{3} N, C^{1}, N^{\prime}\right)\left(\right.$ trimethylsilylet $^{\prime}$ hynyl)rhodium(III) (7d)

To a $20 \mathrm{~mL}$ screw vial were added activated molecular sieves 4A (200 mg), (aqua)(diacetato)(phebox)Rh(III) complex $\mathbf{1 b}(121 \mathrm{mg}, 0.20 \mathrm{mmol})$, trimethylsilylacetylene 
3d $(277 \mu \mathrm{L}, 2.00 \mathrm{mmol}, 10.0$ equiv) and toluene $(4.0 \mathrm{~mL})$ in a glove box and the mixture was heated on a hot plate at $60{ }^{\circ} \mathrm{C}$ for $48 \mathrm{~h}$. The crude mixture was directly purified by column chromatography using hexane/EtOAc $=1 / 4$ to EtOAc/MeOH $=20 / 1$ as eluent to give $7 \mathbf{d}$ as a yellow solid ( $41.1 \mathrm{mg}, 32 \%$ yield).

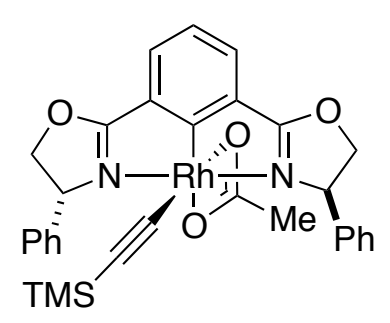

${ }^{1} \mathrm{H}$ NMR $\left(500 \mathrm{MHz}, \mathrm{CDCl}_{3}\right) \delta 7.64(\mathrm{~d}, J=7.5 \mathrm{~Hz}, 1 \mathrm{H}), 7.60(\mathrm{~d}$, $J=7.5 \mathrm{~Hz}, 1 \mathrm{H}), 7.50(\mathrm{~d}, J=7.5 \mathrm{~Hz}, 2 \mathrm{H}), 7.35-7.24(\mathrm{~m}, 10 \mathrm{H})$, $5.26-5.11(\mathrm{~m}, 4 \mathrm{H}), 4.65(\mathrm{t}, J=7.5 \mathrm{~Hz}, 2 \mathrm{H}), 4.58(\mathrm{t}, J=8.0 \mathrm{~Hz}, 2 \mathrm{H})$, $1.30(\mathrm{~s}, 3 \mathrm{H}),-0.08(\mathrm{~s}, 9 \mathrm{H}) ;{ }^{13} \mathrm{C}\left\{{ }^{1} \mathrm{H}\right\}$ NMR $\left(125 \mathrm{MHz}, \mathrm{CDCl}_{3}\right)$ $\delta 185.39\left(\mathrm{~d}, J_{C-R h}=25.1 \mathrm{~Hz}\right), 183.51,171.62\left(\mathrm{~d}, J_{C-R h}=4.9 \mathrm{~Hz}\right)$

$171.42\left(\mathrm{~d}, J_{C-R h}=4.9 \mathrm{~Hz}\right), 137.48,136.36,129.01,128.87,127.06,127.01,126.66,126.63$, $126.31,126.13,126.02,125.71,120.66,107.35\left(\mathrm{~d}, J_{C-R h}=48.1 \mathrm{~Hz}\right), 105.50\left(\mathrm{~d}, J_{C-R h}=7.3\right.$ Hz), 77.25, 76.65, 64.85, 64.45, 21.52, 0.00. IR (KBr) 3245, 2935, 2043, 1616, 1589, 1551, $1485,1454,1397,1329,1244,1147,1061,1026,972,937,860,837 \mathrm{~cm}^{-1}$. HRMS (ESI-TOF) $m / z$ calcd. for $\mathrm{C}_{33} \mathrm{H}_{34} \mathrm{~N}_{4} \mathrm{O}_{2} \mathrm{RhSi}\left[\mathrm{M}^{+}-{ }^{-} \mathrm{OAc}+2 \mathrm{MeCN}\right] 649.1506$, found 649.1513. Anal. calcd. for $\mathrm{C}_{31} \mathrm{H}_{31} \mathrm{~N}_{2} \mathrm{O}_{4} \mathrm{RhSi}$ : C, $59.42 \%$; $\mathrm{H}, 4.99 \%$; $\mathrm{N}, 4.47 \%$; found: $\mathrm{C}$, $60.50 \% ; \mathrm{H}, 4.89 \%$; N, $4.48 \%$.

(Acetato- $\left.\kappa^{2} O, O^{\prime}\right)\left(2,6-b i s((S)-4-b e n z y l o x a z o l i n-2-y l) p h e n y l-\kappa^{3} N, C^{1}, N^{\prime}\right)($ phenylethynyl)r hodium(III) (13a)

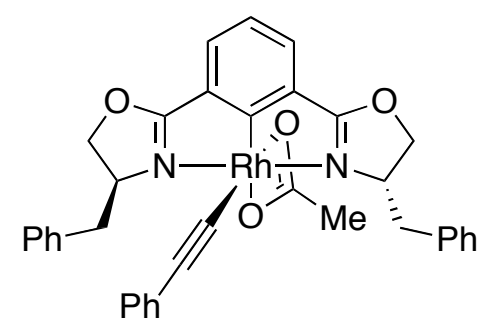

To a $100 \mathrm{~mL}$ flask were added (aqua)(diacetato) (2,6-bis((S)-4-benzyloxazolin-2-yl)phenyl- $\left.\kappa^{3} N, C^{1}, N^{\prime}\right) \mathrm{Rh}(\mathrm{III})$ complex 1c (127 mg, $0.200 \mathrm{mmol})$, phenylacetylene (3a) $(110 \mu \mathrm{L}, 1.00 \mathrm{mmol}, 5.00$ equiv) and toluene $(20 \mathrm{~mL})$ and the mixture was heated in an oil bath at $60^{\circ} \mathrm{C}$ for $40 \mathrm{~h}$. The crude mixture was directly purified by column chromatography using hexane/EtOAc $=1 / 4$ to $0 / 1$ as eluent to give $13 \mathbf{a}$ as a yellow solid ( $122.5 \mathrm{mg}$, $93 \%$ yield).

${ }^{1} \mathrm{H}$ NMR (500 MHz, $\left.\mathrm{CDCl}_{3}\right) \delta 7.54(\mathrm{~d}, J=7.5 \mathrm{~Hz}, 2 \mathrm{H}), 7.34-7.21(\mathrm{~m}, 11 \mathrm{H}), 7.07-7.05$ (m, 4H), 7.00-6.97 (m, 1H), 4.77-4.64 (m, 4H), 4.53-4.46 (m, 2H), 3.84 (dd, $J=14.0,3.0 \mathrm{~Hz}$, 1H), 3.61 (dd, $J=14.0,3.0 \mathrm{~Hz}, 1 \mathrm{H}), 2.90$ (dd, $J=14.0,10.0 \mathrm{~Hz}, 1 \mathrm{H}$ ), 2.77 (dd, $J=14.0$, $10.0 \mathrm{~Hz}, 1 \mathrm{H}), 2.05(\mathrm{~s}, 3 \mathrm{H}) ;{ }^{13} \mathrm{C}\left\{{ }^{1} \mathrm{H}\right\} \mathrm{NMR}\left(125 \mathrm{MHz}, \mathrm{CDCl}_{3}\right) \delta 186.68,186.16\left(\mathrm{~d}, J_{C-R h}=\right.$ 
$25.1 \mathrm{~Hz}), 172.71\left(\mathrm{~d}, J_{C-R h}=4.6 \mathrm{~Hz}\right), 172.18\left(\mathrm{~d}, J_{C-R h}=4.8 \mathrm{~Hz}\right), 137.26,136.54,131.48$, $130.68,129.25,129.22,128.92,128.88,127.76,127.59,127.59,127.57,126.99,126.80$, $125.01,122.46,102.50\left(\mathrm{~d}, J_{C-R h}=9.0 \mathrm{~Hz}\right), 83.84\left(\mathrm{~d}, J_{C-R h}=52.3 \mathrm{~Hz}\right), 75.72,75.51,64.18$, 63.52, 40.56, 39.40, 23.99. IR (KBr) 3360, 3059, 3024, 2960, 2949, 2922, 2120, 1618, $1591,1553,1485,1398,1329,1213,1148,968,949 \mathrm{~cm}^{-1}$. HRMS (ESI-TOF) $\mathrm{m} / z$ calcd. for $\mathrm{C}_{36} \mathrm{H}_{31} \mathrm{~N}_{2} \mathrm{O}_{4} \mathrm{Rh}\left[\mathrm{M}^{+}-{ }^{-} \mathrm{OAc}+\mathrm{MeCN}\right] 640.1471$, found 640.1470 .

\section{(Acetato-k $\left.\kappa^{2} O, O^{\prime}\right)\left(2,6-b i s((S)-4-b e n z y l o x a z o l i n-2-y l) p h e n y l-\kappa^{3} N, C^{1}, N^{\prime}\right)($ trimethylsilylet} hynyl)rhodium(III) (13d)

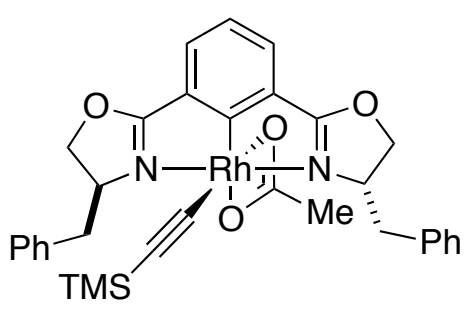

To a $20 \mathrm{~mL}$ screw vial were added activated molecular sieves $\quad 4 \mathrm{~A} \quad(200 \quad \mathrm{mg}), \quad$ (aqua)(diacetato) (2,6-bis((S)-4-benzyloxazolin-2-yl)phenyl- $\left.{ }^{3} N, C^{1}, N^{\prime}\right) \operatorname{Rh}(\mathrm{III})$ complex 1c (127 mg, $0.20 \mathrm{mmol})$, trimethylsilylacetylene 3d (277 $\mu \mathrm{L}, 2.00 \mathrm{mmol}, 10.0$ equiv) and toluene $(4.0 \mathrm{~mL})$ in a glove box and the mixture was heated on a hot plate at $60{ }^{\circ} \mathrm{C}$ for $48 \mathrm{~h}$. The crude mixture was directly purified by column chromatography using hexane/EtOAc $=1 / 4$ to EtOAc/ $\mathrm{MeOH}=20 / 1$ as eluent to give $\mathbf{1 3 d}$ as a yellow solid ( $80.6 \mathrm{mg}, 64 \%$ yield).

${ }^{1} \mathrm{H}$ NMR (500 MHz, $\left.\mathrm{CDCl}_{3}\right) \delta 7.53(\mathrm{~d}, J=5.0 \mathrm{~Hz}, 1 \mathrm{H}), 7.51(\mathrm{~d}, J=5.0 \mathrm{~Hz}, 1 \mathrm{H}) 7.36-7.19$ (m, 11H), 4.74-4.61 (m, 4H), $4.49(\mathrm{~m}, 2 \mathrm{H}), 3.75(\mathrm{~d}, J=10.0 \mathrm{~Hz}, 1 \mathrm{H}), 3.57$ (d, $J=10.0 \mathrm{~Hz}$, 1H), 2.83 (dd, $J=14.5,9.5 \mathrm{~Hz}, 1 \mathrm{H}$ ), 2.73 (dd, $J=14.5,9.5 \mathrm{~Hz}, 1 \mathrm{H}$ ), 2.01 (s, $3 \mathrm{H}$ ), -0.09 (s, $9 \mathrm{H}) ;{ }^{13} \mathrm{C}\left\{{ }^{1} \mathrm{H}\right\} \operatorname{NMR}\left(125 \mathrm{MHz}, \mathrm{CDCl}_{3}\right) \delta 185.55\left(\mathrm{~d}, J_{\mathrm{C}-R h}=25.4 \mathrm{~Hz}\right), 185.30,172.62(\mathrm{~d}$, $\left.J_{C-R h}=4.9 \mathrm{~Hz}\right), 172.16\left(\mathrm{~d}, J_{C-R h}=5.0 \mathrm{~Hz}\right), 137.18,136.65,130.75,130.69,129.19,129.17$, 128.83, 127.42, 127.35, 126.88, 126.78, 122.46, 109.01 (d, $\left.J_{C-R h}=49.1 \mathrm{~Hz}\right), 108.27$ (d, $\left.J_{C-R h}=6.9 \mathrm{~Hz}\right), 75.70,75.45,63.83,63.62,40.69,39.50,24.29,1.47$. IR (KBr) 3379, 2953, 2041, 1618, 1559, 1485, 1452, 1397, 1331, 1294, 1244, 1213, 1147, 968, 949, 860, 837 $\mathrm{cm}^{-1}$. HRMS (ESI-TOF) $\mathrm{m} / z$ calcd. for $\mathrm{C}_{33} \mathrm{H}_{35} \mathrm{~N}_{3} \mathrm{O}_{2} \mathrm{RhSi}\left[\mathrm{M}^{+}-{ }^{-} \mathrm{OAc}+\mathrm{MeCN}\right]$ 636.1554, found 636.1552: Anal. calcd. for $\mathrm{C}_{33} \mathrm{H}_{35} \mathrm{~N}_{2} \mathrm{O}_{4} \mathrm{RhSi}$ : C, 60.55\%; H, 5.39\%; N, 4.28\%; found: C, $60.36 \%$; H, $5.42 \%$; N, $4.25 \%$.

\section{Determination of X-Ray Crystallographic Structure of (Alkynyl)Rh(III) Complex 7a}


Molecular structure of complex $7 \mathbf{a}$ with thermal ellipsoids at $50 \%$ probability was shown Figure S1. The crystal data and data collection parameters for $7 \mathbf{a}$ were summarized in Table S1. Selected bond distance $(\AA)$ and angles (degree) were as follows: C9-Rh (1.912), N1-Rh (2.042), N2-Rh (2.071), C26-Rh (1.960), O4-Rh (2.138), O5-Rh (2.274), C26-C27 (1.209), N1-Rh-N2 (159.50), C9-Rh-C27 (85.20), O4-Rh-O5 (59.61).

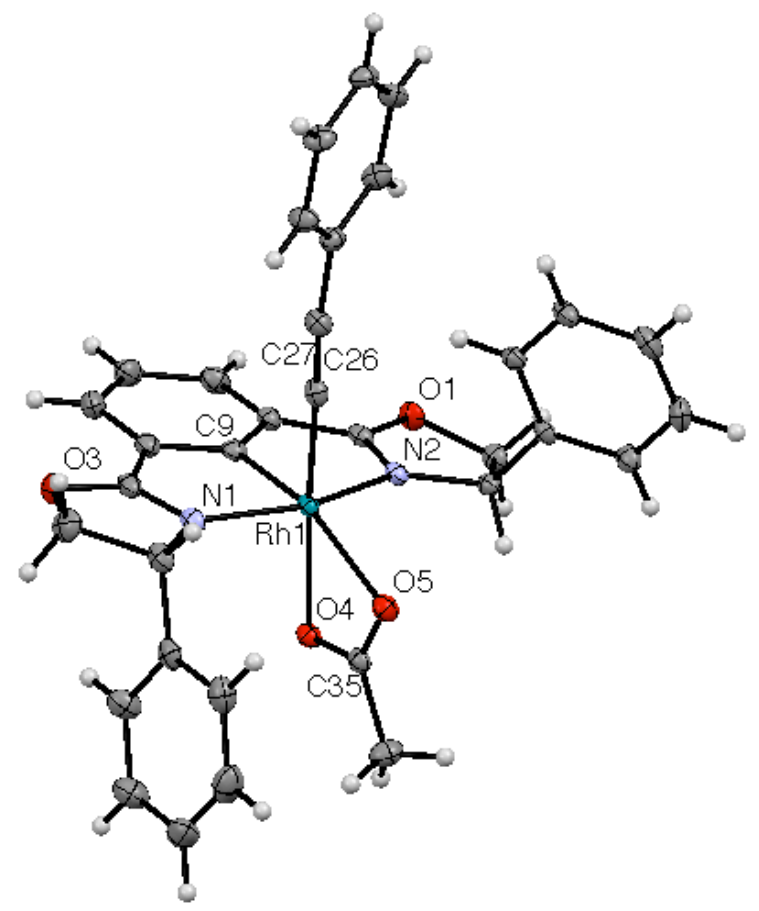

Figure S1. X-ray crystallographic structure of 7a. 
Table S1. Crystal Data and Data Collection Parameters for 7a

\begin{tabular}{|c|c|}
\hline empirical formula & $\mathrm{C}_{34} \mathrm{H}_{27} \mathrm{~N}_{2} \mathrm{O}_{4} \mathrm{Rh}$ \\
\hline formula weight & 630.49 \\
\hline crystal system & $P 2_{1} 2_{1} 2_{1}$ (No. 19) \\
\hline space group & orthorhombic \\
\hline$a, \AA$ & $10.7300(14)$ \\
\hline$b, \AA$ & $14.3916(19)$ \\
\hline$c, \AA$ & $18.130(2)$ \\
\hline$\alpha$, deg. & 90.00 \\
\hline$\beta$, deg. & 90.00 \\
\hline$\gamma$, deg. & 90.00 \\
\hline$V, \AA^{3}$ & 2799.7(6) \\
\hline$Z$ & 4 \\
\hline$D_{\text {calcd }}, \mathrm{g} \mathrm{cm}^{-3}$ & 1.496 \\
\hline$\mu[\mathrm{Mo}-K \alpha], \mathrm{mm}^{-1}$ & 0.653 \\
\hline$T, \mathrm{~K}$ & 90 \\
\hline$\theta$ for data collection, deg. & $1.81-28.40$ \\
\hline no. of reflections & 15770 \\
\hline unique data $\left(R_{\text {int }}\right)$ & 0.0220 \\
\hline data / restraints / parameters & $6352 / 0 / 371$ \\
\hline Flack parameter & $-0.010(13)$ \\
\hline$R 1(I>2.0 \sigma(I))$ & 0.0175 \\
\hline $\mathrm{w} R 2(I>2.0 \sigma(I))$ & 0.0451 \\
\hline$R 1$ (all data) & 0.0178 \\
\hline $\mathrm{w} R 2$ (all data) & 0.0453 \\
\hline GOF on $F^{2}$ & 1.050 \\
\hline$\Delta \rho$, e $\AA^{-3}$ & $0.711,-0.372$ \\
\hline
\end{tabular}




\section{Identification of the Proton Source}

Stoichiometric Reaction of (Alkynyl)Rh(III) Complex 5a and $\alpha$-Ketiminoester 2 with Acetic Acid (Equation 3)

To an NMR sample tube were added a 0.10 M solution of (alkynyl)Rh(III) complex 5a in toluene- $d_{8}(0.20 \mathrm{~mL}, 0.020 \mathrm{mmol})$, a $0.20 \mathrm{M}$ solution of $\alpha$-ketiminoester 2 in toluene- $d_{8}$ $\left(0.10 \mathrm{~mL}, 0.020 \mathrm{mmol}, 1.0\right.$ equiv) and toluene- $d_{8}(0.30 \mathrm{~mL})$ in a glove box and the tube was sealed with a rubber septum. To the tube was added a $0.20 \mathrm{M}$ solution of acetic acid in toluene- $d_{8}(0.10 \mathrm{~mL}, 0.020 \mathrm{mmol}, 1.0$ equiv $)$ via a syringe with a stainless steel needle. After 10 min of the addition, the crude mixture was analyzed by ${ }^{1} \mathrm{H}$ NMR and ${ }^{19} \mathrm{~F}$ NMR spectroscopy.

The ${ }^{19} \mathrm{~F}$ NMR spectrum of the mixture shown in Figure S2 below indicated that no formation of product $\mathbf{4 a}$ at $-75.8 \mathrm{ppm}$ occurred and most of 2 remained intact. The new species generated at $-79.0 \mathrm{ppm}$ and $-81.5 \mathrm{ppm}$ were determined to be an acetic acid adduct of 2 (confirmed by treatment of ketiminoester 2 with 5 equivalents of acetic acid in $\mathrm{CDCl}_{3}$, giving the peak at $-79.0 \mathrm{ppm}$ ) and hemiaminal 2' (prepared by condensation of benzyl carbamate with ethyl trifluoropyruvate; ${ }^{1} \mathrm{H}$ NMR $\left(500 \mathrm{MHz}, \mathrm{CDCl}_{3}\right) \delta 7.39-7.25(\mathrm{~m}, 5 \mathrm{H})$, 5.99 (br, 1H), $5.36(\mathrm{br}, 1 \mathrm{H}) 5.13(\mathrm{~m}, 2 \mathrm{H}), 4.37-4.36(\mathrm{~m}, 2 \mathrm{H}), 1.30(\mathrm{t}, J=7.0 \mathrm{~Hz}, 3 \mathrm{H})$; ${ }^{13} \mathrm{C}\left\{{ }^{1} \mathrm{H}\right\}$ NMR $\left(125 \mathrm{MHz}, \mathrm{CDCl}_{3}\right) \delta 165.66,154.54,135.06,128.63,128.36,121.44$ (q, $\left.J_{C-F}=287 \mathrm{~Hz}\right), 81.02\left(\mathrm{q}, J_{C-F}=32.5 \mathrm{~Hz}\right), 67.95,64.68,13.68 ;{ }^{19} \mathrm{~F} \mathrm{NMR}\left(360 \mathrm{MHz}, \mathrm{CDCl}_{3}\right)$ $\delta-81.5)$. These results suggest that acetic acid does not act as the proton source to generate product 4a from (alkynyl)Rh(III) complex 5a under stoichiometric reaction conditions. 


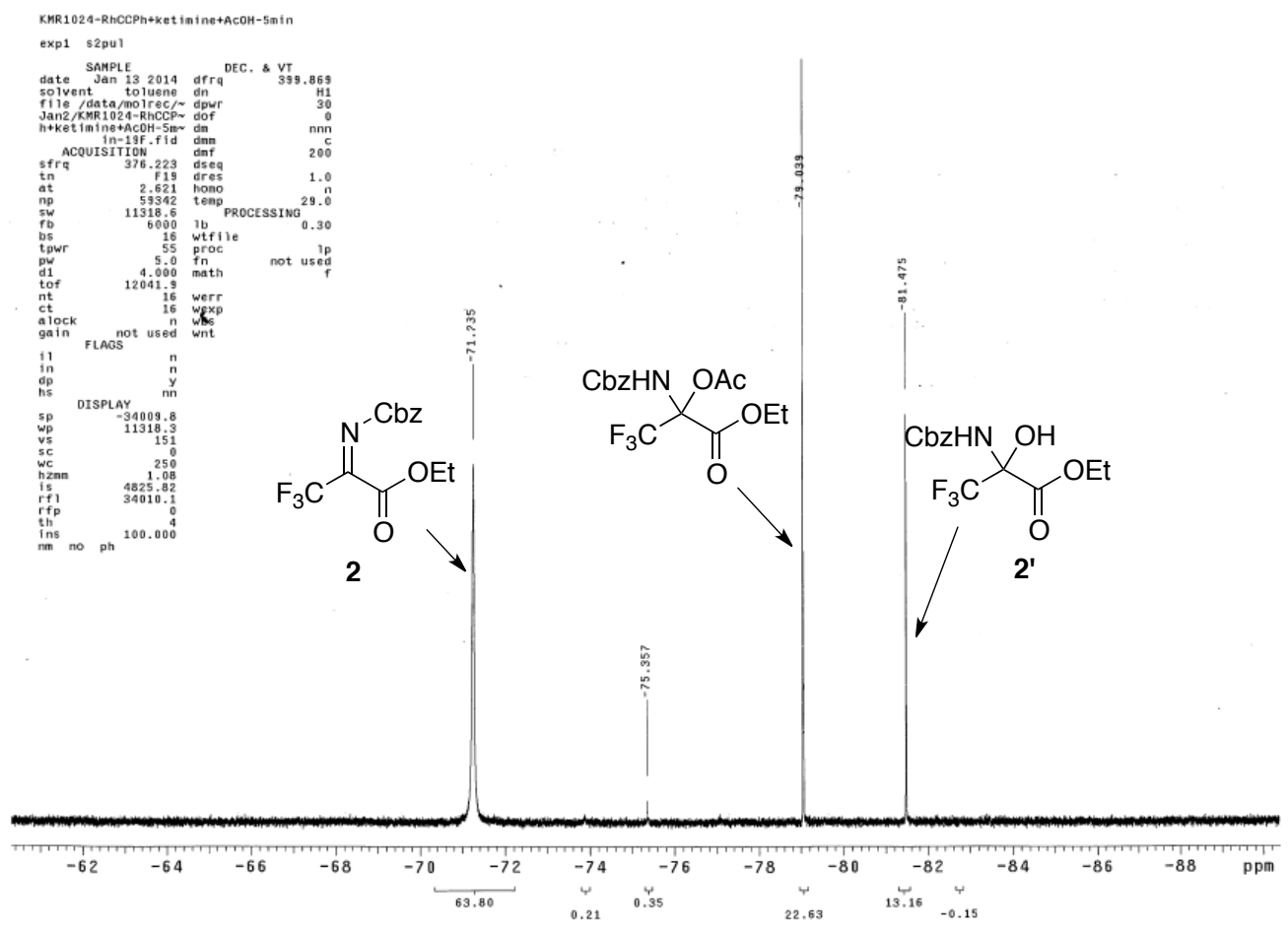

Figure S2. ${ }^{19} \mathrm{~F}$ NMR spectrum of the mixture of (alkynyl)Rh(III) complex 5a, $\alpha$-ketiminoester $\mathbf{2}$ and acetic acid.

\section{Stoichiometric Reaction of (Alkynyl)Rh(III) Complex 5a and $\alpha$-Ketiminoester 2 with Terminal Alkyne 3a (Equation 4)}

To an NMR sample tube were added a 0.10 M solution of (alkynyl)Rh(III) complex 5a in toluene- $d_{8}(0.20 \mathrm{~mL}, 0.020 \mathrm{mmol})$, a $0.20 \mathrm{M}$ solution of $\alpha$-ketiminoester 2 in toluene- $d_{8}$ $(0.10 \mathrm{~mL}, 0.020 \mathrm{mmol}, 1.0$ equiv $)$ and toluene- $d_{8}(0.30 \mathrm{~mL})$ in a glove box and the tube was sealed with a rubber septum. To the tube was added a $0.20 \mathrm{M}$ solution of ethynylbenzene (3a) in toluene- $d_{8}(0.10 \mathrm{~mL}, 0.020 \mathrm{mmol}, 1.0$ equiv) via a syringe with a stainless steel needle. The tube was kept at room temperature for $15 \mathrm{~min}$ and analyzed by ${ }^{1} \mathrm{H}$ NMR and ${ }^{19} \mathrm{~F}$ NMR spectroscopy. After $5.5 \mathrm{~h}$, the crude mixture was purified by short pad silica gel column chromatography using EtOAc as eluent to give $8.7 \mathrm{mg}$ of the mixture of product $\mathbf{4 a}$ and hemiaminal $\mathbf{2}$ ' (ratio of $\mathbf{4 a}: \mathbf{2}$ ' $=74: 26)$ and $10.1 \mathrm{mg}$ of $\mathbf{5 a}(72 \%$ 
recovery).

The ${ }^{19} \mathrm{~F}$ NMR spectrum of the crude mixture shown in Figure S3 below showed the formation of $4 \mathbf{a}$.

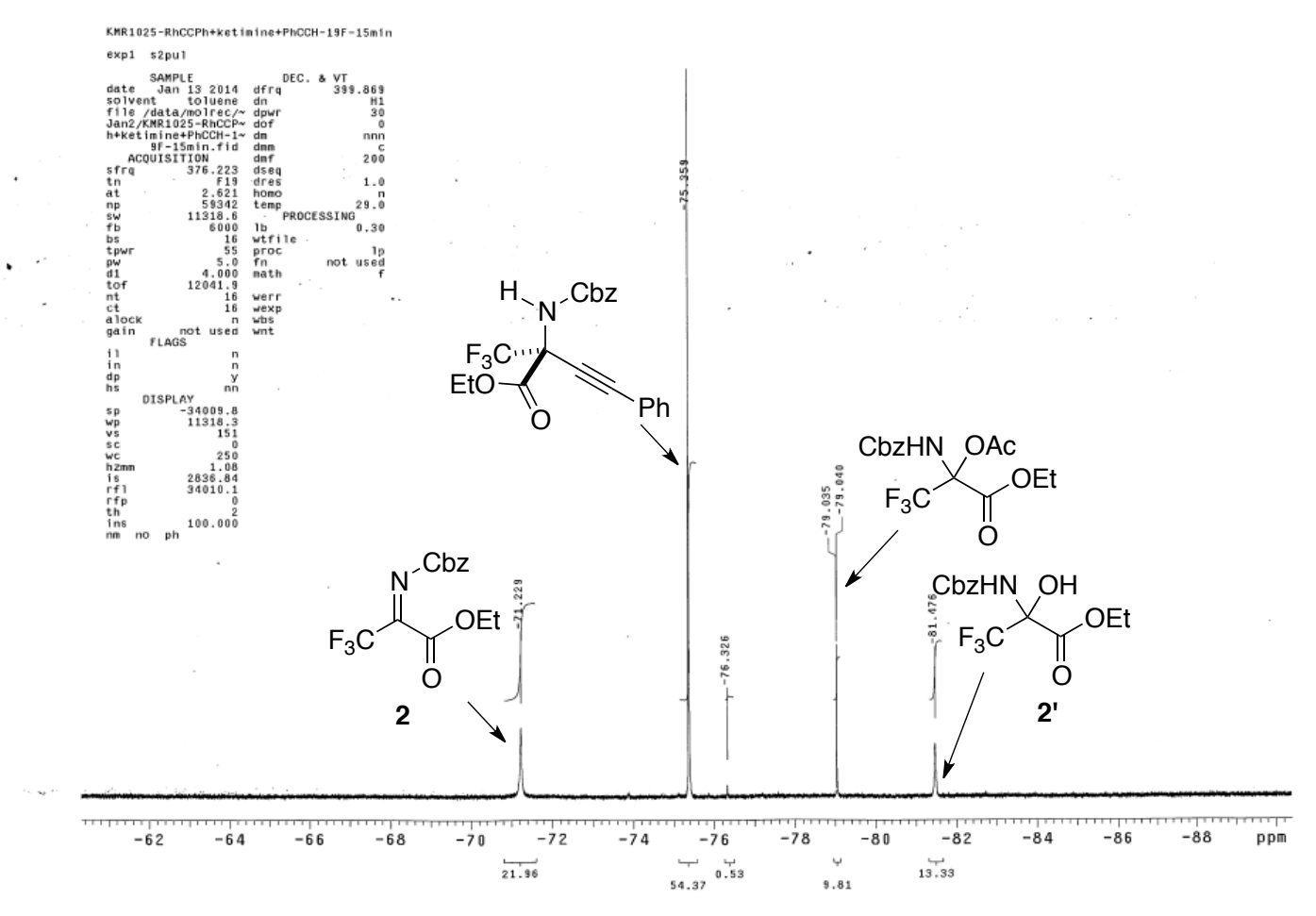

Figure S3. ${ }^{19} \mathrm{~F}$ NMR spectrum of the mixture of (alkynyl)Rh(III) complex 5a, $\alpha$-ketiminoester $\mathbf{2}$ and alkyne $\mathbf{3 a}$.

Reaction of $\alpha$-Ketiminoester 2 with Terminal Alkyne 3a in the Presence of a Catalytic Amount of (Alkynyl)Rh(III) Complex 5a (Equation 5)

A Schlenk tube equipped with a magnetic stir bar was dried under vacuum using heat gun and refilled with argon. To the tube were added (alkynyl)Rh(III) complex 5a (1.75 mg, $2.5 \mu \mathrm{mol}, 2.5 \mathrm{~mol} \%)$, toluene $(0.40 \mathrm{~mL})$ and alkyne 3a (16 $\mu \mathrm{L}, 0.15 \mathrm{mmol}, 1.5$ equiv). After the mixture was stirred for $10 \mathrm{~min}$ at room temperature, $\alpha$-ketiminoester $2(24 \mu \mathrm{L}$, $0.10 \mathrm{mmol}$ ) was added and the mixture was stirred at room temperature for $0.5 \mathrm{~h}$. The mixture was analyzed by ${ }^{19} \mathrm{~F}$ NMR spectroscopy to determine the yield of $4 \mathbf{a}$ based on the 
relative integration values of the peaks at $-71.1 \mathrm{ppm}$ (for 2 ) and $-75.6 \mathrm{ppm}$ (for $\mathbf{4 a}$ ). The NMR sample was purified by preparative TLC using hexane/EtOAc $=10 / 1$ as eluent. Product 4a on the TLC plate was collected and extracted with 2-propanol. Enantiomeric excess of $\mathbf{4 a}$ was determined by chiral HPLC analysis.

\section{Reaction Profiles of (Alkynyl)Rh(III) Complex 5a in the Absence or Presence of Acetic Acid (Figure 2)}

(a) In the absence of acetic acid

To an NMR sample tube were added a $6.3 \mathrm{mM}$ solution of (alkynyl)Rh(III) complex 5a in toluene $(0.60 \mathrm{~mL}, 3.8 \mu \mathrm{mol}, 2.5 \mathrm{~mol} \%)$ and $\alpha$-ketiminoester $2(36 \mu \mathrm{L}, 0.15 \mathrm{mmol})$ in a glove box and the tube was sealed with a rubber septum. To the tube was added ethynylbenzene (3a) (25 $\mu \mathrm{L}, 0.23 \mathrm{mmol}, 1.5$ equiv) via a syringe and a stainless steel needle, and the mixture was analyzed by ${ }^{19} \mathrm{~F}$ NMR spectroscopy to determine the yield of 4a based on the relative integration values of the peaks at -71.12 (for 2) and -75.62 ppm (for $\mathbf{4 a}$ ) at the specified time.

\section{(b) In the presence of $2.5 \mathrm{~mol} \%$ of acetic acid}

To an NMR sample tube were added $18.7 \mathrm{mM}$ solution of (alkynyl)Rh(III) complex 5a in toluene $(0.20 \mathrm{~mL}, 3.8 \mu \mathrm{mol}, 2.5 \mathrm{~mol} \%)$ and $\alpha$-ketiminoester $2(36 \mu \mathrm{L}, 0.15 \mathrm{mmol})$ in a glove box and the tube was sealed with a rubber septum. To the tube were added $5.6 \mathrm{mM}$ solution of acetic acid in toluene $(0.40 \mathrm{~mL}, 0.0038 \mathrm{mmol}, 2.5 \mathrm{~mol} \%)$ and ethynylbenzene (3a) $(25 \mu \mathrm{L}, 0.23 \mathrm{mmol}, 1.5$ equiv) via a syringe and a stainless steel needle, and the mixture was analyzed by ${ }^{19} \mathrm{~F}$ NMR spectroscopy similar to the above procedure.

The results were summarized in Table S2 below and plotted in Figure 2.

Table S2. Reaction Profiles of (Alkynyl)Rh(III) Complex 5a in the Absence or Presence of Acetic Acid

\begin{tabular}{|r|r|r|r|r|r|}
\hline $\begin{array}{c}\text { time } \\
(\mathrm{min})\end{array}$ & $\begin{array}{c}\text { yield (\%) } \\
\text { w/o AcOH }\end{array}$ & $\begin{array}{c}\text { yield (\%) } \\
\text { w/ } 2.5 \mathrm{~mol} \% \\
\text { of AcOH }\end{array}$ & $\begin{array}{c}\text { time } \\
(\mathrm{min})\end{array}$ & $\begin{array}{c}\text { yield (\%) } \\
\text { w/o AcOH }\end{array}$ & $\begin{array}{c}\text { yield (\%) } \\
\text { w/ } / 2.5 \mathrm{~mol} \% \\
\text { of AcOH }\end{array}$ \\
\hline 0 & 0.0 & 0.0 & 23 & 84.4 & 85.2 \\
\hline 1 & 6.3 & 6.9 & 24 & 86.2 & 86.5 \\
\hline
\end{tabular}




\begin{tabular}{|r|r|r|r|r|r|}
\hline 2 & 13.0 & 14.5 & 25 & 87.5 & 87.7 \\
\hline 3 & 19.3 & 21.2 & 26 & 88.9 & - \\
\hline 4 & 25.3 & 27.4 & 27 & 89.9 & - \\
\hline 5 & 31.1 & 32.9 & 28 & 91.3 & - \\
\hline 6 & 36.2 & 38.2 & 29 & 92.4 & - \\
\hline 7 & 40.6 & 42.8 & 30 & 93.4 & 91.9 \\
\hline 8 & 45.4 & 47.7 & 31 & 94.2 & - \\
\hline 9 & 49.5 & 51.5 & 32 & 95.1 & - \\
\hline 10 & 53.7 & 55.7 & 33 & 95.8 & - \\
\hline 11 & 57.1 & 59.0 & 34 & 96.5 & - \\
\hline 12 & 60.6 & 62.1 & 35 & 97.1 & - \\
\hline 13 & 63.1 & 65.2 & 36 & 97.3 & - \\
\hline 14 & 66.1 & 67.8 & 37 & 98.0 & - \\
\hline 15 & 68.7 & 70.4 & 38 & 98.1 & - \\
\hline 16 & 71.6 & 72.8 & 39 & 98.6 & - \\
\hline 17 & 73.6 & 74.9 & 40 & 98.9 & 96.8 \\
\hline 18 & 75.9 & 76.8 & 41 & 99.3 & - \\
\hline 19 & 78.0 & 78.8 & 42 & 99.5 & - \\
\hline 20 & 80.0 & 80.8 & 43 & 99.6 & - \\
\hline 21 & 81.4 & 82.3 & 44 & 99.8 & - \\
\hline 22 & 83.2 & 83.9 & 45 & 99.9 & - \\
\hline
\end{tabular}

\section{Kinetic Experiments (1): Comparison of Reactivity of Rh(III) Complexes}

Comparison of the Reaction Profile of (Alkynyl)Rh(III) Complex 5a with (Diacetato)Rh(III) Complex 1a (Figure 3a)

Procedure for the analysis of reaction profile of (diacetato) $\mathrm{Rh}$ (III) complex 1a

To an NMR sample tube were added a $3.75 \mathrm{mM}$ solution of (diacetato) $\mathrm{Rh}$ (III) complex 1a in toluene ( $0.60 \mathrm{~mL}, 2.3 \mu \mathrm{mol}, 1.5 \mathrm{~mol} \%)$ and $\alpha$-ketiminoester $2(36 \mu \mathrm{L}, 0.15 \mathrm{mmol})$ in a glove box and the tube was sealed with a rubber septum. To the mixture was added ethynylbenzene (3a) $(25 \mu \mathrm{L}, 0.23 \mathrm{mmol}, 1.5$ equiv) via a syringe and a stainless steel needle, and the mixture was analyzed by ${ }^{19} \mathrm{~F}$ NMR spectroscopy to determine the yield of 4a based on the relative integration values of the peaks at -71.12 (for 2 ) and -75.62 (for $\mathbf{4 a}$ ) 
at the specified time.

Procedure for the analysis of reaction profile of (diacetato) $\mathrm{Rh}(\mathrm{III})$ complex $\mathbf{5 a}$

The reaction profile of (alkynyl)Rh(III) complex 5a was determined as described above using 5a instead of 1a.

Procedure for the analysis of reaction profile of (diacetato) $\mathrm{Rh}(\mathrm{III})$ complex 1a under same [e] conditions

The reaction profile of (diacetato) $\mathrm{Rh}(\mathrm{III})$ complex 1a under same $[e]$ condition was determined following the procedure for 1a except for varying the amount of $\alpha$-ketiminoester $2\left(22 \mu \mathrm{L}, 0.090 \mathrm{mmol} ;[2]_{0}=0.15 \mathrm{M}\right)$ and alkyne 3a $(17 \mu \mathrm{L}, 0.165 \mathrm{mmol}$, 1.8 equiv; $\left.[\mathbf{3 a}]_{0}=0.275 \mathrm{M} ;[e]=[\mathbf{3 a}]_{0}-[2]_{0}=0.125 \mathrm{M}\right)$.

The results were summarized in Table S3 below and plotted in Figure 3.

Table S3. Reaction Profiles of (a) (Diacetato)Rh(III) Complex 1a and (b) (Alkynyl)Rh(III) Complex 5a

(a) Data for (Diacetato)Rh(III) Complex 1a

\begin{tabular}{|r|r|r|r|r|r|}
\hline \multicolumn{1}{c|}{$\begin{array}{c}\text { time } \\
(\mathrm{min})\end{array}$} & $\begin{array}{c}\text { yield (\%) } \\
{[\mathbf{2}]_{0}=0.25 \mathrm{M}}\end{array}$ & $\begin{array}{c}\text { yield (\%) } \\
{[\mathbf{2}]_{0}=0.15 \mathrm{M}}\end{array}$ & $\begin{array}{c}\text { time } \\
(\mathrm{min})\end{array}$ & $\begin{array}{c}\text { yield (\%) } \\
{[\mathbf{2}]_{0}=0.25 \mathrm{M}}\end{array}$ & $\begin{array}{c}\text { yield (\%) } \\
{[\mathbf{2}]_{0}=0.15 \mathrm{M}}\end{array}$ \\
\hline 0 & 0 & 0 & 120 & 8.9 & 22.1 \\
\hline 6 & 0 & 0.3 & 240 & 20.9 & 57.3 \\
\hline 12 & 0.3 & 0.9 & 360 & 40.3 & 67.5 \\
\hline 18 & 0.6 & 1.7 & 480 & 62.1 & 75.3 \\
\hline 24 & 0.8 & 2.1 & 600 & 76.0 & 81.5 \\
\hline 30 & 1.3 & 3.1 & 720 & 86.3 & 86.3 \\
\hline 90 & 5.7 & 17.4 & 840 & 91.4 & 90.9 \\
\hline
\end{tabular}

(b) Data for (Alkynyl)Rh(III) Complex 5a

\begin{tabular}{|r|r|r|r|r|r|}
\hline time (min) & yield (\%) & time (min) & yield (\%) & time (min) & yield (\%) \\
\hline 0 & 0 & 31 & 73.6 & 61 & 94.2 \\
\hline 1 & 3.8 & 32 & 74.1 & 62 & 94.5 \\
\hline 2 & 8.5 & 33 & 74.7 & 63 & 94.8 \\
\hline 3 & 12.4 & 34 & 76.0 & 64 & 95.2 \\
\hline 4 & 16.3 & 35 & 76.9 & 65 & 95.6 \\
\hline 5 & 19.9 & 36 & 77.9 & 66 & 95.9 \\
\hline 6 & 23.5 & 37 & 79.3 & 67 & 96.3 \\
\hline
\end{tabular}




\begin{tabular}{|r|r|r|r|r|r|}
\hline 7 & 26.9 & 38 & 80.2 & 68 & 96.4 \\
\hline 8 & 30.0 & 39 & 80.9 & 69 & 96.7 \\
\hline 9 & 33.3 & 40 & 81.7 & 70 & 97.1 \\
\hline 10 & 36.1 & 41 & 81.7 & 71 & 97.2 \\
\hline 11 & 39.0 & 42 & - & 72 & 97.5 \\
\hline 12 & 41.6 & 43 & 83.8 & 73 & 97.8 \\
\hline 13 & 44.1 & 44 & 84.5 & 74 & 98.1 \\
\hline 14 & 46.6 & 45 & 85.2 & 75 & 98.0 \\
\hline 15 & 48.9 & 46 & 86.1 & 76 & 98.4 \\
\hline 16 & 51.1 & 47 & 86.7 & 77 & 98.6 \\
\hline 17 & 53.2 & 48 & 87.4 & 78 & 98.8 \\
\hline 18 & 55.3 & 49 & 88.0 & 79 & 98.8 \\
\hline 19 & 57.3 & 50 & 88.7 & 80 & 98.9 \\
\hline 20 & 59.0 & 51 & 89.1 & 81 & 99.0 \\
\hline 21 & 61.2 & 52 & 89.8 & 82 & 99.2 \\
\hline 22 & 62.9 & 53 & 90.3 & 83 & 99.3 \\
\hline 23 & 64.6 & 54 & 90.8 & 84 & 99.4 \\
\hline 24 & 66.2 & 55 & 91.6 & 85 & 99.6 \\
\hline 25 & 67.6 & 56 & 92.0 & 86 & 99.6 \\
\hline 26 & 68.7 & 57 & 92.2 & 87 & 99.6 \\
\hline 27 & 70.0 & 58 & 92.8 & 88 & 99.7 \\
\hline 28 & 71.1 & 59 & 93.2 & 89 & 99.7 \\
\hline 29 & 72.0 & 60 & 93.7 & 90 & 99.8 \\
\hline 30 & 72.8 & & & & \\
\hline & & & & & \\
\hline
\end{tabular}

\section{Kinetic Experiments (2): Initial Rate Kinetic Studies of the Catalytic Cycle}

Initial Rate Kinetic Experiments with Variable Initial Concentration of the (Alkynyl)Rh(III) Complex 5a

General procedure for initial rate kinetic experiments

To an NMR sample tube were added a $37.5 \mathrm{mM}$ solution of (alkynyl)Rh(III) complex 5a $(50 \mu \mathrm{L}, 1.9 \mu \mathrm{mol}, 1.3 \mathrm{~mol} \%, 3.1 \mathrm{mM}), \alpha$-ketiminoester $2(36 \mu \mathrm{L}, 0.15 \mathrm{mmol}, 0.25 \mathrm{M})$ and toluene $(0.55 \mathrm{~mL})$ in a glove box and the tube was sealed with a rubber septum. To the 
tube was added ethynylbenzene (3a) $(24 \mu \mathrm{L}, 0.23 \mathrm{mmol}, 1.5$ equiv, $0.375 \mathrm{M})$ via a syringe and a stainless steel needle, and the mixture was analyzed by ${ }^{19} \mathrm{~F}$ NMR spectroscopy to determine the yield of $\mathbf{4 a}$ based on the relative integration values of the peaks at -71.12 (for 2) and $-75.62 \mathrm{ppm}$ (for $\mathbf{4 a}$ ) at the specified time.

The reactions with different concentrations of $\mathbf{5 a}$ at $0.78,1.6,3.1$ (the standard condition) and $4.7 \mathrm{mM}$ were performed according to the general procedure described above except for varying the amount of 5a added, and the results were summarized in the Table S4 and Figure S5 below.

Table S4. Initial Rates of Varying Concentrations of (Alkynyl)Rh(III) Complex 5a

\begin{tabular}{|r|r|}
\hline$[\mathbf{5 a}]\left(10^{-3} \mathrm{M}\right)$ & $d[\mathbf{4 a}] / d t\left(10^{-4} \mathrm{M} \cdot \mathbf{s}^{-1}\right)$ \\
\hline 0.78 & 0.262 \\
\hline 1.6 & 0.415 \\
\hline 3.1 & 1.14 \\
\hline 4.7 & 1.74 \\
\hline
\end{tabular}

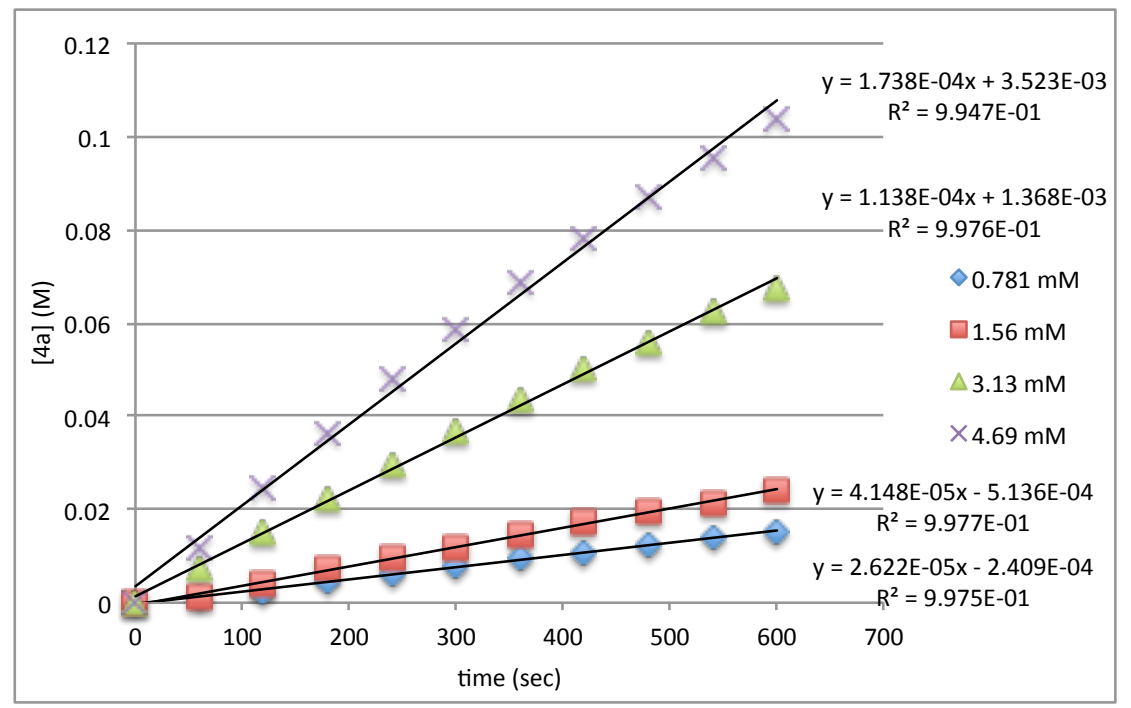




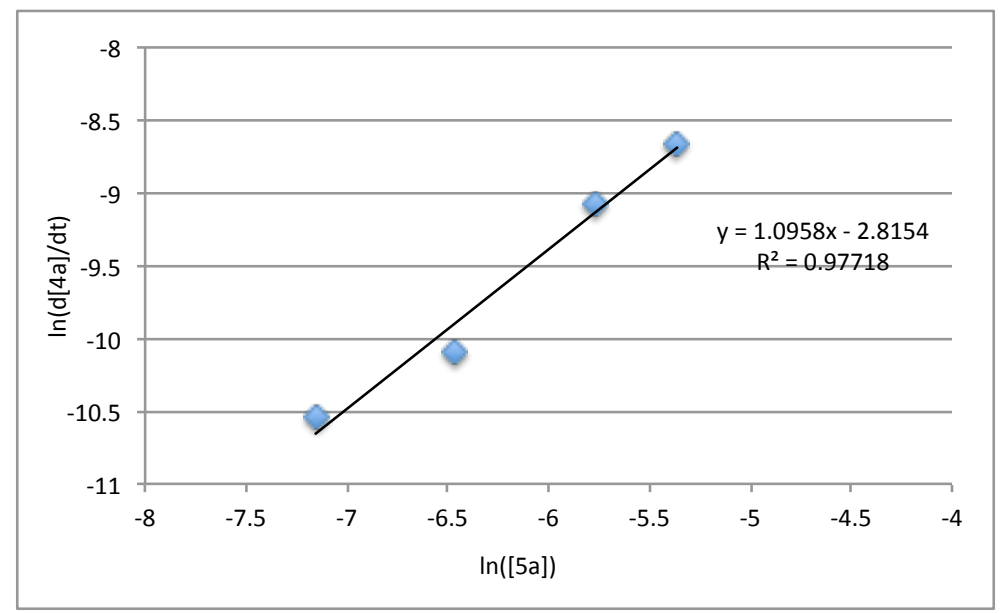

Figure S5. (a) Initial rate kinetic experiments for (alkynyl)Rh(III) complex 5a. (b) Plot of $\ln (d[\mathbf{4 a}] / d t)$ versus $\ln ([\mathbf{5 a}])$.

Initial Rate Kinetic Experiments with Variable Initial Concentration of the Alkyne 3a

The reactions with different concentrations of ethynylbenzene (3a) at 0.188, 0.281, 0.375 (the standard condition), 0.469 and $0.563 \mathrm{M}$ were performed according to the general procedure described above except for varying the amount of $\mathbf{3 a}$ added, and the results were summarized in the Table S5 and Figure S6 below.

Table S5. Initial Rates of Varying Concentrations of Alkyne 3a

\begin{tabular}{|r|r|}
\hline$[3 a](\mathrm{M})$ & $d[\mathbf{4 a}] / d t\left(10^{-4} \mathrm{M} \cdot \mathrm{s}^{-1}\right)$ \\
\hline 0.188 & 0.528 \\
\hline 0.281 & 0.861 \\
\hline 0.375 & 0.907 \\
\hline 0.469 & 1.22 \\
\hline 0.563 & 1.54 \\
\hline
\end{tabular}



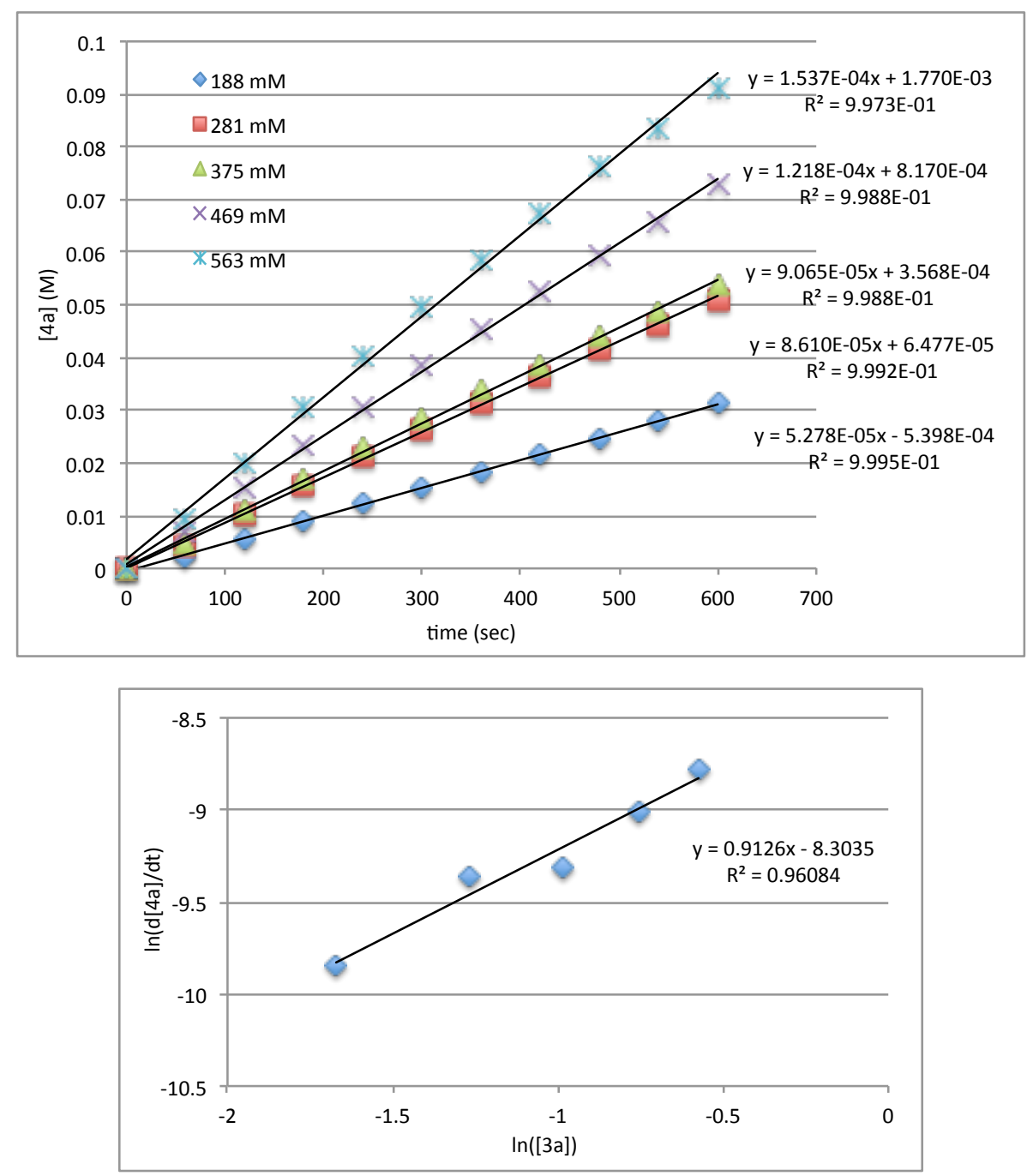

Figure S6. (a) Initial rate kinetic experiments for alkyne 3a. (b) Plot of $\ln (d[\mathbf{4 a}] / d t)$ versus $\ln ([\mathbf{3 a}])$.

\section{Initial Rate Kinetic Experiments with Variable Initial Concentration of the $\alpha$-Ketiminoester 2}

The reactions with different concentrations of $\alpha$-ketiminoester 2 at $0.125,0.188,0.250$ (the standard condition), 0.313 and $0.375 \mathrm{M}$ were performed according to the general procedure described above except for varying the amount of $\mathbf{2}$ added, and the results were summarized in Table S6 and Figure S7 below.

The same experiments were performed at lower concentrations of $2(41.7,83.3$ and 125 
$\mathrm{mM}$ ) using $6.3 \mathrm{mM}(2.5 \mathrm{~mol} \%$ ) of (alkynyl) $\mathrm{Rh}(\mathrm{III})$ complex 5a to obtain sufficient reaction rate in the initial rate kinetic experiments, and the results were summarized in Table $\mathrm{S} 7$ and Figure S8 below.

\section{Table S6. Initial Rates of Varying Concentrations of $\boldsymbol{\alpha}$-Ketiminoester 2}

\begin{tabular}{|r|r|}
\hline$\alpha$-ketiminoester [2] (M) & $d[\mathbf{4 a}] / d t\left(10^{-4} \mathbf{M} \cdot \mathbf{s}^{-1}\right)$ \\
\hline 0.125 & 0.922 \\
\hline 0.188 & 1.24 \\
\hline 0.250 & 1.15 \\
\hline 0.313 & 0.847 \\
\hline 0.375 & 0.723 \\
\hline
\end{tabular}
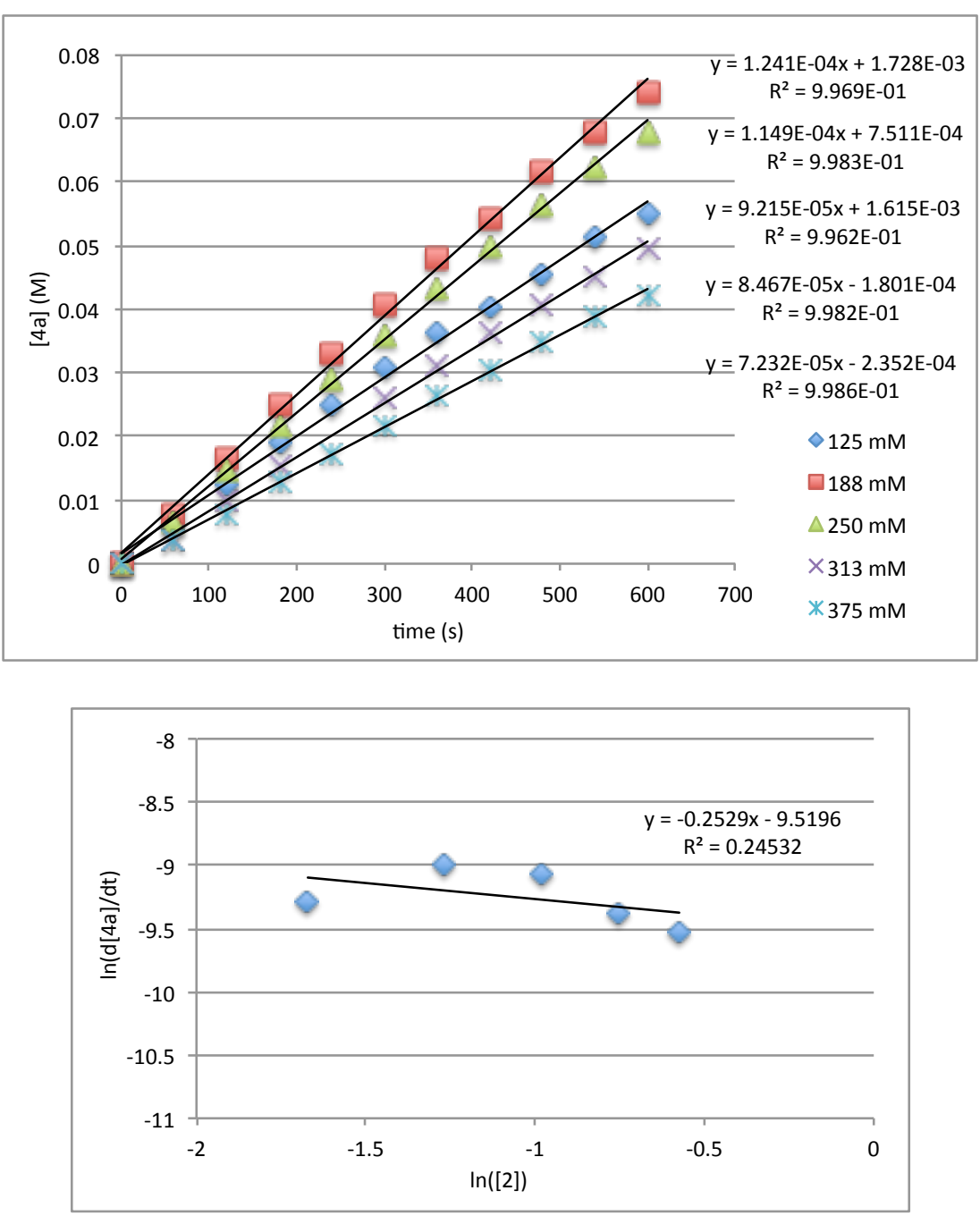

Figure S7. (a) Initial rate kinetic experiments for $\alpha$-ketiminoester 2. (b) Plot of $\ln$ $(d[\mathbf{4} \mathbf{a}] / d t)$ versus $\ln ([\mathbf{2}])$. 
Table S7. Initial Rates at Lower Concentrations of $\alpha$-Ketiminoester 2

\begin{tabular}{|r|r|}
\hline$\alpha$-ketiminoester [2] $(\mathrm{M})$ & $d[\mathbf{4 a}] / d t\left(10^{-4} \mathrm{M} \cdot \mathrm{s}^{-1}\right)$ \\
\hline 0.0417 & 0.494 \\
\hline 0.0833 & 0.850 \\
\hline 0.125 & 1.17 \\
\hline
\end{tabular}
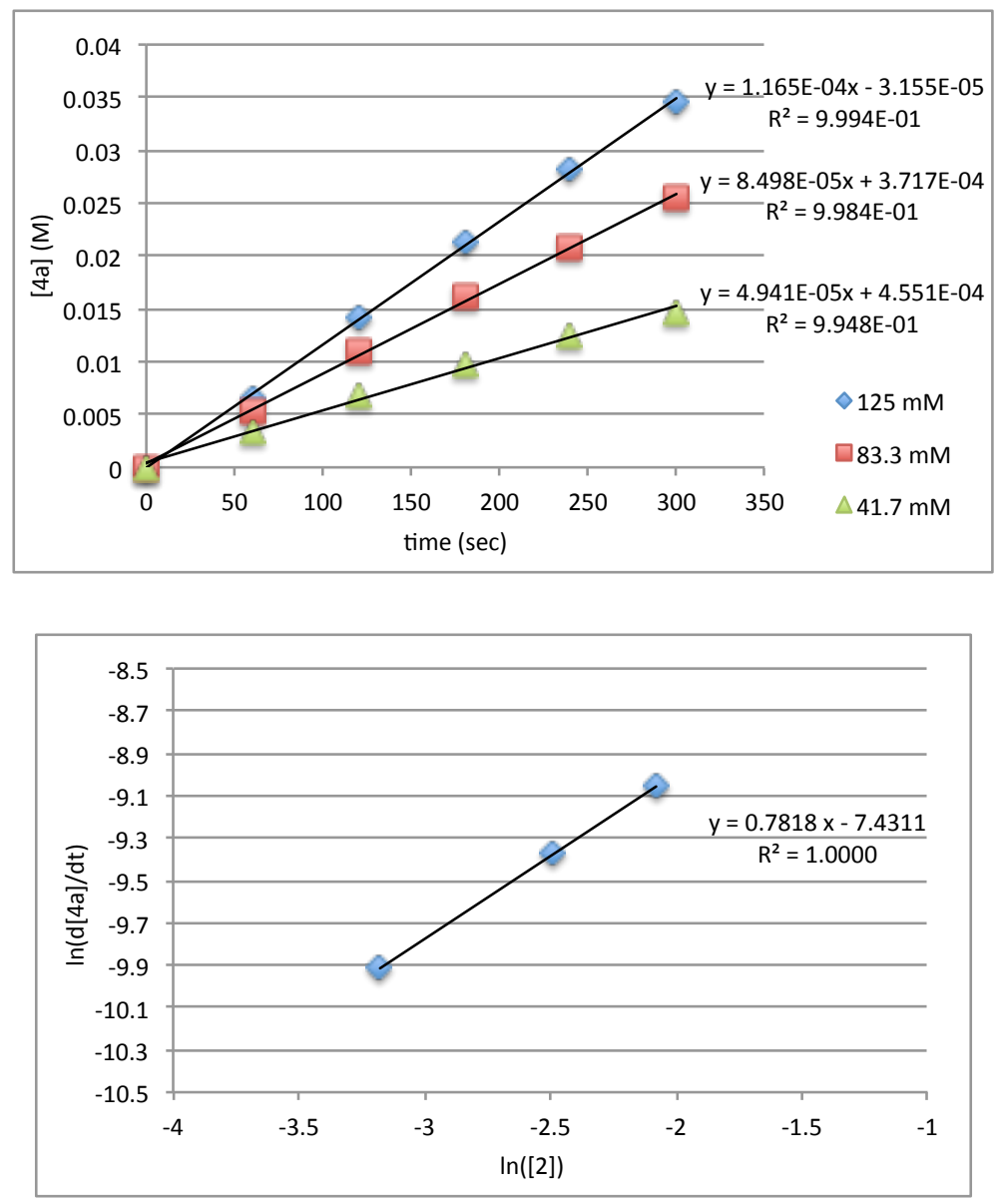

Figure S8. (a) Initial rate kinetic experiments for $\alpha$-ketiminoester 2 at lower concentrations. (b) Plot of $\ln (d[\mathbf{4 a}] / d t)$ versus $\ln ([\mathbf{2}])$.

\section{Kinetic Experiments (3): Reaction Progress Kinetic Analysis ${ }^{5}$}

To an NMR sample tube were added a $37.5 \mathrm{mM}$ solution of (alkynyl)Rh(III) complex 5a in toluene $(0.10 \mathrm{~mL}, 3.8 \mu \mathrm{mol}, 2.5 \mathrm{~mol} \%, 6.3 \mathrm{mM}), \alpha$-ketiminoester $2(36 \mu \mathrm{L}, 0.15$ $\left.\mathrm{mmol} ;[2]_{0}=0.25 \mathrm{M}\right)$ and toluene $(0.50 \mathrm{~mL})$ in a glove box and the tube was sealed with a rubber septum. To the tube was added ethynylbenzene (3a) $(24 \mu \mathrm{L}, 0.23 \mathrm{mmol}, 1.5$ equiv; 
$\left.[\mathbf{3 a}]_{0}=0.375 \mathrm{M} ;[e]=[\mathbf{3 a}]_{0}-[\mathbf{2}]_{0}=0.125 \mathrm{M}\right)$ via a syringe and a stainless steel needle, and the mixture was analyzed by ${ }^{19}$ F NMR spectroscopy to determine the yield of $\mathbf{4 a}$ based on the relative integration values of the peaks at -71.12 (for 2) and $-75.62 \mathrm{ppm}$ (for $\mathbf{4 a}$ ) at the specified time, and the data were summarized in Table S8 below (standard condition) and plotted as blue diamond in Figures $4 \mathrm{a}$ and $4 \mathrm{~b}$.

To perform a different $[e]$ experiment, the above procedure was followed except for varying ethynylbenzene (3a) $\left(30 \mu \mathrm{L}, 0.27 \mathrm{mmol}, 1.8\right.$ equiv; $[\mathbf{3 a}]_{0}=0.45 \mathrm{M}$; $[e]=[\mathbf{3 a}]_{0}-$ $[2]_{0}=0.20 \mathrm{M}$ ), and the data were summarized in Table S8 below (different $[e]$ condition) and plotted as red square in Figure 4a. The raw concentration-time data under the conditions of different $[e]$ experiments were plotted in Figure S9 below. The rate $d[4 \mathbf{4 a}] / d t$ was obtained by calculating the slope using 7 data points around each time point $n$ (i.e., $n-$ $3, n-2, n-1, n, n+1, n+2, n+3) ; 3$ or 5 data points were used for calculating the rate at the beginning and at the end of the reaction.

To perform a same $[e]$ experiment, the same procedure was followed except for varying the amount of $\alpha$-ketiminoester $2\left(22 \mu \mathrm{L}, 0.090 \mathrm{mmol}\right.$; $\left.[2]_{0}=0.15 \mathrm{M}\right)$ and alkyne $3 \mathbf{a}(17 \mu \mathrm{L}$, $0.165 \mathrm{mmol}, 1.8$ equiv; $\left.[\mathbf{3 a}]_{0}=0.275 \mathrm{M} ;[e]=[\mathbf{3 a}]_{0}-[\mathbf{2}]_{0}=0.125 \mathrm{M}\right)$, and the data were summarized in Table S8 below (same $[e]$ condition) and plotted as red square in Figures $4 \mathrm{~b}$ (time-scale was adjusted as $t+420 \mathrm{~s}$ for $[2]_{0}=0.15 \mathrm{M}$ ).

Table S8. Data for Reaction Progress Kinetic Analysis

\begin{tabular}{|r|r|r|r|}
\hline \multirow{2}{*}{ time (s) } & \multicolumn{1}{|c|}{$[\mathbf{2}](\mathrm{M})$} & \multicolumn{2}{c|}{$[\mathbf{2}](\mathrm{M})$} \\
& standard condition & different $[e]$ condition & same $[e]$ condition \\
\hline 0 & 0.25 & 0.25 & 0.15 \\
\hline 60 & 0.2343 & 0.230225 & 0.139095 \\
\hline 120 & 0.217525 & 0.20965 & 0.126765 \\
\hline 180 & 0.20165 & 0.190975 & 0.11592 \\
\hline 240 & 0.1867 & 0.173 & 0.10593 \\
\hline 300 & 0.17225 & 0.1572 & 0.096495 \\
\hline 360 & 0.1594 & 0.14265 & 0.08784 \\
\hline 420 & 0.1484 & 0.1295 & 0.079695 \\
\hline 480 & 0.1364 & 0.117525 & 0.072285 \\
\hline
\end{tabular}




\begin{tabular}{|c|c|c|c|}
\hline 540 & 0.126275 & 0.106425 & 0.065355 \\
\hline 600 & 0.115825 & 0.095725 & 0.059265 \\
\hline 660 & 0.107275 & 0.084875 & 0.05334 \\
\hline 720 & 0.098425 & 0.076525 & 0.04836 \\
\hline 780 & 0.092375 & 0.06775 & 0.04299 \\
\hline 840 & 0.08485 & 0.06045 & 0.038565 \\
\hline 900 & 0.078175 & 0.05415 & 0.034875 \\
\hline 960 & 0.071025 & 0.048275 & 0.030675 \\
\hline 1020 & 0.06605 & 0.042775 & 0.02727 \\
\hline 1080 & 0.060275 & 0.038 & 0.02418 \\
\hline 1140 & 0.0551 & 0.032825 & 0.021165 \\
\hline 1200 & 0.050125 & 0.029175 & 0.0186 \\
\hline 1260 & 0.046575 & 0.02475 & 0.015975 \\
\hline 1320 & 0.042025 & 0.02165 & 0.01431 \\
\hline 1380 & 0.038925 & 0.0186 & 0.012405 \\
\hline 1440 & 0.0345 & 0.0158 & 0.010005 \\
\hline 1500 & 0.031275 & 0.013275 & 0.008955 \\
\hline 1560 & 0.027675 & 0.01145 & 0.00801 \\
\hline 1620 & 0.025175 & 0.008975 & 0.006315 \\
\hline 1680 & 0.021825 & 0.007775 & 0.005205 \\
\hline 1740 & 0.019125 & 0.005875 & 0.004545 \\
\hline 1800 & 0.01655 & 0.0049 & 0.003945 \\
\hline 1860 & 0.0144 & 0.003725 & 0.00261 \\
\hline 1920 & 0.0123 & 0.00255 & 0.00288 \\
\hline 1980 & 0.01055 & 0.001775 & 0.00198 \\
\hline 2040 & 0.0088 & 0.00145 & 0.001665 \\
\hline 2100 & 0.007225 & 0.000875 & 0.00132 \\
\hline 2160 & 0.00665 & - & 0.000855 \\
\hline 2220 & 0.005025 & - & 0.00066 \\
\hline 2280 & 0.004875 & - & - \\
\hline 2340 & 0.003475 & - & - \\
\hline 2400 & 0.0028 & - & - \\
\hline
\end{tabular}




\begin{tabular}{|r|r|r|r|}
\hline 2460 & 0.00175 & - & - \\
\hline 2520 & 0.001225 & - & - \\
\hline 2580 & 0.001075 & - & - \\
\hline 2640 & 0.000475 & - & - \\
\hline 2700 & 0.00035 & - & - \\
\hline
\end{tabular}

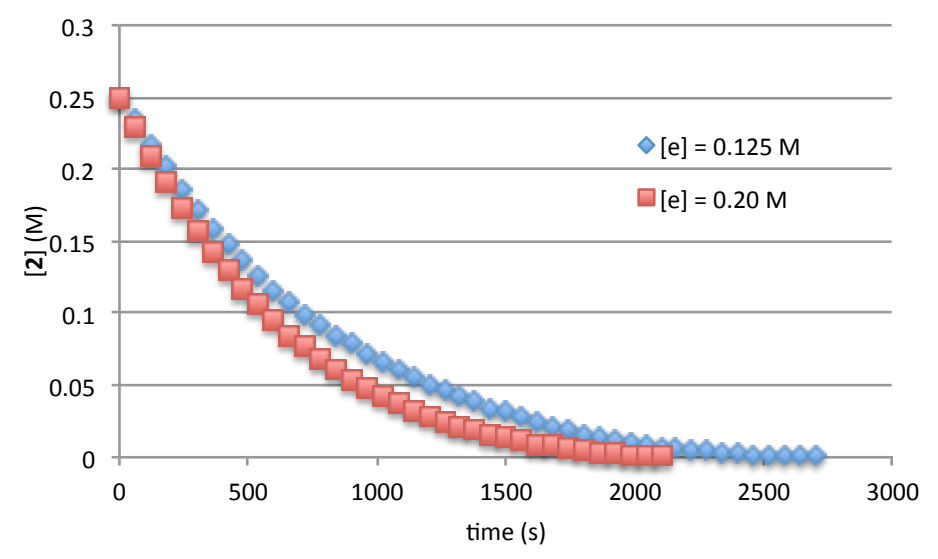

Figure S9. Concentration-time plot under the conditions of different $[e]$ experiments.

\section{Kinetic Experiments (4): Kinetic Isotope Effects}

To an NMR sample tube were added a $37.5 \mathrm{mM}$ solution of (alkynyl)Rh(III) complex 5a in toluene (50 $\mu \mathrm{L}, 1.9 \mu \mathrm{mol}, 1.3 \mathrm{~mol} \%)$, $\alpha$-ketiminoester $2(36 \mu \mathrm{L}, 0.15 \mathrm{mmol})$ and toluene $(0.55 \mathrm{~mL})$ in a glove box and the tube was sealed with a rubber septum. To the tube was added ethynylbenzene (3a) ( $24 \mu \mathrm{L}, 0.23 \mathrm{mmol}, 1.5$ equiv) via a syringe and a stainless steel needle, and the mixture was analyzed by ${ }^{19} \mathrm{~F}$ NMR spectroscopy to determine the yield of $4 \mathbf{a}$ based on the relative integration values of the peaks at -71.12 (for 2) and $-75.62 \mathrm{ppm}$ (for $\mathbf{4 a}$ ) at the specified time. The same experiment was repeated and an average of yields of the two runs was used to calculate kinetic isotope effects.

The reactions with mono-deuterated phenylacetylene (3a- $d$ ) were performed independently under the identical reaction conditions. The results were summarized in Table S9 and Figure 5. Based on the slopes in Figure 5, the value of kinetic isotope effect was calculated to be $k_{\mathrm{H}} / k_{\mathrm{D}}=1.225 / 1.448=0.846$, suggesting the presence of inverse kinetic isotope effects. 
Table S9. Comparison of Initial Rates of 3a and 3a-d

\begin{tabular}{|r|r|r|}
\hline \multicolumn{1}{c|}{$\begin{array}{c}\text { time } \\
(\mathrm{min})\end{array}$} & $\begin{array}{l}\text { average yield } \\
(\%) \text { w/ 3a }\end{array}$ & $\begin{array}{l}\text { average yield } \\
(\%) \text { w/ 3a- } d\end{array}$ \\
\hline 1 & 2.8 & 3.3 \\
\hline 2 & 5.9 & 7.1 \\
\hline 3 & 8.8 & 10.5 \\
\hline 4 & 11.7 & 14 \\
\hline 5 & 14.5 & 17.2 \\
\hline
\end{tabular}

\section{Kinetic Experiments (5): Eyring Plots}

(a) Effects of temperature on activation energies

To a Schlenk tube were added a $6.3 \mathrm{mM}$ solution of (alkynyl)Rh(III) complex $\mathbf{5 a}$ in toluene $(1.2 \mathrm{~mL}, 7.5 \mu \mathrm{mol}, 2.5 \mathrm{~mol} \%, 6.3 \mathrm{mM})$ and $\alpha$-ketiminoester $2(72 \mu \mathrm{L}, 0.30 \mathrm{mmol}$, $0.25 \mathrm{M}$ ), and the mixture was stirred at $29{ }^{\circ} \mathrm{C}$. To the tube was added alkyne 3a (49 $\mu \mathrm{L}$, $0.45 \mathrm{mmol}, 1.5$ equiv, $0.38 \mathrm{M}$ ), and an aliquot (about $0.1 \mathrm{~mL}$ ) of the reaction mixture was taken at the specified time via a syringe charged with a mixture of $0.5 \mathrm{~mL}$ of $\mathrm{CDCl}_{3}$ and 0.1 $\mathrm{mL}$ of $\mathrm{AcOH}$. The sample was analyzed by ${ }^{19} \mathrm{~F}$ NMR spectroscopy to determine the yield of $\mathbf{4 a}$ based on the relative integration values of the peaks at -71.12 (for 2 ), -75.62 (for $\mathbf{4 a}$ ) and $-78.87 \mathrm{ppm}$ (acetic acid adduct of 2 ).

The same procedure was repeated at $0,-10,-20$ and $-30{ }^{\circ} \mathrm{C}$ and the results were summarized in Table S10 and plotted in Figure S10.

Table S10. Effects of Temperature for the Rate of Formation of Product 4a

\begin{tabular}{|r|r|r|r|r|r|}
\hline \multicolumn{1}{c|}{$\begin{array}{c}\text { time } \\
(\mathrm{min})\end{array}$} & $\begin{array}{c}\text { yield }(\%) \\
\text { at } 29{ }^{\circ} \mathrm{C}\end{array}$ & $\begin{array}{c}\text { yield }(\%) \\
\text { at } 0{ }^{\circ} \mathrm{C}\end{array}$ & $\begin{array}{c}\text { yield }(\%) \\
\text { at }-10^{\circ} \mathrm{C}\end{array}$ & $\begin{array}{c}\text { yield }(\%) \\
\text { at }-20{ }^{\circ} \mathrm{C}\end{array}$ & $\begin{array}{c}\text { yield (\%) } \\
\text { at }-30{ }^{\circ} \mathrm{C}\end{array}$ \\
\hline 1 & 9.9 & 5.1 & 2.3 & 0.5 & 0.1 \\
\hline 2 & 22.3 & 9.6 & 4.8 & 1.7 & 0.5 \\
\hline 3 & 33 & 13.3 & 7.2 & 2.7 & 0.9 \\
\hline 4 & - & 16.7 & 9.7 & 4.1 & 1.3 \\
\hline 5 & - & 19.2 & 12.3 & 5.2 & 1.7 \\
\hline
\end{tabular}




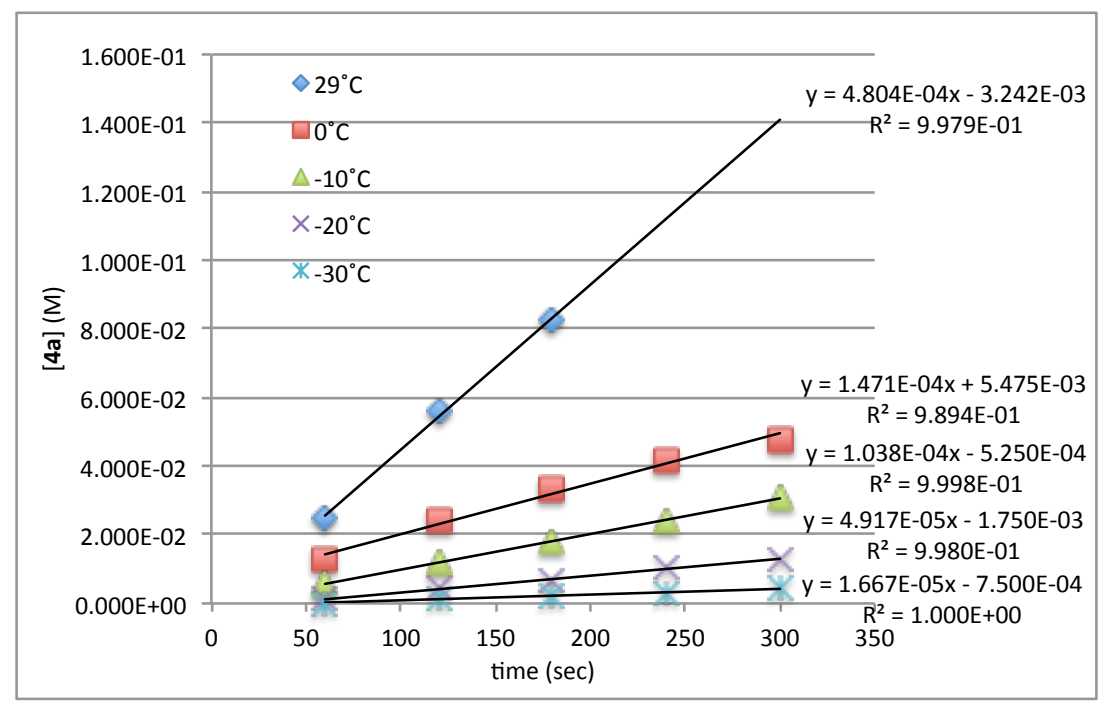

Figure S10. Plots of initial rates of the formation of product 4 a versus time.

Based on the results, second order rate constant $k\left(\mathrm{M}^{-1} \mathrm{~s}^{-1}\right)$ was calculated as follows: $k$ $=(d[\mathbf{4 a}] / d t) /([\mathbf{5 a}][\mathbf{3 a}])$ where $[\mathbf{5 a}]=0.0063 \mathrm{M}$ and $[\mathbf{3 a}]=0.38 \mathrm{M}$, and the results were summarized in Table S11 and plotted according to the equation for Eyring plot, $\ln (k / T)=$ $-\Delta H^{\ddagger} / R T+\ln \left(k_{\mathrm{b}} / h\right)+\Delta S^{\ddagger} / R$, where $R$ is gas constant $\left(8.314 \mathrm{~J} \mathrm{~K}^{-1} \mathrm{~mol}^{-1}\right), k_{\mathrm{b}}$ is Boltzmann constant $\left(1.381 \times 10^{-23} \mathrm{~J} \mathrm{~K}^{-1}\right)$, and $h$ is Plank constant $\left(6.626 \times 10^{-34} \mathrm{~J} \mathrm{~s}\right)$ (Figure 6a). From the plot, two linear lines were obtained from -30 to $-10{ }^{\circ} \mathrm{C}$ and from $-10{ }^{\circ} \mathrm{C}$ to $29{ }^{\circ} \mathrm{C}$, respectively, and each of the lines yielded the following activation parameters: $\Delta H^{\star}=11.1$ $\mathrm{kcal} \mathrm{mol}^{-1}, \Delta S^{\ddagger}=-21.8 \mathrm{cal} \mathrm{mol}^{-1} \mathrm{~K}^{-1}$ and $\Delta G^{\ddagger}=17.8 \mathrm{kcal} \mathrm{mol}^{-1}$ at $29{ }^{\circ} \mathrm{C}$ from the former line and $\Delta H^{\ddagger}=5.7 \mathrm{kcal} \mathrm{mol}^{-1}, \Delta S^{\star}=-42.5 \mathrm{cal} \mathrm{mol}^{-1} \mathrm{~K}^{-1}$ and $\Delta G^{\ddagger}=18.6 \mathrm{kcal} \mathrm{mol}^{-1}$ at $29^{\circ} \mathrm{C}$ from the latter line.

Table S11. Summary of Data for Eyring Plot Analysis

\begin{tabular}{|r|r|r|c|c|c|r|}
\hline$T\left({ }^{\circ} \mathrm{C}\right)$ & $T(\mathrm{~K})$ & $1 / T\left(\mathrm{~K}^{-1}\right)$ & $\begin{array}{c}d[\mathbf{4 a}] / d t \\
\left(\mathrm{M} \mathrm{s}^{-1}\right)\end{array}$ & $k\left(\mathrm{M}^{-1} \mathrm{~s}^{-1}\right)$ & $\begin{array}{c}k / T \\
\left(\mathrm{M}^{-1} \mathrm{~s}^{-1} \mathrm{~K}^{-1}\right)\end{array}$ & $\ln (k / T)$ \\
\hline 29 & 302 & $3.31 \times 10^{-3}$ & $4.80 \times 10^{-4}$ & $2.05 \times 10^{-1}$ & $6.79 \times 10^{-4}$ & -7.30 \\
\hline 0 & 273 & $3.66 \times 10^{-3}$ & $1.47 \times 10^{-4}$ & $6.28 \times 10^{-2}$ & $2.30 \times 10^{-4}$ & -8.38 \\
\hline-10 & 263 & $3.80 \times 10^{-3}$ & $1.04 \times 10^{-4}$ & $4.43 \times 10^{-2}$ & $1.68 \times 10^{-4}$ & -8.69 \\
\hline-20 & 253 & $3.95 \times 10^{-3}$ & $4.92 \times 10^{-5}$ & $2.10 \times 10^{-2}$ & $8.29 \times 10^{-5}$ & -9.40 \\
\hline-30 & 243 & $4.12 \times 10^{-3}$ & $1.67 \times 10^{-5}$ & $7.11 \times 10^{-3}$ & $2.93 \times 10^{-5}$ & -10.4 \\
\hline
\end{tabular}




\section{(b) Effects of temperature for ee of product $4 \mathbf{a}$}

The NMR samples of the above Eyring plot experiments were purified by preparative TLC using hexane/EtOAc $=10 / 1$ as eluent. Product 4a on the TLC plate was collected and extracted with 2-propanol. Enantiomeric excess of 4a was determined by chiral HPLC analysis. The results were summarized in Table S12 and plotted in Figure 6b. From the plot, the following differential activation parameters were obtained: $\Delta \Delta H^{\sharp}=-4.1 \mathrm{kcal} \mathrm{mol}^{-1}$, $\Delta \Delta S^{\sharp}=-7.3 \mathrm{cal} \mathrm{mol}^{-1} \mathrm{~K}^{-1}$ and $\Delta \Delta G^{\sharp}=-1.9 \mathrm{kcal} \mathrm{mol}^{-1}$ at $300 \mathrm{~K}$ according to the equation for Eyring plot, $\ln \left(k_{\mathrm{S}} / k_{\mathrm{R}}\right)=-\Delta \Delta H^{\ddagger} / R T+\Delta \Delta S^{\ddagger} / R$.

Table S12. Effects of Temperature for the Ee of Product 4a and Summary of Data for Eyring Analysis

\begin{tabular}{|r|r|r|r|r|}
\hline$T\left({ }^{\circ} \mathrm{C}\right)$ & $T(\mathrm{~K})$ & \multicolumn{1}{|l|}{$1 / T\left(\mathrm{~K}^{-1}\right)$} & ee $(\%)$ & \multicolumn{1}{l|}{$\left(k_{\mathrm{S}} / k_{\mathrm{R}}\right)$} \\
\hline 27 & 300 & $3.33 \times 10^{-3}$ & 92.09 & 3.19 \\
\hline 0 & 273 & $3.66 \times 10^{-3}$ & 96.24 & 3.95 \\
\hline-10 & 263 & $3.80 \times 10^{-3}$ & 97.15 & 4.23 \\
\hline-20 & 253 & $3.95 \times 10^{-3}$ & 97.85 & 4.52 \\
\hline-30 & 243 & $4.11 \times 10^{-3}$ & 98.46 & 4.86 \\
\hline
\end{tabular}

\section{Reversibility of Reaction Steps}

\section{Reversibility Between (Alkynyl)Rh(III) Complex 5a and (Diacetato)Rh(III) Complex} 1a

(a) Reaction of (alkynyl)Rh(III) complex 5a with acetic acid (Equation 8)

To an NMR sample tube were added a 0.10 M solution of (alkynyl)Rh(III) complex 5a in toluene- $d_{8}(0.20 \mathrm{~mL}, 0.020 \mathrm{mmol})$ and toluene- $d_{8}(0.30 \mathrm{~mL})$ in a glove box and the tube was sealed with a rubber septum. To the tube was added a $0.10 \mathrm{M}$ solution of acetic acid $(0.20 \mathrm{~mL}, 0.020 \mathrm{mmol}, 1.0$ equiv) via a syringe with a stainless steel needle at room temperature, and the mixture was analyzed by ${ }^{1} \mathrm{H}$ NMR spectroscopy (Figure S11). After 2 $\mathrm{h}$, additional acetic acid ( $6 \mu \mathrm{L}, 0.1 \mathrm{mmol}, 5$ equiv) was added and the mixture was analyzed by ${ }^{1} \mathrm{H}$ NMR spectroscopy. In both cases, 1a was not observed on ${ }^{1} \mathrm{H}$ NMR analysis. After 2 $\mathrm{h}$ at room temperature, the mixture was concentrated in vacuo and directly purified by flash silica gel column chromatography using AcOEt as eluent to give the starting complex 5a (11.9 $\mathrm{mg}, 85 \%$ recovery). 

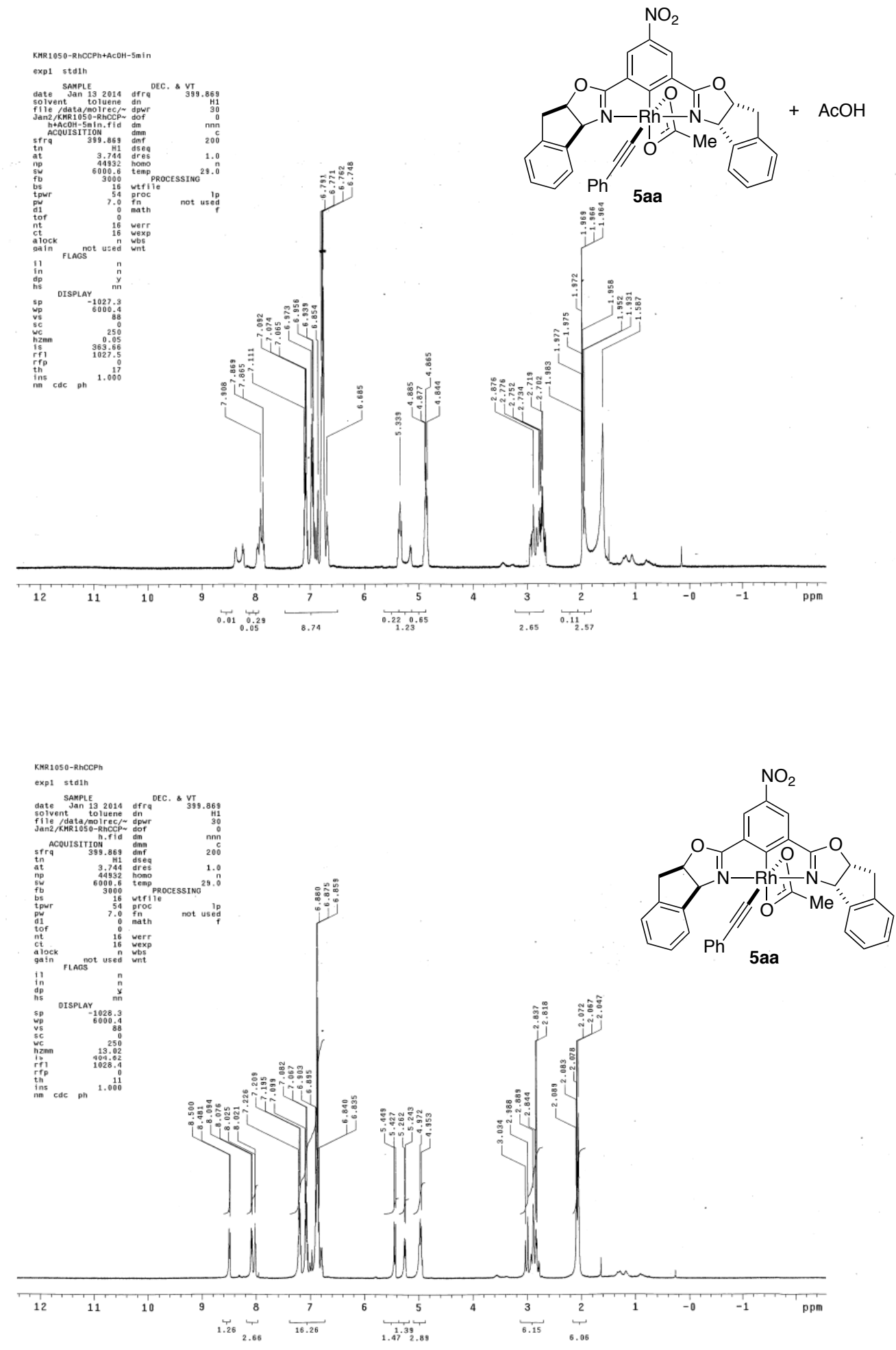

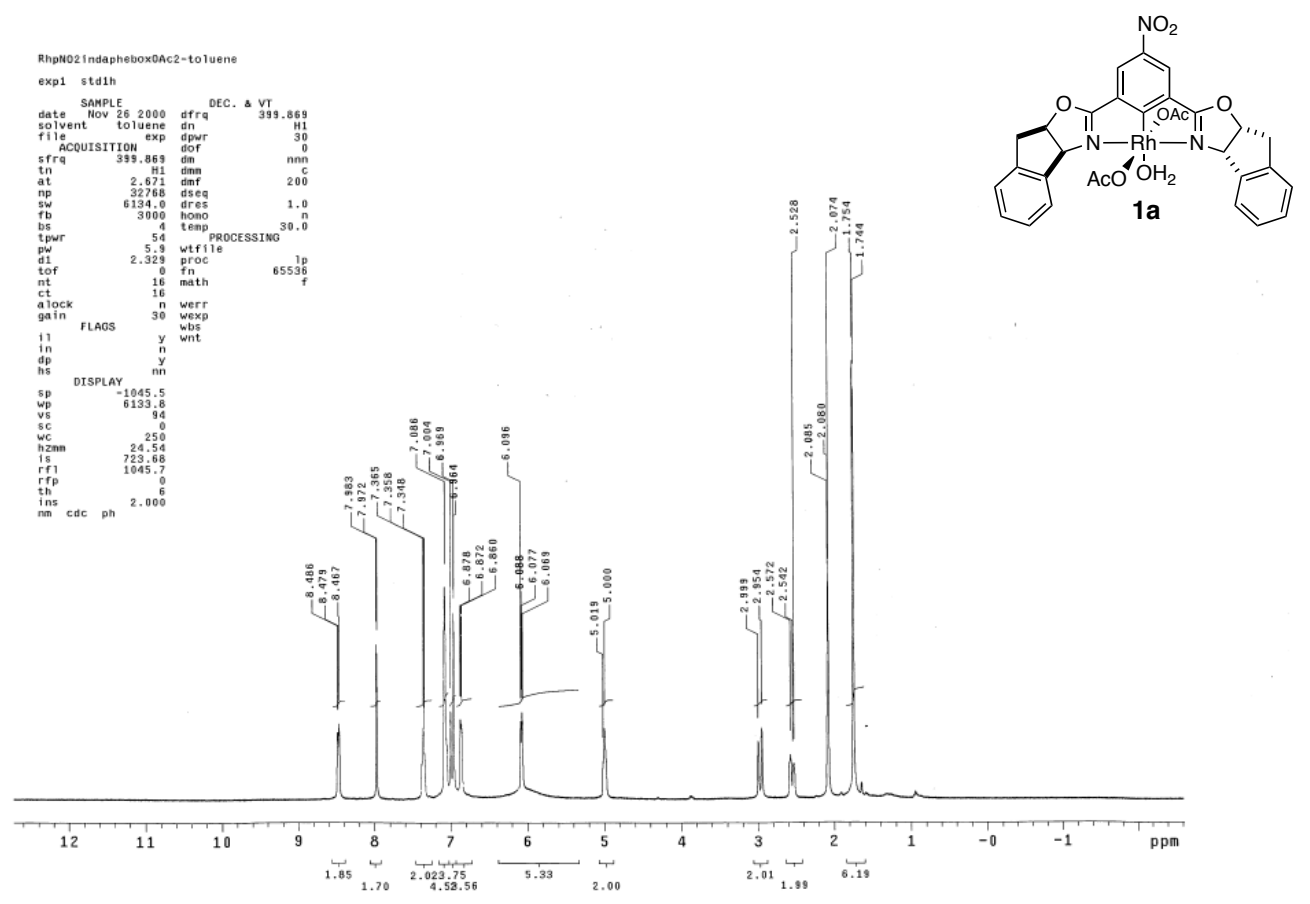

Figure S11. ${ }^{1} \mathrm{H}$ NMR spectrum of the mixture of (alkynyl)Rh(III) complex 5a and acetic acid, (alkynyl)Rh(III) complex 5a and (aqua)(diacetato)Rh(III) 1a.

(b) Reaction of (diacetato)Rh(III) complex 1a and alkyne 3a in the absence or presence of water

To a test tube were added (aqua)(diacetato)(phebox)Rh(III) complex 1a (13.5 mg, $0.0200 \mathrm{mmol})$, phenylacetylene 3a $(11 \mu \mathrm{L}, 0.10 \mathrm{mmol}, 5.0$ equiv) and toluene $(0.80 \mathrm{~mL})$. The mixture was heated at $60{ }^{\circ} \mathrm{C}$ for $50 \mathrm{~min}$. An aliquot (about $0.05 \mathrm{~mL}$ ) of the reaction mixture was taken via a syringe with a stainless steel needle from the test tube and placed to a vial. After evaporation of the volatiles under reduced pressure, the crude mixture was analyzed by ${ }^{1} \mathrm{H}$ NMR spectroscopy to give $28 \%$ conversion from $\mathbf{1 a}$ to $\mathbf{5 a}$.

The above procedure was followed with the addition of $\mathrm{H}_{2} \mathrm{O}(4 \mu \mathrm{L}, 0.22 \mathrm{mmol}, 11$ equiv). ${ }^{1} \mathrm{H}$ NMR analysis of the crude mixture showed $9 \%$ conversion from $\mathbf{1 a}$ to $\mathbf{5 a}$.

(c) Reaction of (diacetato)Rh(III) complex 1a and alkyne 3a in the presence of additional 
alkyne 3a

The above procedure was followed with the addition of additional 3a $(11 \mu \mathrm{L}, 0.10 \mathrm{mmol}$, 5.0 equiv, 10 equiv in total). ${ }^{1} \mathrm{H}$ NMR analysis of the crude mixture showed $45 \%$ conversion from $\mathbf{1 a}$ to $\mathbf{5 a}$.

\section{Reversibility Between (Alkynyl)Rh(III) Complex 5 and (Amido)Rh(III) Complex 6 (Equation 9)}

To an NMR sample tube were added a $6.3 \mathrm{mM}$ solution of (alkynyl)Rh(III) complex 5a in toluene- $d_{8}(0.40 \mathrm{~mL}, 0.0025 \mathrm{mmol})$, a $22 \mathrm{mM}$ solution of product $4 \mathbf{b}$ in toluene- $d_{8}(93 \%$ ee, $109 \mu \mathrm{L}, 0.0025 \mathrm{mmol}, 1.0$ equiv) and toluene- $d_{8}(91 \mu \mathrm{L})$ in a glove box. After $30 \mathrm{~min}$ at room temperature, the mixture was analyzed by ${ }^{1} \mathrm{H}$ NMR and ${ }^{19} \mathrm{~F}$ NMR spectroscopy. To the mixture was added ethynylbenzene (3a) ( $3 \mu \mathrm{L}, 0.03 \mathrm{mmol}, 11$ equiv) via syringe with a stainless steel needle. After $18 \mathrm{~h}$ at $60{ }^{\circ} \mathrm{C}$, the mixture was analyzed by ${ }^{1} \mathrm{H}$ NMR and ${ }^{19} \mathrm{~F}$ NMR spectroscopy, and no crossover product 4a was detected (Figure S12). This result suggests that deprotonation of the coordinated alkyne 3a is irreversible.

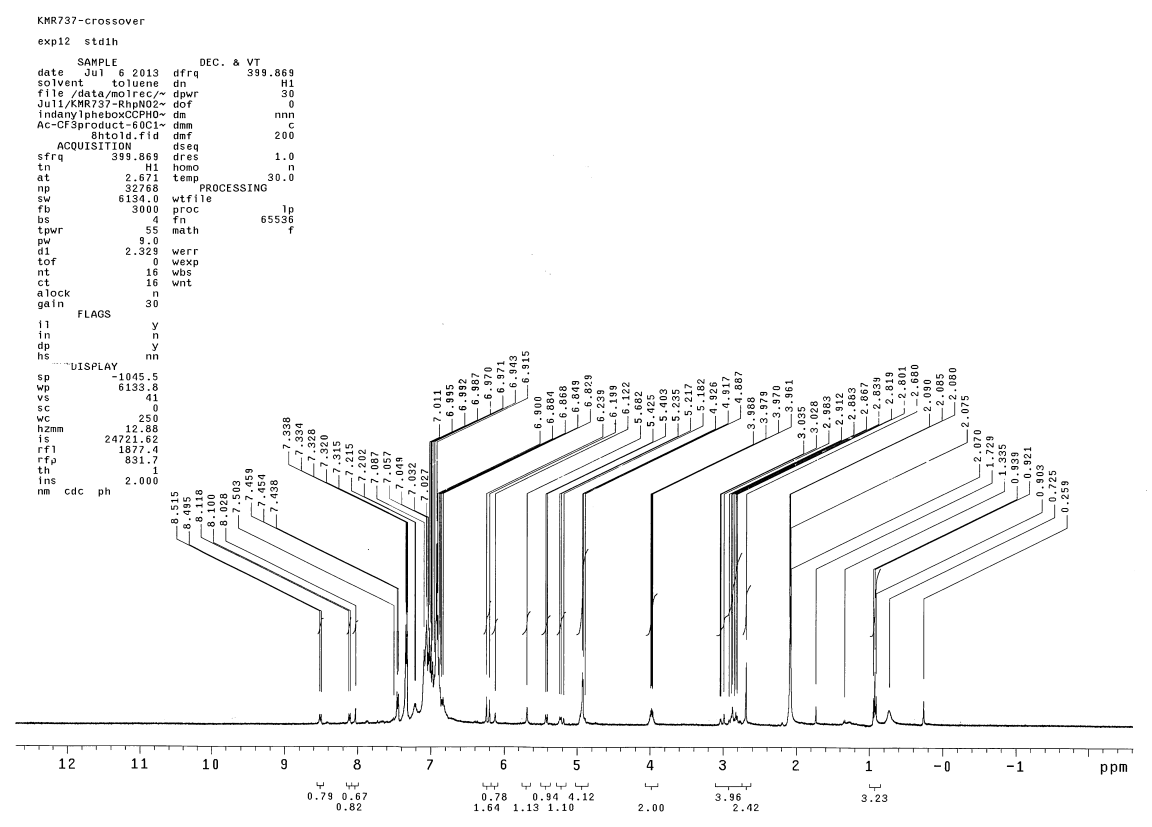




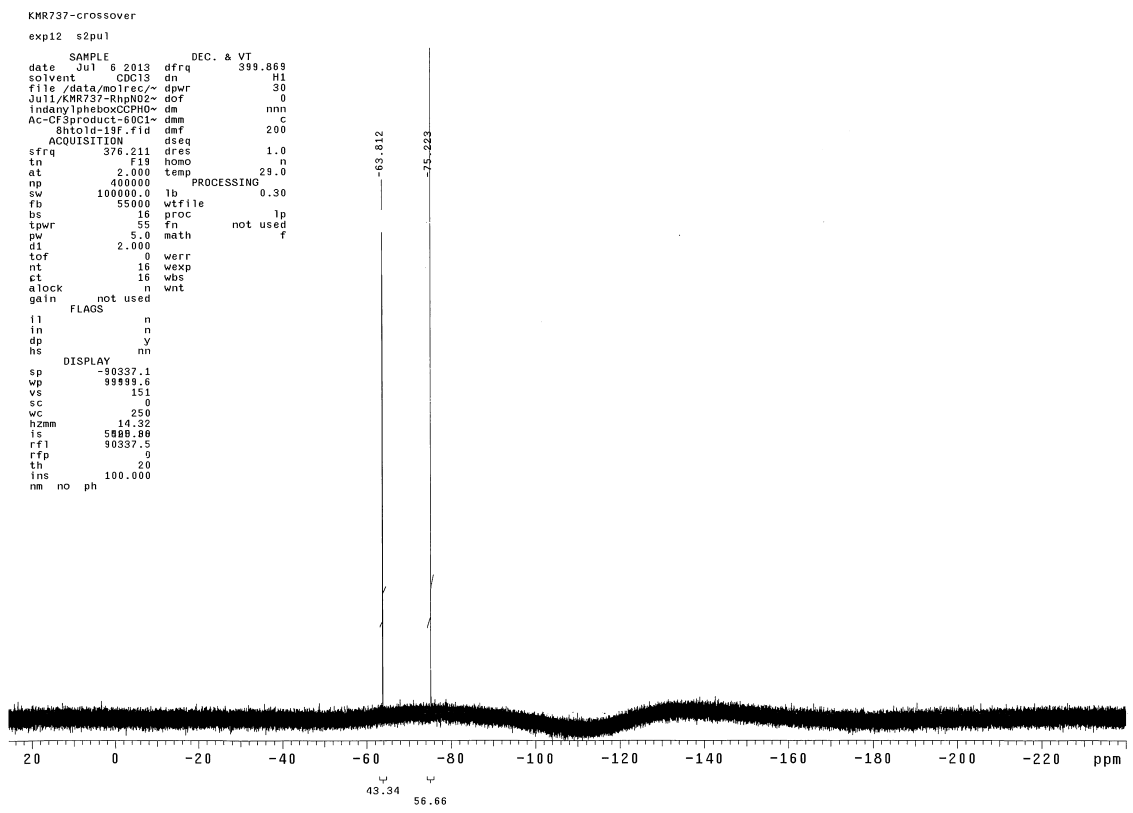

Figure S12. ${ }^{1} \mathrm{H}$ and ${ }^{19} \mathrm{~F}$ NMR spectra of the mixture of (alkynyl)Rh(III) complex 5a, product $\mathbf{4 b}$ and alkyne $\mathbf{3 a}$.

\section{Non-Linear Effects}

To a $4 \mathrm{~mL}$ screw vial equipped with a stirring bar were added a $37.5 \mathrm{mM}$ solution of (alkynyl)Rh(III) complex (3aS,8aR)-5a in toluene ( $80 \mu \mathrm{L}, 0.0030 \mathrm{mmol}, 2.0 \mathrm{~mol} \%)$, a 37.5 $\mathrm{mM}$ solution of $(3 \mathrm{a} R, 8 \mathrm{a} S)-5 \mathrm{a}$ in toluene $(20 \mu \mathrm{L}, 0.00075 \mathrm{mmol}, 0.50 \mathrm{~mol} \%)$, $\alpha$-ketiminoester 2 (36 $\mu \mathrm{L}, 0.15 \mathrm{mmol})$, ethynylbenzene (3a) (24 $\mu \mathrm{L}, 0.225 \mathrm{mmol}, 1.5$ equiv) and toluene $(0.50 \mathrm{~mL})$ in a glove box. The mixture with $60 \%$ ee of $\mathbf{5 a}$ was stirred at room temperature for $2 \mathrm{~h}$, and the crude mixture was diluted with dichloromethane and purified by preparative TLC using hexane/EtOAc $=10 / 1$ as eluent. Product 4a on the TLC plate was collected and extracted with 2-propanol. Enantiomeric excess of 4a was determined by chiral HPLC analysis.

The reactions with $0 \%, 20 \%, 40 \%, 80 \%$ and $>99 \%$ ee of $\mathbf{5 a}$ were performed in a similar manner except for changing the ratio of $(3 \mathrm{a} S, 8 \mathrm{a} R)-\mathbf{5 a}$ and $(3 \mathrm{a} R, 8 \mathrm{a} S)-\mathbf{5 a}$. The results were summarized in Table S13 and plotted in Figure S13. 
Table S13. Relationship Between Ee of (Alkynyl)Rh(III) Complex 5a and Ee of

Product 4a

\begin{tabular}{|r|r|}
\hline $\begin{array}{c}\text { ee (\%) of } \\
\text { (alkynyl)Rh(III) complex 5a }\end{array}$ & $\begin{array}{c}\text { ee (\%) of } \\
\text { product 4a }\end{array}$ \\
\hline 0 & 1 \\
\hline 20 & 19 \\
\hline 40 & 33 \\
\hline 60 & 54 \\
\hline 80 & 73 \\
\hline$>99$ & 93 \\
\hline
\end{tabular}

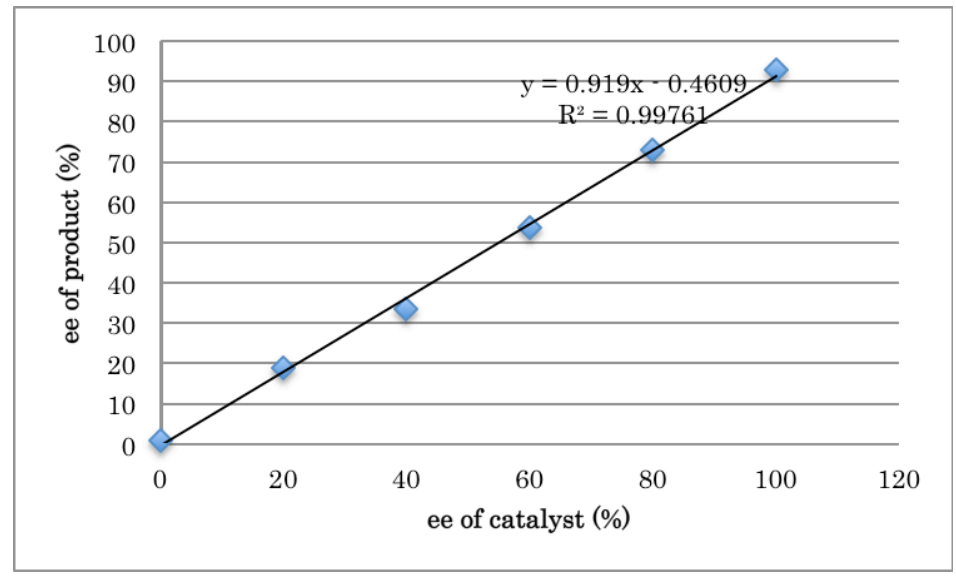

Figure S13. Plot of non-linear effects.

\section{Computational Analysis of the Transition States}

All calculation reported in the present study were carried out using density functional theory with the B3PW91 functional ${ }^{6}$, as implemented in the Gaussian09 program package. ${ }^{7}$ (alkynyl)Rh(III) complex 5a', methoxycarbonyl $\alpha$-ketiminoester 2a' and phenylacetylene (3a) were used as model substrates. For geometry optimizations, the 6-31G(d) basis set was used for the H, C, N, O, F elements, and the LANL2DZ ${ }^{8}$ pseudopotential for Rh. Based on these optimized geometries, single-point energy calculations were performed using the M06 ${ }^{9}$ functional with the $6-311+\mathrm{G}(2 \mathrm{~d}, 2 \mathrm{p})$ basis set for the $\mathrm{H}, \mathrm{C}, \mathrm{N}, \mathrm{O}, \mathrm{F}$ elements, and the $\mathrm{SDD}^{10}$ pseudopotential for $\mathrm{Rh}$ with solvents effects simulated by the $\mathrm{SMD}^{11}$ solvent model 
(toluene). The stationary points were confirmed as minima (no imaginary frequencies) or transition state (only one imaginary frequency) by analytical frequency calculations at the same theory level as the geometry optimizations. The reported energies are Gibbs free energies that include zero-point vibrational corrections, thermal corrections at $298.15 \mathrm{~K}$, and solvation free energies. All frequencies of the calculation data were scaled by an empirical factor of 0.958 and used for the calculations of the thermodynamic parameters.

\section{Optimized Structures and Cartesian Coordinates of Stationary Points (alkynyl)Rh(III) complex 5a'}

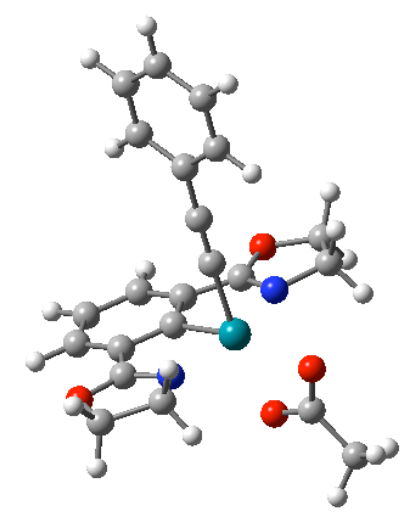

B3PW91/6-311+G(2d,2p) (SDD for Rh) energy: -1370.271206500 a.u.

ZPE: 0.35555 a.u.

Thermal Correction to Gibbs Free Energy: 0.293029 a.u.

Solvation energy: $-10.42 \mathrm{kcal} \mathrm{mol}^{-1}$

$\begin{array}{cccc}\mathrm{Rh} & 0.90025400 & -0.00223800 & -0.57402500 \\ \mathrm{C} & 0.92338300 & 0.00700600 & 1.34604600 \\ \mathrm{~N} & 0.91117100 & -2.03400600 & -0.18170600 \\ \mathrm{C} & 0.91994900 & -2.30703800 & 1.08857400 \\ \mathrm{O} & 0.87220700 & -3.59734700 & 1.42512300 \\ \mathrm{C} & 0.87227500 & -4.34698600 & 0.17821300 \\ \mathrm{C} & 0.77029900 & -3.27429200 & -0.92994400\end{array}$




\begin{tabular}{|c|c|c|c|}
\hline $\mathrm{N}$ & 0.91941600 & 2.03224300 & -0.20195600 \\
\hline $\mathrm{C}$ & 0.93160900 & 2.31842700 & 1.06524900 \\
\hline $\mathrm{O}$ & 0.89541500 & 3.61252400 & 1.38826600 \\
\hline $\mathrm{C}$ & 0.89924300 & 4.34914700 & 0.13347500 \\
\hline $\mathrm{C}$ & 0.79307300 & 3.26559300 & -0.96390400 \\
\hline $\mathrm{C}$ & 0.95894500 & -1.20760200 & 2.03735600 \\
\hline C & 1.02751400 & -1.20484400 & 3.43360000 \\
\hline C & 1.06064700 & 0.02054500 & 4.10936600 \\
\hline $\mathrm{C}$ & 1.03319400 & 1.23926200 & 3.42125700 \\
\hline $\mathrm{C}$ & 0.96444200 & 1.22837100 & 2.02513200 \\
\hline $\mathrm{O}$ & 3.00903800 & -0.00424300 & -1.14508100 \\
\hline $\mathrm{C}$ & 2.72644200 & -0.01180100 & -2.39009300 \\
\hline $\mathrm{O}$ & 1.52589100 & -0.01049600 & -2.78101100 \\
\hline $\mathrm{H}$ & 1.06560700 & 2.17754900 & 3.96788300 \\
\hline $\mathrm{H}$ & 1.11254700 & 0.02590500 & 5.19361600 \\
\hline $\mathrm{H}$ & 1.05535800 & -2.13774600 & 3.98961200 \\
\hline $\mathrm{C}$ & 3.85775200 & -0.05049300 & -3.38932000 \\
\hline $\mathrm{H}$ & 1.83323500 & 4.91573900 & 0.08948700 \\
\hline $\mathrm{H}$ & 0.05460800 & 5.04026400 & 0.15465700 \\
\hline $\mathrm{H}$ & 1.58770400 & 3.33891900 & -1.71140900 \\
\hline $\mathrm{H}$ & 0.02489800 & -5.03441500 & 0.20692000 \\
\hline $\mathrm{H}$ & 1.80401300 & -4.91776700 & 0.13950900 \\
\hline $\mathrm{H}$ & -0.19539500 & -3.28048900 & -1.44659500 \\
\hline $\mathrm{H}$ & 3.50936500 & 0.26522200 & -4.37390000 \\
\hline $\mathrm{H}$ & 4.23760800 & -1.07498900 & -3.46467800 \\
\hline $\mathrm{H}$ & 4.68277800 & 0.58158300 & -3.05276500 \\
\hline $\mathrm{H}$ & -0.16986100 & 3.27519300 & -1.48551600 \\
\hline $\mathrm{H}$ & 1.56024200 & -3.36339700 & -1.68066100 \\
\hline$C$ & -1.05280900 & 0.00047300 & -0.47874300 \\
\hline C & -2.27720600 & 0.00143000 & -0.46065300 \\
\hline
\end{tabular}




$$
\begin{array}{llll}
\mathrm{C} & -3.70306300 & 0.00239400 & -0.47867200 \\
\mathrm{C} & -4.41022500 & 0.00131900 & -1.69707400 \\
\mathrm{C} & -4.44358100 & 0.00435900 & 0.71946300 \\
\mathrm{C} & -5.80100500 & 0.00215800 & -1.71244100 \\
\mathrm{H} & -3.84882300 & -0.00018700 & -2.62668200 \\
\mathrm{C} & -5.83441700 & 0.00518300 & 0.69677600 \\
\mathrm{H} & -3.90904400 & 0.00526500 & 1.66485600 \\
\mathrm{C} & -6.52142500 & 0.00408100 & -0.51748800 \\
\mathrm{H} & -6.32680700 & 0.00130400 & -2.66398400 \\
\mathrm{H} & -6.38626000 & 0.00671300 & 1.63348200 \\
\mathrm{H} & -7.60792800 & 0.00473000 & -0.53249600
\end{array}
$$

\section{$\alpha$-ketiminoester 2' ((E)-methyl}

\section{3,3,3-trifluoro-2-((methoxycarbonyl)imino)propanoate)}

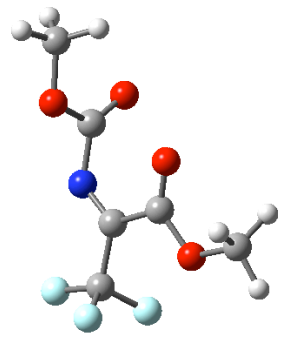

M06/6-311+G(2d,2p) energy: -887.28344039 a.u.

ZPE: 0.126711 a.u.

Thermal Correction to Gibbs Free Energy: 0.082763 a.u.

Solvation energy: $-0.45 \mathrm{kcal} \mathrm{mol}^{-1}$

$\begin{array}{lrrr}\mathrm{C} & -0.00363100 & 0.04290000 & -0.02254200 \\ \mathrm{C} & -0.00580800 & 0.02143600 & 1.50601100 \\ \mathrm{C} & 1.31719600 & 0.06810300 & 2.25094400 \\ \mathrm{O} & 2.33781600 & 0.34568700 & 1.44553600\end{array}$




$\begin{array}{lrrr}\mathrm{N} & -1.12179400 & -0.04883600 & 2.09042600 \\ \mathrm{C} & -1.27959000 & 0.00827600 & 3.49118900 \\ \mathrm{O} & 1.39203300 & -0.12384900 & 3.44181000 \\ \mathrm{O} & -1.42829600 & 1.04604800 & 4.08656500 \\ \mathrm{O} & -1.41307700 & -1.22450900 & 3.98031100 \\ \mathrm{C} & 3.62305400 & 0.41708300 & 2.08328300 \\ \mathrm{H} & 3.87033600 & -0.54307700 & 2.54139200 \\ \mathrm{H} & 4.32775900 & 0.66043500 & 1.29018200 \\ \mathrm{H} & 3.61911000 & 1.19266500 & 2.85214100 \\ \mathrm{C} & -1.66374700 & -1.27741300 & 5.39082000 \\ \mathrm{H} & -2.56274500 & -0.71038100 & 5.64255000 \\ \mathrm{H} & -1.79781200 & -2.33346800 & 5.62194500 \\ \mathrm{H} & -0.81245200 & -0.86977400 & 5.94056900 \\ \mathrm{~F} & 0.75836900 & -0.95921400 & -0.49341500 \\ \mathrm{~F} & 0.49627200 & 1.20237400 & -0.47617100 \\ \mathrm{~F} & -1.23226200 & -0.10224100 & -0.51278400\end{array}$




\section{Phenylacetylene (3a)}

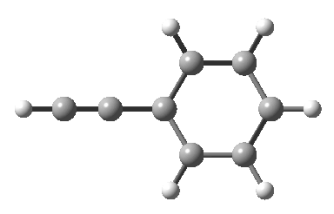

M06/6-311+G(2d,2p) energy: -308.239656884 a.u.

ZPE: 0.105147 a.u.

Thermal Correction to Gibbs Free Energy: 0.074563 a.u.

Solvation energy: $-3.04 \mathrm{kcal} \mathrm{mol}^{-1}$

$\begin{array}{lrrr}\mathrm{C} & 1.51058200 & 1.20667400 & -0.00009600 \\ \mathrm{C} & 0.12019600 & 1.21149600 & -0.00001000 \\ \mathrm{C} & -0.59199800 & 0.00000000 & 0.00003600 \\ \mathrm{C} & 0.12019400 & -1.21149800 & -0.00000500 \\ \mathrm{C} & 1.51058000 & -1.20667800 & -0.00009100 \\ \mathrm{C} & 2.20975200 & -0.00000300 & -0.00013600 \\ \mathrm{H} & 2.05101200 & 2.14900200 & -0.00013100 \\ \mathrm{H} & -0.42868800 & 2.14789400 & 0.00002100 \\ \mathrm{H} & -0.42869100 & -2.14789500 & 0.00003000 \\ \mathrm{H} & 2.05100800 & -2.14900700 & -0.00012200 \\ \mathrm{H} & 3.29599700 & -0.00000400 & -0.00020300 \\ \mathrm{C} & -2.01954400 & 0.00000100 & 0.00012300 \\ \mathrm{C} & -3.23043300 & 0.00000200 & 0.00019800 \\ \mathrm{H} & -4.29661800 & 0.00000300 & 0.00026300\end{array}$




\section{TS1}

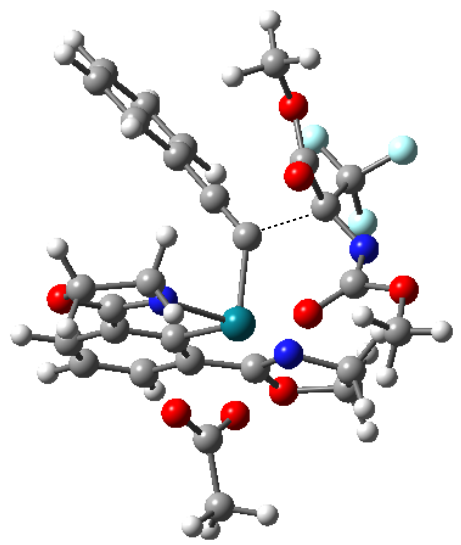

M06/6-311+G(2d,2p) (SDD for Rh) energy: -2257.54750026 a.u.

ZPE: 0.48275 a.u.

Thermal Correction to Gibbs Free Energy: 0.401698 a.u.

Solvation energy: $-9.55 \mathrm{kcal} \mathrm{mol}^{-1}$

$\begin{array}{crrr}\text { Rh } & 1.16631600 & -0.04565400 & -0.03686300 \\ \mathrm{C} & 1.04352500 & 1.76775300 & 0.60317300 \\ \mathrm{C} & 0.98111800 & 2.81055600 & -0.32464900 \\ \mathrm{C} & 0.95435700 & 4.13246200 & 0.12851700 \\ \mathrm{C} & 1.06338600 & 3.33724400 & 2.43791700 \\ \mathrm{C} & 1.09558900 & 2.01765800 & 1.97547800 \\ \mathrm{H} & 1.10642200 & 3.55420400 & 3.50159500 \\ \mathrm{H} & 0.91624200 & 4.95946400 & -0.57495600 \\ \mathrm{C} & -1.34261600 & -2.08608200 & 0.11873700 \\ \mathrm{C} & -2.35174800 & -2.16055300 & 1.28660300 \\ \mathrm{~F} & -1.73090900 & -1.92780000 & 2.45694400 \\ \mathrm{~F} & -2.87860300 & -3.39521200 & 1.34080000 \\ \mathrm{~F} & -3.37795400 & -1.30128300 & 1.21363400 \\ \mathrm{C} & -1.91713600 & -2.19351100 & -1.30061100 \\ \mathrm{O} & -1.24494300 & -2.49886600 & -2.25721000\end{array}$




\begin{tabular}{|c|c|c|c|}
\hline $\mathrm{O}$ & -3.23154200 & -1.95111600 & -1.35245400 \\
\hline $\mathrm{C}$ & -0.86644700 & -0.23094900 & 0.01624400 \\
\hline $\mathrm{C}$ & -1.84907700 & 0.54549200 & -0.01809800 \\
\hline $\mathrm{N}$ & -0.32904300 & -2.90278800 & 0.38668200 \\
\hline $\mathrm{C}$ & 0.75183700 & -3.02591400 & -0.40344100 \\
\hline $\mathrm{O}$ & 1.46122100 & -2.09799000 & -0.83162400 \\
\hline $\mathrm{O}$ & 1.12684900 & -4.29976100 & -0.57974700 \\
\hline$C$ & 1.22416200 & 0.76117500 & 2.69708000 \\
\hline $\mathrm{N}$ & 1.25335800 & -0.34851100 & 2.02618100 \\
\hline $\mathrm{O}$ & 1.34237400 & 0.66449700 & 4.02431000 \\
\hline$C$ & -2.95219900 & 1.42243000 & -0.06066800 \\
\hline$C$ & -3.57943900 & 1.84767800 & 1.13066800 \\
\hline$C$ & -3.42931100 & 1.90911000 & -1.29708300 \\
\hline $\mathrm{C}$ & -4.64783100 & 2.73185500 & 1.08048900 \\
\hline $\mathrm{H}$ & -3.21610300 & 1.46542800 & 2.07846700 \\
\hline C & -4.49470300 & 2.79831500 & -1.33616400 \\
\hline $\mathrm{H}$ & -2.94345300 & 1.58298100 & -2.21109500 \\
\hline $\mathrm{C}$ & -5.10600000 & 3.20951000 & -0.14971500 \\
\hline $\mathrm{H}$ & -5.12725800 & 3.05299000 & 2.00062300 \\
\hline $\mathrm{H}$ & -4.85299900 & 3.17297500 & -2.29065200 \\
\hline $\mathrm{H}$ & -5.94106300 & 3.90372200 & -0.18363800 \\
\hline $\mathrm{C}$ & 1.01072800 & 2.26296700 & -1.67298000 \\
\hline $\mathrm{N}$ & 1.04403200 & 0.97588200 & -1.81452400 \\
\hline $\mathrm{O}$ & 1.04688300 & 2.98976700 & -2.79175100 \\
\hline $\mathrm{C}$ & 1.12019900 & 0.64858200 & -3.22954100 \\
\hline $\mathrm{H}$ & 1.98958900 & 0.01095800 & -3.39215700 \\
\hline $\mathrm{H}$ & 0.20919400 & 0.12507500 & -3.54000300 \\
\hline $\mathrm{C}$ & 1.28420400 & 2.04036800 & -3.87474100 \\
\hline $\mathrm{H}$ & 0.56309800 & 2.25799000 & -4.66518100 \\
\hline $\mathrm{H}$ & 2.30027800 & 2.21264200 & -4.23664200 \\
\hline
\end{tabular}




$\begin{array}{lrrr}\mathrm{C} & 1.49662900 & -1.44936200 & 2.94914900 \\ \mathrm{H} & 0.76309200 & -2.24258300 & 2.79215400 \\ \mathrm{H} & 2.49833100 & -1.85571900 & 2.76817600 \\ \mathrm{C} & 1.36914700 & -0.76066400 & 4.32378500 \\ \mathrm{H} & 2.21071700 & -0.94142000 & 4.99460700 \\ \mathrm{H} & 0.43335600 & -1.00417000 & 4.83505000 \\ \mathrm{C} & 2.34831700 & -4.48740500 & -1.29588500 \\ \mathrm{H} & 3.18008300 & -3.99187600 & -0.78842400 \\ \mathrm{H} & 2.50593600 & -5.56620900 & -1.32309600 \\ \mathrm{H} & 2.26885000 & -4.09004900 & -2.31091000 \\ \mathrm{C} & -3.82221900 & -2.08735800 & -2.64918700 \\ \mathrm{H} & -4.88386000 & -1.88586500 & -2.51020600 \\ \mathrm{H} & -3.38334900 & -1.37170500 & -3.34902200 \\ \mathrm{H} & -3.66825500 & -3.09780300 & -3.03536900 \\ \mathrm{C} & 0.98713800 & 4.37912600 & 1.50666200 \\ \mathrm{H} & 0.96487900 & 5.40514800 & 1.86104300 \\ \mathrm{O} & 3.23674200 & 0.07901900 & 0.11683500 \\ \mathrm{C} & 4.06822900 & 0.30041600 & -0.85659100 \\ \mathrm{O} & 3.80682400 & 0.54419100 & -2.03334300 \\ \mathrm{C} & 5.51992400 & 0.21692300 & -0.38804900 \\ \mathrm{H} & 6.19079900 & 0.51768200 & -1.19417600 \\ \mathrm{H} & 5.67635300 & 0.85290800 & 0.48772800 \\ \mathrm{H} & 5.74982300 & -0.81064200 & -0.08856100\end{array}$




\section{TS2}

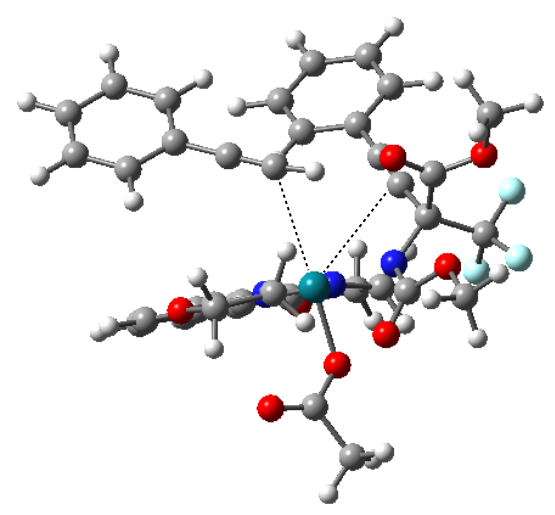

M06/6-311+G(2d,2p) (SDD for Rh) energy: -2565.80529659 a.u.

ZPE: 0.590662 a.u.

Thermal Correction to Gibbs Free Energy: 0.499831 a.u.

Solvation energy: $-11.88 \mathrm{kcal} \mathrm{mol}^{-1}$

$\begin{array}{crrr}\text { Rh } & -0.14275000 & -0.95466200 & 0.53049300 \\ \mathrm{C} & 1.69466100 & -1.35901700 & 1.04252300 \\ \mathrm{O} & -0.86464000 & -2.15980400 & 1.90909600 \\ \mathrm{C} & 2.26425600 & -0.72424600 & 2.14464700 \\ \mathrm{C} & 2.42419900 & -2.28060400 & 0.29253100 \\ \mathrm{C} & -0.69970200 & -3.46655800 & 1.92222700 \\ \mathrm{C} & 3.58393500 & -1.01354900 & 2.51353700 \\ \mathrm{C} & 1.29247400 & 0.16524400 & 2.75465700 \\ \mathrm{C} & 3.73954100 & -2.58060200 & 0.64747300 \\ \mathrm{C} & 1.59219800 & -2.79323300 & -0.78356500 \\ \mathrm{C} & -1.69951700 & -4.09074300 & 2.88470700 \\ \mathrm{O} & 0.09530500 & -4.13070200 & 1.28230000 \\ \mathrm{C} & 4.30569700 & -1.94163800 & 1.75909100 \\ \mathrm{H} & 4.03882900 & -0.53072400 & 3.37387000 \\ \mathrm{O} & 1.49983700 & 0.80339400 & 3.90700400\end{array}$




$\begin{array}{lrrr}\mathrm{N} & 0.13212600 & 0.32962400 & 2.18971900 \\ \mathrm{H} & 4.31723600 & -3.30330900 & 0.07850000 \\ \mathrm{O} & 1.99687200 & -3.70368800 & -1.66496300 \\ \mathrm{~N} & 0.38386900 & -2.34140400 & -0.91597100 \\ \mathrm{H} & -2.65579800 & -4.17046800 & 2.35907700 \\ \mathrm{H} & -1.85133500 & -3.47019900 & 3.77006800 \\ \mathrm{H} & -1.35369700 & -5.08604100 & 3.16732000 \\ \mathrm{H} & 5.32802500 & -2.17462700 & 2.04039200 \\ \mathrm{C} & 0.31253600 & 1.61392300 & 4.13532100 \\ \mathrm{C} & -0.70798200 & 1.09417500 & 3.10856800 \\ \mathrm{C} & 0.83285500 & -4.02576000 & -2.48106300 \\ \mathrm{C} & -0.25432600 & -3.02728200 & -2.03362100 \\ \mathrm{H} & 0.59252400 & 2.65746500 & 3.96315000 \\ \mathrm{H} & 0.01460000 & 1.47932600 & 5.17619600 \\ \mathrm{H} & -1.45453800 & 0.42328900 & 3.54649000 \\ \mathrm{H} & -1.23041500 & 1.89379800 & 2.58525600 \\ \mathrm{H} & -2.57205200 & -5.06568300 & -2.27112800 \\ \mathrm{H} & -1.12774300 & -3.92309800 & -3.52734400 \\ \mathrm{H} & -1.17248400 & -3.50165500 & -1.68304300 \\ \mathrm{H} & -0.51283300 & -2.30147700 & -2.81228500 \\ \mathrm{C} & -2.17693700 & -0.41812100 & -0.23083300 \\ \mathrm{C} & -2.65443600 & 0.93120500 & -0.46174600 \\ \mathrm{C} & -2.94150100 & -1.44869900 & -0.65193400 \\ \mathrm{C} & -3.81750100 & 1.32975900 & 0.51270800 \\ \mathrm{H} & -3.00564100 & 1.17097000 & -1.96362000 \\ \mathrm{H} & -1.58130500 & 1.90519900 & -0.19476500 \\ \mathrm{H} & -4.01692200 & -1.07678100 & -1.42475500 \\ \mathrm{H} & -2.73717200 & -2.63839800 & -0.42161000 \\ \mathrm{H} & -324700 & 1.08728400 & 1.78312800 \\ \mathrm{H} & -2.64458000 & 0.43520200\end{array}$




\begin{tabular}{|c|c|c|c|}
\hline $\mathrm{F}$ & -4.95509800 & 0.66220600 & 0.31808800 \\
\hline $\mathrm{O}$ & -4.15276600 & 1.81624000 & -2.15415300 \\
\hline $\mathrm{O}$ & -2.24800900 & 0.85190500 & -2.85378400 \\
\hline $\mathrm{C}$ & -0.74826300 & 2.76507000 & 0.00233600 \\
\hline $\mathrm{C}$ & -4.87707200 & -2.13518400 & -1.81764000 \\
\hline $\mathrm{C}$ & -4.48466600 & 2.07576600 & -3.52273900 \\
\hline $\mathrm{C}$ & 0.23090800 & 3.76427400 & 0.27949200 \\
\hline $\mathrm{H}$ & -5.71860100 & -1.66295100 & -2.32835600 \\
\hline $\mathrm{H}$ & -4.36943000 & -2.82940700 & -2.49434200 \\
\hline $\mathrm{H}$ & -5.23055500 & -2.69661200 & -0.94862500 \\
\hline $\mathrm{H}$ & -4.59190100 & 1.13674600 & -4.07080100 \\
\hline $\mathrm{H}$ & -3.70732700 & 2.67895500 & -3.99796400 \\
\hline $\mathrm{H}$ & -5.43055600 & 2.61595200 & -3.49722800 \\
\hline $\mathrm{C}$ & -0.15129600 & 5.10965200 & 0.42609700 \\
\hline $\mathrm{C}$ & 1.58575400 & 3.41646600 & 0.42628800 \\
\hline $\mathrm{C}$ & 0.80176100 & 6.08105700 & 0.71375000 \\
\hline $\mathrm{H}$ & -1.19712900 & 5.37807300 & 0.31262700 \\
\hline $\mathrm{C}$ & 2.53066400 & 4.39601200 & 0.71612300 \\
\hline $\mathrm{H}$ & 1.88058400 & 2.37963800 & 0.30326900 \\
\hline $\mathrm{C}$ & 2.14367200 & 5.72881500 & 0.86084600 \\
\hline $\mathrm{H}$ & 0.49541900 & 7.11758400 & 0.82382100 \\
\hline $\mathrm{H}$ & 3.57429400 & 4.11564900 & 0.82895800 \\
\hline $\mathrm{H}$ & 2.88494200 & 6.49024400 & 1.08675700 \\
\hline $\mathrm{C}$ & 0.83798000 & 0.51180900 & -2.19596000 \\
\hline $\mathrm{C}$ & 2.05056000 & 0.59687900 & -2.23179000 \\
\hline $\mathrm{H}$ & -0.23403600 & 0.48926700 & -2.29764700 \\
\hline $\mathrm{C}$ & 3.47146900 & 0.70209900 & -2.30566900 \\
\hline $\mathrm{C}$ & 4.09773300 & 1.95624400 & -2.18786900 \\
\hline $\mathrm{C}$ & 4.26365600 & -0.43807100 & -2.53085600 \\
\hline $\mathrm{C}$ & 5.48081800 & 2.06154600 & -2.28844300 \\
\hline
\end{tabular}




$$
\begin{array}{llrl}
\mathrm{H} & 3.48653100 & 2.83791000 & -2.02298500 \\
\mathrm{C} & 5.64594500 & -0.32272900 & -2.62933600 \\
\mathrm{H} & 3.78347100 & -1.40653800 & -2.62677100 \\
\mathrm{C} & 6.25884700 & 0.92468500 & -2.50870800 \\
\mathrm{H} & 5.95361100 & 3.03574700 & -2.19930300 \\
\mathrm{H} & 6.24794400 & -1.21009200 & -2.80416200 \\
\mathrm{H} & 7.33890700 & 1.01080800 & -2.58877400
\end{array}
$$

\section{TS3}

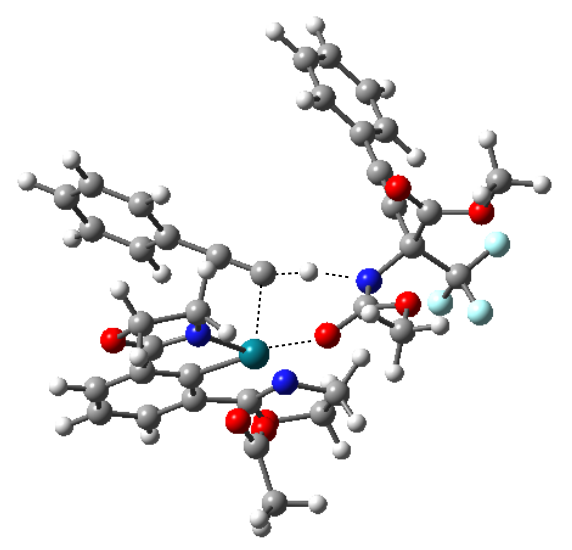

M06/6-311+G(2d,2p) (SDD for Rh) energy: -2565.81255710 a.u.

ZPE: 0.589232 a.u.

Thermal Correction to Gibbs Free Energy: 0.495251 a.u.

Solvation energy: $-12.79 \mathrm{kcal} \mathrm{mol}^{-1}$

$\begin{array}{cccc}\mathrm{Rh} & 1.59844900 & -0.75977800 & 0.14541300 \\ \mathrm{C} & 2.97457800 & 0.39597400 & 0.84625300 \\ \mathrm{O} & 2.68051700 & -2.35495200 & 0.79496600 \\ \mathrm{C} & 2.96256700 & 0.72360500 & 2.20415300 \\ \mathrm{C} & 4.00940600 & 0.81240500 & 0.00509800 \\ \mathrm{C} & 3.34482400 & -3.17186700 & 0.02285700 \\ \mathrm{C} & 3.99381200 & 1.50746300 & 2.73212400\end{array}$




\begin{tabular}{|c|c|c|c|}
\hline $\mathrm{C}$ & 1.81584500 & 0.10020900 & 2.84885100 \\
\hline $\mathrm{C}$ & 5.04688200 & 1.59092600 & 0.52615100 \\
\hline $\mathrm{C}$ & 3.79165700 & 0.27860700 & -1.33180400 \\
\hline $\mathrm{C}$ & 3.87156600 & -4.36380300 & 0.81601900 \\
\hline $\mathrm{O}$ & 3.54923800 & -3.07339600 & -1.18151800 \\
\hline $\mathrm{C}$ & 5.02152200 & 1.93533200 & 1.88327300 \\
\hline $\mathrm{H}$ & 4.00699400 & 1.77200000 & 3.78584900 \\
\hline $\mathrm{O}$ & 1.53804500 & 0.19065500 & 4.15144200 \\
\hline $\mathrm{N}$ & 1.00876500 & -0.61705800 & 2.12997100 \\
\hline $\mathrm{H}$ & 5.86525500 & 1.92093100 & -0.10745700 \\
\hline $\mathrm{O}$ & 4.62973300 & 0.43043500 & -2.35865700 \\
\hline $\mathrm{N}$ & 2.72284000 & -0.42311800 & -1.55033700 \\
\hline $\mathrm{H}$ & 4.29413000 & -4.04361200 & 1.77137800 \\
\hline $\mathrm{H}$ & 3.04143200 & -5.04363000 & 1.03384700 \\
\hline $\mathrm{H}$ & 4.62145900 & -4.89446500 & 0.22743900 \\
\hline $\mathrm{H}$ & 5.82611300 & 2.54014400 & 2.29027300 \\
\hline $\mathrm{C}$ & 0.27082900 & -0.50711500 & 4.34077900 \\
\hline $\mathrm{C}$ & 0.01696700 & -1.23089500 & 3.00279600 \\
\hline $\mathrm{C}$ & 4.12186900 & -0.43797900 & -3.41603100 \\
\hline $\mathrm{C}$ & 2.72188300 & -0.86938300 & -2.93589300 \\
\hline $\mathrm{H}$ & 0.38645900 & -1.17343400 & 5.19692000 \\
\hline $\mathrm{H}$ & -0.48640300 & 0.24992500 & 4.56476900 \\
\hline $\mathrm{H}$ & -0.98874600 & -1.08268300 & 2.60618500 \\
\hline $\mathrm{H}$ & 0.20941900 & -2.30847600 & 3.05784600 \\
\hline $\mathrm{H}$ & 4.81061800 & -1.28234500 & -3.49727800 \\
\hline $\mathrm{H}$ & 4.12384400 & 0.13758400 & -4.34335000 \\
\hline $\mathrm{H}$ & 2.58798400 & -1.95083900 & -2.95651500 \\
\hline $\mathrm{H}$ & 1.90707800 & -0.38047000 & -3.48246700 \\
\hline $\mathrm{N}$ & -1.81943700 & -1.23797600 & -0.05659400 \\
\hline $\mathrm{C}$ & -3.26157400 & -1.21361300 & -0.12591400 \\
\hline
\end{tabular}




\begin{tabular}{|c|c|c|c|}
\hline $\mathrm{C}$ & -1.11581300 & -2.22351000 & -0.58797500 \\
\hline$\Omega$ & -3.86890700 & -1.97862000 & 1.08612100 \\
\hline $\mathrm{C}$ & -3.81191700 & -1.69588700 & -1.50594400 \\
\hline $\mathrm{C}$ & -3.71807900 & 0.18410000 & -0.01200700 \\
\hline $\mathrm{O}$ & -1.79102600 & -3.28932300 & -1.09231200 \\
\hline $\mathrm{O}$ & 0.13985000 & -2.23958900 & -0.65505800 \\
\hline $\mathrm{F}$ & -5.20296600 & -1.84982800 & 1.16200800 \\
\hline $\mathrm{F}$ & -3.36872300 & -1.46903000 & 2.23510600 \\
\hline $\mathrm{F}$ & -3.57432300 & -3.28263700 & 1.07332800 \\
\hline$\Omega$ & -4.80182700 & -2.58648200 & -1.39502300 \\
\hline$\Omega$ & -3.40251900 & -1.25449400 & -2.55115800 \\
\hline $\mathrm{C}$ & -4.10545800 & 1.33052700 & 0.05595000 \\
\hline $\mathrm{C}$ & -1.00391700 & -4.29580900 & -1.72159500 \\
\hline $\mathrm{C}$ & -5.35349600 & -3.02894100 & -2.63825800 \\
\hline C & -4.55668100 & 2.68155000 & 0.12650600 \\
\hline $\mathrm{H}$ & -0.25695000 & -4.69743600 & -1.03291800 \\
\hline $\mathrm{H}$ & -0.49062600 & -3.90060800 & -2.60256500 \\
\hline $\mathrm{H}$ & -1.70711600 & -5.07712200 & -2.01508400 \\
\hline $\mathrm{H}$ & -4.58337000 & -3.50558300 & -3.24967100 \\
\hline $\mathrm{H}$ & -5.77412900 & -2.18643800 & -3.19315500 \\
\hline $\mathrm{H}$ & -6.13331000 & -3.74370900 & -2.37621700 \\
\hline 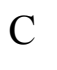 & -5.34733300 & 3.11688500 & 1.20459500 \\
\hline 4 & -4.21900300 & 3.59703900 & -0.88609400 \\
\hline 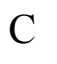 & -5.78675300 & 4.43508400 & 1.26458700 \\
\hline 11 & -5.61042500 & 2.41029200 & 1.98566100 \\
\hline 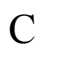 & -4.66289000 & 4.91317700 & -0.81742800 \\
\hline 11 & -3.61034400 & 3.26004800 & -1.71929900 \\
\hline 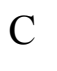 & -5.44695300 & 5.33691800 & 0.25593000 \\
\hline $\mathrm{H}$ & -6.39841700 & 4.75998500 & 2.10181800 \\
\hline & -4.39662300 & 5.61158800 & -1.60610300 \\
\hline
\end{tabular}




$\begin{array}{llll}\mathrm{H} & -5.79286900 & 6.36569100 & 0.30549600 \\ \mathrm{C} & 0.03713500 & 0.75742300 & -0.30906700 \\ \mathrm{C} & 0.60174900 & 1.82648400 & -0.56375700 \\ \mathrm{H} & -0.86341000 & 0.03602700 & -0.16336900 \\ \mathrm{C} & 1.26440000 & 3.03818800 & -0.89263100 \\ \mathrm{C} & 1.50853900 & 3.36384200 & -2.24131200 \\ \mathrm{C} & 1.66382300 & 3.93614700 & 0.11586700 \\ \mathrm{C} & 2.13713200 & 4.55903400 & -2.56898500 \\ \mathrm{H} & 1.18902100 & 2.67273500 & -3.01477700 \\ \mathrm{C} & 2.29468900 & 5.12714800 & -0.22097400 \\ \mathrm{H} & 1.47476500 & 3.68120700 & 1.15335300 \\ \mathrm{C} & 2.53143900 & 5.44012500 & -1.56078500 \\ \mathrm{H} & 2.31967100 & 4.80653900 & -3.61065900 \\ \mathrm{H} & 2.60201300 & 5.81549400 & 0.56083400 \\ \mathrm{H} & 3.02311800 & 6.37374000 & -1.81982000\end{array}$

\section{Improvement of the Catalytic Activity (1): Development of Precatalyst} Alkynyl Ligand Exchange Using (Phenylethynyl)Rh(III) Complex 5a

(a) With aryl-substituted alkyne $\mathbf{3 b}$ (Equation 10)

To an NMR sample tube were added a $6.3 \mathrm{mM}$ solution of (phenylethynyl) $\mathrm{Rh}$ (III) complex $5 \mathbf{a}$ in toluene- $d_{8}(0.40 \mathrm{~mL}, 0.0025 \mathrm{mmol})$, a $21 \mathrm{mM}$ solution of alkyne $\mathbf{3 b}$ in toluene- $d_{8}(60 \mu \mathrm{L}, 0.013 \mathrm{mmol}, 5.0$ equiv $)$ and toluene- $d_{8}(140 \mu \mathrm{L})$ in a glove box. After 30 min at room temperature, the mixture was analyzed by ${ }^{1} \mathrm{H}$ and ${ }^{19} \mathrm{~F}$ NMR spectroscopy that showed the starting complex 5a was consumed to give (4-trifluoromethylphenylethynyl)Rh(III) complex 5b. The mixture was concentrated in vacuo and directly purified by flash silica gel column chromatography using AcOEt as eluent to give $\mathbf{5 b}$ ( $1.9 \mathrm{mg}, 99 \%$ yield).

(b) With alkyl-substituted alkyne 3c (Equation 11)

To an NMR sample tube were added a $6.3 \mathrm{mM}$ solution of (phenylethynyl)Rh(III) 
complex 5a in toluene- $d_{8}(0.40 \mathrm{~mL}, 0.0025 \mathrm{mmol})$, a $21 \mathrm{mM}$ solution of alkyne 3c in toluene- $d_{8}\left(60 \mu \mathrm{L}, 0.013 \mathrm{mmol}, 5.0\right.$ equiv) and toluene- $d_{8}(140 \mu \mathrm{L})$ in a glove box. After 2 $\mathrm{h}$ at $80{ }^{\circ} \mathrm{C}$, the mixture was analyzed by ${ }^{1} \mathrm{H}$ NMR spectroscopy. The ${ }^{1} \mathrm{H}$ NMR spectrum showed that the starting complex 5a remained intact and formation of the desired complex 5c was not detected.

\section{Alkynyl Ligand Exchange Using (Trimethylsilylethynyl)Rh(III) Complex 5d}

(a) With aryl-substituted alkyne 3a (Equation 12)

To an NMR sample tube were added a $16.6 \mathrm{mM}$ solution of (trimethylsilylethynyl)Rh(III) complex $5 \mathbf{d}$ in toluene- $d_{8}(0.30 \mathrm{~mL}, 0.0050 \mathrm{mmol})$ and an $83.3 \mathrm{mM}$ solution of aryl-substituted alkyne $\mathbf{3 a}$ in toluene- $d_{8}(0.30 \mathrm{~mL}, 0.025 \mathrm{mmol}, 5.0$ equiv) via a syringe and a stainless steel needle. After $10 \mathrm{~min}$ at room temperature, the mixture was analyzed by ${ }^{1} \mathrm{H}$ NMR spectroscopy to determine the yield of (phenylethynyl)Rh(III) complex 5a as $75 \%$ by comparing the integration values of the peaks at 8.44 ppm (5d) and $8.52 \mathrm{ppm}(\mathbf{5 a})$. The ratio of $\mathbf{5 d}$ to $\mathbf{5 a}$ did not change after $3.5 \mathrm{~h}$. Based on the result, equilibrium constant $K_{\text {eq }}$ was determined to be 0.529 and conversion of $\mathbf{5 d}$ to $5 \mathbf{a}$ under the actual reaction conditions using $1.25 \mathrm{mM}$ of $\mathbf{5 d}$ and $0.375 \mathrm{M}$ of $\mathbf{3 a}$ was estimated to be $99 \%$.

(b) With alkyl-substituted alkyne 3c (Equation 13)

To an NMR sample tube were added a $12.5 \mathrm{mM}$ solution of (trimethylsilylethynyl)Rh(III) complex 5d in toluene- $d_{8}(0.40 \mathrm{~mL}, 0.0050 \mathrm{mmol})$ and an $125 \mathrm{mM}$ solution of alkyl-substituted alkyne $3 \mathbf{c}$ in toluene- $d_{8}(0.20 \mathrm{~mL}, 0.025 \mathrm{mmol}, 5.0$ equiv) via a syringe and a stainless steel needle. After $10 \mathrm{~min}$ at room temperature, the mixture was analyzed by ${ }^{1} \mathrm{H}$ NMR spectroscopy to determine the yield of (4-phenyl-1-butynyl)Rh(III) complex $\mathbf{5 c}$ as $38 \%$ by comparing the integration values of the peaks at $8.44 \mathrm{ppm}(\mathbf{5 d})$ and $8.50 \mathrm{ppm}(\mathbf{5 c})$. The ratio of $\mathbf{5 d}$ to $\mathbf{5 c}$ did not change after $1.25 \mathrm{~h}$. To the mixture was added alkyne $3 \mathbf{c}(7 \mu \mathrm{L}, 0.050 \mathrm{mmol}, 10.0$ equiv) via a syringe and a stainless steel needle. After $10 \mathrm{~min}$ at room temperature, the mixture was analyzed by ${ }^{1} \mathrm{H}$ NMR spectroscopy to determine the yield of (4-phenyl-1-butynyl)Rh(III) complex 5c as 
$57 \%$. Based on these results, equilibrium constant $K_{\text {eq }}$ was determined to be 0.051 and conversion of $\mathbf{5 d}$ to $\mathbf{5 c}$ under the actual reaction conditions using $6.3 \mathrm{mM}$ of $\mathbf{5 d}$ and $0.75 \mathrm{M}$ of $3 \mathbf{c}$ was estimated to be $87 \%$.

\section{Comparison of the Reactivity of (Trimethylsilylethynyl)Rh(III) Complex 5d with (Diacetato)Rh(III) Complex 5a (Scheme 5)}

(a) With aryl-substituted alkyne 3a (Equation 14)

A Schlenk tube equipped with a magnetic stir bar was dried under vacuum using heat gun and refilled with argon. To the tube were added a $1.25 \mathrm{mM}$ solution of (alkynyl)Rh(III) complex $5 \mathbf{d}$ in toluene $(0.40 \mathrm{~mL}, 0.50 \mu \mathrm{mol}, 0.50 \mathrm{~mol} \%)$ and aryl-substituted alkyne 3a (16 $\mu \mathrm{L}, 0.15 \mathrm{mmol}, 1.5$ equiv). After the mixture was stirred for $10 \mathrm{~min}$ at room temperature, $\alpha$-ketiminoester $2(24.0 \mu \mathrm{L}, 0.10 \mathrm{mmol})$ was added and the mixture was stirred at room temperature for $6 \mathrm{~h}$. The crude mixture was analyzed by ${ }^{19} \mathrm{~F}$ NMR spectroscopy and the yield of $\mathbf{4 a}$ was determined to be $97 \%$. The product $\mathbf{4 d}$ was not detected based on ${ }^{19} \mathrm{~F}$ NMR analysis of the crude mixture. The crude mixture was purified by preparative TLC using hexane/EtOAc $=10 / 1$ as eluent. Product $4 \mathbf{a}$ on the TLC plate was collected and extracted with 2-propanol. Enantiomeric excess of $\mathbf{4 a}$ was determined to be $92 \%$ by chiral HPLC analysis.

For (diacetato) $\mathrm{Rh}(\mathrm{III})$ complex 1a, the same procedure was followed except for using a $1.25 \mathrm{mM}$ solution of $1 \mathrm{a}$ in toluene $(0.40 \mathrm{~mL}, 0.50 \mu \mathrm{mol}, 0.50 \mathrm{~mol} \%)$ as catalyst and the product $4 \mathbf{a}$ was obtained in $8 \%$ yield and $92 \%$ ee.

\section{(b) With alkyl-substituted alkyne 3c (Equation 15)}

A Schlenk tube equipped with a magnetic stir bar and molecular sieves 4A (20 mg) was dried under vacuum using heat gun and refilled with argon. To the tube were added 25.0 $\mathrm{mM}$ solution of (alkynyl)Rh(III) complex $\mathbf{5 d}$ in toluene ( $0.10 \mathrm{~mL}, 2.5 \mu \mathrm{mol}, 2.5 \mathrm{~mol} \%)$ and alkyl-substituted alkyne $3 \mathbf{c}(42.0 \mu \mathrm{L}, 0.30 \mathrm{mmol}, 3.0$ equiv). The mixture was stirred for 10 min at room temperature and then cooled to $0{ }^{\circ} \mathrm{C}$, and $\alpha$-ketiminoester $2(24 \mu \mathrm{L}, 0.10$ mmol) was added and the mixture was stirred at $0{ }^{\circ} \mathrm{C}$ for $48 \mathrm{~h}$. The crude mixture was directly purified by flash silica gel column chromatography using hexane/AcOEt $=10 / 1$ as 
eluent to give product $4 \mathbf{c}$ as a colorless oil ( $41.8 \mathrm{mg}, 96 \%$ yield, $87 \%$ ee). The product $\mathbf{4 d}$ was not detected based on ${ }^{19} \mathrm{~F}$ NMR analysis of the crude mixture.

For (diacetato)Rh(III) complex 1a, the same procedure was followed except for using 1a $(1.7 \mathrm{mg}, 2.5 \mu \mathrm{mol}, 2.5 \mathrm{~mol} \%)$ and toluene $(0.10 \mathrm{~mL})$ instead of solution of $\mathbf{5 d}$ as catalyst and the product $\mathbf{4} \mathbf{c}$ was obtained in $8 \%$ yield and $89 \%$ ee.

\section{Ethyl (S)-2-(((benzyloxy)carbonyl)amino)-6-phenyl-2-(trifluoromethyl)hex-3-ynoate} $(4 \mathrm{c})$

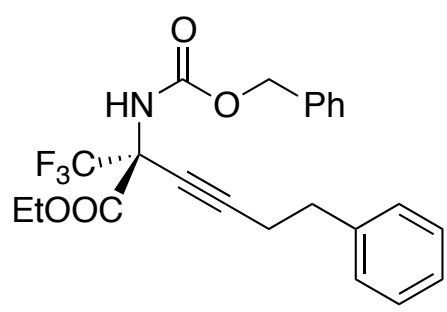

${ }^{1} \mathrm{H}$ NMR $\left(500 \mathrm{MHz}, \mathrm{CDCl}_{3}\right) \delta 7.36-7.19(\mathrm{~m}, 10 \mathrm{H}), 5.60$ (br, 1H), 5.16-5.09 (m, 2H), 4.32-4.25 (m, 2H), $2.83(\mathrm{t}, J=$ $7.2 \mathrm{~Hz}, 2 \mathrm{H}), 2.54$ (t, $J=7.2 \mathrm{~Hz}, 2 \mathrm{H}), 1.26(\mathrm{t}, J=7.2 \mathrm{~Hz}, 3 \mathrm{H})$; ${ }^{13} \mathrm{C}\left\{{ }^{1} \mathrm{H}\right\}$ NMR $\left(125 \mathrm{MHz}, \mathrm{CDCl}_{3}\right) \delta 162.98,153.53,139.91$, $135.61,128.53,128.41,128.37,128.25,126.44,122.23$ (q, $\left.J_{C-F}=285 \mathrm{~Hz}\right), 88.21,70.65,67.57,63.75,60.00\left(\mathrm{q}, J_{C-F}=32 \mathrm{~Hz}\right), 34.09,20.88,13.68 ;{ }^{19} \mathrm{~F}$ NMR $\left(360 \mathrm{MHz}, \mathrm{CDCl}_{3}\right) \delta-75.57$. HPLC conditions: DAICEL CHIRALCEL AD-3, eluent: Hexane/2-Propanol 90.0/10.0, flow: $1.0 \mathrm{~mL} / \mathrm{min}$, detection: $220 \mathrm{~nm}, t_{\mathrm{R}}: 9.32 \mathrm{~min}$ (major), $13.03 \mathrm{~min}$ (minor). $[\alpha]_{\mathrm{D}}^{27}=-7.9\left(\mathrm{c} 1.8, \mathrm{CHCl}_{3}\right)$.

\section{Improvement of the Catalytic Activity (2): Substrate Scope}

\section{Alkynylation of $\alpha$-Ketiminoesters 2 with Aryl-Substituted Alkynes 3 (Table 1)}

Ethyl (S)-2-(((benzyloxy)carbonyl)amino)-4-phenyl-2-(trifluoromethyl)but-3-ynoate (4a)<smiles>CCOC(=O)[C@](C#Cc1ccccc1)(NC(=O)OCc1ccccc1)C(F)(F)F</smiles>

A Schlenk tube equipped with a magnetic stir bar was dried under vacuum using heat gun and refilled with argon. To the tube were added a $1.25 \mathrm{mM}$ solution of (alkynyl)Rh(III) complex 5d in toluene $(0.40 \mathrm{~mL}, 0.50 \mu \mathrm{mol}, 0.50 \mathrm{~mol} \%)$ and aryl-substituted alkyne 3a (16 $\mu \mathrm{L}, 0.15 \mathrm{mmol}, 1.5$ equiv). After the mixture was stirred for $10 \mathrm{~min}$ at room temperature, $\alpha$-ketiminoester $2(24 \mu \mathrm{L}, 0.10 \mathrm{mmol})$ was added and the mixture was stirred at room temperature for $12 \mathrm{~h}$. The crude mixture was directly purified by flash silica gel column chromatography using hexane/AcOEt $=10 / 1$ as eluent to 
give product 4a as a colorless oil (38.2 $\mathrm{mg}, 94 \%$ yield, $92 \%$ ee).

${ }^{1} \mathrm{H}$ NMR (500 MHz, $\left.\mathrm{CDCl}_{3}\right) \delta 7.47(\mathrm{~d}, J=7.0 \mathrm{~Hz}, 2 \mathrm{H}) 7.40-7.31(\mathrm{~m}, 8 \mathrm{H}), 5.80$ (br, 1H), 5.19-5.13 (m 2H), 4.39-4.34 (m, 2H), $1.32(\mathrm{t}, J=7.0 \mathrm{~Hz}, 3 \mathrm{H}),{ }^{13} \mathrm{C}\left\{{ }^{1} \mathrm{H}\right\} \mathrm{NMR}(125$ $\left.\mathrm{MHz}_{2} \mathrm{CDCl}_{3}\right) \delta 162.91,153.60,135.57,132.17,129.55,128.56,128.40,128.33,128.30$, $122.24\left(\mathrm{q}, J_{C-F}=285 \mathrm{~Hz}\right), 120.76,87.51,78.20,67.71,64.08,60.61\left(\mathrm{q}, J_{C-F}=33 \mathrm{~Hz}\right)$, 13.75; ${ }^{19} \mathrm{~F}$ NMR $\left(360 \mathrm{MHz}, \mathrm{CDCl}_{3}\right) \delta-75.76$. HPLC conditions: DAICEL CHIRALCEL OD-3, eluent: Hexane/2-Propanol 90.0/10.0, flow: $1.0 \mathrm{~mL} / \mathrm{min}$, detection: $254 \mathrm{~nm}, t_{\mathrm{R}}: 7.77$ $\min$ (minor), 10.68 min (major). $[\alpha]^{25}=-14.3$ (c 1.21, $\mathrm{CHCl}_{3}$ ).

For alkynes $\mathbf{3 b}$ to $\mathbf{3 l}$, the above procedure for 3a was followed except for the use of alkyne $\mathbf{3 b}$ to $\mathbf{3 l}$ instead of alkyne $\mathbf{3} \mathbf{a}$ unless otherwise noted.

\section{Ethyl}

(S)-2-(((benzyloxy)carbonyl)amino)-4-(4-methylphenyl)-2-(trifluoromethyl)but-3-ynoa te (4e)

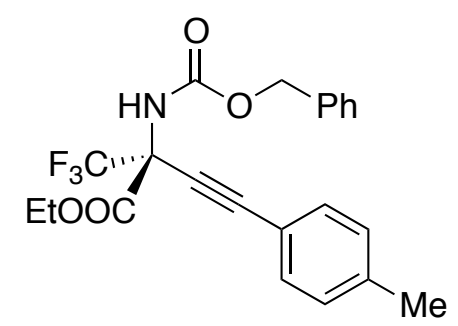

Reaction was performed according to the general procedure, and the crude mixture was purified using hexane/AcOEt $=10 / 1$ as eluent to give $4 \mathbf{e}$ as a colorless oil $(35.3 \mathrm{mg}, 84 \%$ yield, $93 \%$ ee).

${ }^{1} \mathrm{H}$ NMR $\left(500 \mathrm{MHz}, \mathrm{CDCl}_{3}\right) \delta$ 7.38-7.32 (m, 7H), $7.12(\mathrm{~d}$, $J=8.0 \mathrm{~Hz}, 2 \mathrm{H}), 5.79(\mathrm{br}, 1 \mathrm{H}), 5.19-5.12(\mathrm{~m}, 2 \mathrm{H}), 4.38-4.33(\mathrm{~m}, 2 \mathrm{H}), 2.35(\mathrm{~s}, 3 \mathrm{H}), 1.31(\mathrm{t}$, $J=7.0 \mathrm{~Hz}, 3 \mathrm{H}) ;{ }^{13} \mathrm{C}\left\{{ }^{1} \mathrm{H}\right\} \mathrm{NMR}\left(125 \mathrm{MHz}, \mathrm{CDCl}_{3}\right) \delta 162.95,153.61,139.90,135.58$, $132.05,129.07,128.55,128.28,128.28,122.27$ (q, $\left.J_{C-F}=285 \mathrm{~Hz}\right), 117.63,87.74,77.56$, 67.68, 63.97, 60.59 (q, $\left.J_{C-F}=33 \mathrm{~Hz}\right), 21.54,13.74 ;{ }^{19} \mathrm{~F}$ NMR $\left(360 \mathrm{MHz}, \mathrm{CDCl}_{3}\right) \delta-76.01$. HPLC conditions: DAICEL CHIRALCEL OD-3, eluent: Hexane/2-Propanol 90.0/10.0, flow: $1.0 \mathrm{~mL} / \mathrm{min}$, detection: $254 \mathrm{~nm}, t_{\mathrm{R}}: 6.92 \mathrm{~min}$ (minor), $8.82 \min$ (minor). $[\alpha]_{\mathrm{D}}^{26}=$ $-17.0\left(\mathrm{c} 0.81, \mathrm{CHCl}_{3}\right)$.

\section{Ethyl}

(S)-2-(((benzyloxy)carbonyl)amino)-4-(4-methoxyphenyl)-2-(trifluoromethyl)but-3-yn 
oate (4f)

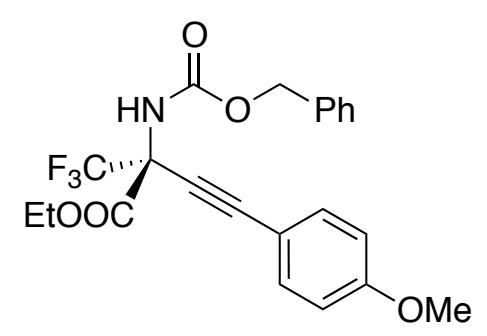

Reaction was performed according to the general procedure, and the crude mixture was purified using hexane $/ \mathrm{CH}_{2} \mathrm{Cl}_{2}=10 / 1$ as eluent to give $\mathbf{4 f}$ as a colorless oil (39.3 mg, 90\% yield, $92 \%$ ee).

${ }^{1} \mathrm{H}$ NMR (500 MHz, $\left.\mathrm{CDCl}_{3}\right) \delta 7.41-7.31(\mathrm{~m}, 7 \mathrm{H}), 6.83(\mathrm{~d}$, $J=8.5 \mathrm{~Hz}, 2 \mathrm{H}), 5.80(\mathrm{br}, 1 \mathrm{H}), 5.19-5.12(\mathrm{~m}, 2 \mathrm{H}), 4.38-4.33(\mathrm{~m}, 2 \mathrm{H}), 3.81(\mathrm{~s}, 3 \mathrm{H}), 1.31(\mathrm{t}$, $J=7.5 \mathrm{~Hz}, 3 \mathrm{H}) ;{ }^{13} \mathrm{C}\left\{{ }^{1} \mathrm{H}\right\} \mathrm{NMR}\left(125 \mathrm{MHz}, \mathrm{CDCl}_{3}\right) \delta 163.00,160.55,153.63,135.60$, $133.74,128.54,128.37,128.28,122.30\left(\mathrm{q}, J_{C-F}=286 \mathrm{~Hz}\right), 113.97,112.69,87.65,67.66$, 63.94, 60.61 (q, $\left.J_{C-F}=32 \mathrm{~Hz}\right), 55.31,13.75 ;{ }^{19} \mathrm{~F} \mathrm{NMR}\left(360 \mathrm{MHz}, \mathrm{CDCl}_{3}\right) \delta-76.01$. HPLC conditions: DAICEL CHIRALCEL OD-3, eluent: Hexane/2-Propanol 90.0/10.0, flow: 1.0 $\mathrm{mL} / \mathrm{min}$, detection: $254 \mathrm{~nm}, t_{\mathrm{R}}: 10.06 \mathrm{~min}$ (minor), $13.47 \mathrm{~min}$ (major). $[\alpha]_{\mathrm{D}}^{25}=-7.7$ (c 1.43 , $\left.\mathrm{CHCl}_{3}\right)$.

\section{Ethyl}

(S)-2-(((benzyloxy)carbonyl)amino)-4-(4-bromophenyl)-2-(trifluoromethyl)but-3-ynoa te (4g)

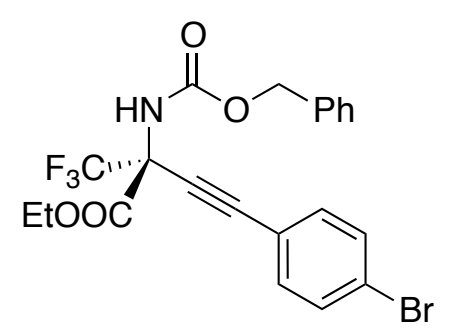

Reaction was performed according to the general procedure, and the crude mixture was purified using hexane/EtOAc $=10 / 1$ as eluent to give $\mathbf{4 g}$ as a colorless oil $(47.2 \mathrm{mg}, 97 \%$ yield, $94 \%$ ee).

${ }^{1} \mathrm{H}$ NMR (500 MHz, $\left.\mathrm{CDCl}_{3}\right) \delta 7.46(\mathrm{~d}, J=9.0 \mathrm{~Hz}, 2 \mathrm{H})$ 7.46-7.31 (m, 7H), 5.81 (br, 1H), $5.16(\mathrm{~m}, 2 \mathrm{H}), 4.40-4.34(\mathrm{~m}, 2 \mathrm{H}), 1.32$ (t, $J=7.0 \mathrm{~Hz}$, $3 \mathrm{H}) ;{ }^{13} \mathrm{C}\left\{{ }^{1} \mathrm{H}\right\} \mathrm{NMR}\left(125 \mathrm{MHz}, \mathrm{CDCl}_{3}\right) \delta 162.78,153.57,135.52,133.55,131.66,128.57$, 128.43, 128.31, 124.05, 122.11 (q, $\left.J_{C-F}=286 \mathrm{~Hz}\right), 119.73,86.45,79.31,67.76,64.25$, $60.67\left(\mathrm{q}, J_{C-F}=33 \mathrm{~Hz}\right), 13.75 ;{ }^{19} \mathrm{~F}$ NMR $\left(360 \mathrm{MHz}, \mathrm{CDCl}_{3}\right) \delta-75.72$. HPLC conditions: DAICEL CHIRALCEL OD-3, eluent: Hexane/2-Propanol 90.0/10.0, flow: $1.0 \mathrm{~mL} / \mathrm{min}$, detection: $254 \mathrm{~nm}, t_{\mathrm{R}}$ : $9.91 \mathrm{~min}$ (minor), $10.75 \min$ (major). $[\alpha]_{\mathrm{D}}^{25}=-7.3$ (c $0.63, \mathrm{CHCl}_{3}$ ).

\section{Ethyl}


(S)-2-(((benzyloxy)carbonyl)amino)-4-(4-trifluorophenyl)-2-(trifluoromethyl)but-3-yn oate (4b)

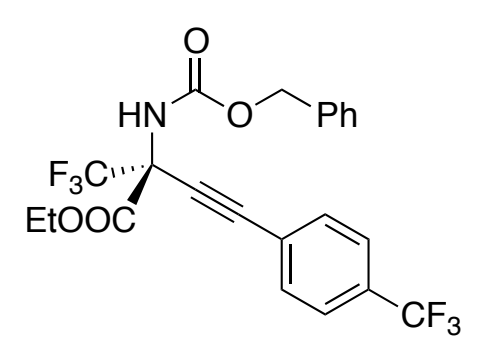

\author{
Reaction was performed according to the general \\ procedure, and the crude mixture was purified using \\ hexane $/$ EtOAc $=10 / 1$ as eluent to give $\mathbf{4 b}$ as a colorless oil \\ (44.8 $\mathrm{mg}, 95 \%$ yield, $93 \%$ ee). \\ ${ }^{1} \mathrm{H}$ NMR $\left(500 \mathrm{MHz}, \mathrm{CDCl}_{3}\right) \delta 7.60-7.55(\mathrm{~m}, 4 \mathrm{H})$,
} 7.38-7.33 (m, 5H), 5.85 (br, 1H), 5.20-5.14 (m, 2H), 4.41-4.35 (m, 2H), 1.33 (t, $J=7.5 \mathrm{~Hz}$, $3 \mathrm{H}) ;{ }^{13} \mathrm{C}\left\{{ }^{1} \mathrm{H}\right\}$ NMR $\left(125 \mathrm{MHz}, \mathrm{CDCl}_{3}\right) \delta 162.72,153.59,135.47,132.47,131.26$ (q, $J_{C-F}=$ $33 \mathrm{~Hz}), 128.59,128.47,128.33,125.26\left(\mathrm{q}, J_{C-F}=3.9 \mathrm{~Hz}\right), 124.62,123.67\left(\mathrm{q}, J_{C-F}=271\right.$ $\mathrm{Hz}), 122.08\left(\mathrm{q}, J_{C-F}=286 \mathrm{~Hz}\right), 85.99,80.50,67.81,64.40,60.69\left(\mathrm{q}, J_{C-F}=33 \mathrm{~Hz}\right), 13.75$; ${ }^{19} \mathrm{~F}$ NMR $\left(360 \mathrm{MHz}, \mathrm{CDCl}_{3}\right) \delta-64.14,-75.68$. HPLC conditions: DAICEL CHIRALCEL AD-3, eluent: Hexane/2-Propanol 95.0/5.0, flow: $1.0 \mathrm{~mL} / \mathrm{min}$, detection: $254 \mathrm{~nm}, t_{\mathrm{R}}: 20.05$ $\min$ (major), $32.76 \mathrm{~min}$ (minor). $[\alpha]_{\mathrm{D}}^{26}=-5.1$ (c $0.86, \mathrm{CHCl}_{3}$ ).

\title{
Ethyl
}

(S)-2-(((benzyloxy)carbonyl)amino)-4-(2-methylphenyl)-2-(trifluoromethyl)but-3-ynoa te $(4 h)$

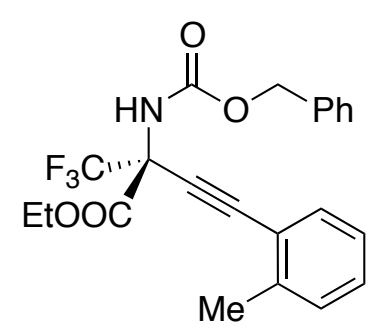

Reaction was performed according to the general procedure, and the crude mixture was purified using hexane/AcOEt $=10 / 1$ as eluent to give $4 \mathrm{~h}$ as a white solid ( $37.2 \mathrm{mg}, 89 \%$ yield, $88 \%$ ee).

${ }^{1} \mathrm{H}$ NMR $\left(500 \mathrm{MHz}, \mathrm{CDCl}_{3}\right) \delta 7.41(\mathrm{~d}, J=8.0 \mathrm{~Hz}, 1 \mathrm{H})$, $7.38-7.32(\mathrm{~m}, 5 \mathrm{H}), 7.19$ (d, $J=7.3 \mathrm{~Hz}, 1 \mathrm{H}), 7.13$ (t, $J=8.0 \mathrm{~Hz}, 1 \mathrm{H})$, $5.82(\mathrm{br}, 1 \mathrm{H}), 5.19-5.13(\mathrm{~m}, 2 \mathrm{H}), 4.38-4.33(\mathrm{~m}, 2 \mathrm{H}), 2.42(\mathrm{~s}, 3 \mathrm{H}), 1.32(\mathrm{t}, J=7.0 \mathrm{~Hz}, 3 \mathrm{H})$; ${ }^{13} \mathrm{C}\left\{{ }^{1} \mathrm{H}\right\}$ NMR $\left(125 \mathrm{MHz}, \mathrm{CDCl}_{3}\right) \delta 163.04,153.60,141.37,135.57,132.35,129.53$, $129.52,128.55,128.39,128.30,125.52,132.27$ (q, $\left.J_{C-F}=286 \mathrm{~Hz}\right), 120.59,86.67,81.94$, 67.69, 64.06, 60.72 (q, $\left.J_{C-F}=32 \mathrm{~Hz}\right), 20.33,13.77 ;{ }^{19} \mathrm{~F}$ NMR $\left(360 \mathrm{MHz}, \mathrm{CDCl}_{3}\right) \delta-75.82$. HPLC conditions: DAICEL CHIRALCEL OD-3, eluent: Hexane/2-Propanol 90.0/10.0, flow: $1.0 \mathrm{~mL} / \mathrm{min}$, detection: $254 \mathrm{~nm}, t_{\mathrm{R}}$ : $6.61 \mathrm{~min}$ (minor), $10.32 \mathrm{~min}$ (major). $[\alpha]^{26}{ }_{\mathrm{D}}=$ $-12.2\left(\mathrm{c} 1.43, \mathrm{CHCl}_{3}\right)$. 


\section{Ethyl}

(S)-2-(((benzyloxy)carbonyl)amino)-4-(thiophen-3-yl)-2-(trifluoromethyl)but-3-ynoate (4i)

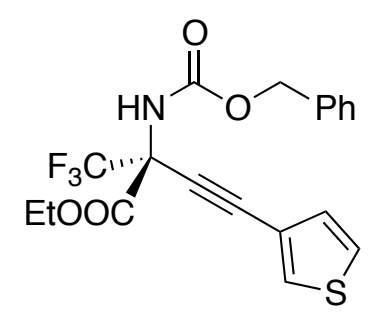

Reaction was performed according to the general procedure, and the crude mixture was purified using hexane/AcOEt $=10 / 1$ as eluent to give $4 \mathbf{i}$ as a colorless oil (37.2 $\mathrm{mg}, 90 \%$ yield, 93\% ee).

${ }^{1} \mathrm{H}$ NMR $\left(500 \mathrm{MHz}, \mathrm{CDCl}_{3}\right) \delta 7.55(\mathrm{~d}, J=2.5 \mathrm{~Hz}, 1 \mathrm{H})$, $7.37-7.32(\mathrm{~m}, 5 \mathrm{H}), 7.27(\mathrm{dd}, J=5.0,3.0 \mathrm{~Hz}, 1 \mathrm{H}), 7.13(\mathrm{dd}, J=5.0$, $1.0 \mathrm{~Hz}, 1 \mathrm{H}), 5.79$ (br, 1H), 5.19-5.12 (m, 2H), 4.39-4.33 (m, 2H), $1.31(\mathrm{t}, J=7.2 \mathrm{~Hz}, 3 \mathrm{H})$; ${ }^{13} \mathrm{C}\left\{{ }^{1} \mathrm{H}\right\}$ NMR $\left(125 \mathrm{MHz}, \mathrm{CDCl}_{3}\right) \delta 162.87,153.59,135.56,131.17,129.88,128.56$, 128.40, 128.29, 125.59, 122.20 (q, $\left.J_{C-F}=286 \mathrm{~Hz}\right), 119.81,82.86,77.93,67.71$, 64.10, $60.65\left(\mathrm{q}, J_{C-F}=33 \mathrm{~Hz}\right), 13.75 ;{ }^{19} \mathrm{~F}$ NMR $\left(360 \mathrm{MHz}, \mathrm{CDCl}_{3}\right) \delta-75.69$. HPLC conditions: DAICEL CHIRALCEL OD-3, eluent: Hexane/2-Propanol 90.0/10.0, flow: $1.0 \mathrm{~mL} / \mathrm{min}$, detection: $254 \mathrm{~nm}, t_{\mathrm{R}}$ : $9.38 \mathrm{~min}$ (minor), $12.04 \mathrm{~min}$ (major). $[\alpha]_{\mathrm{D}}^{27}=-14.8$ (c 1.07, $\mathrm{CHCl}_{3}$ ).

\section{tert-Butyl}

(S)-5-(3-(((benzyloxy)carbonyl)amino)-3-(ethoxycarbonyl)-4,4,4-trifluorobut-1-yn-1-yl )-1H-indole-1-carboxylate $(4 \mathrm{j})$

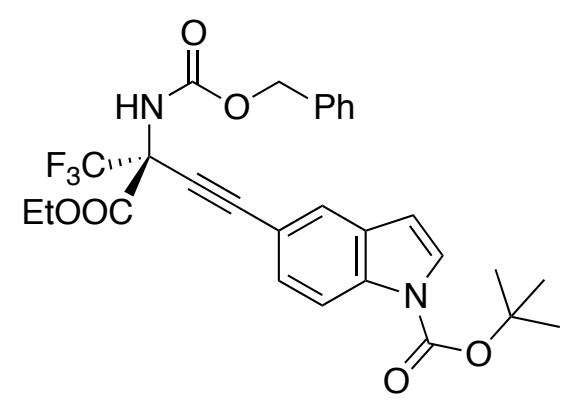

$$
\begin{aligned}
& \text { Reaction was performed according to the general } \\
& \text { procedure and the crude mixture was purified using } \\
& \text { hexane/AcOEt }=10 / 1 \text { as eluent to give } 4 \mathbf{j} \text { as a colorless } \\
& \text { oil ( } 49.9 \mathrm{mg}, 92 \% \text { yield, } 95 \% \text { ee). } \\
& { }^{1} \mathrm{H} \mathrm{NMR}\left(500 \mathrm{MHz}, \mathrm{CDCl}_{3}\right) \delta 8.10(\mathrm{~d}, J=8.5 \mathrm{~Hz} \text {, } \\
& 1 \mathrm{H}), 7.69(\mathrm{~s}, 1 \mathrm{H}), 7.61(\mathrm{~d}, J=4.0 \mathrm{~Hz}, 1 \mathrm{H}), 7.40-7.33
\end{aligned}
$$
$(\mathrm{m}, 6 \mathrm{H}), 6.53(\mathrm{~d}, J=4.0 \mathrm{~Hz}, 1 \mathrm{H}), 5.83(\mathrm{br}, 1 \mathrm{H}), 5.20-5.13(\mathrm{~m}, 2 \mathrm{H}), 4.38-4.35(\mathrm{~m}, 2 \mathrm{H})$, $1.59(\mathrm{~s}, 9 \mathrm{H}), 1.33(\mathrm{t}, J=7.5 \mathrm{~Hz}, 3 \mathrm{H}),{ }^{13} \mathrm{C}\left\{{ }^{1} \mathrm{H}\right\} \mathrm{NMR}\left(125 \mathrm{MHz}, \mathrm{CDCl}_{3}\right) \delta 163.02,153.64$, $149.35,135.62,135.48,130,33,128.55,128.37,128.29,128.00,127.05,125.32,122.33$ (q, $\left.J_{C-F}=286 \mathrm{~Hz}\right), 115.17,114.72,106.97,88.34,84.23,76.88,67.68,63.98,60.64\left(\mathrm{q}, J_{C-F}=\right.$ $32 \mathrm{~Hz}), 28.13,13.78 ;{ }^{19} \mathrm{~F}$ NMR $\left(360 \mathrm{MHz}, \mathrm{CDCl}_{3}\right) \delta-75.74$. HPLC conditions: DAICEL 
CHIRALCEL OD-3, eluent: Hexane/2-Propanol 90.0/10.0, flow: $1.0 \mathrm{~mL} / \mathrm{min}$, detection: $254 \mathrm{~nm}, t_{\mathrm{R}}: 9.78 \mathrm{~min}$ (minor), $12.69 \min$ (major). $[\alpha]_{\mathrm{D}}^{27}=-14.6$ (c $0.94, \mathrm{CHCl}_{3}$ ).

\section{Ethyl}

(S)-2-(((benzyloxy)carbonyl)amino)-4-(4-formylphenyl)-2-(trifluoromethyl)but-3-ynoa te $(4 k)$

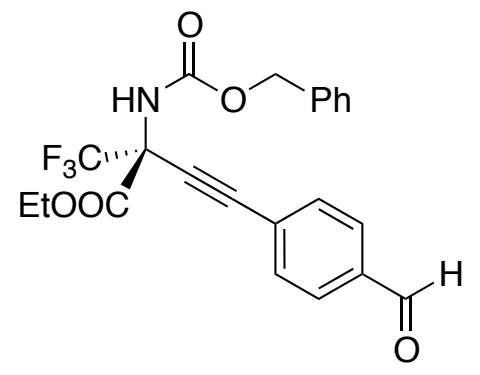

Reaction was performed according to the general procedure using $1.0 \mathrm{~mol} \%$ of $\mathbf{5 d}$ as catalyst and the crude mixture was purified using hexane/AcOEt $=20 / 1$ to $4 / 1$ as eluent to give $4 \mathbf{k}$ as a colorless oil (37.4 $\mathrm{mg}, 86 \%$ yield, $95 \%$ ee).

${ }^{1} \mathrm{H}$ NMR $\left(500 \mathrm{MHz}, \mathrm{CDCl}_{3}\right) \delta 10.02(\mathrm{~s}, 1 \mathrm{H}), 7.84(\mathrm{~d}, J=$ $8.0 \mathrm{~Hz}, 2 \mathrm{H}), 7.61$ (d, $J=8.0 \mathrm{~Hz}, 2 \mathrm{H}), 7.38-7.33(\mathrm{~m}, 5 \mathrm{H}), 5.91$ (br, 1H), 5.20-5.14 (m, 2H), 4.21-4.36 (m, 2H), 1.33 (t, $J=7.0 \mathrm{~Hz}, 3 \mathrm{H}) ;{ }^{13} \mathrm{C}\left\{{ }^{1} \mathrm{H}\right\}$ NMR (125 MHz, $\left.\mathrm{CDCl}_{3}\right) \delta$ 191.29, $162.667,153.60,136.36,135.47,132.74,129.39,128.57,128.45,128.30,126.87,122.04$ $\left(\mathrm{q}, J_{C-F}=286 \mathrm{~Hz}\right), 86.31,81.78,67.79,64.41,60.76\left(\mathrm{q}, J_{C-F}=33 \mathrm{~Hz}\right), 13.74 ;{ }^{19} \mathrm{~F}$ NMR $\left(360 \mathrm{MHz}, \mathrm{CDCl}_{3}\right) \delta-75.33$. HPLC conditions: DAICEL CHIRALCEL OD-3, eluent: Hexane/2-Propanol 90.0/10.0, flow: $1.0 \mathrm{~mL} / \mathrm{min}$, detection: $254 \mathrm{~nm}, t_{\mathrm{R}}: 19.95 \mathrm{~min}$ (minor), $24.20 \min$ (major). $[\alpha]^{26}=-6.1$ (c $\left.0.64, \mathrm{CHCl}_{3}\right)$.

\section{Ethyl}

(S)-4-(4-((((9H-fluoren-9-yl)methoxy)carbonyl)amino)phenyl)-2-(((benzyloxy)carbonyl )amino)-2-(trifluoromethyl)but-3-ynoate (4I)

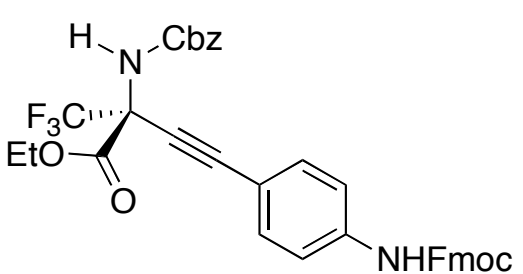

NHFmoc

${ }^{1} \mathrm{H}$ NMR (500 MHz, $\left.\mathrm{CDCl}_{3}\right) \delta 7.76(\mathrm{~d}, J=8.0 \mathrm{~Hz}, 2 \mathrm{H}), 8.58(\mathrm{~d}, J=8.0 \mathrm{~Hz}, 2 \mathrm{H})$, 7.41-7.29 (m, 13H), 7.24 (br, 1H), 5.87 (br, 1H), 5.18-5.11 (m, 2H), 4.53 (d, J = 6.5 Hz,

Reaction was performed according to the general procedure using $1.0 \mathrm{~mol} \%$ of $\mathbf{5 d}$ as catalyst and dichloromethane as solvent and the crude mixture was purified using hexane/AcOEt $=5 / 1$ as eluent to give $4 \mathbf{l}$ as a colorless oil ( $59.2 \mathrm{mg}, 92 \%$ yield, $92 \%$ ee). 
2H), 4.37-4.25 (m, 2H), $4.23(\mathrm{t}, J=6.5 \mathrm{~Hz}, 1 \mathrm{H}), 1.30(\mathrm{t}, J=7.0 \mathrm{~Hz}, 3 \mathrm{H}) ;{ }^{13} \mathrm{C}\left\{{ }^{1} \mathrm{H}\right\} \mathrm{NMR}$ $\left(125 \mathrm{MHz}, \mathrm{CDCl}_{3}\right) \delta 162.95,153.66,152.99,143.55,141.33,138.95,135.52,133.09$, 128.53, 128.37, 128.25, 127.81, 127.12, 124.83, 122.25 (q, $\left.J_{C-F}=286 \mathrm{~Hz}\right), 120.04,118.08$, $115.30,87.33,77.62,67.69,66.97,64.04,60.63\left(\mathrm{q}, J_{C-F}=32 \mathrm{~Hz}\right), 47.00,13.73 ;{ }^{19} \mathrm{~F}$ NMR $\left(360 \mathrm{MHz}, \mathrm{CDCl}_{3}\right) \delta-75.91$. HPLC conditions: DAICEL CHIRALCEL AS-3, eluent: Hexane/2-Propanol 80.0/20.0, flow: $1.0 \mathrm{~mL} / \mathrm{min}$, detection: $254 \mathrm{~nm}, t_{\mathrm{R}}: 25.04 \mathrm{~min}$ (minor), $39.26 \min$ (major). $[\alpha]^{26}=-9.5$ (c $2.28, \mathrm{CHCl}_{3}$ ).

\section{Alkynylation of $\alpha$-Ketiminoesters 2 with Alkyl-Substituted Alkynes 3 (Table 2)}

For alkynes $\mathbf{3 m}$ to $\mathbf{3 s}$, the procedure for Equation 15 was followed except for the use of alkynes $\mathbf{3 m}$ to $3 \mathrm{~s}$ unless otherwise noted.

\section{Ethyl}

(S)-2-(((benzyloxy)carbonyl)amino)-4-cyclopropyl-2-(trifluoromethyl)but-3-ynoate (4m)

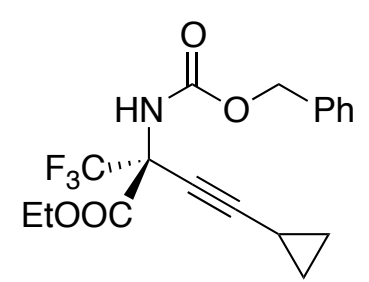

Reaction was performed according to the general procedure, and the crude mixture was purified using hexane/AcOEt $=10 / 1$ as eluent to give $\mathbf{4 m}$ as a colorless oil ( $40.0 \mathrm{mg}, 93 \%$ yield, $91 \%$ ee).

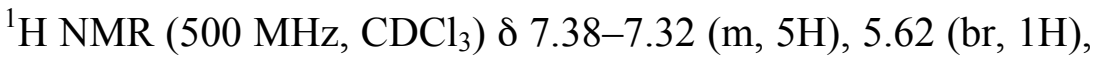
5.16-5.09 (m, 2H), 4.33-4.28 (m, 2H), 1.32-1.27 (m, 4H), 0.84-0. $0.73(\mathrm{~m}, 4 \mathrm{H}) ;{ }^{13} \mathrm{C}\left\{{ }^{1} \mathrm{H}\right\}$ NMR $\left(125 \mathrm{MHz}, \mathrm{CDCl}_{3}\right) \delta 163.09,153.54,135.63,128.54,128.36$, $128.26,122.25$ (q, $\left.J_{C-F}=285 \mathrm{~Hz}\right), 92.12,76.68,67.56,64.55,63.72,60.03\left(\mathrm{q}, J_{C-F}=32\right.$ $\mathrm{Hz}), 13.70,8.42,-0.62 ;{ }^{19} \mathrm{~F}$ NMR $\left(360 \mathrm{MHz}, \mathrm{CDCl}_{3}\right) \delta-75.99$. HPLC conditions: DAICEL CHIRALCEL AD-3, eluent: Hexane/2-Propanol 90.0/10.0, flow: $1.0 \mathrm{~mL} / \mathrm{min}$, detection: $220 \mathrm{~nm}, t_{\mathrm{R}}: 8.79 \mathrm{~min}$ (major), $10.72 \mathrm{~min}$ (minor). $[\alpha]^{27}{ }_{\mathrm{D}}=-10.9$ (c $1.91, \mathrm{CHCl}_{3}$ ).

\section{Ethyl}

(S)-2-(((benzyloxy)carbonyl)amino)-4-(cyclohex-1-en-1-yl)-2-(trifluoromethyl)but-3-yn oate (4n)

Reaction was performed according to the general procedure, and the crude mixture was 
purified using hexane/AcOEt $=10 / 1$ as eluent to give $\mathbf{4 n}$ as a colorless oil $(35.3 \mathrm{mg}, 86 \%$ yield, $93 \%$ ee).

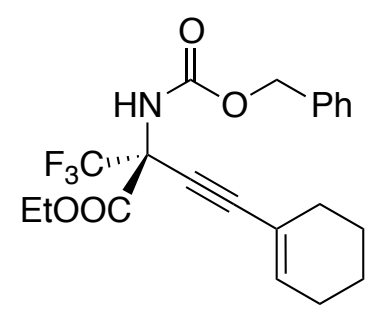

${ }^{1} \mathrm{H}$ NMR $\left(500 \mathrm{MHz}, \mathrm{CDCl}_{3}\right) \delta 7.37-7.31(\mathrm{~m}, 5 \mathrm{H}), 6.23(\mathrm{t}, J=$ $1.5 \mathrm{~Hz}, 1 \mathrm{H}), 5.68(\mathrm{br}, 1 \mathrm{H}), 5.17-5.10(\mathrm{~m}, 2 \mathrm{H}), 4.34-4.29(\mathrm{~m}, 2 \mathrm{H})$, 2.11-2.08 (m, 4H), 1.63-1.56 (m, 4H), 1.29 (t, $J=7.0 \mathrm{~Hz}, 3 \mathrm{H})$; ${ }^{13} \mathrm{C}\left\{{ }^{1} \mathrm{H}\right\}$ NMR $\left(125 \mathrm{MHz}, \mathrm{CDCl}_{3}\right) \delta 163.03,153.53,138.49,135.62$, $128.51,128.33,128.25,122.27\left(\mathrm{q}, J_{C-F}=285 \mathrm{~Hz}\right), 118.85,89.32$, $75.54,67.58,63.77,60.44$ (q, $\left.J_{C-F}=32 \mathrm{~Hz}\right), 28.29,25.60,21.94,21.19,13.70 ;{ }^{19} \mathrm{~F}$ NMR $\left(360 \mathrm{MHz}, \mathrm{CDCl}_{3}\right) \delta-75.60$. HPLC conditions: DAICEL CHIRALCEL AD-3, eluent: Hexane/2-Propanol 95.0/5.0, flow: $1.0 \mathrm{~mL} / \mathrm{min}$, detection: $220 \mathrm{~nm}, t_{\mathrm{R}}: 9.93 \mathrm{~min}$ (major), $12 / 15 \min$ (minor). $[\alpha]^{27}=-8.9\left(\mathrm{c} 0.84, \mathrm{CHCl}_{3}\right)$.

\section{Ethyl}

(S)-2-(((benzyloxy)carbonyl)amino)-5,5-diethoxy-2-(trifluoromethyl)pent-3-ynoate (40)<smiles>CCOC(=O)[C@](C#CC(OCC)OCC)(NC(=O)OCc1ccccc1)OCC</smiles>

Reaction was performed according to the general procedure, and the mixture was purified using hexane/AcOEt $=10 / 1$ as eluent to give $4 \mathrm{o}$ as a colorless oil ( $33.2 \mathrm{mg}, 90 \%$ yield, $88 \%$ ee).

${ }^{1} \mathrm{H}$ NMR (500 MHz, $\left.\mathrm{CDCl}_{3}\right) \delta 7.38-7.31(\mathrm{~m}, 5 \mathrm{H}), 5.76(\mathrm{br}, 1 \mathrm{H})$, $5.30(\mathrm{~s}, 1 \mathrm{H}), 5.16-5.10(\mathrm{~m}, 2 \mathrm{H}), 4.36-4.29(\mathrm{~m}, 2 \mathrm{H}), 3.75-3.69(\mathrm{~m}$, 2H), 3.63-3.56 (m, 2H), $1.29(\mathrm{t}, J=7.0 \mathrm{~Hz}, 3 \mathrm{H}) 1.22(\mathrm{t}, J=7.0 \mathrm{~Hz}, 6 \mathrm{H}) ;{ }^{13} \mathrm{C}\left\{{ }^{1} \mathrm{H}\right\} \mathrm{NMR}$ $\left(125 \mathrm{MHz}, \mathrm{CDCl}_{3}\right) \delta 162.42,153.47,135.48,128.55,128.41,128.27,121.97$ (q, $J_{C-F}=286$ $\mathrm{Hz}), 90.91,83.40,74.39,67.73,64.21,61.22,60.00\left(\mathrm{q}, J_{C-F}=33 \mathrm{~Hz}\right), 14.98,13.67 ;{ }^{19} \mathrm{~F}$ NMR $\left(360 \mathrm{MHz}, \mathrm{CDCl}_{3}\right) \delta-75.60$. HPLC conditions: DAICEL CHIRALCEL AD-3, eluent: Hexane/2-Propanol 90.0/10.0, flow: $1.0 \mathrm{~mL} / \mathrm{min}$, detection: $220 \mathrm{~nm}, t_{\mathrm{R}}: 11.10 \mathrm{~min}$ (major), $16.77 \mathrm{~min}$ (minor). $[\alpha]_{\mathrm{D}}^{27}=-5.4\left(\mathrm{c} 1.96, \mathrm{CHCl}_{3}\right)$.

\section{Ethyl}

(S)-2-(((benzyloxy)carbonyl)amino)-5-((tert-butyldimethylsilyl)oxy)-2-(trifluoromethyl )pent-3-ynoate (4p) 
Reaction was performed according to the general procedure, and the crude mixture was purified using hexane/AcOEt $=10 / 1$ as eluent to give $\mathbf{4 p}$ as a colorless oil $(43.5 \mathrm{mg}, 92 \%$ yield, $82 \%$ ee).

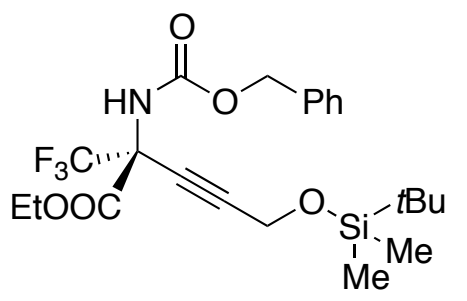

${ }^{1} \mathrm{H}$ NMR (400 MHz, $\left.\mathrm{CDCl}_{3}\right) \delta 7.36-7.34(\mathrm{~m}, 5 \mathrm{H}), 5.70$ (br, $1 \mathrm{H}), 5.17-5.10(\mathrm{~m}, 2 \mathrm{H}), 4.37(\mathrm{~s}, 2 \mathrm{H}), 4.33-4.30(\mathrm{~m}, 2 \mathrm{H}), 1.29$ $(\mathrm{t}, J=7.0 \mathrm{~Hz}, 3 \mathrm{H}), 0.89(\mathrm{~s}, 9 \mathrm{H}), 0.11(\mathrm{~s}, 6 \mathrm{H}) ;{ }^{13} \mathrm{C}\left\{{ }^{1} \mathrm{H}\right\} \mathrm{NMR}$ $\left(100 \mathrm{MHz}, \mathrm{CDCl}_{3}\right) \delta 162.65,153.53,135.53,128.55,128.40$, $128.27,122.09\left(\mathrm{q}, J_{C-F}=285 \mathrm{~Hz}\right), 86.55,73.98,64.00,60.10$ $\left(\mathrm{q}, J_{C-F}=32 \mathrm{~Hz}\right), 51.55,25.63,18.13,13.68,-5.34 ;{ }^{19} \mathrm{~F} \mathrm{NMR}\left(360 \mathrm{MHz}, \mathrm{CDCl}_{3}\right) \delta-75.35$. HPLC conditions: DAICEL CHIRALCEL AD-3, eluent: Hexane/2-Propanol 95.0/5.0, flow: $1.0 \mathrm{~mL} / \mathrm{min}$, detection: $220 \mathrm{~nm}, t_{\mathrm{R}}: 6.41 \mathrm{~min}$ (major), $9.05 \mathrm{~min}$ (minor). $[\alpha]_{\mathrm{D}}^{27}=-4.2$ (c $1.58, \mathrm{CHCl}_{3}$ ).

\section{Ethyl (S)-2,5-bis(((benzyloxy)carbonyl)amino)-2-(trifluoromethyl)pent-3-ynoate (4q)}

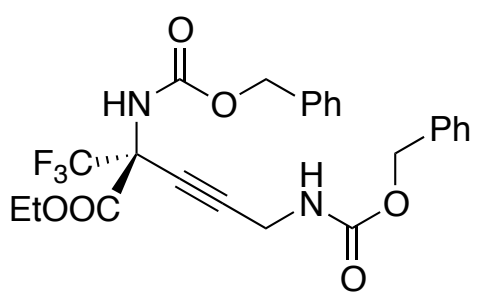

Reaction was performed according to the general procedure on a $0.40 \mathrm{mmol}$ scale, and the crude mixture was purified using hexane/AcOEt $=4 / 1$ to $2 / 1$ as eluent to give $\mathbf{4 q}$ as a colorless oil (182 $\mathrm{mg}, 92 \%$ yield, $87 \%$ ee).

${ }^{1} \mathrm{H}$ NMR (500 MHz, $\left.\mathrm{CDCl}_{3}\right) \delta 7.36-7.31(\mathrm{~m}, 10 \mathrm{H}), 5.94$ (br, $1 \mathrm{H}), 5.23-5.08(\mathrm{~m}, 5 \mathrm{H}), 4.32-4.28(\mathrm{~m}, 2 \mathrm{H}), 4.05-4.04(\mathrm{~m}, 2 \mathrm{H}), 1.26(\mathrm{t}, J=7.0 \mathrm{~Hz}$, $3 \mathrm{H}) ;{ }^{13} \mathrm{C}\left\{{ }^{1} \mathrm{H}\right\}$ NMR $\left(125 \mathrm{MHz}, \mathrm{CDCl}_{3}\right) \delta 162.63,155.73,153.62,136.05,135.44,128.51$, 128.48, 128.38, 128.22, 128.19, 128.08, 121.97 (q, $\left.J_{C-F}=286 \mathrm{~Hz}\right), 83.93,72.82,67.64$, $67.11,64.13,60.05$ (q, $\left.J_{C-F}=32.3 \mathrm{~Hz}\right), 30.93,13.60 ;{ }^{19} \mathrm{~F} \mathrm{NMR}\left(360 \mathrm{MHz}, \mathrm{CDCl}_{3}\right) \delta$ -75.79. HPLC conditions: DAICEL CHIRALCEL AD-3, eluent: Hexane/2-Propanol 80.0/20.0, flow: $1.0 \mathrm{~mL} / \mathrm{min}$, detection: $220 \mathrm{~nm}, t_{\mathrm{R}}: 8.73 \mathrm{~min}$ (major), $10.71 \mathrm{~min}$ (minor). $[\alpha]_{\mathrm{D}}^{25}=-8.1\left(\mathrm{c} 1.24, \mathrm{CHCl}_{3}\right)$.

(S)-Ethyl 2-(((benzyloxy)carbonyl)amino)-7-nitro-2-(trifluoromethyl)hept-3-ynoate (4r)

Reaction was performed according to the general procedure, and the crude mixture was 
purified using hexane/AcOEt $=10 / 1$ to $4 / 1$ as eluent to give $4 \mathbf{r}$ as a colorless oil $(39.4 \mathrm{mg}$, $95 \%$ yield, $80 \%$ ee).

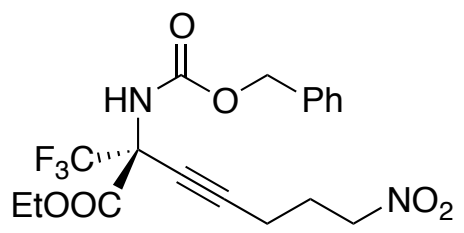

${ }^{1} \mathrm{H}$ NMR $\left(500 \mathrm{MHz}, \mathrm{CDCl}_{3}\right) \delta$ ) 7.39-7.33 (m, 5H), 5.77 (br, $1 \mathrm{H}), 5.16-5.11(\mathrm{~m}, 2 \mathrm{H}), 4.54-4.51(\mathrm{~m}, 2 \mathrm{H}), 4.38-4.31(\mathrm{~m}$, $2 \mathrm{H}), 2.46-2.22(\mathrm{~m}, 2 \mathrm{H}), 2.22-2.18(\mathrm{~m}, 2 \mathrm{H}), 1.31$ (t, $J=7.0$ $\mathrm{Hz}, 3 \mathrm{H}) ;{ }^{13} \mathrm{C}\left\{{ }^{1} \mathrm{H}\right\} \mathrm{NMR}\left(125 \mathrm{MHz}, \mathrm{CDCl}_{3}\right) \delta 163.04,153.54$, 135.56, 128.57, 128.44, 128.31, 122.07 (q, $J_{C-F}=286 \mathrm{~Hz}$ ), 86.00, 73.42, 71.85, 67.66, 64.31, 60.09 (q, $\left.J_{C-F}=33 \mathrm{~Hz}\right), 25.30,15.93,13.72 ;{ }^{19} \mathrm{~F}$ NMR (360 MHz, $\left.\mathrm{CDCl}_{3}\right) \delta-76.15$. IR ( $\mathrm{NaCl}) 3433,3402,3017,2257,1746,1557,1499,1261,1240,1211,1188,1059,1024$ $\mathrm{cm}^{-1}$. HRMS (ESI-TOF) $\mathrm{m} / z$ calcd. For $\mathrm{C}_{18} \mathrm{H}_{20} \mathrm{~F}_{3} \mathrm{~N}_{2} \mathrm{O}_{6}\left[\mathrm{M}+\mathrm{H}^{+}\right]$417.1273, found 417.1275. HPLC conditions: DAICEL CHIRALCEL AD-3, eluent: Hexane/2-Propanol 80.00/20.00, flow: $1.0 \mathrm{~mL} / \mathrm{min}$, detection: $220 \mathrm{~nm}, t_{\mathrm{R}}: 8.89 \min$ (major), $11.96 \min \left(\right.$ minor). $[\alpha]^{27}{ }_{\mathrm{D}}=$ $-1.1\left(\mathrm{c} 0.88, \mathrm{CHCl}_{3}\right)$.

\section{Ethyl}

(S)-2-(((benzyloxy)carbonyl)amino)-5-hydroxy-5-methyl-2-(trifluoromethyl)hex-3-yno ate (4s)

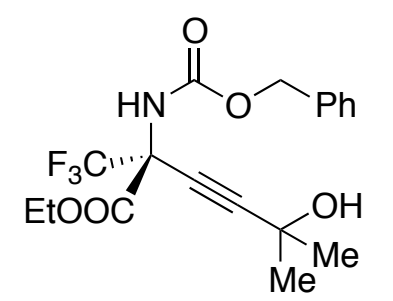

\footnotetext{
Reaction was performed according to the general procedure at room temperature for $96 \mathrm{~h}$, and the crude mixture was purified using hexane $/ \mathrm{AcOEt}=4 / 1$ as eluent to give $4 \mathbf{s}$ as a colorless oil (38.0 mg, $98 \%$ yield, $85 \%$ ee).

${ }^{1} \mathrm{H}$ NMR (500 MHz, $\left.\mathrm{CDCl}_{3}\right) \delta$ 7.38-7.32 (m, 5H), 5.85 (br, 1H),
} 5.16-5.10 (m, 2H), 4.34-4.30 (m, 2H), 2.66 (br, 1H), $1.50(\mathrm{~s}, 3 \mathrm{H}), 1.50(\mathrm{~s}, 3 \mathrm{H}), 1.30$ (t, $J=$ $7.5 \mathrm{~Hz}, 3 \mathrm{H}) ;{ }^{13} \mathrm{C}\left\{{ }^{1} \mathrm{H}\right\}$ NMR $\left(125 \mathrm{MHz}, \mathrm{CDCl}_{3}\right) \delta 162.90,153.61,135.54,128.55,128.46$, $128.42,128.27,122.07$ (q, $\left.J_{C-F}=285 \mathrm{~Hz}\right), 92.62,71.50,67.66,64.91,64.02,59.98$ (q, $J_{C-F}$ $=33 \mathrm{~Hz}), 30.60,30.63,13.68 ;{ }^{19} \mathrm{~F}$ NMR $\left(360 \mathrm{MHz}, \mathrm{CDCl}_{3}\right) \delta-75.91$. HPLC conditions: DAICEL CHIRALCEL AD-3, eluent: Hexane/2-Propanol 80.00/20.00, flow: $1.0 \mathrm{~mL} / \mathrm{min}$, detection: $220 \mathrm{~nm}, t_{\mathrm{R}}: 5.41 \mathrm{~min}$ (major), $6.19 \mathrm{~min}$ (minor). $[\alpha]^{27}{ }_{\mathrm{D}}=-3.0$ (c 1.06, $\mathrm{CHCl}_{3}$ ). 


\section{Expansion of the Substrate Scope (1): $\alpha$-Ketiminophosphonate}

\section{Preparation of $\alpha$-Ketiminophosphonate 9}

$\alpha$-Ketiminophosphonate 9 was prepared according to the literature procedure ${ }^{2 \mathrm{c}}$ with the following modification.

1-Benzyloxycarbonylimino-2,2,2-trifluoroacetimidoyl chloride was synthesized according to the literature procedure. $^{2 \mathrm{c}}$ To a flask were added a 1-benzyloxycarbonylimino-2,2,2-trifluoroacetimidoyl chloride (3.98 g, $15.0 \mathrm{mmol})$ and diethyl ether $(7.5 \mathrm{~mL})$. After the mixture was cooled to $-40{ }^{\circ} \mathrm{C}$, triethyl phosphite $(2.83 \mathrm{~mL}$, $16.5 \mathrm{mmol}, 1.1$ equiv) in diethyl ether $(7.5 \mathrm{~mL})$ was added dropwise over $3 \mathrm{~h}$ and the mixture was stirred overnight at $-40{ }^{\circ} \mathrm{C}$. To the mixture was added $\mathrm{H}_{2} \mathrm{O}(0.81 \mathrm{~mL}, 45 \mathrm{mmol}$, 3.0 equiv). The mixture was warmed to room temperature slowly and stirred for 1 day. The crude mixture was purified by silica gel column chromatography using hexane $/ \mathrm{CH}_{2} \mathrm{Cl}_{2} / \mathrm{AcOEt}=20 / 20 / 1$ to $1 / 1 / 1$ as eluent and recrystallization using hexane/AcOEt $\quad=\quad 10 / 1$ to give benzyl (1-(diethoxyphosphoryl)-2,2,2-trifluoro-1-hydroxyethyl)carbamate as a colorless solid (2.418 g, 42\% yield).

To a flask were added benzyl (1-(diethoxyphosphoryl)-2,2,2-trifluoro-1-hydroxyethyl) carbamate $(4.97 \mathrm{~g}, 15.0 \mathrm{mmol})$, pyridine $(4.84 \mathrm{~mL}, 60.0 \mathrm{mmol}, 4.00$ equiv) and diethyl ether $(30 \mathrm{~mL})$. After the mixture was cooled to $0{ }^{\circ} \mathrm{C}$, trifluoroacetic anhydride $(4.23 \mathrm{~mL}$, $30.0 \mathrm{mmol}, 2.00$ equiv) was added. The mixture was warmed to room temperature and stirred for $3 \mathrm{~h}$. After completion of the reaction, the solvent was removed in vacuo over 30 minutes at $30{ }^{\circ} \mathrm{C}$. To this flask was added dried hexane $(80 \mathrm{~mL})$. The supernatant liquid (50 $\mathrm{mL}$ ) was transferred to other flask and the solvent was removed in vacuo over $1 \mathrm{~h}$ at $30^{\circ} \mathrm{C}$. The residue was purified by distillation under vacuum to give $\alpha$-ketiminophosphonate 9 (1.756 g, 32\% yield).

\section{General Procedure for Direct Catalytic Enantioselective Alkynylation of $\alpha$-Ketiminophosphonate 9 (Table 3)}

A Schlenk tube equipped with a magnetic stir bar and molecular sieves 4A (20 mg) was dried under vacuum using heat gun and refilled with argon. To the tube were added 
(alkynyl)Rh(III) complex 7d (3.1 mg, $0.0050 \mathrm{mmol}, 5.0 \mathrm{~mol} \%)$, toluene $(0.40 \mathrm{~mL})$ and alkyne 3 (0.30 mmol, 3.0 equiv). After the mixture was stirred for $10 \mathrm{~min}$ at room temperature, $\alpha$-ketiminophosphonate $9(29 \mu \mathrm{L}, 0.10 \mathrm{mmol})$ was added and the mixture was stirred at room temperature for $18 \mathrm{~h}$. The crude mixture was directly purified by flash silica gel column chromatography using hexane/AcOEt $=4 / 1$ to $1 / 1$ as eluent to give product $\mathbf{1 0}$.

\section{Benzyl (S)-(2-(diethoxyphosphoryl)-1,1,1-trifluoro-4-phenylbut-3-yn-2-yl)carbamate} (10a)

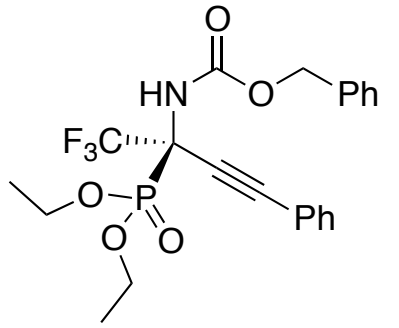

Reaction was performed according to the general procedure using alkyne 3a, and the crude mixture was purified using hexane/AcOEt $=4 / 1$ to $1 / 1$ as eluent to give $\mathbf{1 0 a}$ as a colorless solid (44.9 mg, 96\% yield, $88 \%$ ee).

${ }^{1} \mathrm{H}$ NMR $\left(500 \mathrm{MHz}, \mathrm{CDCl}_{3}\right) \delta 7.47-7.26(\mathrm{~m}, 9 \mathrm{H}), 5.65(\mathrm{~d}$, $\left.J_{P-H}=7.5 \mathrm{~Hz}, 1 \mathrm{H}\right), 5.20-5.13(\mathrm{~m}, 2 \mathrm{H}), 4.43-4.32(\mathrm{~m}, 4 \mathrm{H}), 1.38(\mathrm{~m}, 6 \mathrm{H}) ;{ }^{13} \mathrm{C}\left\{{ }^{1} \mathrm{H}\right\} \mathrm{NMR}$ $\left(125 \mathrm{MHz}, \mathrm{CDCl}_{3}\right) \delta 153.45\left(\mathrm{~d}, J_{C-P}=11 \mathrm{~Hz}\right), 135.70,132.04\left(\mathrm{~d}, J_{C-P}=3 \mathrm{~Hz}\right), 129.36$, 128.57, 128.50, 128.45, 128.34, 128.29, $122.89\left(\mathrm{qd}, J_{C-F}=286 \mathrm{~Hz}, J_{C-P}=4 \mathrm{~Hz}\right), 121.32(\mathrm{~d}$, $\left.J_{C-P}=4 \mathrm{~Hz}\right), 90.22\left(\mathrm{~d}, J_{C-P}=9 \mathrm{~Hz}\right), 76.42\left(\mathrm{~d}, J_{C-P}=9 \mathrm{~Hz}\right), 67.58,66.30\left(\mathrm{~d}, J_{C-P}=8 \mathrm{~Hz}\right)$, $66.79\left(\mathrm{~d}, J_{C-P}=8 \mathrm{~Hz}\right), 56.93\left(\mathrm{qd}, J_{C-F}=33 \mathrm{~Hz}, J_{C-P}=149 \mathrm{~Hz}\right), 16.38\left(\mathrm{~d}, J_{C-P}=6 \mathrm{~Hz}\right) ;{ }^{19} \mathrm{~F}$ NMR (470 MHz, $\mathrm{CDCl}_{3}$ ): $\delta-72.17 ;{ }^{31} \mathrm{P} \mathrm{NMR}\left(202 \mathrm{MHz}, \mathrm{CDCl}_{3}\right): \delta 9.47\left(\mathrm{~d}, J_{P-H}=7.5 \mathrm{~Hz}\right)$. IR $(\mathrm{NaCl})$ 3426, 3393, 3011, 2236, 1751, 1501, 1267, 1200, $12028 \mathrm{~cm}^{-1}$. HRMS (ESI-TOF) $\mathrm{m} / \mathrm{z}$ calcd. for $\mathrm{C}_{22} \mathrm{H}_{23} \mathrm{~F}_{3} \mathrm{NO}_{5} \mathrm{P}\left[\mathrm{M}+\mathrm{H}^{+}\right]$470.1344, found 470.1335. HPLC conditions: DAICEL CHIRALCEL AD-3, eluent: Hexane/2-Propanol 67.0/33.0, flow: 1.0 $\mathrm{mL} / \mathrm{min}$, detection: $254 \mathrm{~nm}, t_{\mathrm{R}}: 5.16 \mathrm{~min}$ (minor), $8.93 \min$ (major). $[\alpha]_{\mathrm{D}}^{26}=18.7$ (c 1.14, $\left.\mathrm{CHCl}_{3}\right)$.

\section{Benzyl (S)-(2-(diethoxyphosphoryl)-1,1,1-trifluoro-4-(p-tolyl)but-3-yn-2-yl)carbamate} (10b)

Reaction was performed according to the general procedure, and the crude mixture was purified using hexane $/ \mathrm{AcOEt}=4 / 1$ to $1 / 1$ as eluent to give $\mathbf{1 0 b}$ as a colorless oil $(44.1 \mathrm{mg}$, $91 \%$ yield, $88 \%$ ee). 


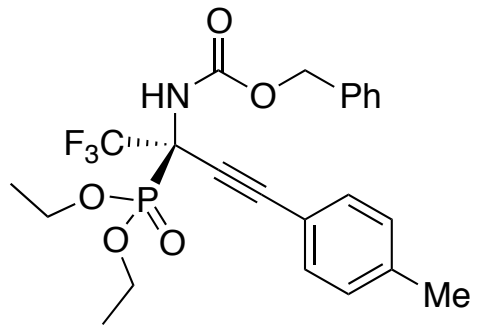

${ }^{1} \mathrm{H}$ NMR $\left(500 \mathrm{MHz}, \mathrm{CDCl}_{3}\right) \delta 7.42(\mathrm{~d}, J=7.5 \mathrm{~Hz}, 1 \mathrm{H})$, $7.37-7.31(\mathrm{~m}, 6 \mathrm{H}), 7.19(\mathrm{~d}, J=7.5 \mathrm{~Hz}, 1 \mathrm{H}), 7.12(\mathrm{t}, J=7.5$ $\mathrm{Hz}, 1 \mathrm{H}), 5.68\left(\mathrm{~d}, J_{P-H}=7.5 \mathrm{~Hz}, 1 \mathrm{H}\right), 5.16(\mathrm{~m}, 2 \mathrm{H}), 4.41-4.27$ (m, 4H), $2.46(\mathrm{~s}, 3 \mathrm{H}), 1.36(\mathrm{~m}, 6 \mathrm{H}) ;{ }^{13} \mathrm{C}\left\{{ }^{1} \mathrm{H}\right\}$ NMR $(125$

$\left.\mathrm{MHz}, \mathrm{CDCl}_{3}\right) \delta 153.38\left(\mathrm{~d}, J_{C-P}=10 \mathrm{~Hz}\right), 141.44\left(\mathrm{~d}, J_{C-P}=3\right.$

$\mathrm{Hz}), 135.74,132.27\left(\mathrm{~d}, J_{C-P}=4 \mathrm{~Hz}\right), 129.55,129.32,128.54,128.44,128.39,128.33$, $125.49,122.98$ (qd, $\left.J_{C-F}=285 \mathrm{~Hz}, J_{C-P}=4 \mathrm{~Hz}\right), 121.17\left(\mathrm{~d}, J_{C-P}=3 \mathrm{~Hz}\right), 89.46\left(\mathrm{~d}, J_{C-P}=8\right.$ $\mathrm{Hz}), 79.97\left(\mathrm{~d}, J_{C-P}=16 \mathrm{~Hz}\right), 67.52,66.14\left(\mathrm{~d}, J_{C-P}=9 \mathrm{~Hz}\right), 65.73\left(\mathrm{~d}, J_{C-P}=8 \mathrm{~Hz}\right), 56.99(\mathrm{qd}$, $\left.J_{C-F}=33 \mathrm{~Hz}, J_{C-P}=149 \mathrm{~Hz}\right), 20.40,16.38\left(\mathrm{~d}, J_{C-P}=4 \mathrm{~Hz}\right), 16.33\left(\mathrm{~d}, J_{C-P}=3 \mathrm{~Hz}\right) ;{ }^{19} \mathrm{~F}$ NMR $\left(470 \mathrm{MHz}, \mathrm{CDCl}_{3}\right) \delta-72.17 ;{ }^{31} \mathrm{P} \mathrm{NMR}\left(202 \mathrm{MHz}, \mathrm{CDCl}_{3}\right) \delta 9.72\left(\mathrm{~d}, J_{P-H}=7.5 \mathrm{~Hz}\right) . \mathrm{IR}$ (NaCl) 3426, 3393, 3017, 2234, 1751, 1501, 1456, 1395, 1265, 1200, 1186, $1028 \mathrm{~cm}^{-1}$. HRMS (ESI-TOF) $m / z$ calcd. for $\mathrm{C}_{23} \mathrm{H}_{25} \mathrm{~F}_{3} \mathrm{NO}_{5} \mathrm{P}\left[\mathrm{M}+\mathrm{H}^{+}\right]$484.1501, found 484.1536. HPLC conditions: DAICEL CHIRALCEL AD-3, eluent: Hexane/2-Propanol 67.0/33.0, flow: $1.0 \mathrm{~mL} / \mathrm{min}$, detection: $254 \mathrm{~nm}, t_{\mathrm{R}}: 5.58 \mathrm{~min}$ (minor), $14.11 \mathrm{~min}$ (major). $[\alpha]^{26}{ }_{\mathrm{D}}=$ 14.2 (c $\left.1.83, \mathrm{CHCl}_{3}\right)$.

\section{Benzyl}

(S)-(2-(diethoxyphosphoryl)-1,1,1-trifluoro-4-(4-methoxyphenyl)but-3-yn-2-yl)carbam ate (10c)

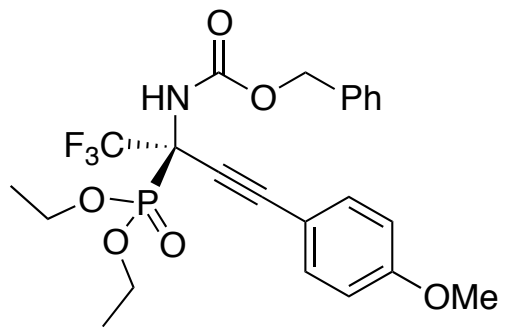

Reaction was performed according to the general procedure, and the crude mixture was purified using hexane/AcOEt $=4 / 1$ to $1 / 1$ as eluent to give 10c as a colorless oil (49.3 mg, 99\% yield, $87 \%$ ee).

${ }^{1} \mathrm{H}$ NMR $\left(500 \mathrm{MHz}, \mathrm{CDCl}_{3}\right) \delta 7.39-7.35(\mathrm{~m}, 7 \mathrm{H})$, 6.84-6.82 (m, 2H), 5.64 (br, 1H), 5.20-5.12 (m, 2H), 4.41-4.30 (m, 4H), $3.80(\mathrm{~s}, 3 \mathrm{H}), 1.37$ $(\mathrm{m}, 6 \mathrm{H}) ;{ }^{13} \mathrm{C}\left\{{ }^{1} \mathrm{H}\right\}$ NMR $\left(125 \mathrm{MHz}, \mathrm{CDCl}_{3}\right) \delta 160.43,153.46\left(\mathrm{~d}, J_{C-p}=10 \mathrm{~Hz}\right), 135.76$, $133.74\left(\mathrm{~d}, J_{C-p}=29 \mathrm{~Hz}\right), 130.96,128.92,128.56,128.45,128.32,128.28,122.95$ (q, $J_{C-F}=$ $286 \mathrm{~Hz}), 113.98,113.35,90.34\left(\mathrm{~d}, J_{C-P}=4 \mathrm{~Hz}\right), 75.06\left(\mathrm{~d}, J_{C-P}=16 \mathrm{~Hz}\right), 67.53,66.27(\mathrm{~d}$, $\left.J_{C-P}=8 \mathrm{~Hz}\right), 65.72\left(\mathrm{~d}, J_{C-P}=7 \mathrm{~Hz}\right), 56.98\left(\mathrm{qd}, J_{C-F}=33 \mathrm{~Hz}, J_{C-P}=149 \mathrm{~Hz}\right), 55.27\left(\mathrm{~d}, J_{C-P}=\right.$ $9 \mathrm{~Hz}), 16.38\left(\mathrm{~d}, J_{C-P}=6 \mathrm{~Hz}\right) ;{ }^{19} \mathrm{~F}$ NMR $\left(470 \mathrm{MHz}, \mathrm{CDCl}_{3}\right) \delta-72.23 ;{ }^{31} \mathrm{P}$ NMR $(202 \mathrm{MHz}$, 
$\left.\mathrm{CDCl}_{3}\right) \delta$ 9.56. IR (NaCl) 3422, 3393, 3019, 2234, 1751, 1607, 1510, 1250, 1211, 1198, $1184,1030 \mathrm{~cm}^{-1}$. HRMS (ESI-TOF) $\mathrm{m} / z$ calcd. for $\mathrm{C}_{23} \mathrm{H}_{25} \mathrm{~F}_{3} \mathrm{NO}_{6} \mathrm{P}\left[\mathrm{M}+\mathrm{H}^{+}\right]$500.1450, found 500.1456. HPLC conditions: DAICEL CHIRALCEL AD-3, eluent: Hexane/2-Propanol 67.0/33.0, flow: $1.0 \mathrm{~mL} / \mathrm{min}$, detection: $254 \mathrm{~nm}, t_{\mathrm{R}}: 6.79 \mathrm{~min}$ (minor), 17.61 min (major). $[\alpha]^{26}=11.3$ (c $1.34, \mathrm{CHCl}_{3}$ ).

\section{Benzyl}

(S)-(2-(diethoxyphosphoryl)-1,1,1-trifluoro-4-(4-(trifluoromethyl)phenyl)but-3-yn-2-yl carbamate (10d)

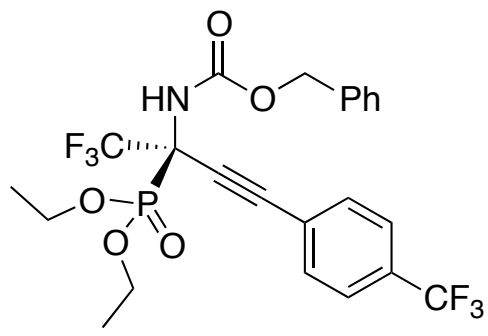

Reaction was performed according to the general procedure, and the crude mixture was purified using hexane/AcOEt $=4 / 1$ to $1 / 1$ as eluent to give $10 d$ as a colorless oil (52.0 mg, 97\% yield, $88 \%$ ee).

${ }^{1} \mathrm{H}$ NMR $\left(500 \mathrm{MHz}, \mathrm{CDCl}_{3}\right) \delta 7.57(\mathrm{~m}, 4 \mathrm{H}), 7.38-7.32$ $(\mathrm{m}, 5 \mathrm{H}), 5.67\left(\mathrm{~d}, J_{P-H}=7.5 \mathrm{~Hz}, 1 \mathrm{H}\right), 5.20-5.13(\mathrm{~m}, 2 \mathrm{H}), 4.40-4.31(\mathrm{~m}, 4 \mathrm{H}), 1.32(\mathrm{~m}, 6 \mathrm{H})$; ${ }^{13} \mathrm{C}\left\{{ }^{1} \mathrm{H}\right\}$ NMR $\left(125 \mathrm{MHz}, \mathrm{CDCl}_{3}\right) \delta 153.46\left(\mathrm{~d}, J_{C-P}=10 \mathrm{~Hz}\right), 135.61,132.33\left(\mathrm{~d}, J_{C-P}=2\right.$ $\mathrm{Hz}), 131.10\left(\mathrm{q}, J_{C-F}=33 \mathrm{~Hz}\right), 128.61,128.49,128.45,128.34,125.11,123.75\left(\mathrm{q}, J_{C-F}=271\right.$ $\mathrm{Hz}), 124.62\left(\mathrm{qd}, J_{C-F}=285 \mathrm{~Hz}, J_{C-P}=3 \mathrm{~Hz}\right), 88.67\left(\mathrm{~d}, J_{C-P}=8 \mathrm{~Hz}\right), 78.97\left(\mathrm{~d}, J_{C-P}=16 \mathrm{~Hz}\right)$, $67.72,66.28\left(\mathrm{~d}, J_{C-P}=8 \mathrm{~Hz}\right), 65.88\left(\mathrm{~d}, J_{C-P}=7 \mathrm{~Hz}\right), 56.90\left(\mathrm{qd}, J_{C-F}=33 \mathrm{~Hz}, J_{C-P}=148 \mathrm{~Hz}\right)$, 29.71, $16.38\left(\mathrm{~d}, J_{C-P}=6 \mathrm{~Hz}\right) ;{ }^{19} \mathrm{~F}$ NMR $\left(470 \mathrm{MHz}, \mathrm{CDCl}_{3}\right) \delta-63.01,-72.07 ;{ }^{31} \mathrm{P}$ NMR $\left(202 \mathrm{MHz}, \mathrm{CDCl}_{3}\right) \delta$ 9.42. IR ( $\left.\mathrm{NaCl}\right) 3426,3393,3017,1751,1499,1323,1267,1211$, $1206,1184,1134,1064,1026 \mathrm{~cm}^{-1}$. HRMS (ESI-TOF) $\mathrm{m} / z$ calcd. for $\mathrm{C}_{23} \mathrm{H}_{22} \mathrm{~F}_{6} \mathrm{NO}_{5} \mathrm{P}[\mathrm{M}+$ $\mathrm{H}^{+}$] 538.1218, found 538.1209. HPLC conditions: DAICEL CHIRALCEL AD-3, eluent: Hexane/2-Propanol 67.0/33.0, flow: $1.0 \mathrm{~mL} / \mathrm{min}$, detection: $254 \mathrm{~nm}, t_{\mathrm{R}}: 5.14 \mathrm{~min}$ (minor), 10.65 min (major). $[\alpha]^{26}=13.3$ (c 1.04, $\mathrm{CHCl}_{3}$ ).

\section{Benzyl (S)-(2-(diethoxyphosphoryl)-1,1,1-trifluoro-4-(o-tolyl)but-3-yn-2-yl)carbamate (10e)}

Reaction was performed according to the general procedure, and the crude mixture was purified using hexane $/ \mathrm{AcOEt}=4 / 1$ to $1 / 1$ as eluent to give 10e as a colorless oil $(47.1 \mathrm{mg}$, 
97\% yield, $93 \%$ ee).

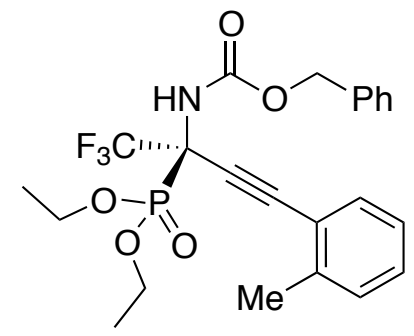

${ }^{1} \mathrm{H}$ NMR $\left(500 \mathrm{MHz}, \mathrm{CDCl}_{3}\right) \delta$ 7.38-7.30 (m, 7H), $7.12(\mathrm{~d}, J=$ $8.0 \mathrm{~Hz}, 2 \mathrm{H}), 5.65\left(\mathrm{~d}, J_{P-H}=7.5 \mathrm{~Hz}, 1 \mathrm{H}\right), 5.20-5.12(\mathrm{~m}, 2 \mathrm{H})$, 4.42-4.30 (m, 2H), $2.35(\mathrm{~s}, 3 \mathrm{H}), 1.32(\mathrm{~m}, 6 \mathrm{H}) ;{ }^{13} \mathrm{C}\left\{{ }^{1} \mathrm{H}\right\}$ NMR $\left(125 \mathrm{MHz}, \mathrm{CDCl}_{3}\right) \delta 153.46\left(\mathrm{~d}, J_{C-P}=11 \mathrm{~Hz}\right), 139.65,135.74$, $131.94\left(\mathrm{~d}, J_{C-P}=3 \mathrm{~Hz}\right), 129.09,128.56,128.45,128.32,128.28$, $122.92\left(\mathrm{qd}, J_{C-F}=285 \mathrm{~Hz}, J_{C-P}=3 \mathrm{~Hz}\right), 118.25\left(\mathrm{~d}, J_{C-P}=4 \mathrm{~Hz}\right), 90.45\left(\mathrm{~d}, J_{C-P}=8 \mathrm{~Hz}\right)$, $75.72\left(\mathrm{~d}, J_{C-P}=15 \mathrm{~Hz}\right), 67.54,66.31\left(\mathrm{~d}, J_{C-P}=8 \mathrm{~Hz}\right), 65.76\left(\mathrm{~d}, J_{C-P}=8 \mathrm{~Hz}\right), 56.96\left(\mathrm{qd}, J_{C-F}\right.$ $\left.=33 \mathrm{~Hz}, J_{C-P}=149 \mathrm{~Hz}\right), 21.57,16.37\left(\mathrm{~d}, J_{C-P}=5 \mathrm{~Hz}\right) ;{ }^{19} \mathrm{~F}$ NMR $\left(470 \mathrm{MHz}, \mathrm{CDCl}_{3}\right) \delta$ -72.20; ${ }^{31} \mathrm{P}$ NMR (202 MHz, $\left.\mathrm{CDCl}_{3}\right) \delta 9.48$ (d, $J_{P-H}=7.5 \mathrm{~Hz}$ ). IR (NaCl) 3426, 3393, 3019, 2988, 2959, 2928, 2234, 1751, 1503, 1265, 1198, $1024 \mathrm{~cm}^{-1}$. HRMS (ESI-TOF) $\mathrm{m} / z$ calcd. for $\mathrm{C}_{23} \mathrm{H}_{25} \mathrm{~F}_{3} \mathrm{NO}_{5} \mathrm{P}\left[\mathrm{M}+\mathrm{H}^{+}\right]$484.1501, found 484.1483. HPLC conditions: DAICEL CHIRALCEL AD-3, eluent: Hexane/2-Propanol 67.0/33.0, flow: $1.0 \mathrm{~mL} / \mathrm{min}$, detection: $254 \mathrm{~nm}, t_{\mathrm{R}}: 4.68 \mathrm{~min}$ (minor), $6.02 \mathrm{~min}$ (major). $[\alpha]_{\mathrm{D}}^{26}=12.7$ (c 1.27, $\mathrm{CHCl}_{3}$ ).

\section{Benzyl}

\section{(S)-(4-cyclopropyl-2-(diethoxyphosphoryl)-1,1,1-trifluorobut-3-yn-2-yl)carbamate} (10f)

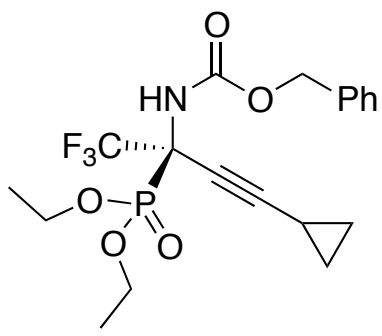

Reaction was performed according to the general procedure using 3.0 equiv of alkyne $3 \mathbf{m}$ and for $24 \mathrm{~h}$, and the crude mixture was purified using hexane/AcOEt $=4 / 1$ to $1 / 1$ as eluent to give 10 as a colorless oil (37.1 mg, 86\% yield, $80 \%$ ee).

${ }^{1} \mathrm{H}$ NMR $\left(500 \mathrm{MHz}, \mathrm{CDCl}_{3}\right) \delta$ 7.37-7.27 (m, 5H), $5.51(\mathrm{~d}$, $\left.J_{P-H}=7.5 \mathrm{~Hz}, 1 \mathrm{H}\right), 5.17-5.10(\mathrm{~m}, 2 \mathrm{H}), 4.37-4.24(\mathrm{~m}, 2 \mathrm{H}), 1.39-1.32(\mathrm{~m}, 6 \mathrm{H}), 0.84-0.76$ $(\mathrm{m}, 4 \mathrm{H}) ;{ }^{13} \mathrm{C}\left\{{ }^{1} \mathrm{H}\right\}$ NMR $\left(125 \mathrm{MHz}, \mathrm{CDCl}_{3}\right) \delta 153.55\left(\mathrm{~d}, J_{C-P}=11 \mathrm{~Hz}\right), 136.00,128.75$, 128.65, 128.62, 128.59, 128.52, 128.44, 123.10(qd, $\left.J_{C-F}=286 \mathrm{~Hz}, J_{C-P}=4 \mathrm{~Hz}\right), 95.00(\mathrm{~d}$, $\left.J_{C-P}=8 \mathrm{~Hz}\right), 67.58,66.28\left(\mathrm{~d}, J_{C-P}=8 \mathrm{~Hz}\right), 65.70\left(\mathrm{~d}, J_{C-P}=16 \mathrm{~Hz}\right), 62.43\left(\mathrm{~d}, J_{C-P}=16 \mathrm{~Hz}\right)$, $56.68\left(\mathrm{dq}, J_{C-P}=150 \mathrm{~Hz}, J_{C-F}=33 \mathrm{~Hz}\right), 16.55\left(\mathrm{~d}, J_{C-P}=4 \mathrm{~Hz}\right), 16.51\left(\mathrm{~d}, J_{C-P}=4 \mathrm{~Hz}\right), 8.54$ $\left(\mathrm{d}, J_{C-P}=2 \mathrm{~Hz}\right),-0.01\left(\mathrm{~d}, J_{C-P}=3 \mathrm{~Hz}\right) ;{ }^{19} \mathrm{~F}$ NMR $\left(470 \mathrm{MHz}, \mathrm{CDCl}_{3}\right) \delta-72.59 ;{ }^{31} \mathrm{P}$ NMR $\left(202 \mathrm{MHz}, \mathrm{CDCl}_{3}\right) \delta$ 9.79. IR (NaCl) 3428, 3395, 3009, 2247, 1751, 1503, 1265, 1200, 
1172, 1051, $1024 \mathrm{~cm}^{-1}$. HRMS (ESI-TOF) $\mathrm{m} / z$ calcd. for $\mathrm{C}_{19} \mathrm{H}_{23} \mathrm{~F}_{3} \mathrm{NO}_{5} \mathrm{P}\left[\mathrm{M}+\mathrm{H}^{+}\right]$ 434.1344, found 434.1323. HPLC conditions: DAICEL CHIRALCEL AD-3, eluent: Hexane/2-Propanol 80.0/20.0, flow: $1.0 \mathrm{~mL} / \mathrm{min}$, detection: $220 \mathrm{~nm}, t_{\mathrm{R}}: 6.00 \mathrm{~min}$ (minor), 6.91 min (major). $[\alpha]^{26}=17.2\left(\mathrm{c} 1.20, \mathrm{CHCl}_{3}\right.$ ).

\section{Comparison of Reactivity Using (Diacetato)Rh(III) Complex 1a for Direct Catalytic} Enantioselective Alkynylation of $\alpha$-Ketiminophosphonate 9

The above procedure was followed with alkyne 3a except for using (diacetato)Rh(III) complex 1a (3.0 mg, $0.0050 \mathrm{mmol}, 5.0 \mathrm{~mol} \%$ ) as catalyst, and $8 \%$ of product 10a was observed at room temperature for $24 \mathrm{~h}$ based on ${ }^{19} \mathrm{~F}$ NMR analysis of the crude mixture.

\section{Expansion of the Substrate Scope (2): Cyclic N-Sulfonyl $\alpha$-Ketiminoesters}

General Procedure for Direct Catalytic Enantioselective Alkynylation of Cyclic $N$-Sulfonyl $\alpha$-Ketiminoesters 11 (Tables 4 and 5)

A Schlenk tube equipped with a magnetic stir bar and molecular sieves 4A $(20 \mathrm{mg})$ was dried under vacuum using heat gun and refilled with argon. To the tube were added (alkynyl)Rh(III) complex 13d (3.3 mg, $0.0050 \mathrm{mmol}, 5.0 \mathrm{~mol} \%)$, toluene $(0.40 \mathrm{~mL})$ and alkyne 3 (3.0 mmol, 3.0 equiv). After the mixture was stirred for $10 \mathrm{~min}$ at room temperature, cyclic $N$-sulfonyl $\alpha$-ketiminoester 11 (24 mg, $0.10 \mathrm{mmol})$ was added and the mixture was stirred at $70{ }^{\circ} \mathrm{C}$ for $48 \mathrm{~h}$. The crude mixture was directly purified by flash silica gel column chromatography to give product $\mathbf{1 2}$.

\section{Ethyl $(S)$-3-(phenylethynyl)-2,3-dihydrobenzo[d] isothiazole-3-carboxylate 1,1-dioxide}

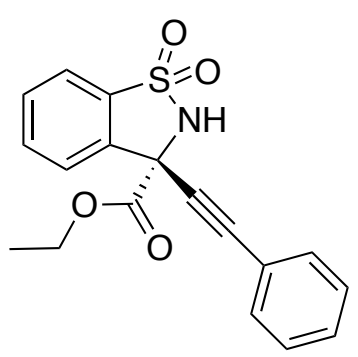

Reaction was performed according to the general procedure using alkyne 3a, and the crude mixture was purified using hexane $/ \mathrm{AcOEt}=4 / 1$ to $1 / 1$ as eluent to give 12a as a colorless oil (33.5 mg, $98 \%$ yield, $85 \%$ ee).

${ }^{1} \mathrm{H}$ NMR (500 MHz, $\left.\mathrm{CDCl}_{3}\right) \delta 7.93(\mathrm{~d}, J=8.0 \mathrm{~Hz}, 1 \mathrm{H}), 7.79$ (d, $J=7.5 \mathrm{~Hz}, 1 \mathrm{H}), 7.73(\mathrm{t}, J=7.5,1 \mathrm{H}), 7.64(\mathrm{t}, J=7.5,1 \mathrm{H}), 7.43(\mathrm{~d}, J$ 
$=7.5 \mathrm{~Hz}, 2 \mathrm{H}), 7.37-7.29(\mathrm{~m}, 3 \mathrm{H}), 5.91(\mathrm{br}, 1 \mathrm{H}), 4.43-4.35(\mathrm{~m}, 2 \mathrm{H}), 1.37(\mathrm{t}, J=7.5 \mathrm{~Hz}$, $3 \mathrm{H}) ;{ }^{13} \mathrm{C}\left\{{ }^{1} \mathrm{H}\right\}$ NMR $\left(125 \mathrm{MHz}, \mathrm{CDCl}_{3}\right) \delta 166.88,135.96,134.25,133.92,132.05,131.09$, 129.41, 128.37, 126.15, 121.33, 121.07, 85.52, 84.28, 64.58, 61.83, 13.97. IR (NaCl) 3287, 3030, 2986, 2928, 2236, 1748, 1492, 1454, 1370, 1319, 1240, 1222, $1173 \mathrm{~cm}^{-1}$. HRMS (ESI-TOF) $\mathrm{m} / z$ calcd. for $\mathrm{C}_{18} \mathrm{H}_{15} \mathrm{NO}_{4} \mathrm{~S}\left[\mathrm{M}+\mathrm{H}^{+}\right]$342.0800, found 342.0796. HPLC conditions: DAICEL CHIRALCEL AD-3, eluent: Hexane/2-Propanol 67.0/33.0, flow: 1.0 $\mathrm{mL} / \mathrm{min}$, detection: $254 \mathrm{~nm}, t_{\mathrm{R}}: 12.51 \min$ (major), $18.36 \min \left(\right.$ minor). $[\alpha]^{26}{ }_{\mathrm{D}}=-48.9(\mathrm{c}$ $\left.1.28, \mathrm{CHCl}_{3}\right)$.

Ethyl (S)-5-methoxy-3-(phenylethynyl)-2,3-dihydrobenzo[d] isothiazole-3-carboxylate 1,1-dioxide (12b)

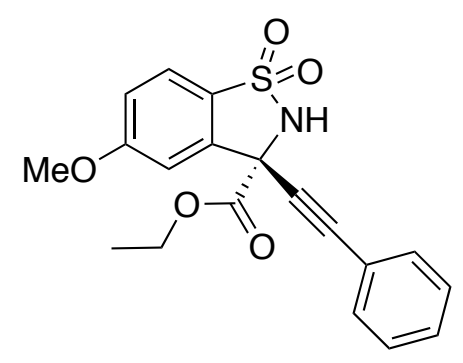

Reaction was performed according to the general procedure, and the crude mixture was purified using hexane/ $\mathrm{AcOEt}=2 / 1$ to $1 / 1$ as eluent to give $\mathbf{1 2 b}$ as a colorless oil $(35.5 \mathrm{mg}, 92 \%$ yield, $86 \%$ ee).

${ }^{1} \mathrm{H}$ NMR $\left(500 \mathrm{MHz}, \mathrm{CDCl}_{3}\right) \delta 7.68(\mathrm{~d}, J=8.5 \mathrm{~Hz}, 1 \mathrm{H})$, $7.44(\mathrm{~d}, J=8.0 \mathrm{~Hz}, 2 \mathrm{H}), 7.37-7.29(\mathrm{~m}, 4 \mathrm{H}), 7.12(\mathrm{~d}, J=8.5$ $\mathrm{Hz}, 1 \mathrm{H}), 5.87$ (br, 1H), 4.42-4.38 (m, 2H), $3.91(\mathrm{~s}, 3 \mathrm{H}), 1.38(\mathrm{t}, J=7.0 \mathrm{~Hz}, 3 \mathrm{H}) ;{ }^{13} \mathrm{C}\left\{{ }^{1} \mathrm{H}\right\}$ NMR (125 MHz, $\left.\mathrm{CDCl}_{3}\right) \delta$ 166.85, 164.12, 138.52, 132.06, 129.39, 128.36, 126.31, 122.72, 121.10, 117.81, 110.51, 85.44, 84.35, 64.52, 61.53, 56.09, 14.00. IR ( NaCl) 3289, 3017, 2236, 1748, 1601, 1485, 1497, 1317, 1246, $1215 \mathrm{~cm}^{-1}$. HRMS (ESI-TOF) $\mathrm{m} / z$ calcd. for $\mathrm{C}_{19} \mathrm{H}_{17} \mathrm{NO}_{5} \mathrm{~S}\left[\mathrm{M}+\mathrm{H}^{+}\right]$372.0906, found 372.0878. HPLC conditions: DAICEL CHIRALCEL AD-3, eluent: Hexane/2-Propanol 67.0/33.0, flow: $1.0 \mathrm{~mL} / \mathrm{min}$, detection: $254 \mathrm{~nm}, t_{\mathrm{R}}: 12.90 \mathrm{~min}$ (major), $18.30 \mathrm{~min}$ (minor). $[\alpha]^{25}=-71.8\left(\mathrm{c} 1.06, \mathrm{CHCl}_{3}\right.$ ).

Ethyl (S)-5-chloro-3-(phenylethynyl)-2,3-dihydrobenzo[d]isothiazole-3-carboxylate

\section{1,1-dioxide (12c)}

Reaction was performed according to the general procedure, and the crude mixture was purified using hexane $/ \mathrm{AcOEt}=4 / 1$ to $2 / 1$ as eluent to give $12 \mathrm{c}$ as a colorless oil $(35.2 \mathrm{mg}$, $90 \%$ yield, $87 \%$ ee). 


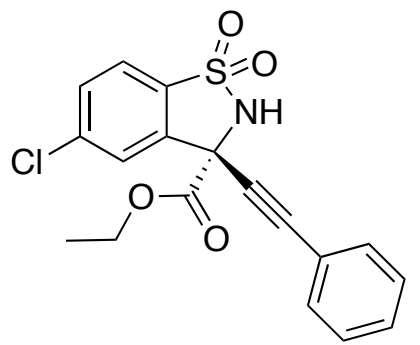

${ }^{1} \mathrm{H}$ NMR $\left(500 \mathrm{MHz}, \mathrm{CDCl}_{3}\right) \delta 7.89(\mathrm{~s}, 1 \mathrm{H}), 7.72(\mathrm{~d}, J=8.5$ $\mathrm{Hz}, 1 \mathrm{H}), 7.61(\mathrm{t}, J=8.0 \mathrm{~Hz}, 1 \mathrm{H}), 7.45(\mathrm{~d}, J=8.0 \mathrm{~Hz}, 2 \mathrm{H})$, 7.37-7.26 (m, 3H), 5.95 (br, 1H), 4.46-4.38 (m, 2H), 1.39 (t, $J=$ $8.0 \mathrm{~Hz}, 3 \mathrm{H}) ;{ }^{13} \mathrm{C}\left\{{ }^{1} \mathrm{H}\right\} \mathrm{NMR}\left(125 \mathrm{MHz}, \mathrm{CDCl}_{3}\right) \delta 166.31,140.33$, $137.90,132.87,132.09,131.63,129.58,128.41,126.34,122.53$, 120.77, 86.04, 83.55, 64.84, 61.33, 13.96. IR ( NaCl) 3283, 3032, 2978, 2236, 1749, 1586, 1323, 1221, $1088 \mathrm{~cm}^{-1}$. HRMS (ESI-TOF) $\mathrm{m} / \mathrm{z}$ calcd. for $\mathrm{C}_{18} \mathrm{H}_{14} \mathrm{ClNO}_{4} \mathrm{~S}\left[\mathrm{M}+\mathrm{H}^{+}\right]$376.0410, found 376.0410. HPLC conditions: DAICEL CHIRALCEL AD-3, eluent: Hexane/2-Propanol 67.0/33.0, flow: $1.0 \mathrm{~mL} / \mathrm{min}$, detection: $254 \mathrm{~nm}, t_{\mathrm{R}}: 8.78 \mathrm{~min}$ (major), $10.19 \min$ (minor). $[\alpha]^{24}=-58.4$ (c 1.27, $\mathrm{CHCl}_{3}$ ).

Ethyl (S)-3-(p-tolylethynyl)-2,3-dihydrobenzo[d] isothiazole-3-carboxylate 1,1-dioxide (12d)

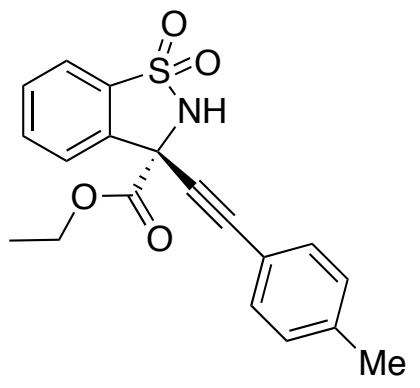

Reaction was performed according to the general procedure, and the crude mixture was purified using hexane/AcOEt $=4 / 1$ to 2/1 as eluent to give 12d as a colorless oil $(35.1 \mathrm{mg}, 99 \%$ yield, $86 \%$ ee).

${ }^{1} \mathrm{H}$ NMR (500 MHz, $\left.\mathrm{CDCl}_{3}\right) \delta 7.93(\mathrm{~d}, J=7.5 \mathrm{~Hz}, 1 \mathrm{H}), 7.78$ $(\mathrm{d}, J=8.0 \mathrm{~Hz}, 1 \mathrm{H}), 7.72(\mathrm{t}, J=8.0 \mathrm{~Hz}, 1 \mathrm{H}), 7.63(\mathrm{t}, J=7.5 \mathrm{~Hz}$, 1H), $7.32(\mathrm{~d}, J=8.0 \mathrm{~Hz}, 2 \mathrm{H}), 7.11(\mathrm{~d}, J=8.0 \mathrm{~Hz}, 2 \mathrm{H}), 5.88(\mathrm{br}, 1 \mathrm{H}), 4.42-4.34(\mathrm{~m}, 2 \mathrm{H})$, 2.34 (s, 3H), 1.37 (t, $J=7.5 \mathrm{~Hz}, 3 \mathrm{H}) ;{ }^{13} \mathrm{C}\left\{{ }^{1} \mathrm{H}\right\}$ NMR $\left(125 \mathrm{MHz}, \mathrm{CDCl}_{3}\right) \delta$ 166.94, 139.71, 136.06, 134.22, 133.87, 131.01, 129.11, 126.19, 121.26, 117.96, 85.76, 83.61, 64.49, 61.89, 21.54, 13.95. IR ( $\mathrm{NaCl}) 3285,3017,2986,2927,2359,2340,2234,1746,1510,1454,1319$, $1242,1225,1209 \mathrm{~cm}^{-1}$. HRMS (ESI-TOF) $\mathrm{m} / z$ calcd. for $\mathrm{C}_{19} \mathrm{H}_{17} \mathrm{NO}_{4} \mathrm{~S}\left[\mathrm{M}+\mathrm{H}^{+}\right] 356.0957$, found 356.0966. HPLC conditions: DAICEL CHIRALCEL AD-3, eluent: Hexane/2-Propanol 67.0/33.0, flow: $1.0 \mathrm{~mL} / \mathrm{min}$, detection: $254 \mathrm{~nm}, t_{\mathrm{R}}: 13.21 \mathrm{~min}$ (major), $19.89 \min$ (minor). $[\alpha]^{26}=-43.4\left(\mathrm{c} 1.48, \mathrm{CHCl}_{3}\right)$.

Ethyl (S)-3-((4-methoxyphenyl)ethynyl)-2,3-dihydrobenzo[d] isothiazole-3-carboxylate 1,1-dioxide (12e) 


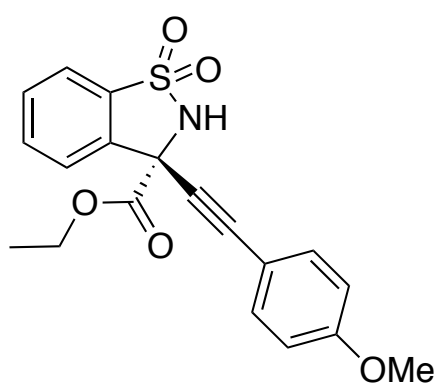

Reaction was performed according to the general procedure, and the crude mixture was purified using hexane $/ \mathrm{AcOEt}=4 / 1$ to $1 / 2$ as eluent to give 12e as a colorless oil $(34.3 \mathrm{mg}, 82 \%$ yield, $87 \%$ ee).

${ }^{1} \mathrm{H}$ NMR $\left(500 \mathrm{MHz}, \mathrm{CDCl}_{3}\right) \delta 7.93(\mathrm{~d}, J=8.0 \mathrm{~Hz}, 1 \mathrm{H})$, $7.78(\mathrm{~d}, J=8.0 \mathrm{~Hz}, 1 \mathrm{H}), 7.72(\mathrm{t}, J=7.5 \mathrm{~Hz}, 1 \mathrm{H}), 7.64(\mathrm{~d}, J=$ $7.5 \mathrm{~Hz}, 1 \mathrm{H}), 7.36$ (d, $J=8.0 \mathrm{~Hz}, 2 \mathrm{H}), 6.82(\mathrm{~d}, J=8.0 \mathrm{~Hz}, 2 \mathrm{H}), 5.89$ (br, $1 \mathrm{H}), 4.42-4.34$ (m, 2H), 3.80 (s, 3H), 1.36 (t, $J=7.5 \mathrm{~Hz}, 3 \mathrm{H}) ;{ }^{13} \mathrm{C}\left\{{ }^{1} \mathrm{H}\right\}$ NMR (125 MHz, $\left.\mathrm{CDCl}_{3}\right) \delta 166.99$, $160.41,136.15,134.21,133.86,133.60,130.98,126.20,121.24,114.00,113.02,85.68$, 83.01, 64.45, 61.95, 55.33, 13.95. IR ( $\mathrm{NaCl}) 3624,3285,3021,2976,2232,1746,1607$, $1510,1319,1250,1219,1173 \mathrm{~cm}^{-1}$. HRMS (ESI-TOF) $\mathrm{m} / z$ calcd. for $\mathrm{C}_{19} \mathrm{H}_{17} \mathrm{NO}_{5} \mathrm{~S}[\mathrm{M}+$ $\mathrm{H}^{+}$] 372.0906, found 372.0920. HPLC conditions: DAICEL CHIRALCEL AD-3, eluent: Hexane/2-Propanol 67.0/33.0, flow: $1.0 \mathrm{~mL} / \mathrm{min}$, detection: $254 \mathrm{~nm}, t_{\mathrm{R}}: 17.98 \mathrm{~min}$ (major), $30.30 \min$ (minor). $[\alpha]_{\mathrm{D}}^{26}=-46.6\left(\mathrm{c} 1.31, \mathrm{CHCl}_{3}\right)$.

\section{Ethyl}

(S)-3-((4-trifluoromethylphenyl)ethynyl)-2,3-dihydrobenzo[d] isothiazole-3-carboxylat

\section{e 1,1-dioxide (12f)}

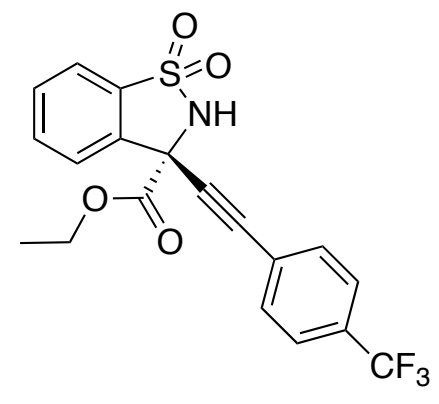

Reaction was performed according to the general procedure
for $96 \mathrm{~h}$, and the crude mixture was purified using
hexane/AcOEt $=10 / 1$ to $1 / 1$ as eluent to give $\mathbf{1 2 f}$ as a colorless
oil ( $36.8 \mathrm{mg}, 90 \%$ yield, $78 \%$ ee).
${ }^{1} \mathrm{H} \mathrm{NMR}\left(500 \mathrm{MHz}, \mathrm{CDCl}_{3}\right) \delta 7.93(\mathrm{~d}, J=8.0 \mathrm{~Hz}, 1 \mathrm{H}), 7.81$
(d, $J=8.0 \mathrm{~Hz}, 1 \mathrm{H}), 7.75(\mathrm{t}, J=8.0 \mathrm{~Hz}, 1 \mathrm{H}), 7.67(\mathrm{t}, J=7.5 \mathrm{~Hz}$, 1H), 7.37-7.29 (m, 4H), 5.96 (br, $1 \mathrm{H}), 4.46-4.39(\mathrm{~m}, 2 \mathrm{H}), 1.39(\mathrm{t}, J=7.5 \mathrm{~Hz}, 3 \mathrm{H}) ;{ }^{13} \mathrm{C}\left\{{ }^{1} \mathrm{H}\right\}$ $\operatorname{NMR}\left(125 \mathrm{MHz}, \mathrm{CDCl}_{3}\right) \delta 166.58,135.60,134.33,134.04,132.37,131.29,131.10$ (q, $J_{C-F}$ $=33 \mathrm{~Hz}), 125.92,125.31$ (q, $\left.J_{C-F}=4 \mathrm{~Hz}\right), 124.91,123.69$ (q, $\left.J_{C-F}=271 \mathrm{~Hz}\right), 121.49,86.63$, 83.85, 64.82, 61.64, 13.98; ${ }^{19} \mathrm{~F}$ NMR (470 MHz, $\left.\mathrm{CDCl}_{3}\right) \delta-63.00$. IR (NaCl) 3281, 3032, 2986, 2359, 2342, 1748, 1454, 1323, 1240, 1173, 1134, $1067 \mathrm{~cm}^{-1}$. HRMS (ESI-TOF) $\mathrm{m} / \mathrm{z}$ calcd. for $\mathrm{C}_{19} \mathrm{H}_{14} \mathrm{~F}_{3} \mathrm{NO}_{4} \mathrm{~S}\left[\mathrm{M}+\mathrm{H}^{+}\right]$410.0674, found 410.0680. HPLC conditions: DAICEL 
CHIRALCEL AD-3, eluent: Hexane/2-Propanol 67.0/33.0, flow: $1.0 \mathrm{~mL} / \mathrm{min}$, detection: $254 \mathrm{~nm}, t_{\mathrm{R}}: 12.73 \min$ (major), $14.28 \mathrm{~min}$ (minor). $[\alpha]^{25}{ }_{\mathrm{D}}=-38.9\left(\mathrm{c} 1.35, \mathrm{CHCl}_{3}\right)$.

Ethyl (S)-3-(o-tolylethynyl)-2,3-dihydrobenzo[d] isothiazole-3-carboxylate 1,1-dioxide (12g)

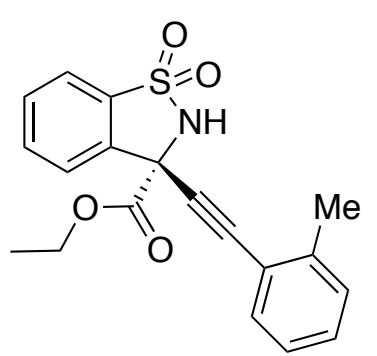

Reaction was performed according to the general procedure, and the mixture was purified using hexane/AcOEt $=4 / 1$ to $1 / 1$ as eluent to give $\mathbf{1 2 g}$ as a colorless oil (34.8 $\mathrm{mg}, 98 \%$ yield, $82 \%$ ee).

${ }^{1} \mathrm{H}$ NMR $\left(500 \mathrm{MHz}, \mathrm{CDCl}_{3}\right) \delta 7.93(\mathrm{~d}, J=7.5 \mathrm{~Hz}, 1 \mathrm{H}), 7.79(\mathrm{~d}$, $J=8.0 \mathrm{~Hz}, 1 \mathrm{H}), 7.72(\mathrm{t}, J=7.5 \mathrm{~Hz}, 1 \mathrm{H}), 7.64(\mathrm{t}, J=7.5 \mathrm{~Hz}, 1 \mathrm{H})$, $7.38(\mathrm{~d}, J=7.5 \mathrm{~Hz}, 1 \mathrm{H}), 7.24(\mathrm{t}, J=7.5 \mathrm{~Hz}, 1 \mathrm{H}), 7.18$ (d, $J=7.5$ $\mathrm{Hz}, 1 \mathrm{H}), 7.12$ (t, $J=7.5 \mathrm{~Hz}, 1 \mathrm{H}), 5.90(\mathrm{br}, 1 \mathrm{H}), 4.45-4.34(\mathrm{~m}, 2 \mathrm{H}), 2.39$ (s, 3H), $1.38(\mathrm{t}, J=$ $7.0 \mathrm{~Hz}, 3 \mathrm{H}) ;{ }^{13} \mathrm{C}\left\{{ }^{1} \mathrm{H}\right\}$ NMR $\left(125 \mathrm{MHz}, \mathrm{CDCl}_{3}\right) \delta 166.94,140.94,136.14,134.30,133.88$, $132.19,131.05,129.53,129.39,126.02,125.61,121.34,120.84,88.10,84.75,64.56,61.93$, 20.51, 13.98. IR (NaCl) 3287, 3030, 3014, 2986, 2232, 1748, 1454, 1370, 1319, 1240, 1172 , 1134, $1065 \mathrm{~cm}^{-1}$. HRMS (ESI-TOF) $m / z$ calcd. for $\mathrm{C}_{19} \mathrm{H}_{17} \mathrm{NO}_{4} \mathrm{~S}\left[\mathrm{M}+\mathrm{H}^{+}\right]$356.0957, found 356.0952. HPLC conditions: DAICEL CHIRALCEL AD-3, eluent: Hexane/2-Propanol 67.0/33.0, flow: $1.0 \mathrm{~mL} / \mathrm{min}$, detection: $254 \mathrm{~nm}, t_{\mathrm{R}}: 10.54 \mathrm{~min}$ (major), $12.19 \mathrm{~min}$ (minor). $[\alpha]^{25}=-50.9\left(\mathrm{c} 1.16, \mathrm{CHCl}_{3}\right)$.

Ethyl (S)-3-(4-phenylbut-1-yn-1-yl)-2,3-dihydrobenzo[d]isothiazole-3-carboxylate 1,1-dioxide (12h)

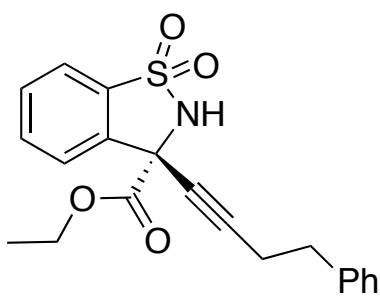

Reaction was performed according to the general procedure, and the mixture was purified using hexane/AcOEt $=4 / 1$ to $1 / 1$ as eluent to give $\mathbf{1 2 h}$ as a colorless oil (34.7 $\mathrm{mg}$, 94\% yield, $82 \%$ ee).

${ }^{1} \mathrm{H}$ NMR (500 MHz, $\left.\mathrm{CDCl}_{3}\right) \delta 7.75(\mathrm{~d}, J=7.5 \mathrm{~Hz}, 1 \mathrm{H}), 7.70$ $(\mathrm{d}, J=7.5 \mathrm{~Hz}, 1 \mathrm{H}), 7.66(\mathrm{td}, J=7.5,1.0 \mathrm{~Hz}, 1 \mathrm{H}), 7.60(\mathrm{td}, J=7.5$, $1.0 \mathrm{~Hz}, 1 \mathrm{H}), 7.28-7.16(\mathrm{~m}, 5 \mathrm{H}), 5.70(\mathrm{br}, 1 \mathrm{H}), 4.31$ (q, J=7.0 Hz, 2H), 2.81 (t, $J=7.5 \mathrm{~Hz}$, 2H), $2.52(\mathrm{t}, J=7.5 \mathrm{~Hz}, 2 \mathrm{H}), 1.31(\mathrm{t}, J=7.0 \mathrm{~Hz}, 3 \mathrm{H}) ;{ }^{13} \mathrm{C}\left\{{ }^{1} \mathrm{H}\right\} \mathrm{NMR}\left(125 \mathrm{MHz}, \mathrm{CDCl}_{3}\right) \delta$ $167.00,140.03,136.35,134.16,133.67,130.81,128.51,128,38,126.42,126.11,121.14$, 
86.21, 64.28, 61.41, 34.22, 20.83, 13.89, 0.00. IR (NaCl) 3279, 3021, 1746, 1454, 1368, $1319,1215,1173,1057,1022 \mathrm{~cm}^{-1}$. HRMS (ESI-TOF) $\mathrm{m} / z$ calcd. for $\mathrm{C}_{20} \mathrm{H}_{19} \mathrm{NO}_{4} \mathrm{~S}[\mathrm{M}+$ $\left.\mathrm{Na}^{+}\right]$392.0927, found 392.0925. HPLC conditions: DAICEL CHIRALCEL AD-3, eluent: Hexane/2-Propanol 67.0/33.0, flow: $1.0 \mathrm{~mL} / \mathrm{min}$, detection: $220 \mathrm{~nm}, t_{\mathrm{R}}: 11.94 \mathrm{~min}$ (major), $14.72 \min$ (minor). $[\alpha]_{\mathrm{D}}^{25}=-37.1$ (c $\left.1.37, \mathrm{CHCl}_{3}\right)$.

\section{Ethyl (S)-3-(cyclopropylethynyl)-2,3-dihydrobenzo[d]isothiazole-3-carboxylate}

\section{1,1-dioxide (12i)}

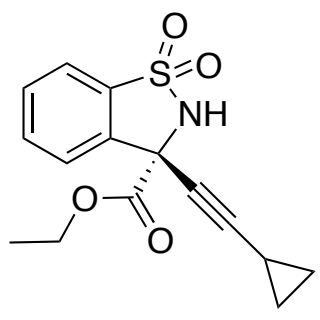

Reaction was performed according to the general procedure, and the mixture was purified using hexane/ $\mathrm{AcOEt}=4 / 1$ to $2 / 1$ as eluent to give $\mathbf{1 2} \mathbf{i}$ as a colorless oil ( $30.0 \mathrm{mg}, 98 \%$ yield, $84 \%$ ee).

${ }^{1} \mathrm{H}$ NMR $\left(500 \mathrm{MHz}, \mathrm{CDCl}_{3}\right) \delta 7.83(\mathrm{~d}, J=8.0 \mathrm{~Hz}, 1 \mathrm{H}), 7.74(\mathrm{~d}, J$ $=8.0 \mathrm{~Hz}, 1 \mathrm{H}), 7.69(\mathrm{t}, J=8.0 \mathrm{~Hz}, 1 \mathrm{H}), 7.61(\mathrm{t}, J=7.5 \mathrm{~Hz}, 1 \mathrm{H}), 5.71$

(br, 1H), 4.35-4.32 (m, 2H), 1.34 (t, J=7.5 Hz,3H), $1.26(\mathrm{~m}, 1 \mathrm{H}), 0.80$ (m, 2H), 0.71 (m, $2 \mathrm{H}) ;{ }^{13} \mathrm{C}\left\{{ }^{1} \mathrm{H}\right\}$ NMR $\left(125 \mathrm{MHz}, \mathrm{CDCl}_{3}\right) \delta 167.64,137.00,134.69,134.27,131.36,126.66$, $121.68,90.63,71.31,64.80,62.12,14.45,9.00,0.00$. IR (NaCl) 3619, 3343, 3285, 3027, 2980, 2243, 1746, 1454, 1358, 1317, 1244, 1221, 1171, $1065 \mathrm{~cm}^{-1}$. HRMS (ESI-TOF) $\mathrm{m} / \mathrm{z}$ calcd. For $\mathrm{C}_{15} \mathrm{H}_{15} \mathrm{NO}_{4} \mathrm{~S}\left[\mathrm{M}+\mathrm{H}^{+}\right]$306.0800, found 306.0798. HPLC conditions: DAICEL CHIRALCEL AD-3, eluent: Hexane/2-Propanol 67.0/33.0, flow: $1.0 \mathrm{~mL} / \mathrm{min}$, detection: $254 \mathrm{~nm}, t_{\mathrm{R}}: 10.08 \mathrm{~min}$ (major), $14.60 \mathrm{~min}$ (minor). $[\alpha]_{\mathrm{D}}^{25}=-53.9\left(\mathrm{c} 1.20, \mathrm{CHCl}_{3}\right)$.

Table S14. Additional Results for the Screening of Rh(III) Catalysts
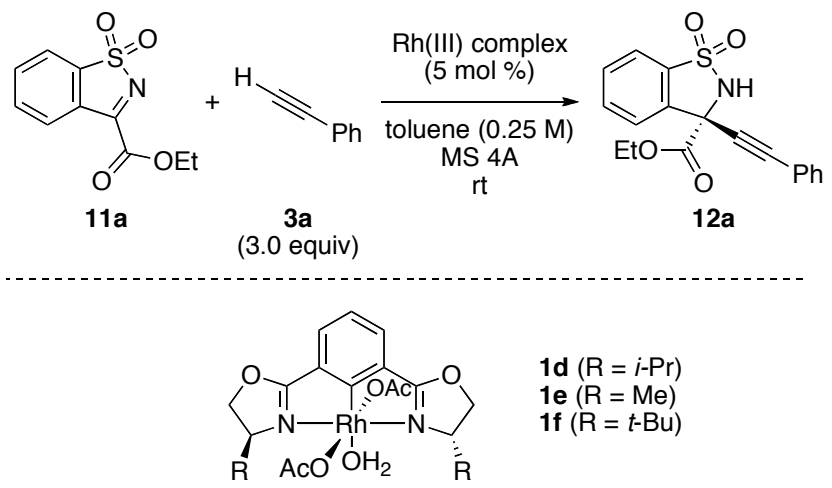


\begin{tabular}{|c|c|c|c|c|}
\hline entry & $\begin{array}{c}\text { Rh(III) } \\
\text { complex }\end{array}$ & $\begin{array}{c}\text { time } \\
(\mathrm{h})\end{array}$ & $\begin{array}{c}\text { yield } \\
(\%)^{a}\end{array}$ & $\begin{array}{c}\text { ee } \\
(\%)^{b}\end{array}$ \\
\hline 1 & 1d & 43 & 12 & 79 \\
\hline 2 & 1e & 45 & 16 & 69 \\
\hline 3 & 1f & 45 & 10 & 51 \\
\hline
\end{tabular}

${ }^{a}$ Yield was determined by ${ }^{1} \mathrm{H}$ NMR analysis of the crude mixture. ${ }^{b}$ Enantiomeric excess was determined by HPLC analysis with chiral stationary phase.

\section{Determination of Absolute Configuration of Products}

\section{Determination of Absolute Configuration of the Product 10a}

Absolute configuration of the product 10a was determined to be $(S)$ by single crystal X-ray crystallography of 10a. The single crystal was obtained by crystallization from hot hexane. The molecular structure of $\mathbf{1 0 a}$ with thermal ellipsoids at $50 \%$ probability was shown Figure S14. The crystal data and data collection parameters for 10a were summarized in Table S15.

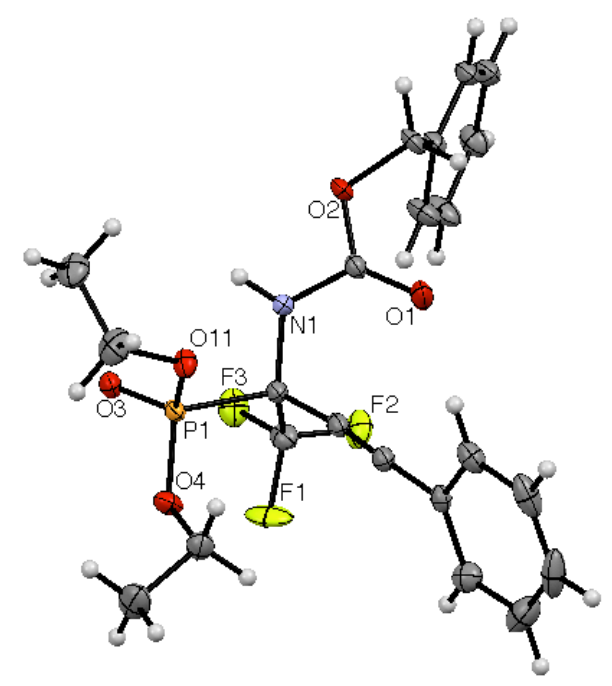

Figure S14. X-ray crystal structure of 10a.

\section{Determination of Absolute Configuration of the Product 12a}

Absolute configuration of the product 12a was determined to be $(S)$ by single crystal 
X-ray crystallography after conversion to the corresponding amide 14a as described below. $^{12}$

A test tube equipped with a magnetic stir bar and $\mathrm{La}(\mathrm{OTf})_{3}(5.9 \mathrm{mg}, 0.10 \mathrm{mmol}, 5.0$ mol \%) was dried under vacuum using a heat gun, and refilled with argon. To the tube were added a $0.50 \mathrm{M}$ solution of $\mathbf{1 2 \mathrm { a }}$ in toluene $(0.40 \mathrm{~mL}, 0.20 \mathrm{mmol})$ and $p$-methoxybenzylamine ( $39 \mu \mathrm{L}, 0.30 \mathrm{mmol}, 1.5$ equiv). The mixture was stirred at room temperature for $12.5 \mathrm{~h}$, and the crude mixture was directly purified by flash silica gel column chromatography to give 14a as white solid (56.4 mg, $65 \%$ yield, $84 \%$ ee).

$(S)-N$-(4-methoxybenzyl)-3-(phenylethynyl)-2,3-dihydrobenzo[d]isothiazole-3-carboxa mide 1,1-dioxide (14a)

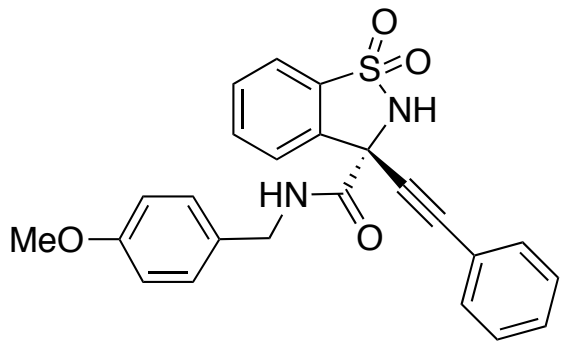

${ }^{1} \mathrm{H}$ NMR $\left(500 \mathrm{MHz}, \mathrm{CDCl}_{3}\right) \delta 8.03(\mathrm{~d}, J=8.0 \mathrm{~Hz}$, $1 \mathrm{H}), 7.74-7.68(\mathrm{~m}, 3 \mathrm{H}), 7.60(\mathrm{t}, J=7.5 \mathrm{~Hz}, 1 \mathrm{H}), 7.50(\mathrm{t}$, $J=5.5 \mathrm{~Hz}, 1 \mathrm{H}), 7.41(\mathrm{~d}, J=7.0 \mathrm{~Hz}, 2 \mathrm{H}), 7.35$ (t, $J=7.5$ $\mathrm{Hz}, 1 \mathrm{H}), 7.30-7.25(\mathrm{~m}, 3 \mathrm{H}), 7.17$ (d, $J=8.5 \mathrm{~Hz}, 2 \mathrm{H})$, $6.83(\mathrm{~d}, J=8.5 \mathrm{~Hz}, 1 \mathrm{H}), 5.72(\mathrm{br}, 1 \mathrm{H}), 4.48-4.29(\mathrm{~m}$, $2 \mathrm{H}), 3.77(\mathrm{~s}, 3 \mathrm{H}) ;{ }^{13} \mathrm{C}\left\{{ }^{1} \mathrm{H}\right\}$ NMR $\left(125 \mathrm{MHz}, \mathrm{CDCl}_{3}\right) \delta$ 165.80, 159.14, 137.94, 134.07, 133.60, 132.07, 130.67, 129.51, 128.34, 126.53, 120.77, 120.63, 114.13, 86.33, 84.40, 62.66, 55.23, 43.93. IR (NaCl) 3410, 3335, 3009, 2959, 2236, $1694,1614,1514,1456,1370,1312,1252,1211,1175,1132,1036,827 \mathrm{~cm}^{-1}$. HRMS (ESI-TOF) $\mathrm{m} / \mathrm{z}$ calcd. for $\mathrm{C}_{24} \mathrm{H}_{21} \mathrm{~N}_{2} \mathrm{O}_{4} \mathrm{~S}\left[\mathrm{M}+\mathrm{H}^{+}\right]$433.1202, found 433.1202. HPLC conditions: DAICEL CHIRALCEL AS-3, eluent: Hexane/2-Propanol 67.0/33.0 + 0.1\% $\mathrm{CF}_{3} \mathrm{CO}_{2} \mathrm{H}$, flow: $1.0 \mathrm{~mL} / \mathrm{min}$, detection: $254 \mathrm{~nm}, t_{\mathrm{R}}: 19.02 \mathrm{~min}$ (major), $41.59 \mathrm{~min}$ (minor). $[\alpha]^{25}=-107.9\left(\mathrm{c} 0.44, \mathrm{CHCl}_{3}\right)$.

The single crystal of amide 14a was obtained by crystallization from hexane/EtOAc. The molecular structure of $14 \mathrm{a}$ with thermal ellipsoids at $50 \%$ probability was shown Figure S15. The crystal data and data collection parameters for 14a were summarized in Table S15. 


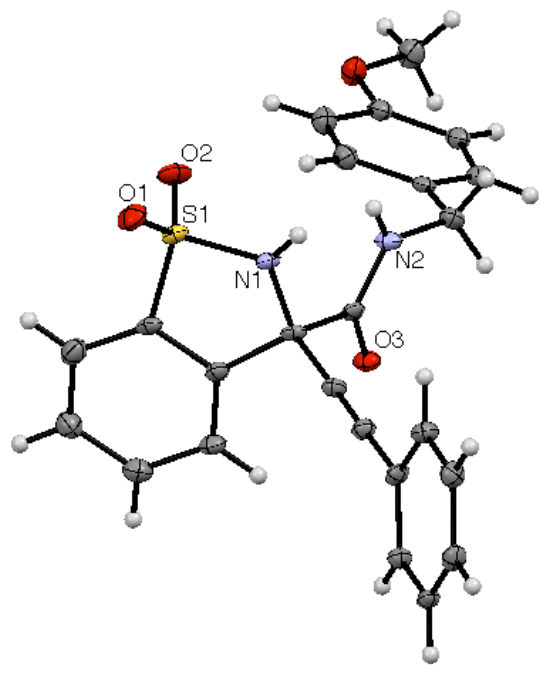

Figure S15. X-ray crystal structure of 14a. 
Table S15. Crystal Data and Data Collection Parameters for 10a and 14a

\begin{tabular}{|c|c|c|}
\hline compound & $10 a$ & $14 a$ \\
\hline empirical formula & $\mathrm{C}_{22} \mathrm{H}_{23} \mathrm{~F}_{3} \mathrm{NO}_{5} \mathrm{P}$ & $\mathrm{C}_{24} \mathrm{H}_{20} \mathrm{~N}_{2} \mathrm{O}_{4} \mathrm{~S}$ \\
\hline formula weight & 469.38 & 432.48 \\
\hline crystal system & $P 1$ (No. 1) & $P 122_{1} 1$ (No. 4) \\
\hline space group & triclinic & monoclinic \\
\hline$a, \AA$ & $9.548(2)$ & $5.7300(8)$ \\
\hline$b, \AA$ & $10.705(3)$ & $20.761(3)$ \\
\hline$c, \AA$ & $12.059(3)$ & $8.5902(12)$ \\
\hline$\alpha$, deg. & $87.745(3)$ & 90.00 \\
\hline$\beta$, deg. & $67.638(2)$ & $100.442(2)$ \\
\hline$\gamma$, deg. & $83.773(3)$ & 90.00 \\
\hline$V, \AA^{3}$ & $1133.1(5)$ & $1005.0(2)$ \\
\hline$Z$ & 2 & 2 \\
\hline$D_{\text {calcd }}, \mathrm{g} \mathrm{cm}^{-3}$ & 1.376 & 1.429 \\
\hline$\mu[\mathrm{Mo}-K \alpha], \mathrm{mm}^{-1}$ & 0.179 & 0.197 \\
\hline$T, \mathrm{~K}$ & 90 & 90 \\
\hline$\theta$ for data collection, deg. & $1.83-28.34$ & $1.96-28.42$ \\
\hline no. of reflections & 6338 & 5645 \\
\hline unique data $\left(R_{\text {int }}\right)$ & 0.0328 & 0.0492 \\
\hline data / restraints / parameters & $5490 / 3 / 581$ & $3984 / 1 / 280$ \\
\hline Flack parameter & $0.05(7)$ & $0.08(9)$ \\
\hline$R 1(I>2.0 \sigma(I))$ & 0.0395 & 0.0640 \\
\hline $\mathrm{w} R 2(I>2.0 \sigma(I))$ & 0.1029 & 0.1806 \\
\hline$R 1$ (all data) & 0.0396 & 0.0663 \\
\hline wR2 (all data) & 0.1030 & 0.1849 \\
\hline GOF on $F^{2}$ & 1.038 & 1.517 \\
\hline$\Delta \rho$, e $\AA^{-3}$ & $0.577,-0.565$ & $0.546,-0.522$ \\
\hline
\end{tabular}




\section{References}

${ }^{1}$ (a) Kanazawa, Y.; Tsuchiya, Y.; Kobayashi, K.; Shiomi, T.; Itoh, J.-i.; Kikuchi, M.; Yamamoto, Y.; Nishiyama, H. Chem. Eur. J. 2006, 12, 63. (b) Shiomi, T.; Ito, J.-i.; Yamamoto, Y.; Nishiyama, H. Eur. J. Org. Chem. 2006, 5594.

${ }^{2}$ For $\alpha$-ketiminoester 2, see: (a) Bravo, P.; Fustero, S.; Guidetti, M.; Volonterio, A.; Zanda, M. J. Org. Chem. 1999, 64, 8731. (b) Burger, K.; Höß, E.; Gaa, K.; Sewald, N.;

Schierlinger, C. Z. Naturforsch. 1991, 46b, 361. For $\alpha$-ketiminophosphonate 9, see: (c) Karimova, N. M.; Vorobyeva, D. V.; Shchetnikov, G. T.; Osipov, S. N. Russ. Chem. Bull., Int. Ed. 2010, 59, 107. For cyclic $N$-sulfonyl $\alpha$-ketiminoesters 11, see: (d) Wang, H.; Jiang, T.; Xu, M.-H. J. Am. Chem. Soc. 2013, 135, 971.

${ }^{3}$ For 3j (Boc-indole), see: (a) Furuya, T.; Strom, A. E.; Ritter, T. J. Am. Chem. Soc. 2009, 131, 1662; (b) Naddo, T.; Che, Y.; Zhang, W.; Balakrishnan, K.; Yang, X.; Yen, M.; Zhao, J.; Moore, J. S.; Zang, L. J. Am. Chem. Soc. 2007, 129, 6978; (c) Abbott Laboratories, WO2012/83170 A1, 2012. For 3k (formyl), see: (d) Ohshima, T.; Kawabata, T.; Takeuchi, Y.; Kakinuma, T.; Iwasaki, T.; Yonezawa, T.; Murakami, H.; Nishiyama, H.; Mashima, K. Angew. Chem., Int. Ed. 2011, 50, 6296. For 31 (Fmoc), see: (e) Achaogen, Inc. WO2008/154642 A2, 2008. For 3q (Cbz), see: (f) Koenig, S. G.; Vandenbossche, C. P.; Zhao, H.; Mousaw, P.; Singh, S. P.; Bakale, R. P. Org. Lett. 2009, 11, 433. For 3r $\left(\mathrm{NO}_{2}\right)$, see: (g) Barber, D. M.; Sanganee, H. J.; Dixon, D. J. Org. Lett. 2012, 14, 5290.

${ }^{4}$ Ito, J.-i.; Kitase, M.; Nishiyama, H. Organometallics 2007, 26, 6412.

5 (a) Blackmond, D. G. Angew. Chem. Int. Ed. 2005, 44, 4302; (b) Mathew, J. S.; Klussman, M.; Iwamura, H.; Valera, F.; Futran, A.; Emanuelsson, E. A. C.; Blackmond, D. G. J. Org. Chem. 2006, 71, 4711. (c) Blackmond, D. G. J. Am. Chem. Soc. 2015, 137, 10852. (d) Baxter, R. D.; Sale, D.; Engle, K. M.; Yu, J.-Q.; Blackmond, D. G. J. Am. Chem. Soc. 2012, 134,4600 .

${ }^{6}$ (a) Becke, A. D. J. Chem. Phys., 1993, 98, 5648. (b) Perdew, J. P. in Electronic Structure of Solids '91, Ed. P. Ziesche and H. Eschring (Akademie Verlag, Berlin, 1991) 11.

${ }^{7}$ Gaussian 09, Revision C.01, Gaussian, Inc., Wallingford CT, 2010.

${ }^{8}$ Hay, P J.; Wadt, W. R. J. Chem. Phys. 1985, 82, 270.

${ }^{9}$ (a) Zhao, Y.; Truhlar, D. G. Theor. Chem. Acc. 2008, 120, 215. (b) Zhao, Y.; Truhlar, D. G. Acc. Chem. Res. 2008, 41, 157. (c) Zhao, Y.; Truhlar, D. G. J. Chem. Theory Comput. 2009, 5, 324. (d) Kulkarni,A. D.; Truhlar, D. G. J. Chem. Theory Comput. 2011, 7, 2325.

${ }^{10}$ (a) Andrae, D.; Häussermann, U.; Dolg, M.; Stoll, H.; Preuss, H.Theor. Chim. Acta 1990, 77, 123. (b) Roy, L. E.; Hay, P. J.; Martin, R.L. J. Chem. Theory Comput. 2008, 4, 1029.

${ }^{11}$ Marenich, A. V.; Cramer, C. J.; Truhlar, D. G. J. Phys, Chem. B 2009, 113, 6378.

${ }^{12}$ Morimoto, H.; Fujiwara, R.; Shimizu, Y.; Morisaki, K.; Ohshima, T. Org. Lett. 2014, 16, 2018. 


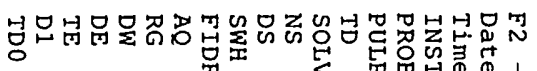

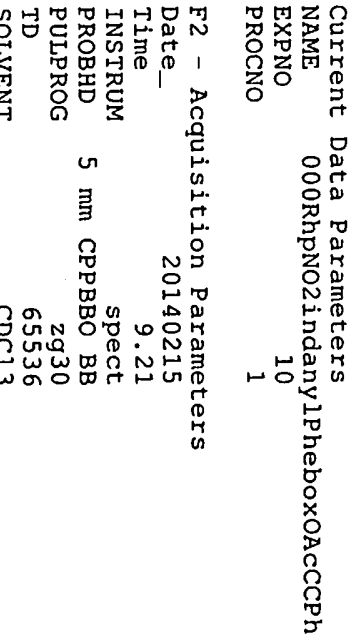

8.391

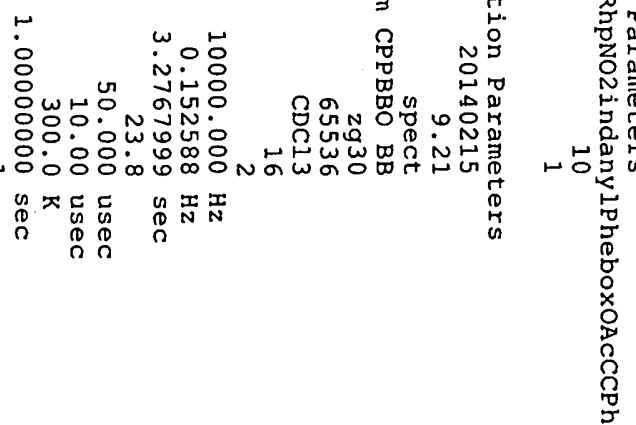

-8.387
-8.348

$-8.344$

$-8.147$

$-8.133$

$-7.899$

$-7.887$

$-7.883$

$-7.336$

7.332

7.325

$-7.318$

7.313

7.298

7.289

7.286

7.275

7.274

0.903

0.887

$0.982 \digamma \infty$

0.987

6.476

$2.962-V$

1.937

0.994

$0.993 \_\sigma$

1.018

1.000
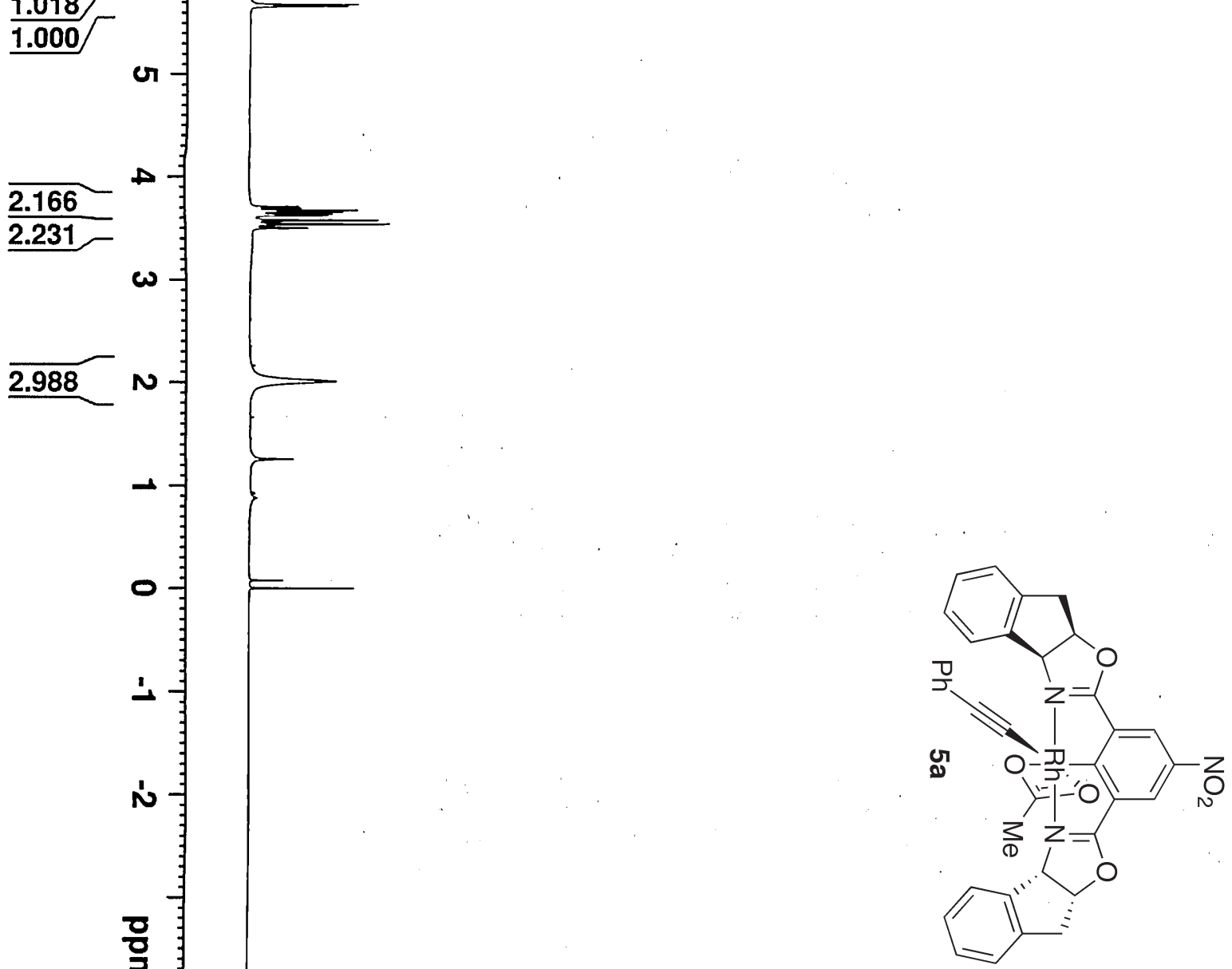

7.248

r 6.953

6.947

F.944

6.936

$-6.925$

$-6.921$

6.587

$3 \amalg-\begin{aligned} & 6.587 \\ & 6.583\end{aligned}$

6.571

6.568

6.080

6.075

6.063

6.058

6.047

6.042

5.998
5.984
5.971

5.968

5.968
-5.854

5.837

5.674

5.658

3.706

3.691

3.670

$-3.655$

3.634

$-3.620$

$-3.573$

$-3.538$

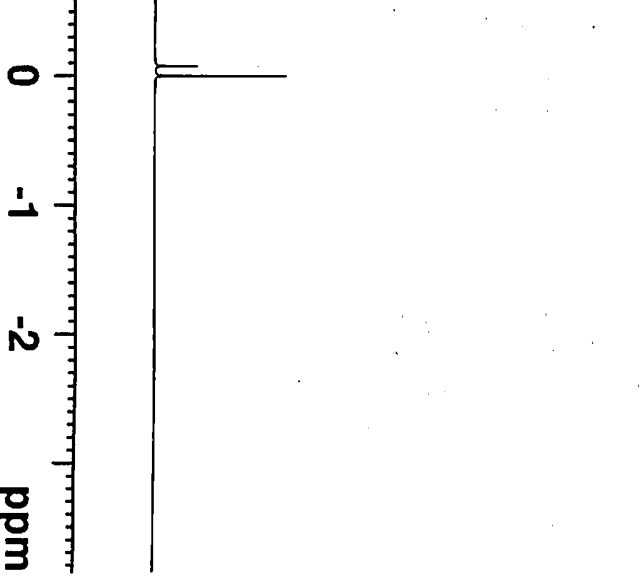

$-3.536$

3.502
3.499

2.007 


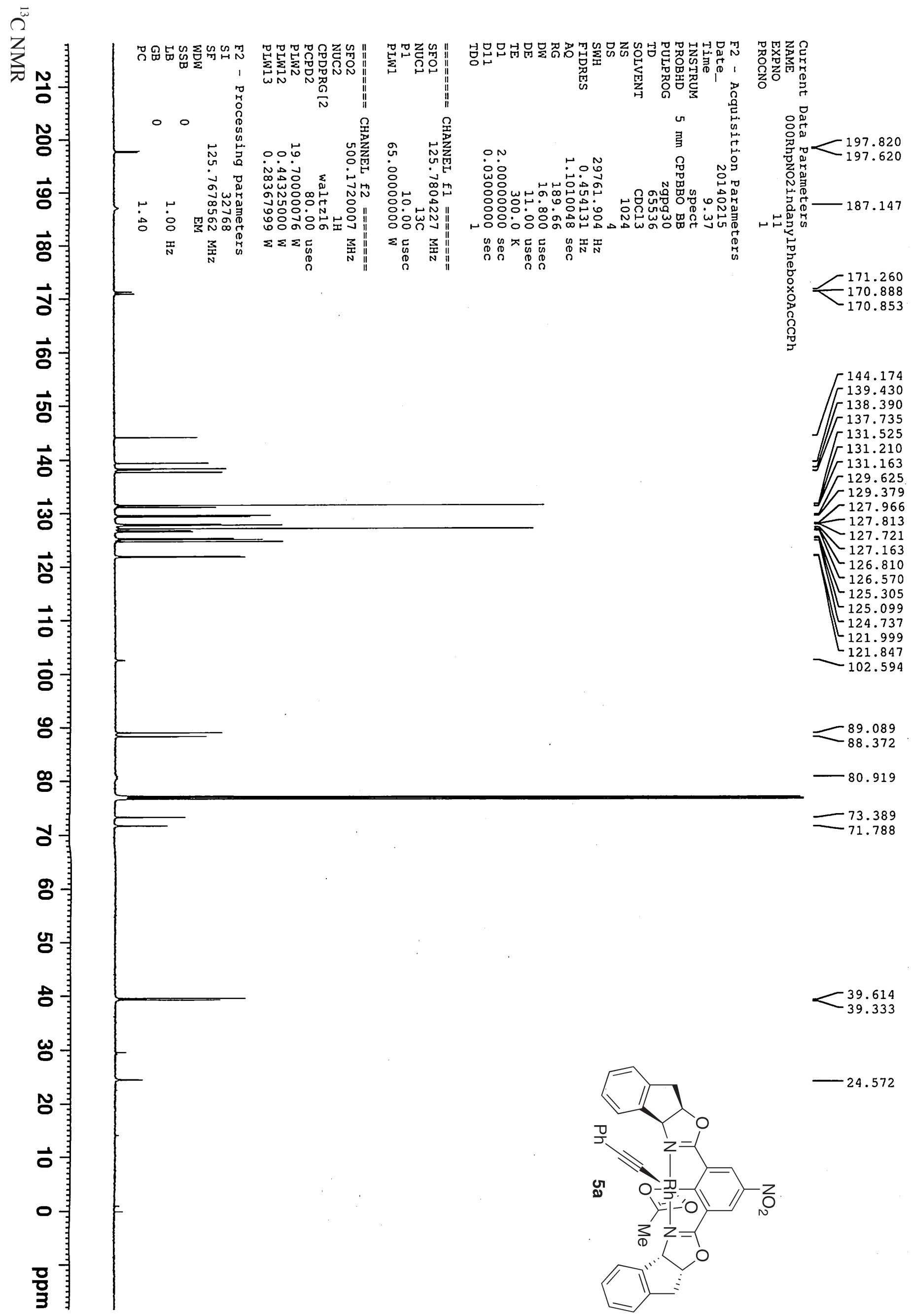




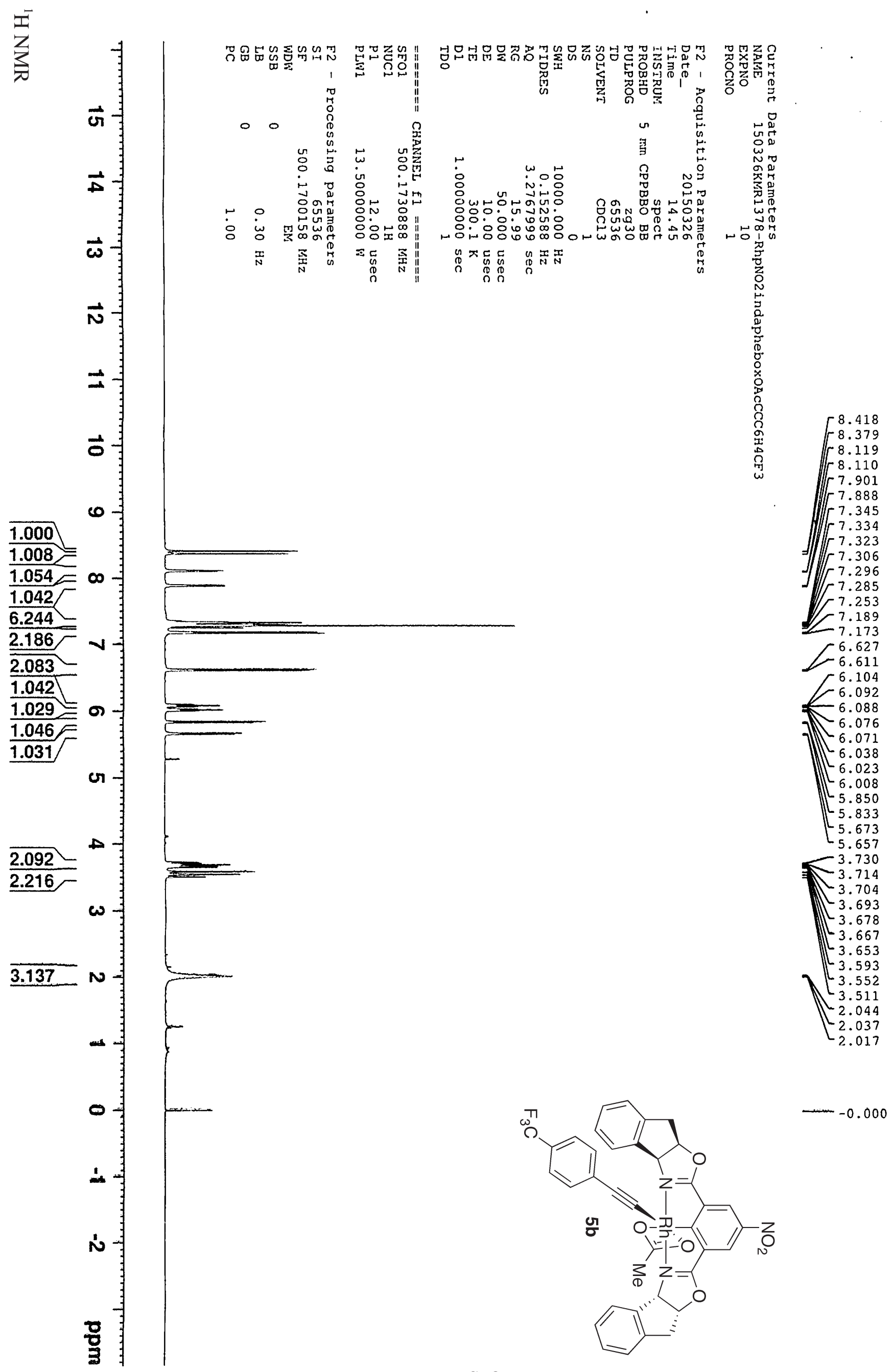




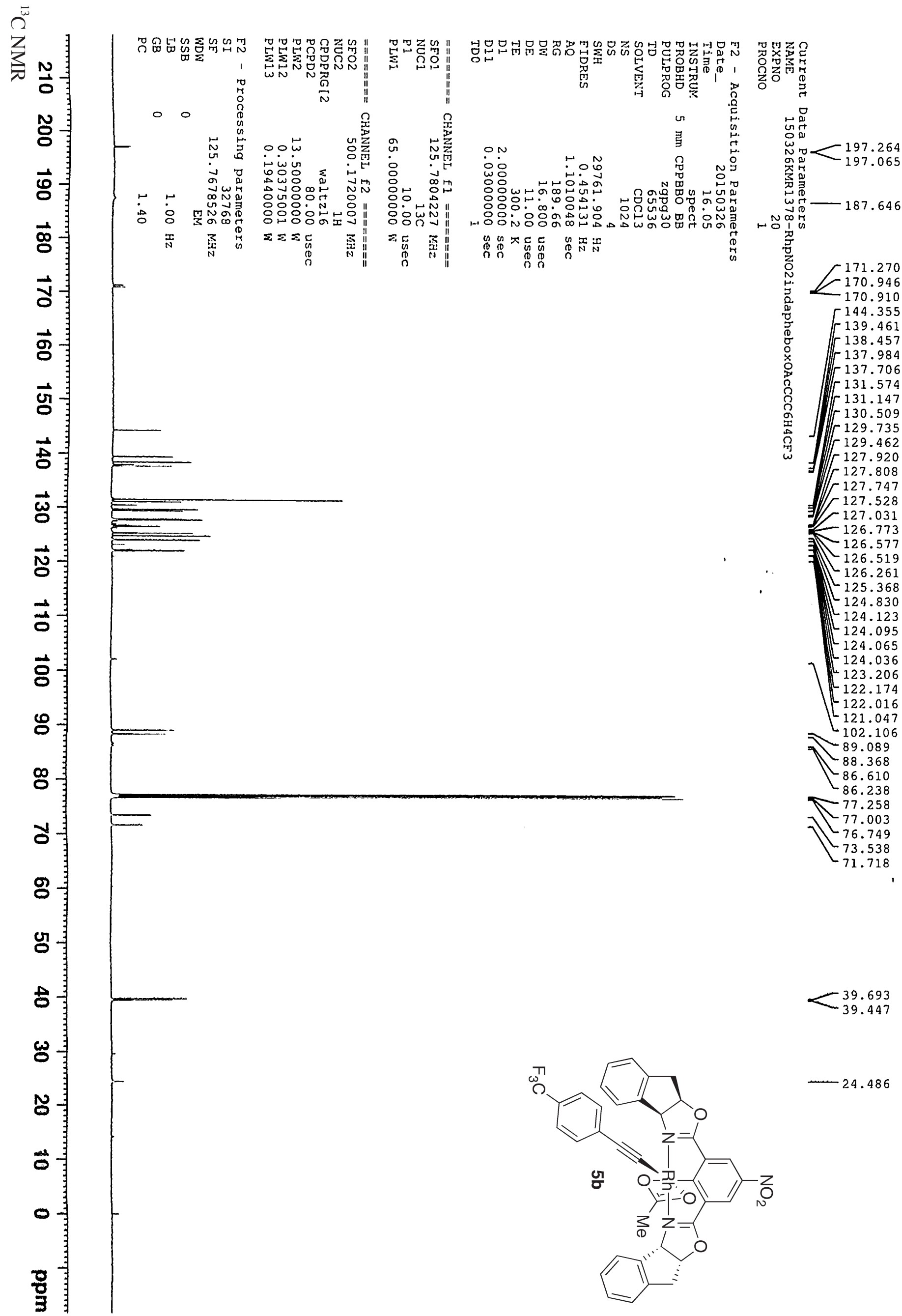




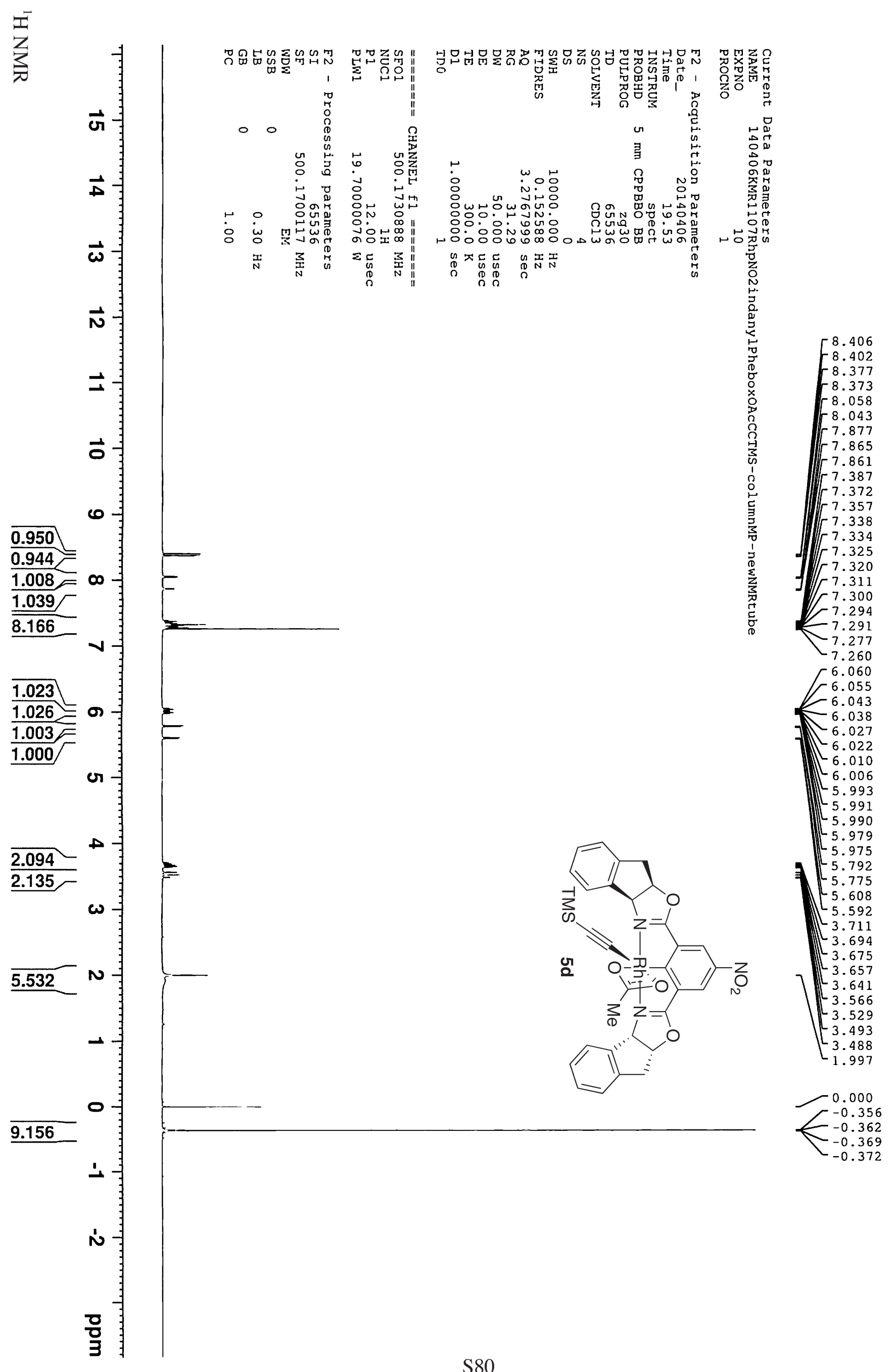




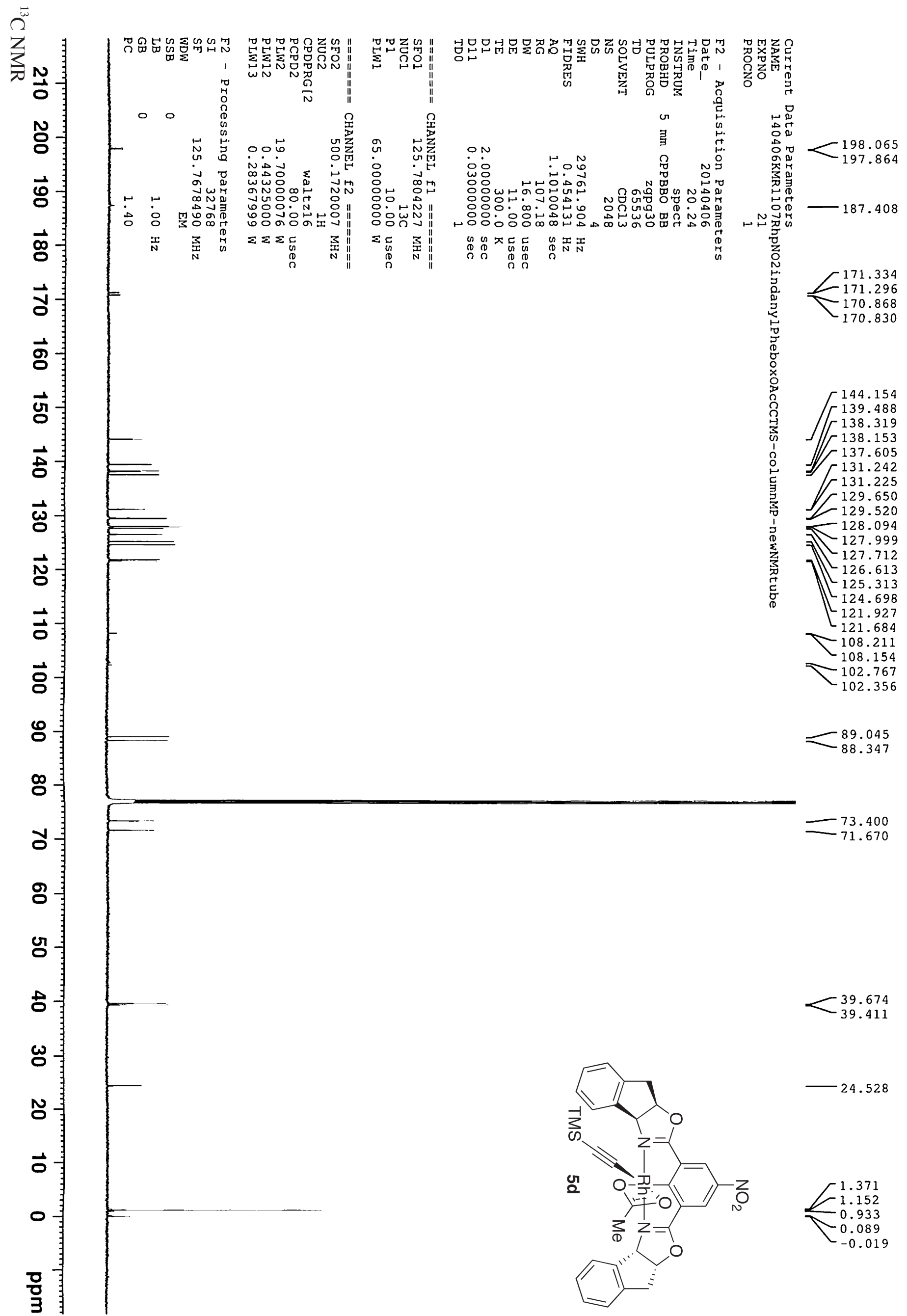


$\frac{1}{Z}$
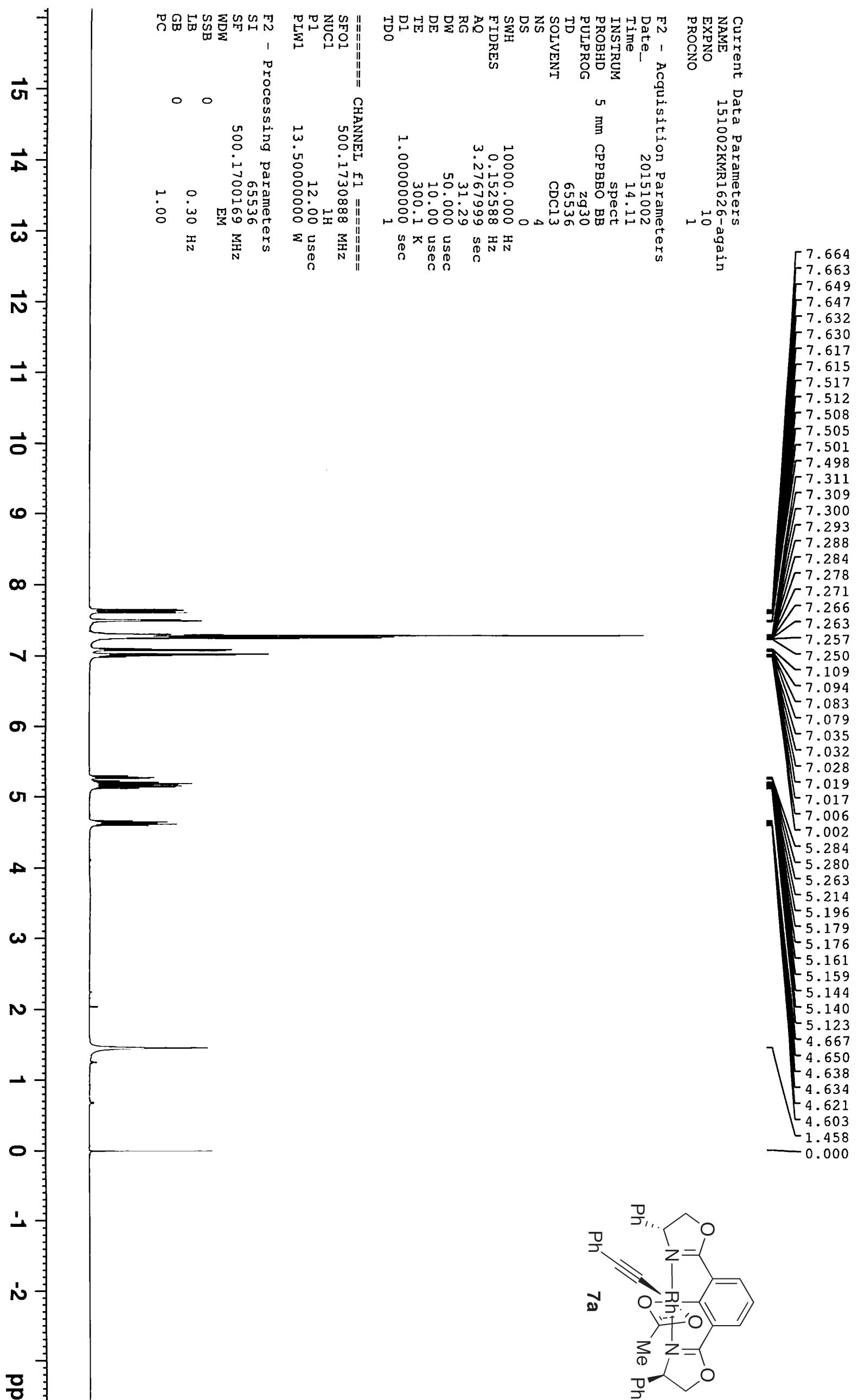


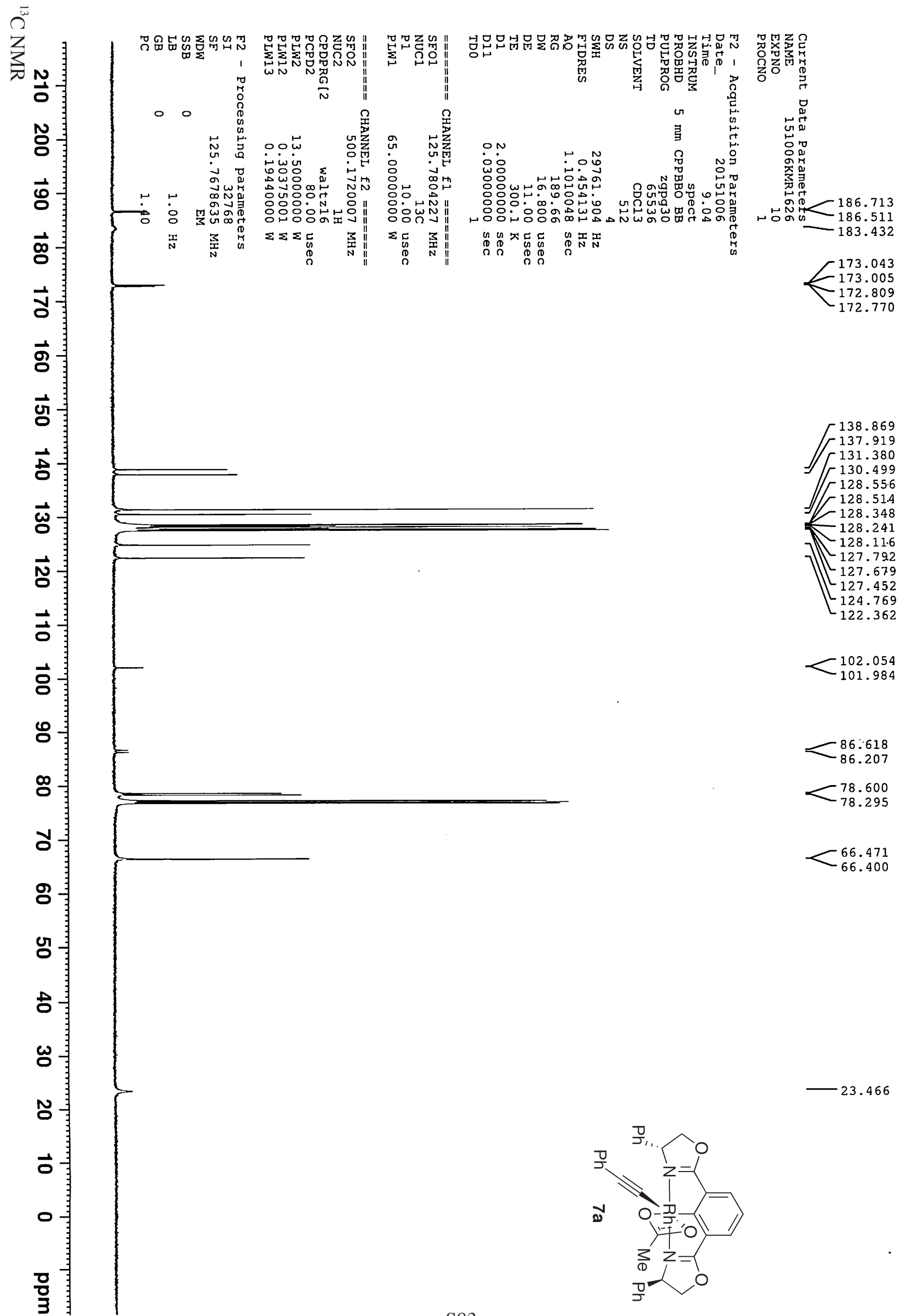




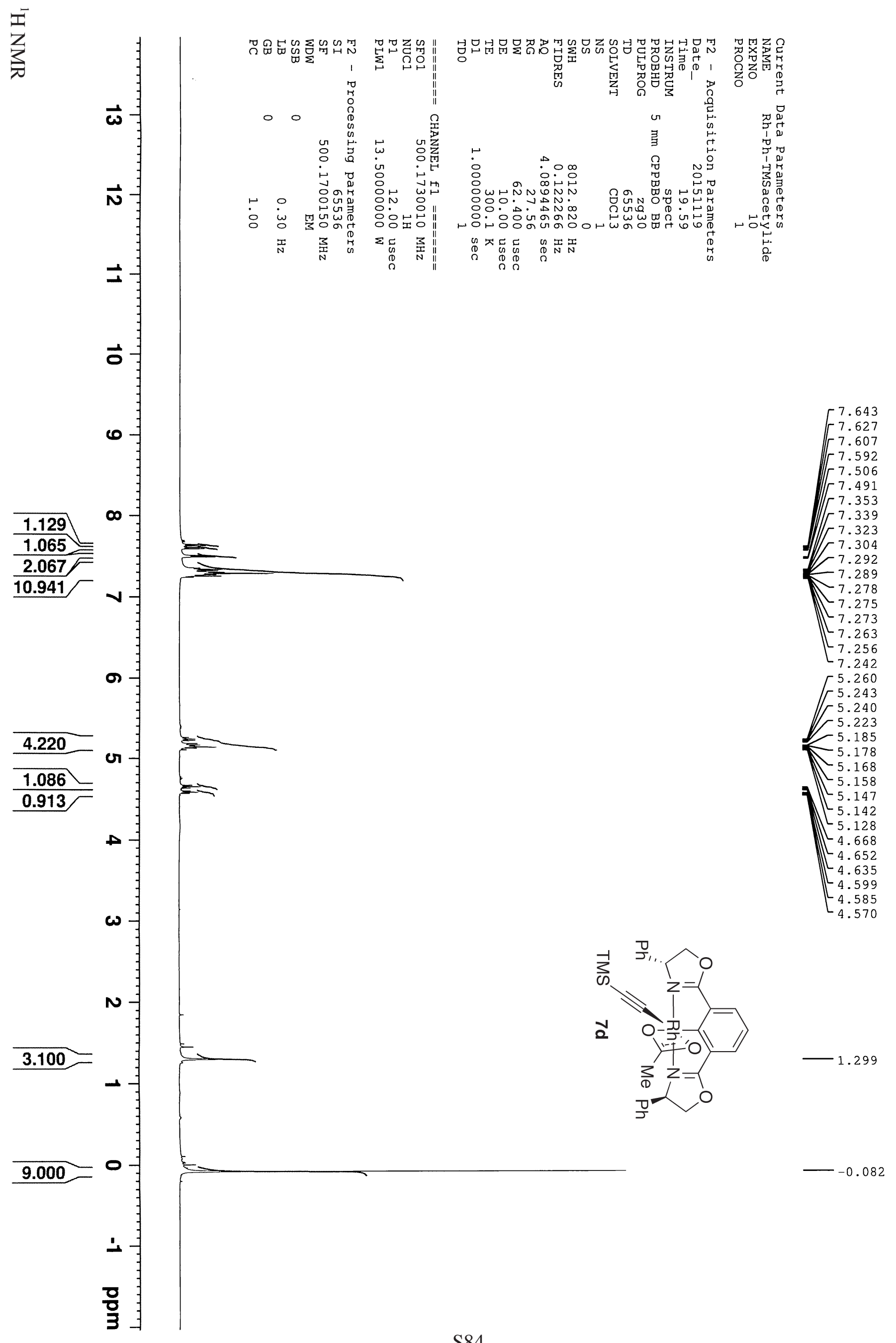




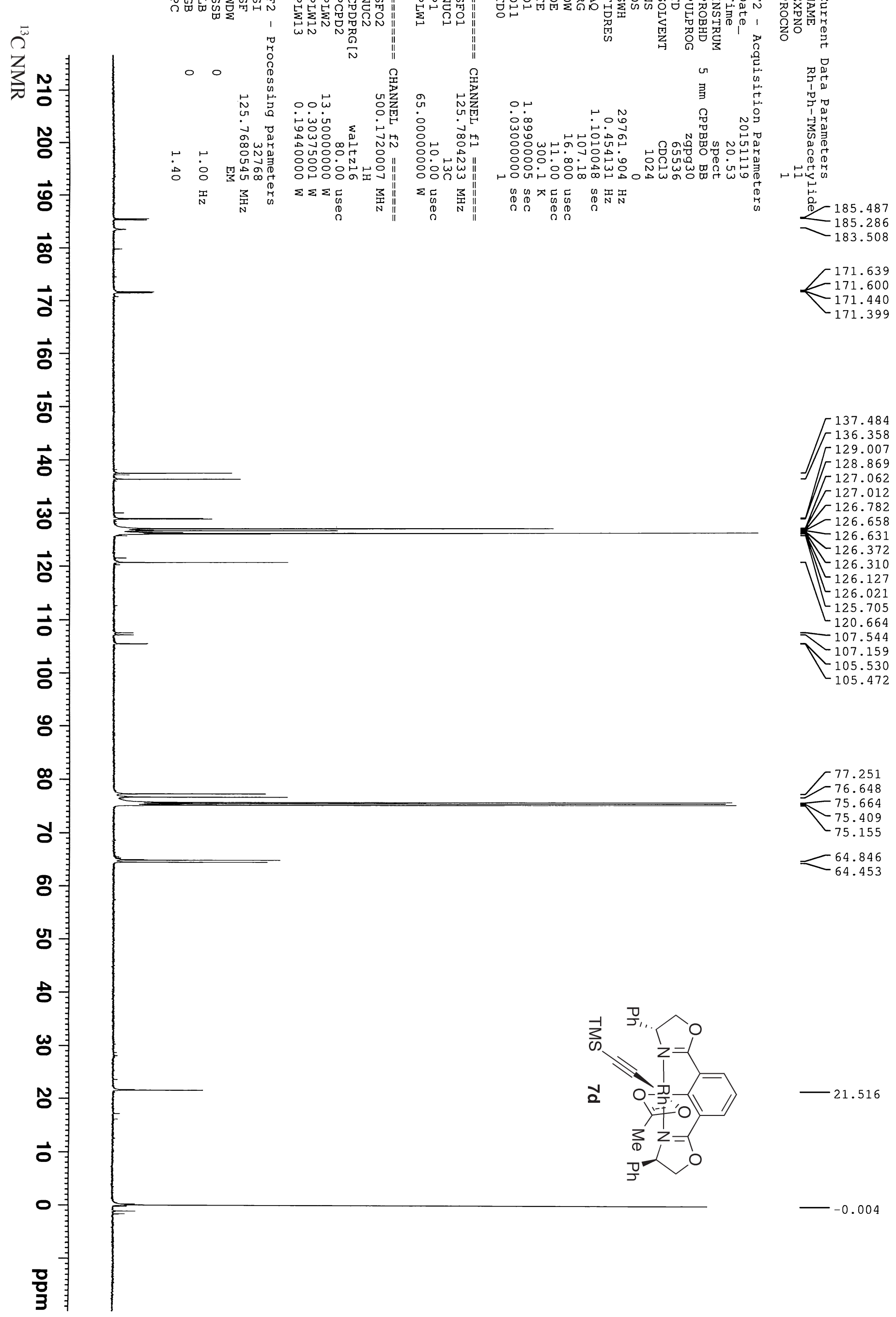




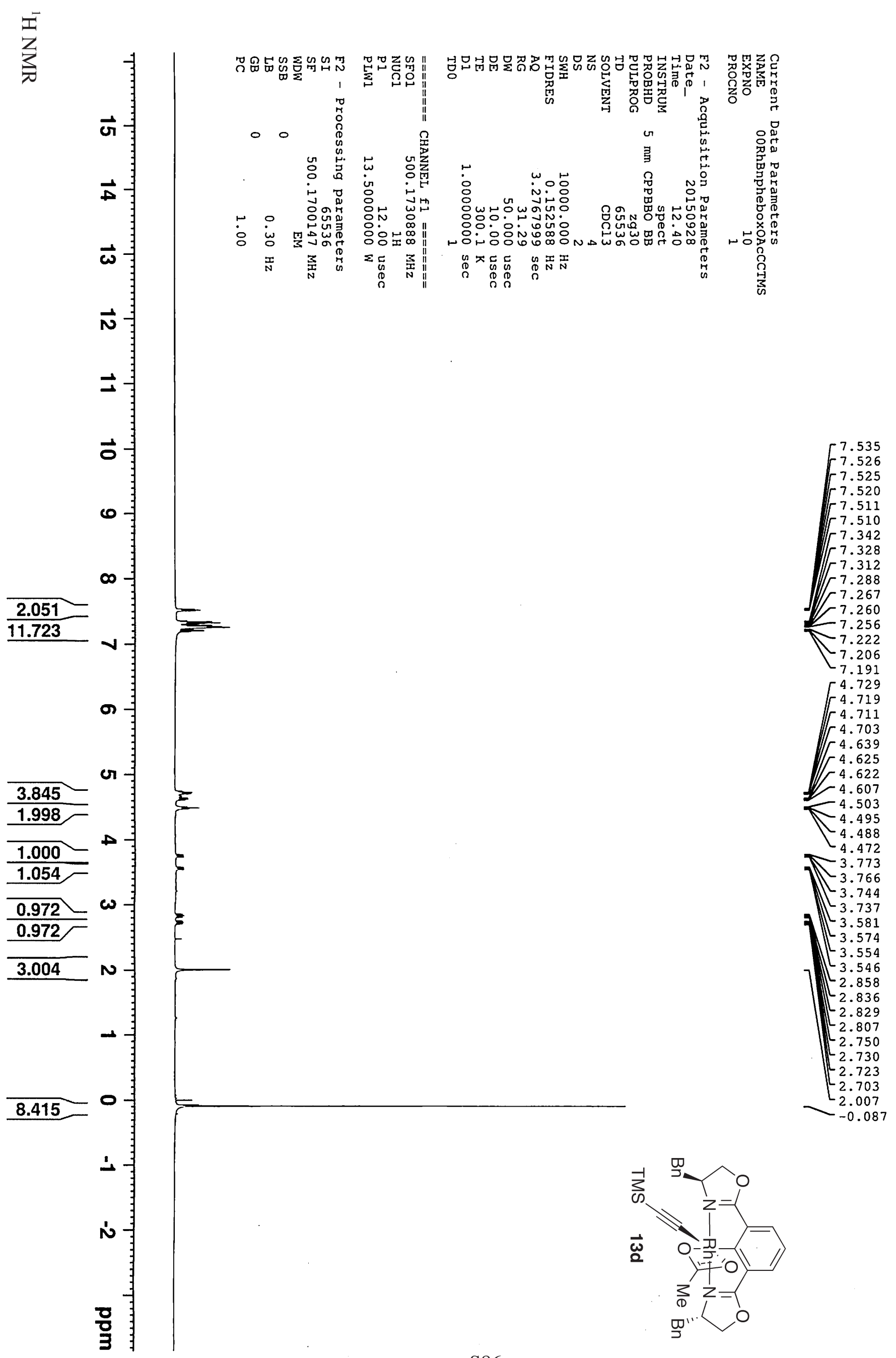




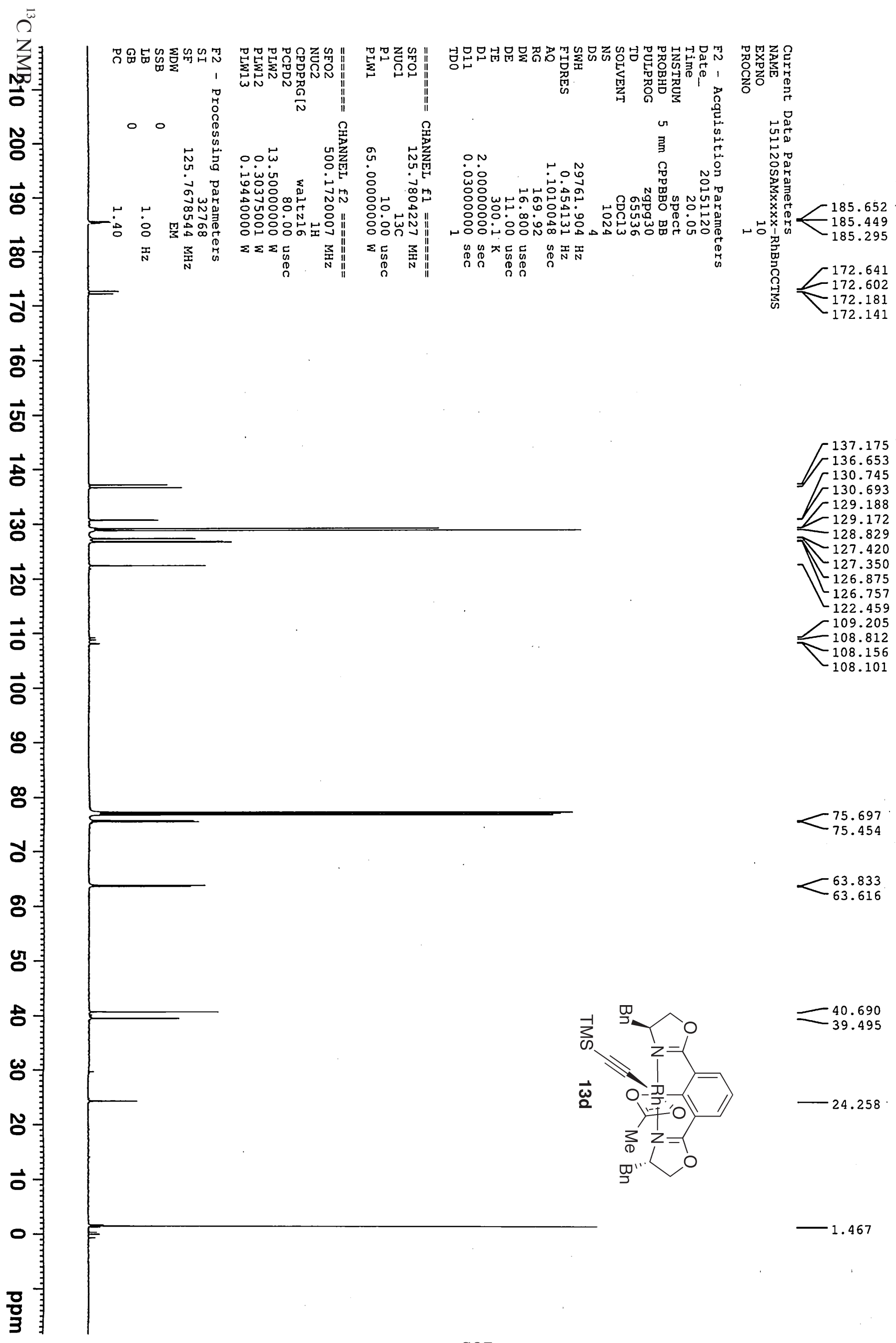


志

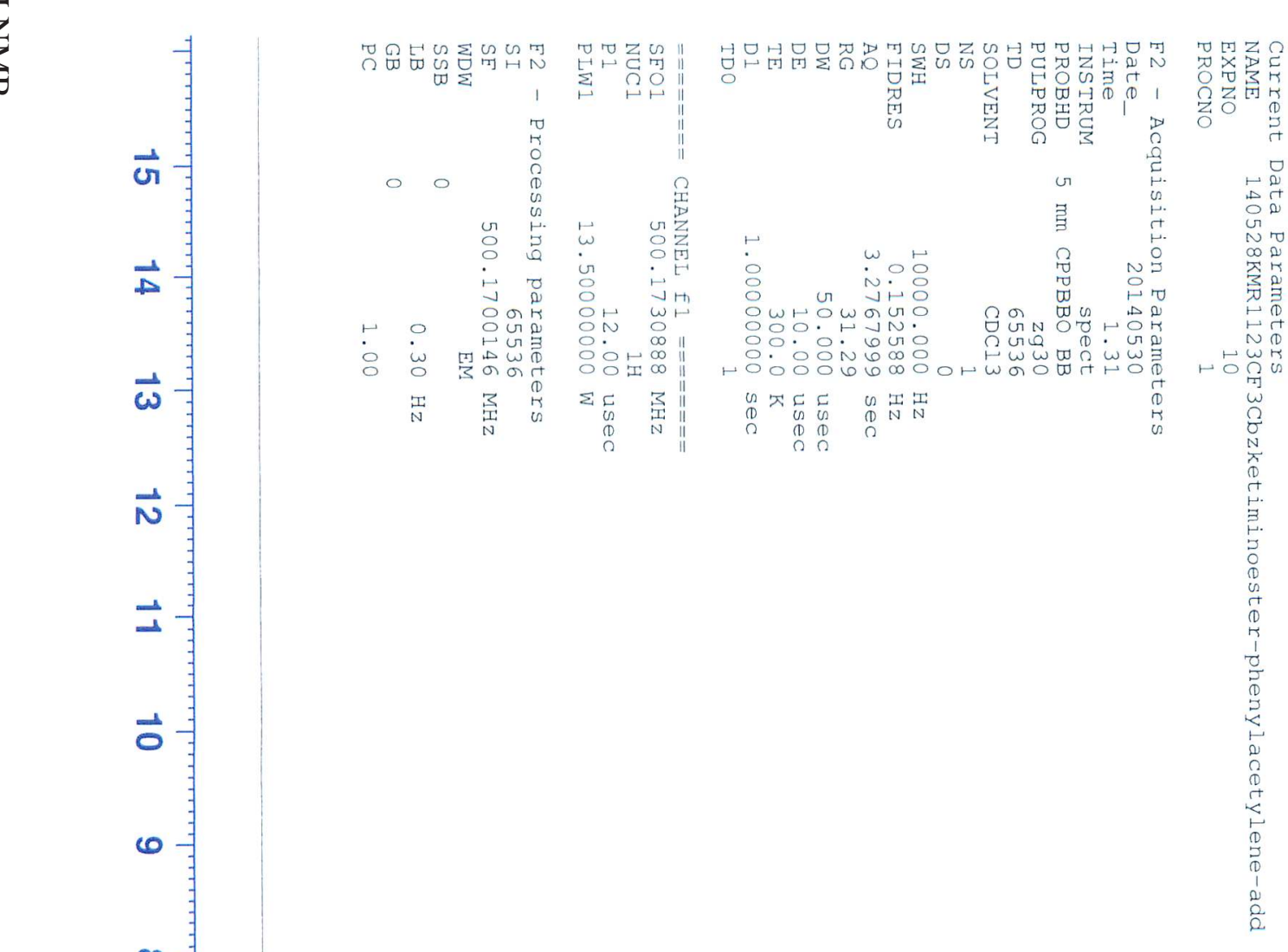

7.472

7.458

7.395

7.392

7.390

- 7.383

7.378

7.366

7.363

$\begin{array}{r}7.352 \\ -7.348 \\ \hline\end{array}$

2.016

7.907

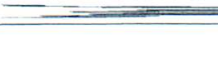

7.335

$-7.330$

7.325

7.322

7.311

7.308

7.306

$[7.255$

5.801
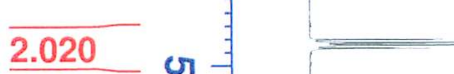

- 5.169

$-5.151$

5.126

$-4.387$

$-4.378$

4.373

4.364

4.359

$-4.350$

4.345

4.336

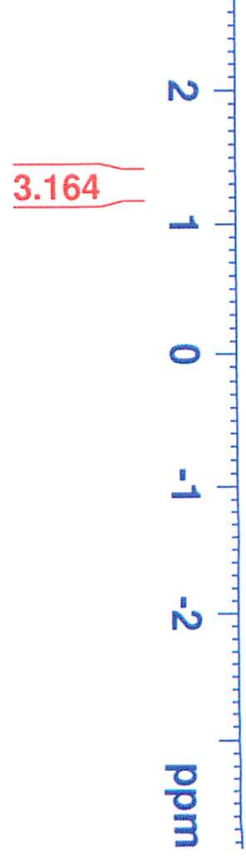

$-1.567$

1.332

$-1.318$

1.304

0.000

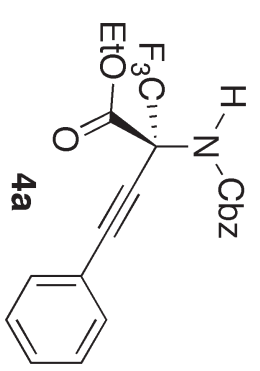




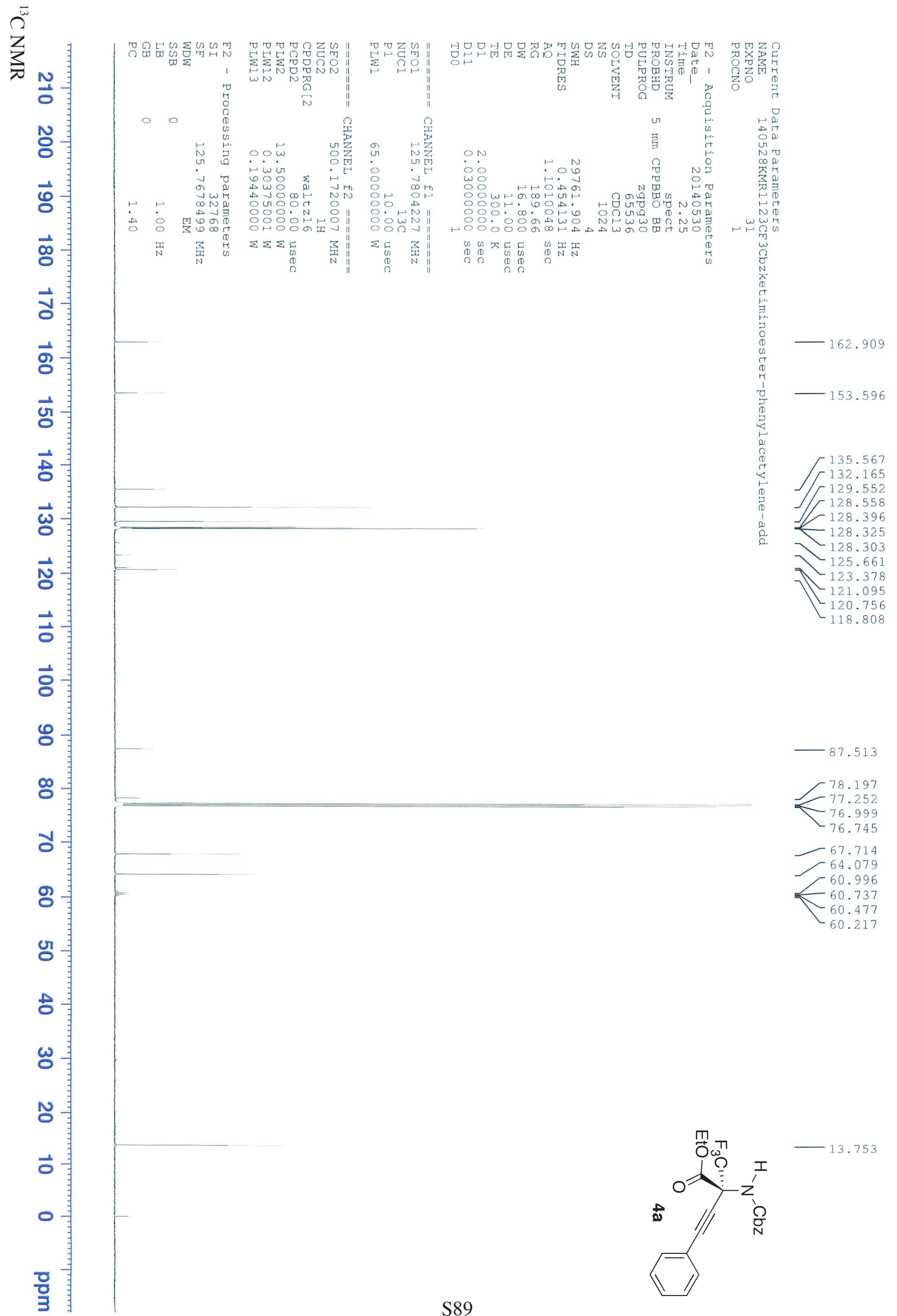


莫

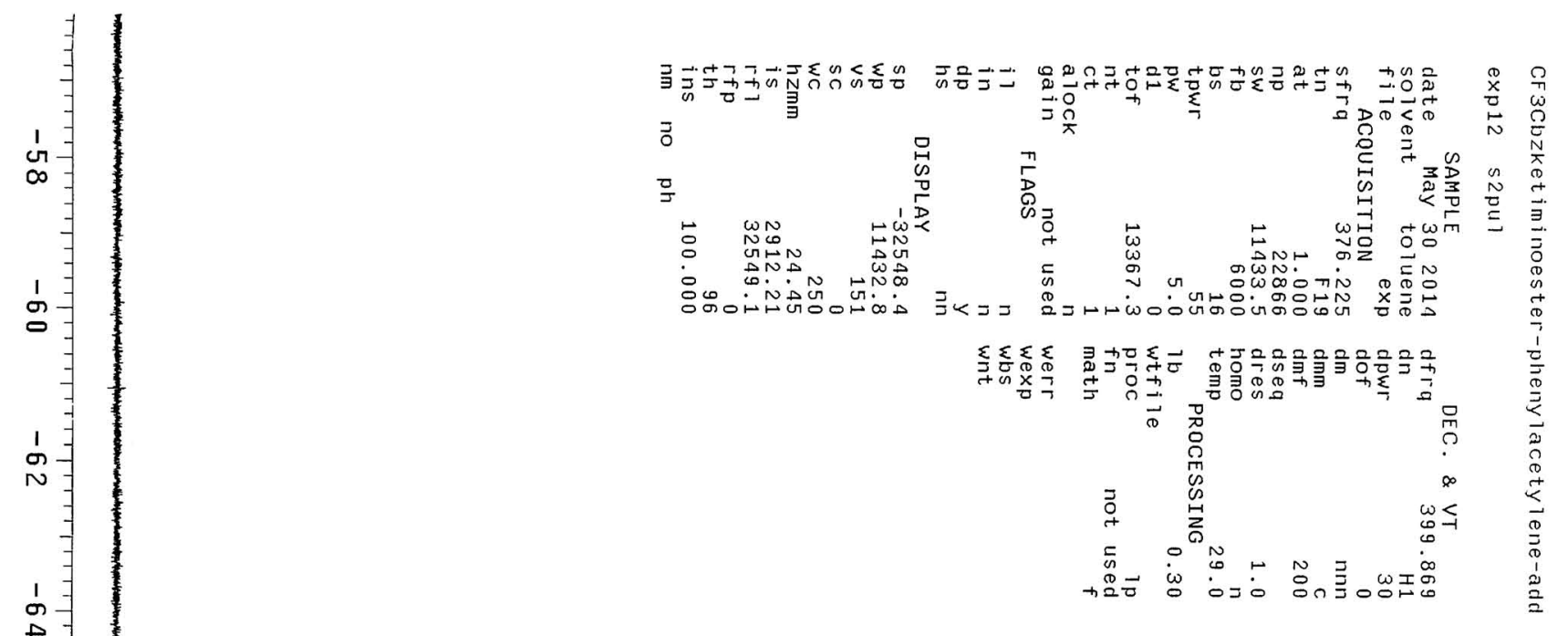

\%

1
5
$\infty$

ำ

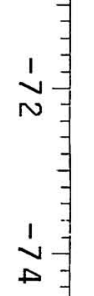

ขั

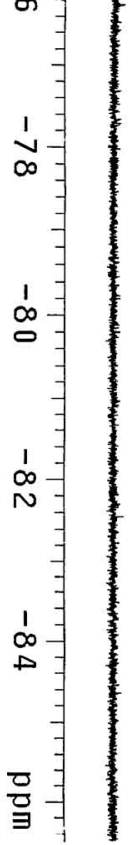

$-75.761$

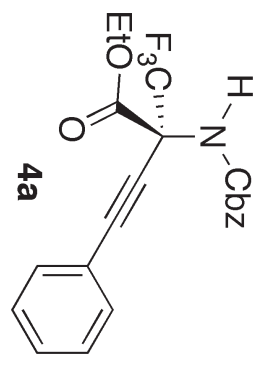




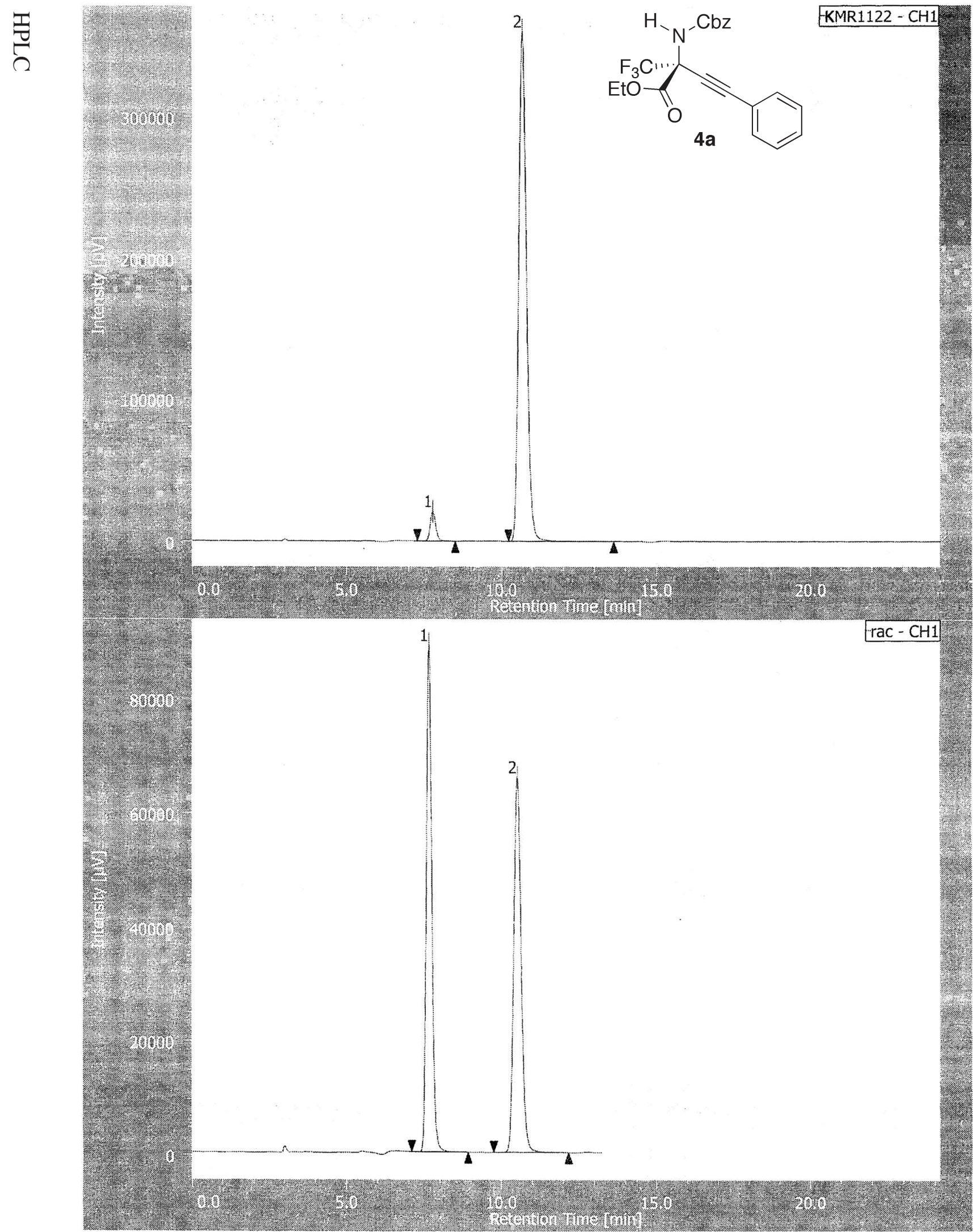

チャンネル情報+ピーク情報

クロマトグラム名

サンプル名

チャンネル名

KMR1122-CH1

\begin{tabular}{|c|c|c|c|c|c|c|c|c|c|c|c|}
\hline ピーク名 & $\mathrm{CH}$ & $\mathrm{tR}[\mathrm{min}]$ & 面積 $[\mu \mathrm{V} \cdot \mathrm{sec}]$ & 高さ $[\mu \mathrm{V}]$ & 面積\% & 高さ\% & 定量値 & NTP & 分離度 & シンメトリー係数 & 警告 \\
\hline Unknown & 1 & 7.767 & 258831 & 21106 & 3.957 & 5.543 & $N / A$ & 9604 & 7.565 & 1.167 & \\
\hline 2 Unknown & 1 & 10.675 & 6282089 & 359647 & \begin{tabular}{|c|}
96.043 \\
\end{tabular} & $\begin{array}{c}94.457 \\
\end{array}$ & $N / A$ & 8849 & $\mathrm{~N} / \mathrm{A}$ & 1.194 & \\
\hline
\end{tabular}

クロマトグラム名

rac- $\mathrm{CH} 1$

$92.09 \% \mathrm{ee}$.

サンプル名

チャンネル名

$\mathrm{CH} 1$

\begin{tabular}{|c|c|c|c|c|c|c|c|c|c|c|c|}
\hline ピーク名 & $\mathrm{CH}$ & $\mathrm{tR}[\mathrm{min}]$ & 面積 [ $\mathrm{V} \mathrm{V} \cdot \mathrm{sec}]$ & 高さ $[\mu \mathrm{V}]$ & 面積\% & 高さ\% & 定量値 & NTP & 分離度 & ジメトリー係数 & \begin{tabular}{|l|} 
鳘告 \\
\end{tabular} \\
\hline 1) Unknown & 1 & 7.633 & 1085325 & 88503 & \begin{tabular}{|c|}
49.747 \\
\end{tabular} & 57.381 & $N / A$ & 9248 & 7.692 & 1.226 & \\
\hline 2 Unknown & 1 & 10.508 & 1096375 & 65734 & 50.253 & 42.619 & $\mathrm{~N} / \mathrm{A}$ & 9466 & $N / A$ & 1.162 & \\
\hline
\end{tabular}




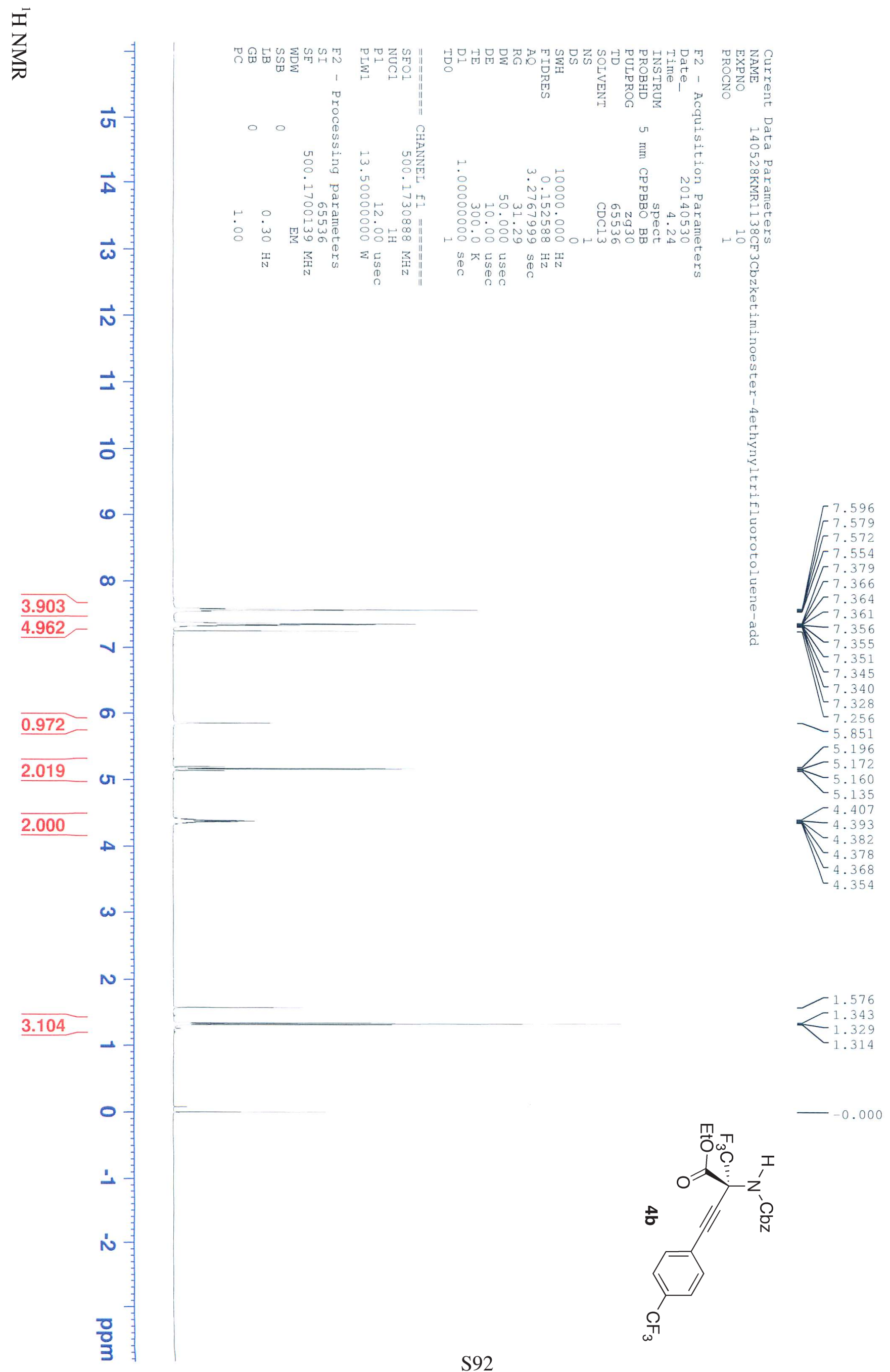




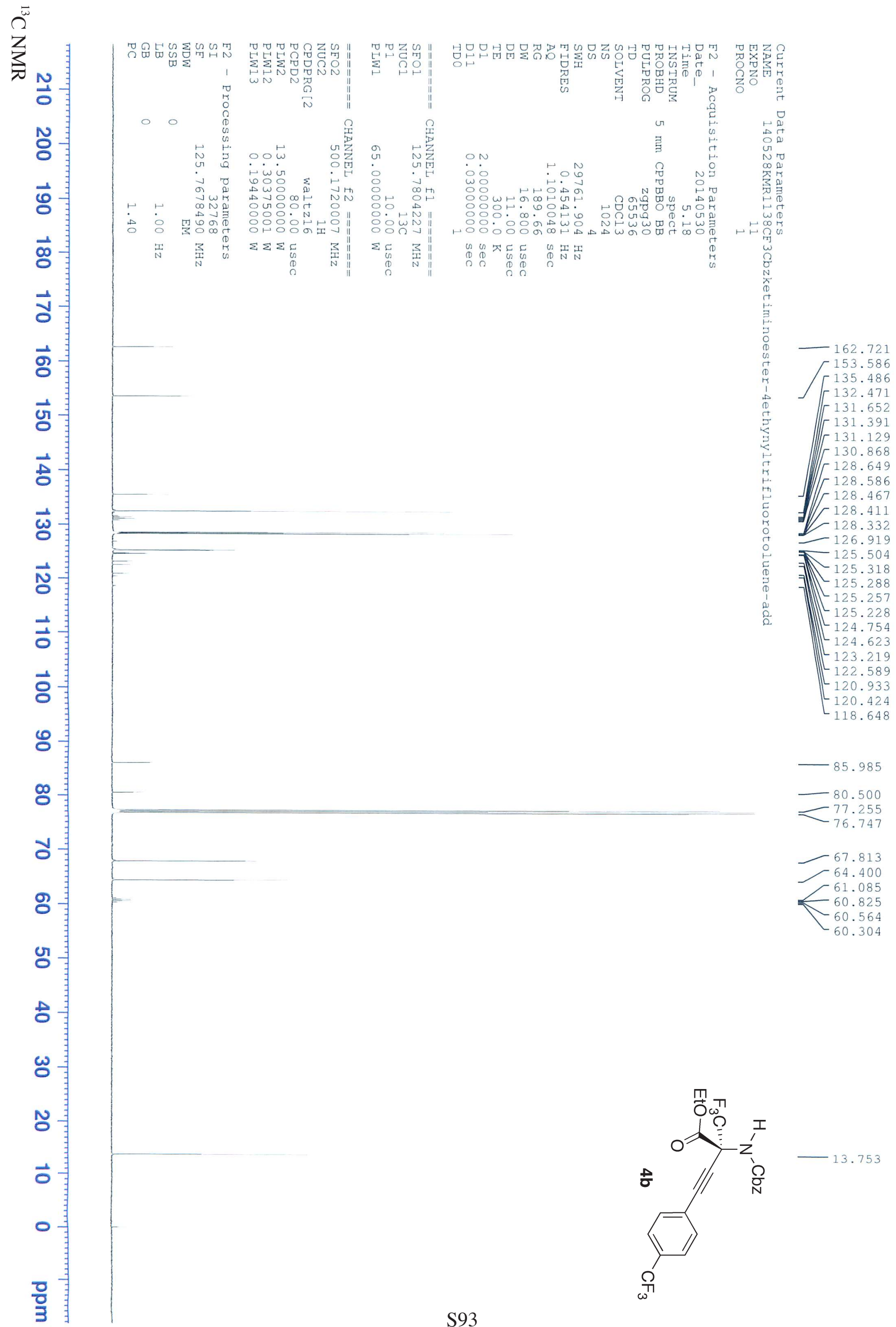


党

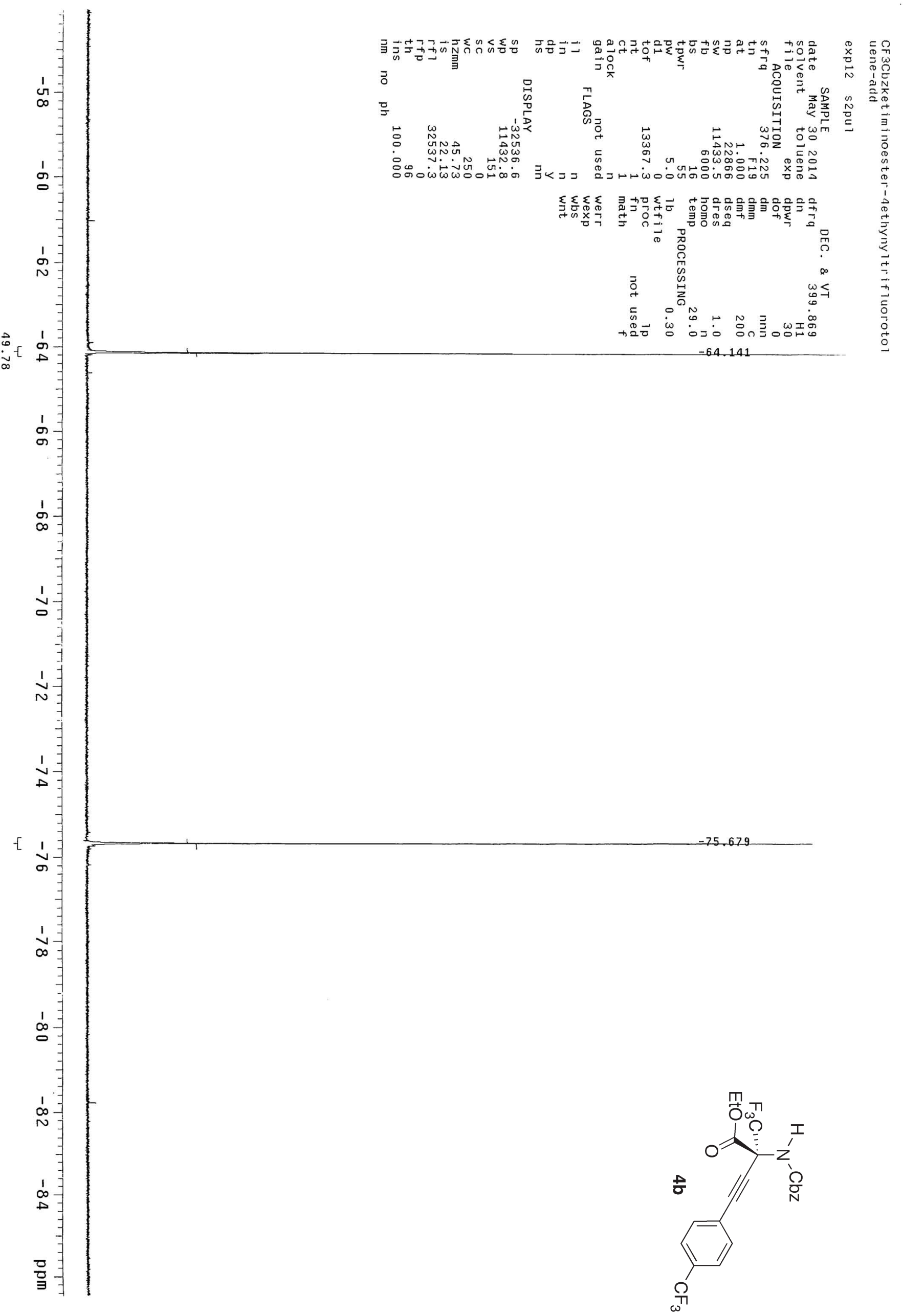




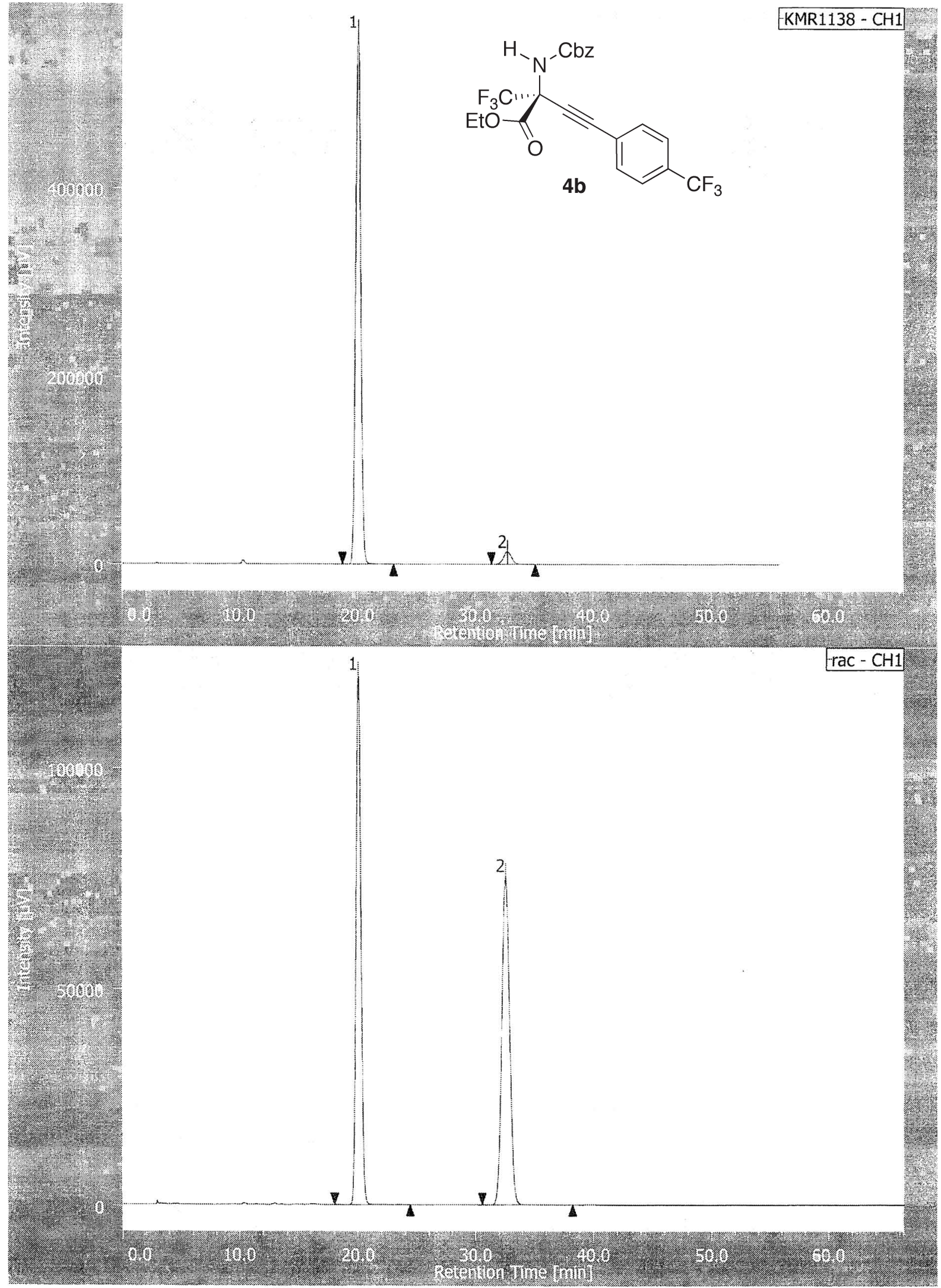

チャンネル情報 ヒピーク情報

クロマトグラム名

KMR1138-CH1

サンプル名

チャンネル名

$\mathrm{CH} 1$

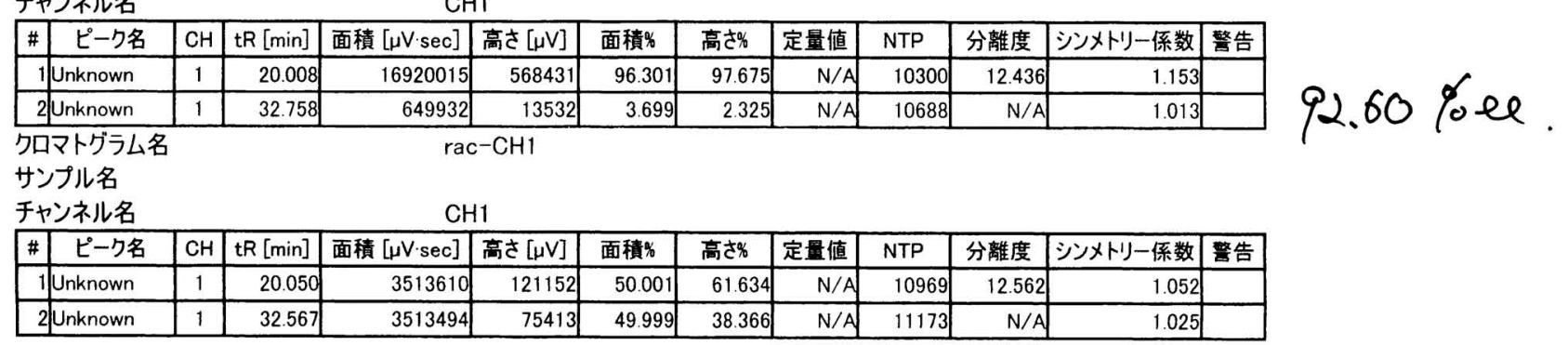




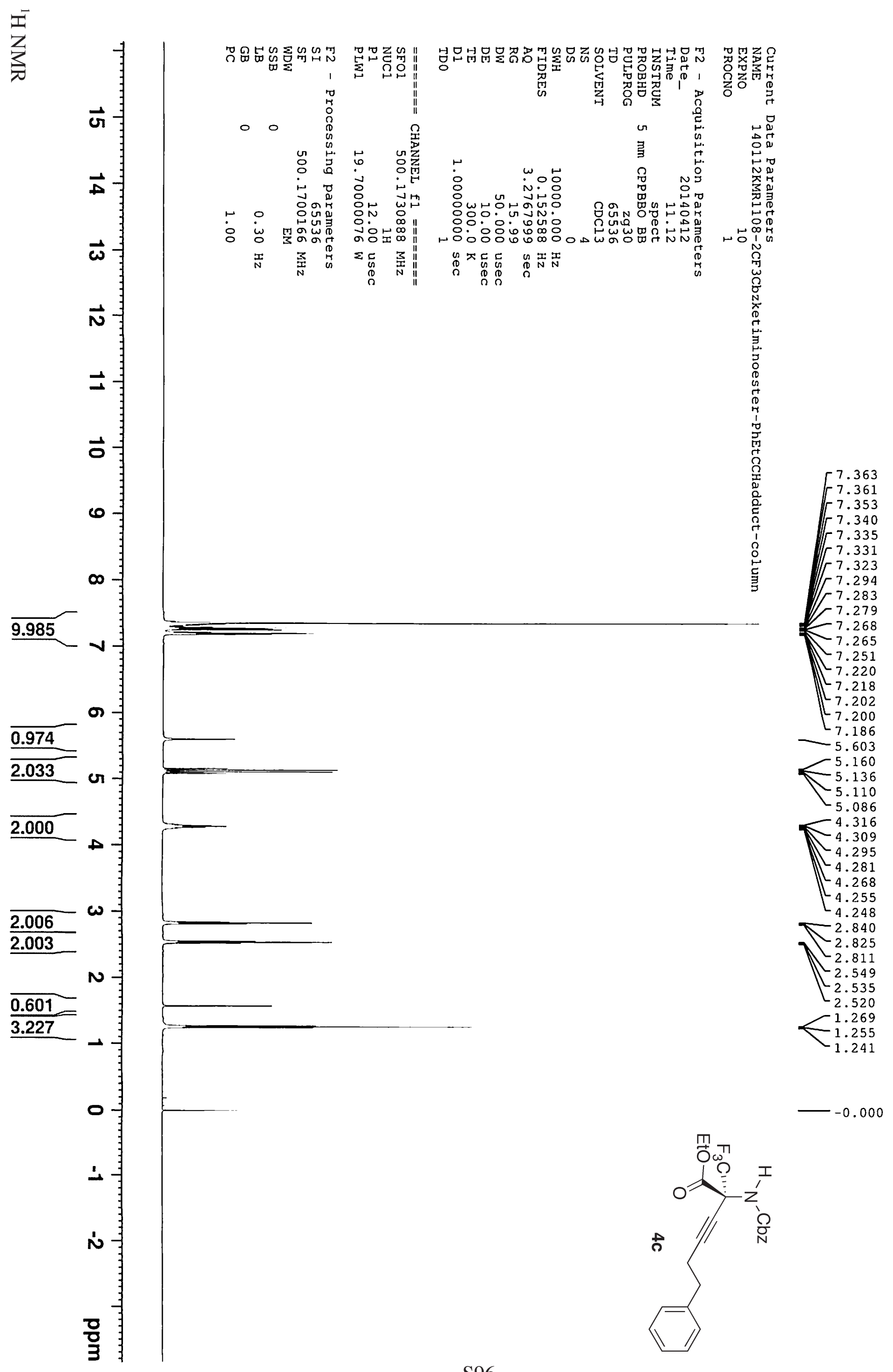




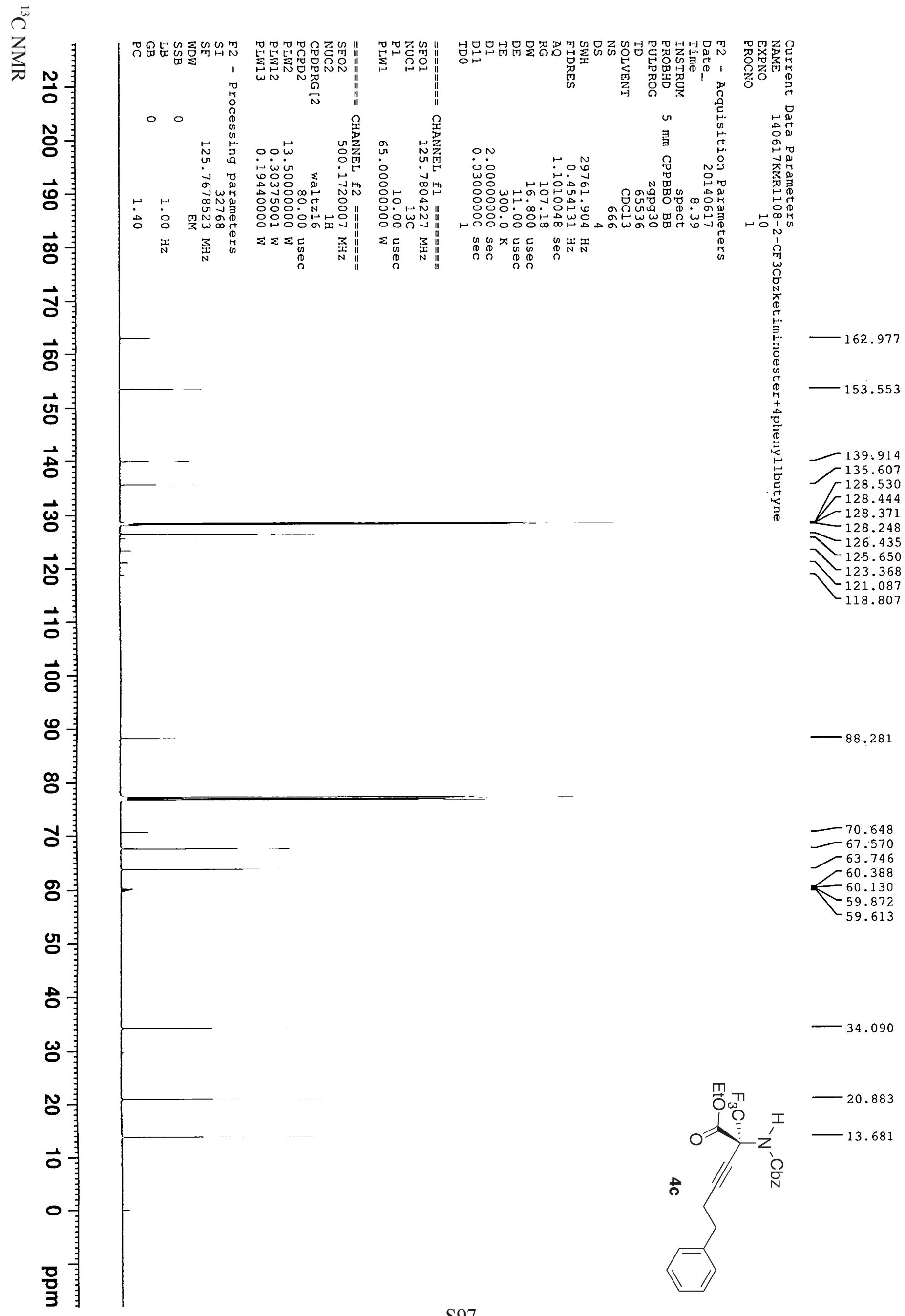



כ

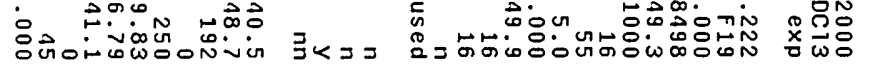

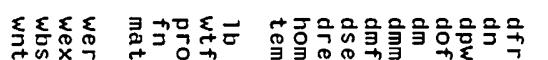

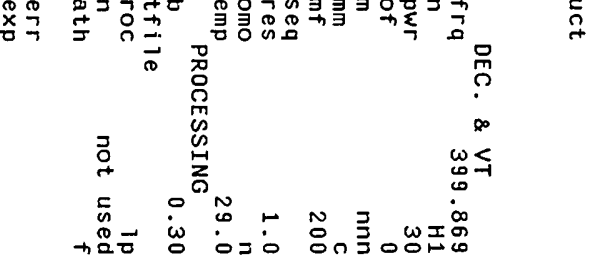

$\stackrel{1}{\square}$

$\stackrel{1}{u}$

$\stackrel{1}{\infty}$

1
$\infty$
$\infty$

$\stackrel{1}{\circ}$

on

1
$\stackrel{5}{\circ}$
0

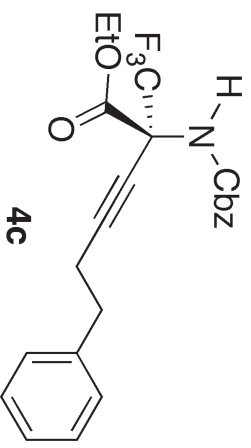




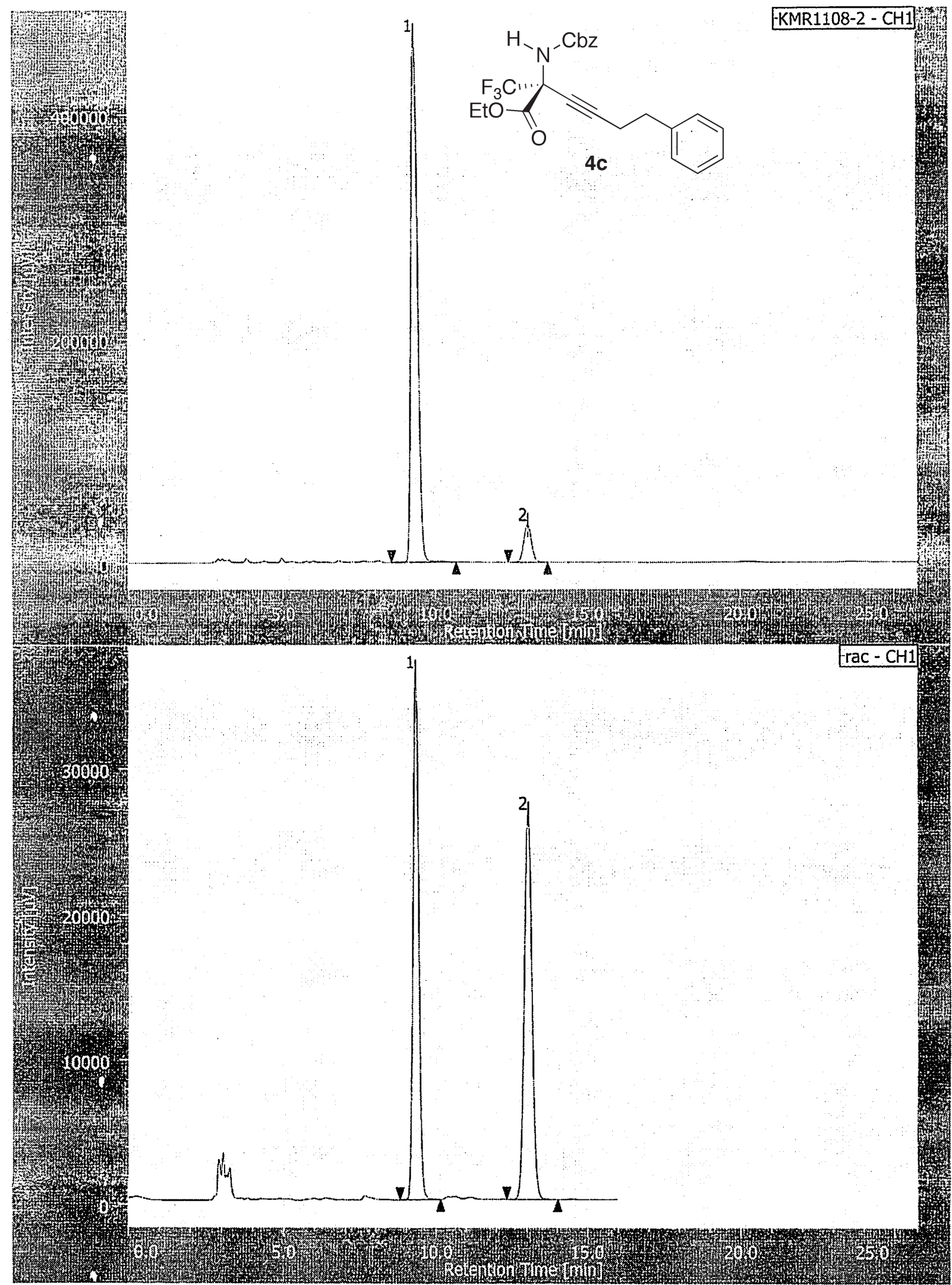

チャンネル情報+ピーク情報

クロマトグラム名

KMR1 108-2-CH1

サンプル名

チャンネル名

$\mathrm{CH} 1$

\begin{tabular}{|c|c|c|c|c|c|c|c|c|c|c|c|}
\hline ピーク名 & $\mathrm{CH}$ & $t R[\mathrm{~min}]$ & 面糟 $[\mu \vee \cdot s e c]$ & \begin{tabular}{|l|} 
\\
\end{tabular} & 面積影 & 高さ为 & 定貫值 & NTP & 分離度 & 历ンメトリー係数 & 㛑告 \\
\hline Unknown & 1 & 9.292 & 16913 & 469449 & 92.370 & 93.450 & $N / A$ & 8383 & 8.571 & 1.259 & \\
\hline 2 Unknown & 1 & 13.042 & 587840 & 2906 & 7.630 & 6.550 & $N / A$ & 12244 & $N / A$ & 1.060 & \\
\hline
\end{tabular}

クロマトグラム名

サンプル名

チャンネル名 rac- $\mathrm{CH} 1$

$\mathrm{CH} 1$

\begin{tabular}{|c|c|c|c|c|c|c|c|c|c|c|c|}
\hline ピーク名 & $\mathrm{CH}$ & $t R[\min ]$ & 而積 $[\mu \mathrm{V} \cdot \mathrm{sec}]$ & 高さ $[\mu \mathrm{V}]$ & 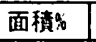 & 高さ为 & 定量值 & NTP & 分㒕度 & シンメトリー係数 & 䇾告 \\
\hline 1 Unknown & 1 & 9.317 & 470092 & 36304 & 50.004 & 57.768 & $N / A$ & 12066 & 9.222 & 1.129 & \\
\hline 2 Unknown & 1 & 13.033 & 470008 & 26540 & 49.996 & 42.232 & N/A & 12362 & $\mathrm{~N} / \mathrm{A}$ & 1.064 & \\
\hline
\end{tabular}




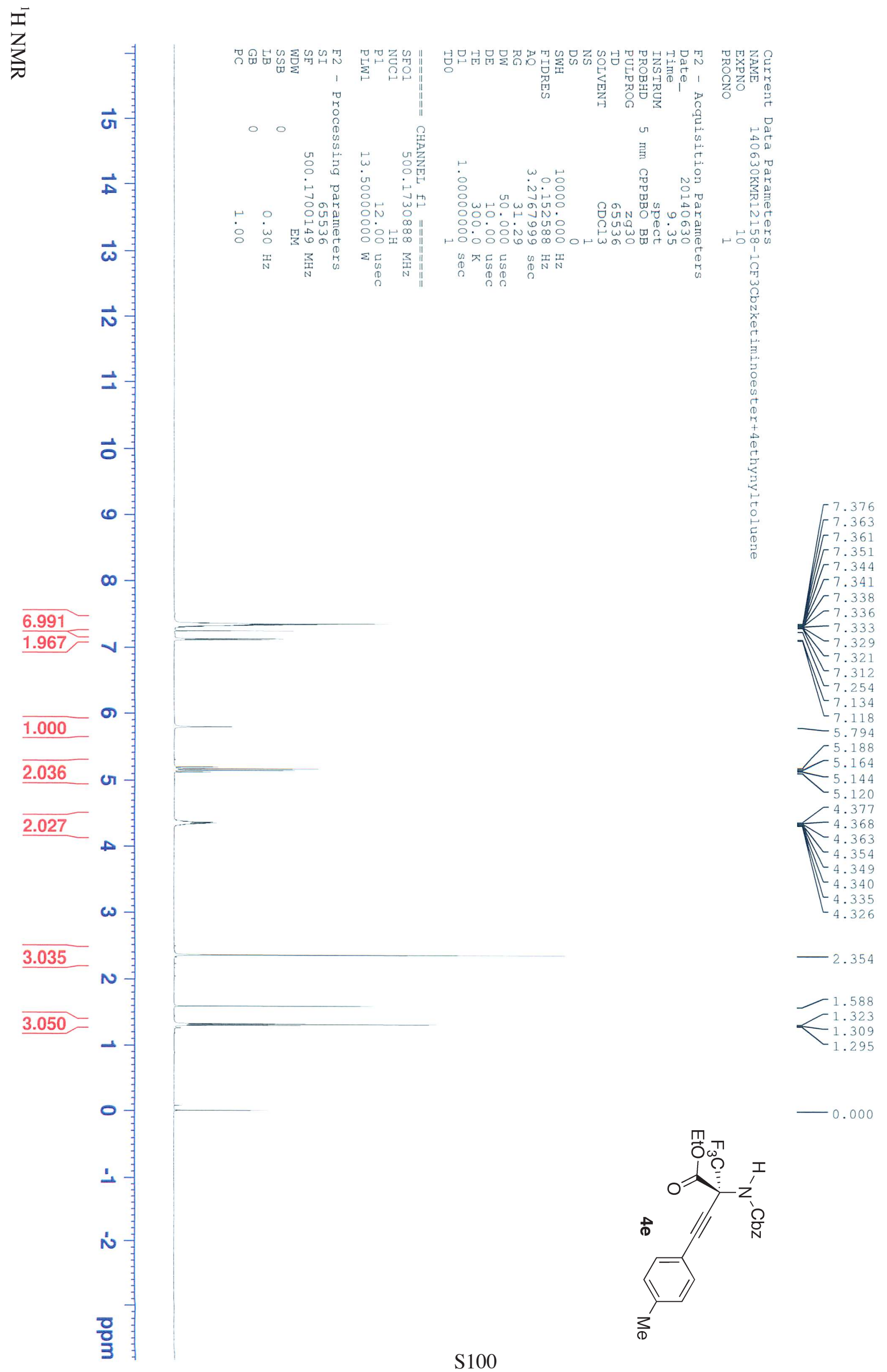




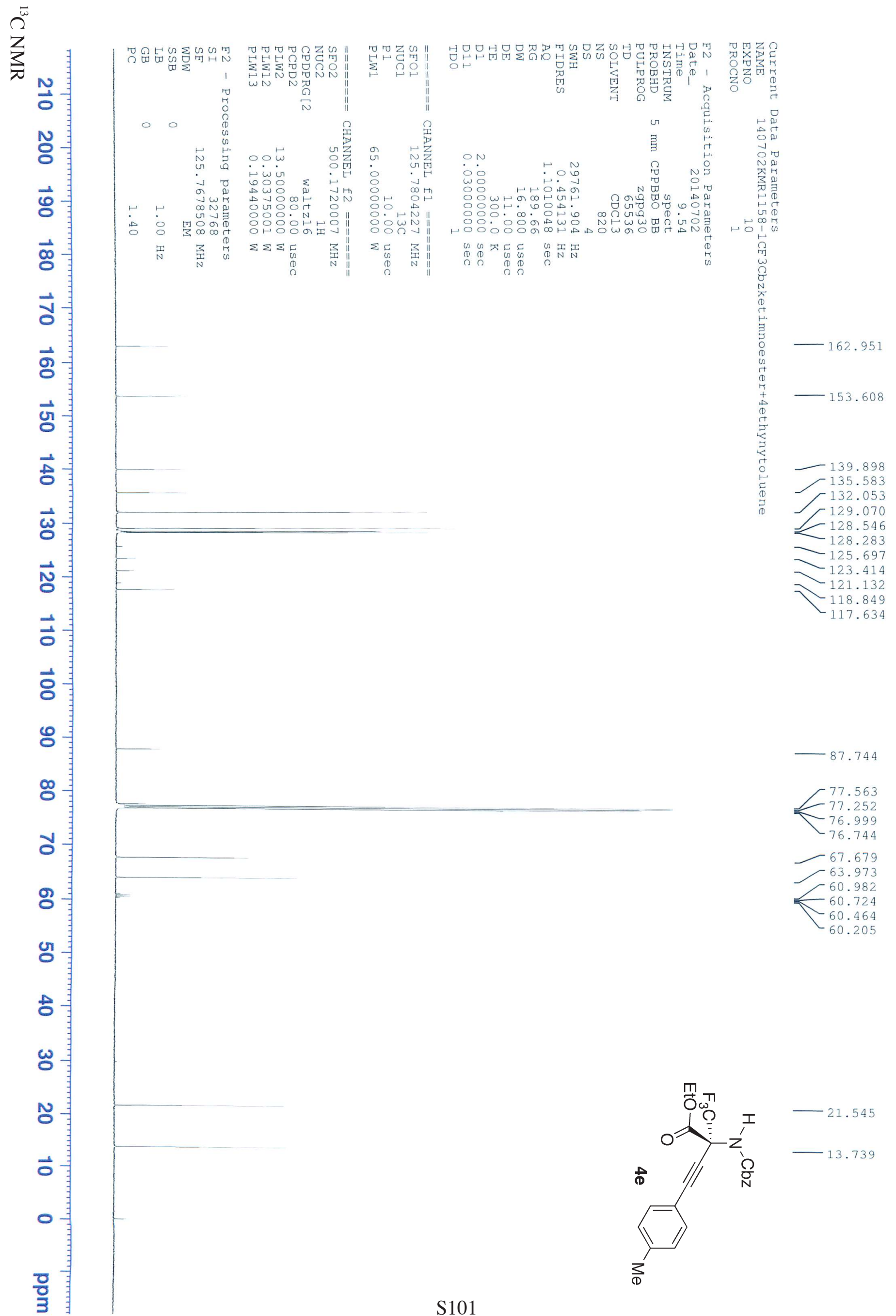




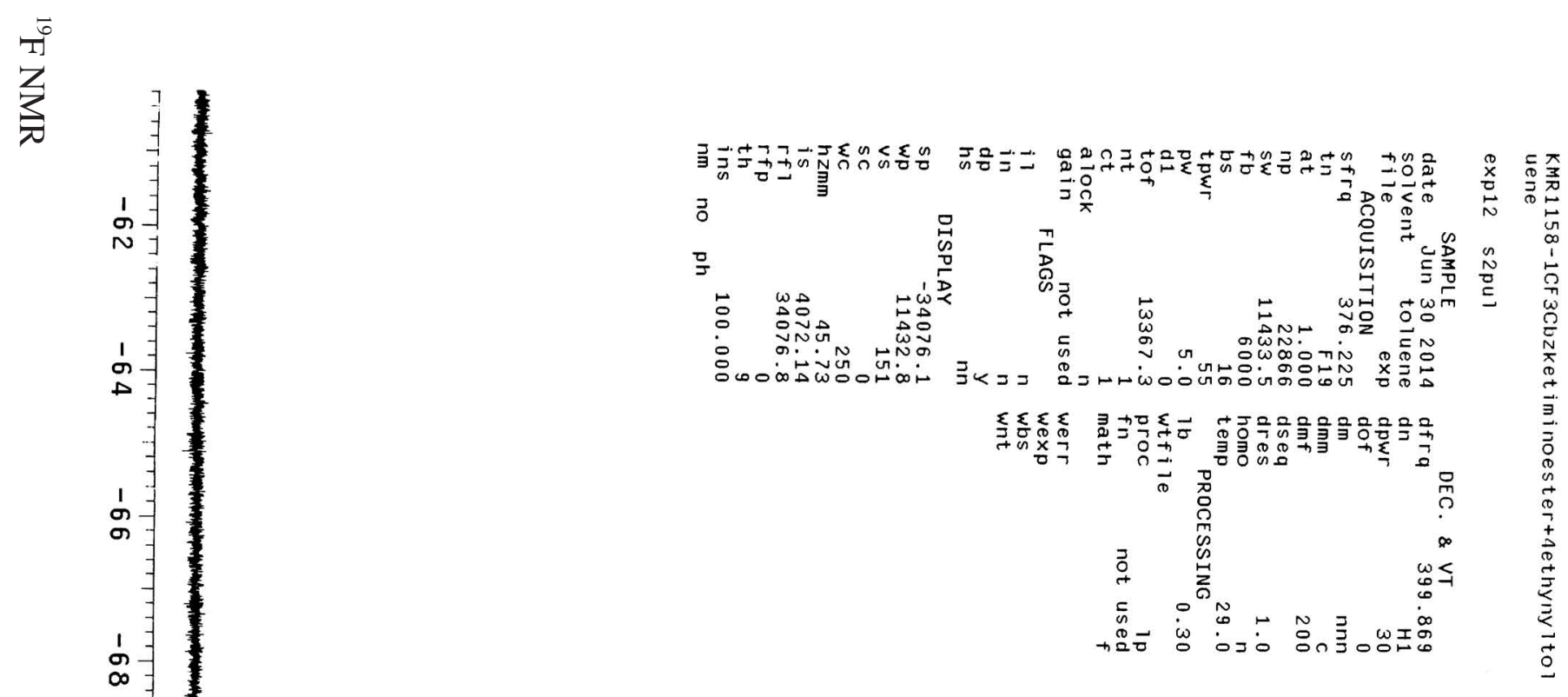

$\frac{1}{0}$

N

1
$\therefore$
+

ป

के

1

D.

1
$\infty$
$\infty$
$\infty$

1
$\infty$
$\infty$
$\infty$

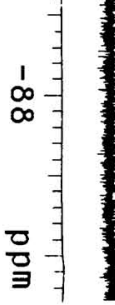

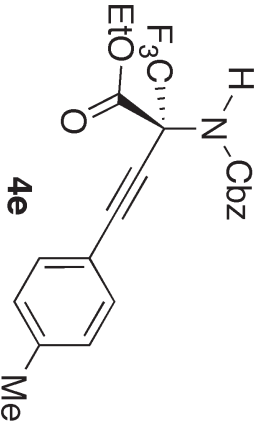




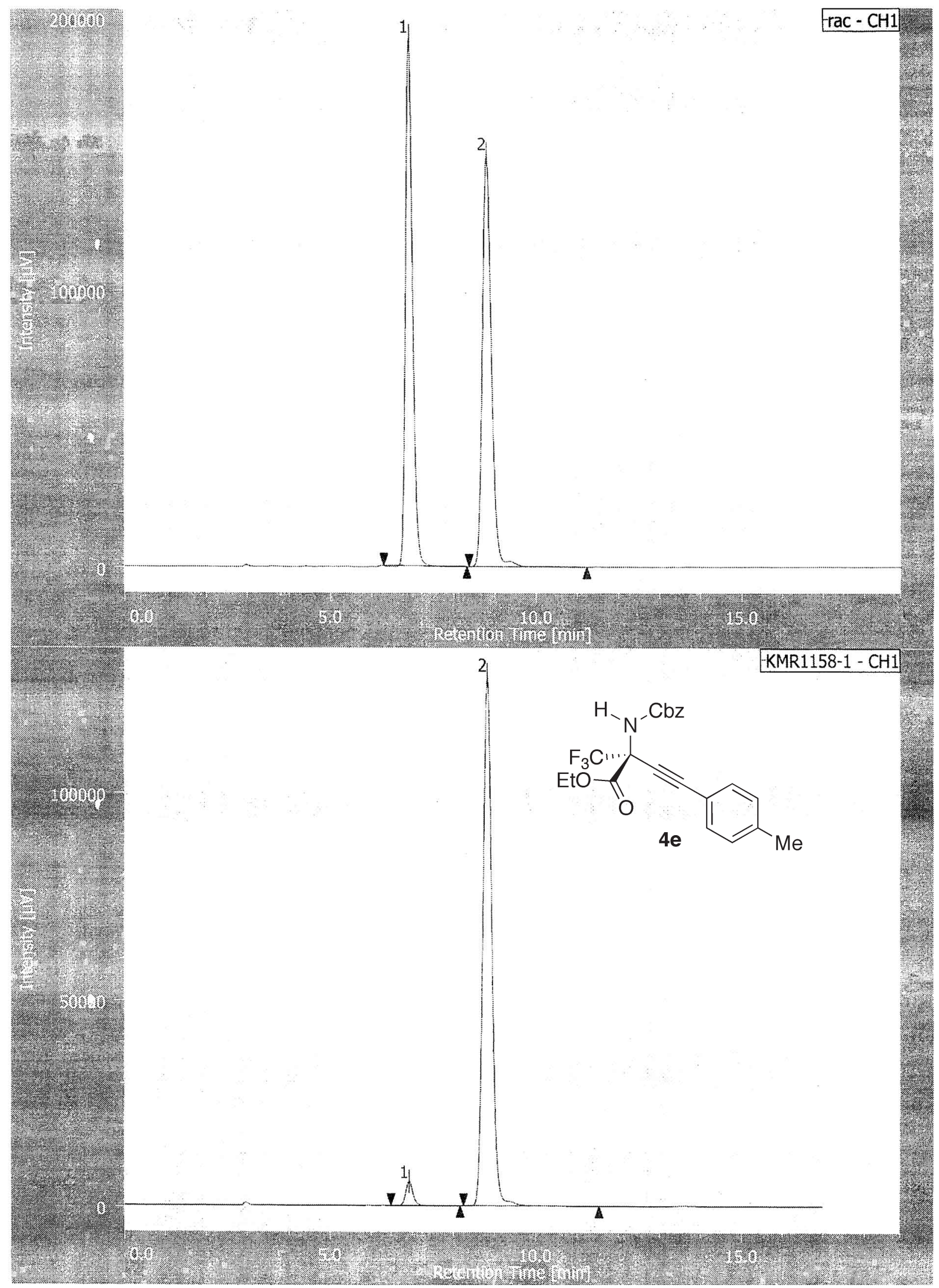

チャンネル情報+ピーク情報

クロマトグラム名

$\mathrm{rac}-\mathrm{CH} 1$

サンプル名

チャンネル名

$\mathrm{CH} 1$

\begin{tabular}{|c|c|c|c|c|c|c|c|c|c|c|c|}
\hline ピーク名 & $\mathrm{CH}$ & $t R[\mathrm{~min}]$ & 面積 [ $\mu \mathrm{V} \cdot \mathrm{sec}]$ & 高さ $[\mu \mathrm{V}]$ & 面積。。 & 高さ\% & 定量値 & NTP & 分離度 & シンメトリー係数 & 警告 \\
\hline Unknown & 1 & 6.908 & 2237083 & 190351 & 49.768 & 56.252 & N/A & 8335 & 5.490 & 1.169 & \\
\hline 2 Unknown & 1 & 8.808 & 2257912 & 148037 & 50.232 & 43.748 & $N / A$ & 8104 & N/A & 1.161 & \\
\hline
\end{tabular}

クロマトグラム名

KMR1158-1-CH

サンプル名

チャンネル名

$\mathrm{CH} 1$

\begin{tabular}{|c|c|c|c|c|c|c|c|c|c|c|c|}
\hline ピーク名 & $\mathrm{CH}$ & $\mathrm{tR}[\mathrm{min}]$ & 面積 [ $[\mu \mathrm{V} \cdot \mathrm{sec}]$ & 高さ $[\mu \mathrm{V}]$ & 面積\% & 高さ\% & 定量値 & NTP & 分離度 & シンメトリー係数 & 警告 \\
\hline Unknown & 1 & 6.917 & 71636 & 6070 & 3.550 & 4.531 & $N / A$ & 8184 & 5.481 & 1.174 & \\
\hline 2 Unknown & 1 & 8.817 & 1946035 & 127897 & 96.450 & 95.469 & $N / A$ & 8204 & $\mathrm{~N} / \mathrm{A}$ & 1.168 & \\
\hline
\end{tabular}




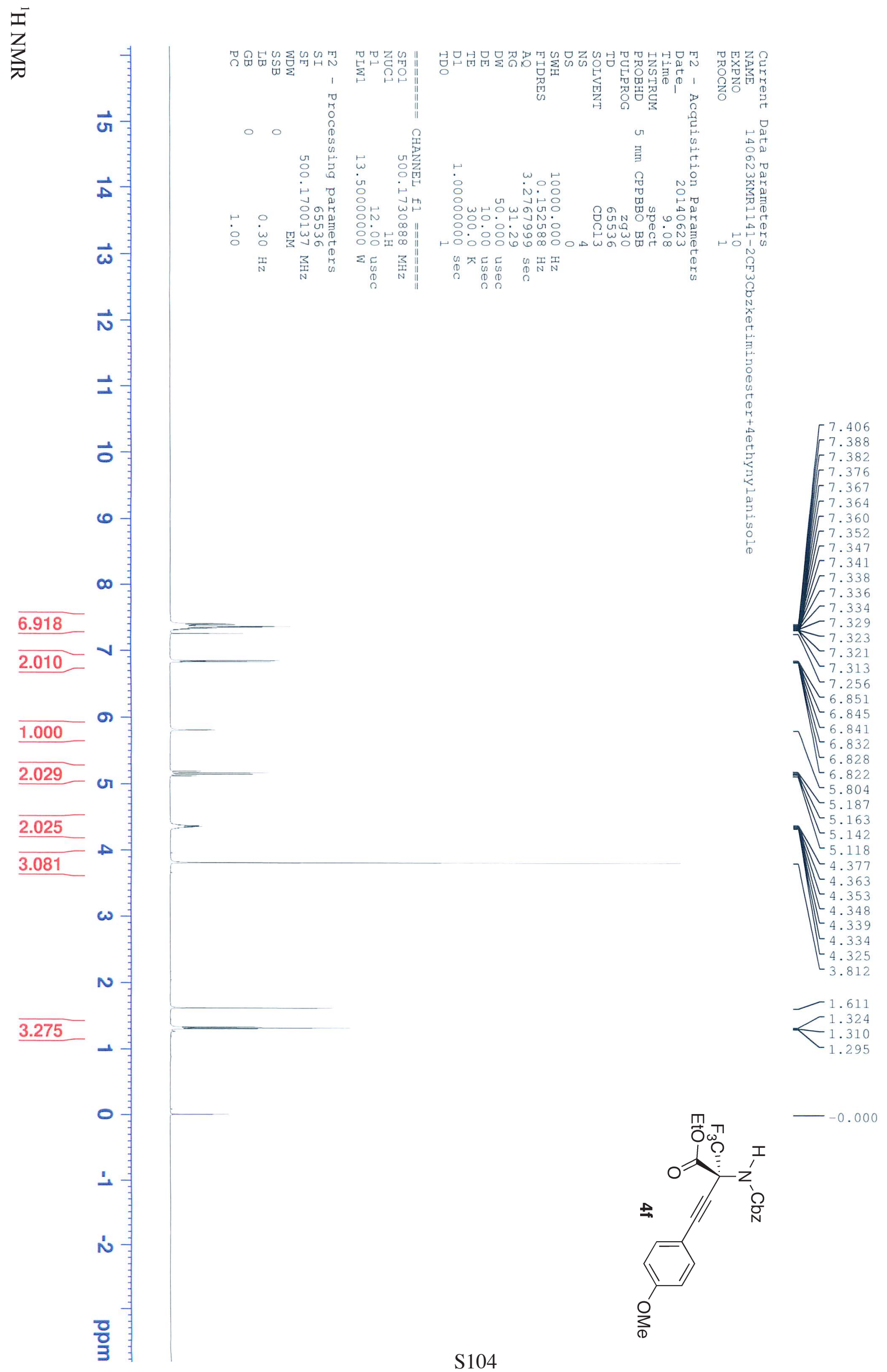




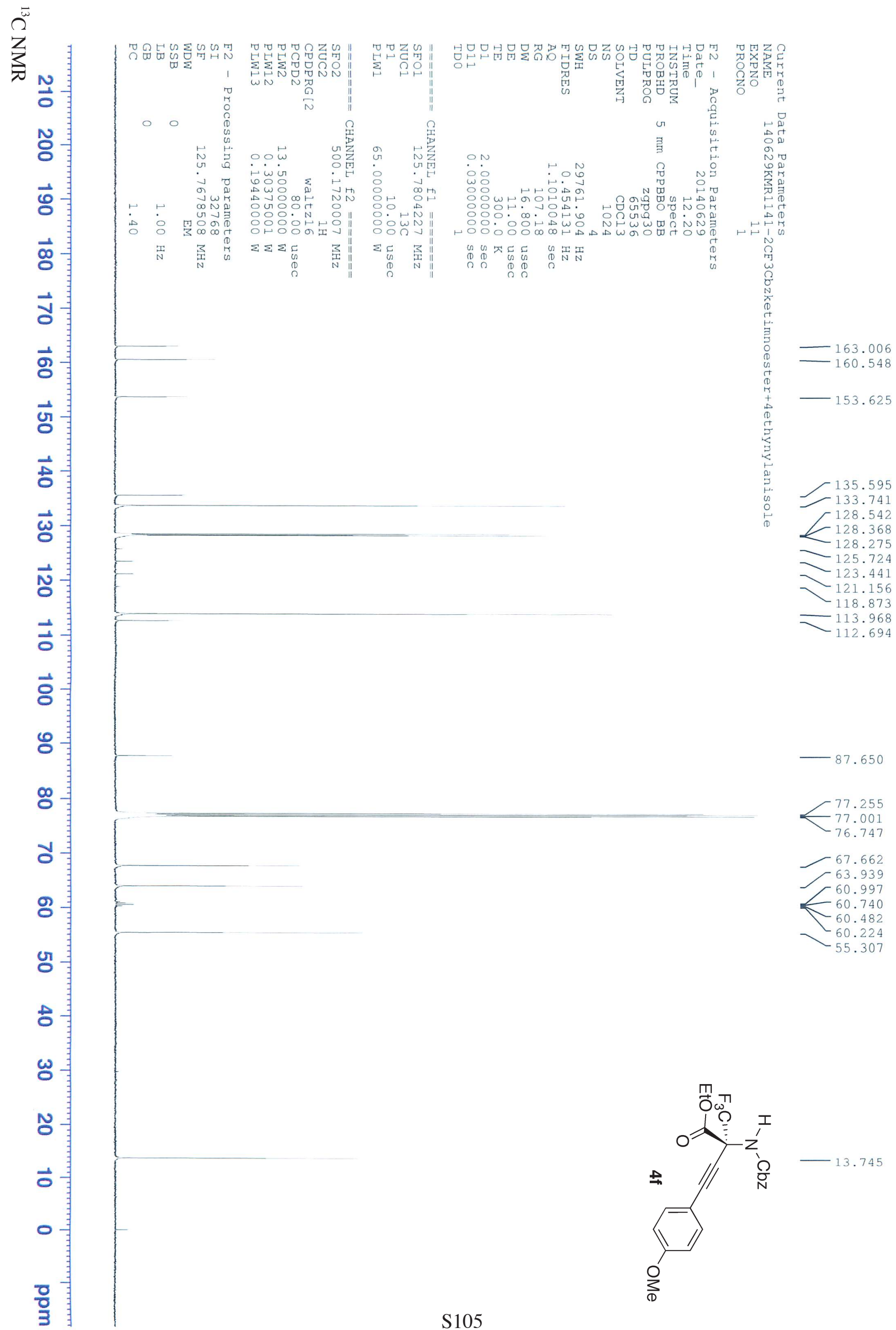


雚

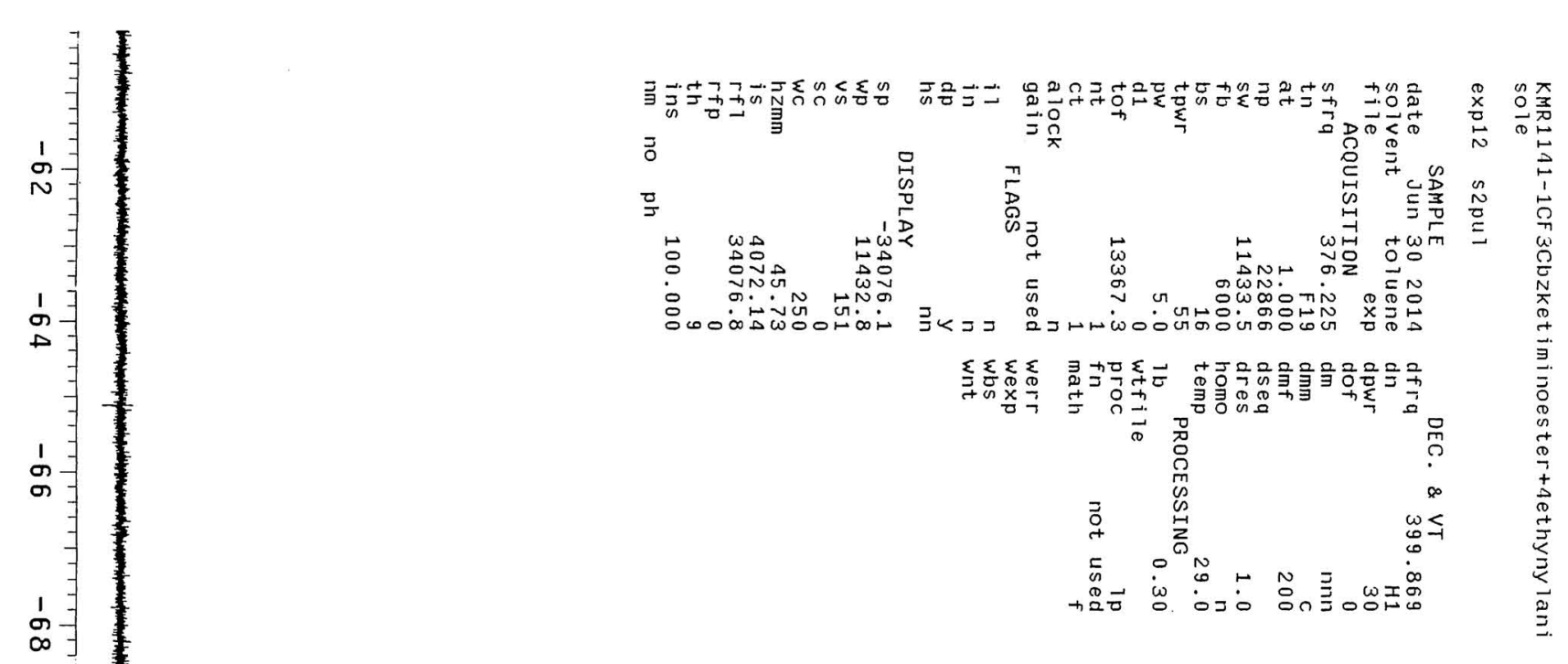

$\stackrel{1}{0}$

걱

$\begin{aligned} & = \\ 1 & = \\ x & = \\ & = \\ 1 & =1\end{aligned}$

2

$\stackrel{1}{\infty}=$

赵

1
$\infty$
$N$

N

1
$\infty$
$\infty$
+

o

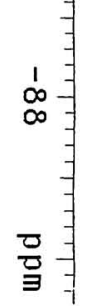

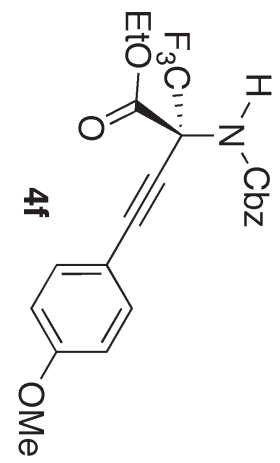




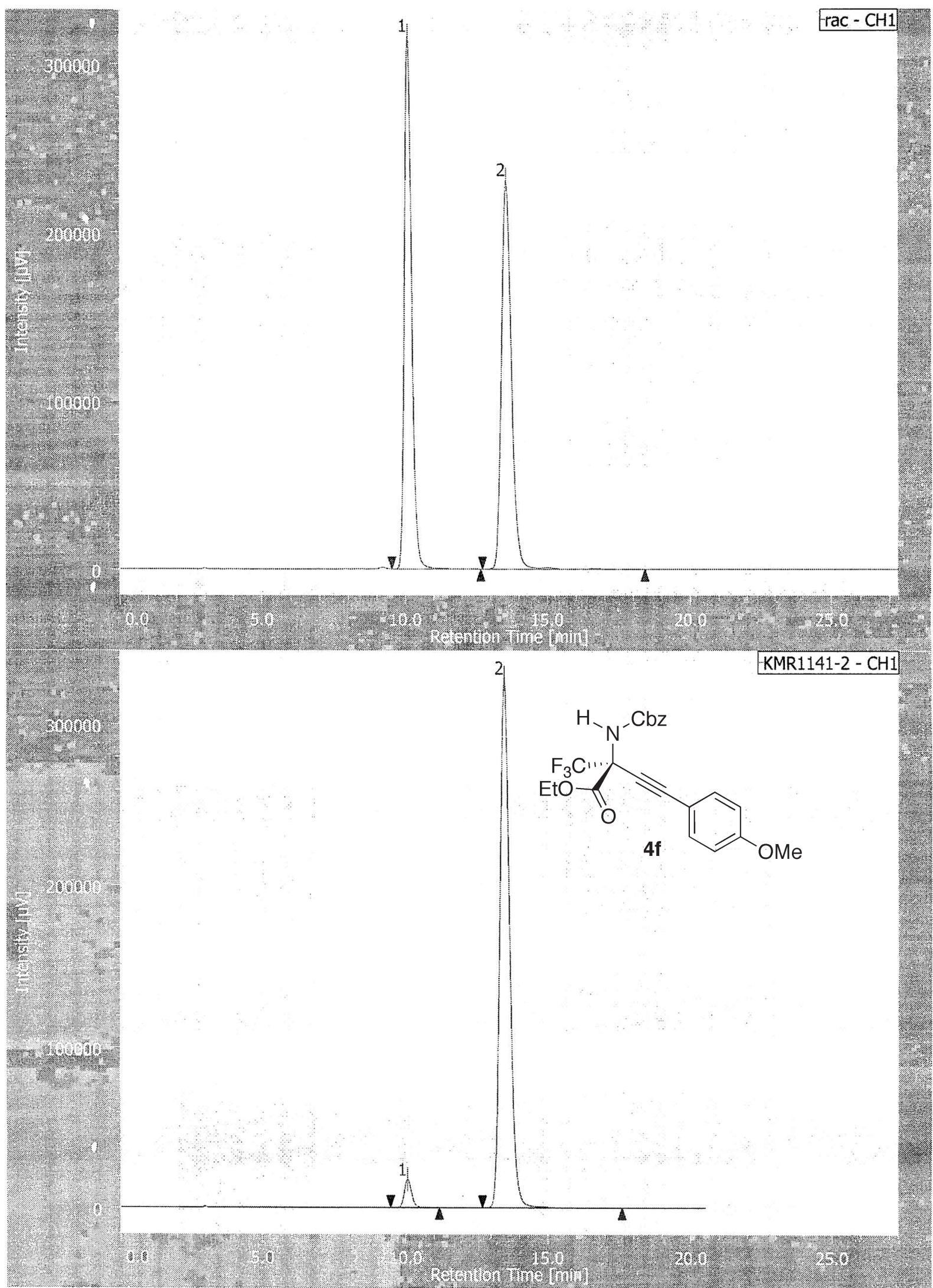

チャンネル情報+ピーク情報

クロマトグラム名

$\mathrm{rac}-\mathrm{CH} 1$

サンプル名

チャンネル名

$\mathrm{CH} 1$

\begin{tabular}{|c|c|c|c|c|c|c|c|c|c|c|c|}
\hline ピーク名 & $\mathrm{CH}$ & $t R[\mathrm{~min}]$ & 面積 $[\mu \mathrm{V} \cdot \mathrm{sec}]$ & 高さ [ [ $\mathrm{V}]$ & 面種为 & 高さ\% & 定量値 & NTP & 分離度 & シンメトリー係数 & 警告 \\
\hline 1 Unknown & 1 & 10.092 & 5554215 & 316049 & 49.848 & 57.794 & $\mathrm{~N} / \mathrm{A}$ & 7951 & 6.471 & 1.213 & \\
\hline 2 Unknown & 1 & 13.558 & 5588153 & 230801 & 50.152 & 42.206 & N/A & 7612 & $N / A$ & 1.198 & \\
\hline
\end{tabular}

クロマトグラム名 KMR1141-2-CH1

サンプル名

チャンネル名

$\mathrm{CH} 1$

\begin{tabular}{|c|c|c|c|c|c|c|c|c|c|c|c|c|}
\hline ピーク名 & $\mathrm{CH}$ & $t R[\mathrm{~min}]$ & 面積 [ $\mathrm{V} \mathrm{V} \cdot \mathrm{sec}]$ & 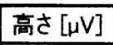 & 面樌\% & 高さ\% & 定量值 & NTP & 分離度 & シンメトリー係数 & 警告 & \\
\hline Unknown & 1 & 10.058 & 310564 & 17721 & 3.817 & 5.133 & N/A & 7911 & 6.39 & 1.190 & & \\
\hline 2) Unknown & 1 & 13.467 & 7825225 & 327479 & 96.183 & 94.867 & $N / A$ & 7621 & $N / A$ & 1.240 & & \\
\hline
\end{tabular}



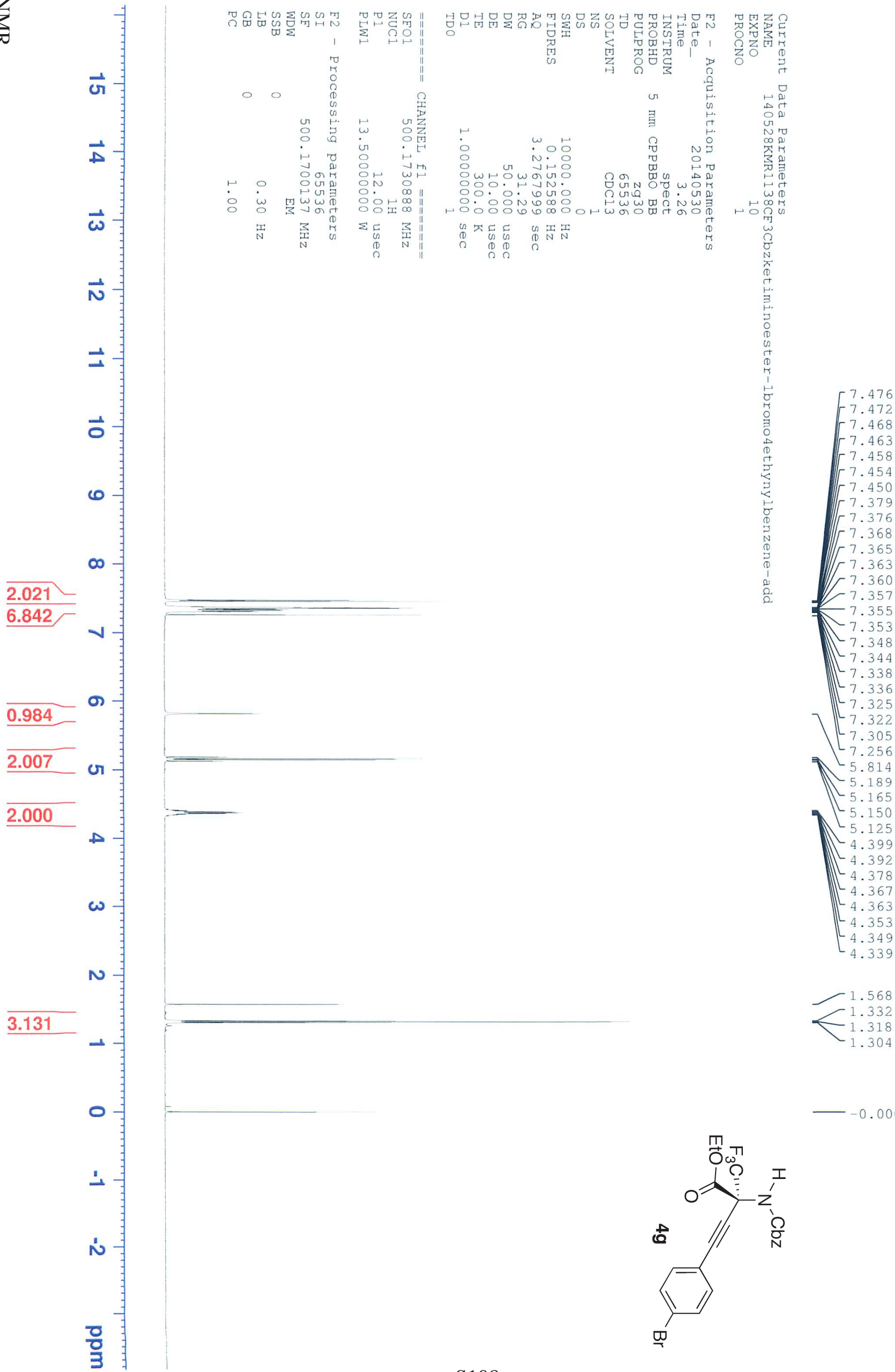

$L_{4.339}$

1.568

$-1.332$

1.318
1.304

$-0.000$

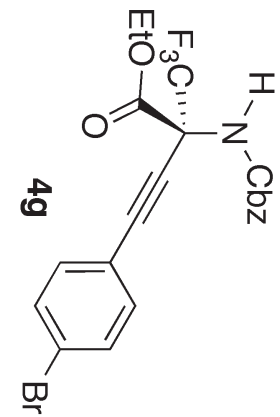




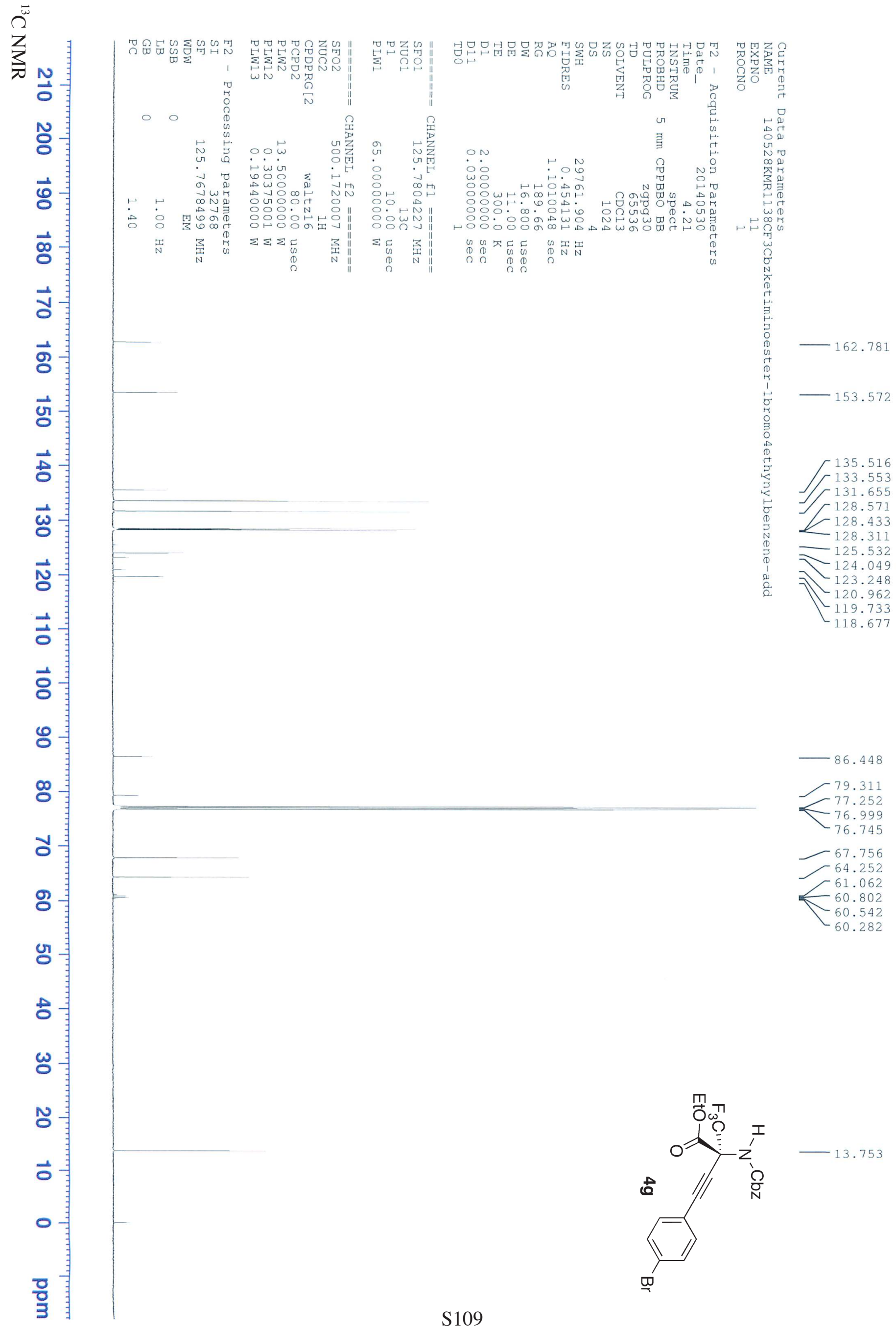




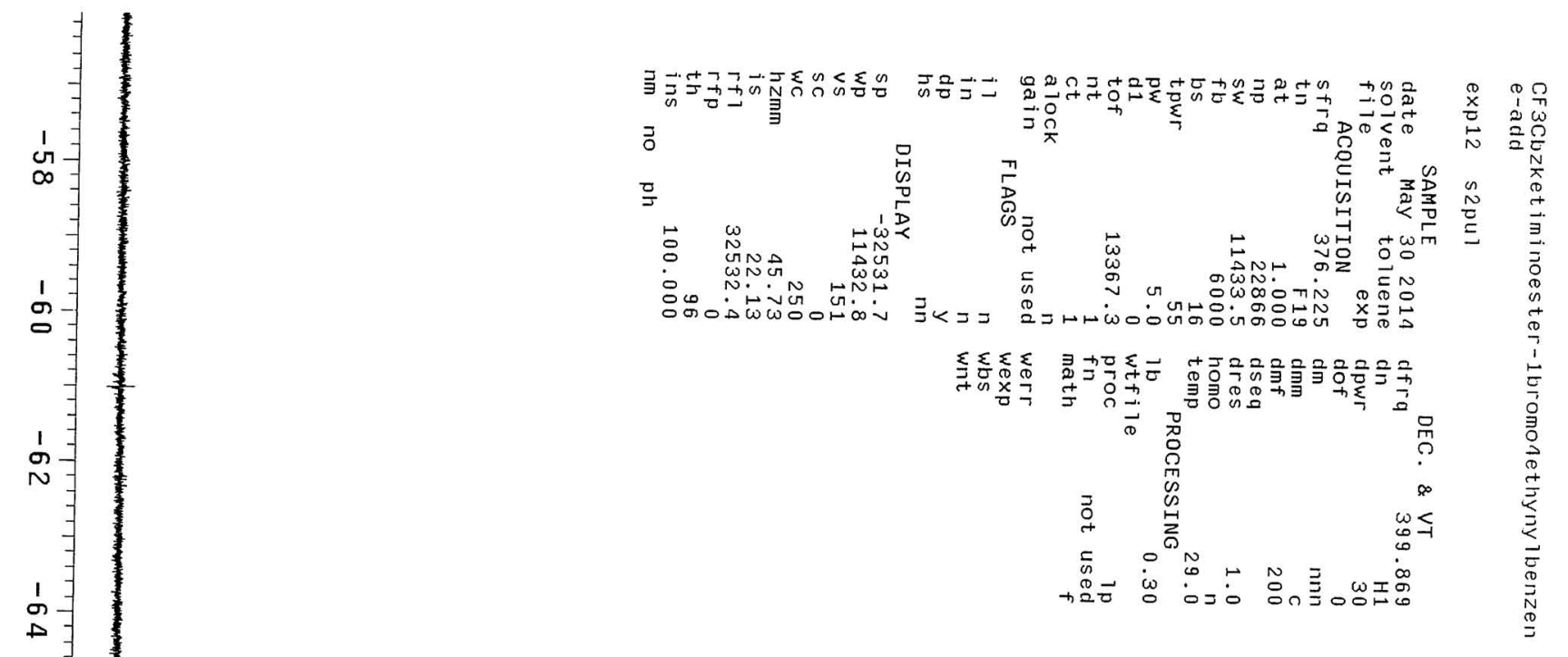

1
5
0

1
5
$\infty$

$\stackrel{1}{0}$

$\stackrel{1}{N}$

$\stackrel{1}{+}$

के

$\frac{1}{\infty}$

$\infty$

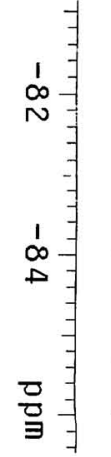

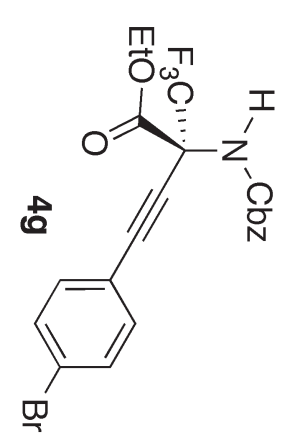




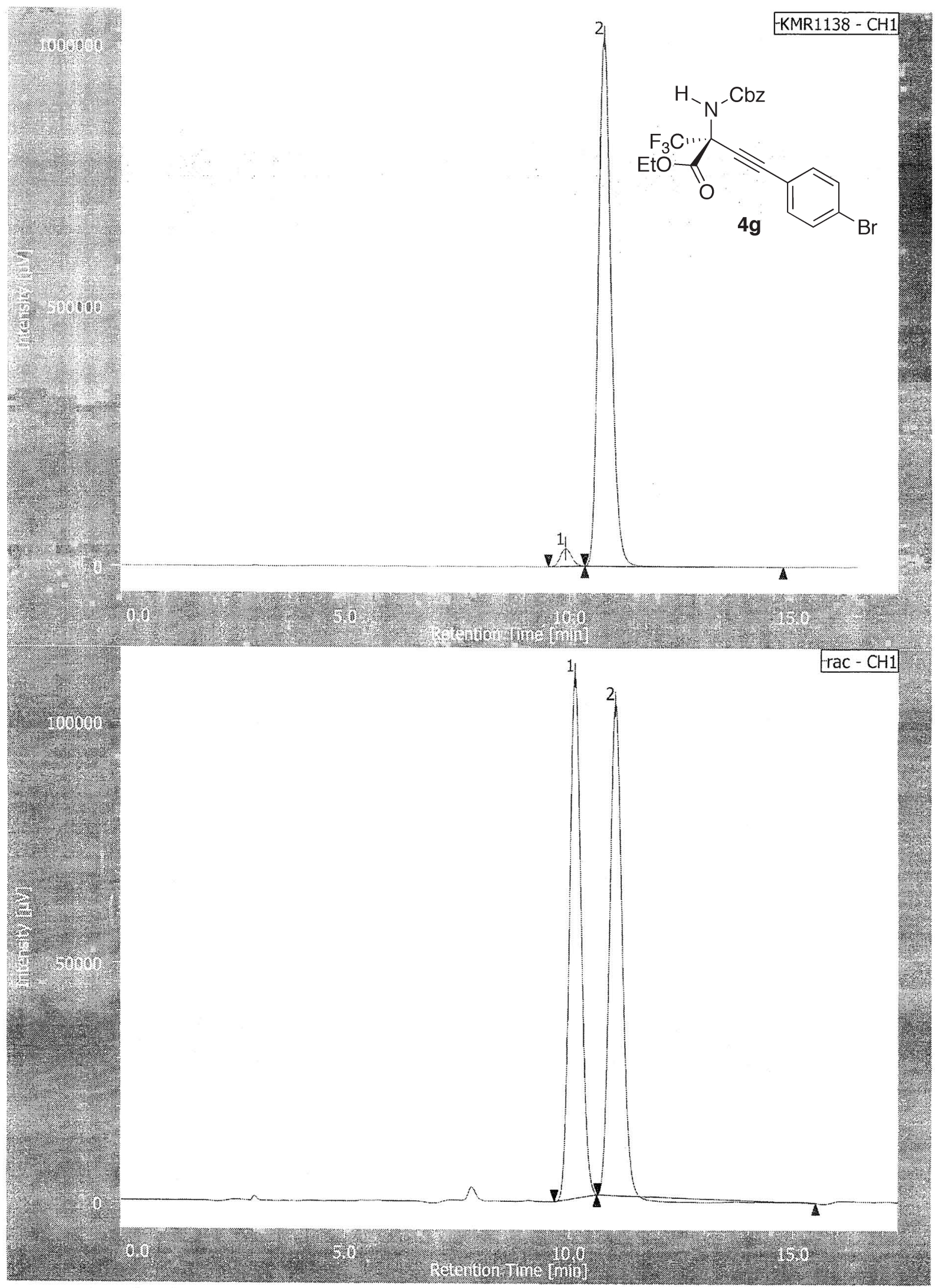

チャンネル情報+ピーク情報

クロマトグラム名

サンプル名

KMR1 138-CH1

チャンネル名

$\mathrm{CH} 1$

\begin{tabular}{|c|c|c|c|c|c|c|c|c|c|c|c|c|}
\hline ピーク名 & $\mathrm{CH}$ & $t R[\mathrm{~min}]$ & 面積 [ $[\mu \mathrm{V} \cdot \mathrm{sec}]$ & 高さ [ $[\mu \mathrm{V}]$ & 面積\% & 高さ\% & 定量値 & NTP & 分離度 & シンメトリー係数 & 警告 & \\
\hline Unknown & 1 & \begin{tabular}{|l|}
9.908 \\
\end{tabular} & 591549 & 34097 & 2.890 & 3.255 & $N / A$ & 7380 & 1.737 & 1.118 & & \\
\hline 2 Unknown & 1 & 10.750 & 19877289 & 1013409 & 97.110 & 96.745 & N/A & 7095 & $\mathrm{~N} / \mathrm{A}$ & 1.248 & & \\
\hline
\end{tabular}

サンプル名

チャンネル名

$\mathrm{CH} 1$

\begin{tabular}{|c|c|c|c|c|c|c|c|c|c|c|c|}
\hline ピーク名 & $\mathrm{CH}$ & $\mathrm{tR}$ [min] & 面積 [ $\mu \mathrm{V} \cdot \mathrm{sec}]$ & 高さ $[\mu \mathrm{V}]$ & 面積\% & 高さ\% & 定量值 & NTP & 分離度 & シンメトリー係数 & 鳘告 \\
\hline 1 Unknown & 1 & 10.125 & 1946450 & 108613 & 49.666 & 51.537 & N/A & 7325 & 1.829 & 1.132 & \\
\hline 2 Unknown & 1 & 11.017 & 1972665 & 102134 & 50.334 & 48.463 & $N / A$ & 7624 & $\mathrm{~N} / \mathrm{A}$ & 1.167 & \\
\hline
\end{tabular}




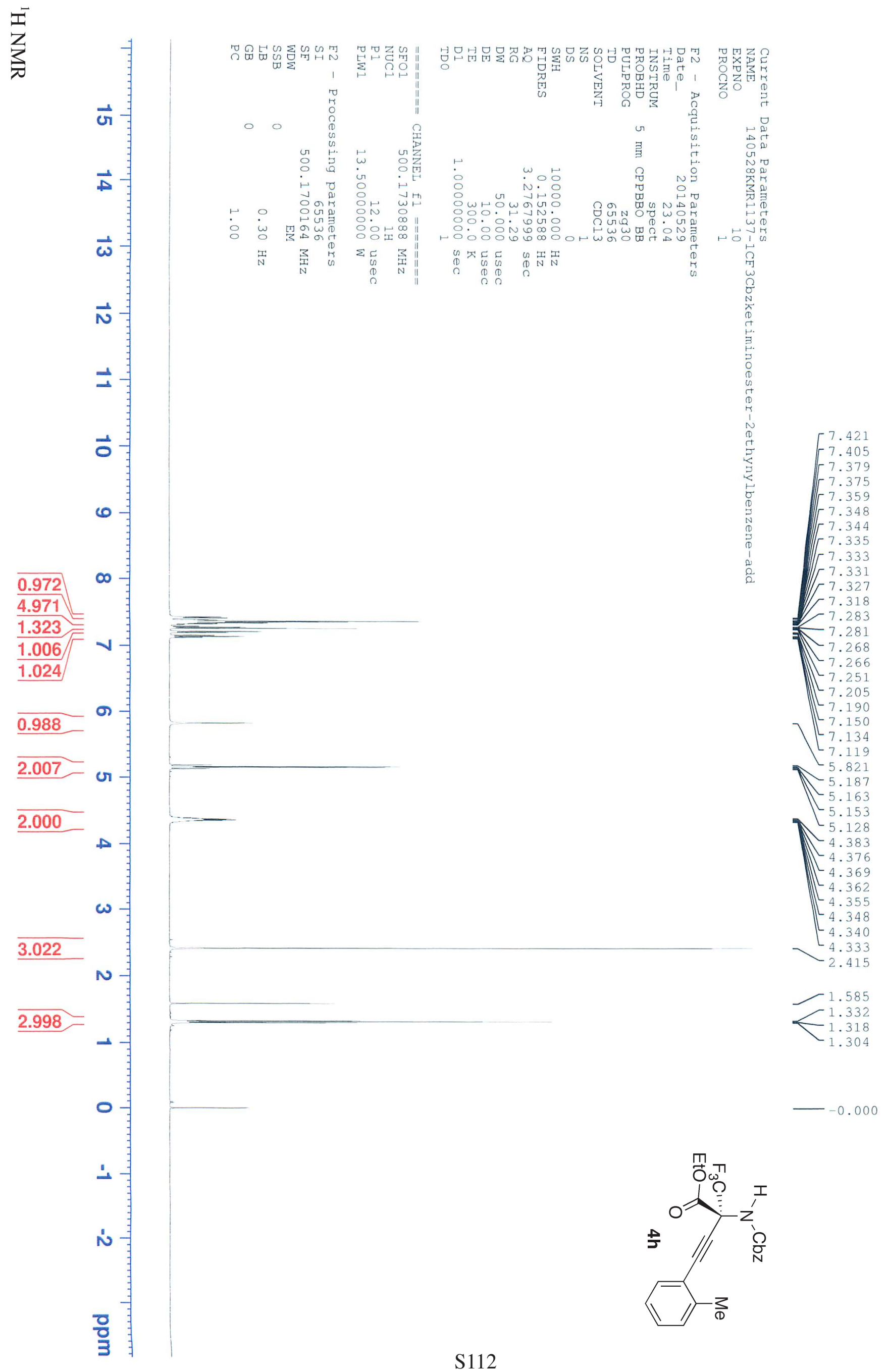




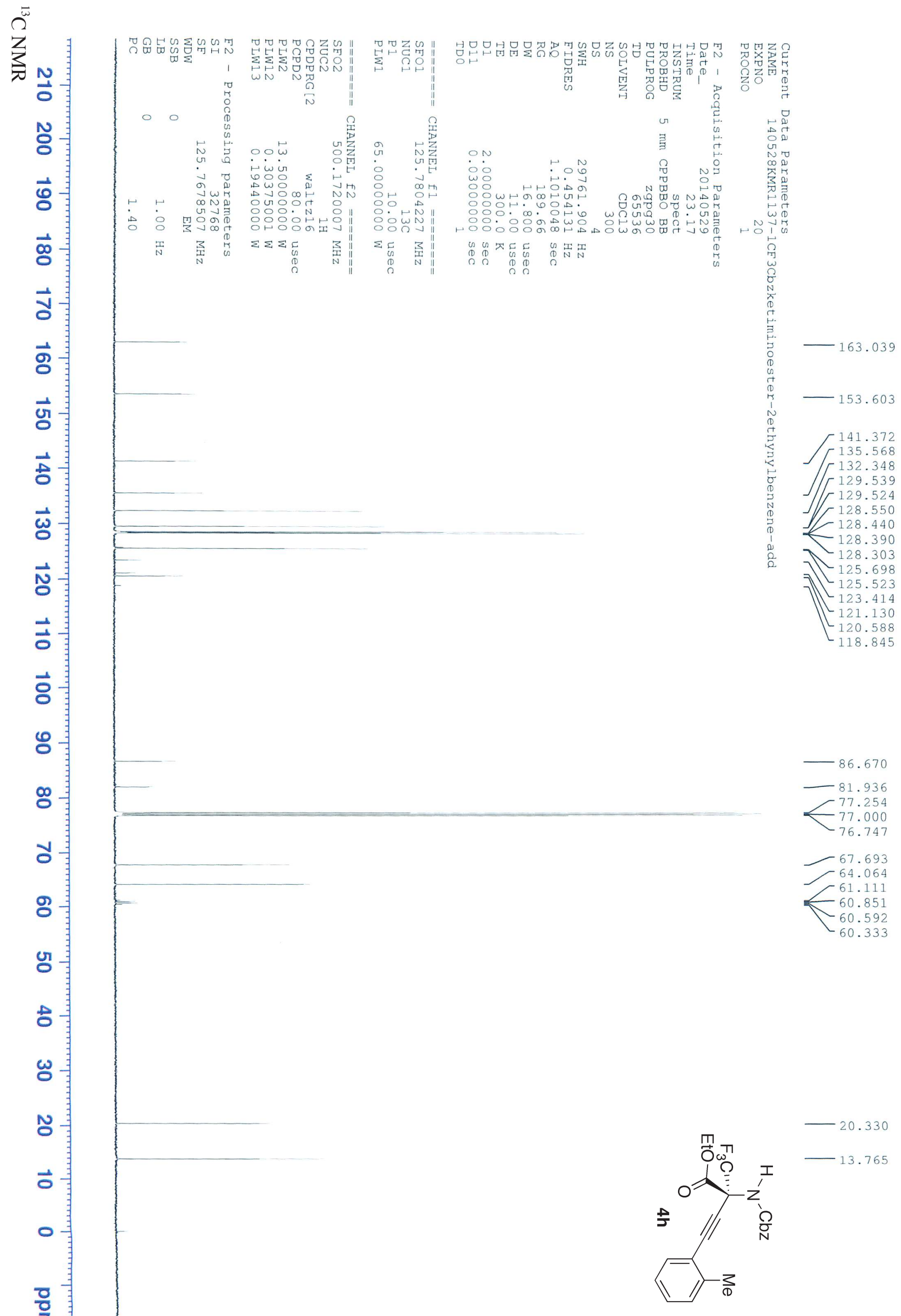




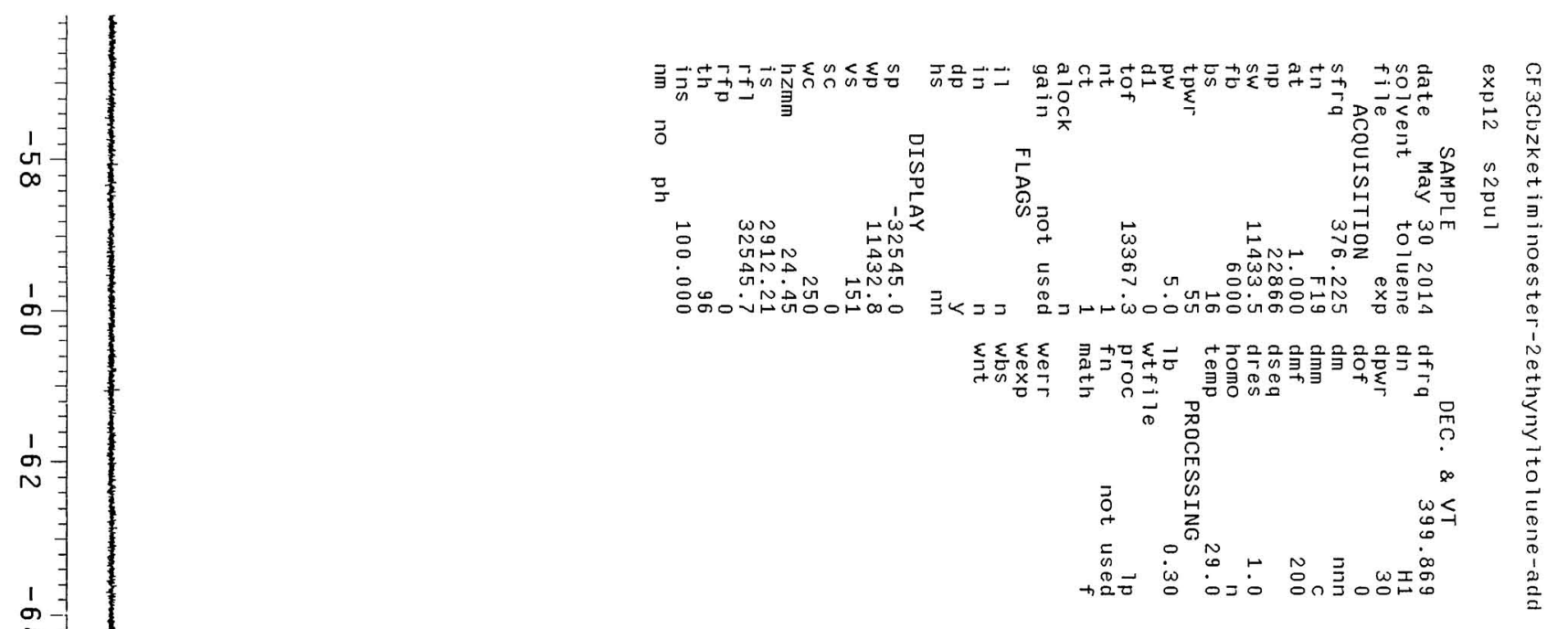

1
5
9
0

a

1
5
$\infty$

1

$\frac{1}{N}$

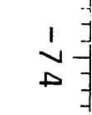

वे-

$\stackrel{1}{\infty}=$

1
0
0
0

1
$\infty$
$N$

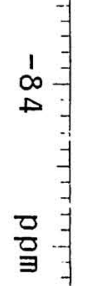

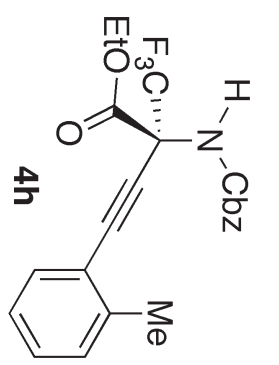




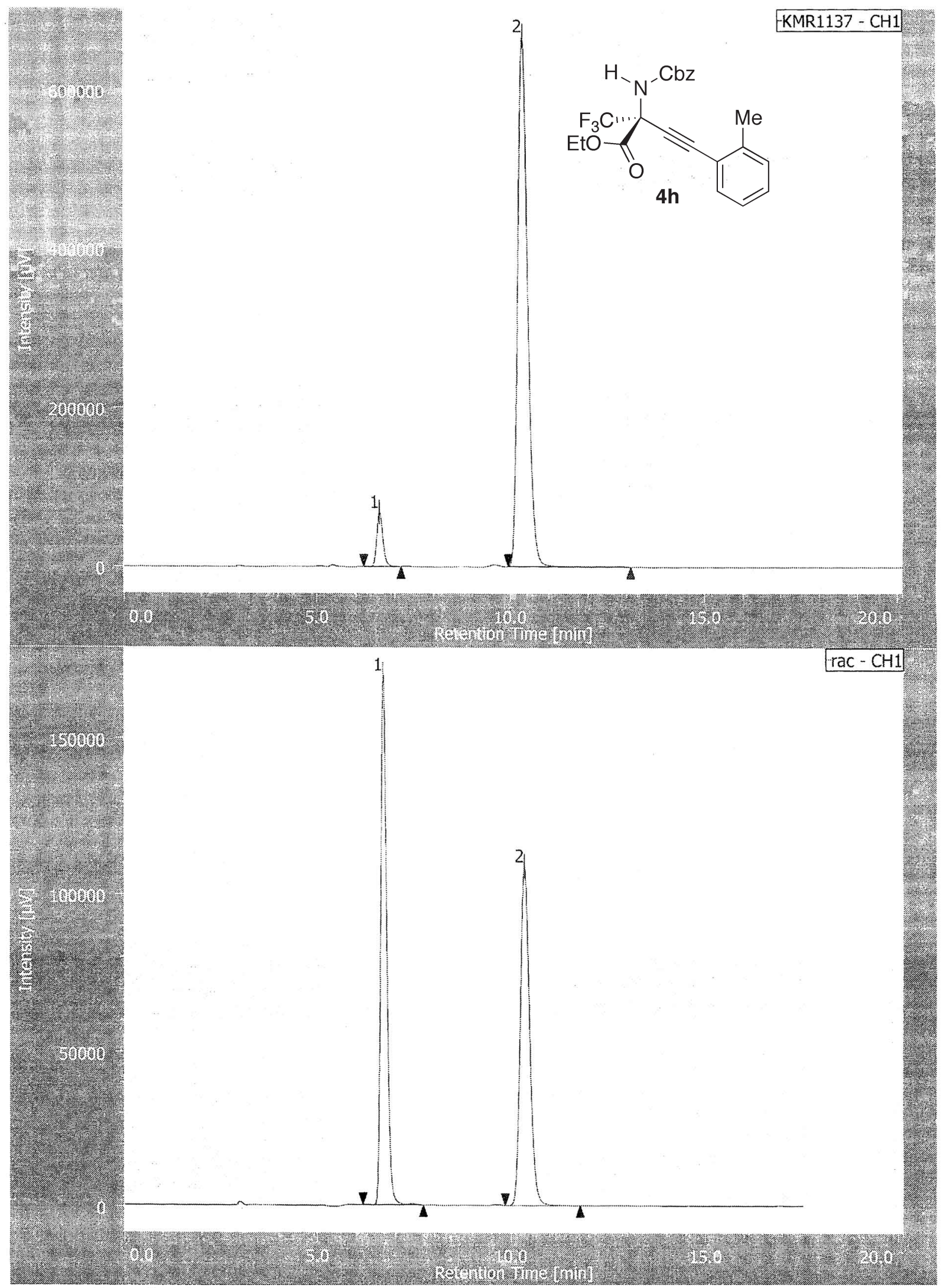

チャンネル情報+ピーク情報

クロマトグラム名

サンプル名

KMR1137-CH1

チャンネル名

$\mathrm{CH} 1$

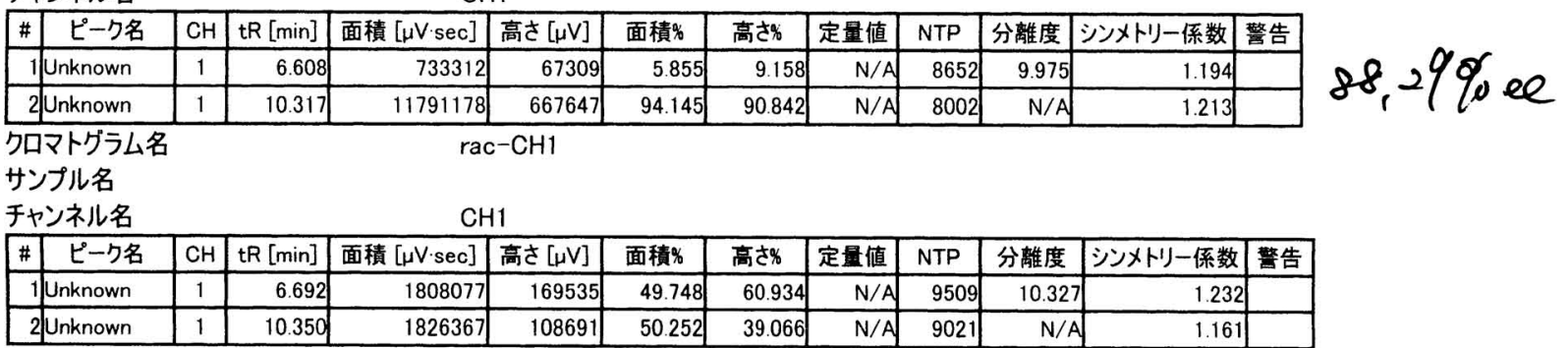




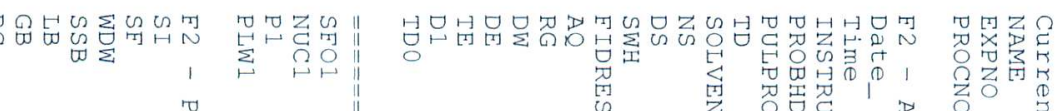

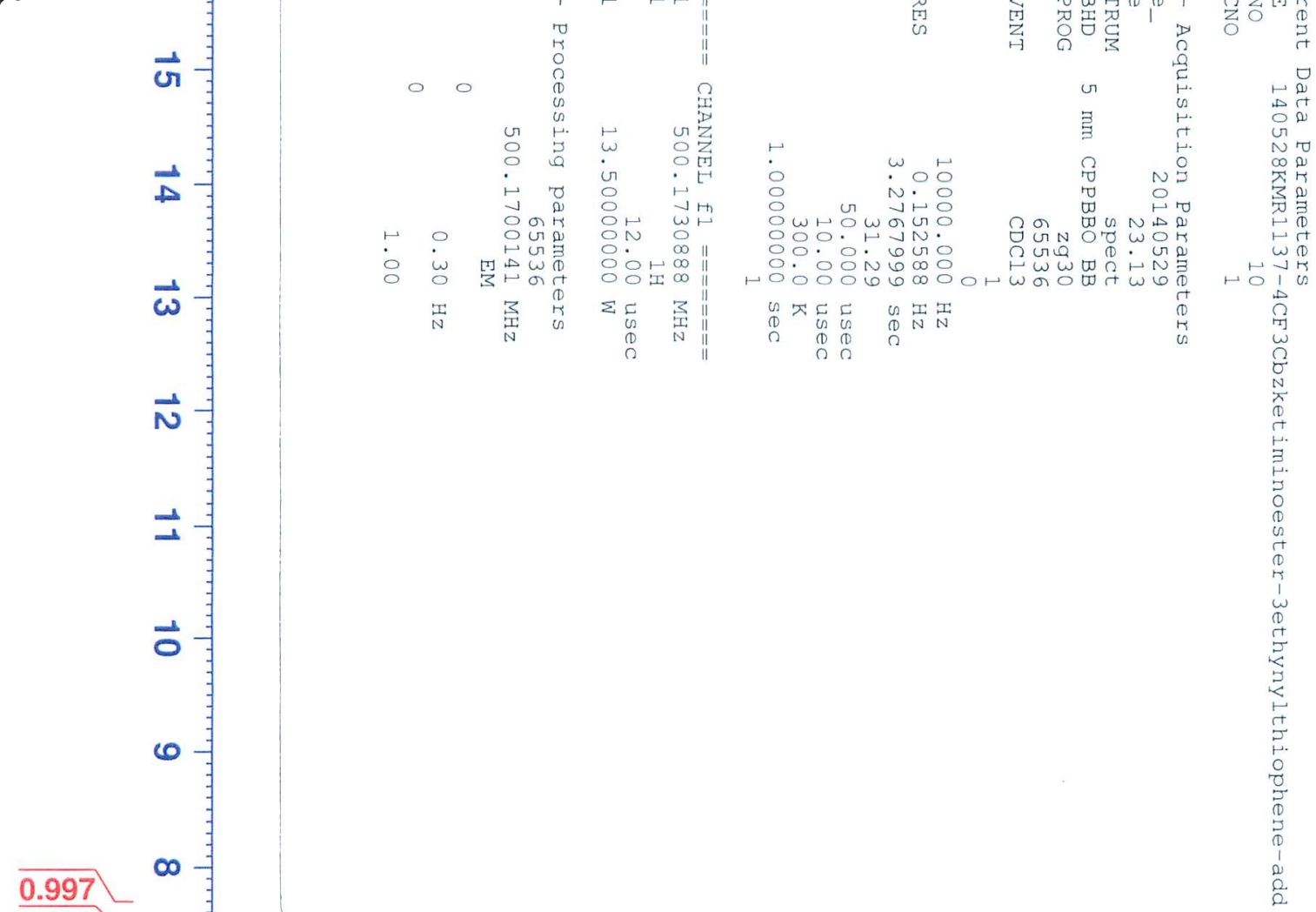

$-7.544$

$-7.376$

7.368

7.365

7.360

$\left[\begin{array}{r}7.354 \\ 7.349 \\ 7.349\end{array}\right.$

$7-7.34$

F 7.338

H 7.336

4.990

$=$

$\frac{1.489}{0.952}$

$\underline{0.982}$

a

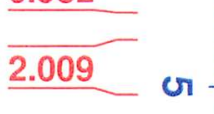

2.000

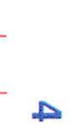

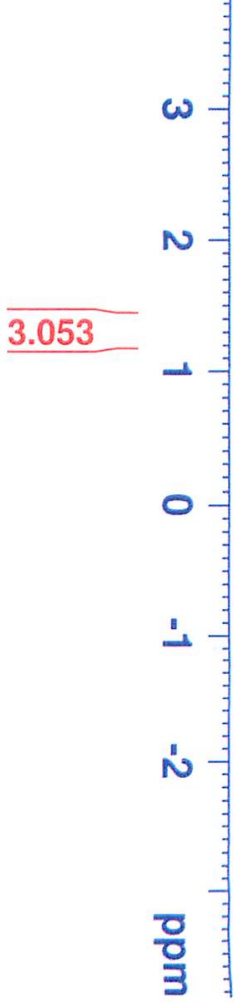

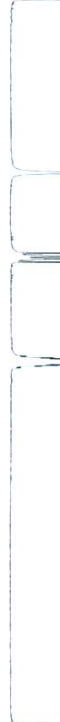

$-7.326$

$-7.324$

$-7.315$

$-7.274$

$-7.264$

7.256

7.132

7.130

7.122

[ 7.120

$-5.792$

$\left[\begin{array}{l}5.165 \\ 5.145\end{array}\right.$

5.120

4.391

4.384

4.370

4.359

4.355

4.345

4.331

$-1.573$

$-1.327$

1.313
1.298

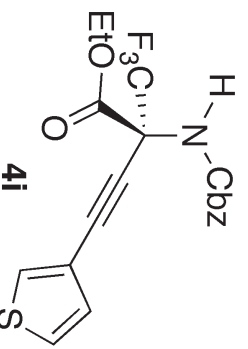




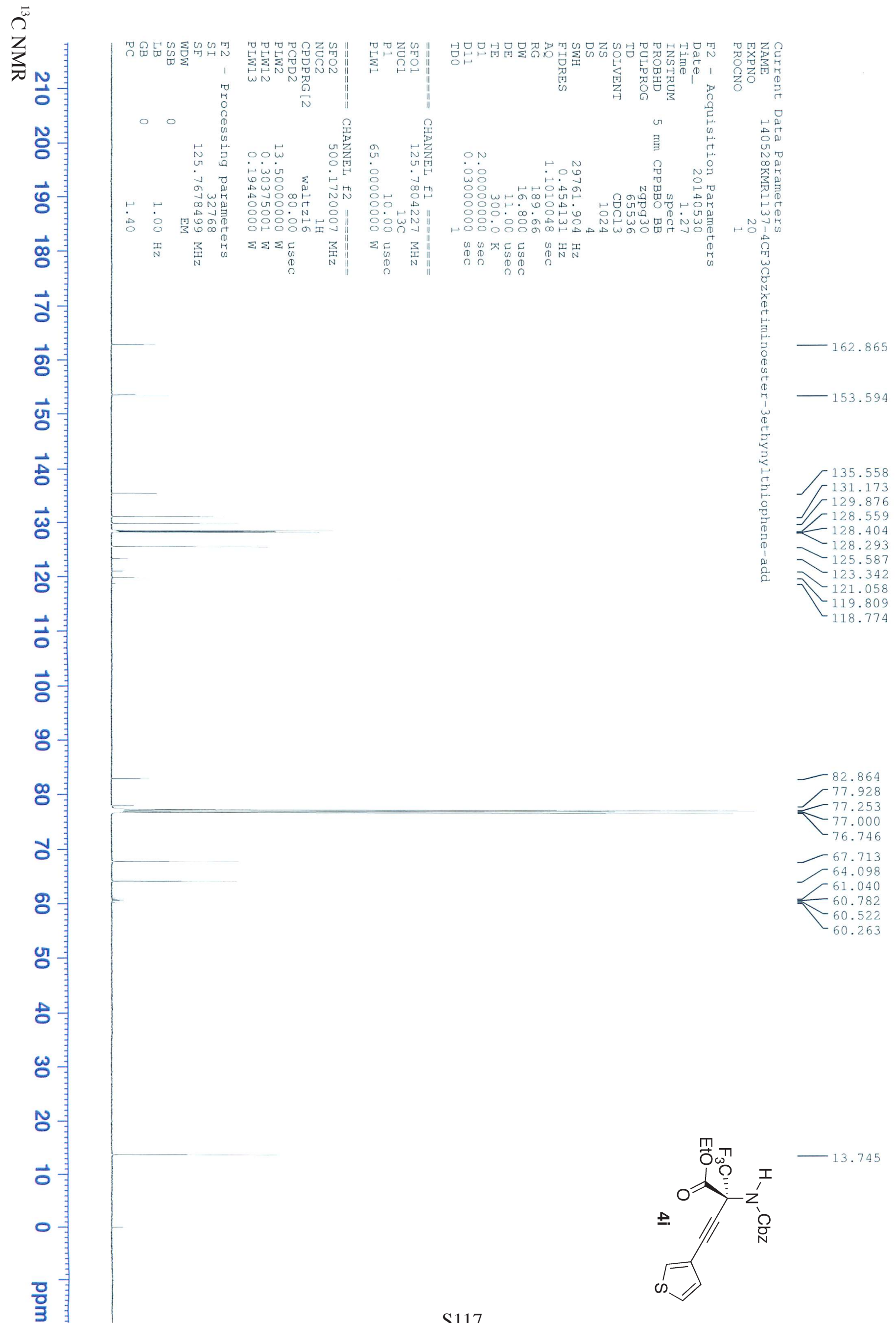


莫

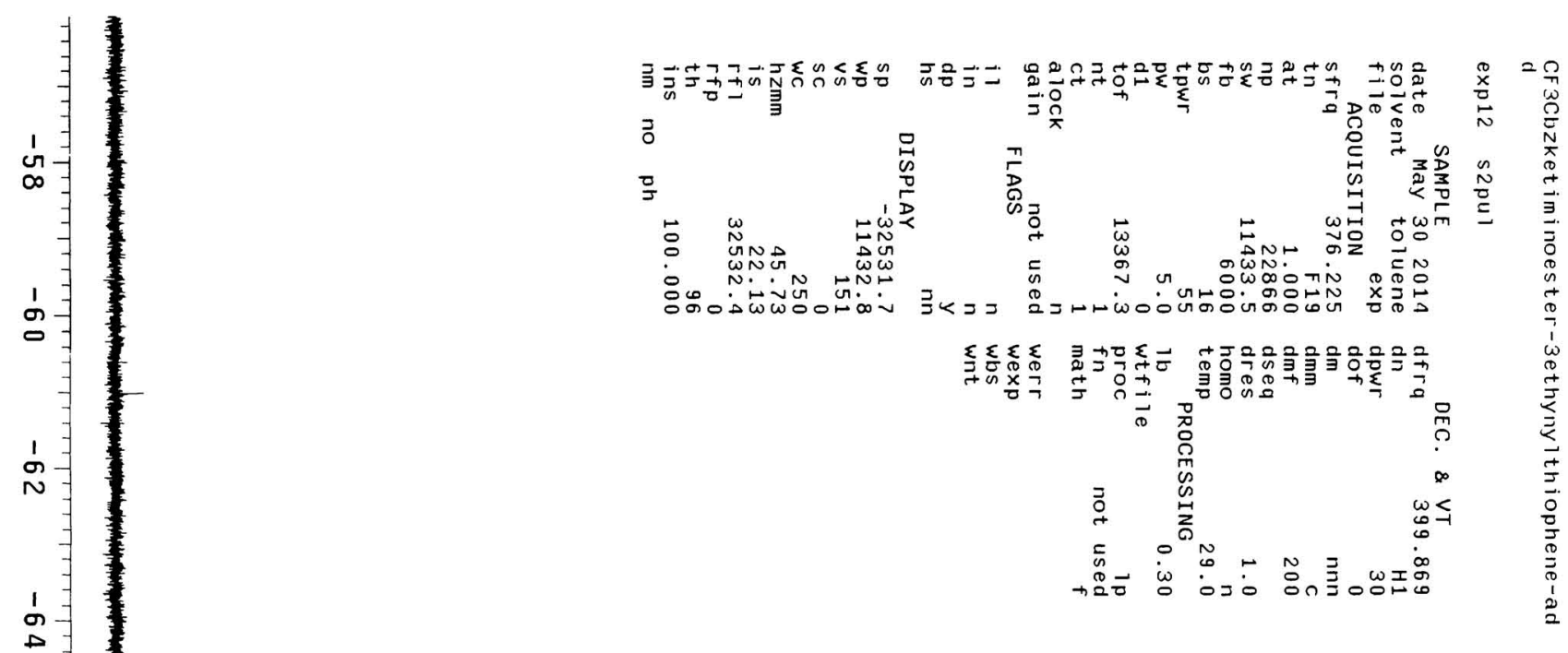

ต

1
$\infty$

ป

$\stackrel{1}{2}$

N

$\stackrel{1}{D}$

17

75690

$\stackrel{1}{\infty}$

$\stackrel{1}{\infty}$

1
$\infty$
$N$

$\stackrel{1}{\infty}$

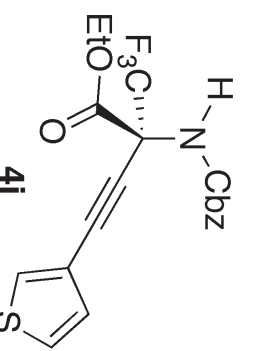




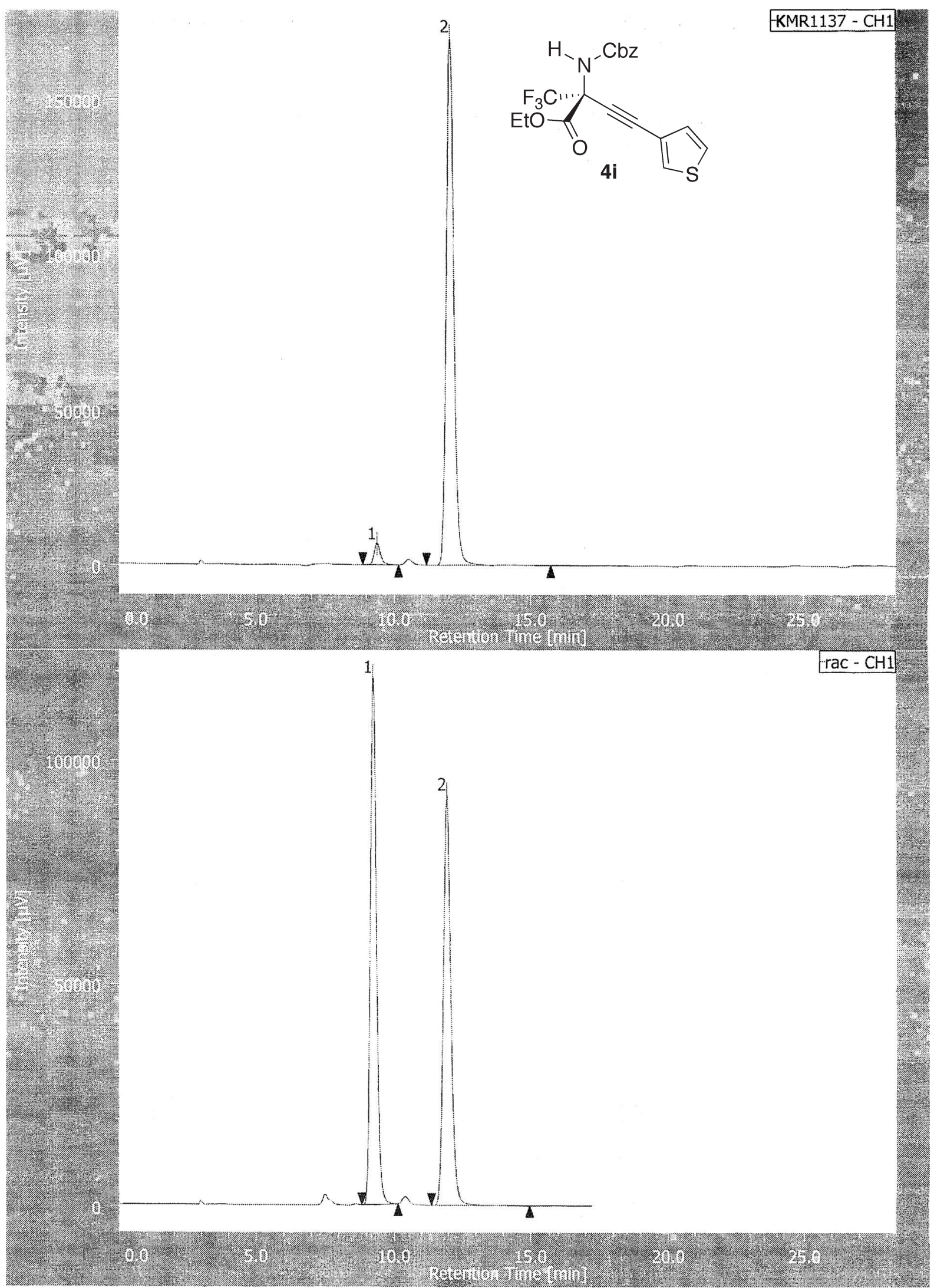

チャンネル情報+ピーク情報

クロマトグラム名

サンプル名

KMR1137-CH1

チャンネル名

$\mathrm{CH} 1$

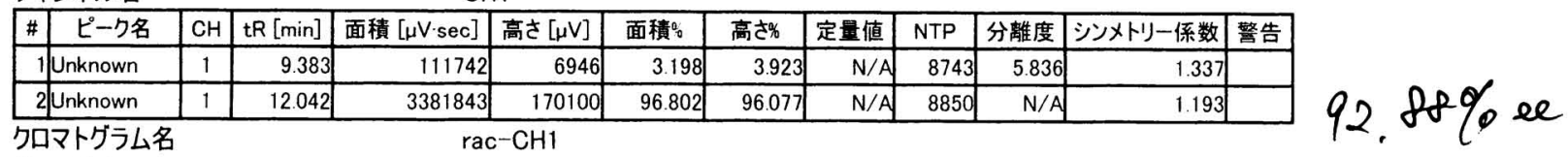

サンプル名

チャンネル名

$\mathrm{CH} 1$

\begin{tabular}{|c|c|c|c|c|c|c|c|c|c|c|c|}
\hline ピーク名 & $\mathrm{CH}$ & $\mathrm{tR}$ [min] & 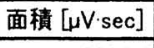 & \begin{tabular}{|l|} 
高さ [ $\mathrm{N} \mathrm{V}]$ \\
\end{tabular} & 面積\% & 高さ\% & 定量值 & NTP & 分離度 & シンメトリー係数 & \begin{tabular}{|l|} 
䇾告 \\
\end{tabular} \\
\hline Unknown & 1 & 9.233 & 1836713 & 118323 & 49.699 & 56.254 & N/A & 8508 & 5.872 & 1.212 & \\
\hline 2 Unknown & 1 & 11.925 & 1858991 & 92014 & 50.301 & 43.746 & $N / A$ & 8454 & N/A & 1.201 & \\
\hline
\end{tabular}




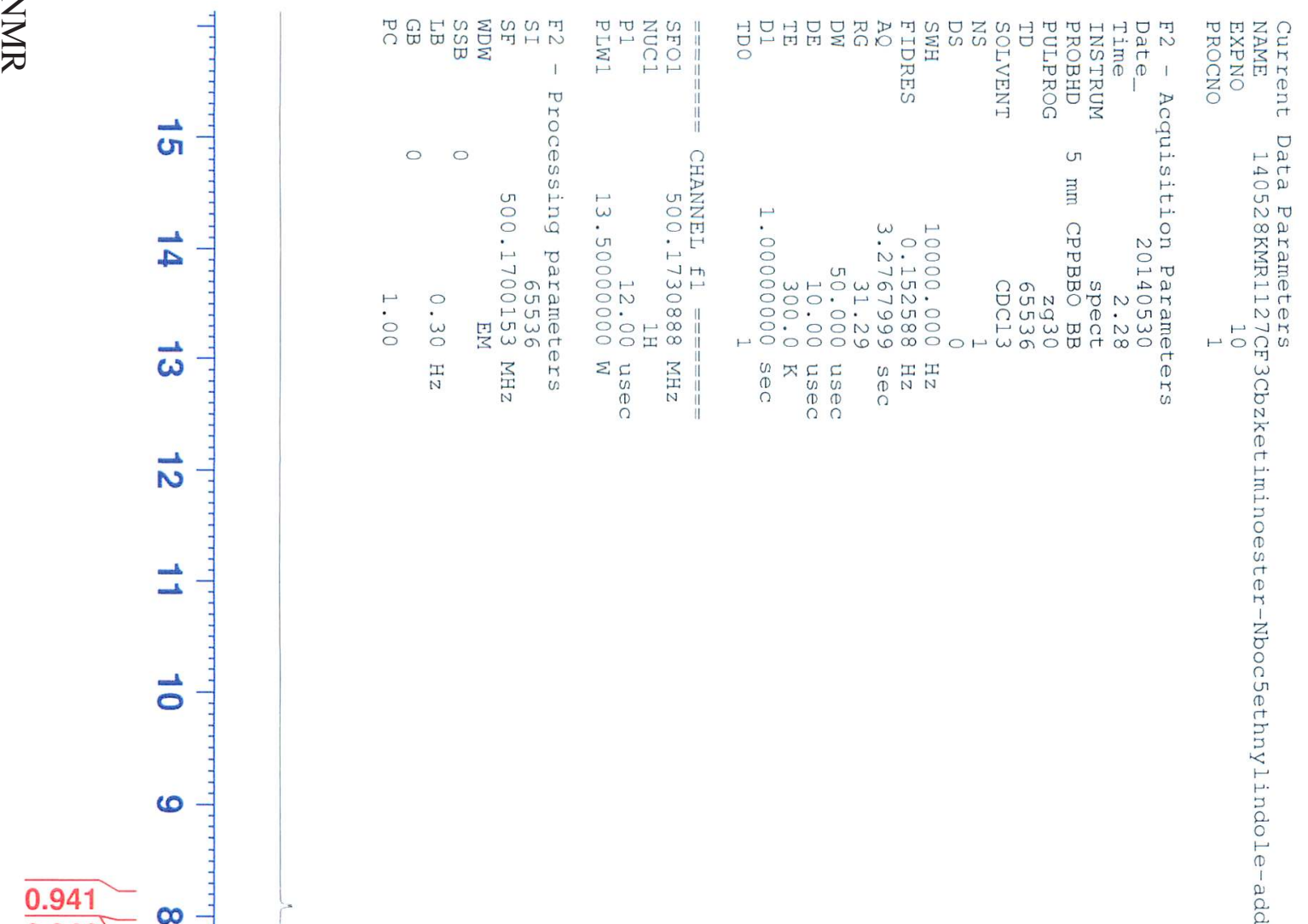

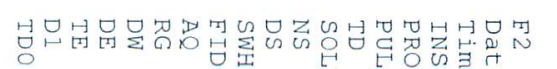

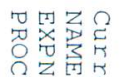

$\left[\begin{array}{l}8.107 \\ 8.090 \\ 7.092\end{array}\right.$

7.692

7.614

7.606

7.402

| 7.400

| 7.384

$\left[\begin{array}{r}7.372 \\ 7.368 \\ 7.3650\end{array}\right.$

$[F=7.368$

$\underline{0.946 \leftarrow} \infty$

0.994

5.945

$\underline{0.969}$

7.360
-7.352
-7.348

$-7.348$

$-7.338$

$-7.333$

]$\left[\begin{array}{l}7.330 \\ 7.328\end{array}\right.$

$\left[\begin{array}{l}7.320 \\ 7.255\end{array}\right.$

6.535

$-6.534$

$[-6.527$

$-5.827$

$-5.201$

$\left[\begin{array}{r}5.177 \\ 5.155\end{array}\right.$

$-5.131$

$-4.381$

$-4.372$

$-4.367$

4.358

4.353

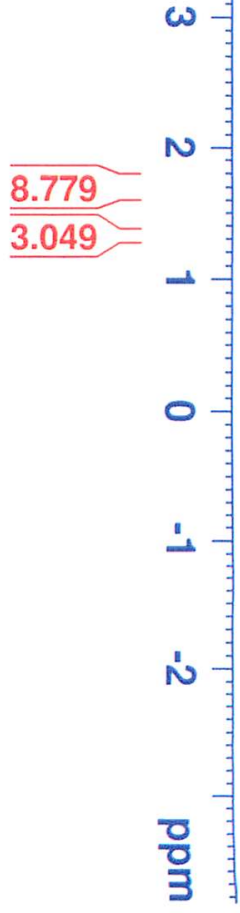

$-1.589$

$-1.340$

1.325

1.311

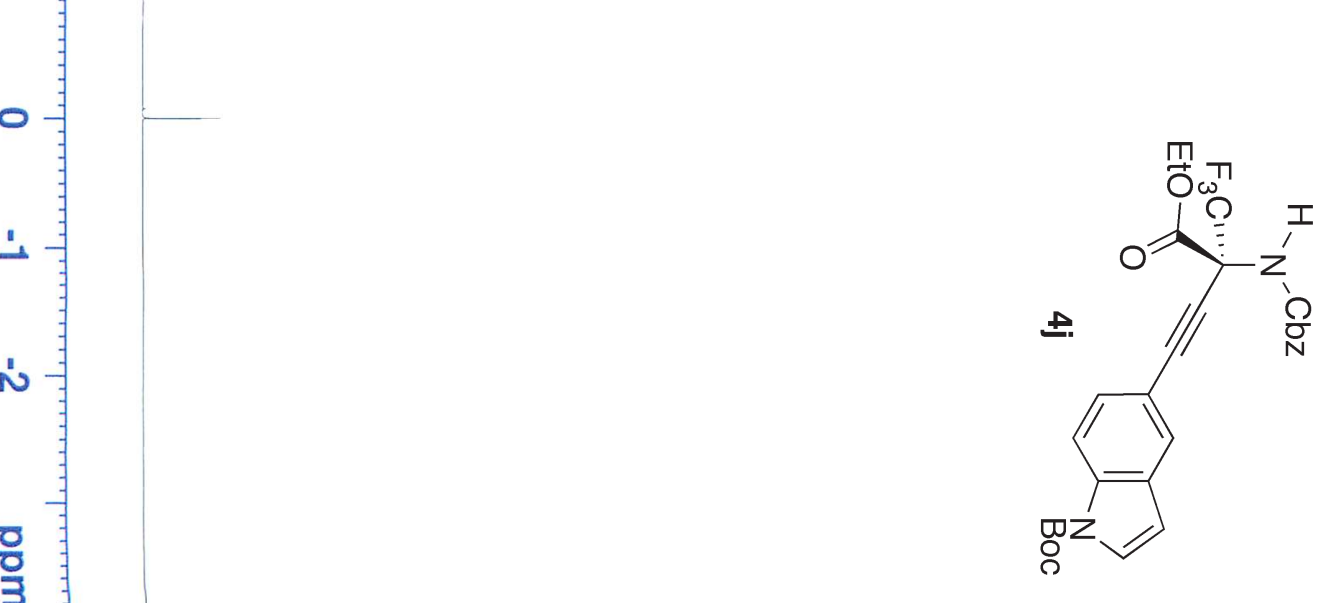

$-0.000$ 


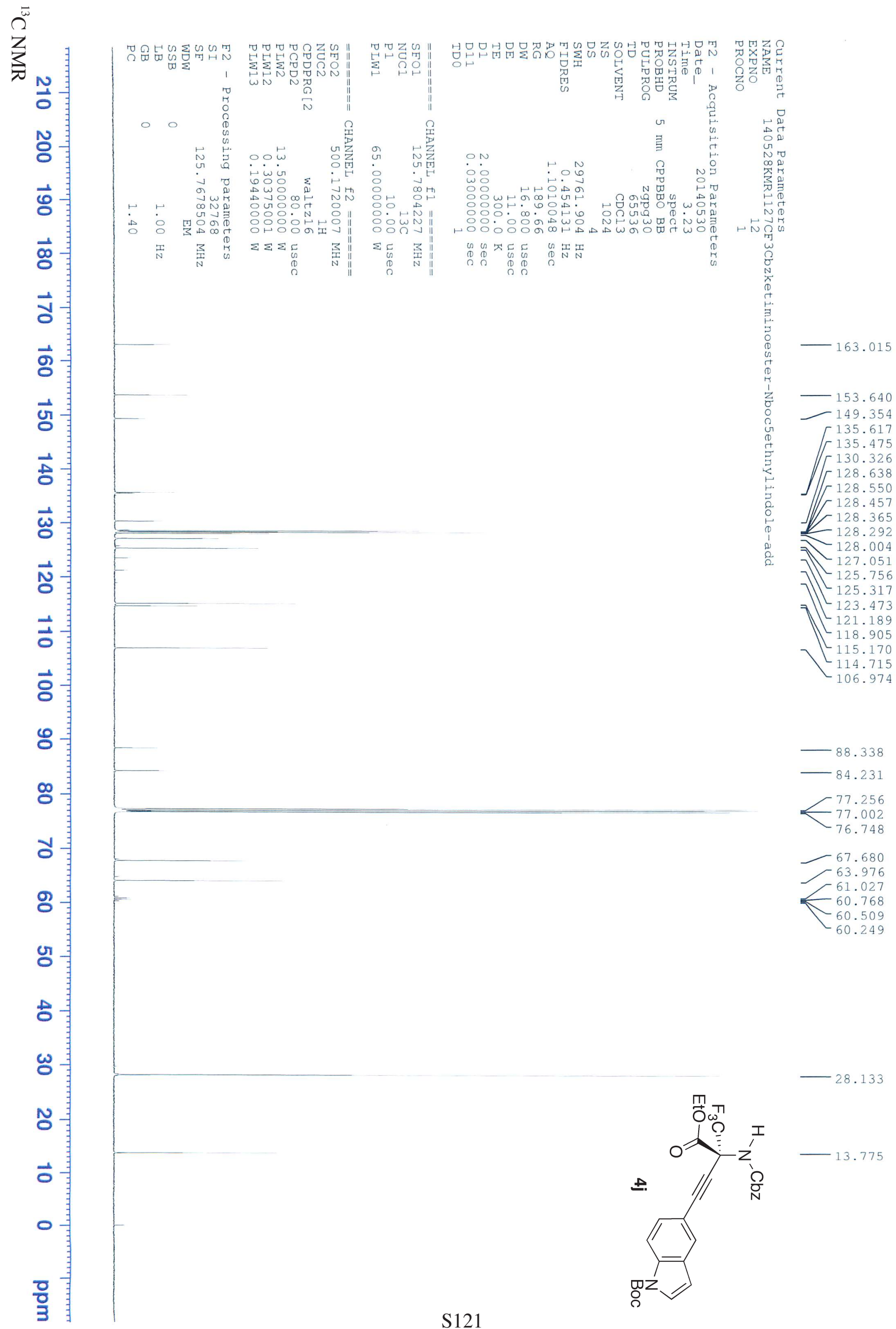




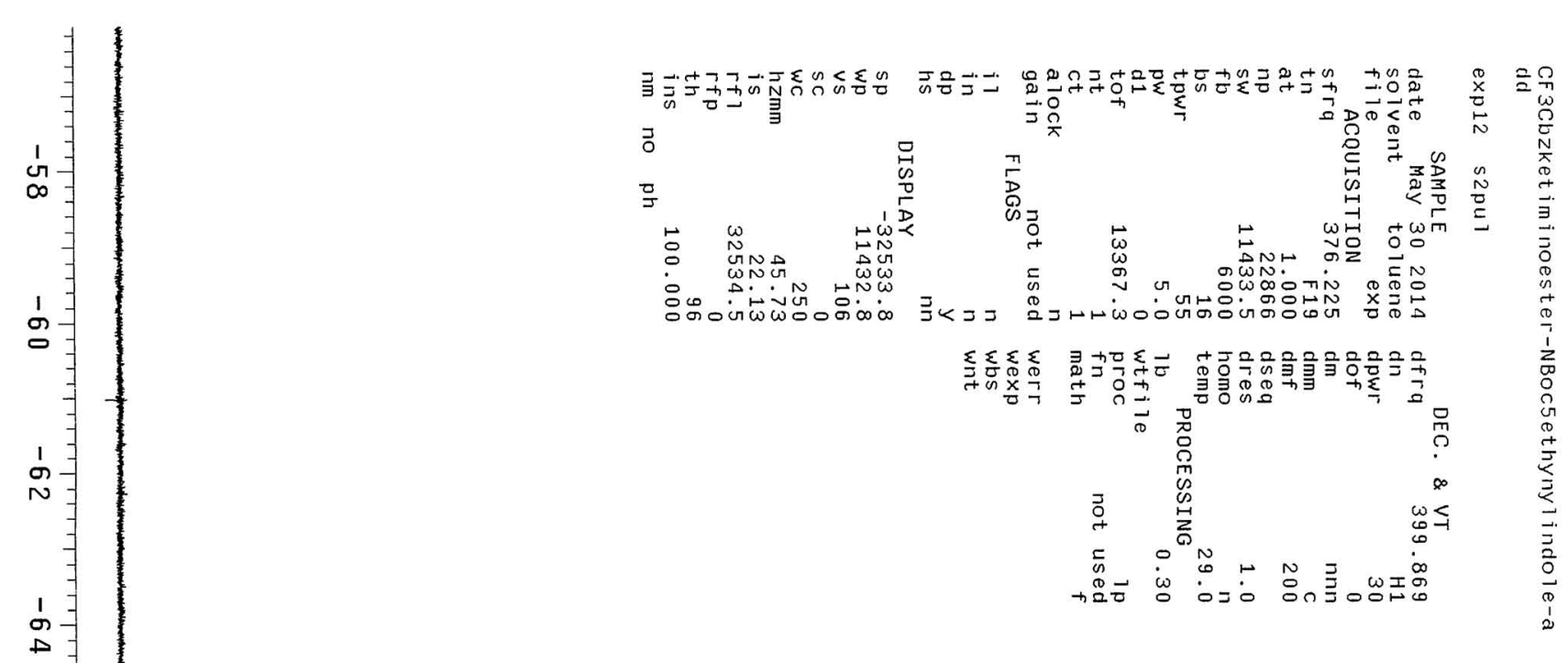

2)

1
$\infty$
$\infty$

ㄴ.

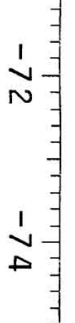

के

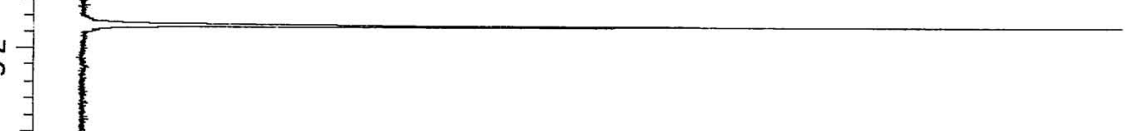

$-75.737$

$\stackrel{1}{\infty}$

1
$\infty$
$\infty$

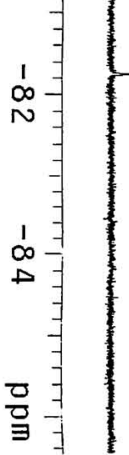

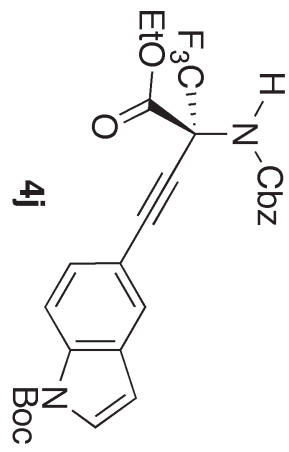




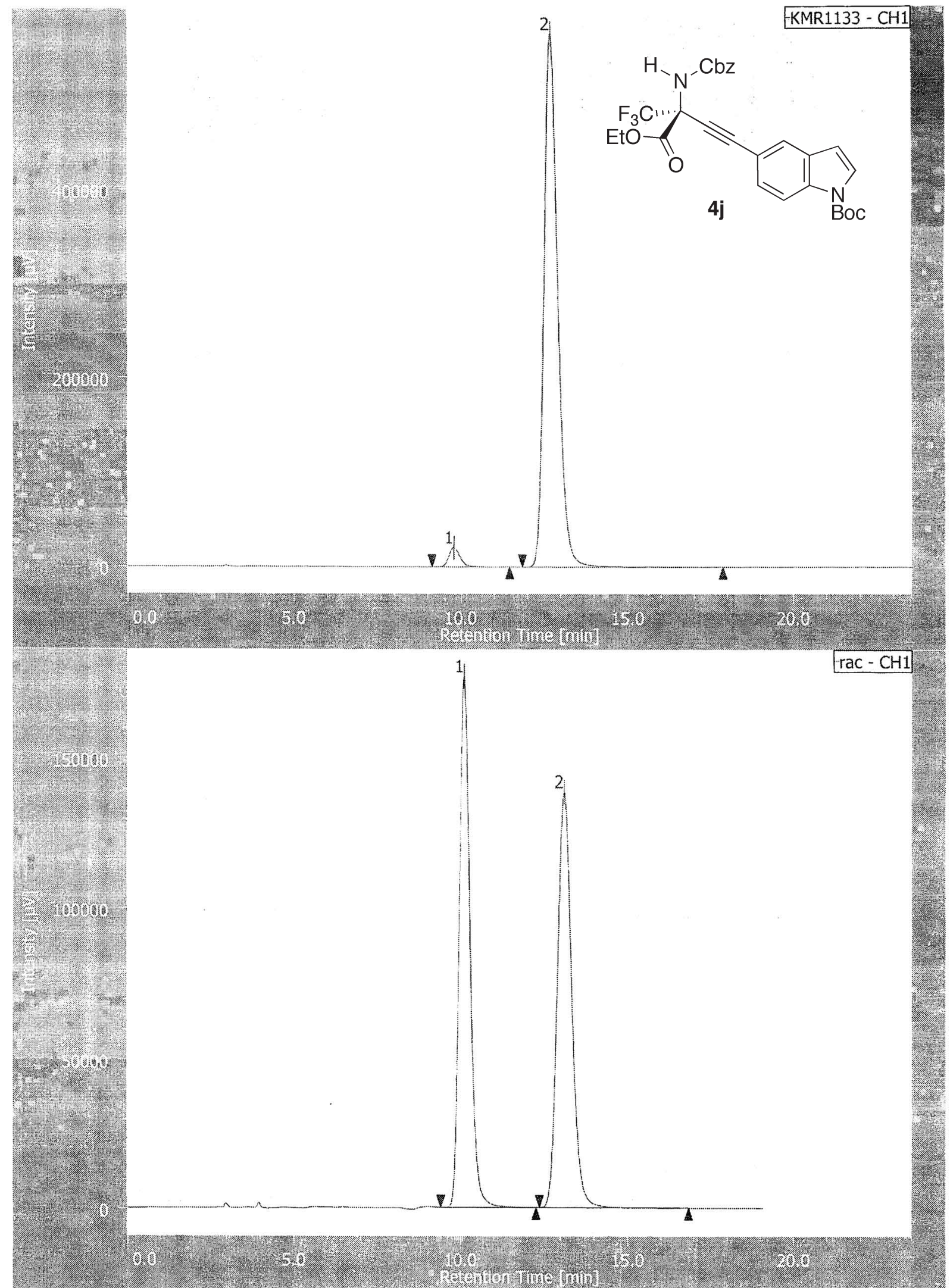

チャンネル情報十ピーク情報

クロマトグラム名

KMR1133-CH1

サンプル名

チャンネル名

$\mathrm{CH} 1$

\begin{tabular}{|c|c|c|c|c|c|c|c|c|c|c|c|c|}
\hline \# & ピーク名 & $\mathrm{CH}$ & $\mathrm{tR}$ [min] & 面積 $[\mu \mathrm{V} \cdot \mathrm{sec}]$ & 高さ $[\mu \mathrm{V}]$ & 面積: & 高さ\% & 定量値 & NTP & 分離度 & シンメトリー係数 & \begin{tabular}{|l|} 
警告 \\
\end{tabular} \\
\hline & Unknown & 1 & 9.783 & 469156 & 20041 & 2.852 & 3.438 & $\mathrm{~N} / \mathrm{A}$ & 4282 & 4.395 & 1.224 & \\
\hline & Unknown & 1 & 12.692 & 15982038 & 562880 & 97.148 & 96.562 & $\mathrm{~N} / \mathrm{A}$ & 4848 & $N / A$ & 1.224 & \\
\hline
\end{tabular}

$94.30 \%$ eel.

クロマトグラム名

$\mathrm{rac}^{-} \mathrm{CH} 1$

サンプル名

チャンネル名

$\mathrm{CH} 1$

\begin{tabular}{|c|c|c|c|c|c|c|c|c|c|c|c|}
\hline ピーク名 & $\mathrm{CH}$ & $t R[\mathrm{~min}]$ & 面積 [ [ $\mathrm{V} \cdot \mathrm{sec}]$ & 高さ $[\mu \mathrm{V}]$ & 面積! & 高さ。 & 定量値 & NTP & 分離度 & シンメトリー係数 & \begin{tabular}{|l|} 
警告 \\
\end{tabular} \\
\hline 1 Unknown & 1 & 10.100 & 5725 & 176459 & 49.646 & 56.025 & $N / A$ & 4650 & 4.413 & 1.229 & \\
\hline 2 Unknown & 1 & 13.100 & 4123739 & 138504 & 50.354 & 43.975 & $N / A$ & 4621 & $\mathrm{~N} / \mathrm{A}$ & 1.172 & \\
\hline
\end{tabular}




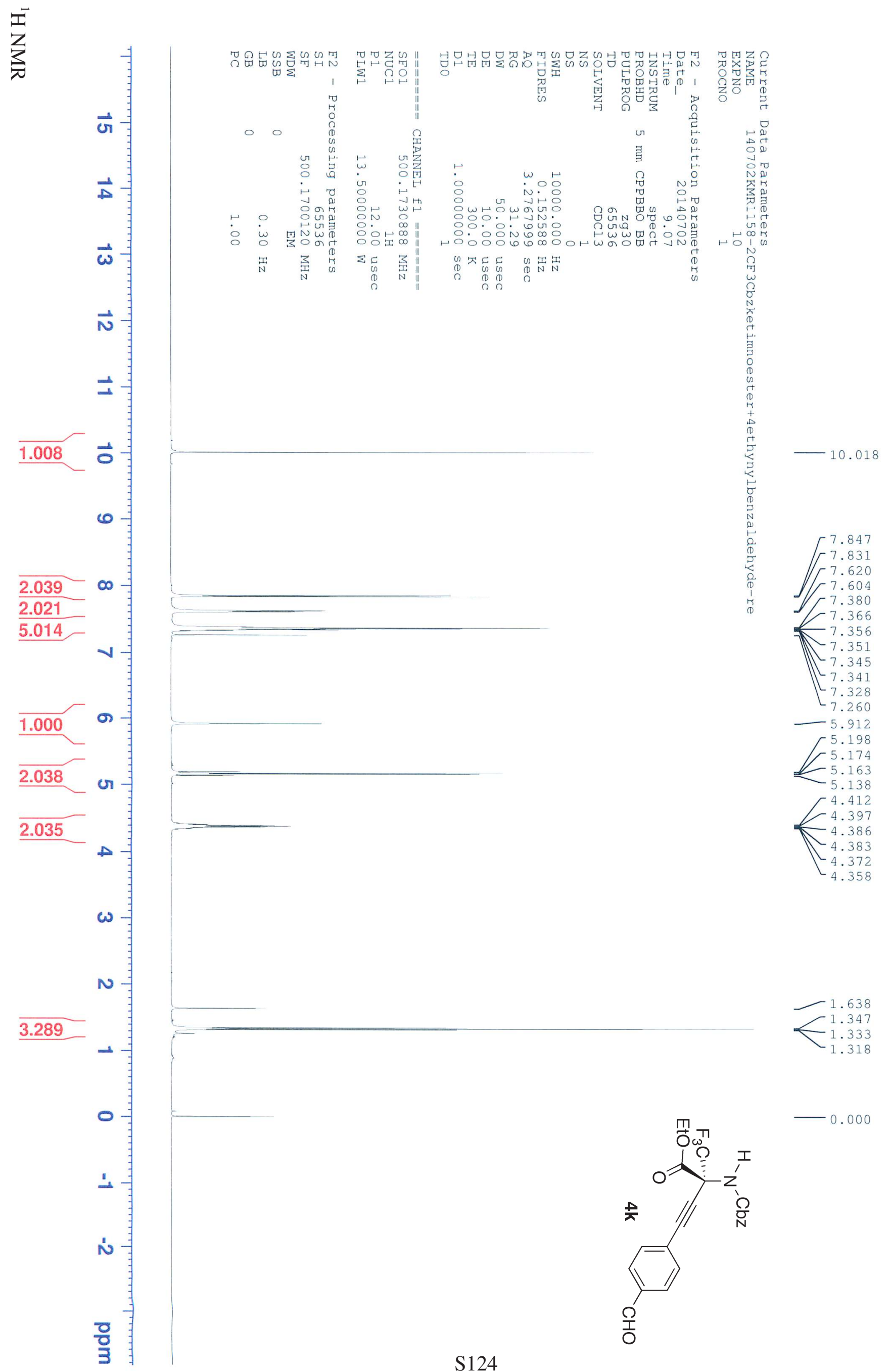




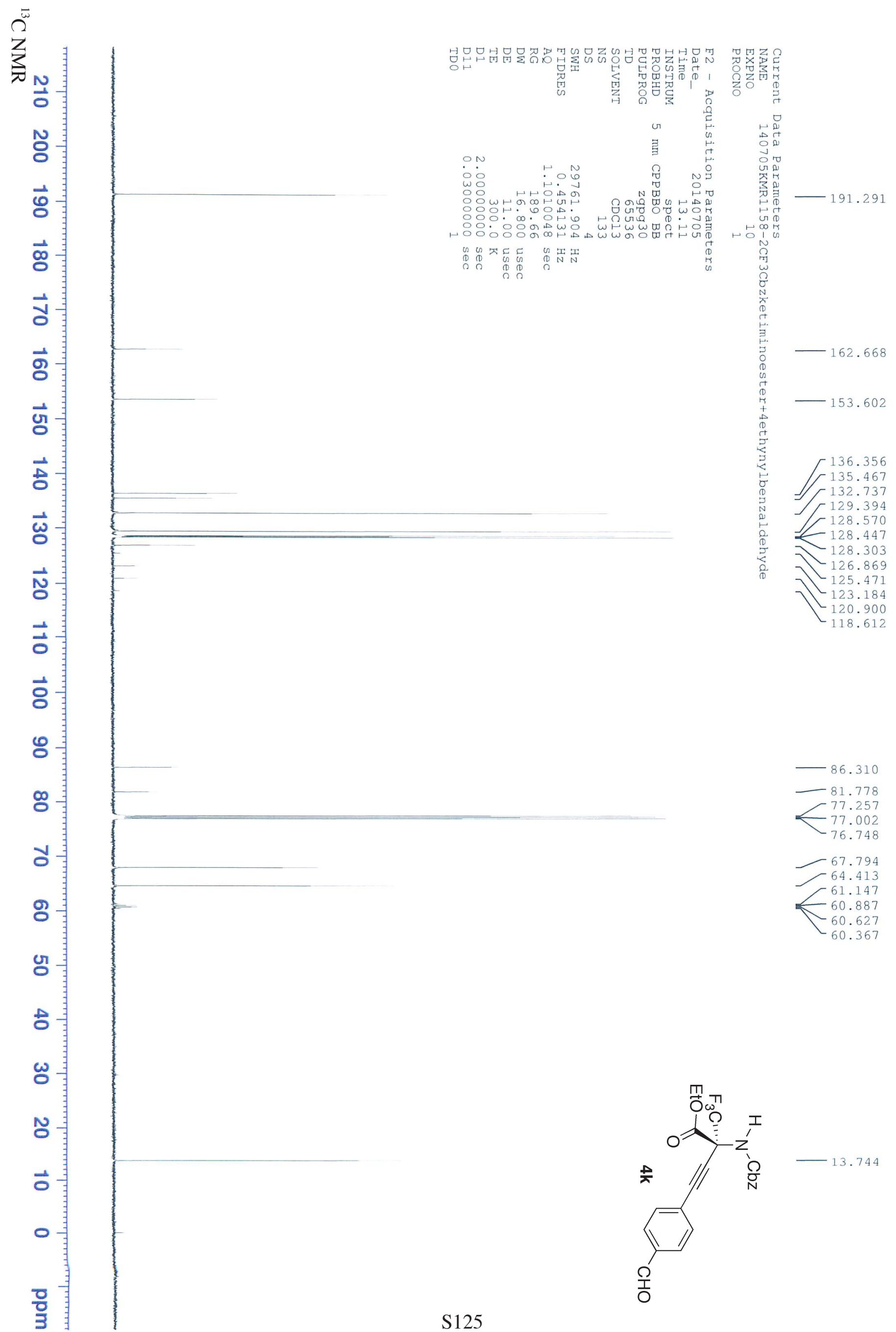


$\stackrel{1}{D}$

के

$\stackrel{1}{\infty}$

1
$\vdots$
$\infty$
0

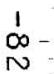

$\stackrel{1}{\infty}$

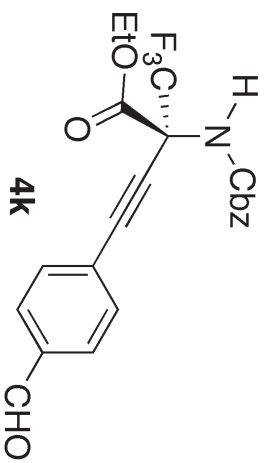

믑 


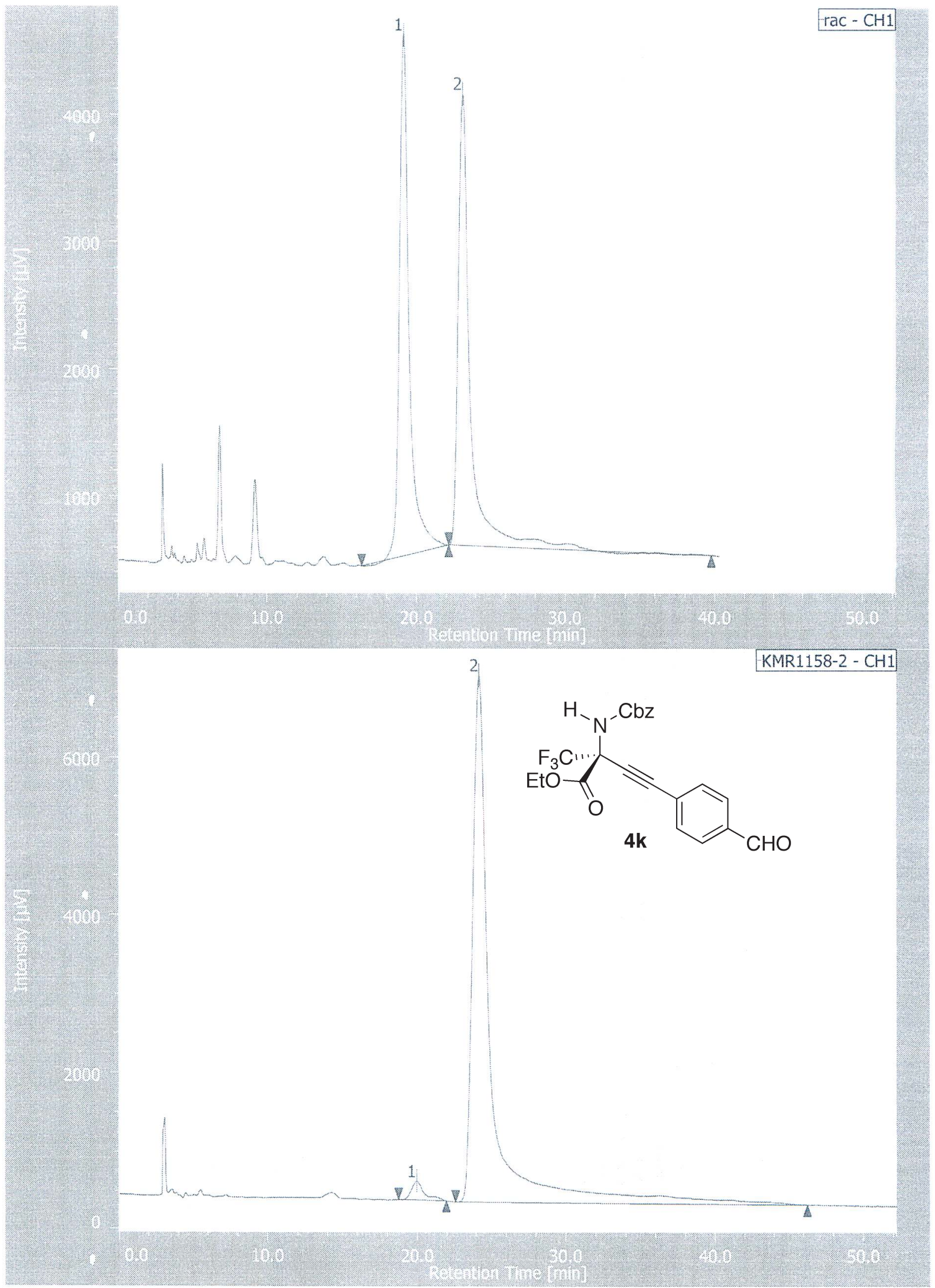

チャンネル情報+゙ピーク情報

クロマトグラム名

$\mathrm{rac}-\mathrm{CH} 1$

サンプル名

チャンネル名

$\mathrm{CH} 1$

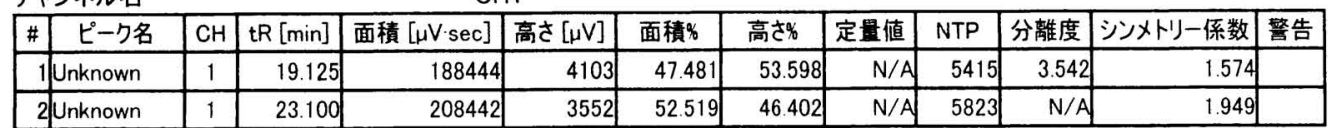

クロマトグラム名 KMR1158-2-CH1

サンプル名

チャンネル名

$\mathrm{CH} 1$

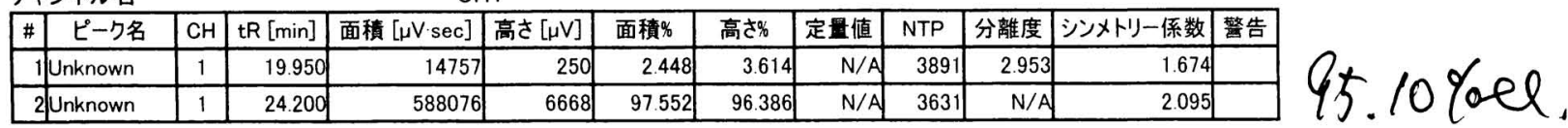




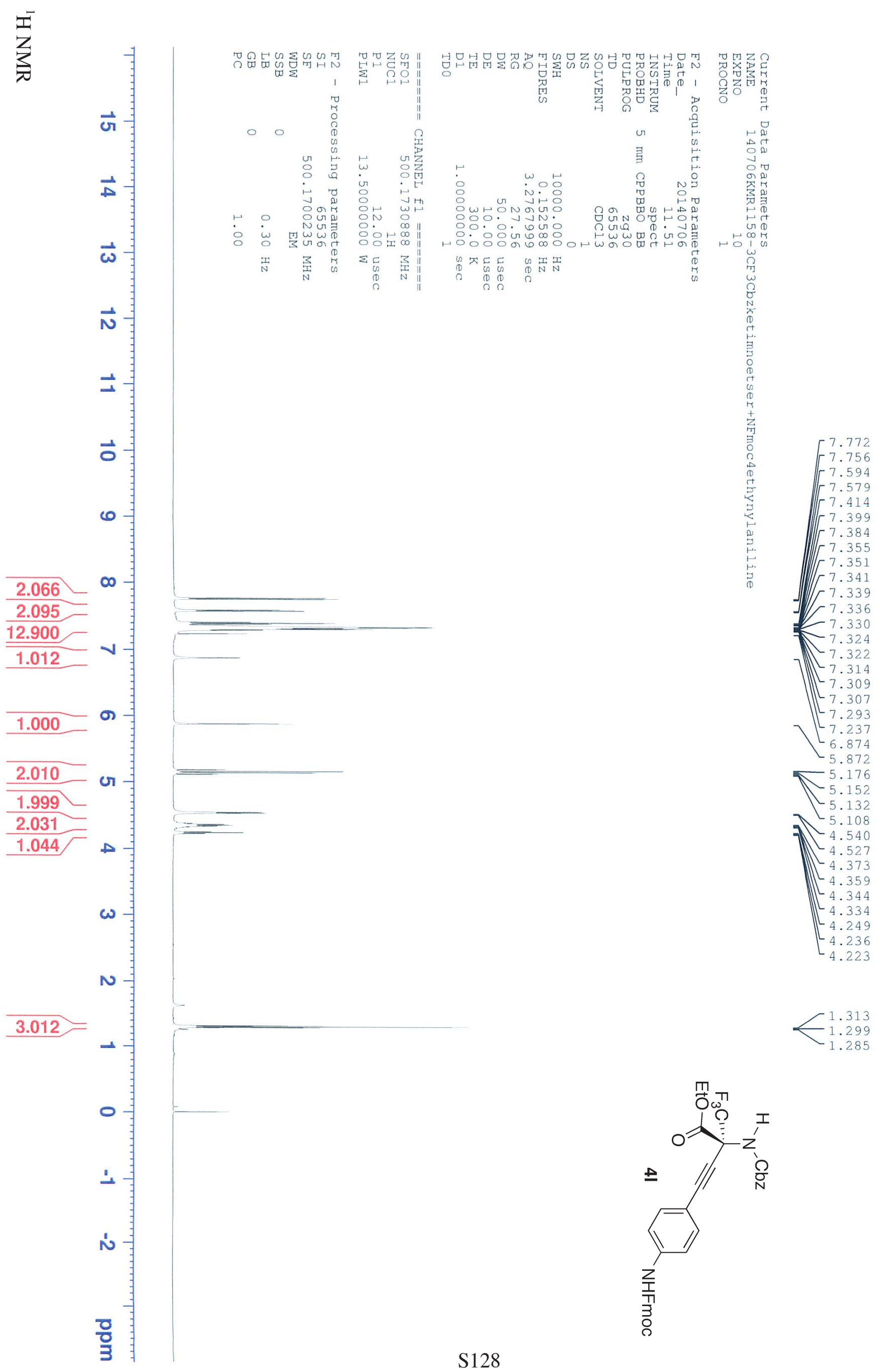




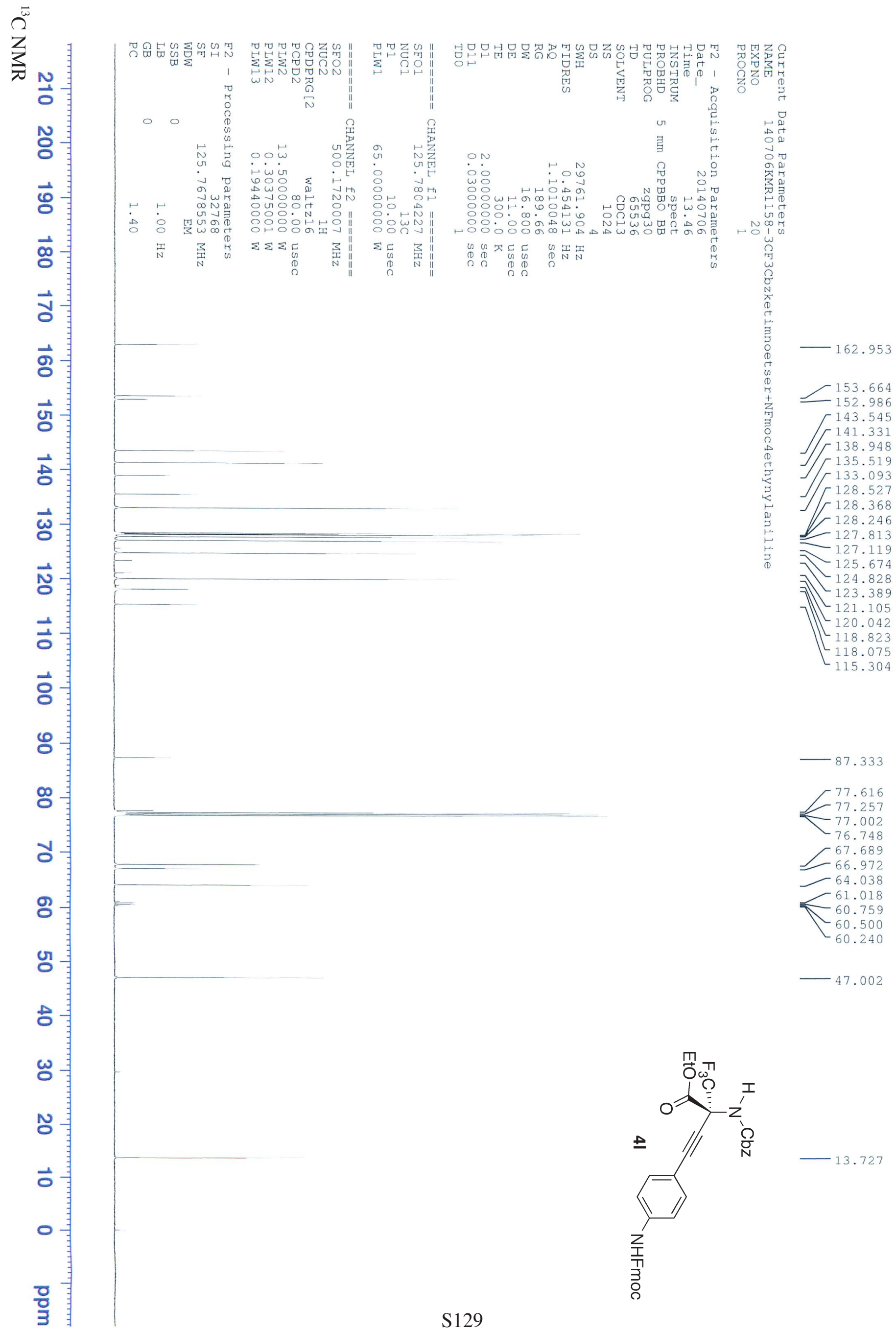


莫

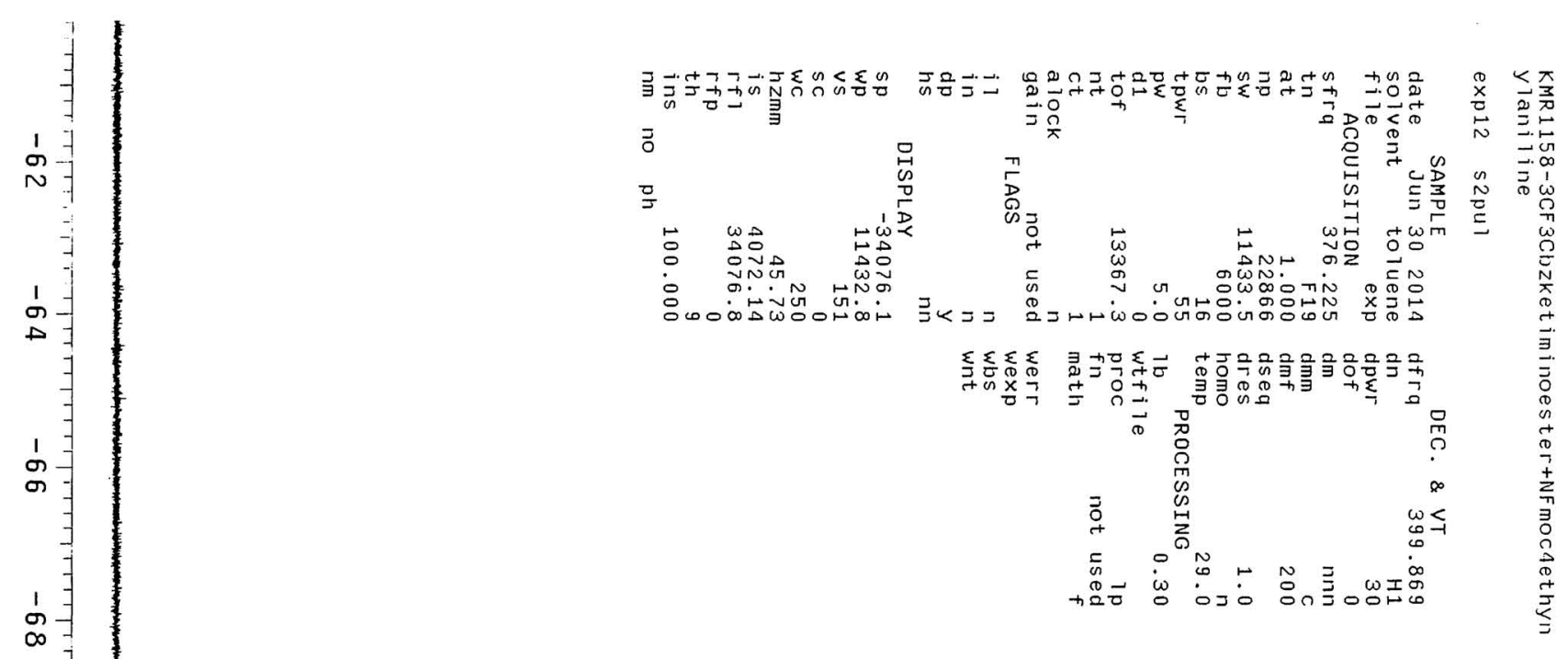

1

1
1
$N$

1
1
$D$
$D$

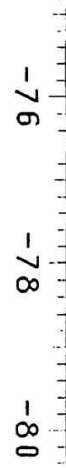

$\stackrel{1}{\infty}$

1
1
$\infty$
$\infty$
-1

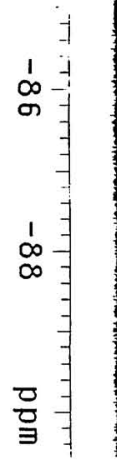

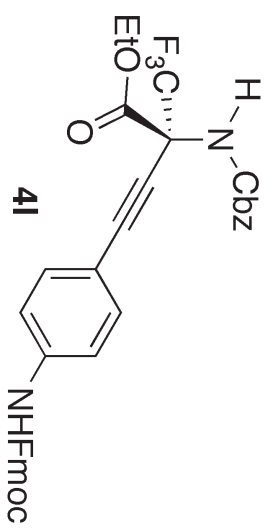




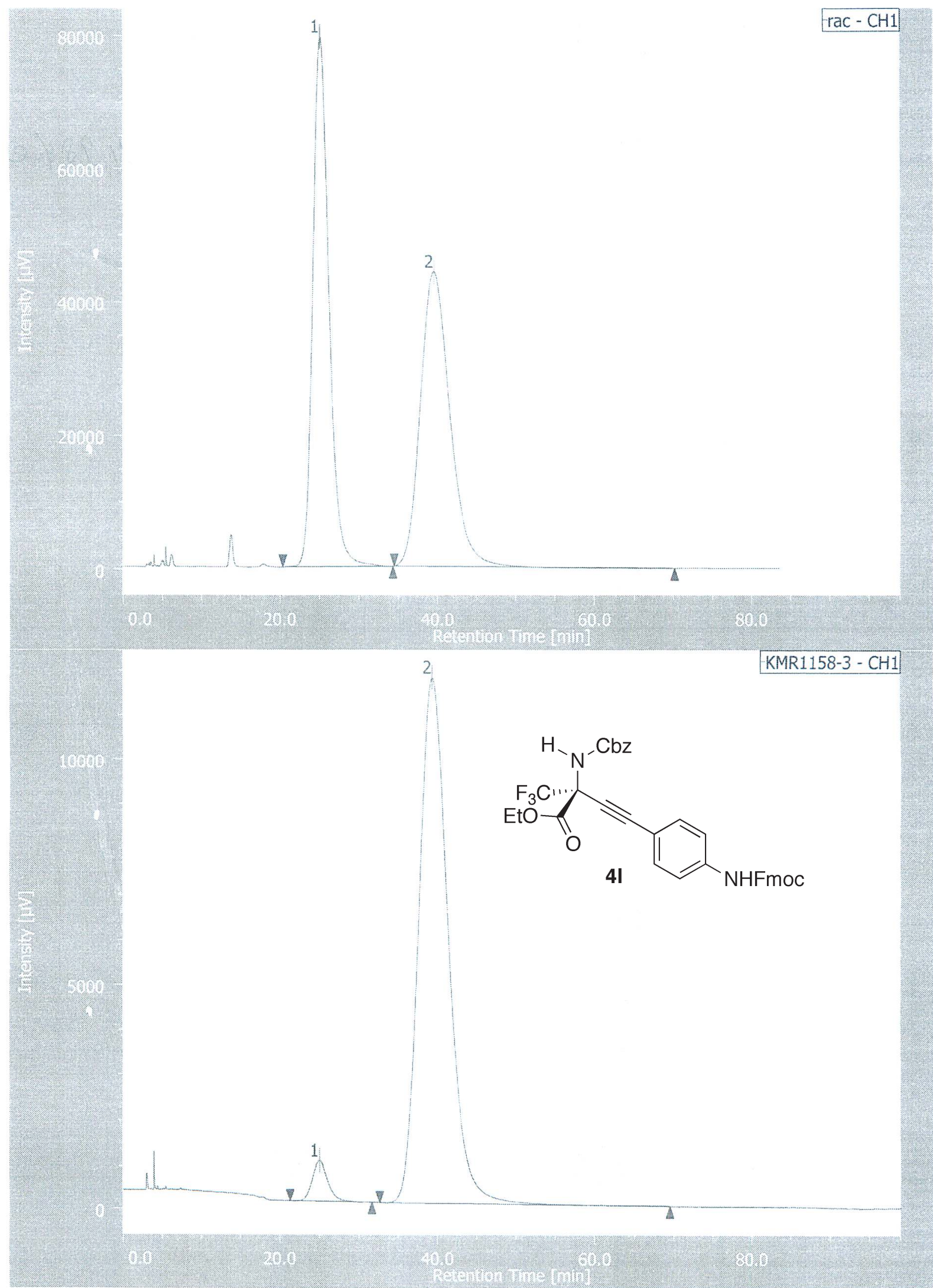

チャンネル情報+ピーク情報

クロマトグラム名

$\mathrm{rac}-\mathrm{CH} 1$

サンプル名

チャンネル名

$\mathrm{CH} 1$

\begin{tabular}{|c|c|c|c|c|c|c|c|c|c|c|c|c|}
\hline \# & ピーク名 & $\mathrm{CH}$ & $t R[\mathrm{~min}]$ & 面積 $[\mu \mathrm{V} \cdot \mathrm{sec}]$ & 高さ $[\mu \mathrm{V}]$ & 面積\% & 高さ\% & 定量値 & NTP & 分離度 & シンメトリー係数 & 警告 \\
\hline & Unknown & 1 & 25.108 & 11249894 & 79471 & 49.383 & 64.318 & N/A & 773 & 2.792 & 1.223 & \\
\hline & Unknown & 1 & 39.533 & 11531182 & 44088 & 50.617 & 35.682 & N/A & 549 & $\mathrm{~N} / \mathrm{A}$ & 1.222 & \\
\hline \multicolumn{13}{|c|}{$\begin{array}{l}\text { クロマトグラム名 } \\
\text { サンプル名 }\end{array}$} \\
\hline \multicolumn{13}{|c|}{ チャンネル名 } \\
\hline$\#$ & ピーク名 & $\mathrm{CH}$ & $\operatorname{tR}[\mathrm{min}]$ & 面栍 $[\mu \mathrm{V} \cdot \mathrm{sec}]$ & 高さ $[\mu \mathrm{V}]$ & 面積\% & 高さ\% & 定量值 & NTP & 分離度 & シンメトリー係数 & 警告 \\
\hline & Unknown & 1 & 25.042 & 127532 & 939 & 4.009 & 7.426 & $N / A$ & 822 & 2.800 & 1.165 & \\
\hline & Unknown & 1 & 39.258 & 3053946 & 11700 & 95.991 & 92.574 & N/A & 552 & $\mathrm{~N} / \mathrm{A}$ & 1.233 & \\
\hline
\end{tabular}




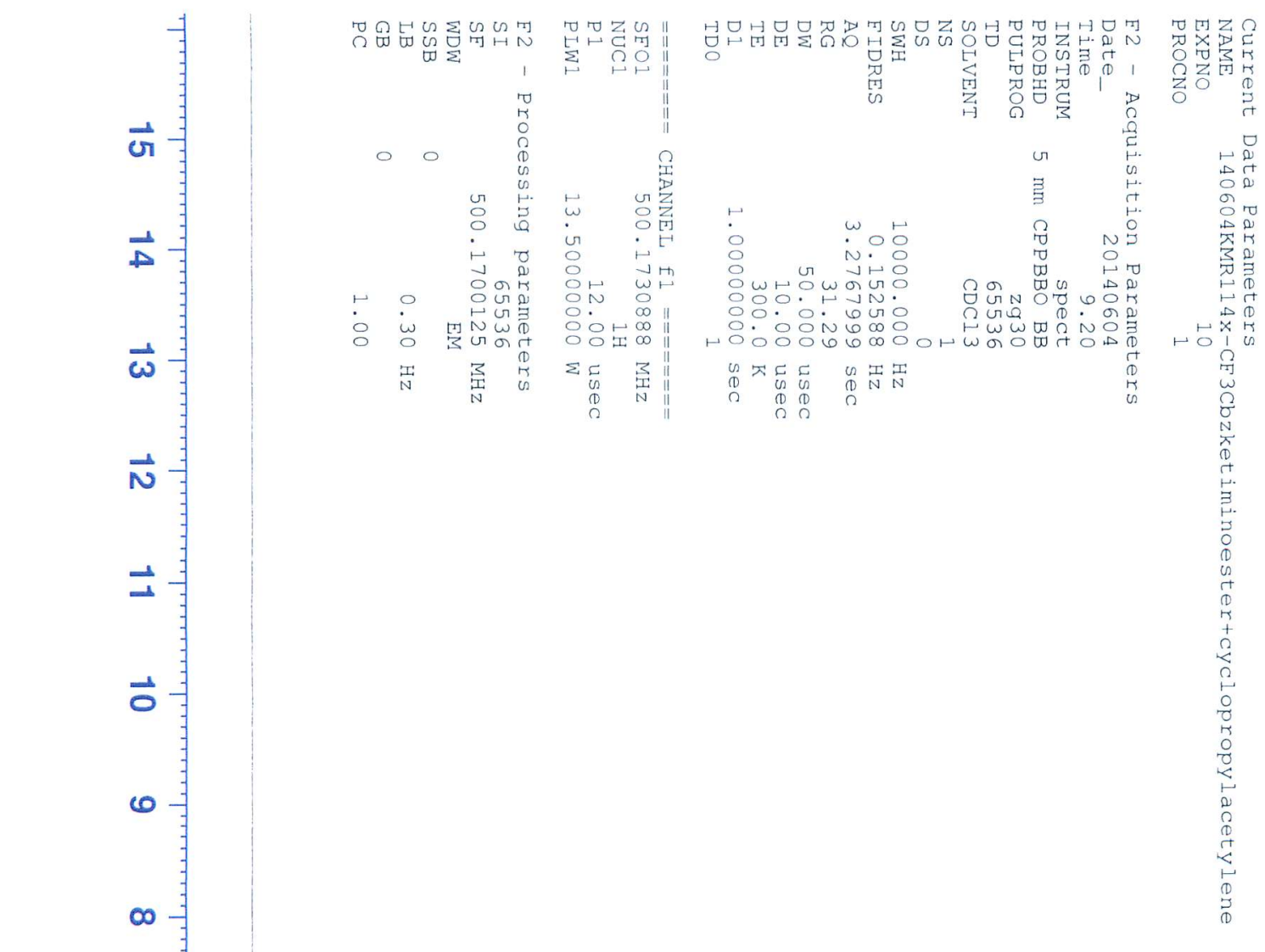

7.380

$\left[\begin{array}{l}7.377 \\ -7.369\end{array}\right.$

7.366

7.365

$\mid \begin{array}{r}7.362 \\ 7.355 \\ 7.350\end{array}$

F 7.355

F 7.342

7.333

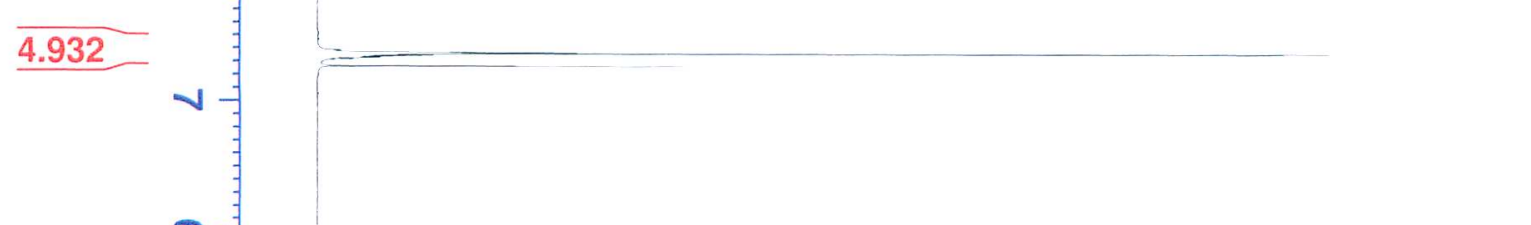

7.3330
-7.328

$-7.325$

7.322

7.315
7.259

厂 5.620

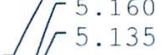

5.114

5.089

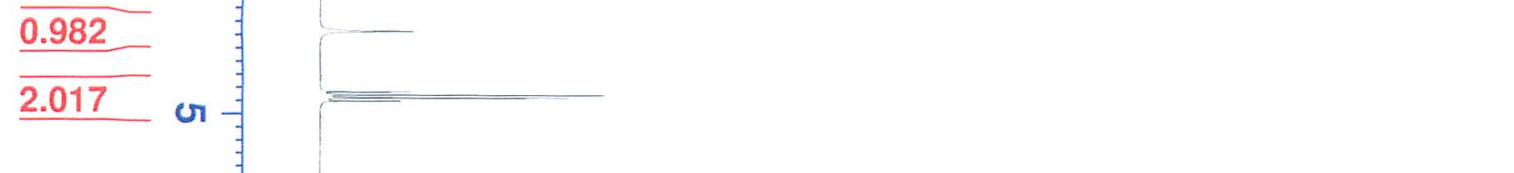

4.329
4.321

4.315

$-4.306$

$-4.300$

4.292

$\left[\begin{array}{r}4.286 \\ 4.278\end{array}\right.$

1.565

1.315

1.305

1.295

1.289

I 1.281

- 1.272

1.266

$r^{0} 0.843$

$-0.835$

$-0.833$

$-0.828$

$\left[\begin{array}{l}0.816 \\ 0.811\end{array}\right.$

0.80

$-0.804$

0.791

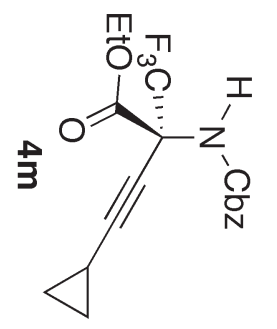

0.775

0.752

$\left[\begin{array}{l}0.752 \\ 0.749 \\ 0.742\end{array}\right.$

0.742

$-0.738$

$\left[\begin{array}{l}0.728 \\ -0.000\end{array}\right.$ 


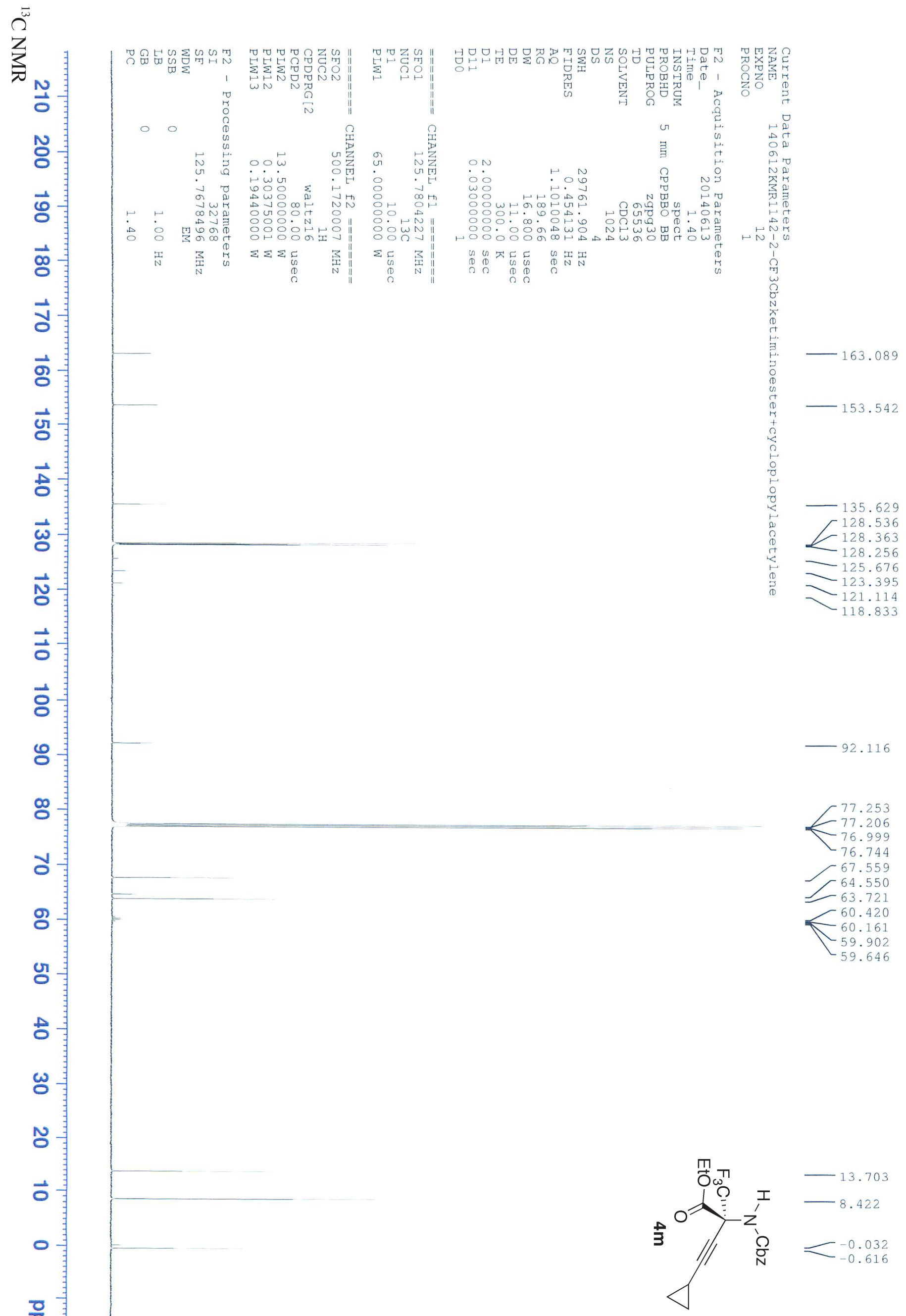


雚

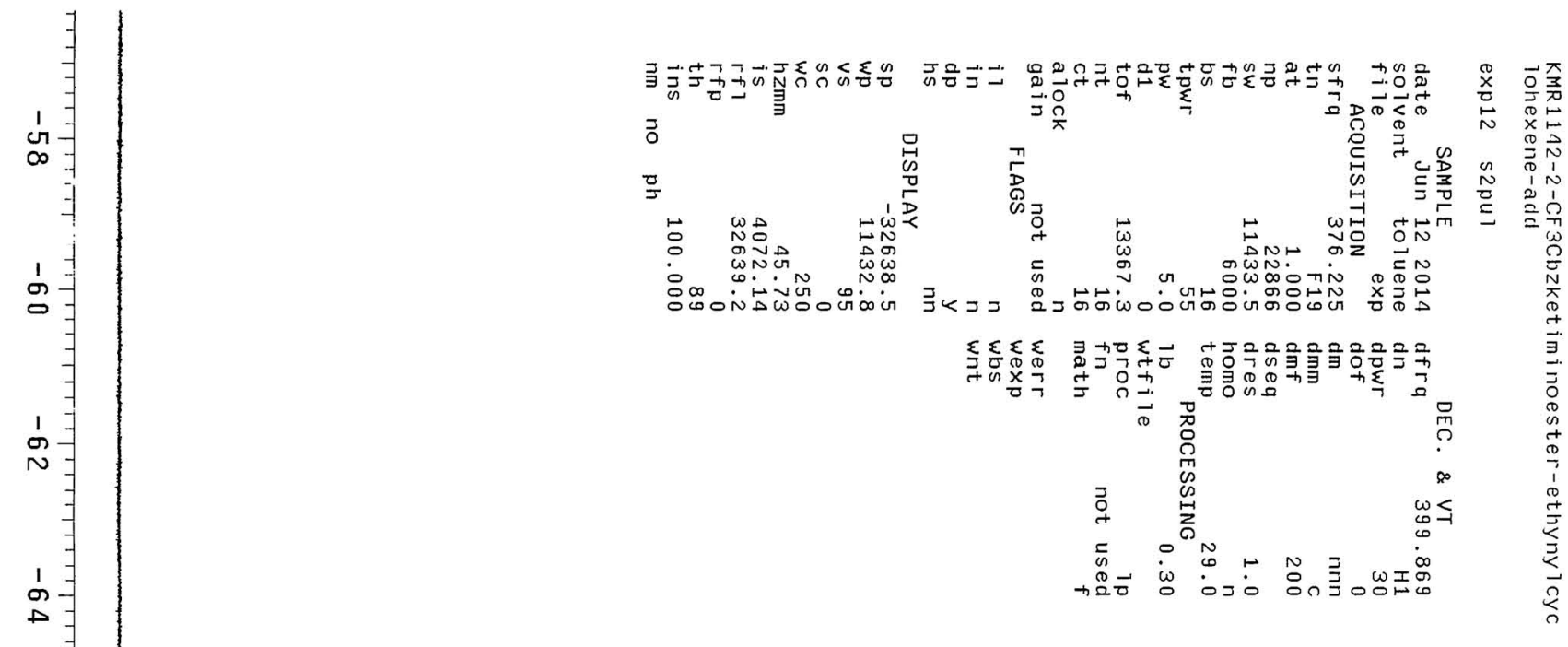

9

o

$\stackrel{1}{\square}$

N

글

1
$\vdots$
0
$\infty$
$\infty$
1
1
$\infty$
0

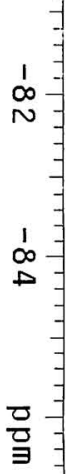

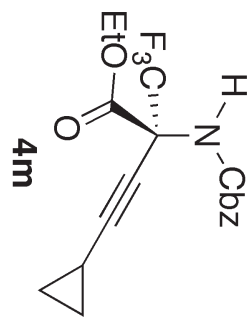



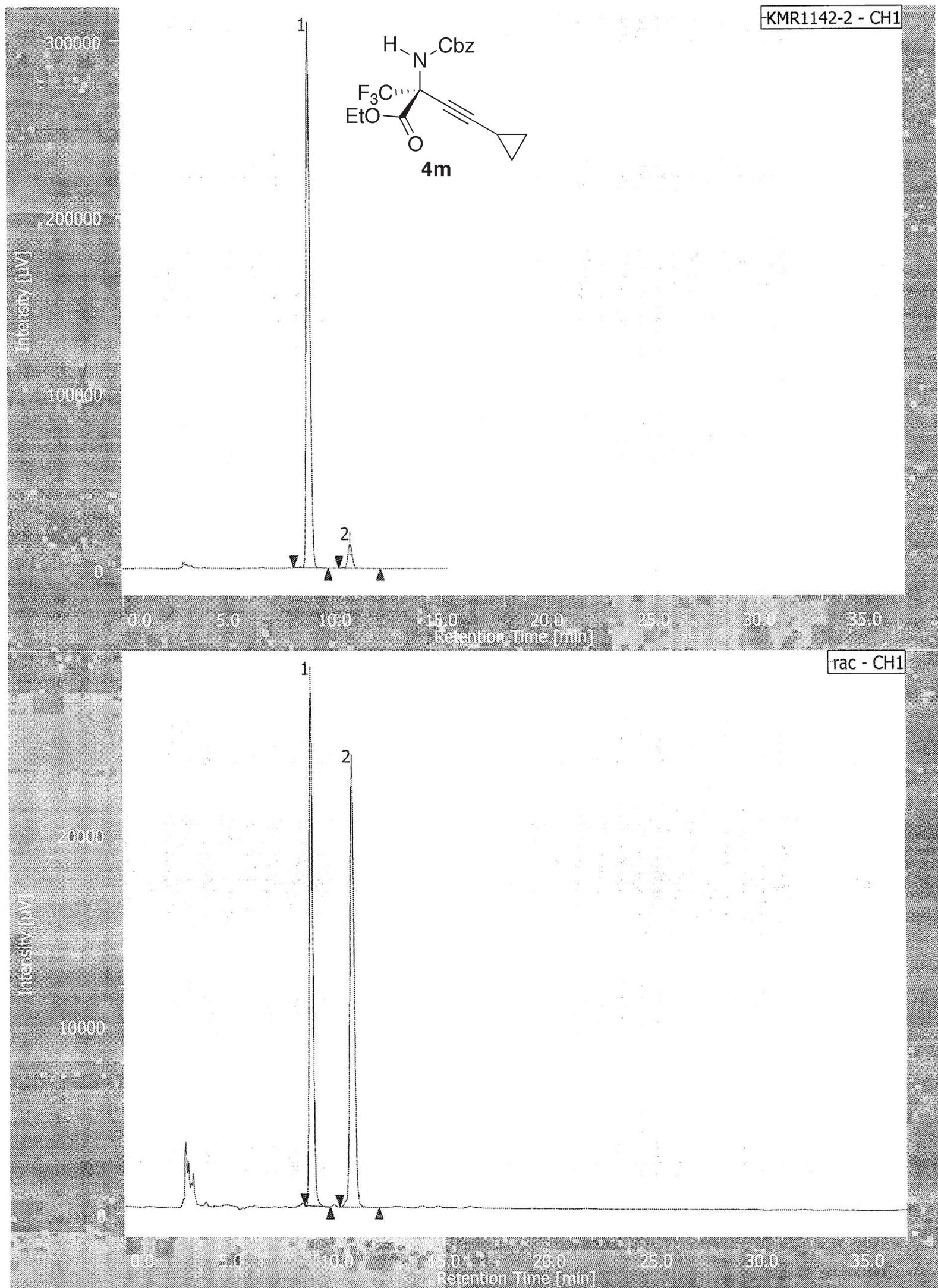

チャンネル情報+ピーク情報

クロマトグラム名

サンプル名

チャンネル名

KMR1142-2-CH1

$\mathrm{CH} 1$

\begin{tabular}{|c|c|c|c|c|c|c|c|c|c|c|c|c|}
\hline \# & ピーク名 & $\mathrm{CH}$ & $\mathrm{tR}[\mathrm{min}]$ & 面積 $[\mu \mathrm{V} \cdot \mathrm{sec}]$ & 高さ $[\mu \mathrm{V}]$ & 面積: & 高さ\% & 定量值 & NTP & 分離度 & シンメトリー係数 & 警告 \\
\hline & Jnknown & 1 & 8.792 & 3736592 & 302442 & 94.926 & 95.642 & $N / A$ & 11630 & 5.523 & 1.155 & \\
\hline & Jnknown & 1 & 10.717 & 199714 & 13780 & 5.074 & 4.358 & N/A & 13221 & $N / A$ & 1.049 & \\
\hline \multicolumn{13}{|c|}{$\begin{array}{l}\text { クロマトグラム名 } \\
\text { サンプル名 }\end{array}$} \\
\hline \multicolumn{13}{|c|}{ チャンネル名 } \\
\hline$\#$ & ピーク名 & $\mathrm{CH}$ & $t R[\mathrm{~min}]$ & 面積 $[\mu \mathrm{V} \cdot \mathrm{sec}]$ & 高さ $[\mu \mathrm{V}]$ & 面積\% & 高さ\% & 定量値 & NTP & 分離度 & シンメトリー係数 & 警告 \\
\hline & Unknown & 1 & 8.817 & 325035 & 27751 & 49.636 & 54.487 & $N / A$ & 13311 & 5.684 & 1.105 & \\
\hline & Unknown & 1 & 10.733 & 329796 & 23180 & 50.364 & 45.513 & N/A & 13426 & N/A & 1.043 & \\
\hline
\end{tabular}




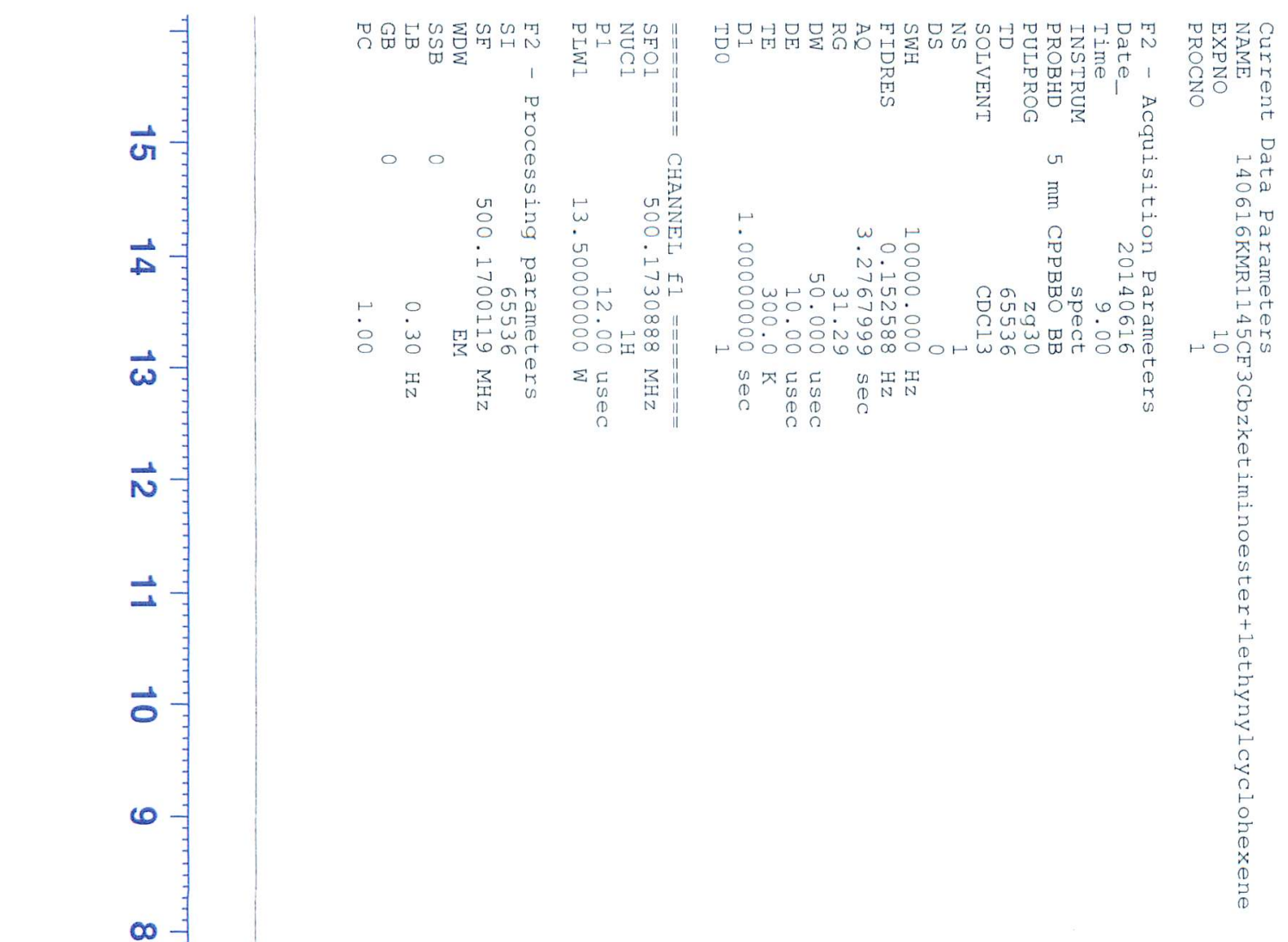

$\left[\begin{array}{r}7.365 \\ 7.362\end{array}\right.$ 7.362

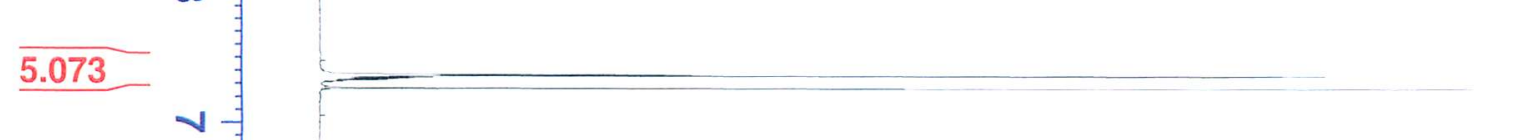

F 7.32

$\begin{array}{r}-7.313 \\ -7.260 \\ \hline\end{array}$ 6.236 6.233 6.229
5.681

5.170

$\int \begin{aligned} & 5.170 \\ & 5.146\end{aligned}$

5.124

5.100

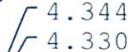

4.320

$-4.315$

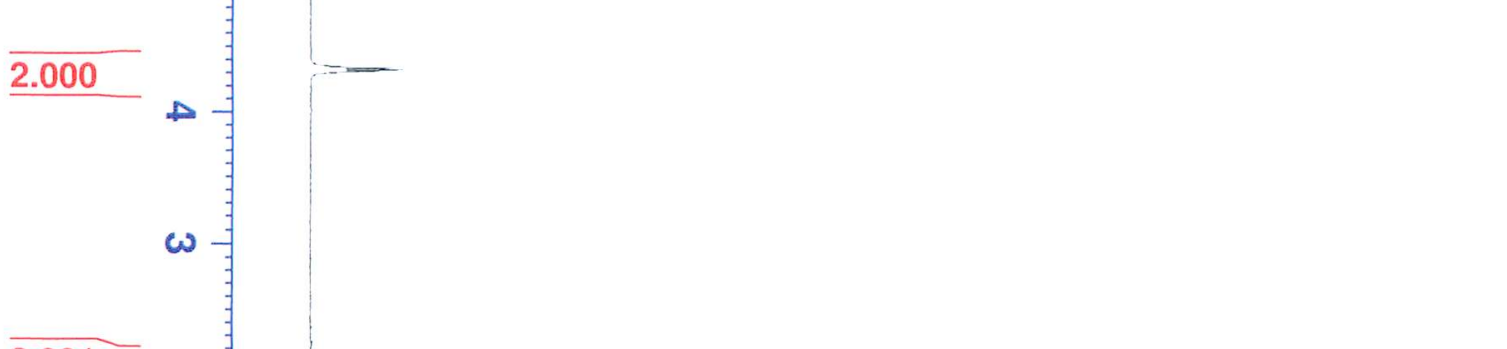

4.306
-4.301

4.301
4.292

2.110

2.101

2.098

2.093

2.089

$r^{2.076}$

$F^{1.633}$

$-1.622$

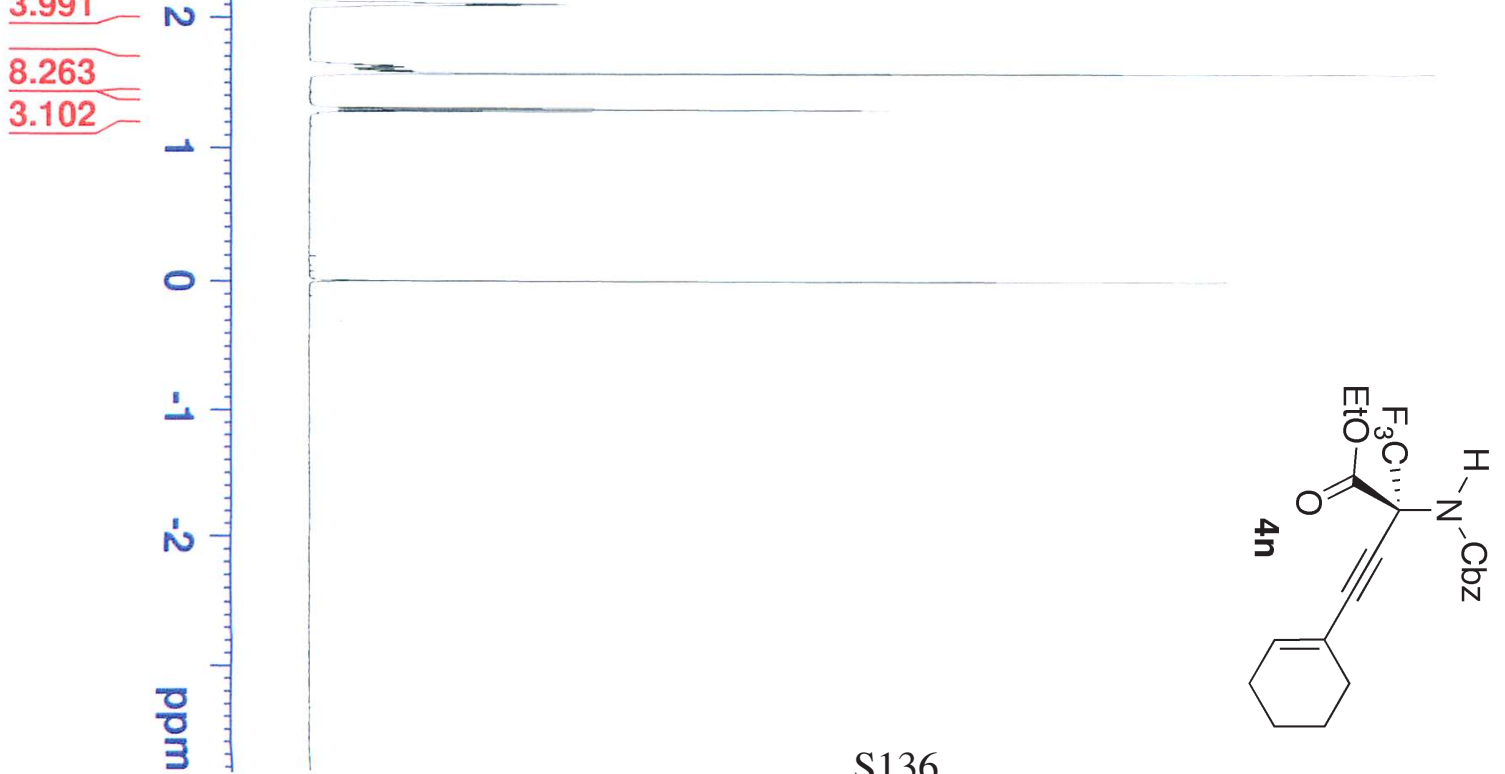

1.622
-1.615

$\mathbb{1}-1.612$

1.603

- 1.593

1. 58

$\left[\begin{array}{l}1.584 \\ 1.580\end{array}\right.$

1.564

1.561

$\left[\begin{array}{l}1.303 \\ 1.3039 \\ 1.289\end{array}\right.$

$\left[\begin{array}{l}1.289 \\ 1.275\end{array}\right.$

0.007

$-0.000$ 옹

$-0.007$ 


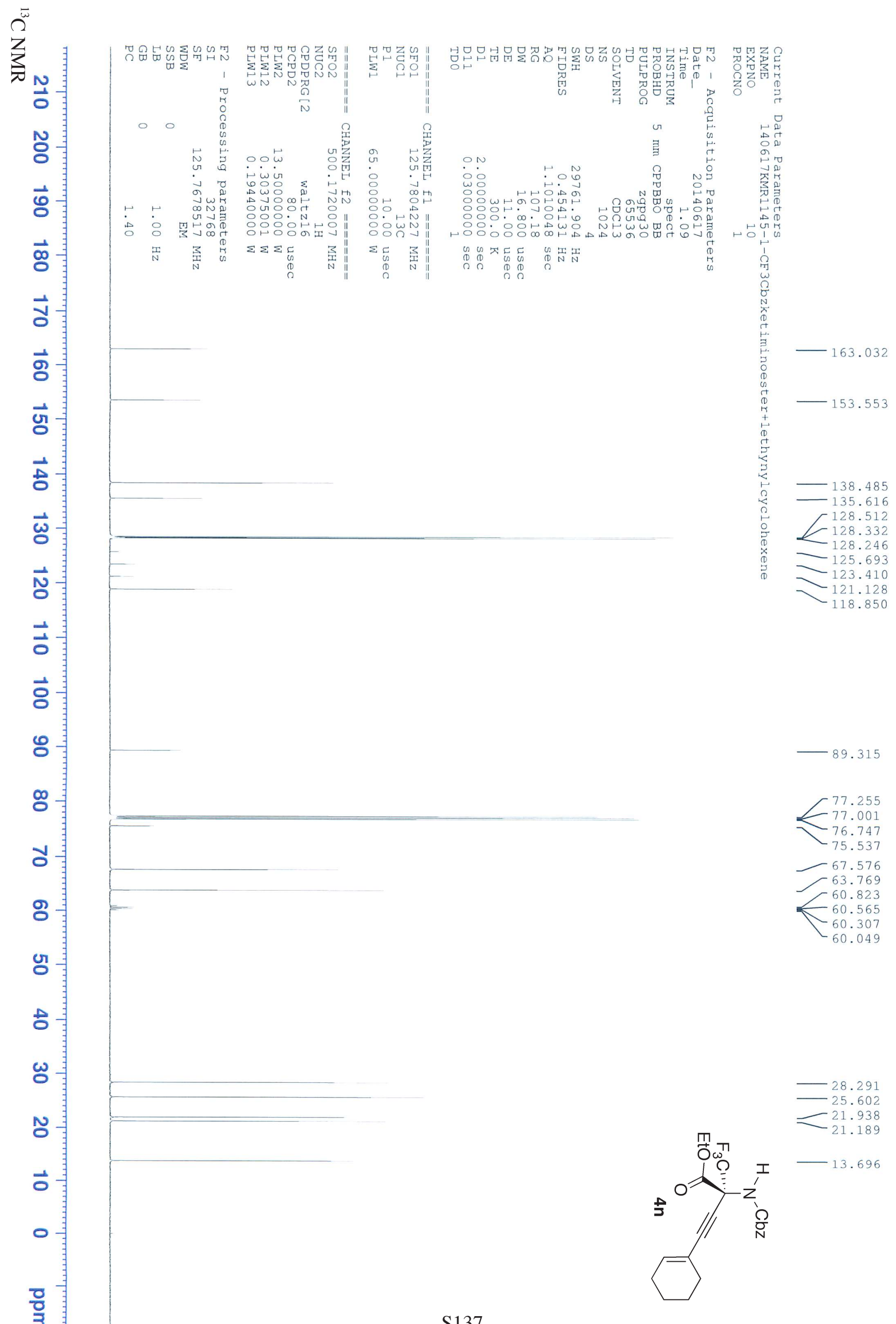



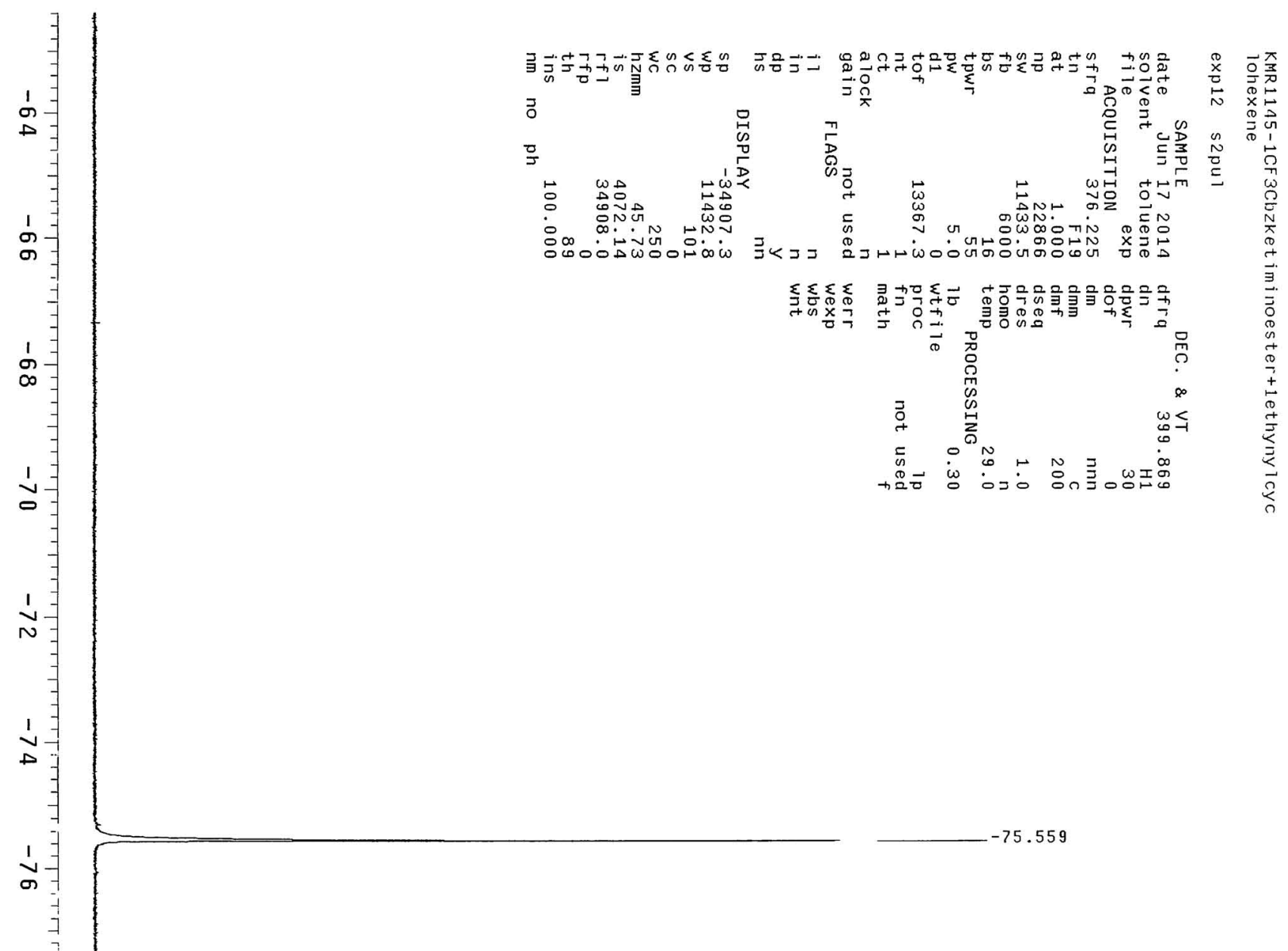

$-75.559$

1
$\infty$
0
1
0
0

1
$N$
$N$

$\stackrel{1}{\infty}$

a

1
$\infty$
$\infty$

1

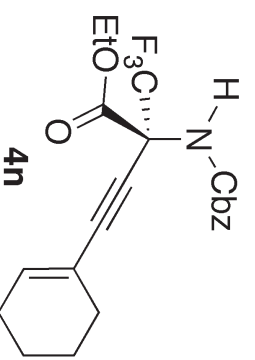


y)
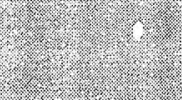

\section{相}

EtO

$\mathrm{H}_{\mathrm{N}^{-}}{ }^{\mathrm{Cbz}}$<smiles>CCOC(=O)[C@](C)(C#CC1=CCCCC1)NC</smiles>

- $v^{2}$

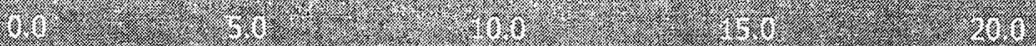

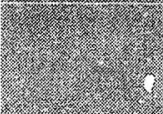

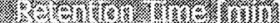

$\mathrm{rac}-\mathrm{CH} 1$

1.t.

$-1 x^{-12}$

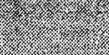

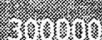

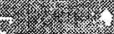

3

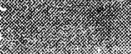

200010
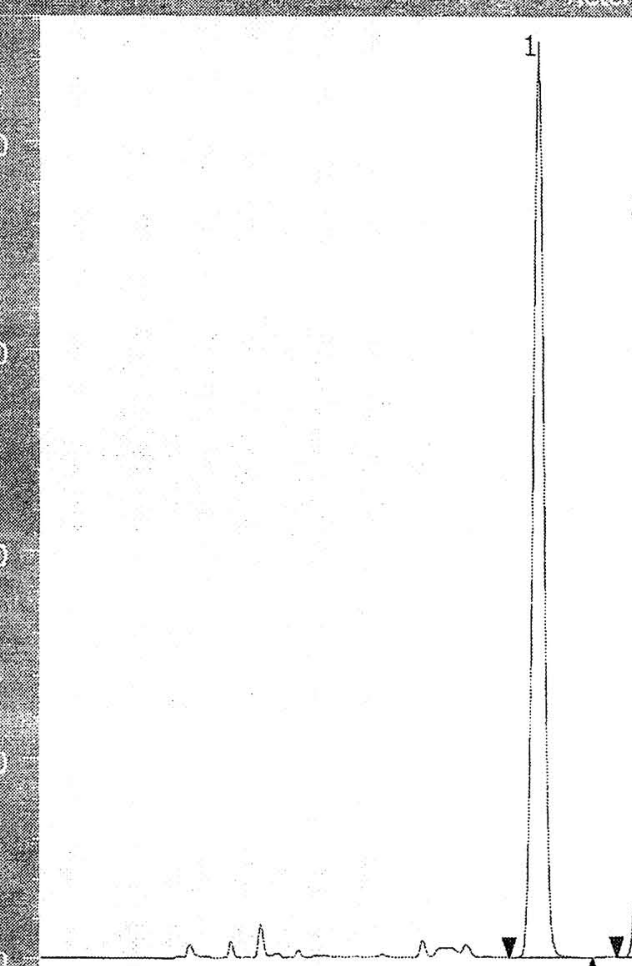

\section{0}

(1)

(6)

チャンネル情報十ピーク情報

クロマトグラム名

サンプル名

KMR1145-1-CH1

チャンネル名

$\mathrm{CH} 1$

\begin{tabular}{|c|c|c|c|c|c|c|c|c|c|c|c|c|}
\hline \# ピーク名 & $\mathrm{CH}$ & \begin{tabular}{|l|l}
$t R[\min ]$ \\
\end{tabular} & 面積 $[\mu \mathrm{V} \cdot \mathrm{sec}]$ & 高さ [uV] & 面皘粪\% & 高さ出 & 定量値 & NTP & 分離度 & シンメトリー係数 & 警告 & \\
\hline 1 Unknown & \begin{tabular}{|l|}
1 \\
\end{tabular} & 9.933 & 18306691 & 1143197 & 96.335 & 96.423 & N/A & 9074 & 5.258 & 1.145 & & 8 \\
\hline 2 Unknown & 1 & 12.150 & 696372 & 42411 & 3.665 & 3.577 & N/A & 12877 & N/A & 1.035 & & \\
\hline
\end{tabular}

サンプル名

チャンネル名

$\mathrm{CH} 1$

\begin{tabular}{|c|c|c|c|c|c|c|c|c|c|c|c|}
\hline ピーク名 & $\mathrm{CH}$ & $\mathrm{tR}$ [min] & 面皘 [ $[\mu \mathrm{V} \cdot \mathrm{sec}]$ & 高さ [uV] & 面栍\% & 高さ\% & 定量值 & NTP & 分離度 & ジメトリーー係数 & 警告 \\
\hline Unknown & 1 & 9.950 & 6648194 & 437298 & 52.219 & 54.910 & N/A & 9637 & 5.132 & 1.054 & \\
\hline 2 Unknown & 1 & 12.133 & 6083260 & 359089 & 47.781 & 45.090 & $N / A$ & 11748 & $N / A$ & 1.051 & \\
\hline
\end{tabular}




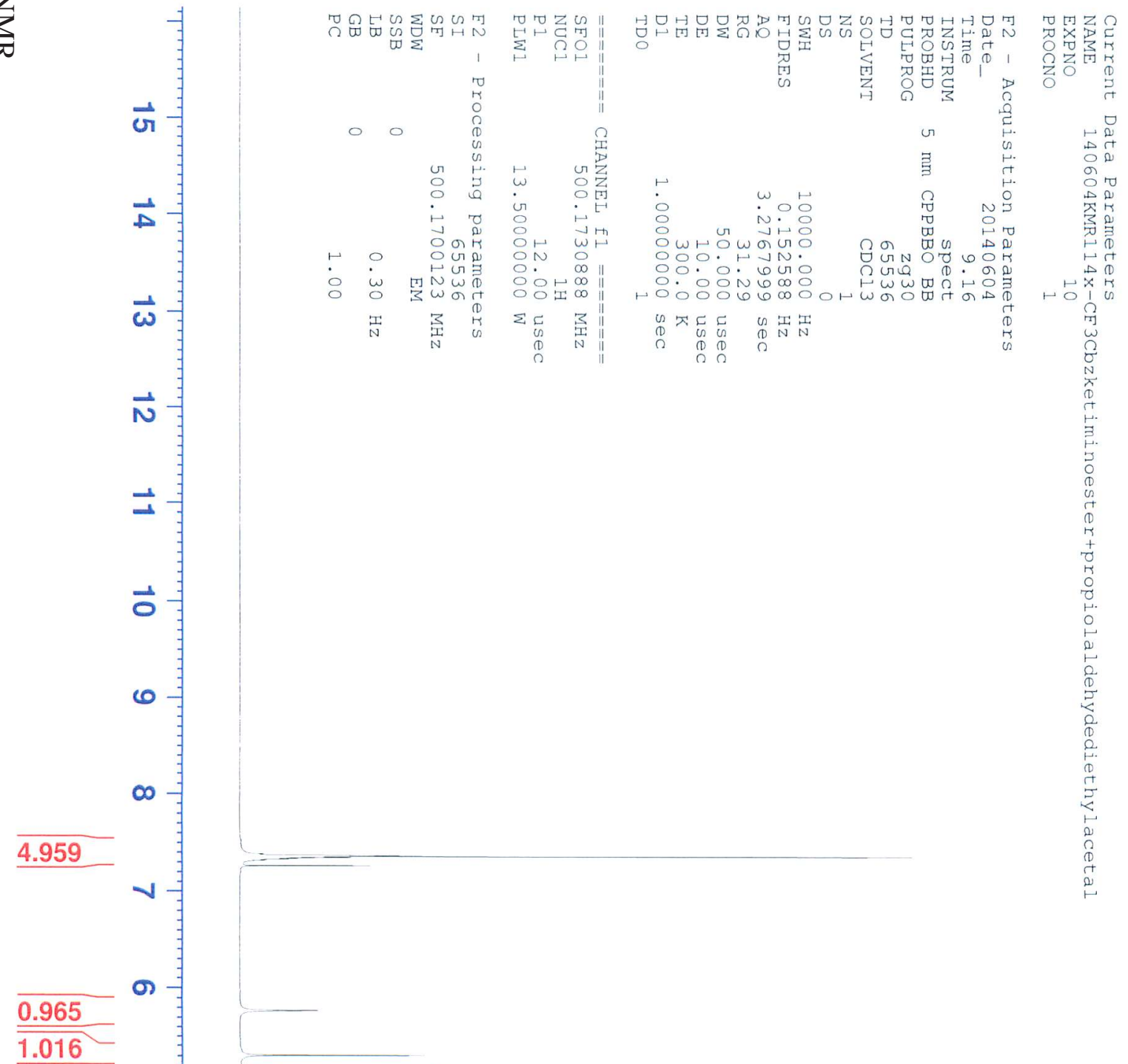

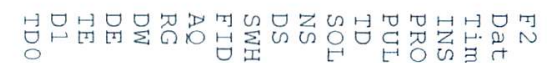

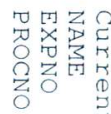

7.381

7.366

7.355

7.348

7.345

$\left[\begin{array}{l}7.340 \\ 7.336\end{array}\right.$

$\left[\begin{array}{l}7.336 \\ 7.330\end{array}\right.$

7.328

7.317

7.311

5.762

5.163

5.139

[5.128

$\lceil 5.103$

$\left[\begin{array}{c}4.358 \\ -4.352\end{array}\right.$

$\left[\begin{array}{r}4.358 \\ -4.337\end{array}\right.$

$\left[\begin{array}{l}4.352 \\ 4.328 \\ 4.328\end{array}\right.$

F. 4.323

] 4.314

F $\begin{array}{r}4.300 \\ 4.293\end{array}$

3.752
3.738

$\frac{\overline{\frac{2.000}{1.981}}}{\underline{1.962}} \omega$
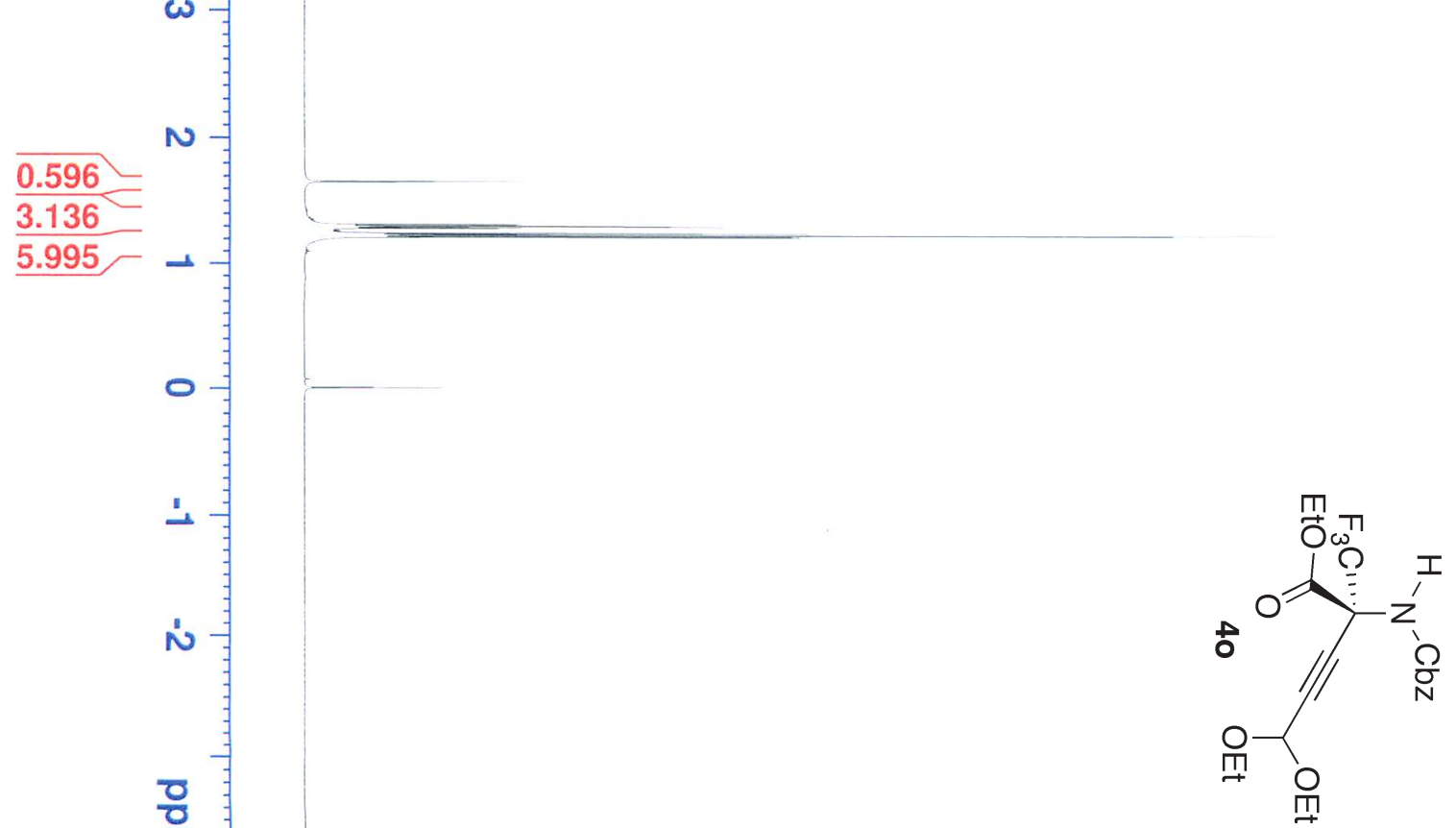

3.738
-3.733

3.733

- 3.724

3.709

3.705

$-3.691$

3.624

-3.61

3.610

3.598

3.596

$\left[\begin{array}{l}3.593 \\ 3.591\end{array}\right.$

$-3.591$

$-3.584$

$-3.577$

$-3.565$

3.563

1.644

$-1.305$

$[1.291$

$\left[\begin{array}{l}1.276 \\ 1.256\end{array}\right.$

L1.235

$\left[\begin{array}{l}1.221 \\ 1.207\end{array}\right.$

L.000

N่

믈 


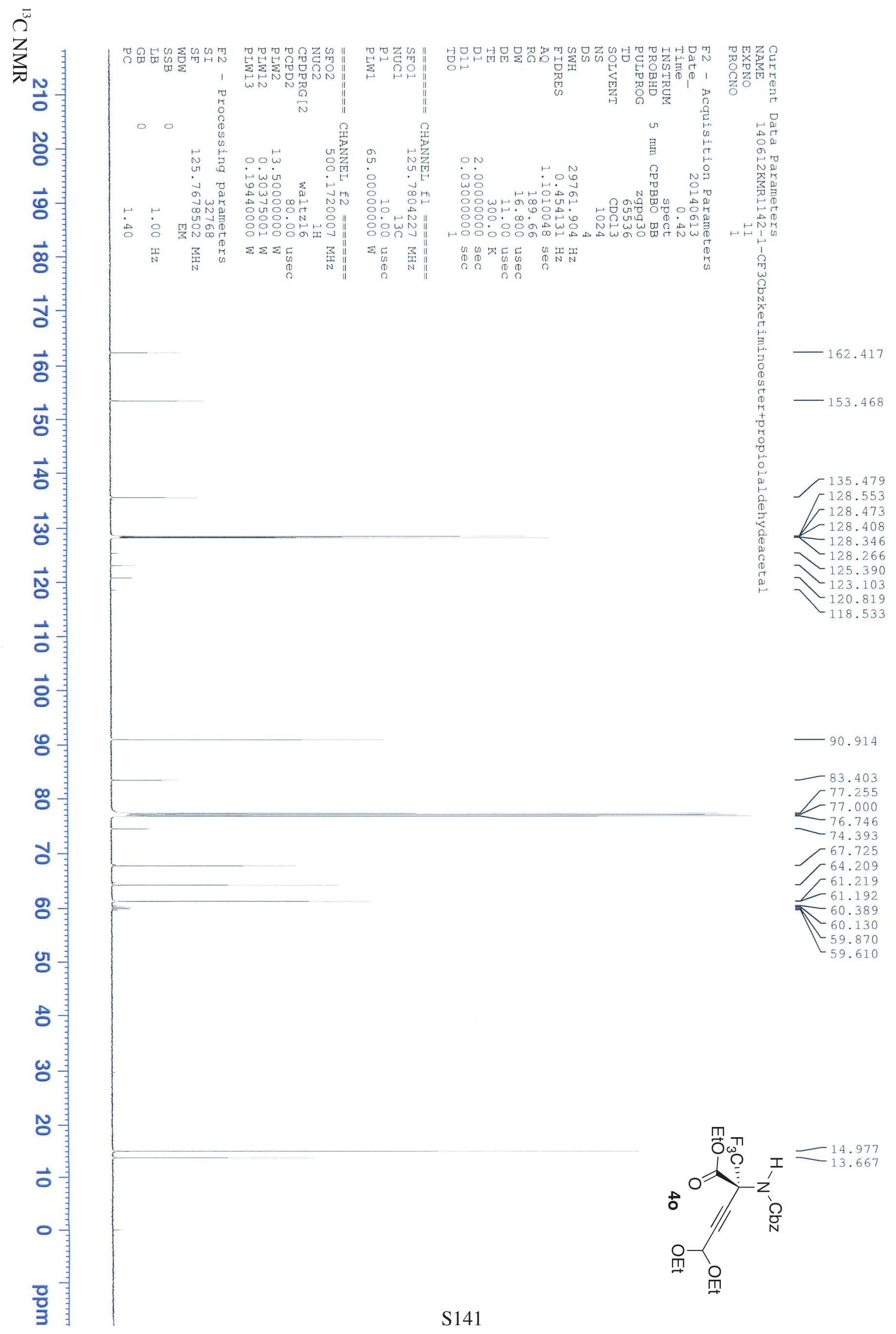




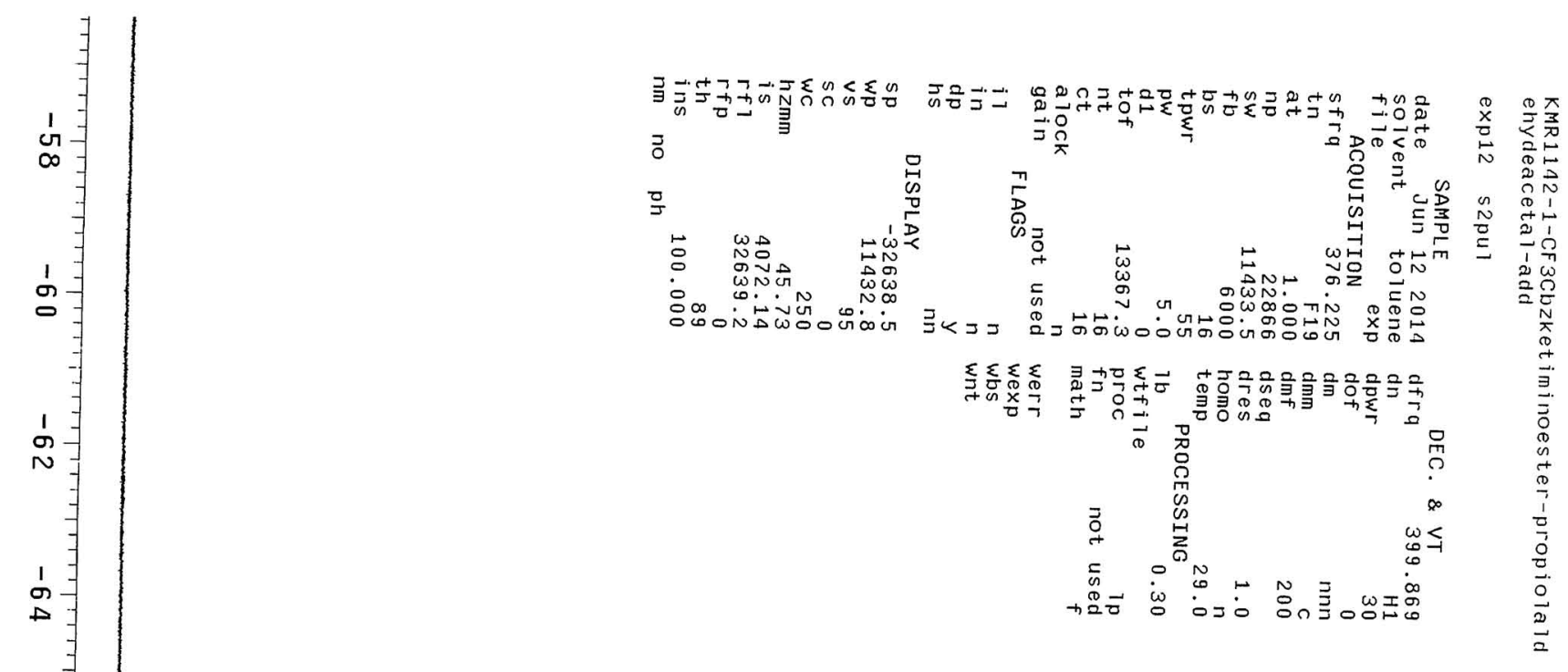

बून

1
$\infty$
$\infty$

ำ-

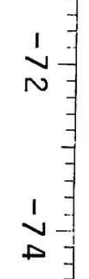

光:

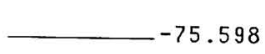

$\stackrel{1}{\infty}$

$\stackrel{1}{\infty}$

1
$\infty$
$\infty$
$\infty$

年

吾音

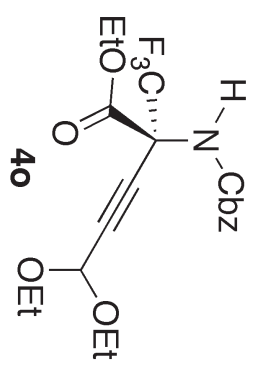




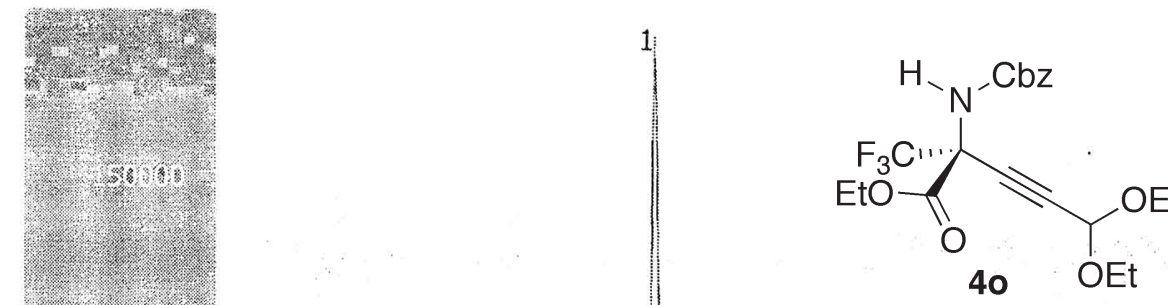

40 Et
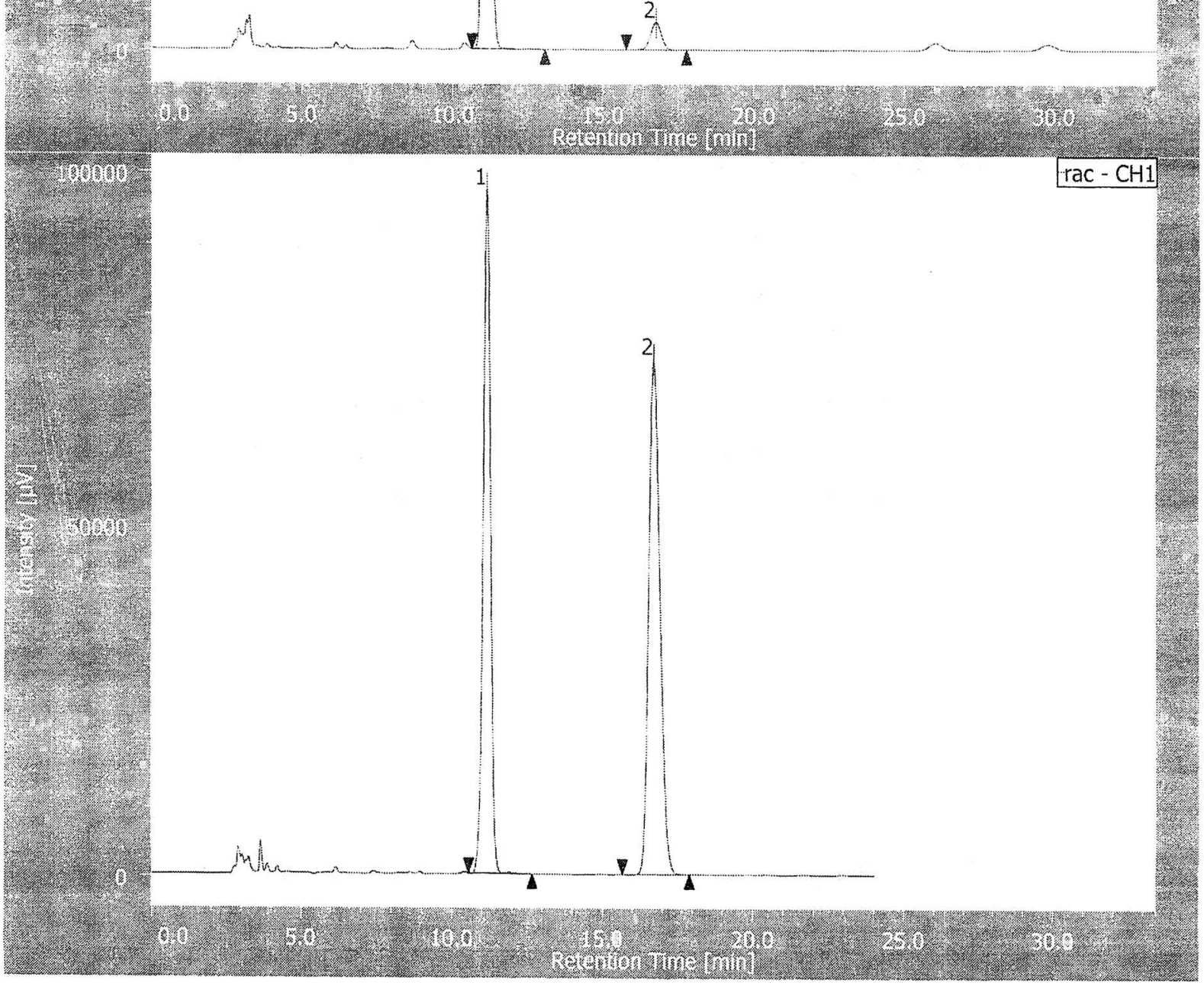

チャンネル情報+ピーク情報

クロマトグラム名

KMR1142-1-CH1

サンプル名

チャンネル名

$\mathrm{CH} 1$

\begin{tabular}{|c|c|c|c|c|c|c|c|c|c|c|c|}
\hline ピーク名 & $\mathrm{CH}$ & $t R$ [min] & 面積 [ $[\mu \mathrm{V} \cdot \mathrm{sec}]$ & \begin{tabular}{|l|} 
高さ $[\mu \mathrm{V}]$ \\
\end{tabular} & 面積\% & 高さ为 & 定量值 & NTP & 分離度 & シンメトリー係数 & 警告 \\
\hline U Unkno & 1 & 11.100 & 4728 & 171332 & 94.228 & 96.076 & N/A & 9700 & 10.171 & 1.137 & \\
\hline 2 Unknown & 1 & 16.767 & 177305 & 6998 & 5.772 & 3.924 & $\mathrm{~N} / \mathrm{A}$ & 10127 & $N / A$ & 1.028 & \\
\hline
\end{tabular}

クロマトグラム名

rac-CH1

$88.46 \% \mathrm{el}$.

サンプル名

$\mathrm{CH} 1$

\begin{tabular}{|c|c|c|c|c|c|c|c|c|c|c|c|}
\hline ピーク名 & $\mathrm{CH}$ & $\operatorname{tR}[\mathrm{min}]$ & 面積 $[\mu \mathrm{V} \cdot \mathrm{sec}]$ & 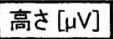 & 面積\% & 高さ\% & 定量値 & NTP & 分離度 & シンメトリー係数 & 警告 \\
\hline Unknown & 1 & 11.142 & 1634499 & 96971 & 46.206 & 57.020 & $\mathrm{~N} / \mathrm{A}$ & 10223 & 9.853 & 1.008 & \\
\hline Unknown & 1 & 16.700 & 1902953 & 73094 & 53.794 & 42.980 & N/A & 9361 & N/A & 1.259 & \\
\hline
\end{tabular}



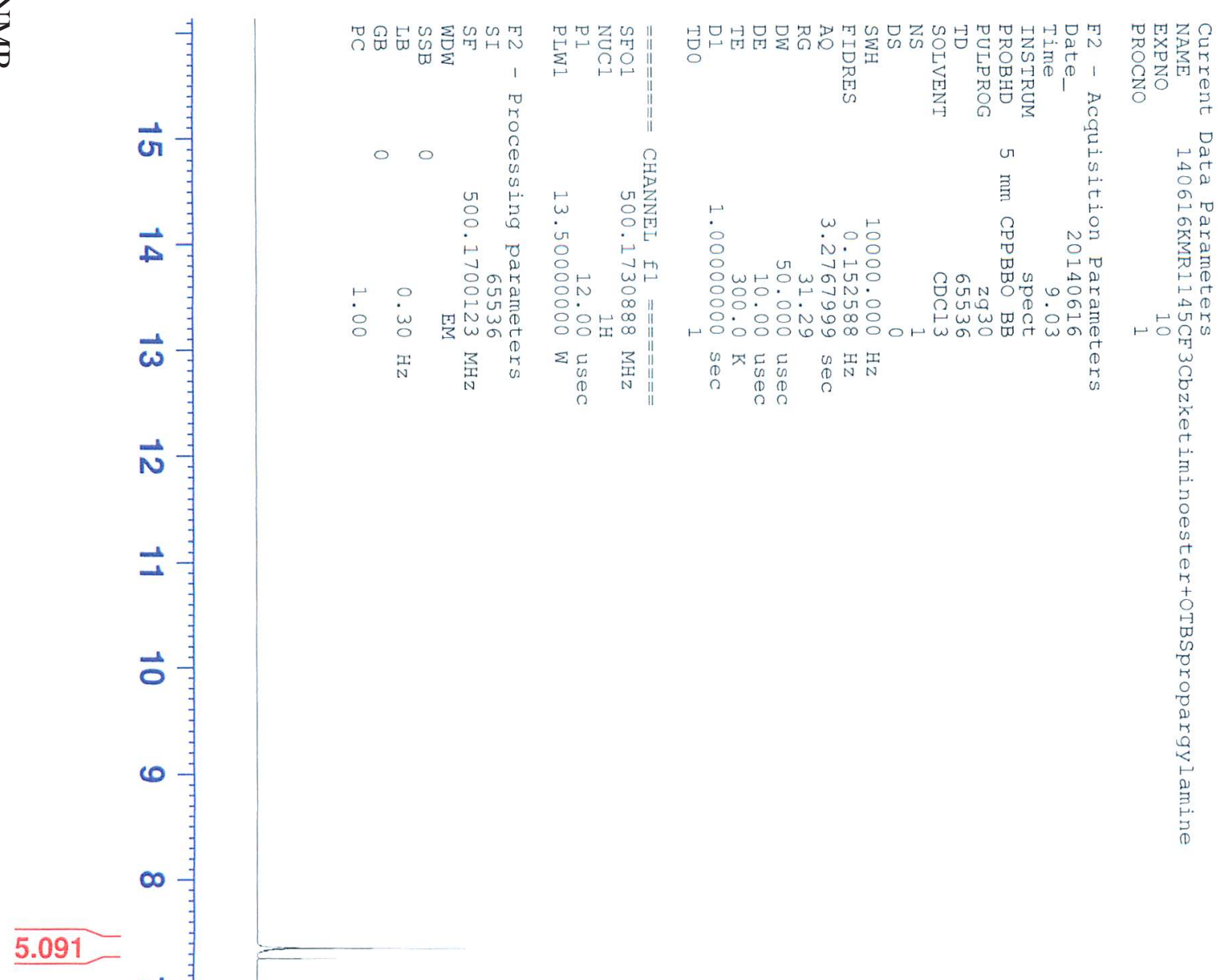

$\infty$
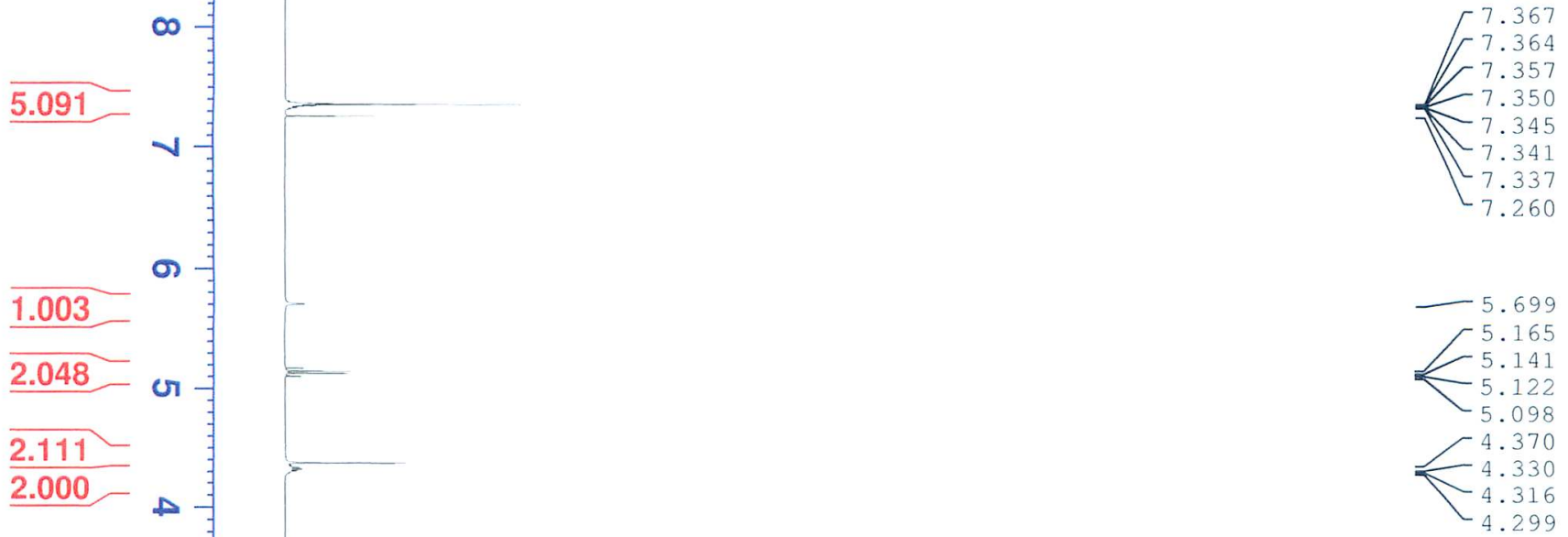


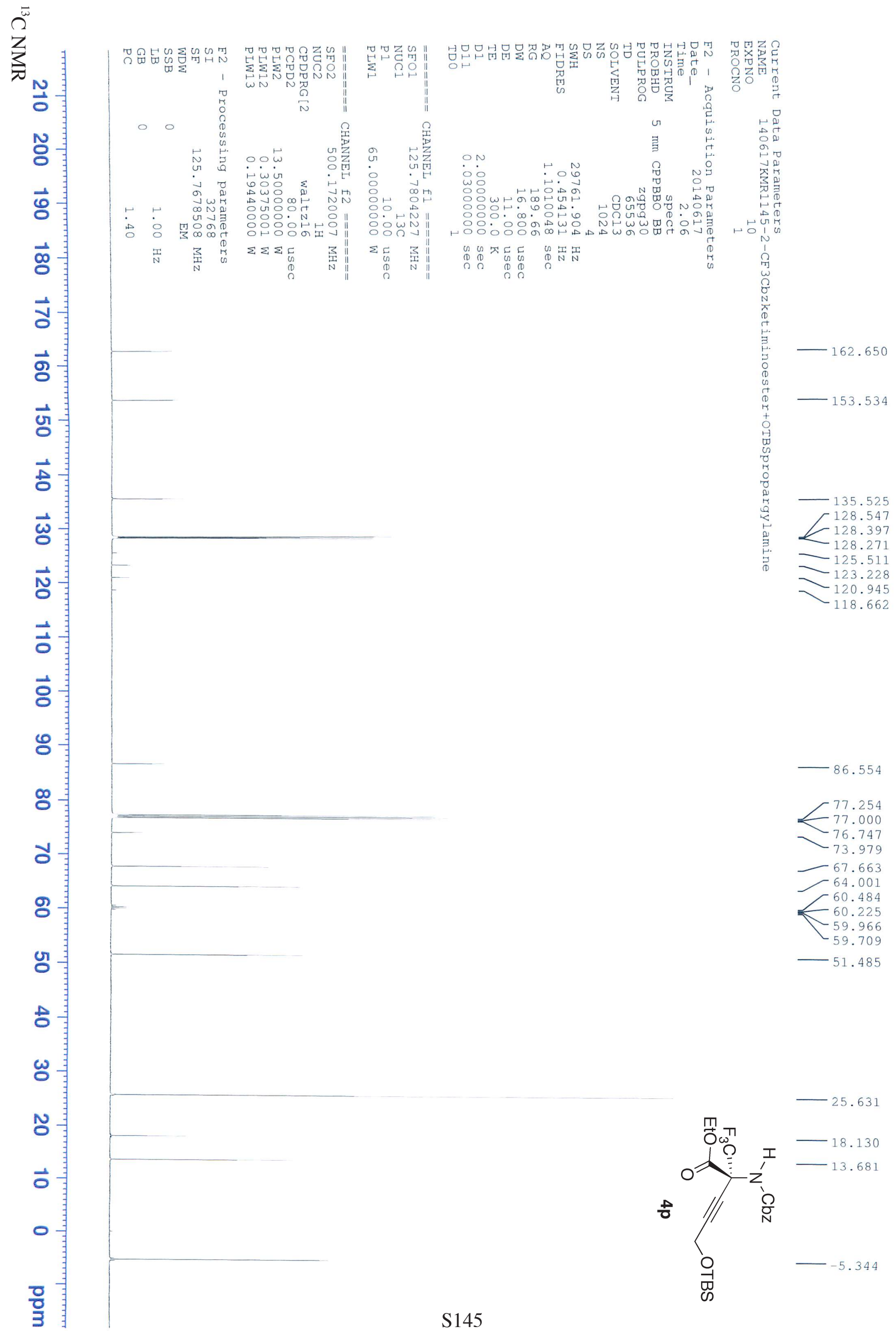


光

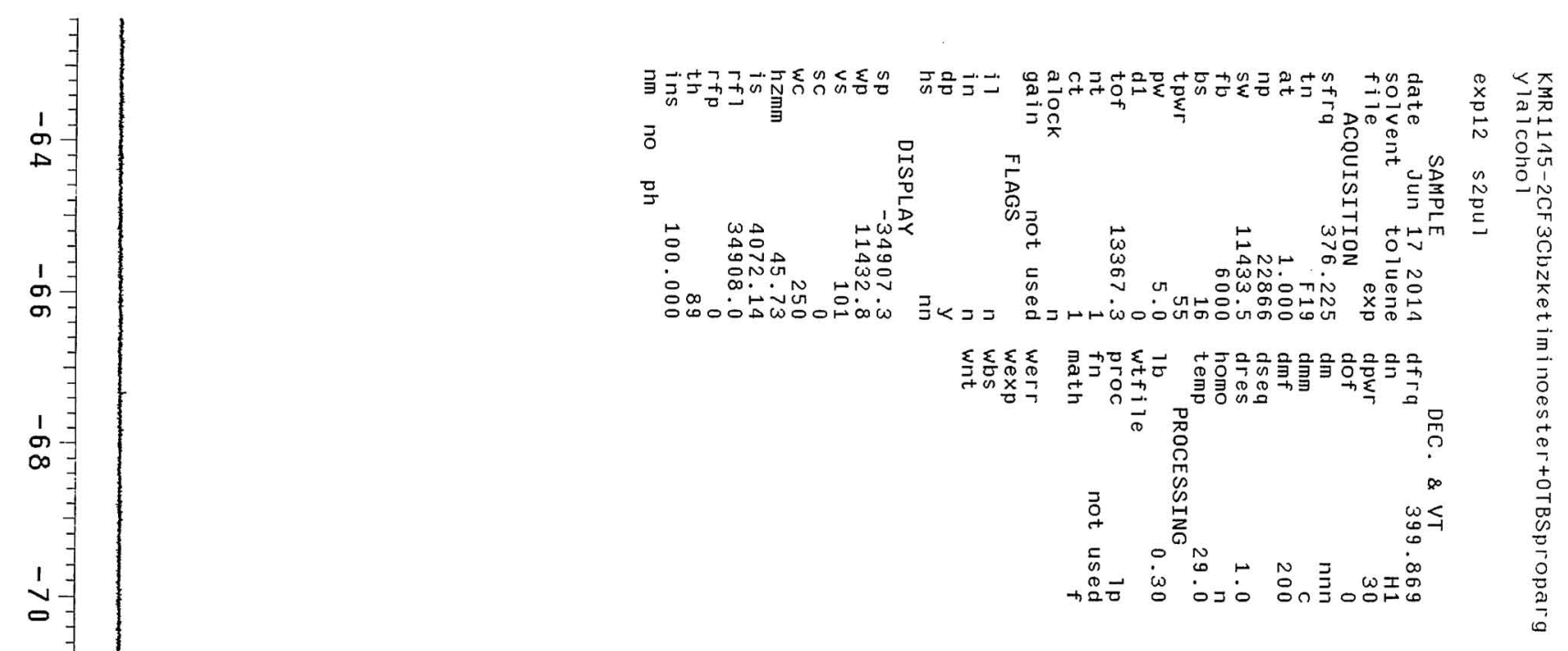

1
$N$
$D$

के

$\stackrel{1}{\infty}$

1

1

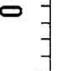

1
$\infty$
$N$

$\stackrel{1}{\infty}$

1
$\infty$
$\infty$
0

1
$\infty$
$\infty$

品

음

$=$
$=$
$=$
$=$

手 

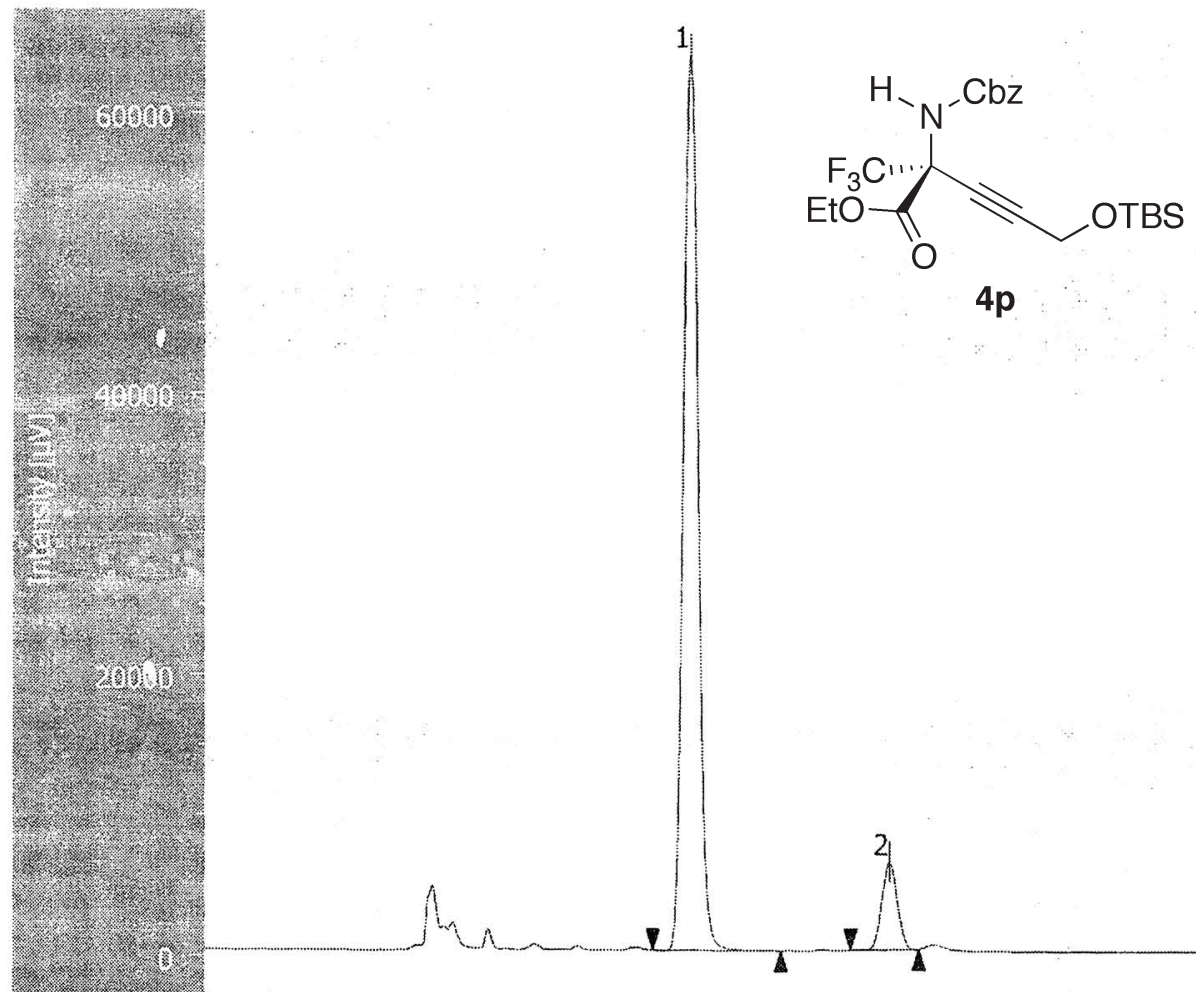

11
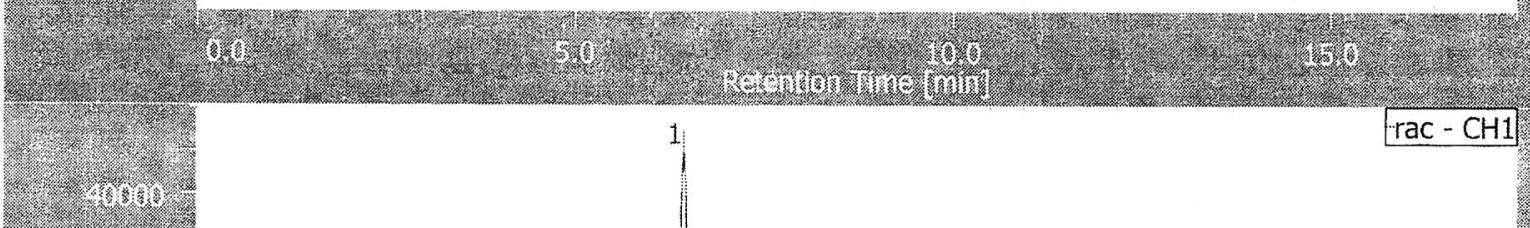

$1+\cos ^{2}$

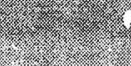

(2:-2.0.

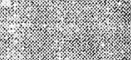

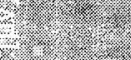

1.200101

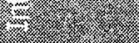

-

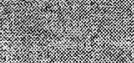

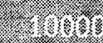

: $: 3.7$

.

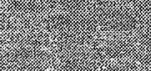

- 1.2

4

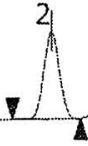

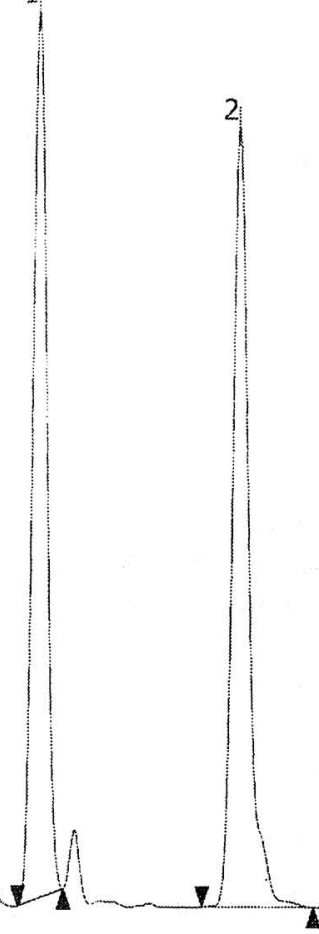

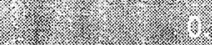

チャンネル情報+ピーク情報

クロマトグラム名

KMR1145-2-CH1

サンプル名

チャンネル名

$\mathrm{CH} 1$

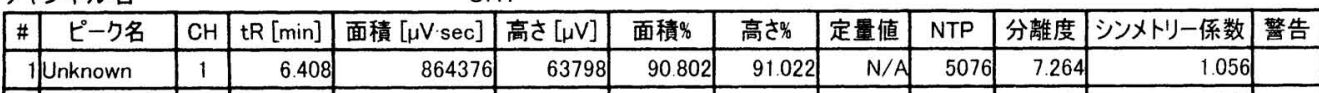

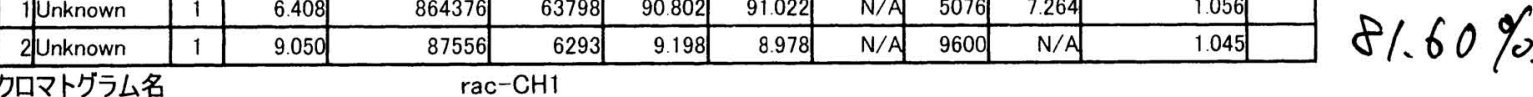

サンプル名

チャンネル名

$\mathrm{CH} 1$

\begin{tabular}{|c|c|c|c|c|c|c|c|c|c|c|c|}
\hline ピーワ名 & $\mathrm{CH}$ & $\operatorname{tR}[\mathrm{min}]$ & 面積 [uV·sec] & 高さ [uV] & 面積\% & 高さ\% & 定量值 & NTP & 分離度 & シンメトリー係数 & 警告 \\
\hline 1 Unknown & 1 & 6.425 & 524917 & 41158 & 49.303 & 53.221 & N/A & 5628 & 7.495 & 1.053 & \\
\hline 2 Unknown & 1 & 9.083 & 539756 & 36176 & 50.697 & 46779 & $N / A$ & 9712 & $\mathrm{~N} / \mathrm{A}$ & 1.417 & \\
\hline
\end{tabular}




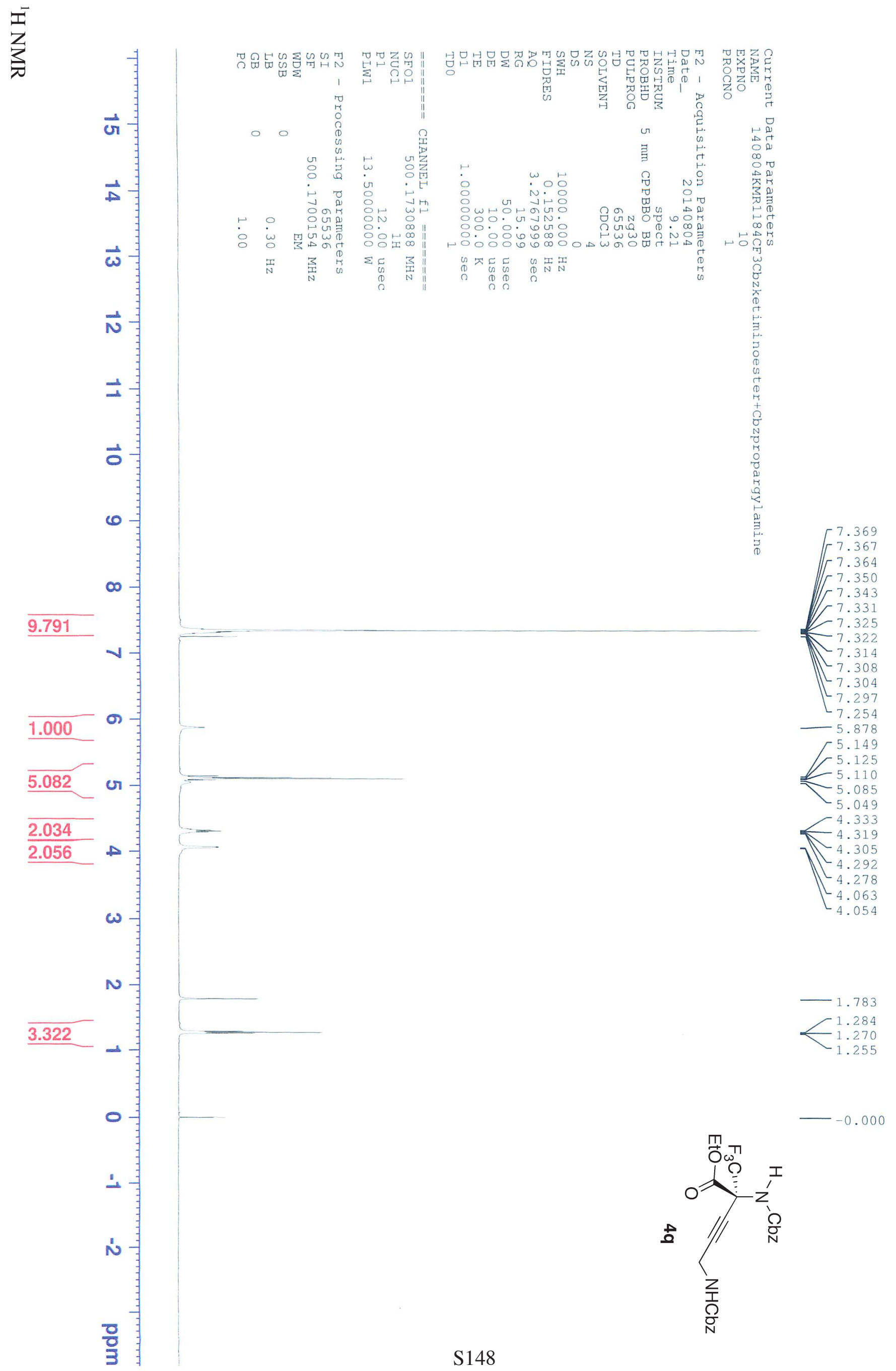




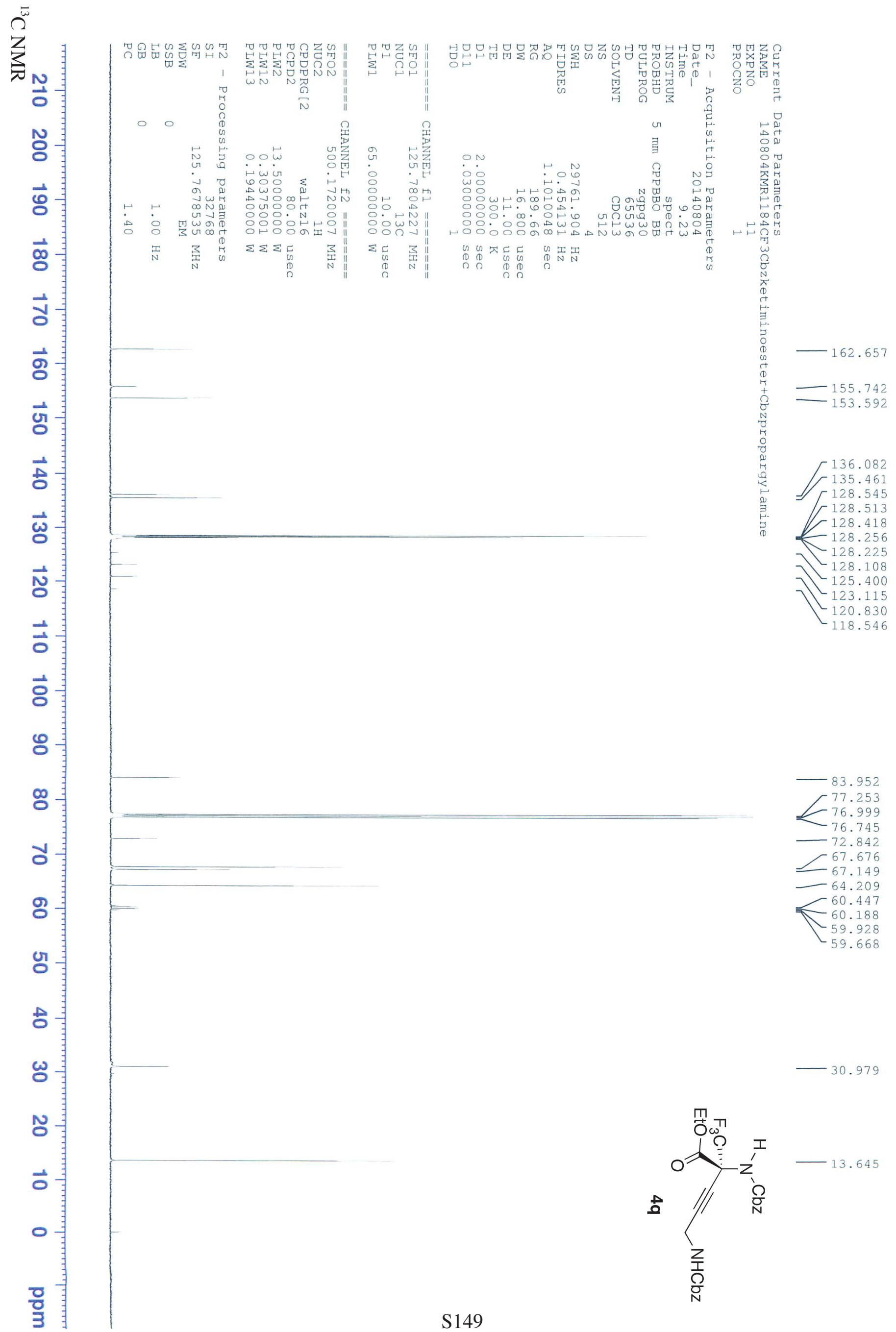


苛

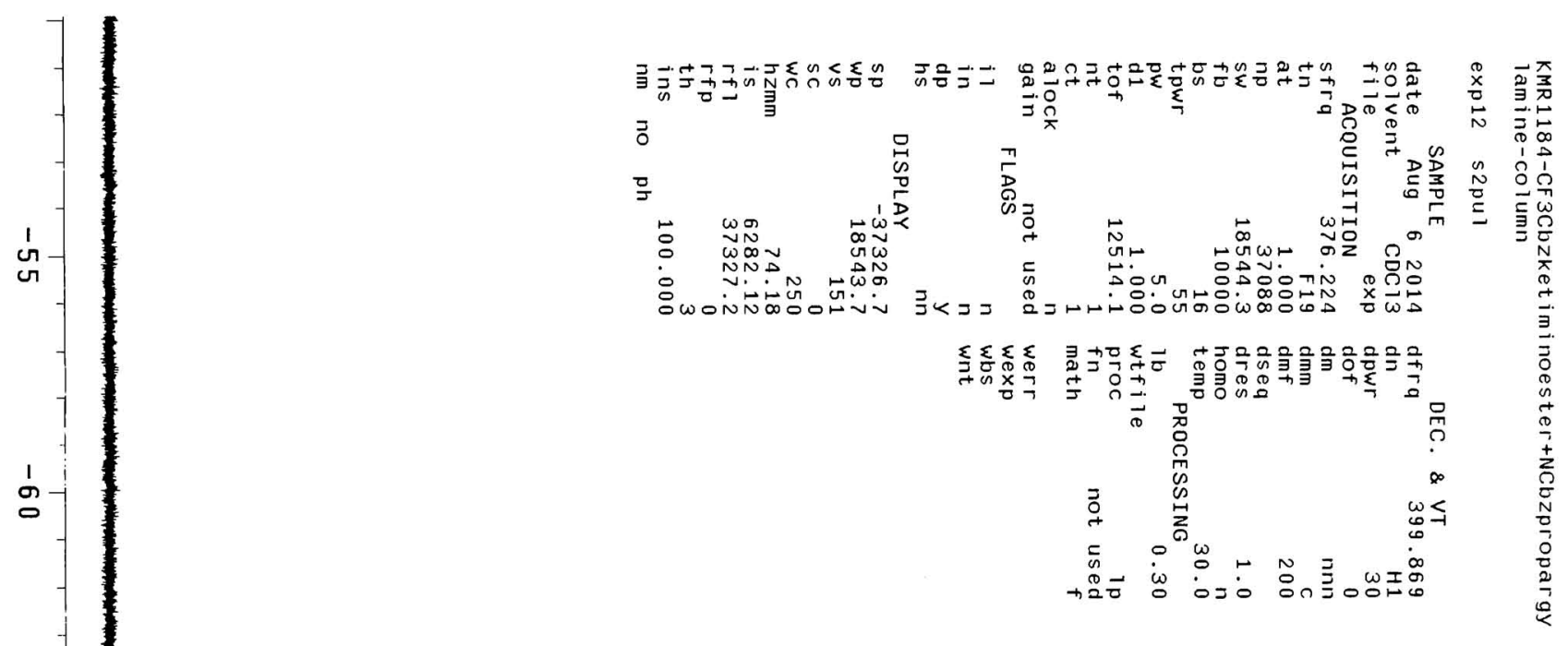

ज

ป

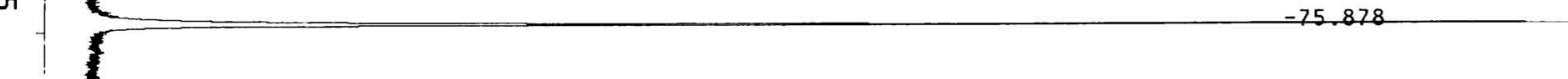

1
0

1
$\infty$
-1

م.

뮼

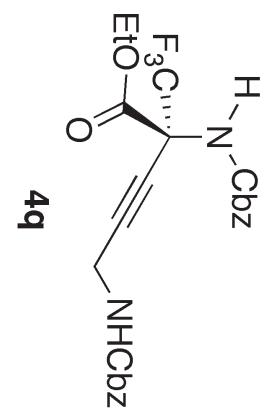




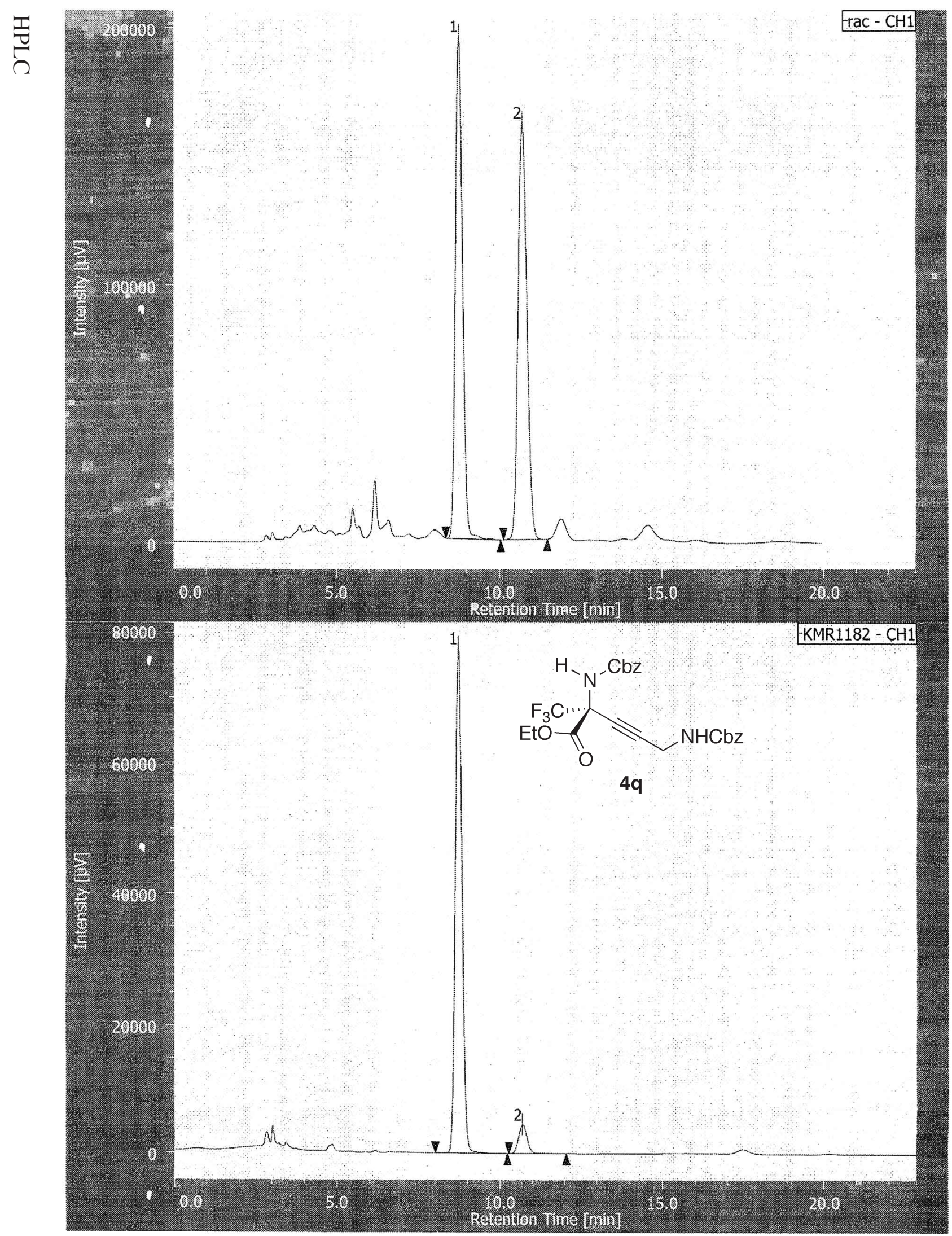

チャンネル情報+ピーク情報

クロマトグラム名

$\mathrm{rac}-\mathrm{CH} 1$

サンプル名

チャンネル名

$\mathrm{CH} 1$

\begin{tabular}{|c|c|c|c|c|c|c|c|c|c|c|c|}
\hline ピーク名 & $\overline{\mathrm{CH}}$ & $t R[\mathrm{~min}]$ & 面積 $[\mu \mathrm{V} \cdot \mathrm{sec}]$ & 高さ $[\mu \mathrm{V}]$ & 面積\% & 高さ\% & 定量值 & NTP & 分離度 & シンメトリー係数 & 警告 \\
\hline 1 Unknown & 1 & 8.758 & 2980515 & 195130 & 49.513 & 54.717 & $\mathrm{~N} / \mathrm{A}$ & 7573 & 4.353 & 1.086 & \\
\hline \begin{tabular}{l|l|}
2 Unknown \\
\end{tabular} & 1 & 10.725 & 3039172 & 161486 & 50.487 & 45.283 & N/A & 7259 & $N / A$ & 1.098 & \\
\hline
\end{tabular}

クロマトグラム名 KMR1182-CH1

サンプル名

チャンネル名

$\mathrm{CH} 1$

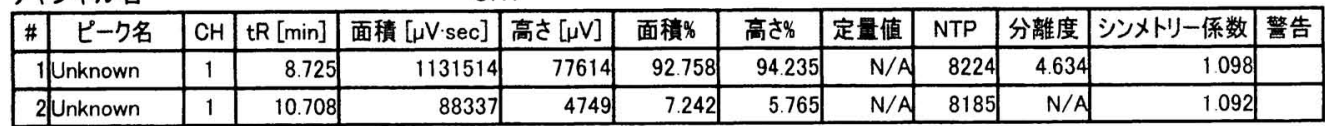




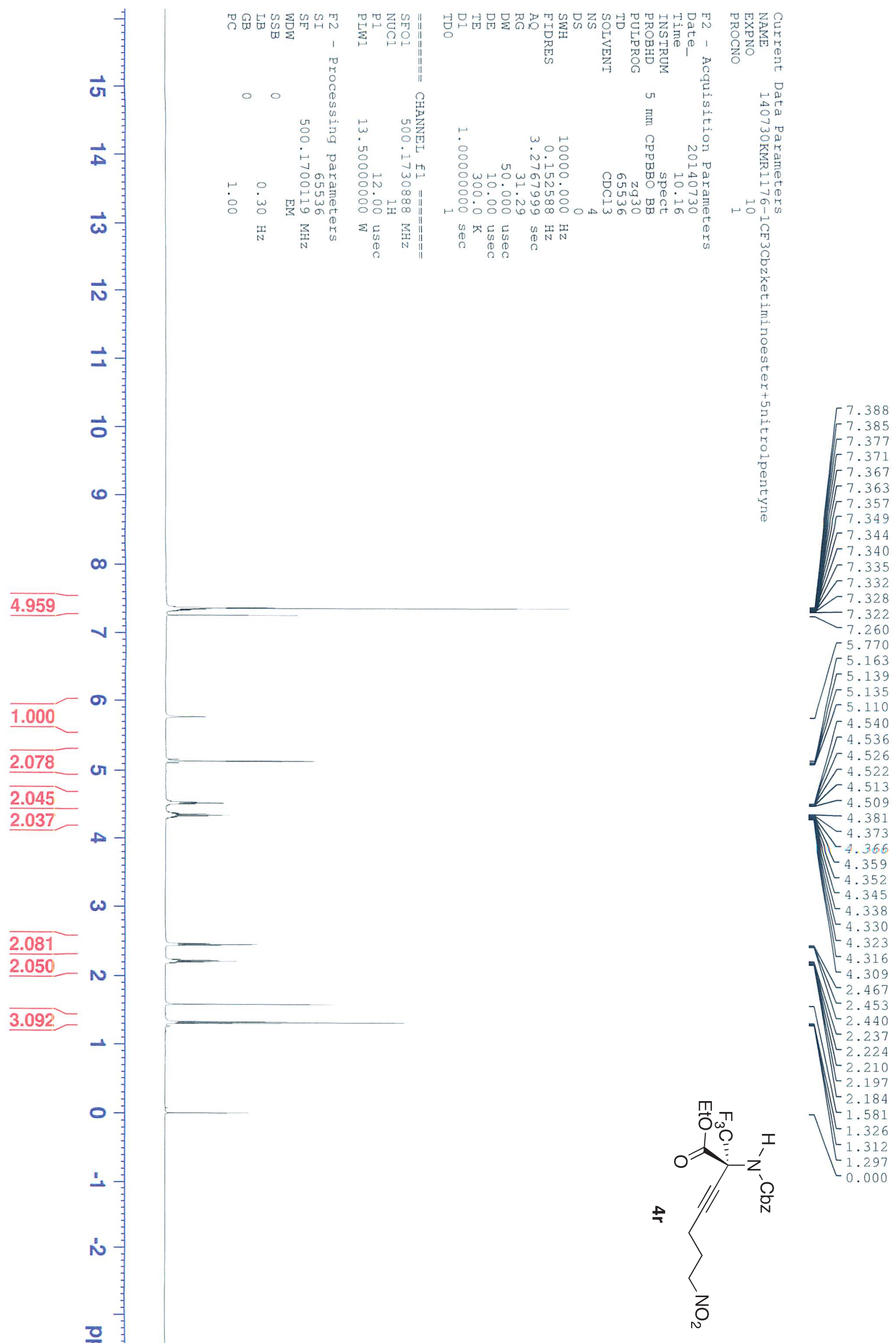




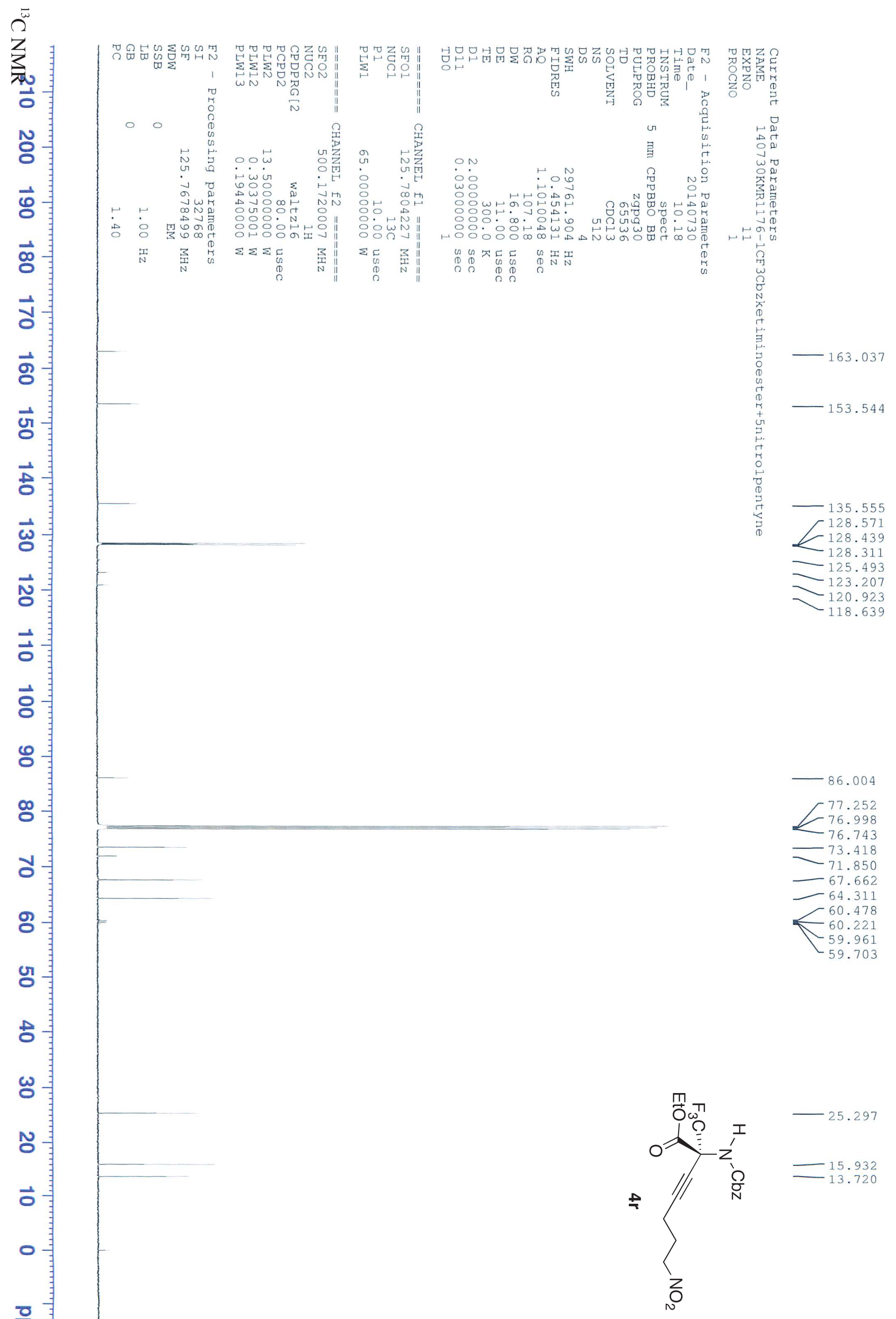


$\overrightarrow{7}$
Z
3

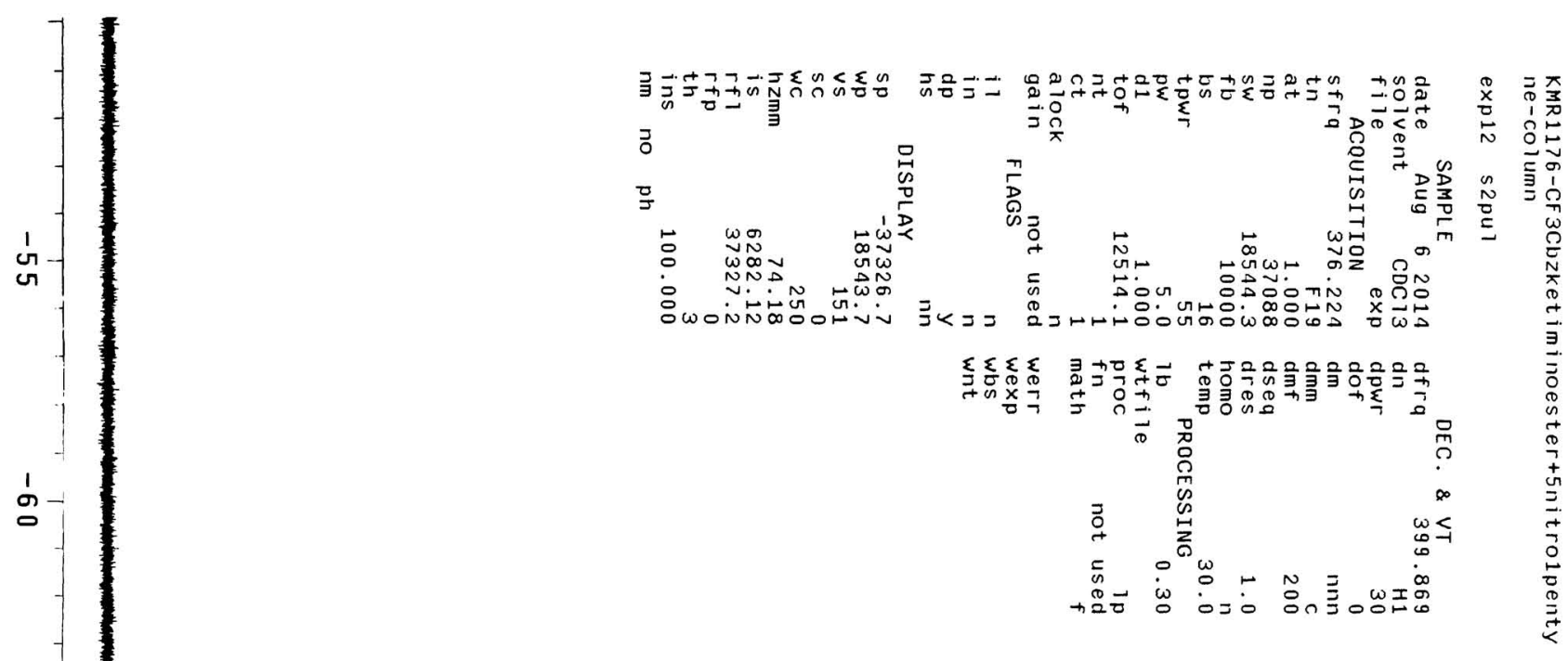

1

$\stackrel{1}{0}$

ज.

$\stackrel{1}{\infty}$

\begin{tabular}{l}
1 \\
$\infty$ \\
\hdashline
\end{tabular}

0

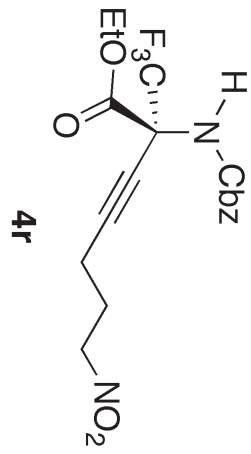




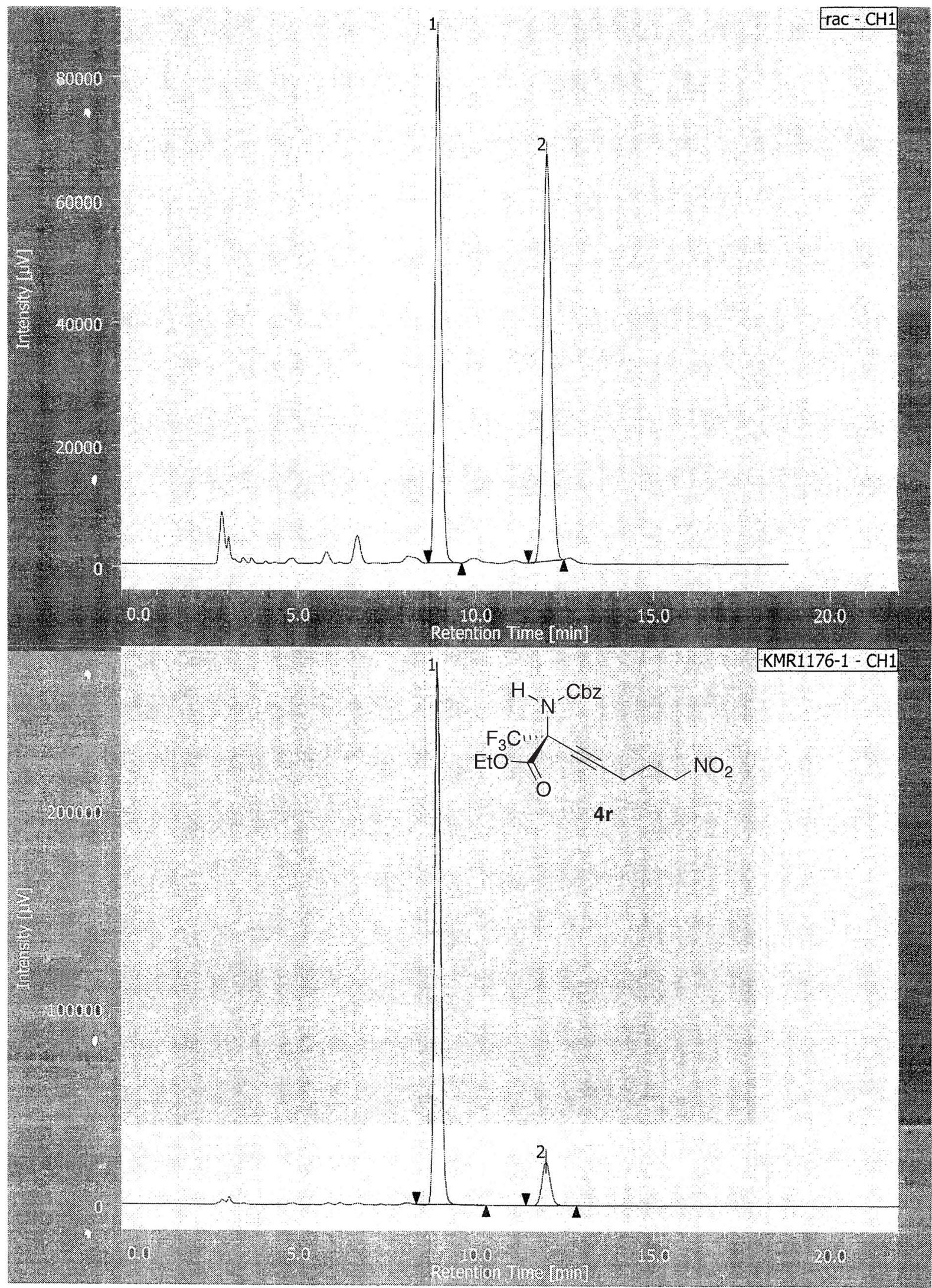

チャンネル情報+ピーク情報

クロマトグラム名

rac- $-\mathrm{CH} 1$

サンプル名

チャンネル名

$\mathrm{CH} 1$

\begin{tabular}{|c|c|c|c|c|c|c|c|c|c|c|c|}
\hline \# ピーク名 & $\mathrm{CH}$ & $\operatorname{tR}[\mathrm{min}]$ & 面皘 [ $[\mu \mathrm{V} \cdot \mathrm{sec}]$ & \begin{tabular}{|l|}
$\operatorname{Da} 丸[\mu \mathrm{V}]$ \\
\end{tabular} & 面積为为 & 高さ为 & 定㽬值 & NTP & 分離度 & ジメトリー係数 & 攺䇾告 \\
\hline Unknown & 1 & 8.933 & 1053871 & 86200 & 49.551 & 56.51 & N/A & 12395 & 8.238 & 1.078 & \\
\hline 2Unknown & 1 & 12.017 & 1072975 & 66332 & 50.449 & 43.48 & $\mathrm{~N} / \mathrm{A}$ & 12518 & $\mathrm{~N} / \mathrm{A}$ & 1.06 & \\
\hline
\end{tabular}

クロマトグラム名 KMR1176-1-CH1

サンプル名

チャンネル名

$\mathrm{CH} 1$

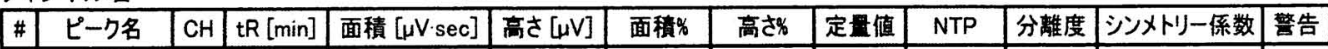

\begin{tabular}{|c|c|c|c|c|c|c|c|c|c|c|}
\hline 1 Unknown & 1 & 8.892 & 3364057 & 268827 & 89.994 & 92.144 & N/A & 11453 & 8.130 & 1.163 \\
\hline Unk & 1 & 11.975 & 374027 & 22920 & 10.006 & 7.856 & N/A & 12511 & $\mathrm{~N} / \mathrm{A}$ & 057 \\
\hline
\end{tabular}




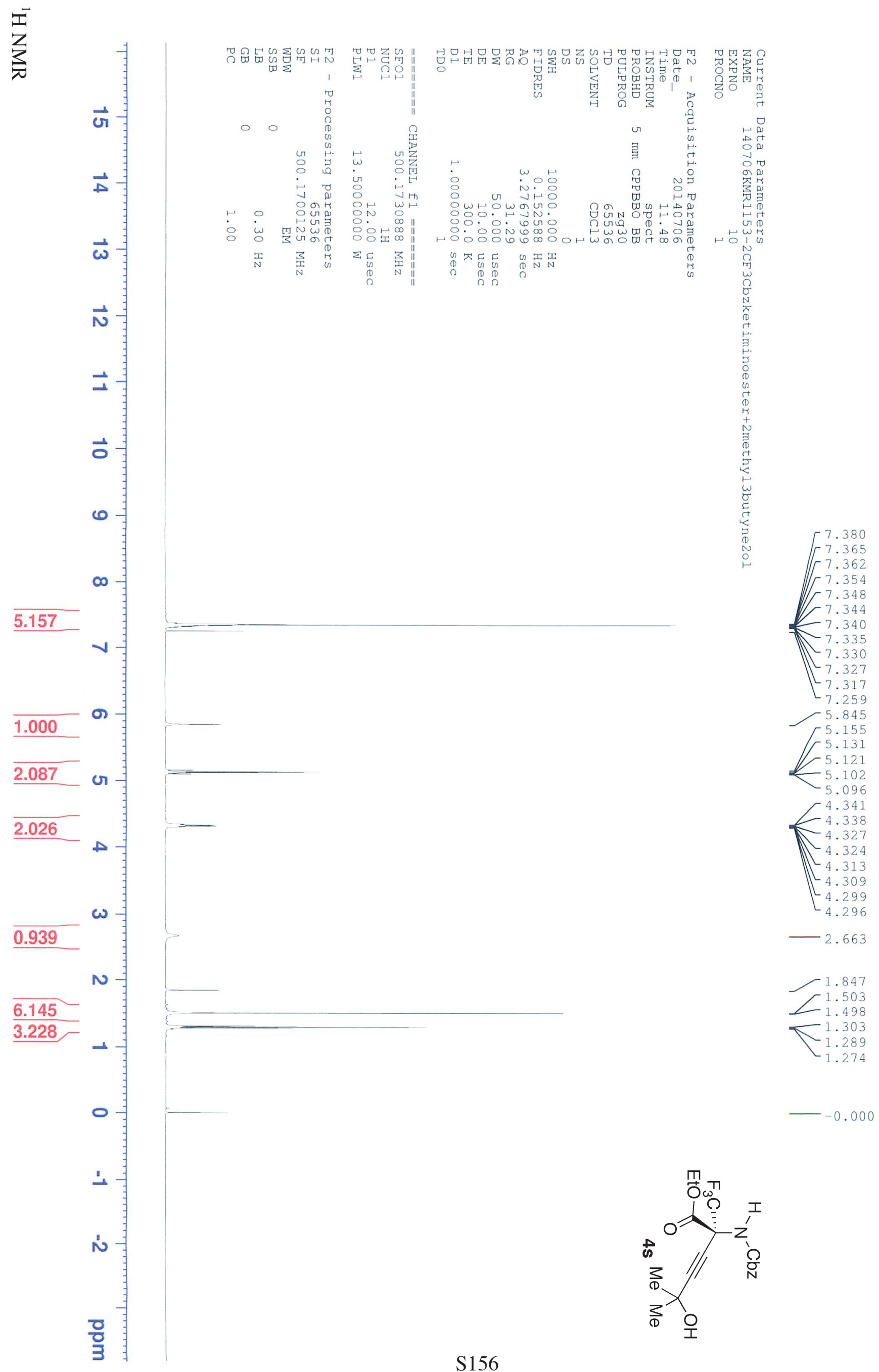




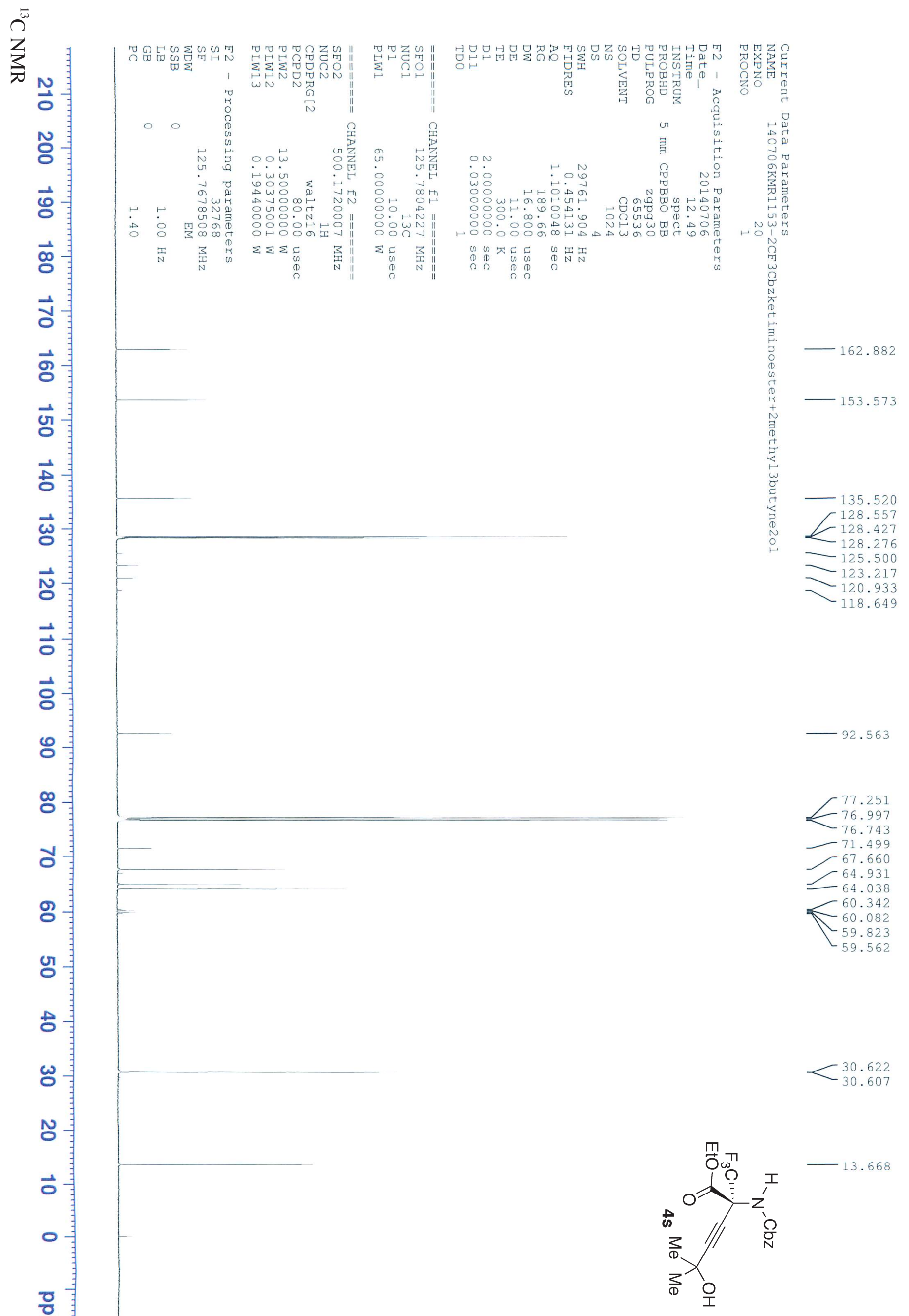



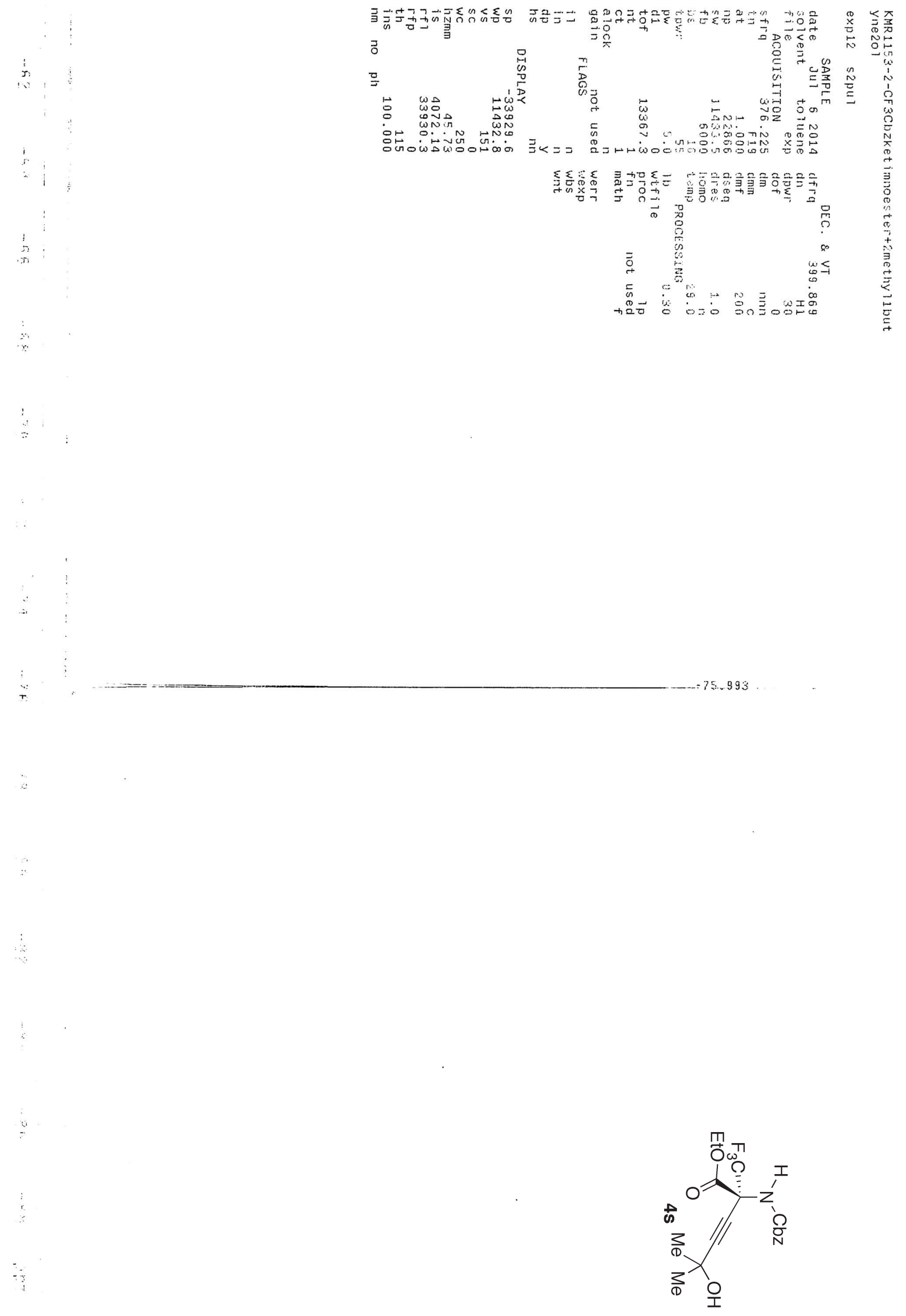


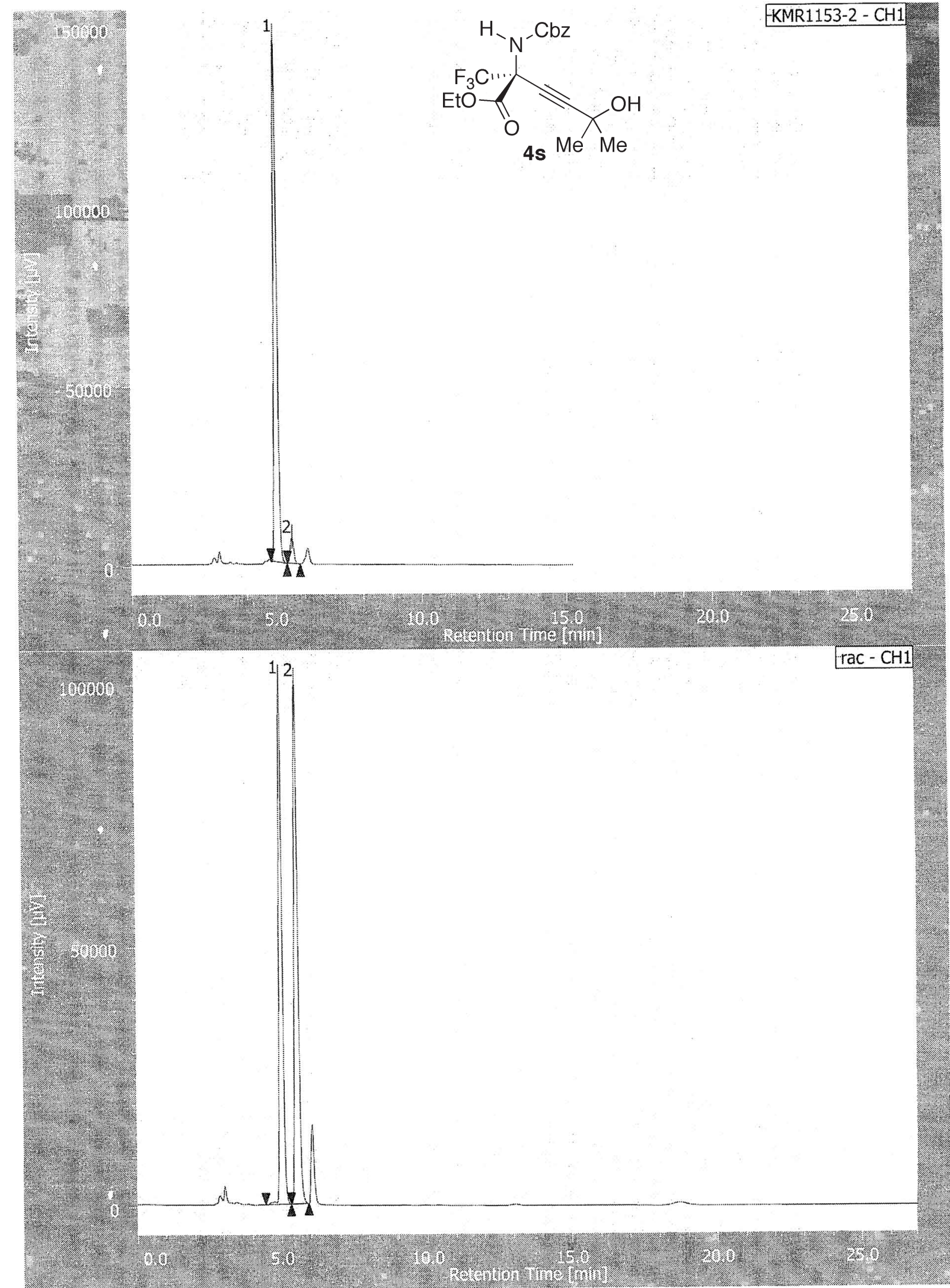

チャンネル情報+ピーク情報

クロマトグラム名

KMR1 153-2-CH1

サンプル名

チャンネル名

$\mathrm{CH} 1$

\begin{tabular}{|c|c|c|c|c|c|c|c|c|c|c|c|}
\hline ピーク名 & $\mathrm{CH}$ & $t R$ [min] & 面積 [ $[\mu \mathrm{V} \cdot \mathrm{sec}]$ & 高さ [uV] & 面積\% & 高さ\% & 定量値 & NTP & 分離度 & シンメトリー係数 & 警 \\
\hline 1 Unknown & 1 & 5.017 & 1189972 & 146569 & 95.060 & 95.168 & N/A & 8830 & 2.388 & 1.129 & \\
\hline 2 Unknown & 1 & 5.533 & 61841 & 7442 & 4.940 & 4.832 & $N / A$ & 10093 & $\mathrm{~N} / \mathrm{A}$ & 1.101 & \\
\hline
\end{tabular}

クロマトグラム名

サンプル名

チャンネル名

$\mathrm{CH} 1$

\begin{tabular}{|c|c|c|c|c|c|c|c|c|c|c|c|c|}
\hline \# & ピーク名 & $\mathrm{CH}$ & $t R[\min ]$ & 面積 [ $[\mu \mathrm{V} \cdot \mathrm{sec}]$ & 高さ [ $\mu \mathrm{V}]$ & 面積\% & 高さ\% & 定量値 & NTP & 分離度 & シンメトリー係数 & \begin{tabular}{|l|} 
警告 \\
\end{tabular} \\
\hline & Unknown & 1 & 5.000 & 817183 & 100888 & 47.471 & 50.154 & N/A & 8982 & 2.346 & 1.104 & \\
\hline & 2 Unknown & 1 & 5.525 & 904237 & 100270 & 52.529 & 49.846 & N/A & 8638 & N/A & 1.157 & \\
\hline
\end{tabular}




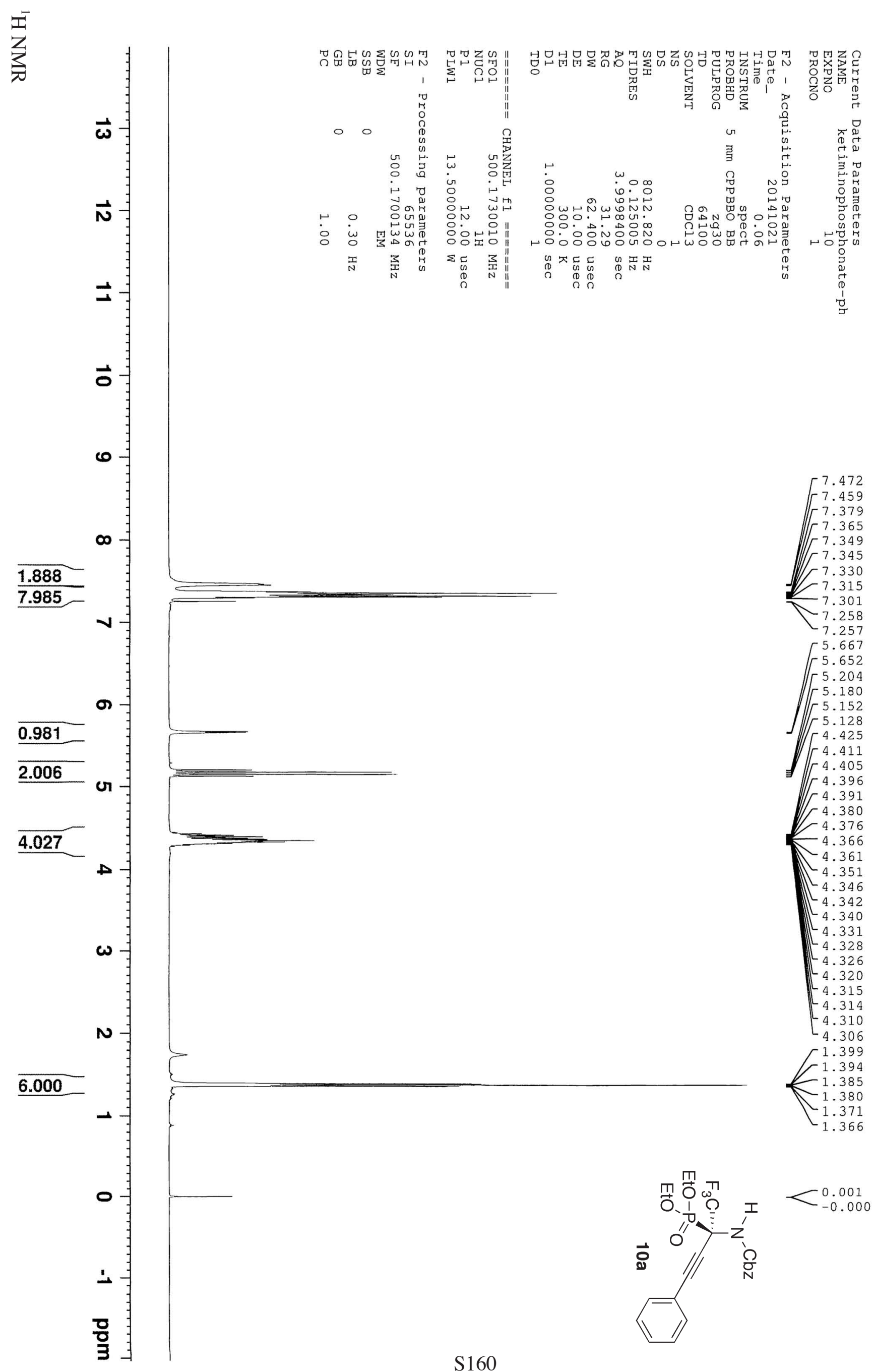




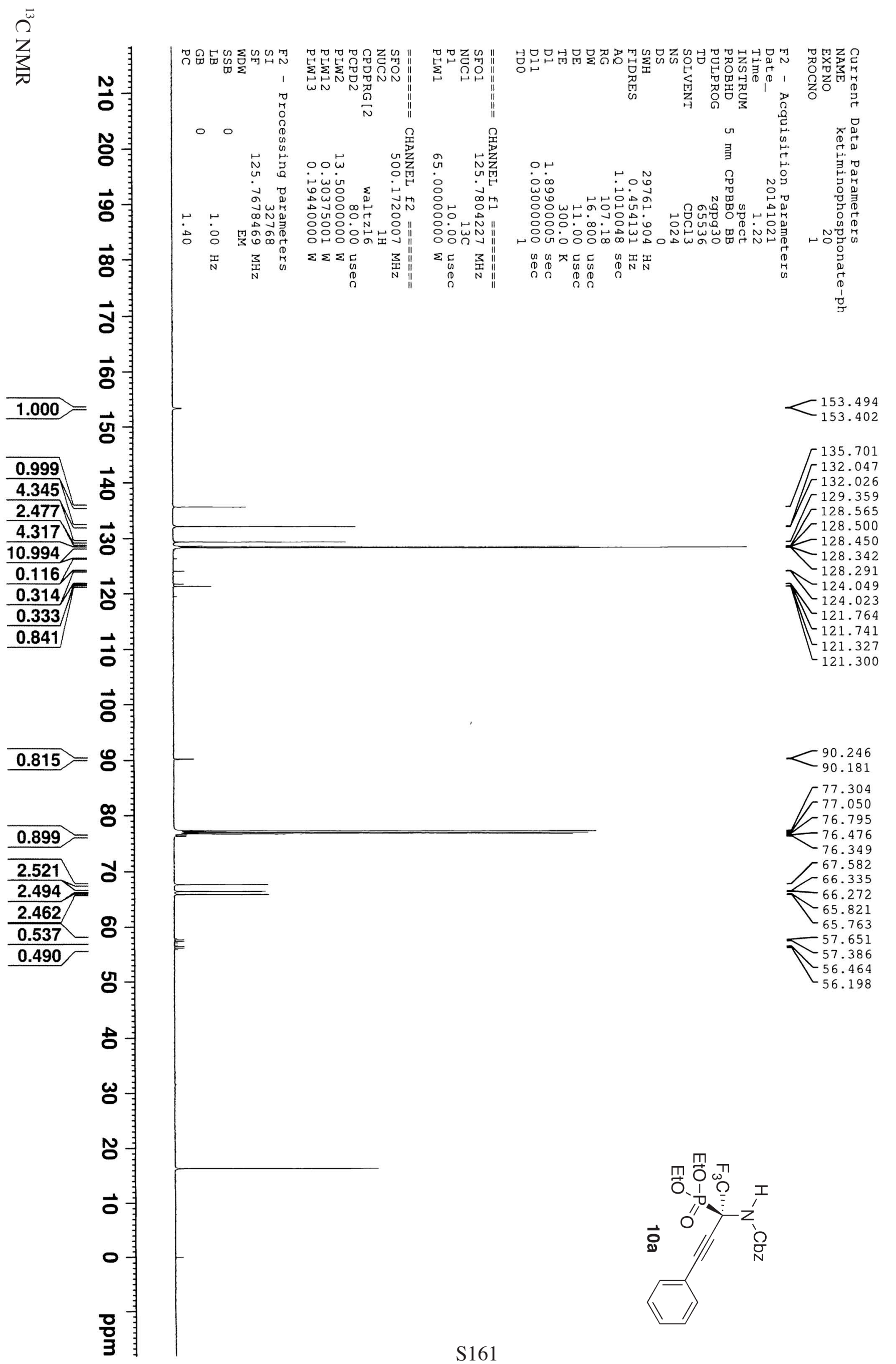




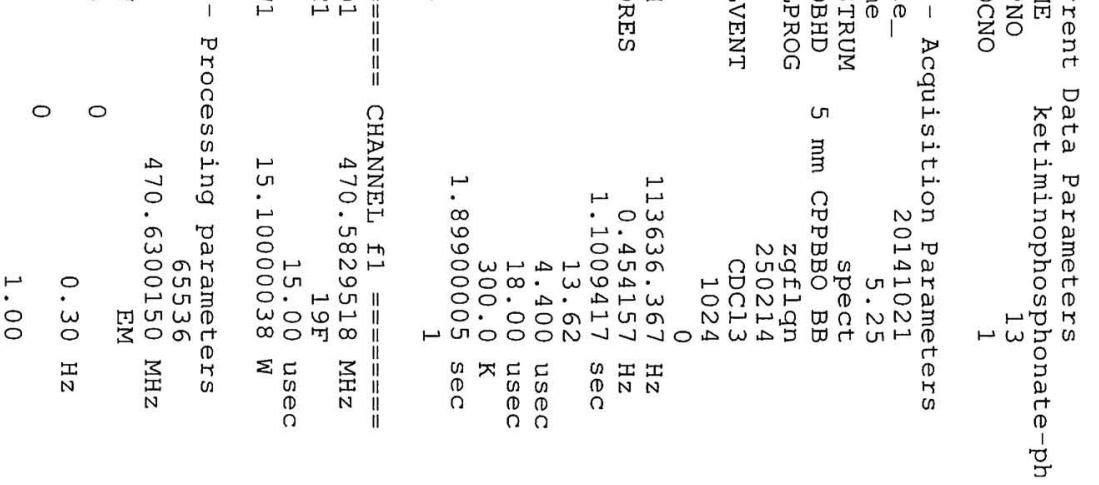

த்

$\$$

ஸे

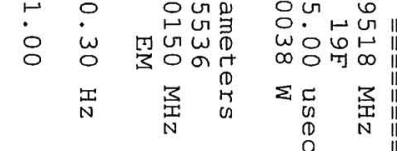
0
0
0
0

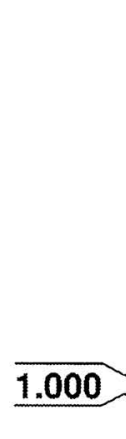

\&
'횽

\begin{tabular}{l}
$\dot{8}$ \\
\hline \\
$\dot{N}$
\end{tabular}

$\frac{1}{\overrightarrow{0}}$

-

官-

̊̊

목

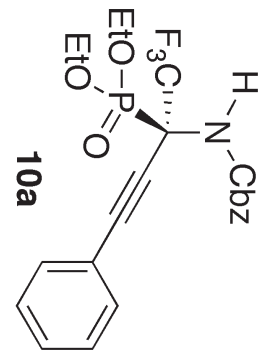




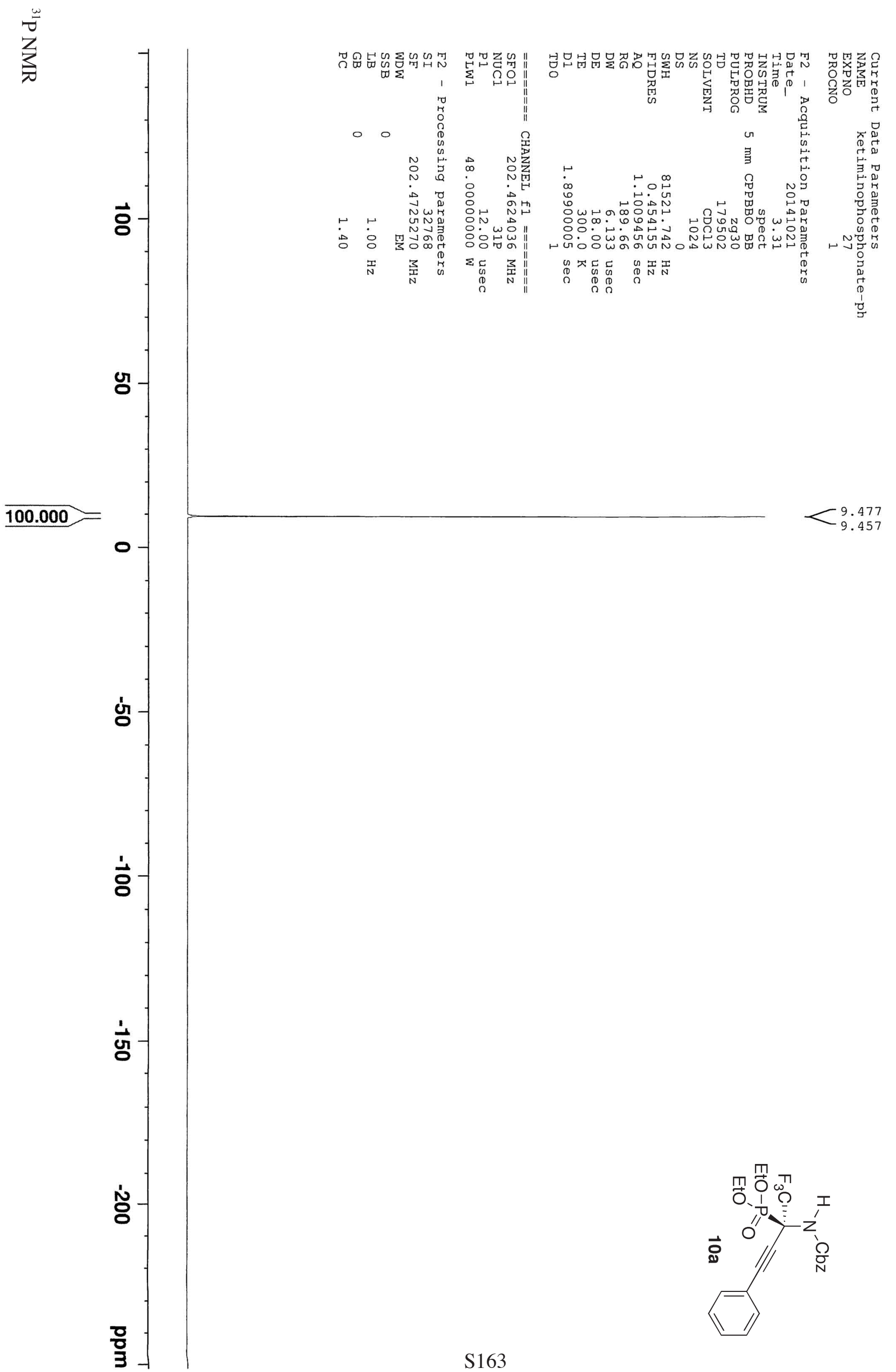




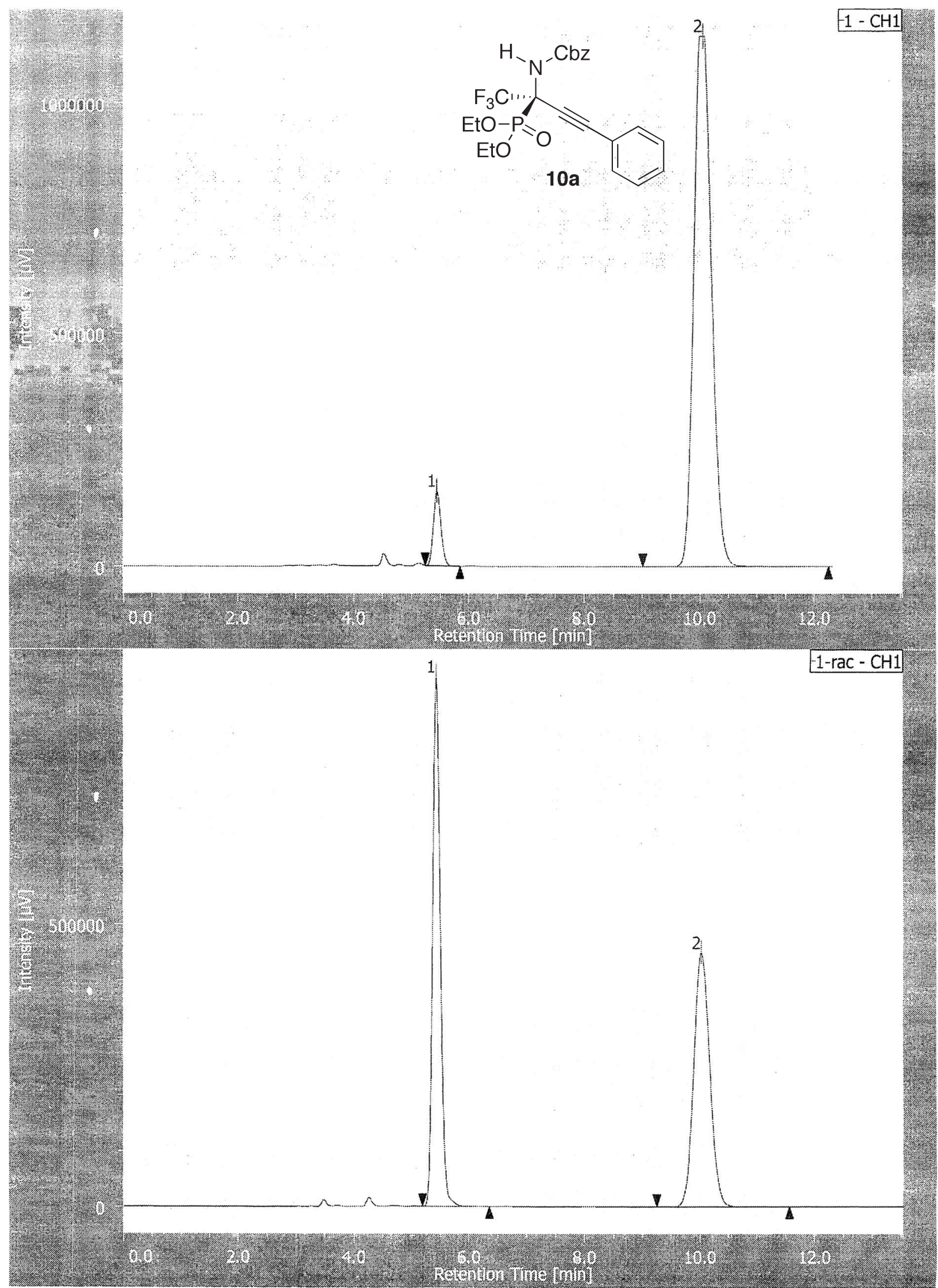

チャンネル情報+ピーク情報

クロマトグラム名

1-CH1

サンプル名

チャンネル名

$\mathrm{CH} 1$

\begin{tabular}{|c|c|c|c|c|c|c|c|c|c|c|c|}
\hline ピーク名 & $\mathrm{CH}$ & $\operatorname{tR}[\mathrm{min}]$ & 面積 $[\mu \mathrm{V} \cdot \mathrm{sec}]$ & 高さ $[\mu \mathrm{V}]$ & 面積\% & 高さ\% & 定盘値 & NTP & 分離度 & シンメトリー係数 & 警告 \\
\hline Unkn & 1 & 5.44 & 353 & 161426 & 5.612 & 12.353 & $\mathrm{~N} / \mathrm{A}$ & 8266 & 11.509 & 1.108 & \\
\hline Unkr & 1 & 10.06 & 24517487 & 1145398 & 94.388 & 87.647 & $\mathrm{~N} / \mathrm{A}$ & 5071 & $N / A$ & 1.093 & \\
\hline
\end{tabular}

クロマトグラム名

1-rac-CH1

サンプル名

チャンネル名

$\mathrm{CH} 1$

\begin{tabular}{|c|c|c|c|c|c|c|c|c|c|c|c|}
\hline ピーク名 & $\mathrm{CH}$ & $\mathrm{tR}$ [min] & 面積 [ $\mathrm{\mu V} \cdot \mathrm{sec}]$ & \begin{tabular}{|l|l} 
高さ $[\mu \mathrm{V}]$ \\
\end{tabular} & 面積\% & 高さ\% & 定量値 & NTP & 分離度 & シンメトリー係数 & 敕告 \\
\hline 1 Unknown & 1 & 5.42 & 8667751 & 935873 & 49.661 & 67.555 & $N / A$ & 7916 & 12.077 & 1.138 & \\
\hline 2 Unknown & 1 & 10.03 & 8786132 & 449478 & 50.339 & 32.445 & $N / A$ & 5949 & $N / A$ & 1.082 & \\
\hline
\end{tabular}




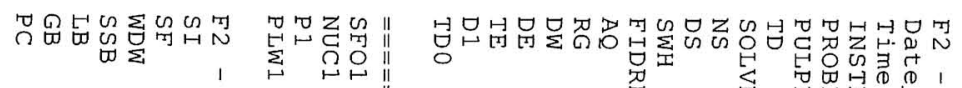

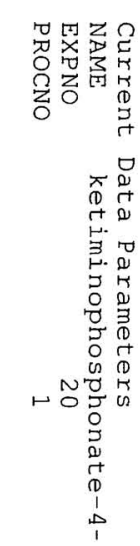

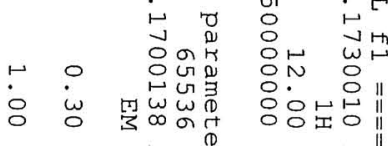

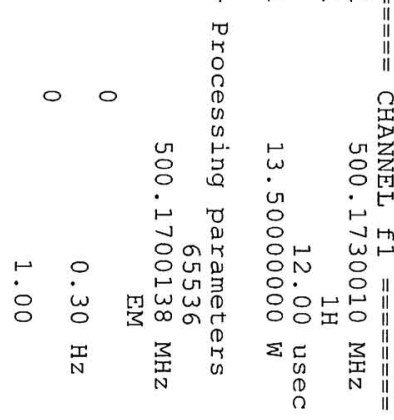

惫璃

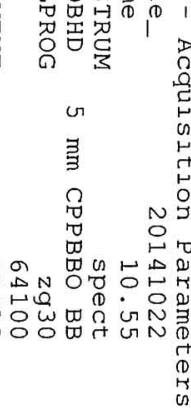

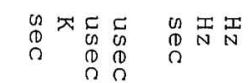

7.379

7.364

7.346

7.053

$\infty$

$\omega$
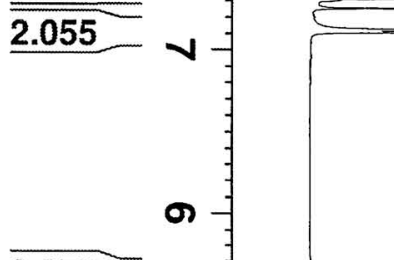

-7.330
-7.317

7.257

$-7.128$

7.112

$\Gamma \begin{array}{r}5.641 \\ 5.626\end{array}$

.

5.6260
5.176

$\left[\begin{array}{r}5.176 \\ 5.150\end{array}\right.$

5.125

4.421
4.406

$\left[\begin{array}{l}4.406 \\ 4.392 \\ 4.386\end{array}\right.$

4.386
4.332

$-4.372$

$-4.360$

4.354

4.344

4.340

4.335

4.330

4.325

4.321

4.310

4.304

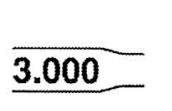

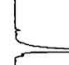

$-2.354$

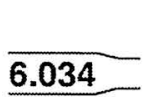

.

1.393

1.393
-1.389

1.379

1.375
1.365

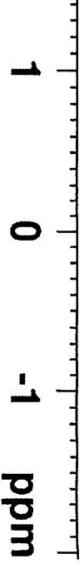

T田

$-1.361$

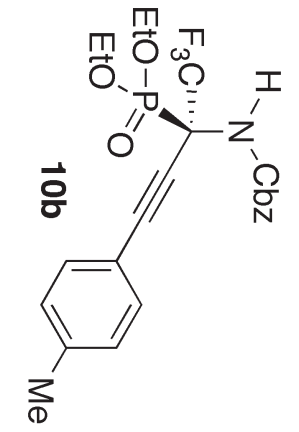

$-0.000$ 


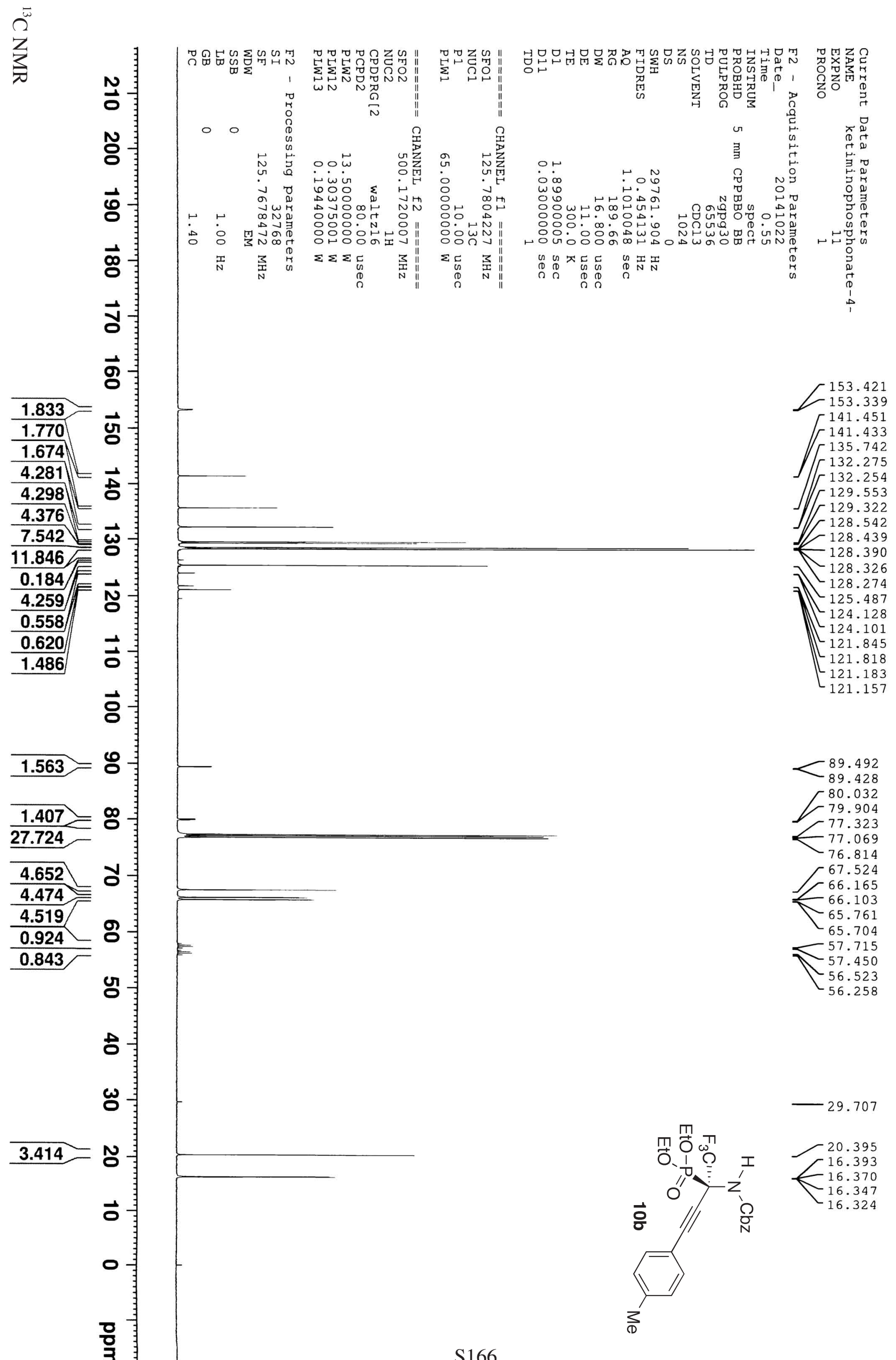




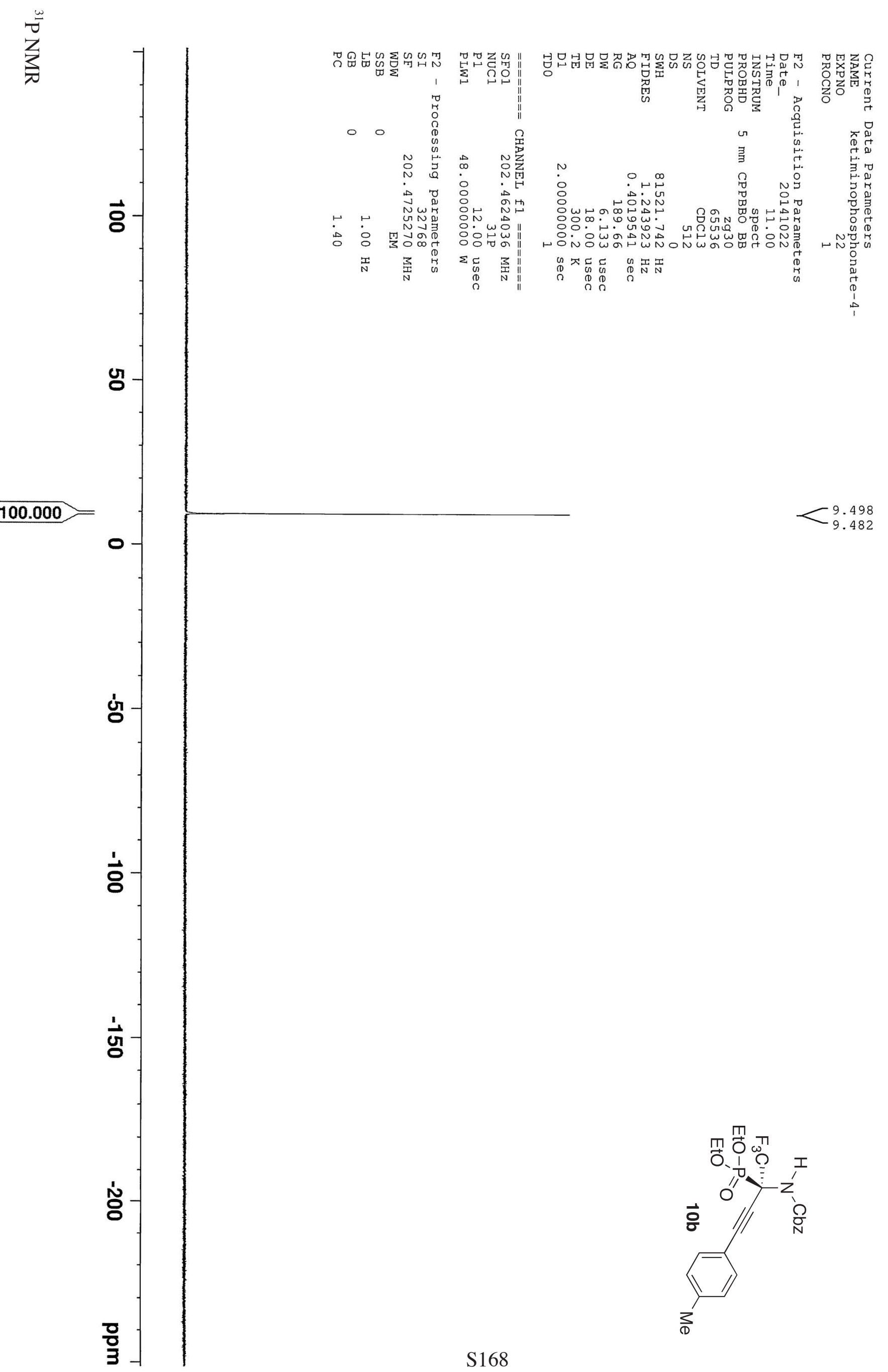




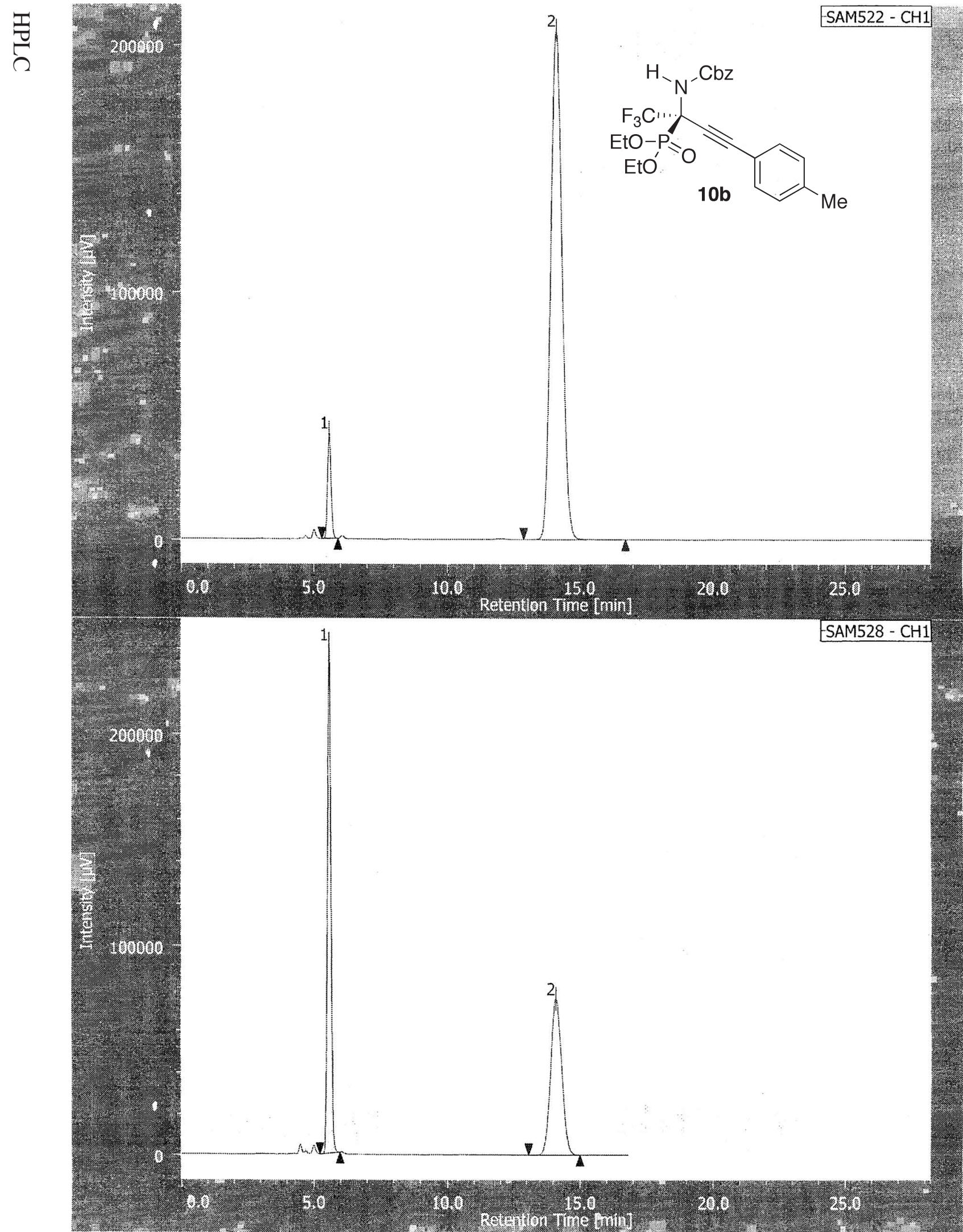

チャンネル情報+ピーク情報

クロマトグラム名

SAM522-CH1

サンプル名

チャンネル名

$\mathrm{CH} 1$

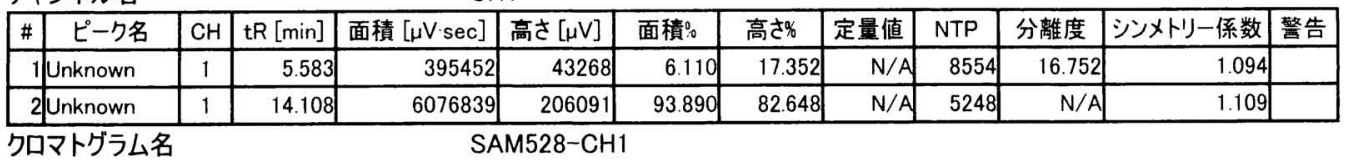

サンプル名

チャンネル名

$\mathrm{CH} 1$

\begin{tabular}{|c|c|c|c|c|c|c|c|c|c|c|c|c|}
\hline$\#$ & ピーク名 & $\mathrm{CH}$ & $t R[\mathrm{~min}]$ & 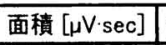 & 高さ $[\mu \mathrm{V}]$ & 面皘\% & 高さ\% & 定量値 & NTP & 分離度 & シンメトリー係数 & 警告 \\
\hline & Unknown & 1 & 5.575 & 2199976 & 241052 & 50.228 & 76.360 & $N / A$ & 8817 & 16.909 & 1.117 & \\
\hline & Unknown & 1 & 14.100 & 2179967 & 74626 & 49.772 & 23.640 & $N / A$ & 5316 & $N / A$ & 1.069 & \\
\hline
\end{tabular}



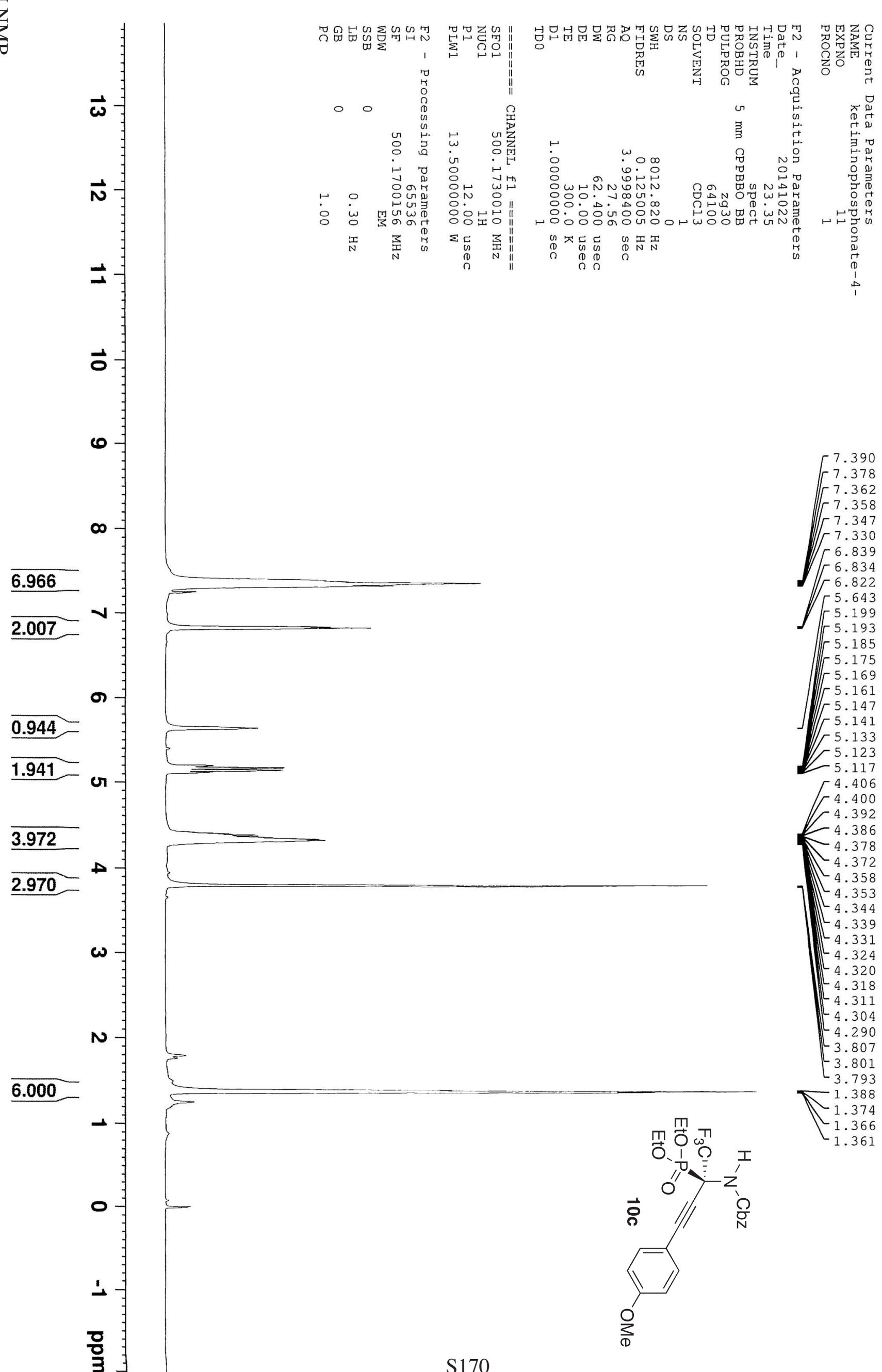
$\stackrel{2}{2}^{\bar{\omega}}$

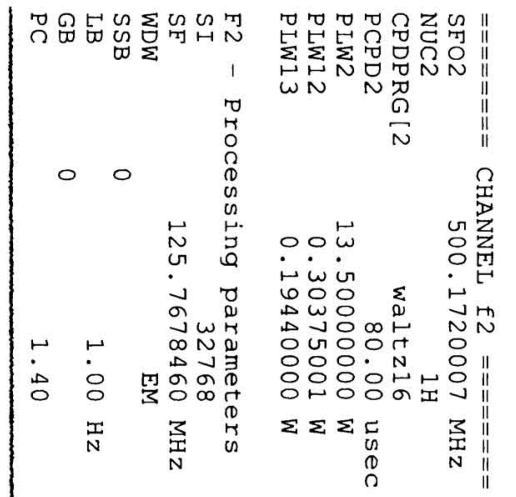

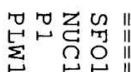

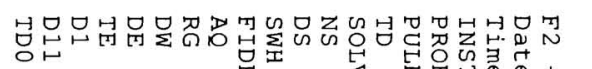

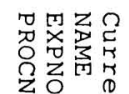

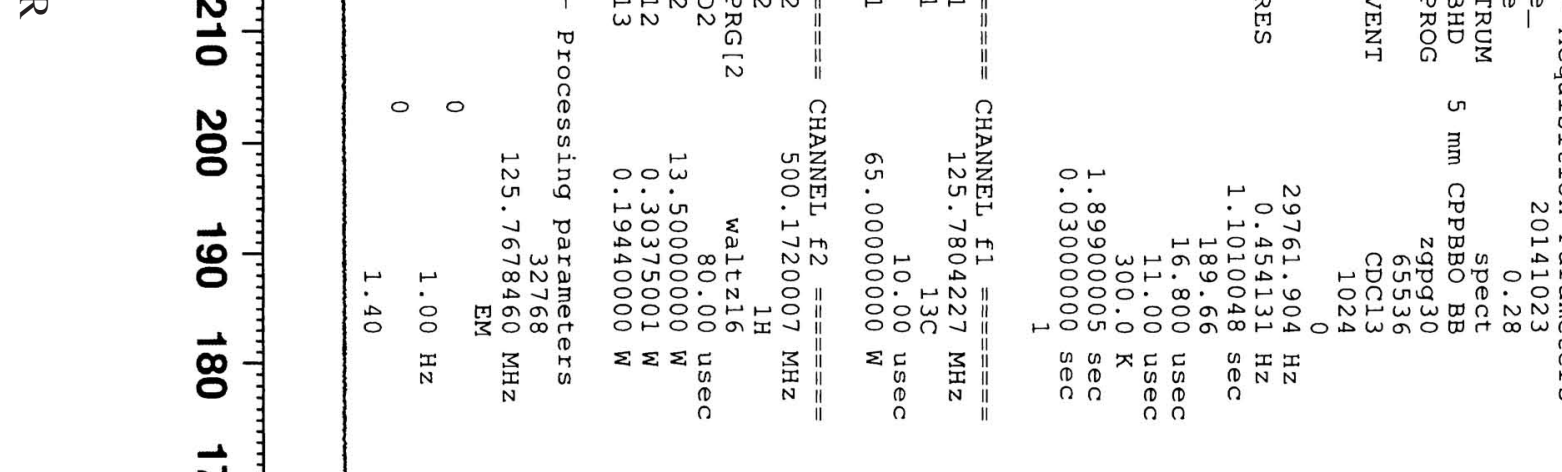
思

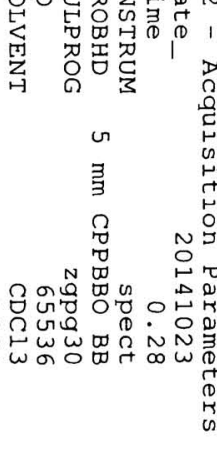

z० 䒿

160.434

$1.000=\vec{g}$

ธั

1.148

ज़ㅇำ

1.095

$\overrightarrow{0}$

$11.721=$

$\vec{\omega}$

0.863

$\vec{\Sigma}$

$\frac{5.037}{1.028}$

ठे
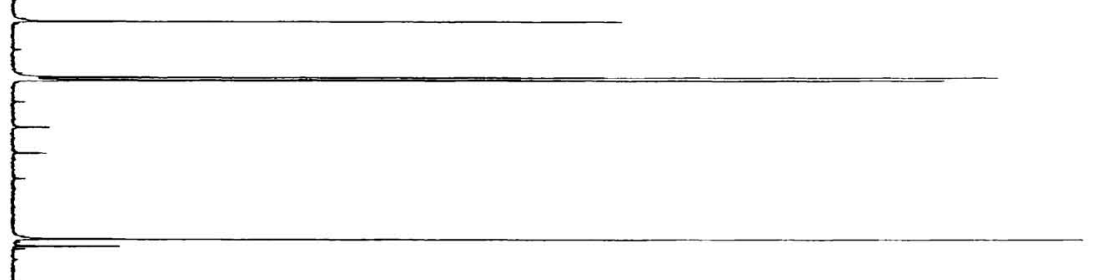

53.502

153.424

135.755

133.845

r 133.615

130.964

128.916

128.564

$-128.453$

$-128.281$

$-126.376$

$-124.093$

$-121.810$

$-119.524$

$-113.983$

$-113.351$

응

$\underline{0.907}=\mathscr{\circ}$

\&

$0.906=$

2.690

d

2.678

0.571 용

0.584

2.578

ำ

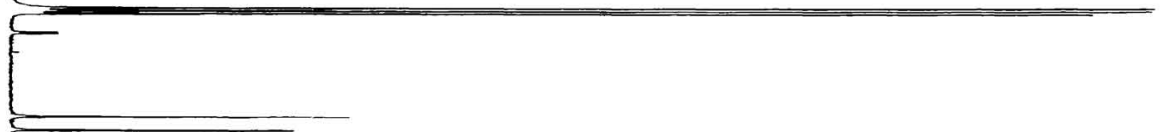

90.374

90.309

77.065

76.811

75.121

74.994

73.274

67.526

66.303

66.241

$-65.751$

65.693

57.976

57.713

$-57.449$

$\left[\begin{array}{r}57.184 \\ 56.784\end{array}\right.$

56.258
55.994

55.994

55.328

55.205
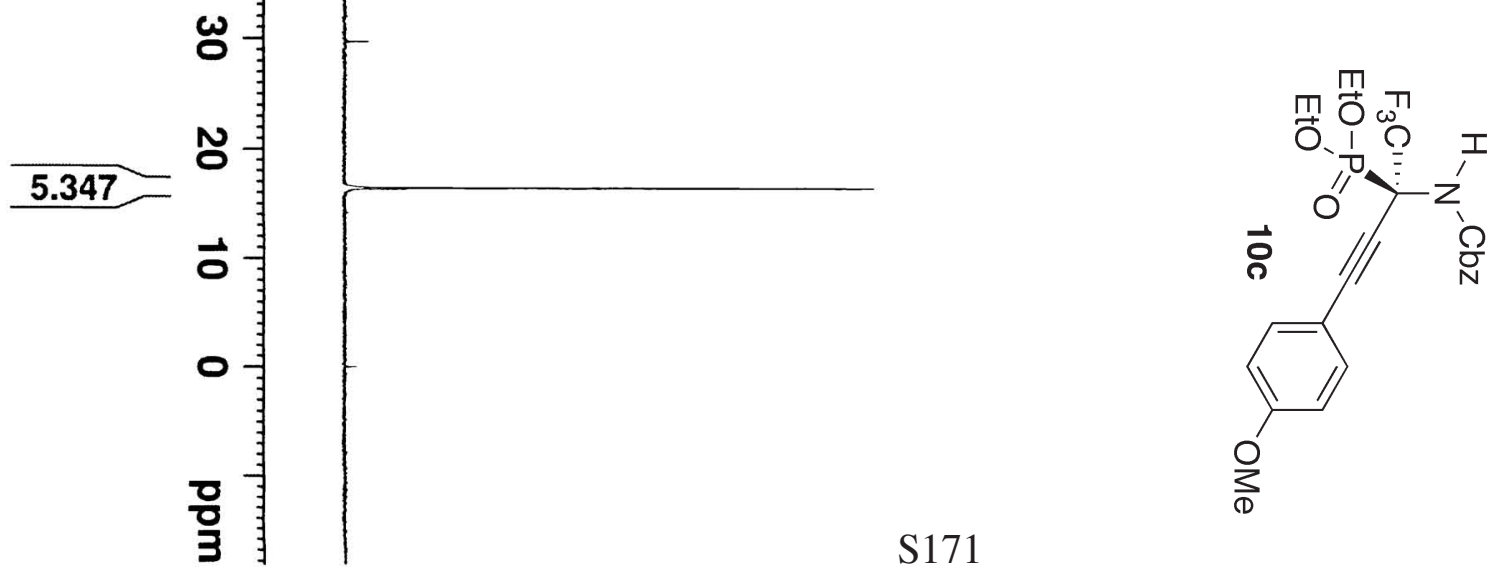

$-29.705$

16.398
-16.353 


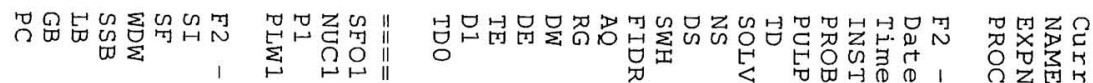

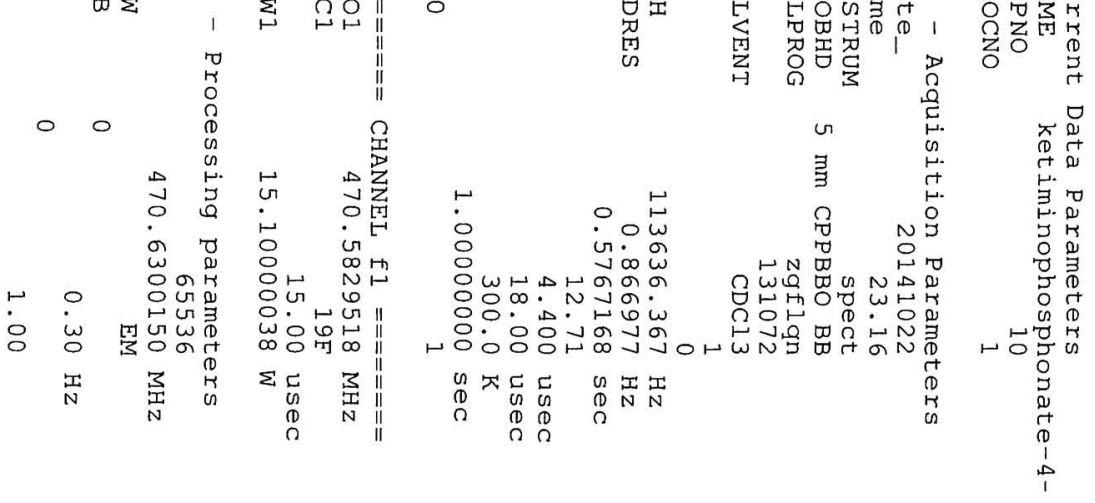




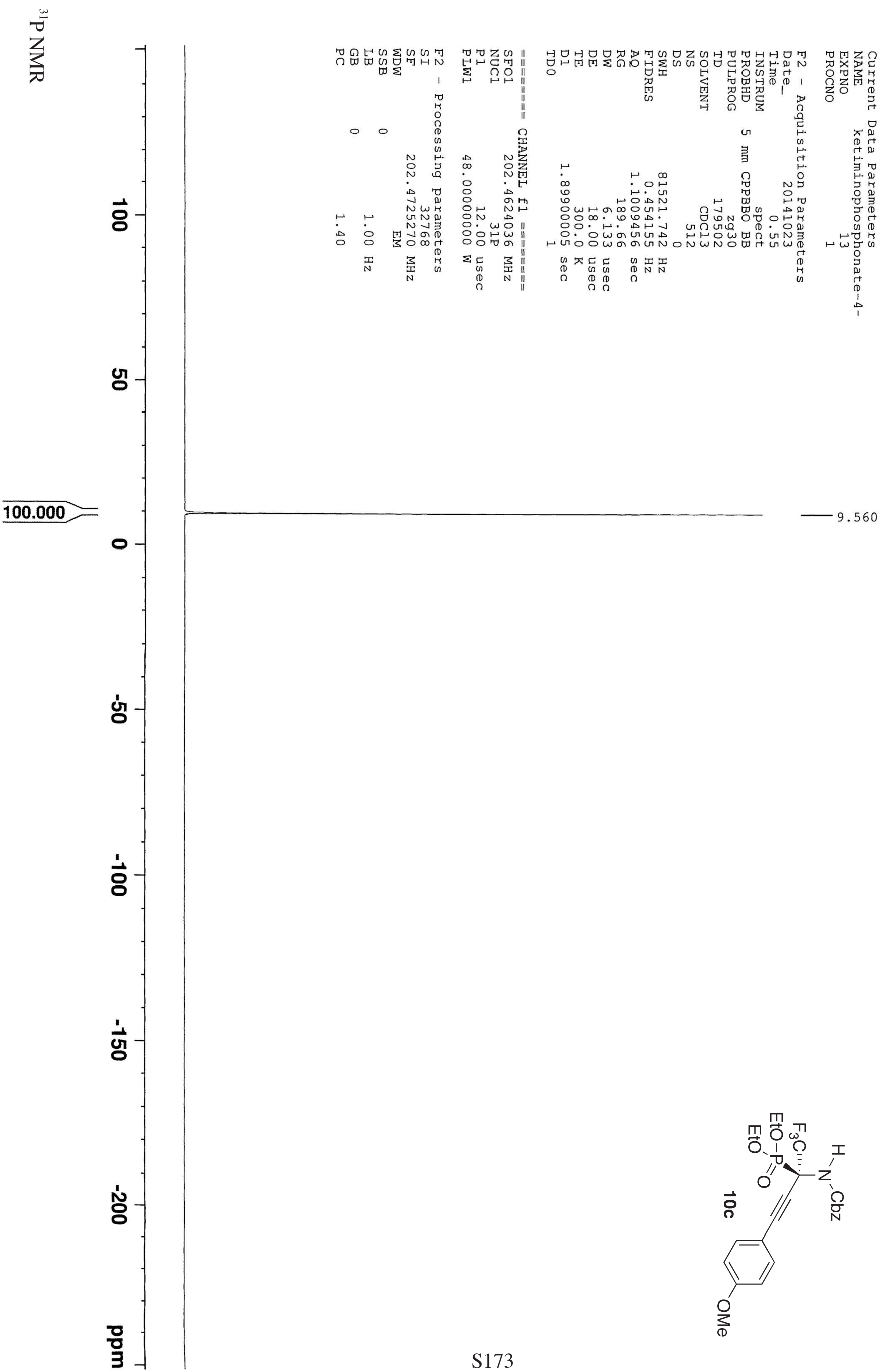




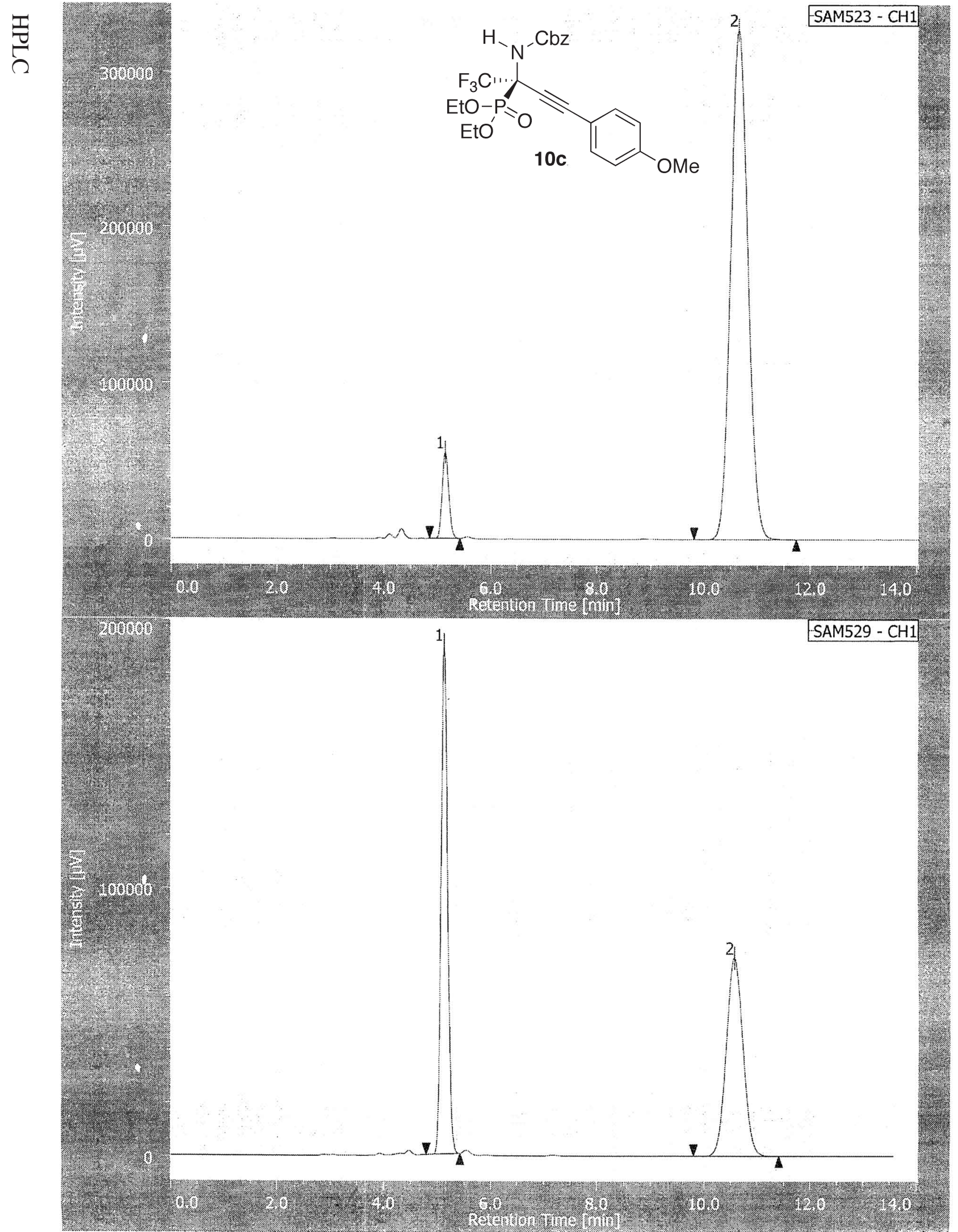

チャンネル情報+ピーク情報

クロマトグラム名

SAM523-CH1

サンプル名

チャンネル名

$\mathrm{CH} 1$

\begin{tabular}{|c|c|c|c|c|c|c|c|c|c|c|c|c|}
\hline & \\
\hline \pm & E-ク名 & $\mathrm{CH}$ & th $[\mathrm{min}]$ & 面䫅 $[\mu \mathrm{V} \cdot \mathrm{sec}\rfloor$ & & 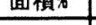 & 高赑 & & Nir & 刀離及 & 策奴 & 물 \\
\hline & Unknown & 1 & 5.142 & 470574 & 55270 & 5.998 & 14.436 & $N / A$ & 8427 & 13.461 & 1.128 & \\
\hline & Unknown & 1 & 10.650 & 7375550 & 327580 & 94.002 & 85.564 & $N / A$ & 5099 & $\mathrm{~N} / \mathrm{A}$ & 1.075 & \\
\hline \multicolumn{13}{|c|}{$\begin{array}{ll}\text { クロマトグラム名 } & \text { SAM529-CH1 } \\
\text { サンプル名 } & \end{array}$} \\
\hline \multicolumn{13}{|c|}{ チャンネル名 } \\
\hline$\#$ & ピーク名 & $\mathrm{CH}$ & tR [min] & 面積 $[\mu \mathrm{V} \cdot \mathrm{sec}]$ & 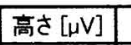 & 面積\% & 高さ\% & 定量値 & NTP & 分離度 & シンメトリー係数 & 警告 \\
\hline & Unknown & 1 & 5.125 & 1615299 & 189770 & 49.739 & 71.960 & $N / A$ & 8346 & 13.501 & 1.137 & \\
\hline & Unknown & 1 & 10.575 & 1632227 & 73946 & 50.261 & 28.040 & N/A & 5226 & $N / A$ & 1.037 & \\
\hline
\end{tabular}




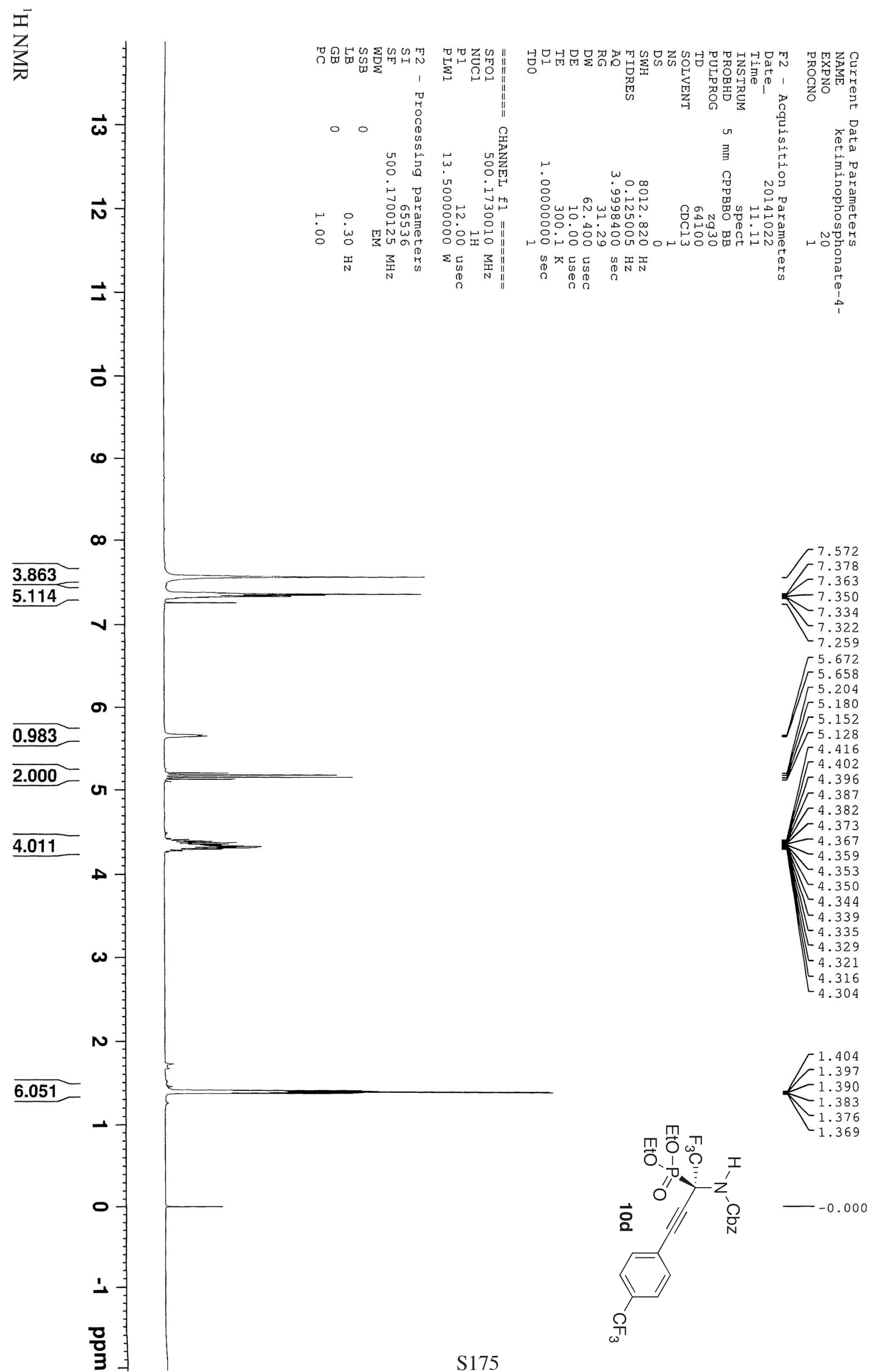




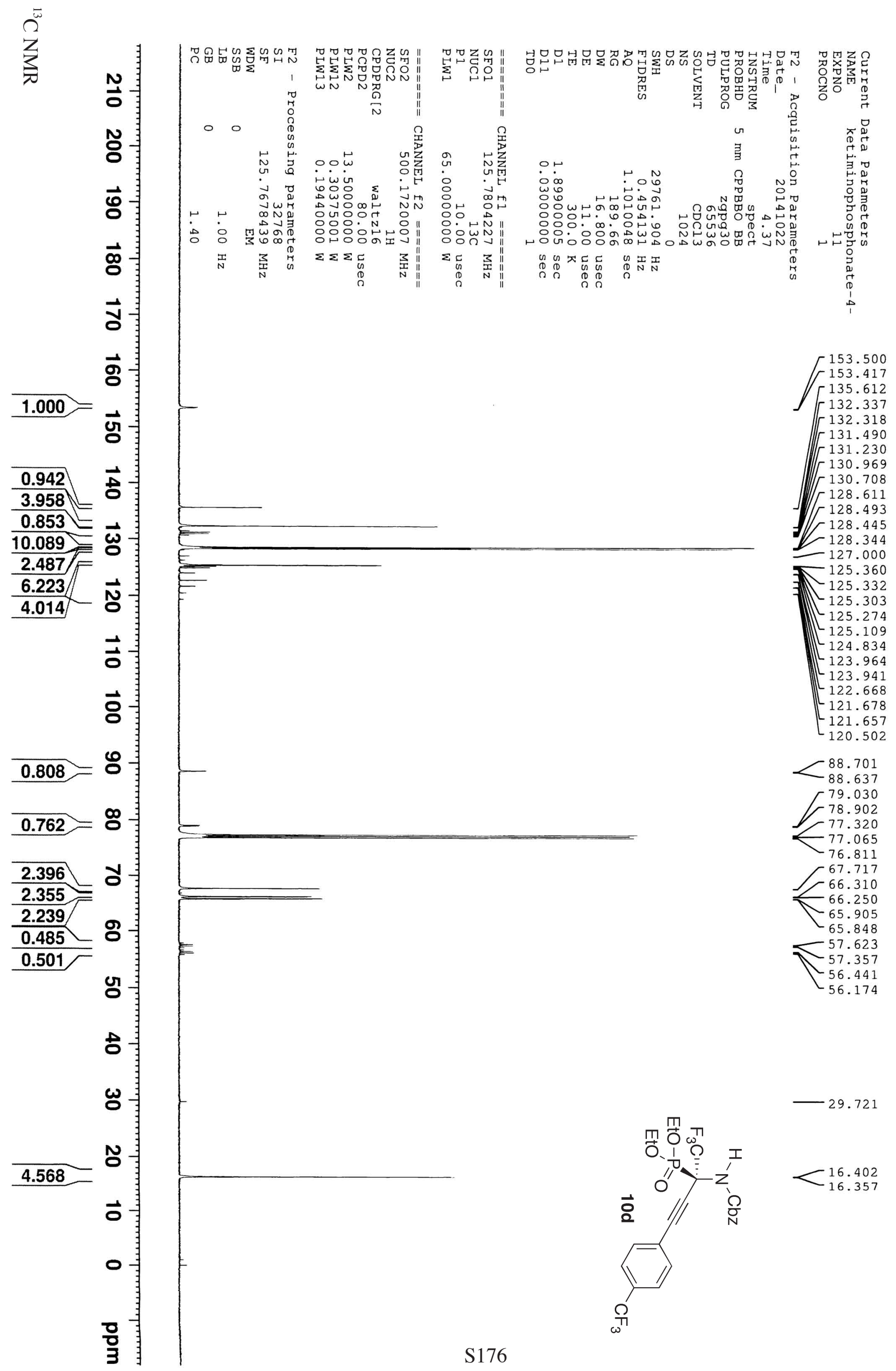




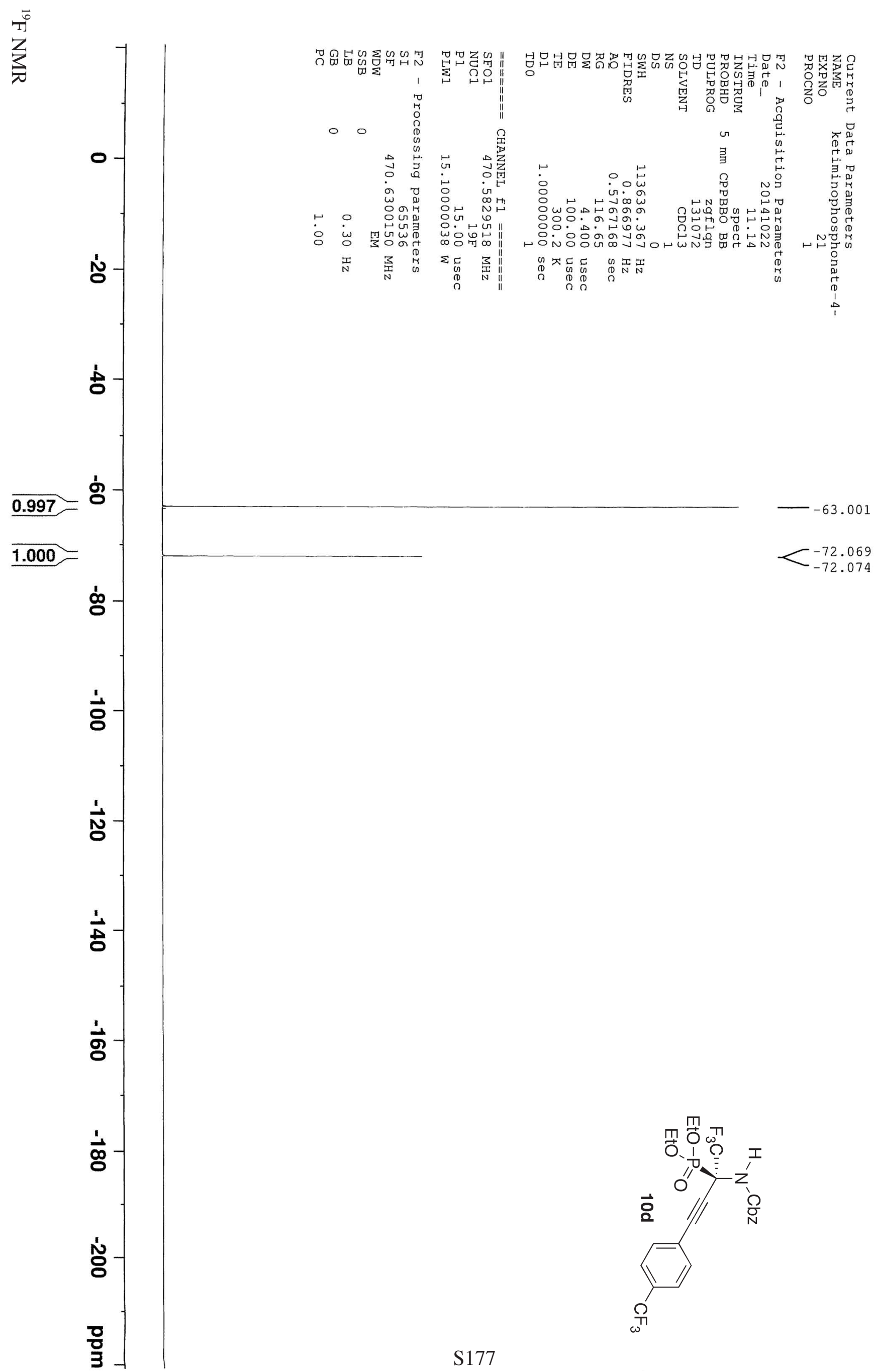




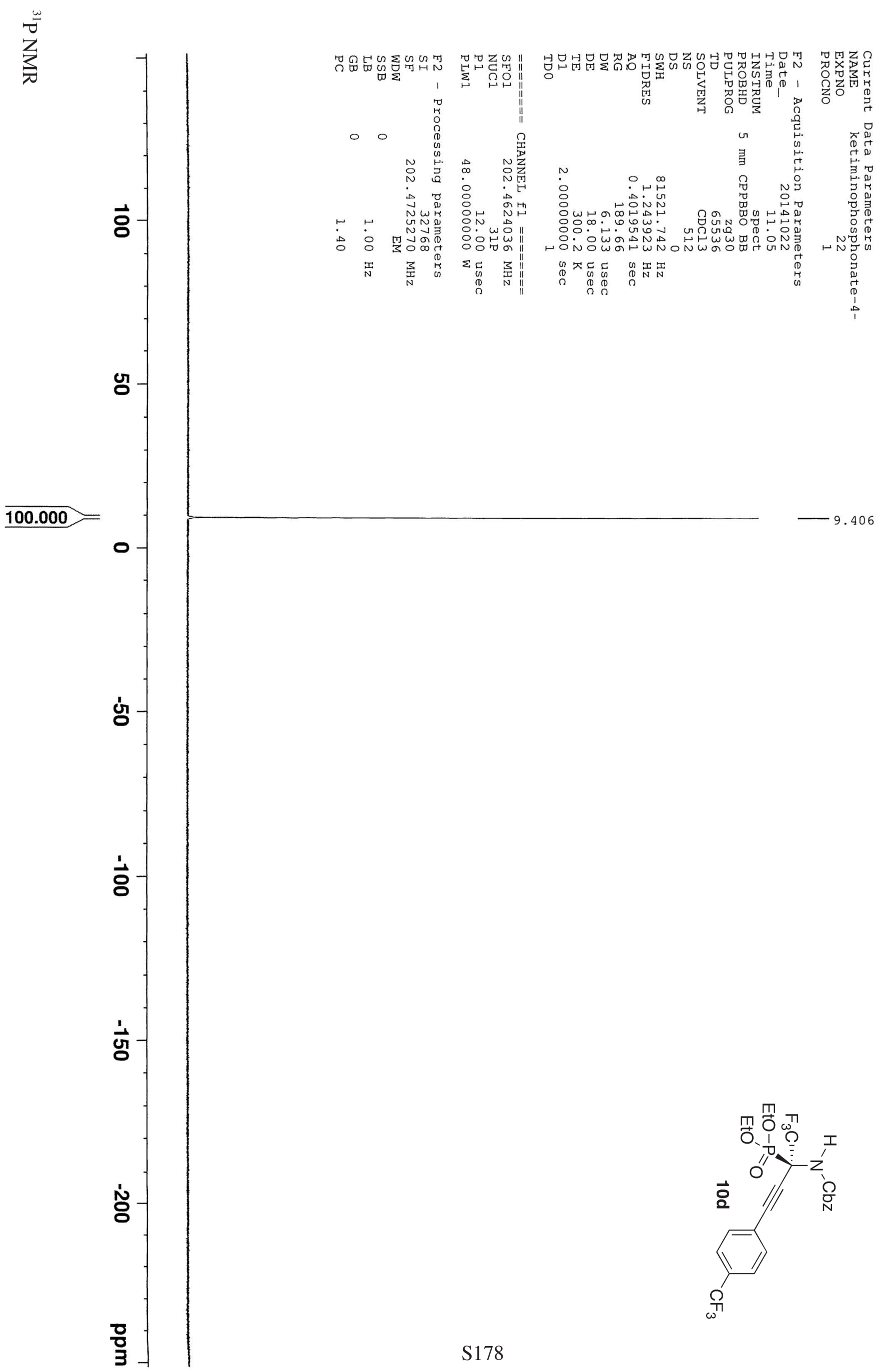




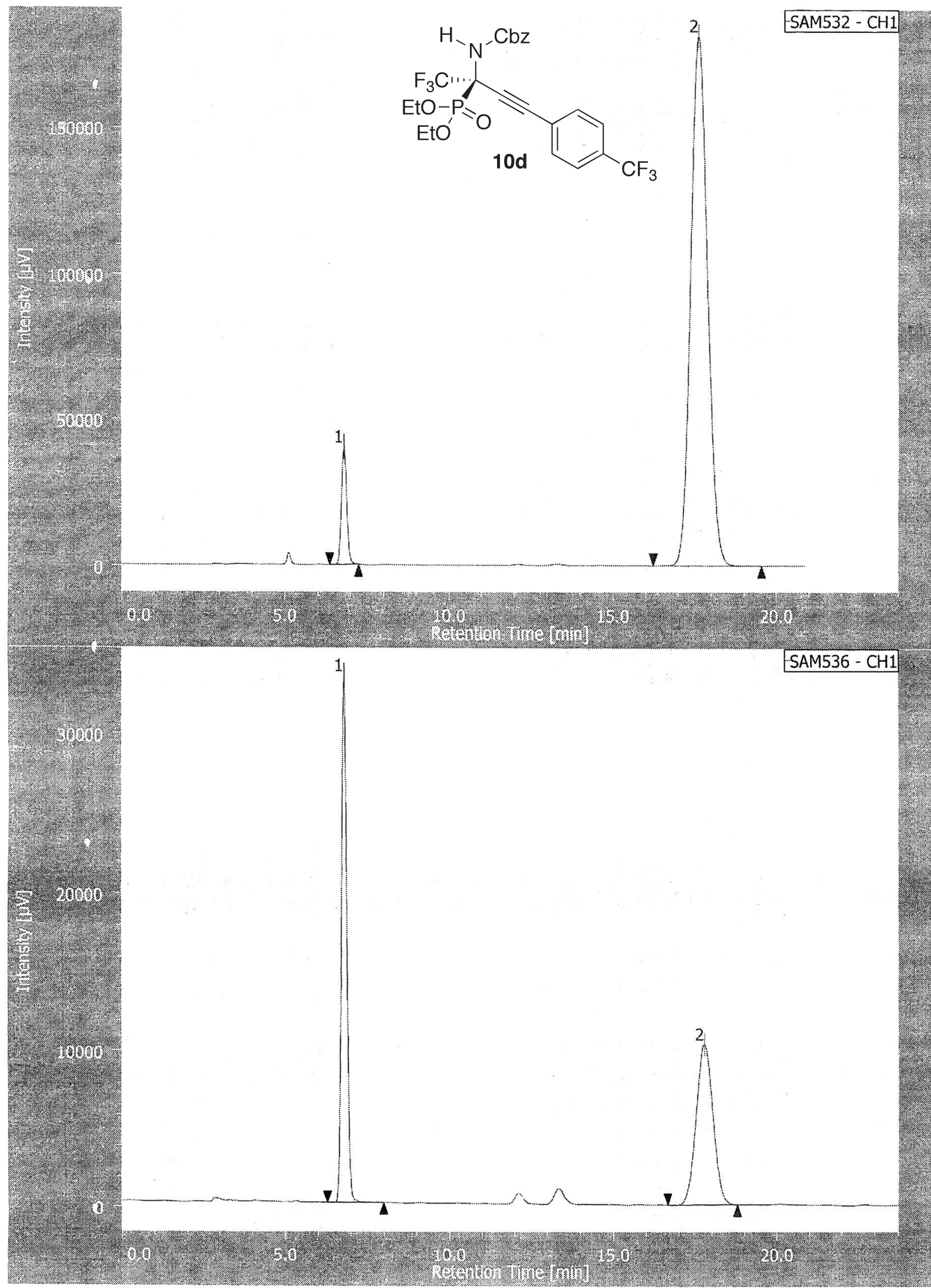

チャンネル情報+ピーク情報

クロマトグラム名

SAM532-CH1

サンプル名

チャンネル名

$\mathrm{CH} 1$

\begin{tabular}{|c|c|c|c|c|c|c|c|c|c|c|c|}
\hline ピーク名 & $\mathrm{CH}$ & $\operatorname{tR}[\mathrm{min}]$ & 面積 [ $\mu \mathrm{V} \cdot \mathrm{sec}]$ & 高さ $[\mu \mathrm{V}]$ & 面積\% & 高さ\% & 定量値 & NTP & 分離度 & シンメトリー係数 & \begin{tabular}{|l|} 
警告 \\
\end{tabular} \\
\hline Unknown & 1 & 6.792 & 454244 & 40499 & 6.567 & 18.256 & N/A & 8635 & 17.575 & 1.105 & \\
\hline 2 Unknown & 1 & 17.608 & 6462538 & 181341 & 93.433 & 81.744 & $N / A$ & 5593 & $\mathrm{~N} / \mathrm{A}$ & 1.098 & \\
\hline
\end{tabular}

クロマトグラム名

SAM536-CH1

サンプル名

チャンネル名

$\mathrm{CH} 1$

\begin{tabular}{|c|c|c|c|c|c|c|c|c|c|c|c|}
\hline ピーク名 & $\mathrm{CH}$ & $t R$ [min] & 面積 [ $\mu \mathrm{V} \cdot \mathrm{sec}]$ & 高さ $[\mu \mathrm{V}]$ & 面積\% & 高さ\% & 定量值 & NTP & 分離度 & シンメトリー係数 & \begin{tabular}{|l|} 
警告 \\
\end{tabular} \\
\hline Unknown & 1 & 6.792 & 374443 & 33419 & 50.042 & 76.435 & $\mathrm{~N} / \mathrm{A}$ & 8566 & 17.492 & 1.080 & \\
\hline \begin{tabular}{l|l|}
2 Unknown
\end{tabular} & 1 & 17.775 & 373819 & 10303 & 49.958 & 23.565 & N/A & 5422 & N/A & 1.021 & \\
\hline
\end{tabular}




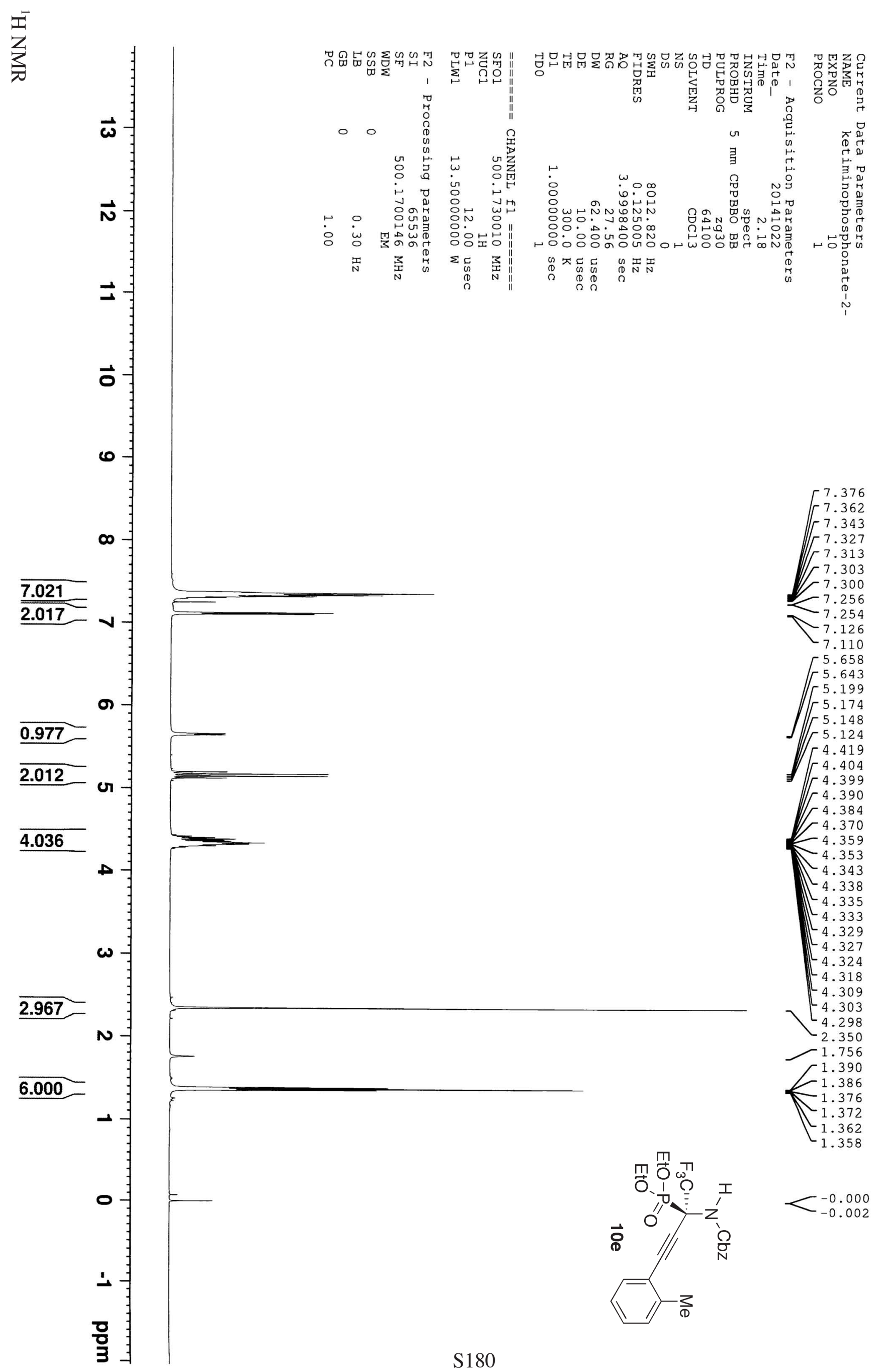




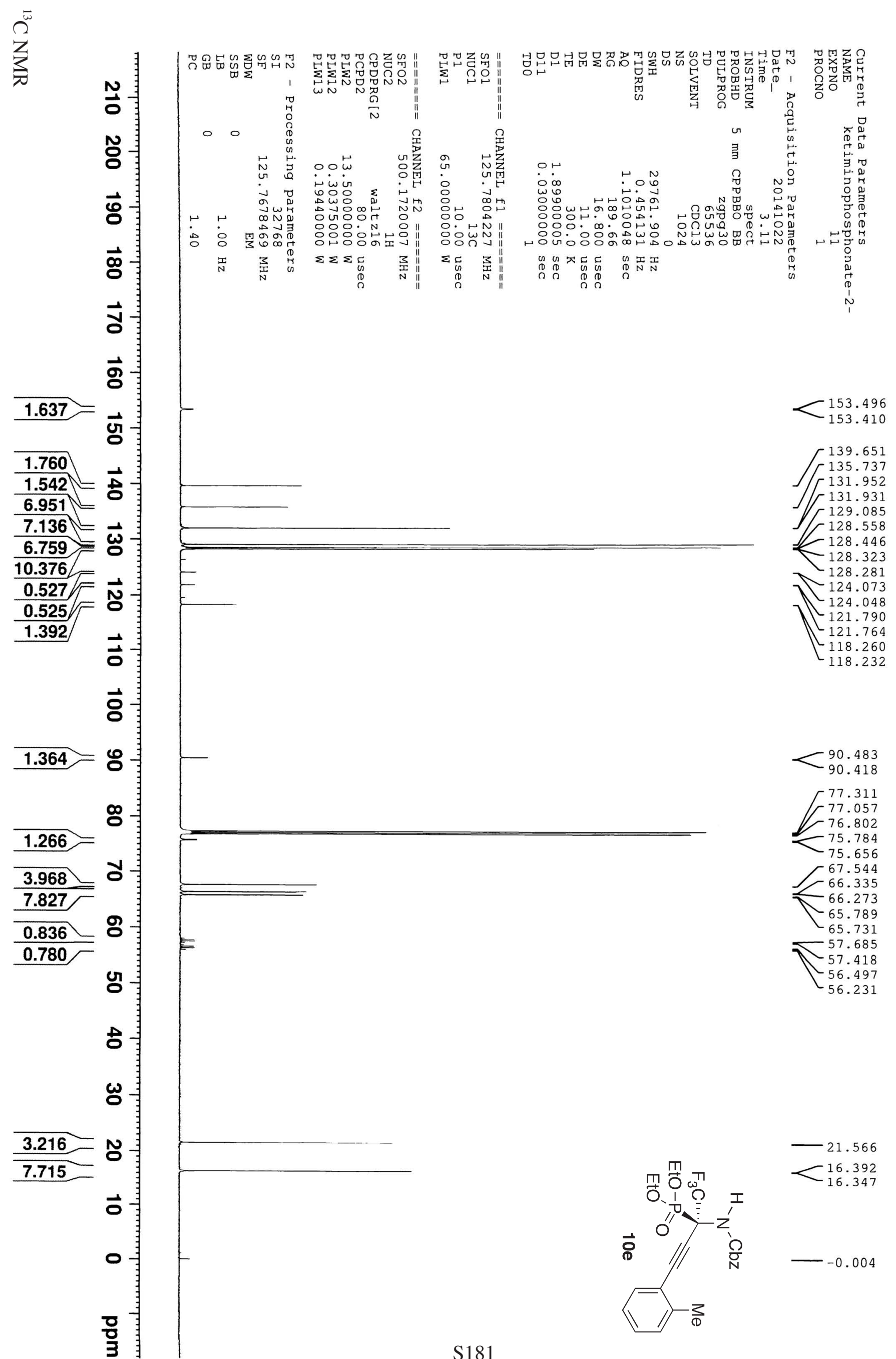




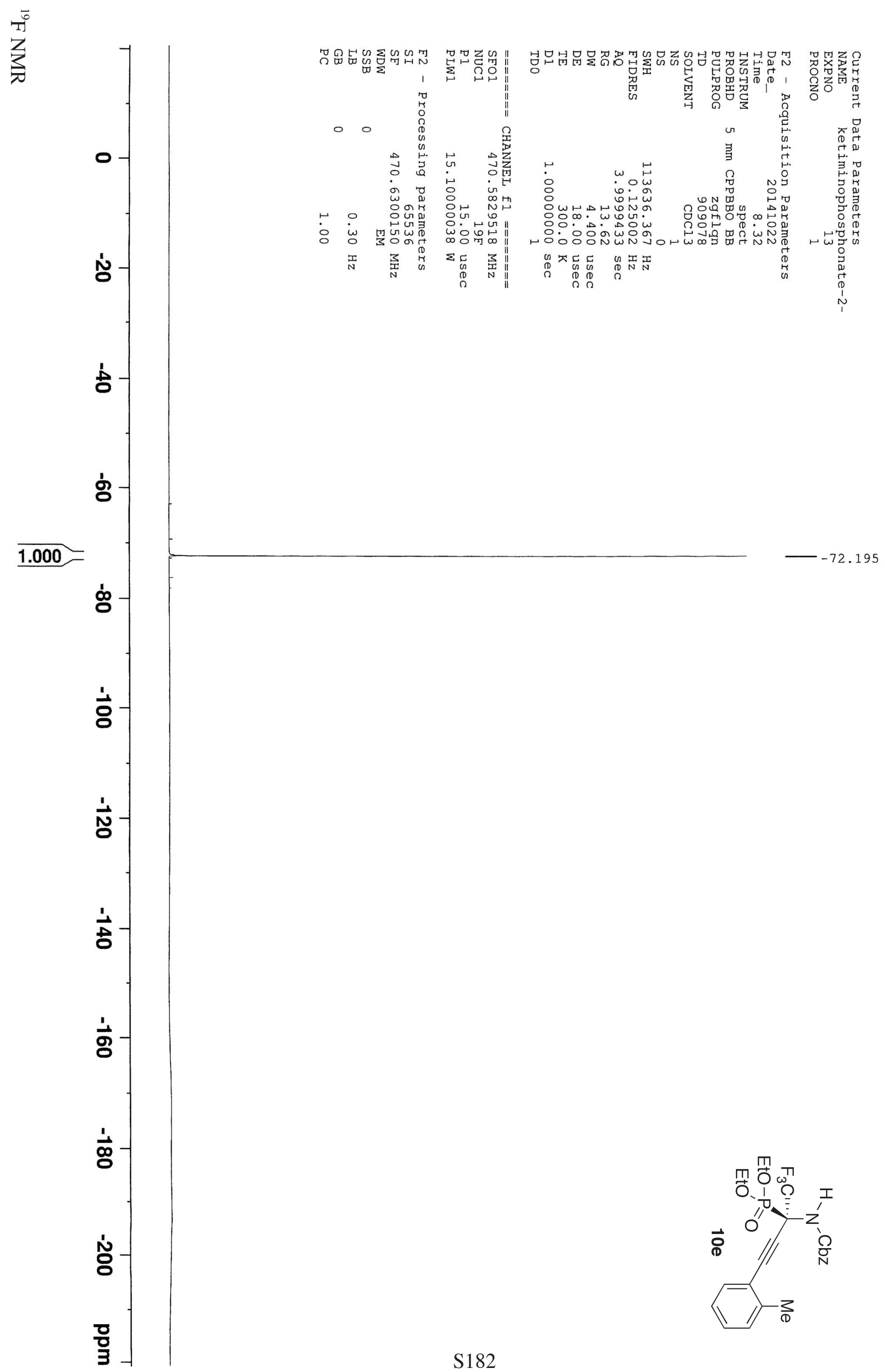




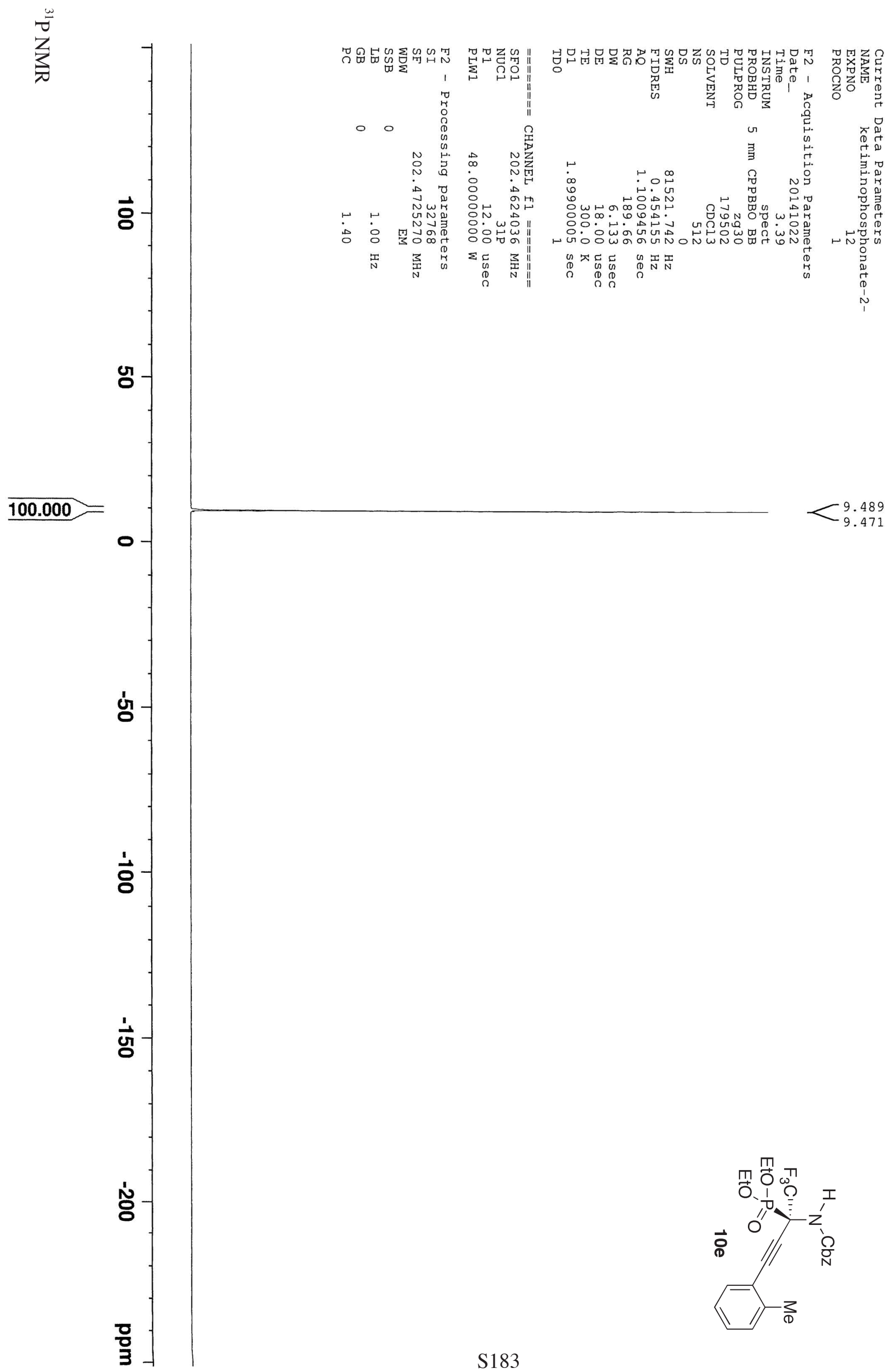




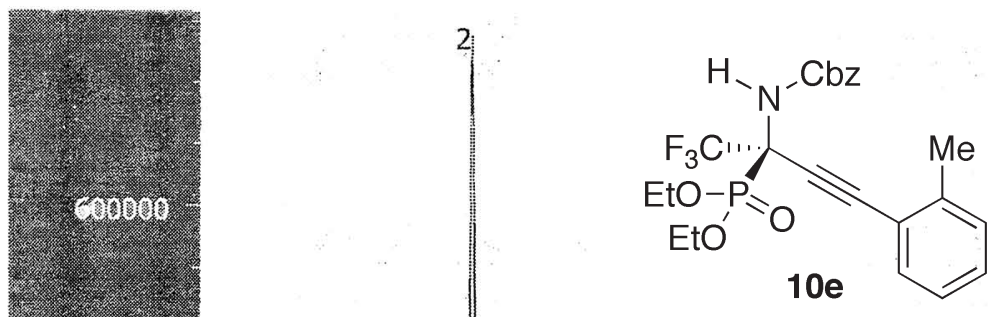

it invert

.

2.0.

-

2.)

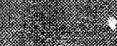

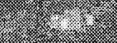
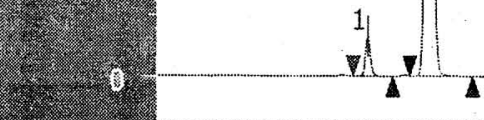

(4)

453

19outaty

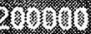
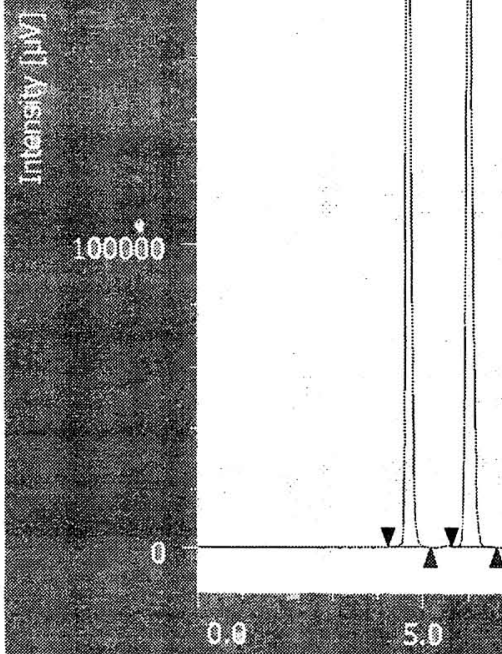

(1)

チャンネル情報十ピーク情報

クロマトグラム名

SAM $538-\mathrm{CH} 1$

サンプル名

チャンネル名

$\mathrm{CH} 1$

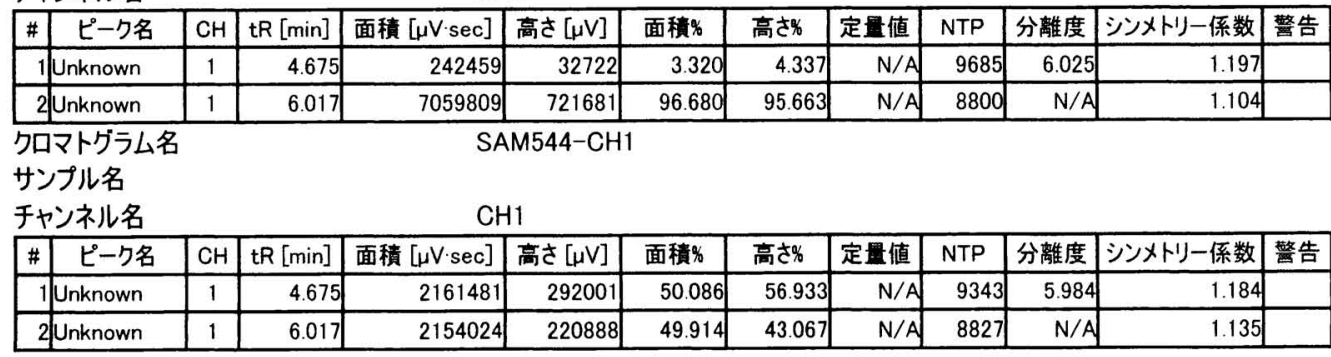




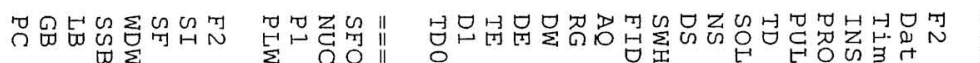

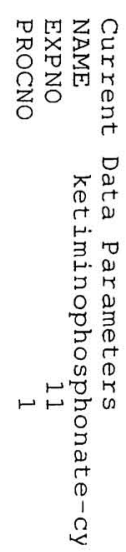

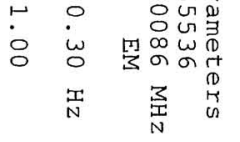
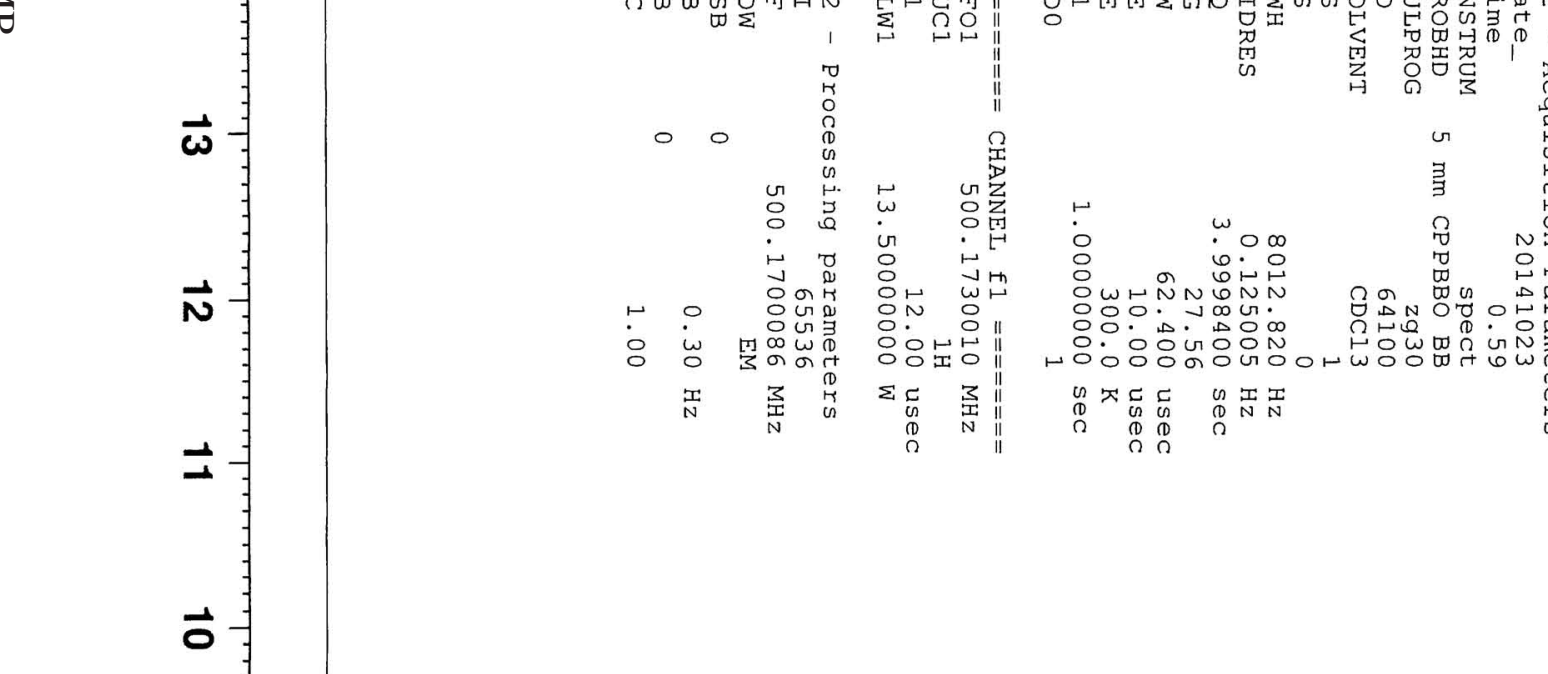

$\left[\begin{array}{l}7.369 \\ 7.361 \\ 7.343\end{array}\right.$

7.343

7.335

4.979

$\infty$

6

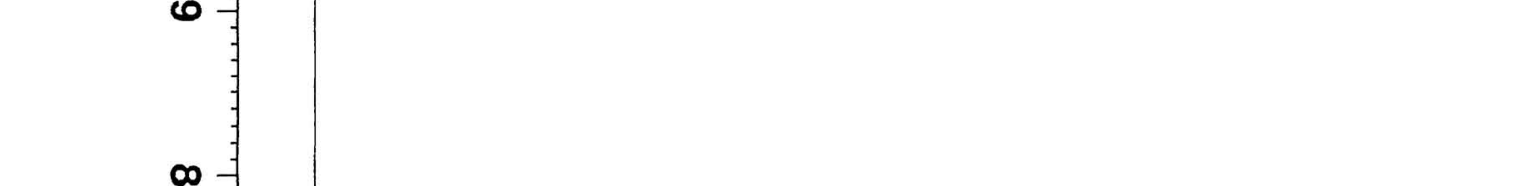

$-7.326$

$-7.319$

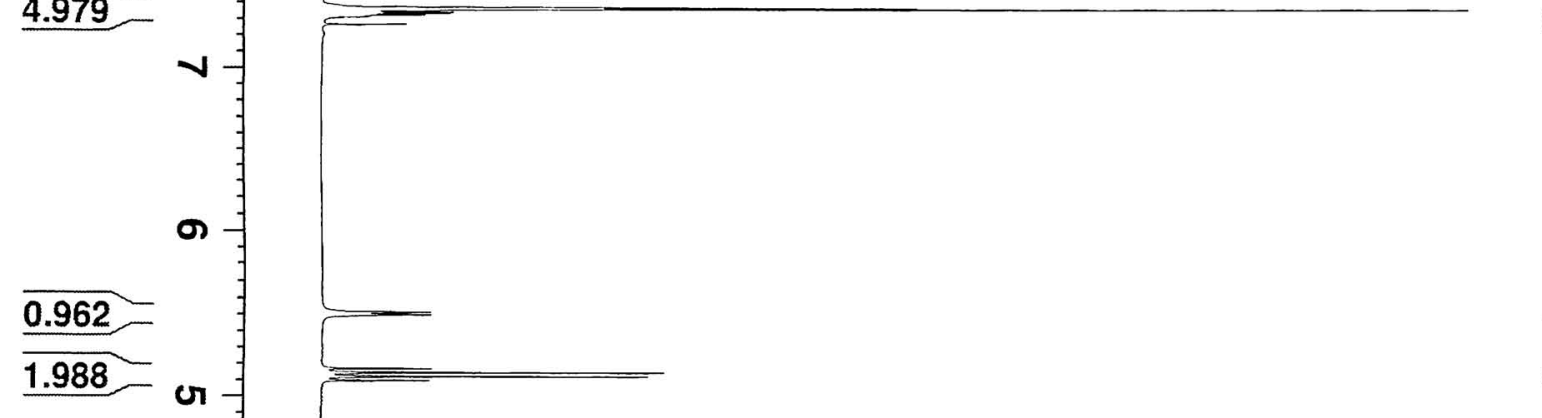

7.319
7.267

7.266

5.515

5.500

5.170

5.145

5.123

5.098

$\left[\begin{array}{r}4.374 \\ 4.360 \\ 4.354\end{array}\right.$

4.354
-3.355

4.345
4.340

F $\begin{aligned} & 4.345 \\ & 4.340 \\ & 4.325\end{aligned}$

4.325

$-4.313$

-4.310
-.302

4.299

4.295

4.289

4.279

4.275

4.263

4.248

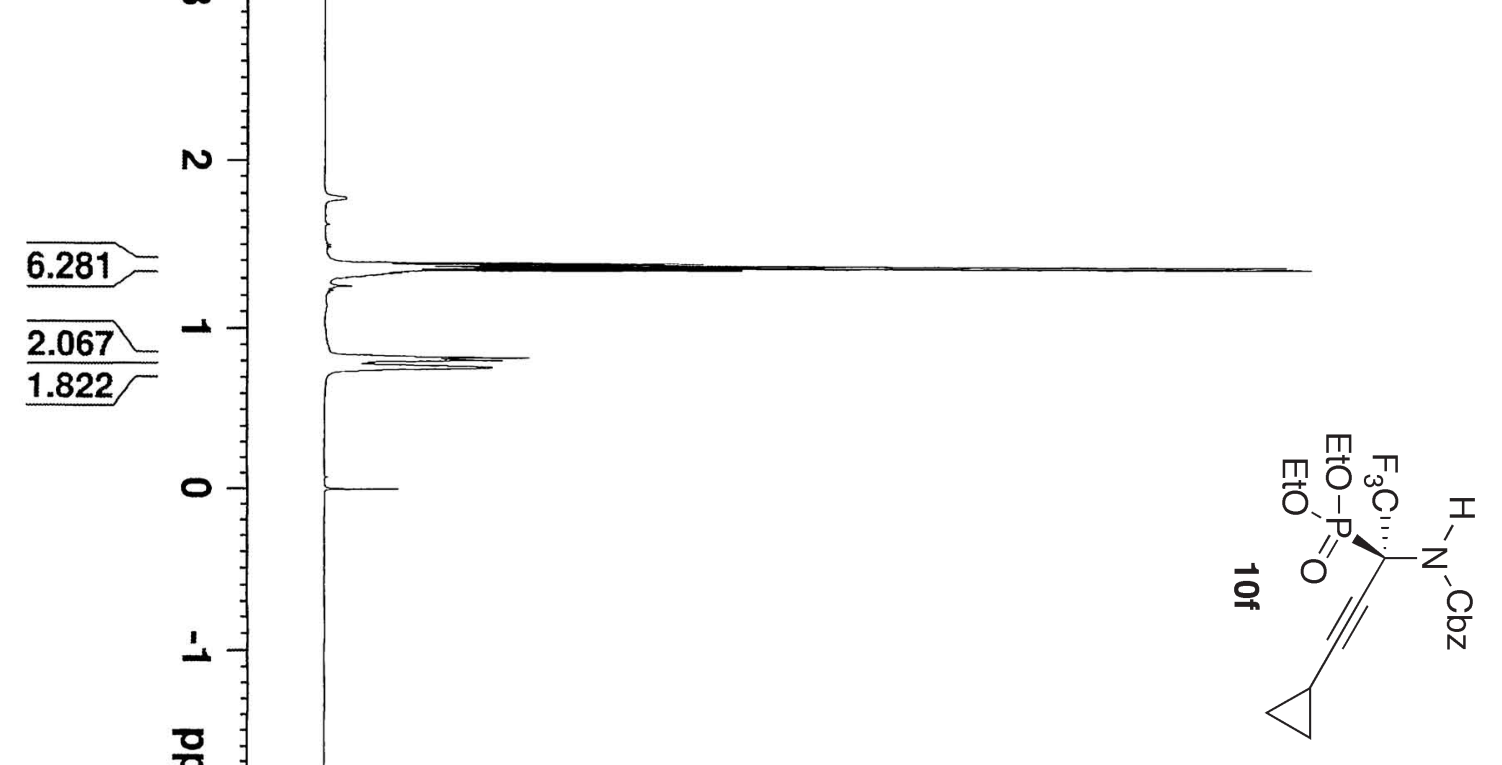

r 1.389

1.375

1.363

1.349

1.340

1.331
-1.323

1.315

0.839

0.824

$-0.817$

$\left[\begin{array}{l}0.808 \\ 0.802 \\ 0.802\end{array}\right.$

$7[0.791$

- 0.767

$\left[\begin{array}{l}0.762 \\ -0.001\end{array}\right.$

$-0.000$ 


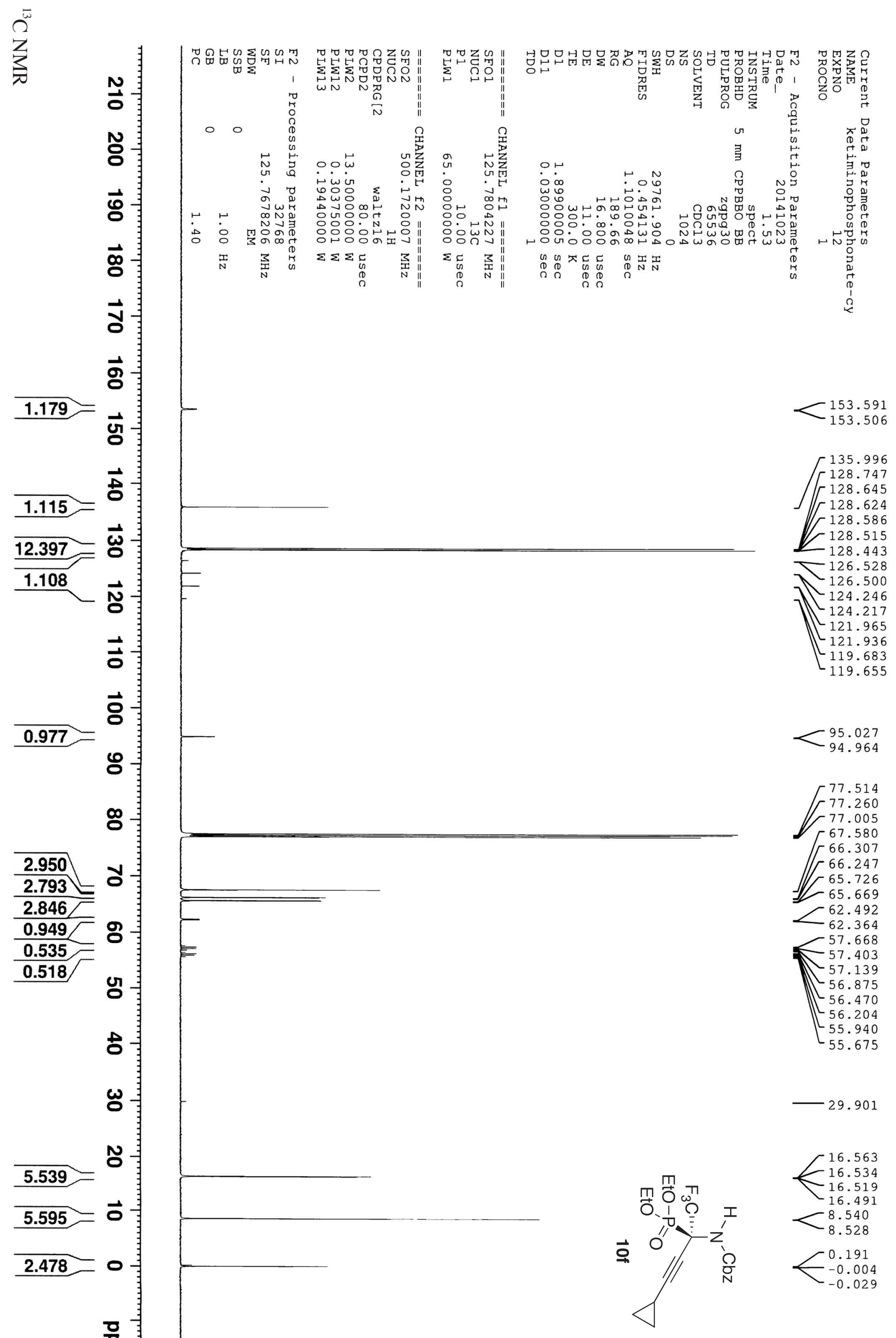




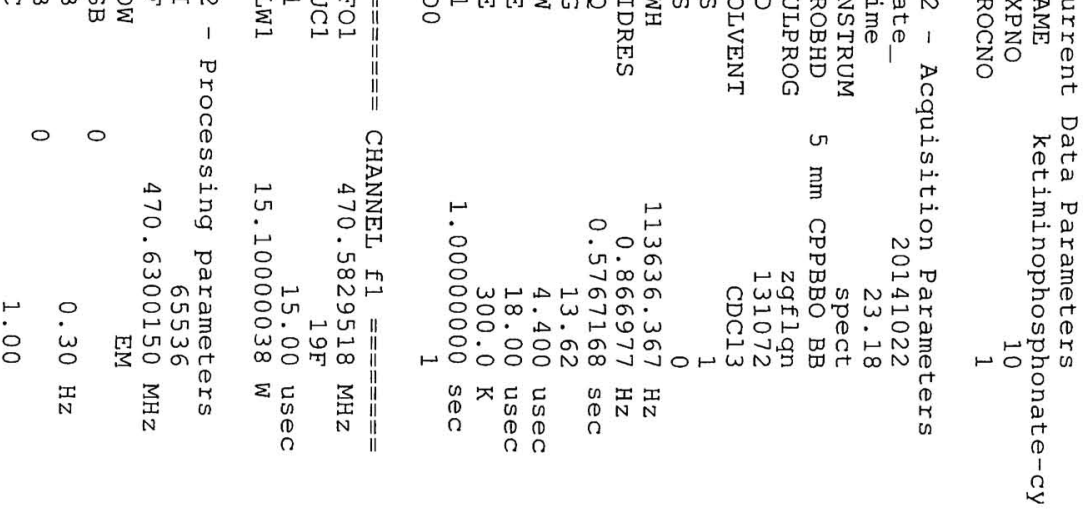




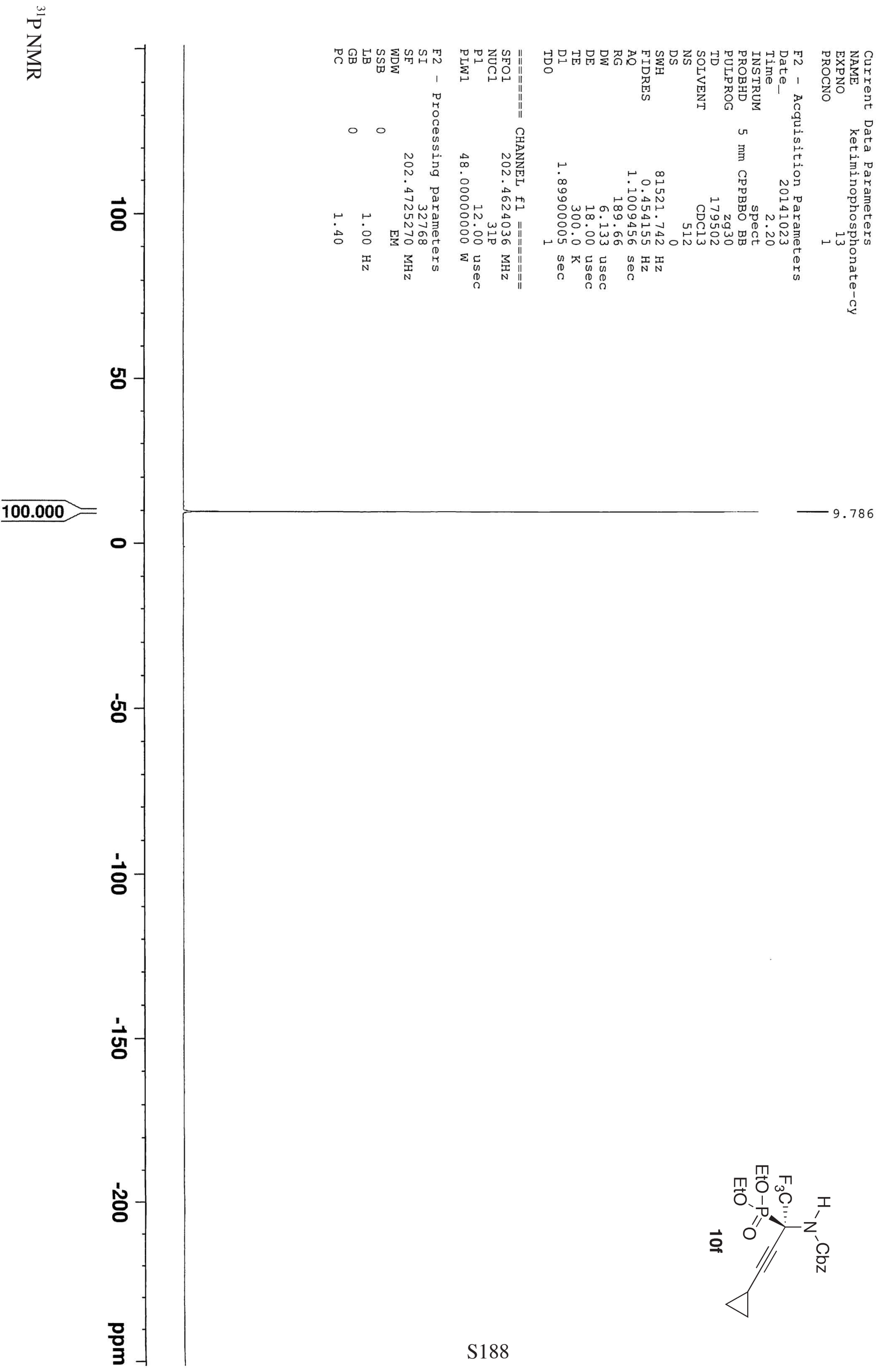




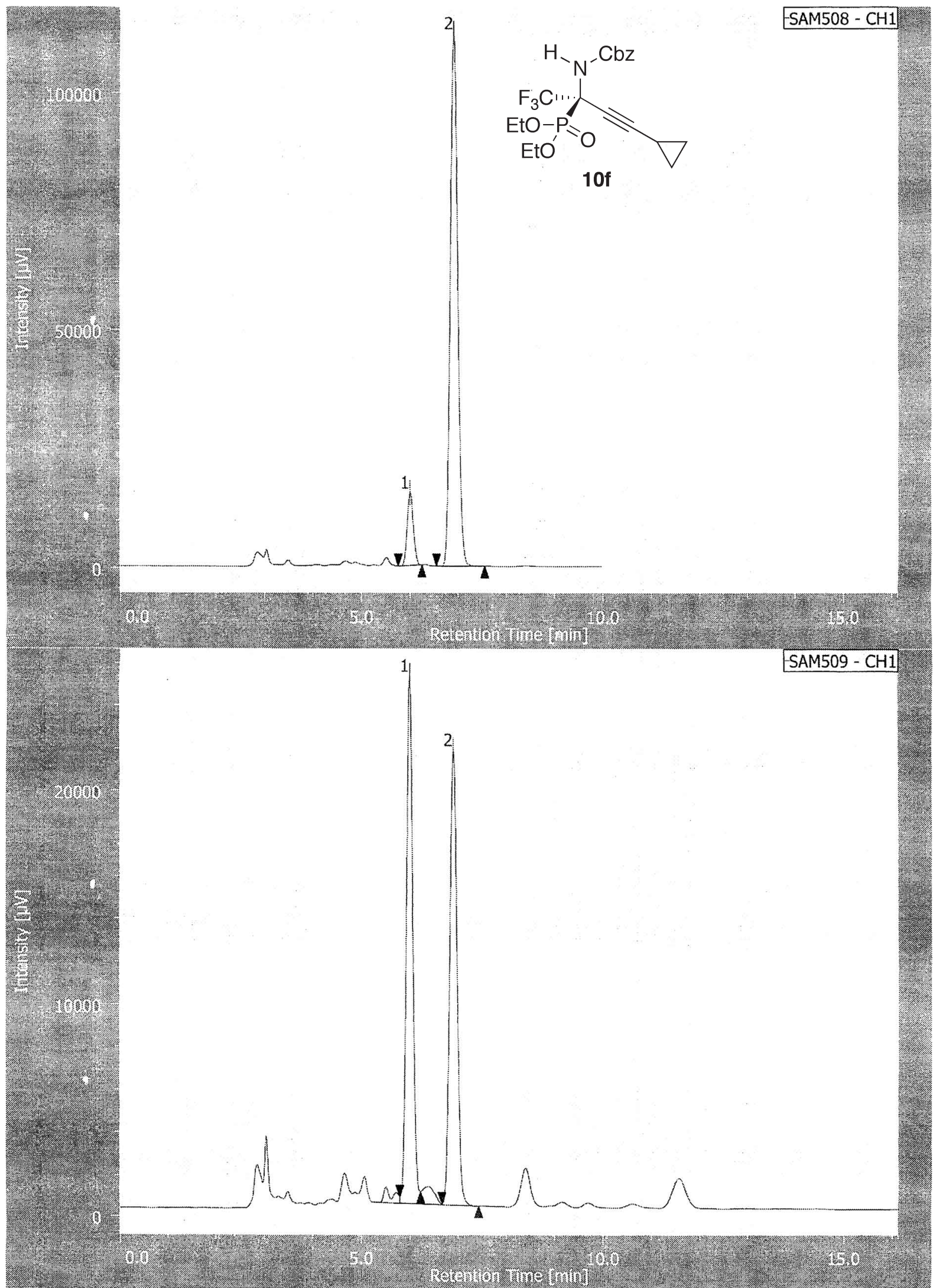

チャンネル情報+ピーク情報

クロマトグラム名

サンプル名

SAM $508-\mathrm{CH} 1$

チャンネル名

$\mathrm{CH} 1$

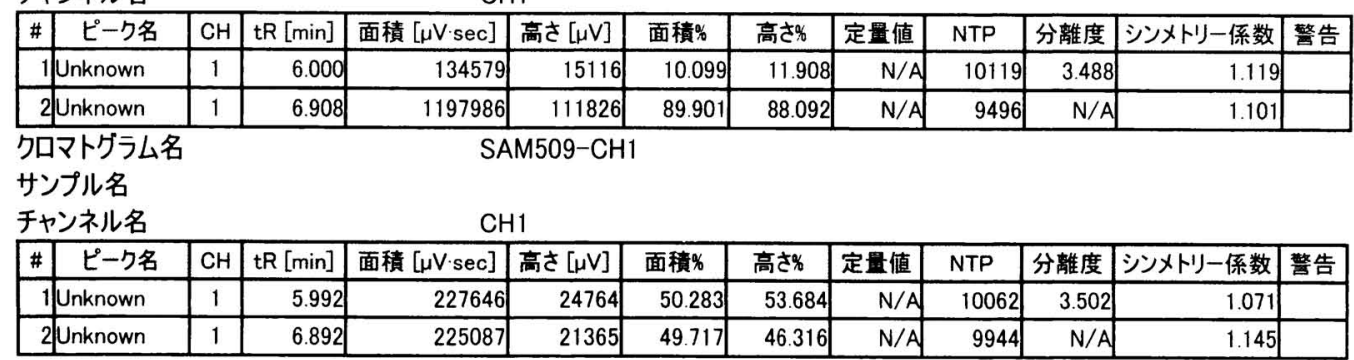




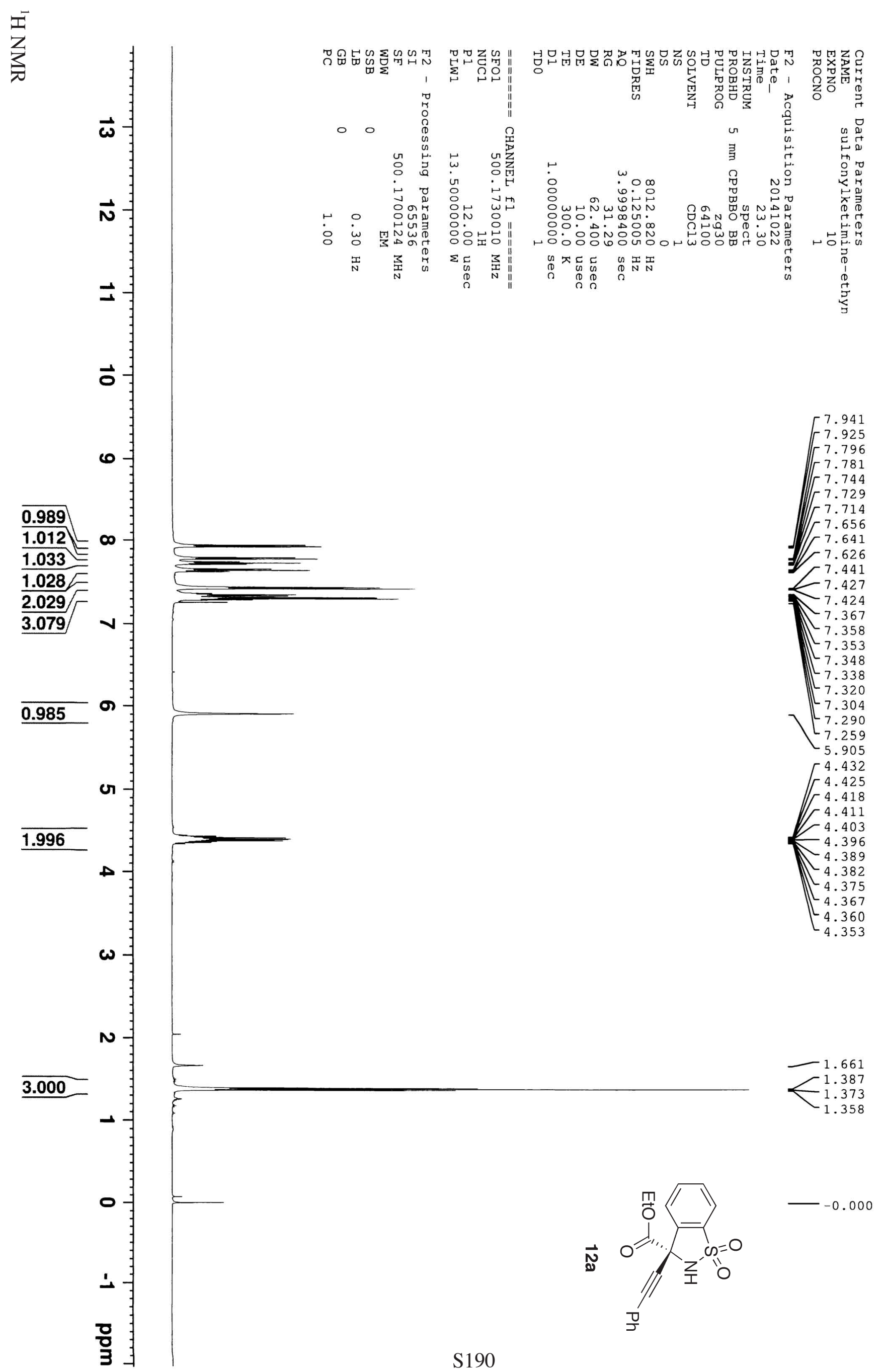




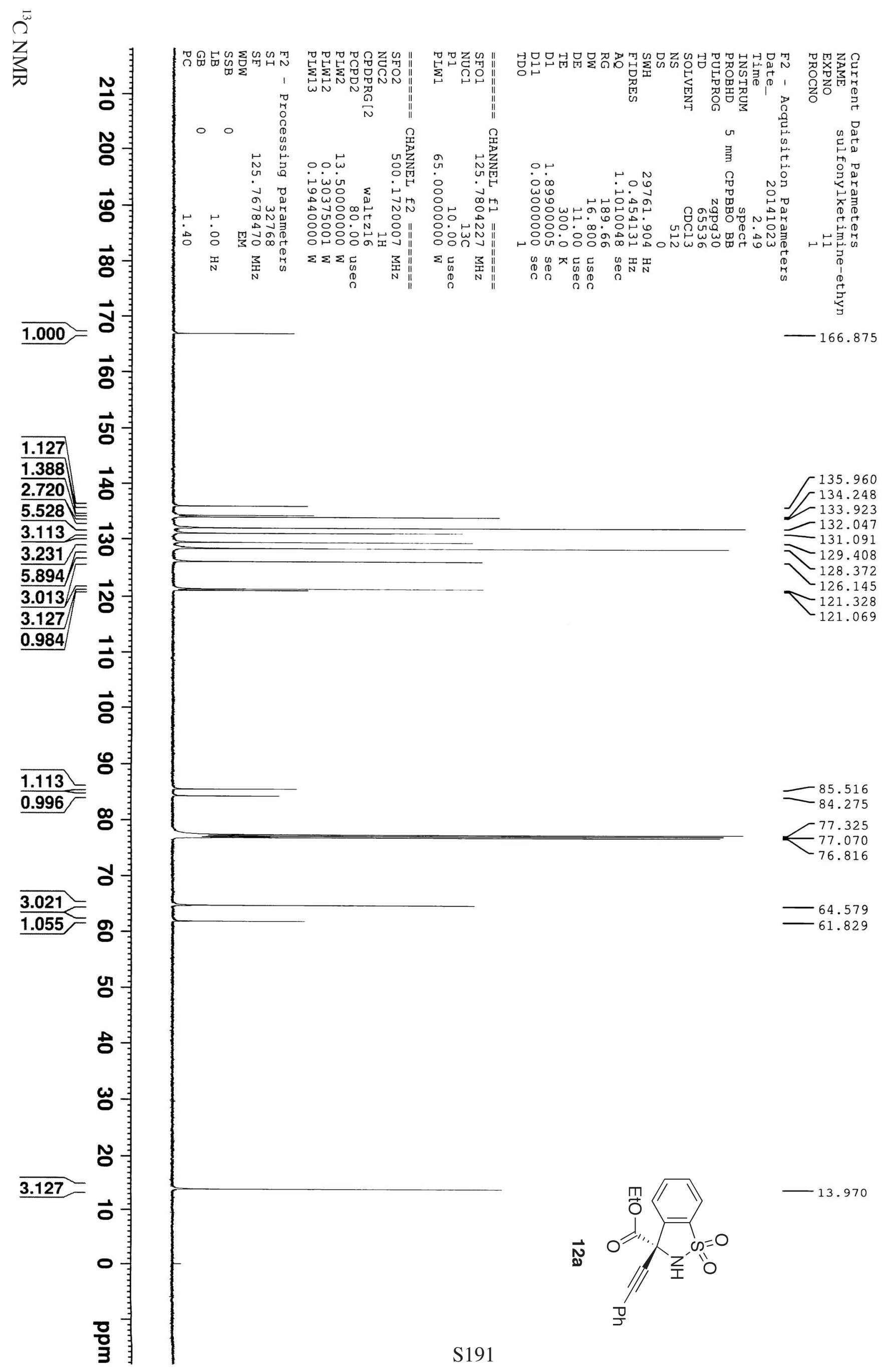




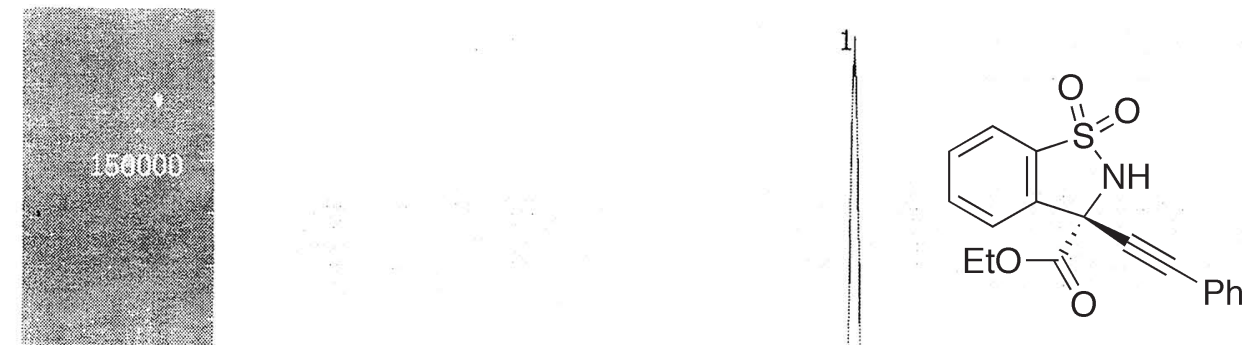

$12 a$

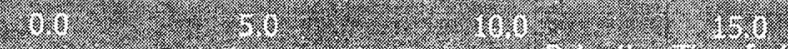

; 2 .

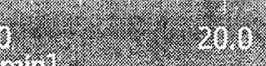

26.
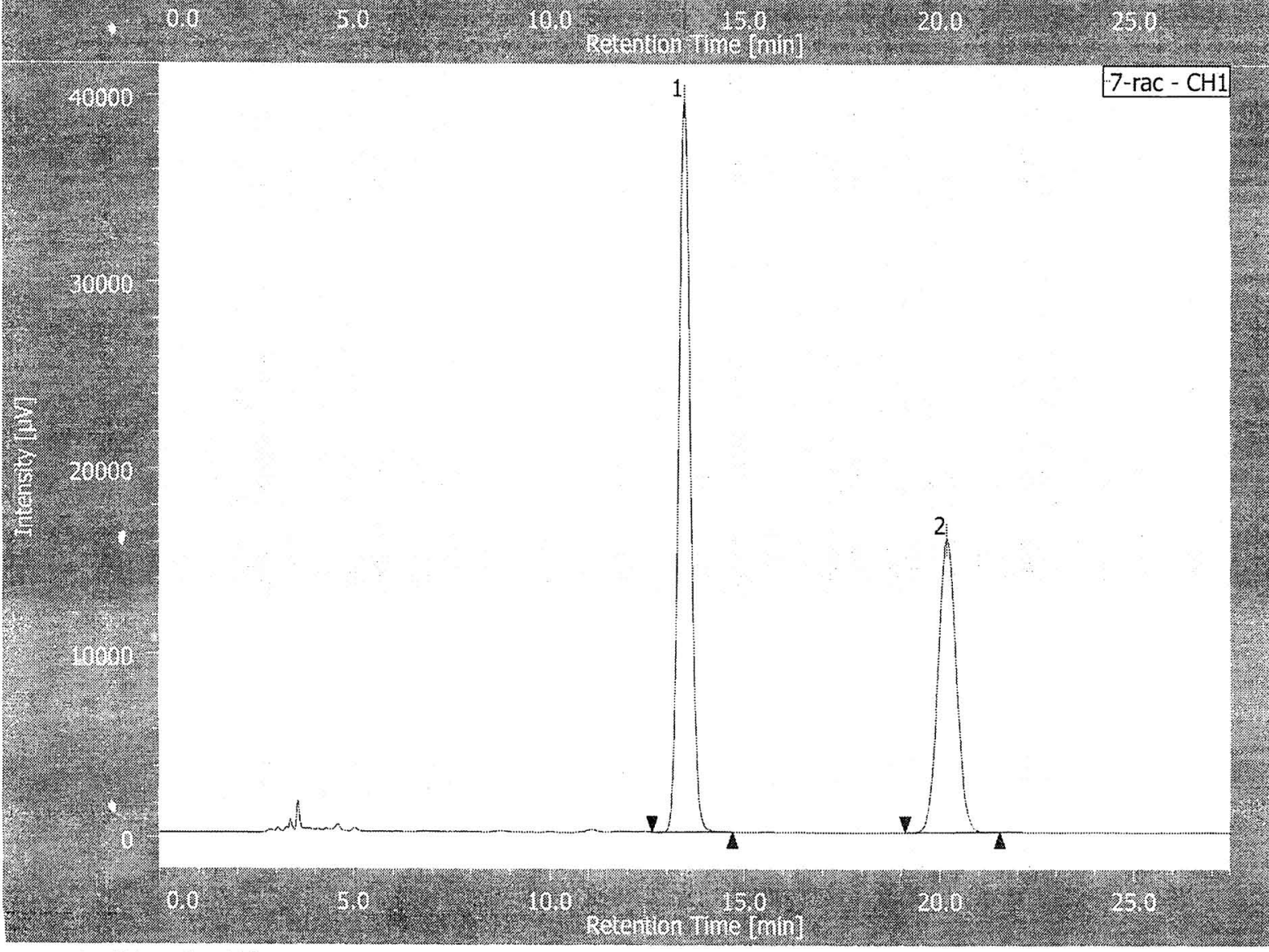

チャンネル情報+ピーク情報

クロマトグラム名

7-CH1

サンプル名

チャンネル名

$\mathrm{CH} 1$

\begin{tabular}{|c|c|c|c|c|c|c|c|c|c|c|c|}
\hline ピーク名 & $\mathrm{CH}$ & $t R$ [min] & 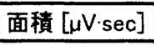 & 高さ [ $\mu \mathrm{V}]$ & 面積\% & 高さ\% & 定量値 & NTP & 分離度 & シンメトリー係数 & 警告 \\
\hline Unknown & 1 & 13.333 & 3644450 & 170322 & 92.286 & 94.862 & $\mathrm{~N} / \mathrm{A}$ & 9175 & 9.290 & 1.111 & \\
\hline 2 Unknown & 1 & 19.942 & 304633 & 9225 & 7.714 & 5.138 & $\mathrm{~N} / \mathrm{A}$ & 8412 & $\mathrm{~N} / \mathrm{A}$ & 1.066 & \\
\hline
\end{tabular}

クロマトグラム名 7-rac- $\mathrm{CH} 1$

サンプル名

チャンネル名

$\mathrm{CH} 1$

\begin{tabular}{|c|c|c|c|c|c|c|c|c|c|c|c|}
\hline ピーク名 & $\mathrm{CH}$ & $\operatorname{tR}$ [min] & 面積 $[\mu \mathrm{V} \cdot \mathrm{sec}]$ & 高さ $[\mu \mathrm{V}]$ & 面積。。 & 高さ\% & 定贉値 & NTP & 分離度 & シンメトリー保数 & 警告 \\
\hline 1 Unknown & 1 & 13.467 & 834722 & 39344 & 61.384 & 71.243 & N/A & 9275 & 9.367 & 1.076 & \\
\hline 2 Unknown & 1 & 20.167 & 525111 & 15881 & 38.616 & 28.757 & N/A & 8498 & $N / A$ & 1.049 & \\
\hline
\end{tabular}




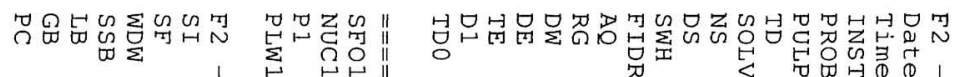

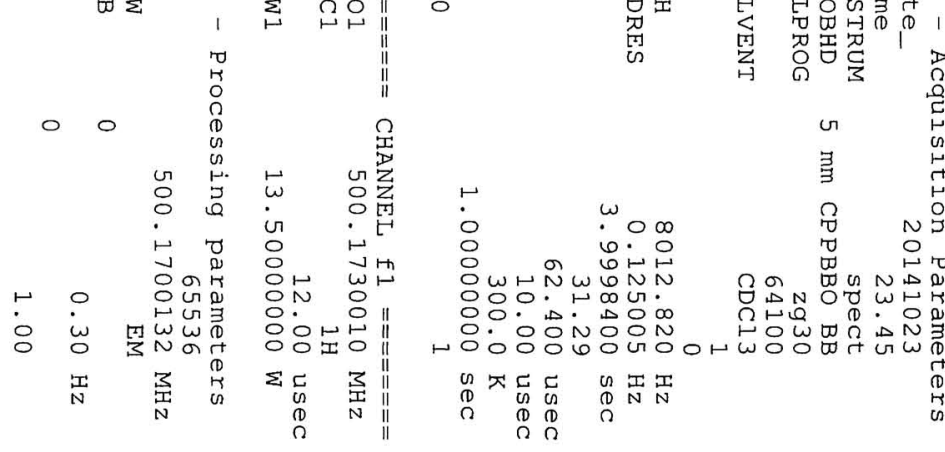

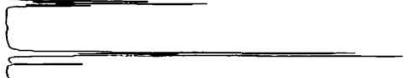

$-7.705$

$-7.647$

7.631

1.957 F

1.963

$\overline{0.974} \sigma$

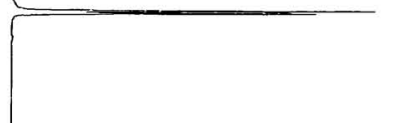

-7. 73

7.314

$\left[\begin{array}{l}7.314 \\ 7.258\end{array}\right.$

7.117

7.101

5.879
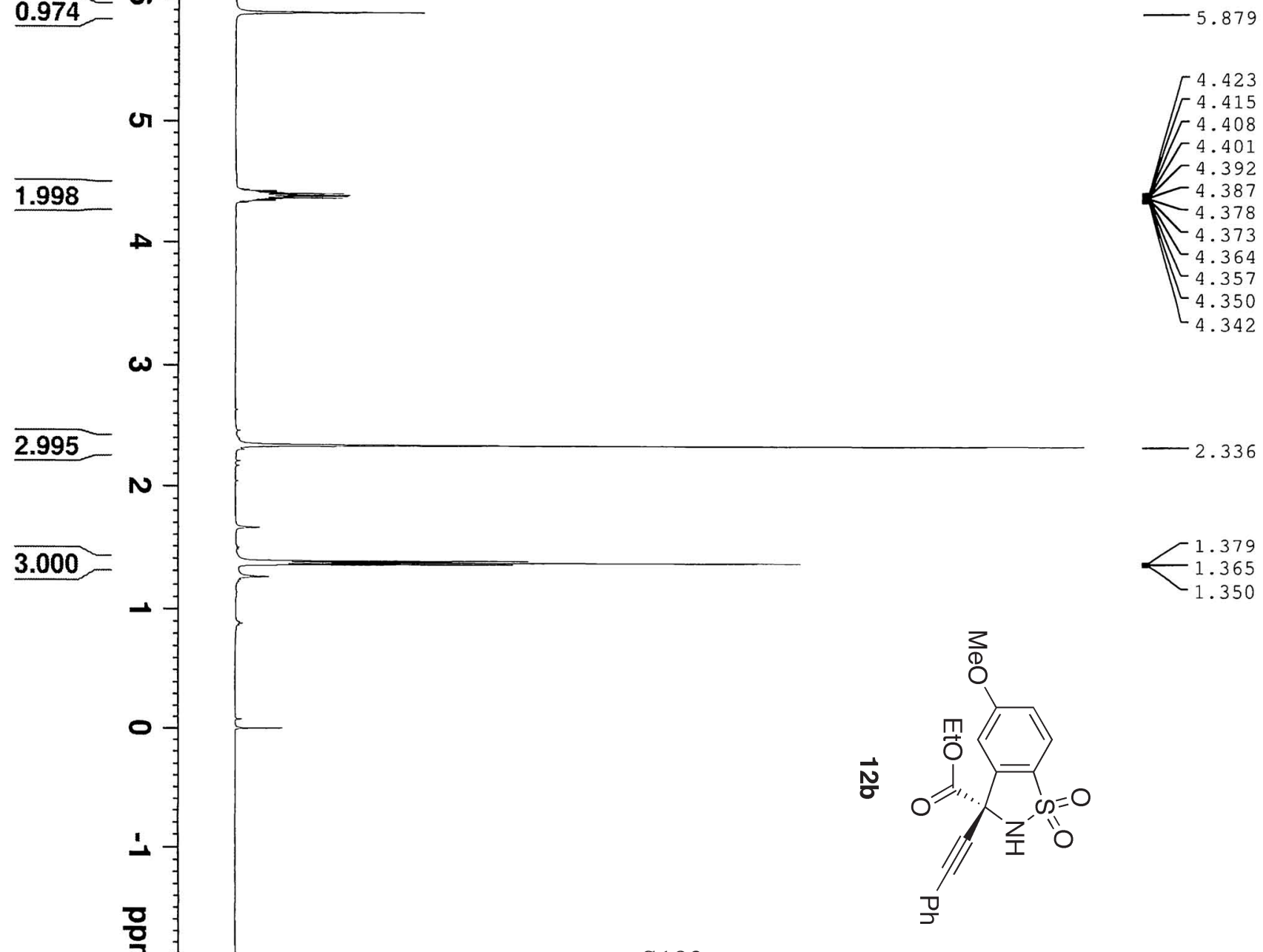


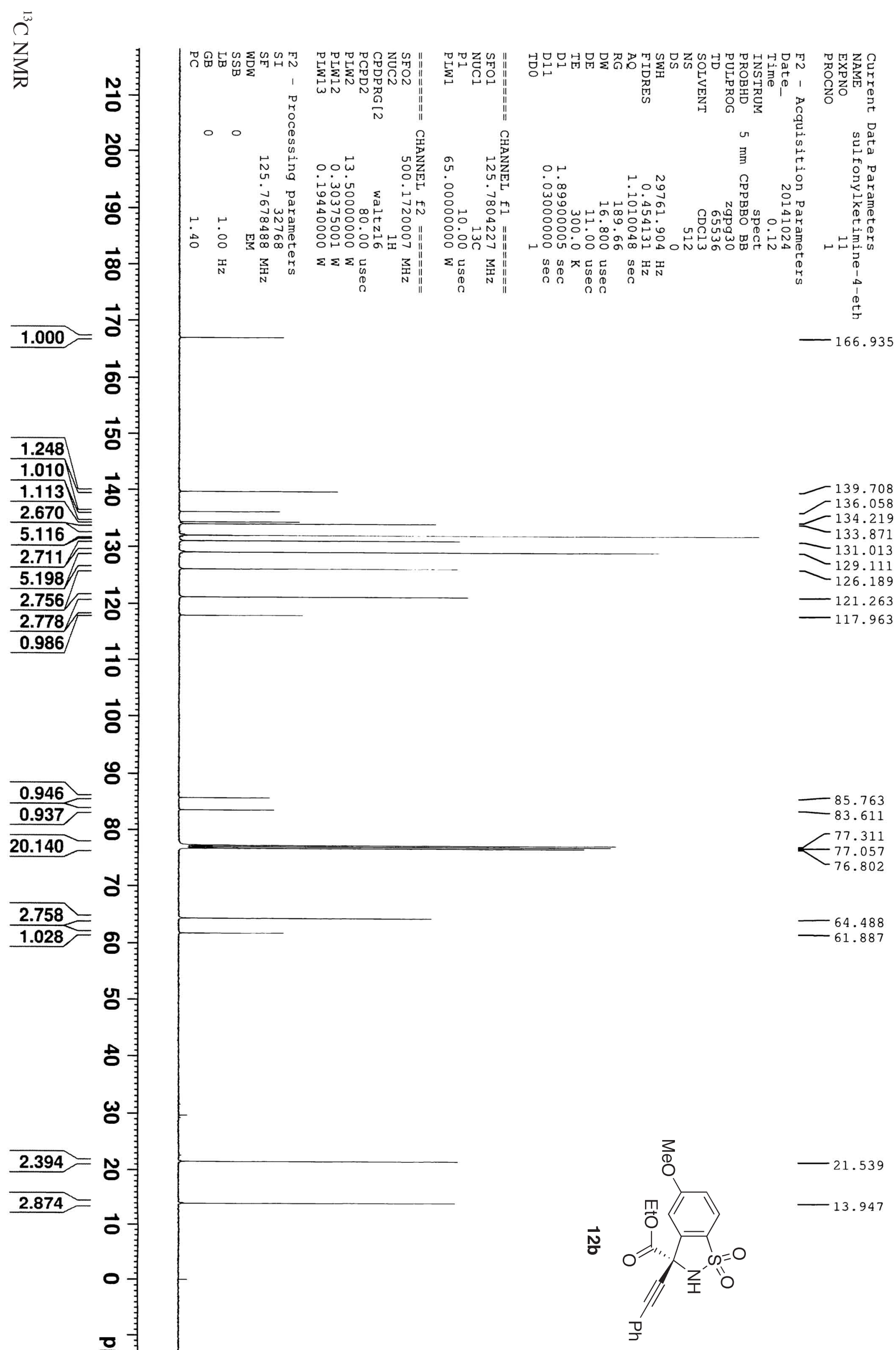




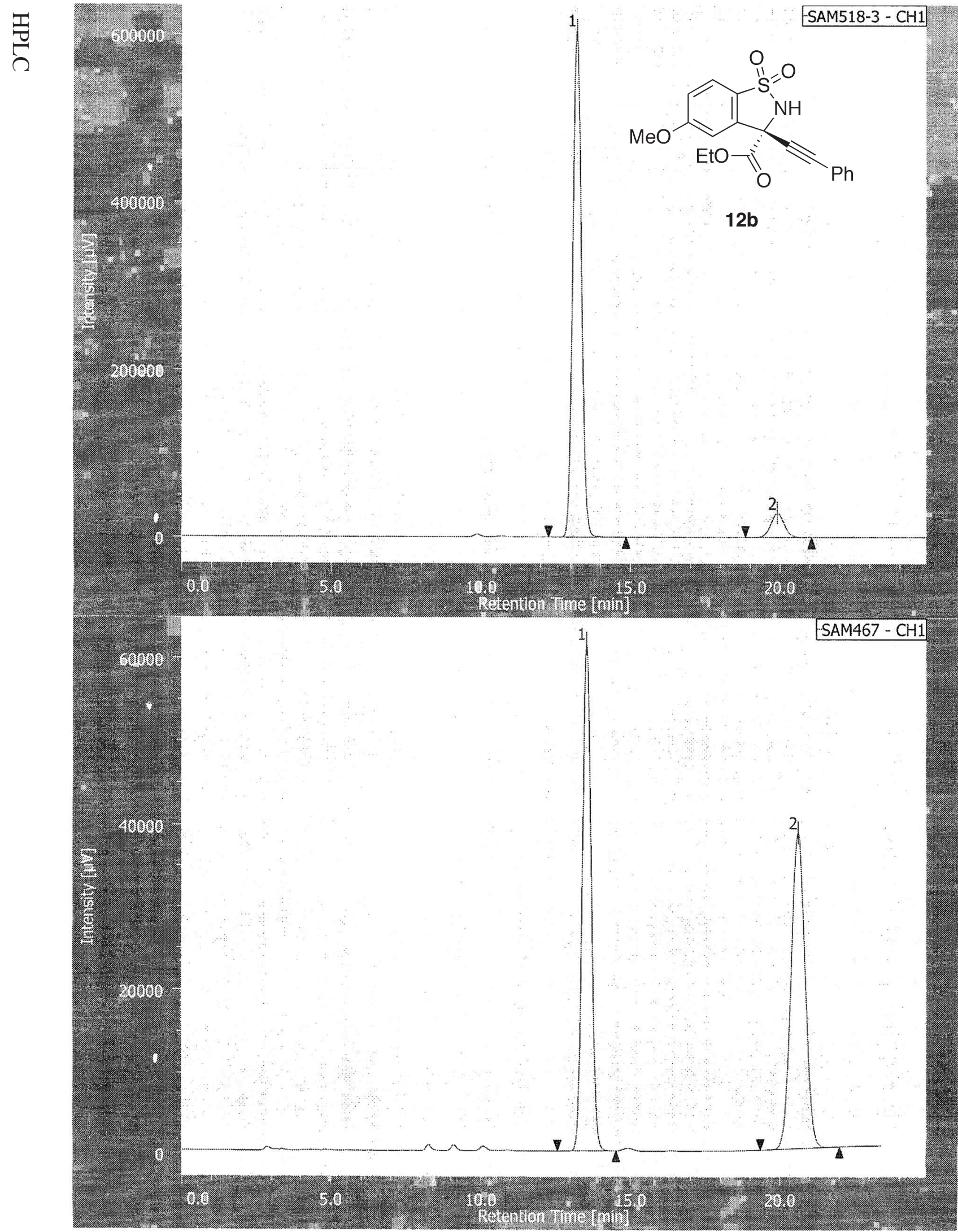

チャンネル情報+ピーク情報

クロマトグラム名

SAM518-3-CH1

サンプル名

チャンネル名

$\mathrm{CH} 1$

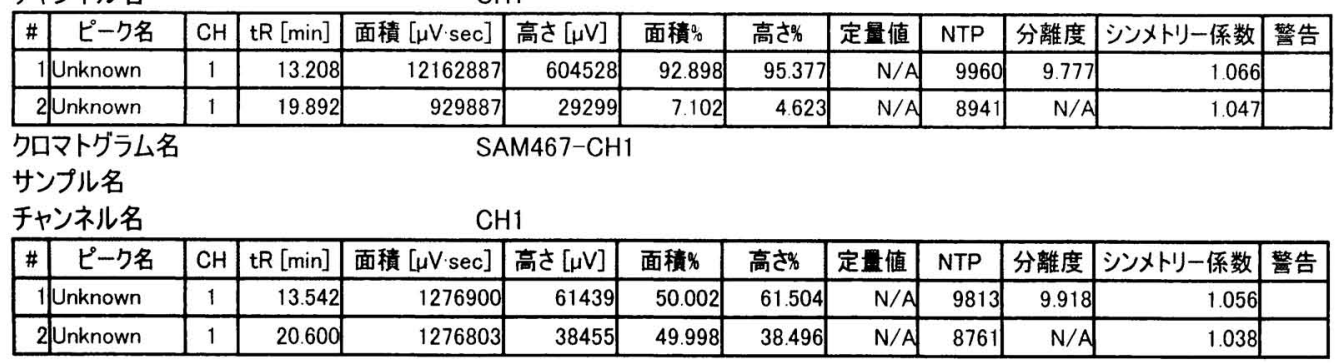




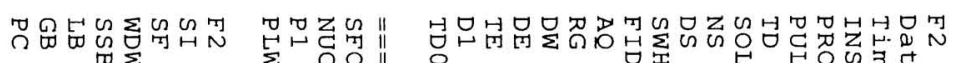
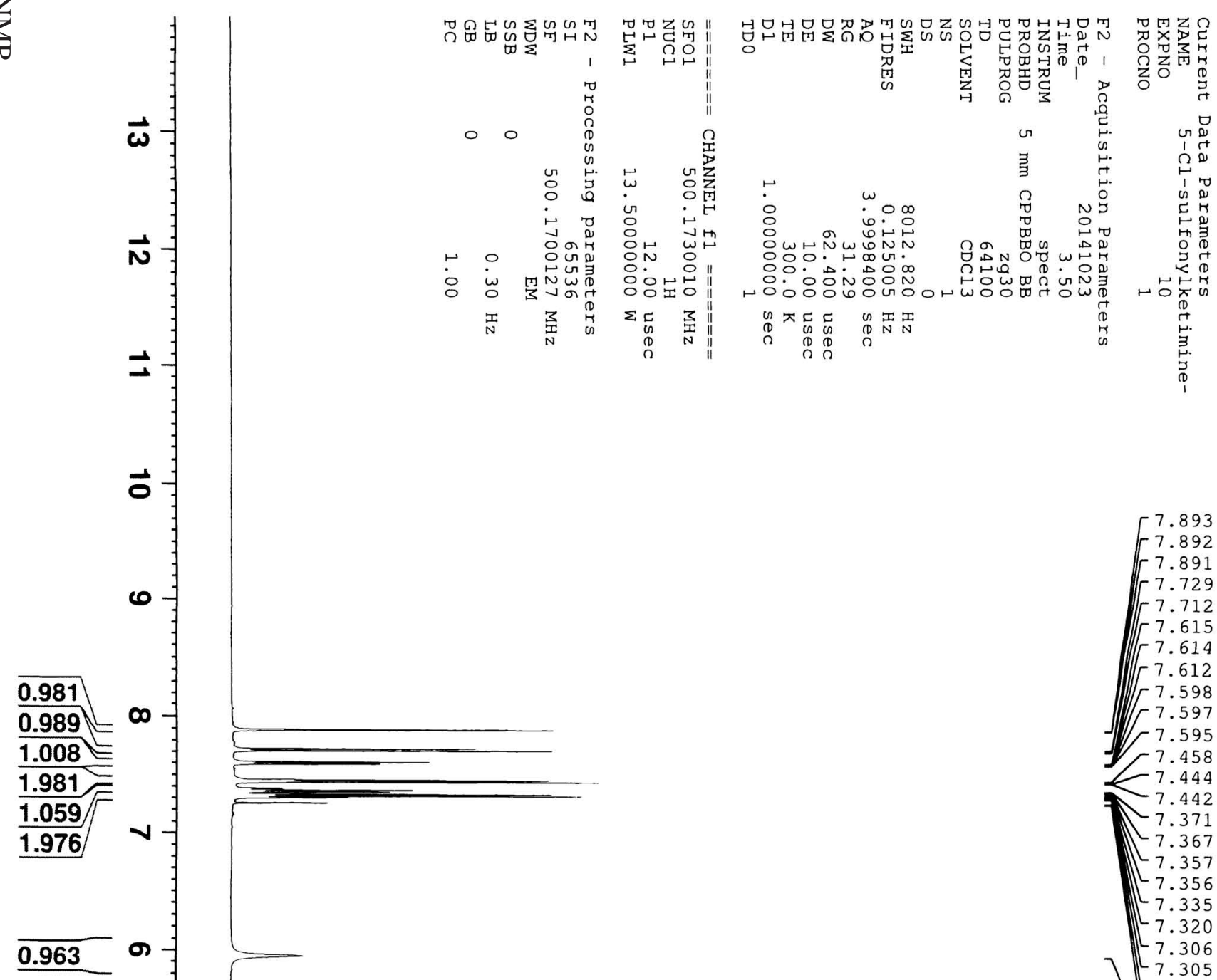

$\overrightarrow{0}$

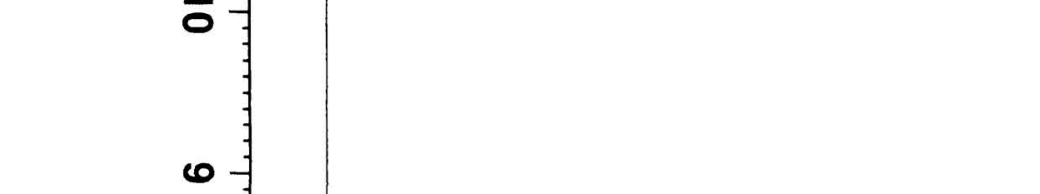

7.893

7.892

7.891

7.729

7.712

7.615

7.614

F.612

$r^{7.598}$

7.597

7.595

7.458

7.444

7.442

7.371
7.367
7.357

7.357

7.356

7.335

7.320

7.306

7.305

$[7.259$

7.258

5.954

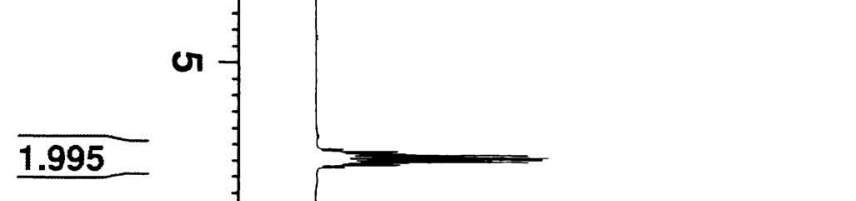

4.461

$r^{4.453}$

4.446

$-4.439$

$-4.425$

4.413

4.400

4.393

4.385

4.378

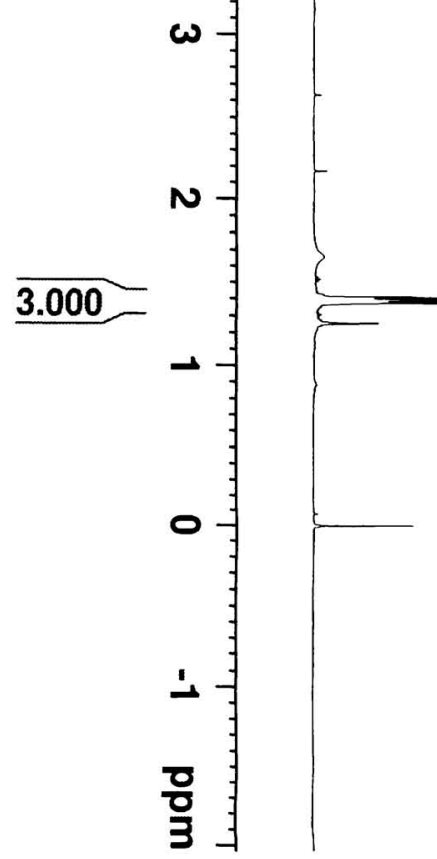

1.394

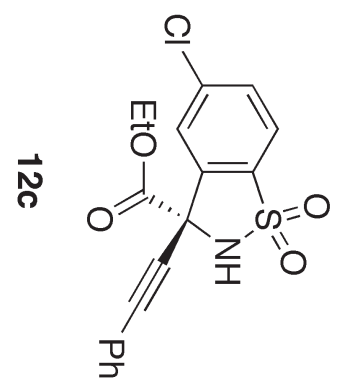




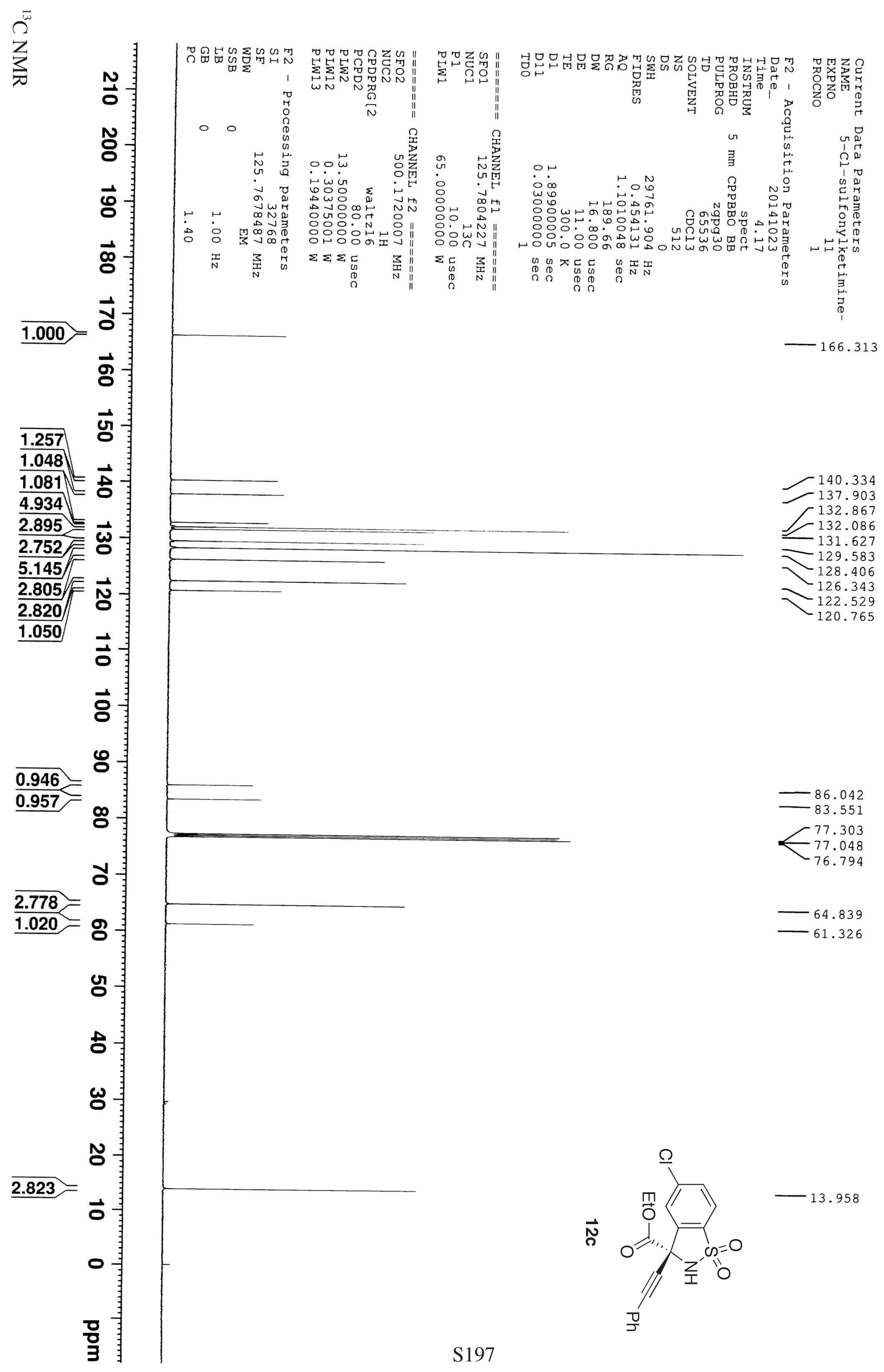




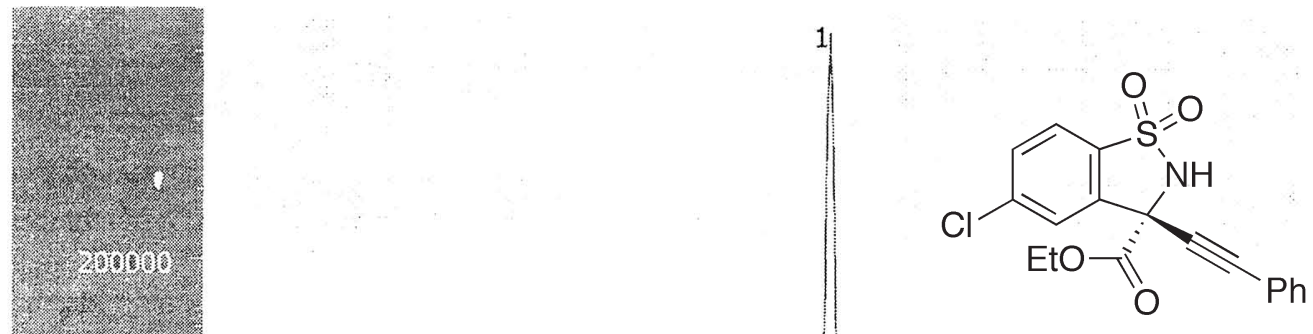

12c

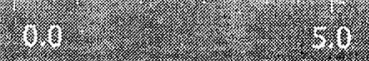

, 1

nit 6

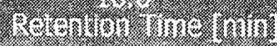

t.t. 6

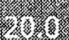
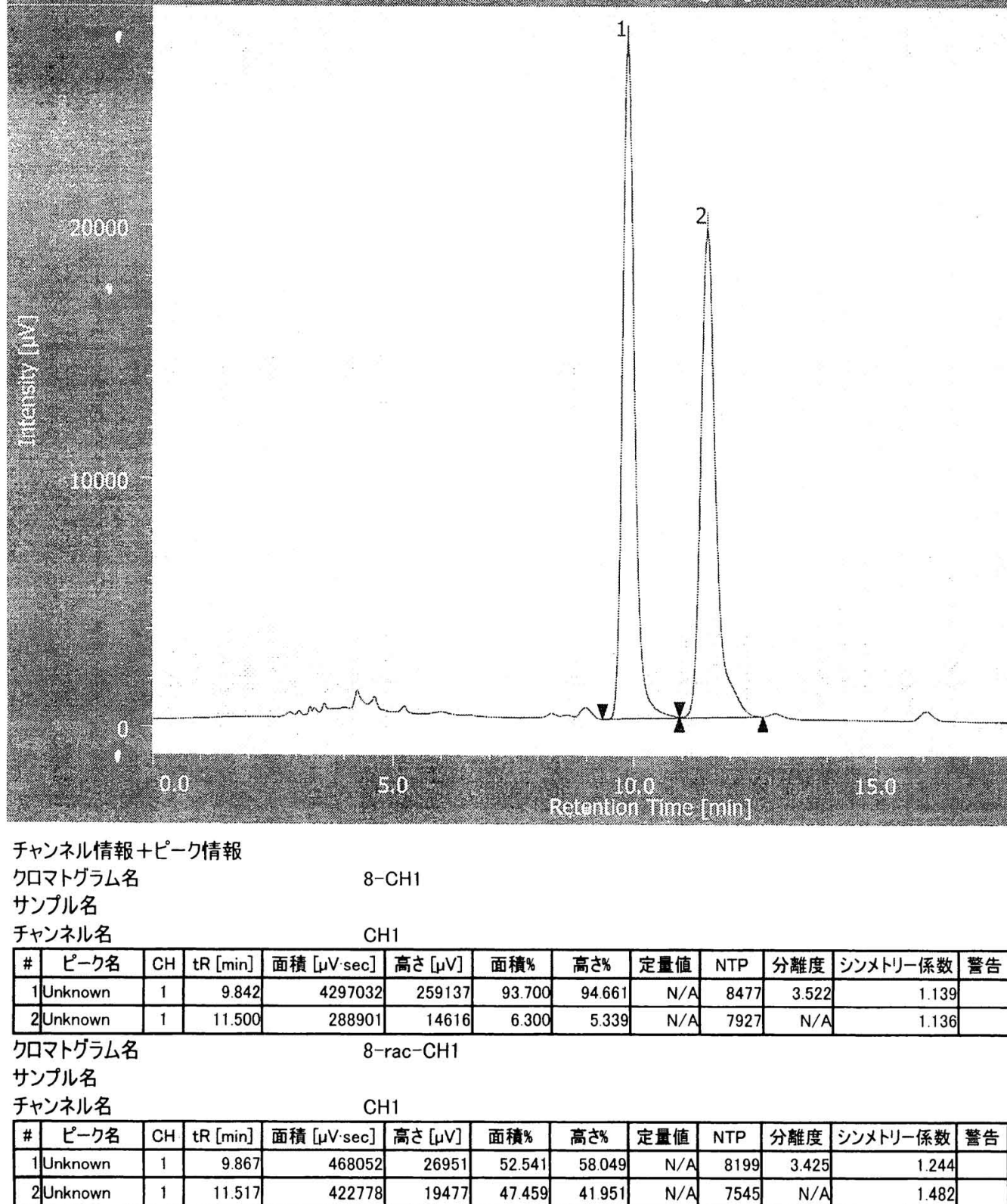

クロマトグラム名

サンプル名

チャンネル名

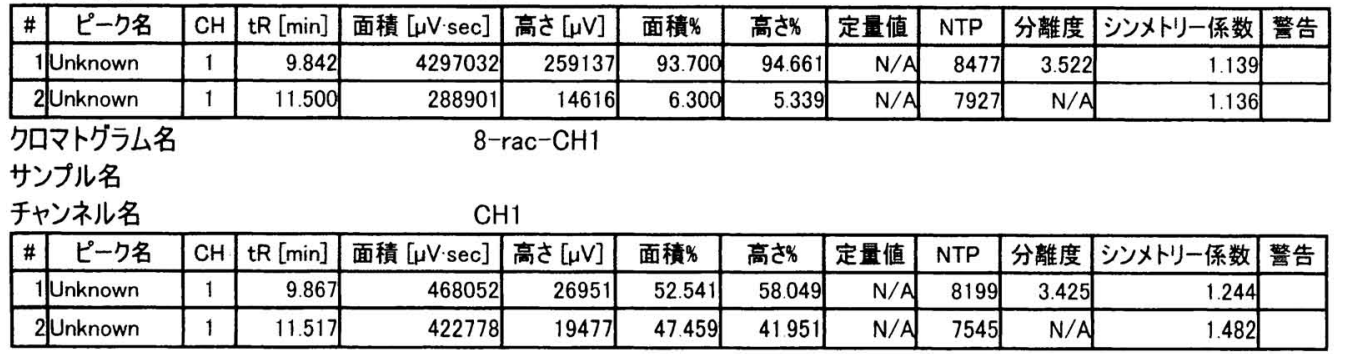




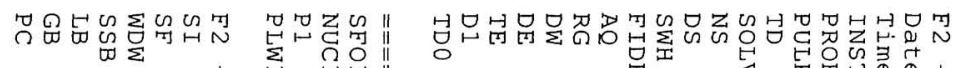

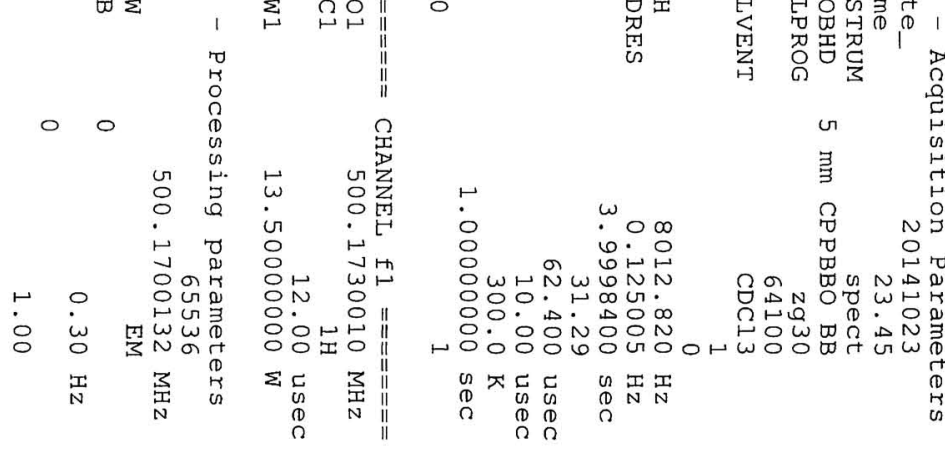

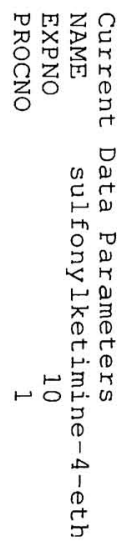

\subsection{6}

7.936
7.921 7.787 7.771

7.736
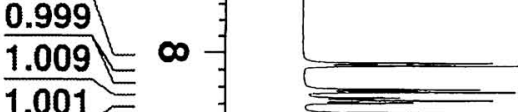

7.721

7.705

7.647

7.631

7.616

$-7.330$

7.314

7.258

7.117

7.101

5.879

$\overline{0.974}$ o

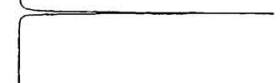

4.423

4.415

4.408

4.401

4.392
-.387
-3.378

4.387
-4.378
-4.373

$-4.373$

$-4.364$

$-4.357$

4.350

4.342
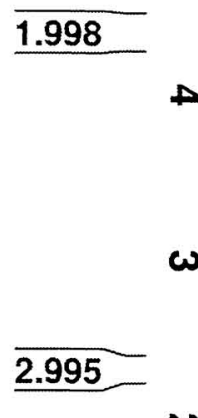

$\omega$

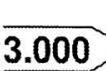

.
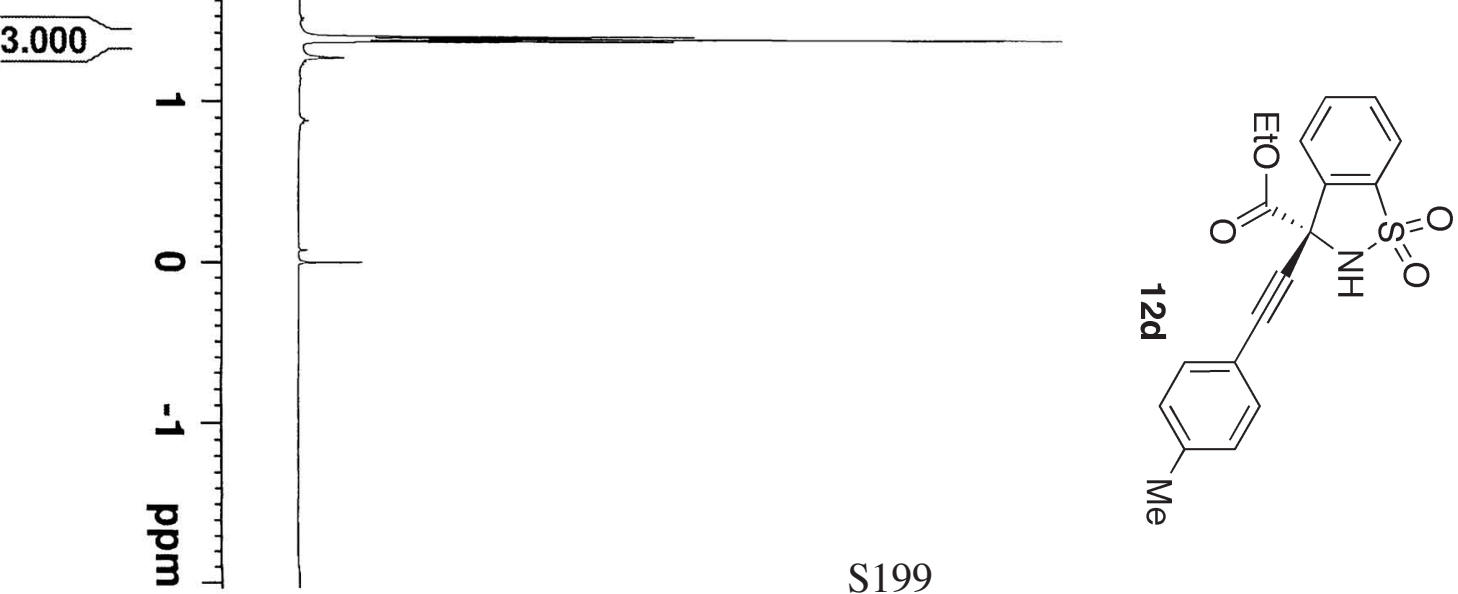

1.379
$\leftarrow 1.365$

1.350 


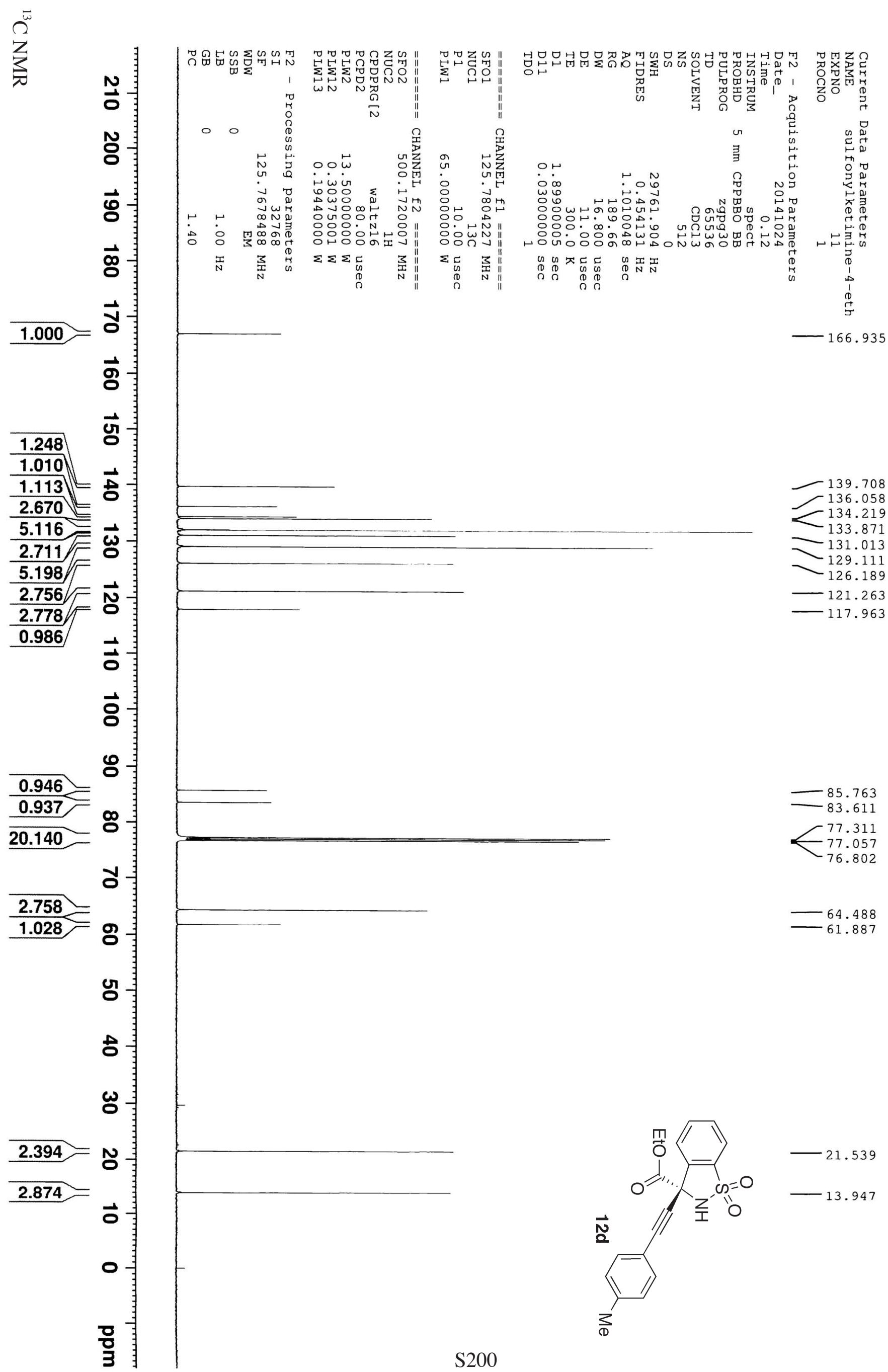




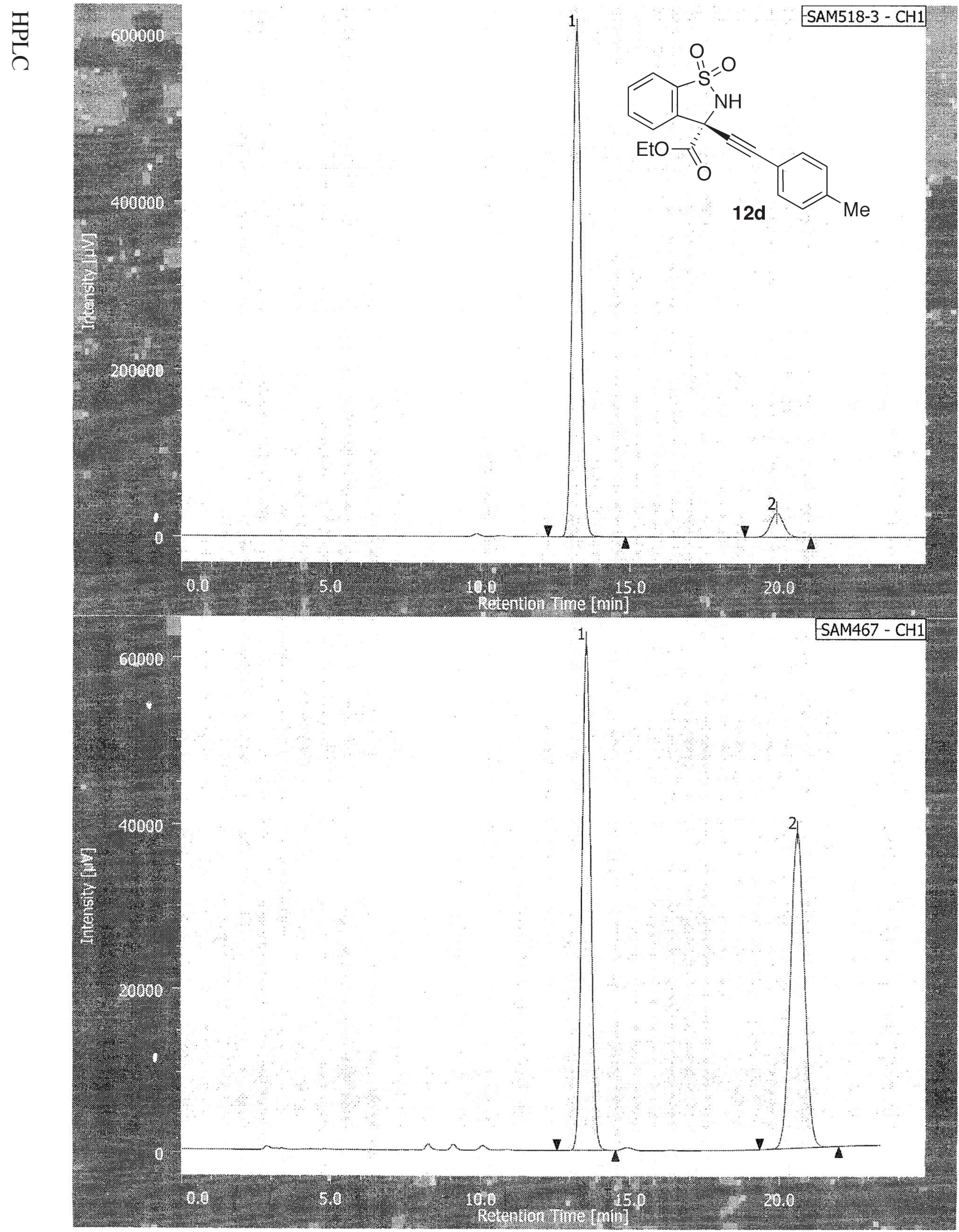

チャンネル情報+ピーク情報

クロマトグラム名

SAM518-3-CH1

サンプル名

チャンネル名

$\mathrm{CH} 1$

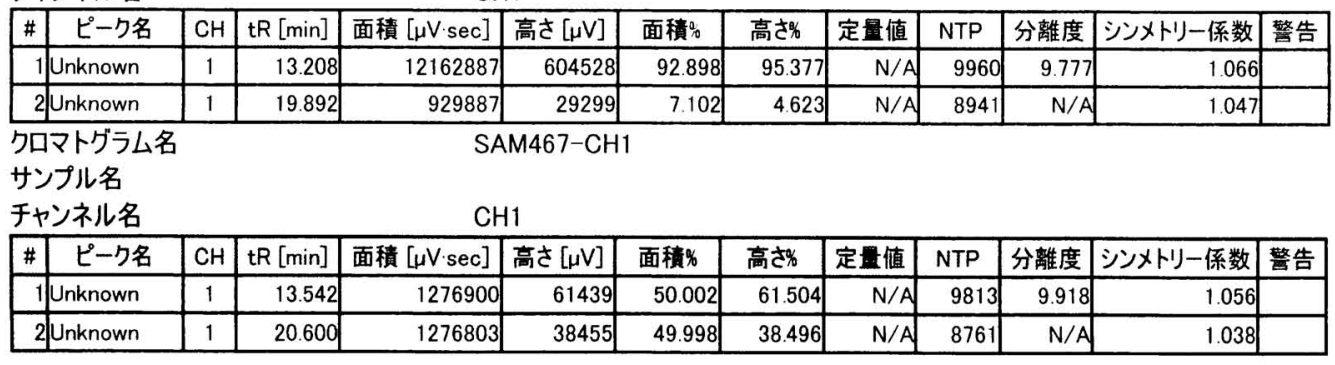



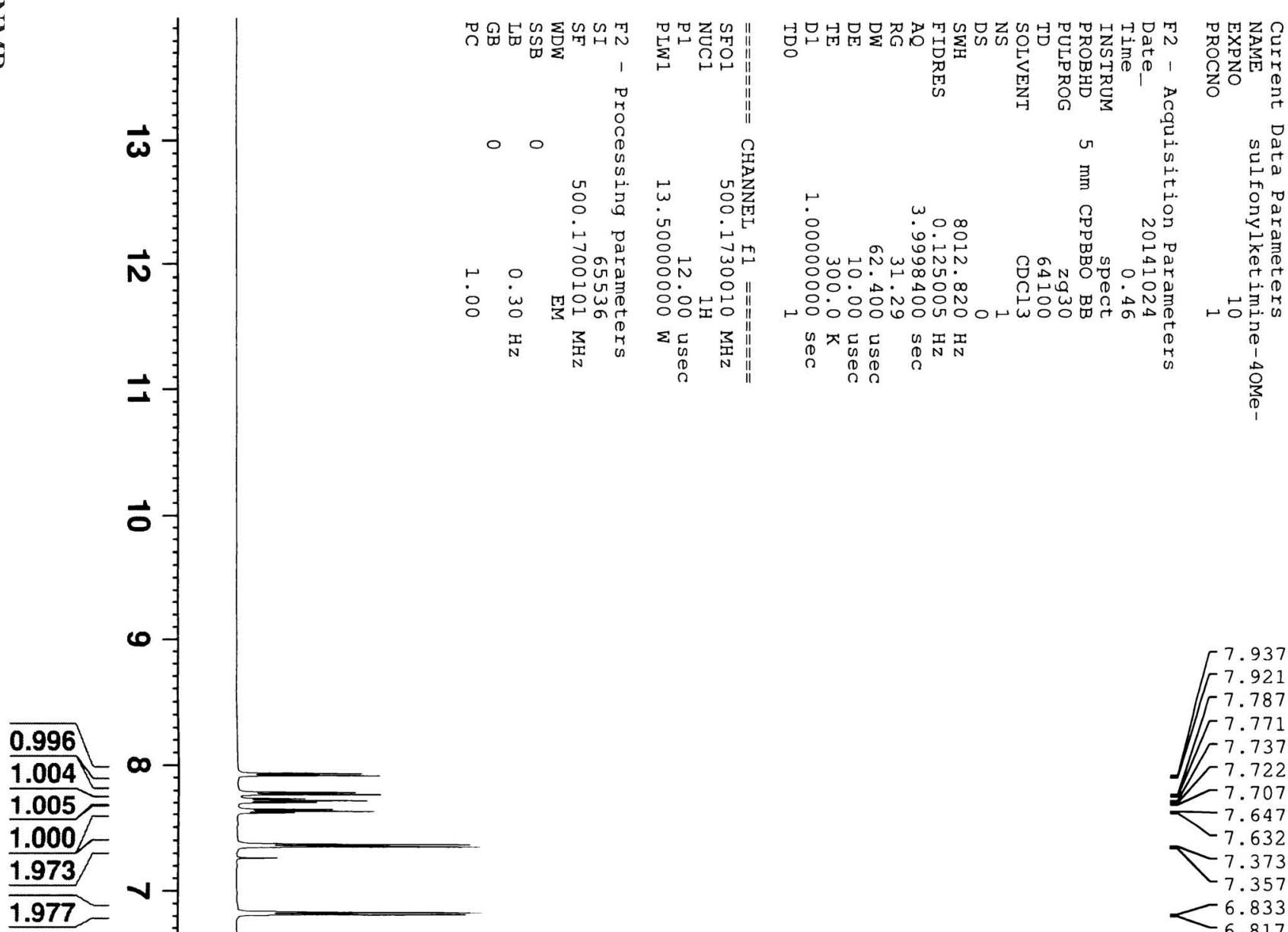

$\boldsymbol{0}$
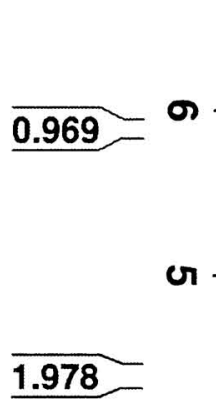

$\overrightarrow{0}$

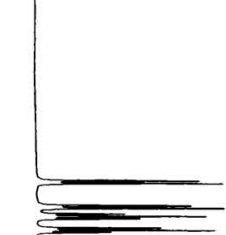

7.937

F.921

7.787

F 7.737

F 7.737 


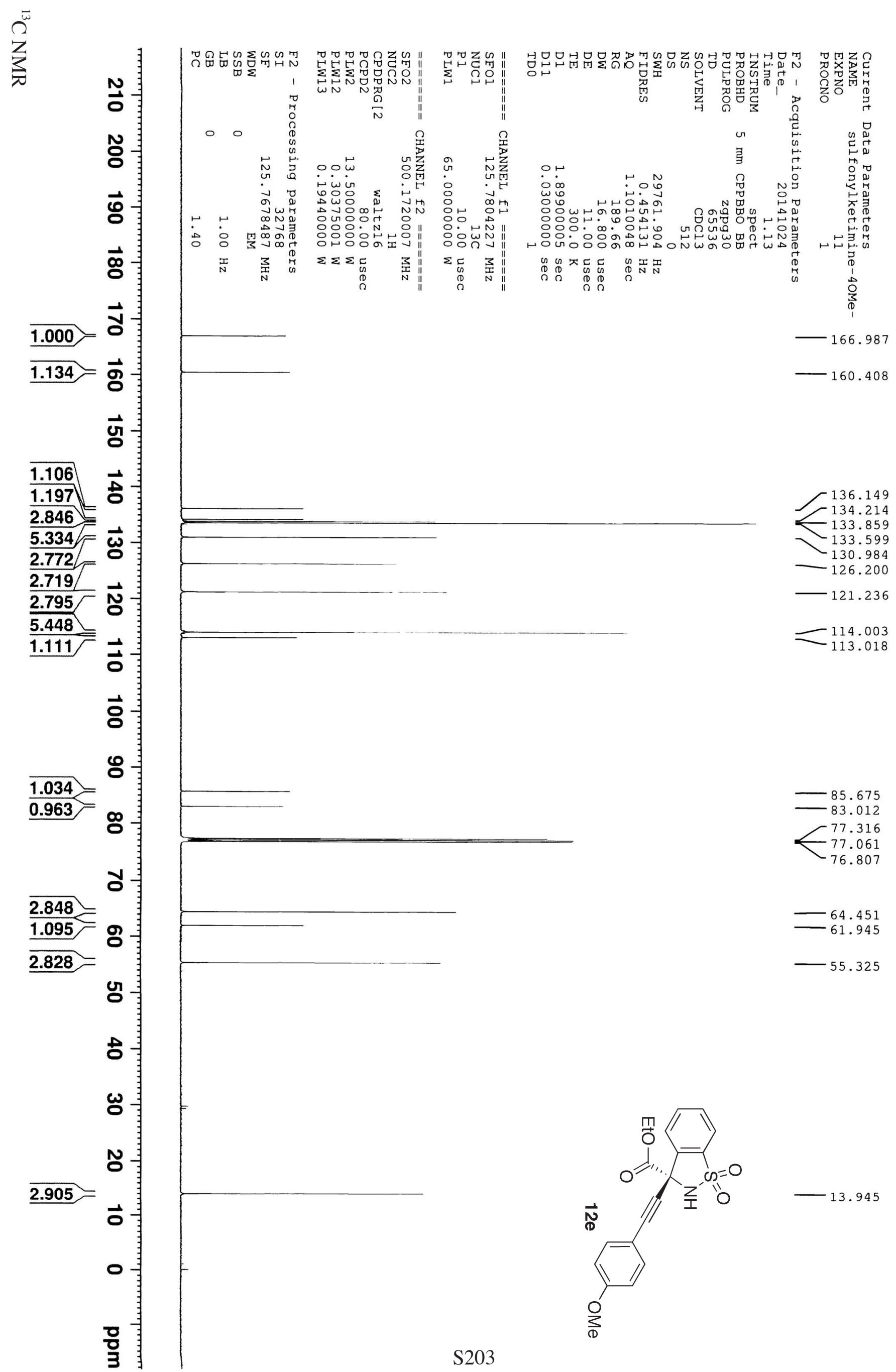




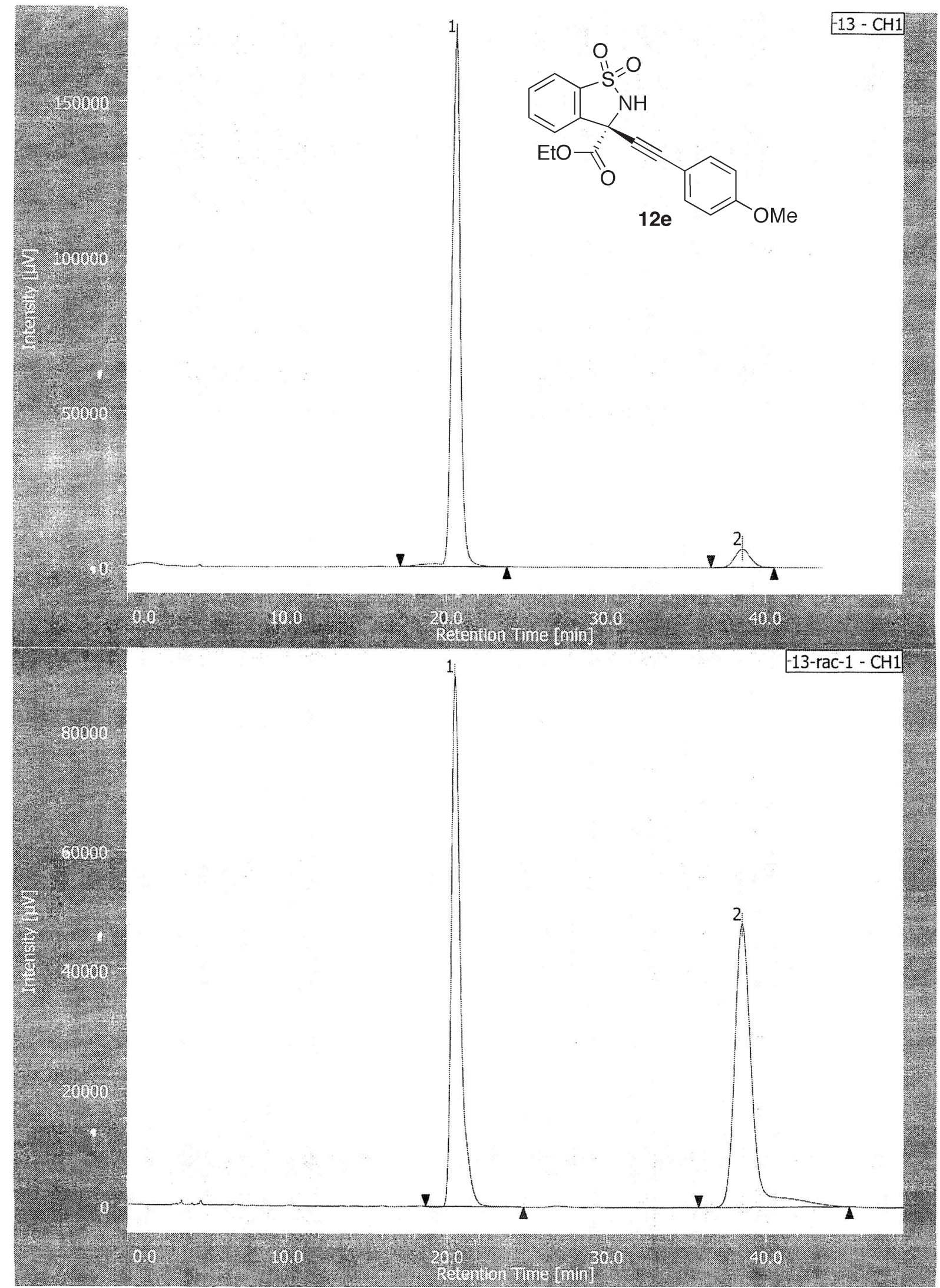

チャンネル情報+ピーク情報

クロマトグラム名

13- $\mathrm{CH} 1$

サンプル名

チャンネル名

$\mathrm{CH} 1$

\begin{tabular}{|c|c|c|c|c|c|c|c|c|c|c|c|c|}
\hline$\pi$ & ピーク名 & $\mathrm{CH}$ & $\operatorname{tR}[\mathrm{min}]$ & 面積 $[\mu \mathrm{V} \cdot \mathrm{sec}]$ & 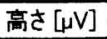 & 面皘\% & 高さ\% & 定量値 & NTP & 分離度 & シンメトリー係数 & 警告 \\
\hline & Unknown & 1 & 20.583 & 6290629 & 171474 & 93.929 & 96.683 & $N / A$ & 7812 & 13.107 & 1.099 & \\
\hline & Unknown & 1 & 38.542 & 406588 & 5884 & 6.071 & 3.317 & $\mathrm{~N} / \mathrm{A}$ & 7206 & N/A & 1.086 & \\
\hline
\end{tabular}

クロマトグラム名

13-rac-1-CH1

サンプル名

チャンネル名

$\mathrm{CH} 1$

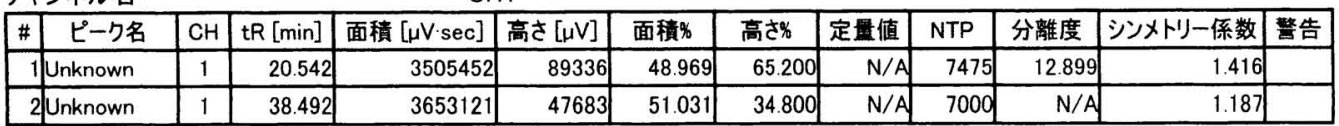




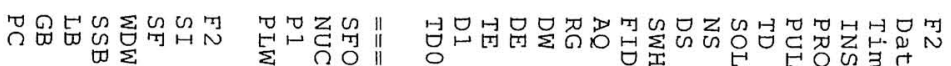
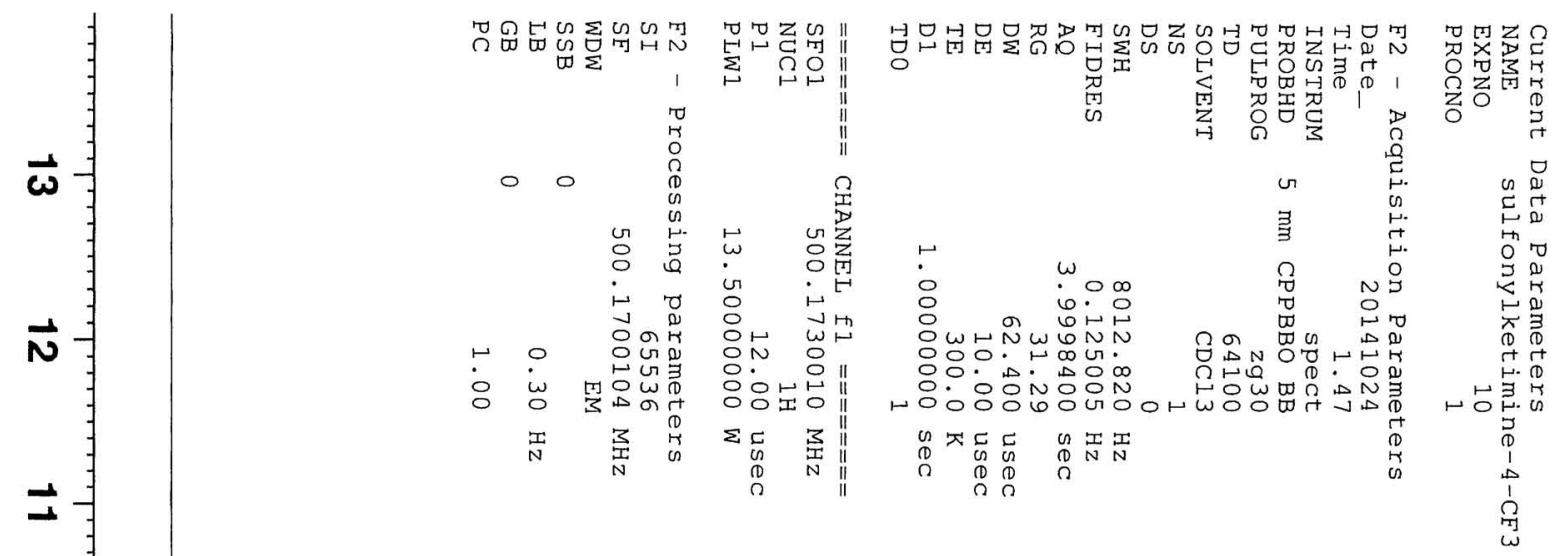

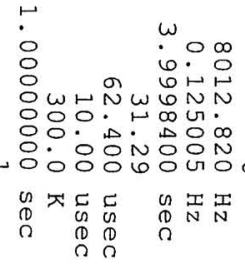

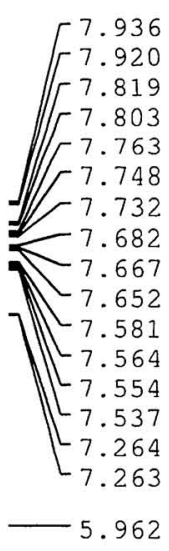

$1.031 \leq \infty$

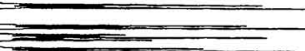

1.066

0

$\overrightarrow{0}$

$\infty$

4.021

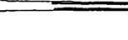

4.456

4.449

4.442

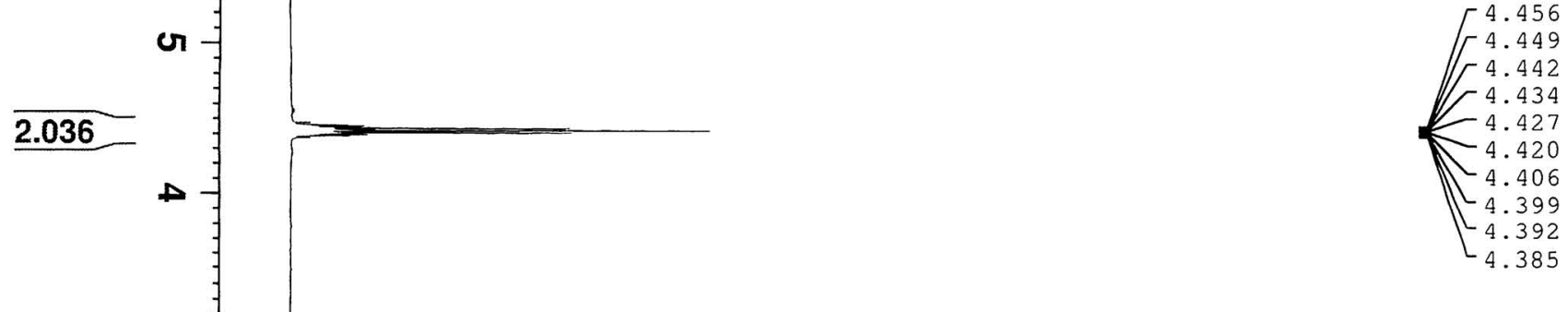

0.937

(1)

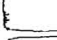

1.403

1.389

1.374

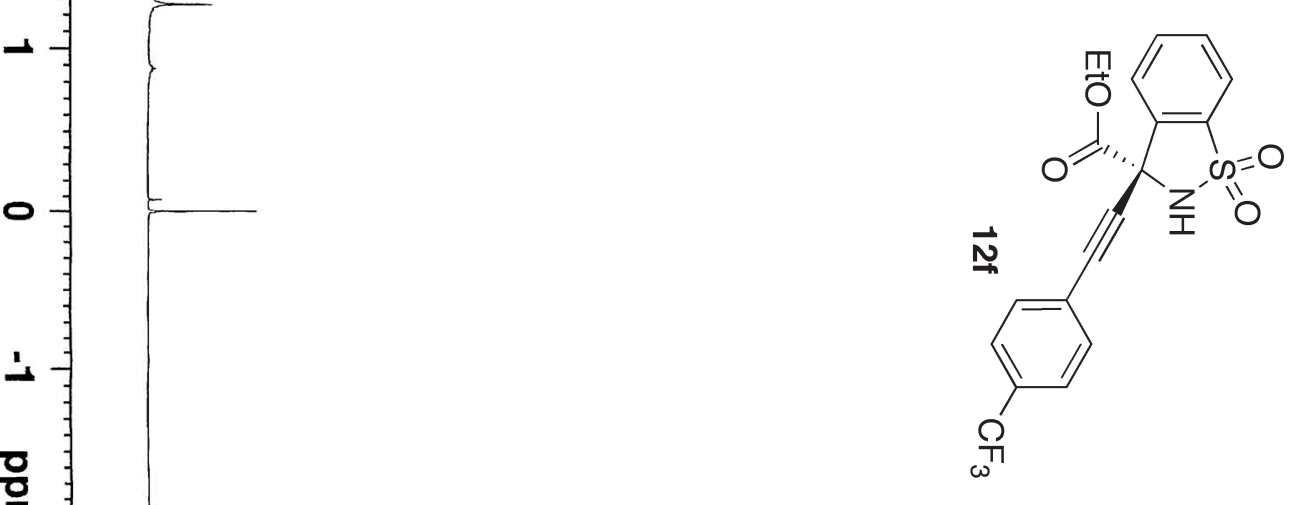




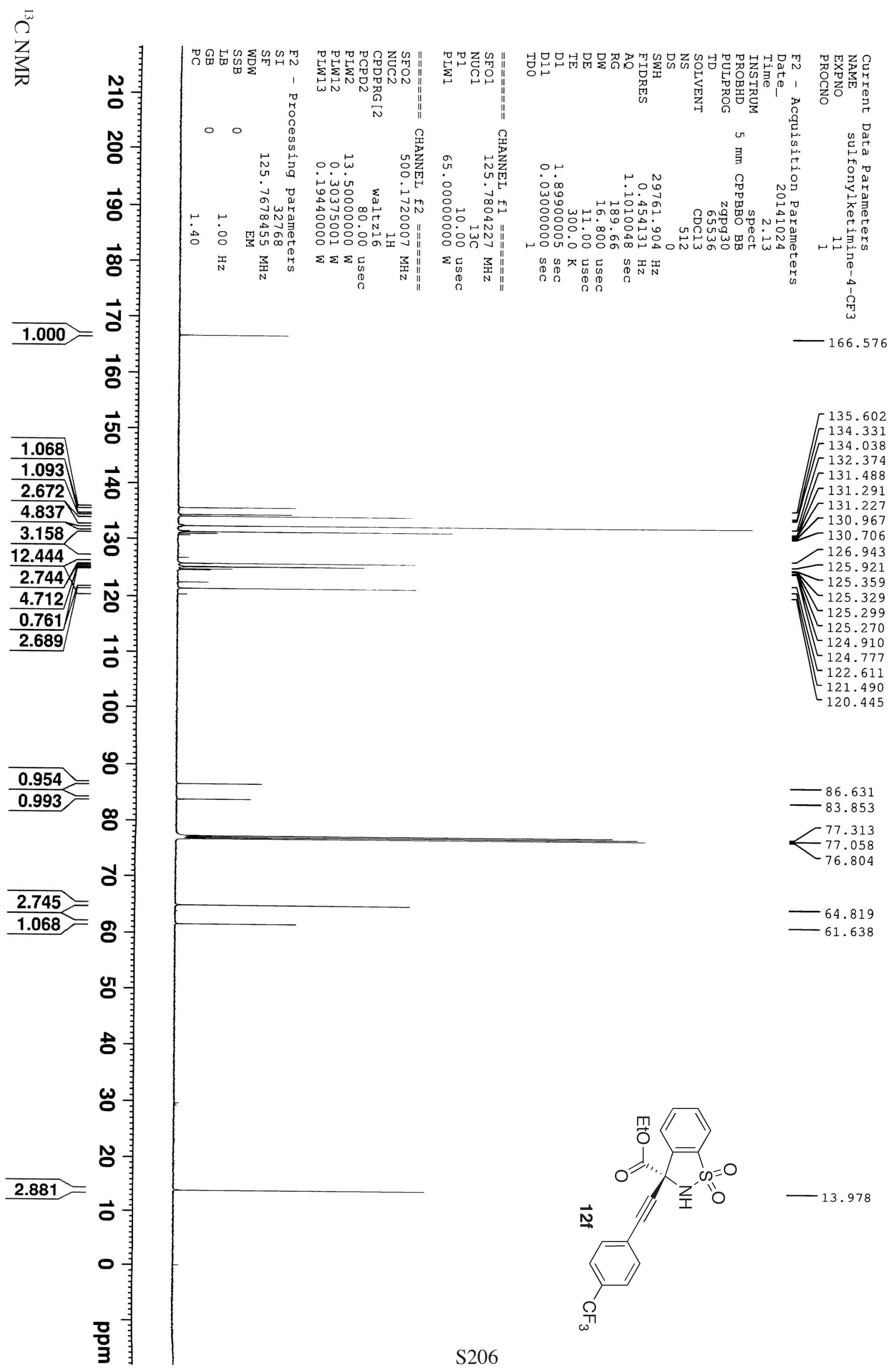




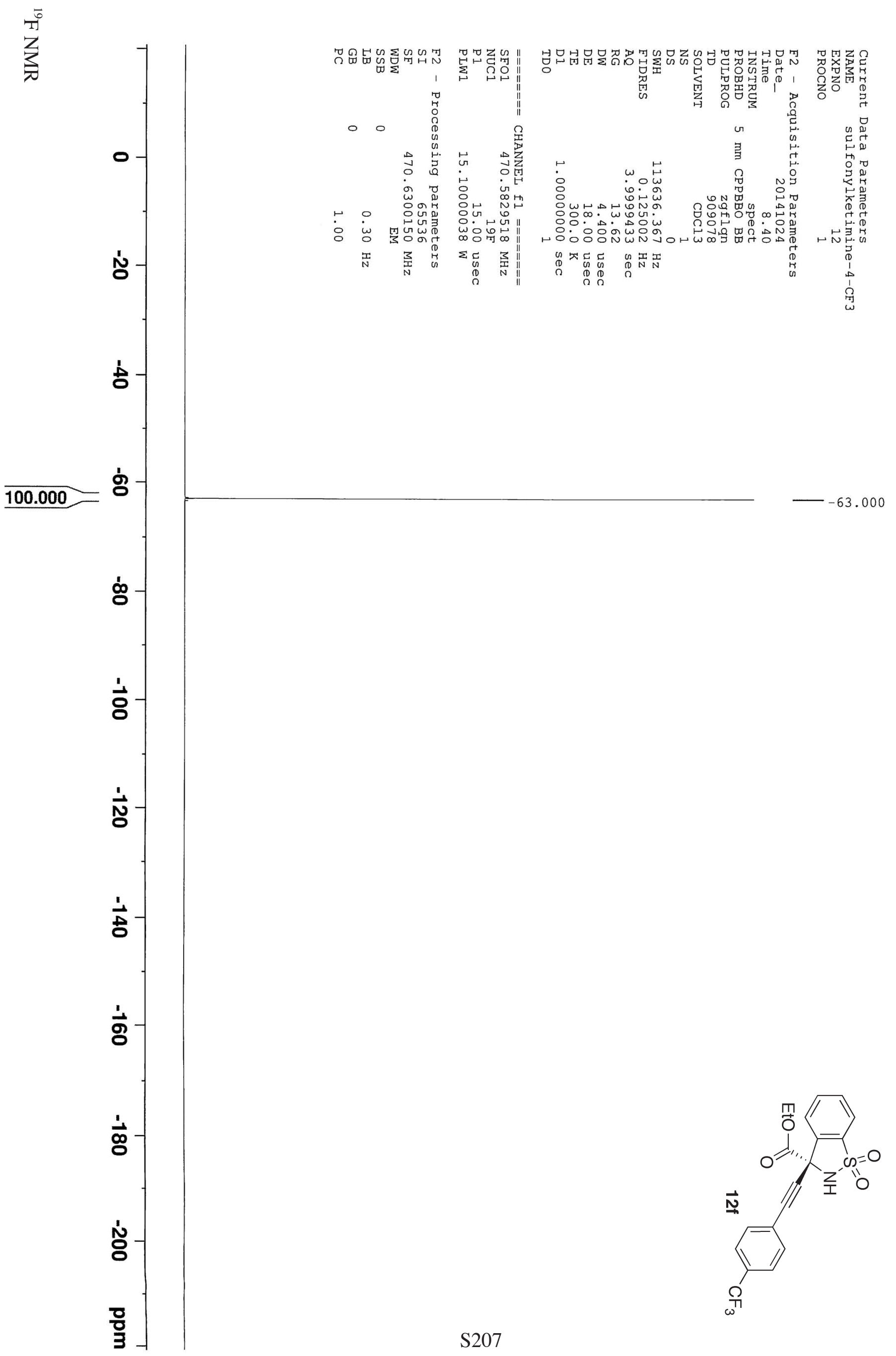



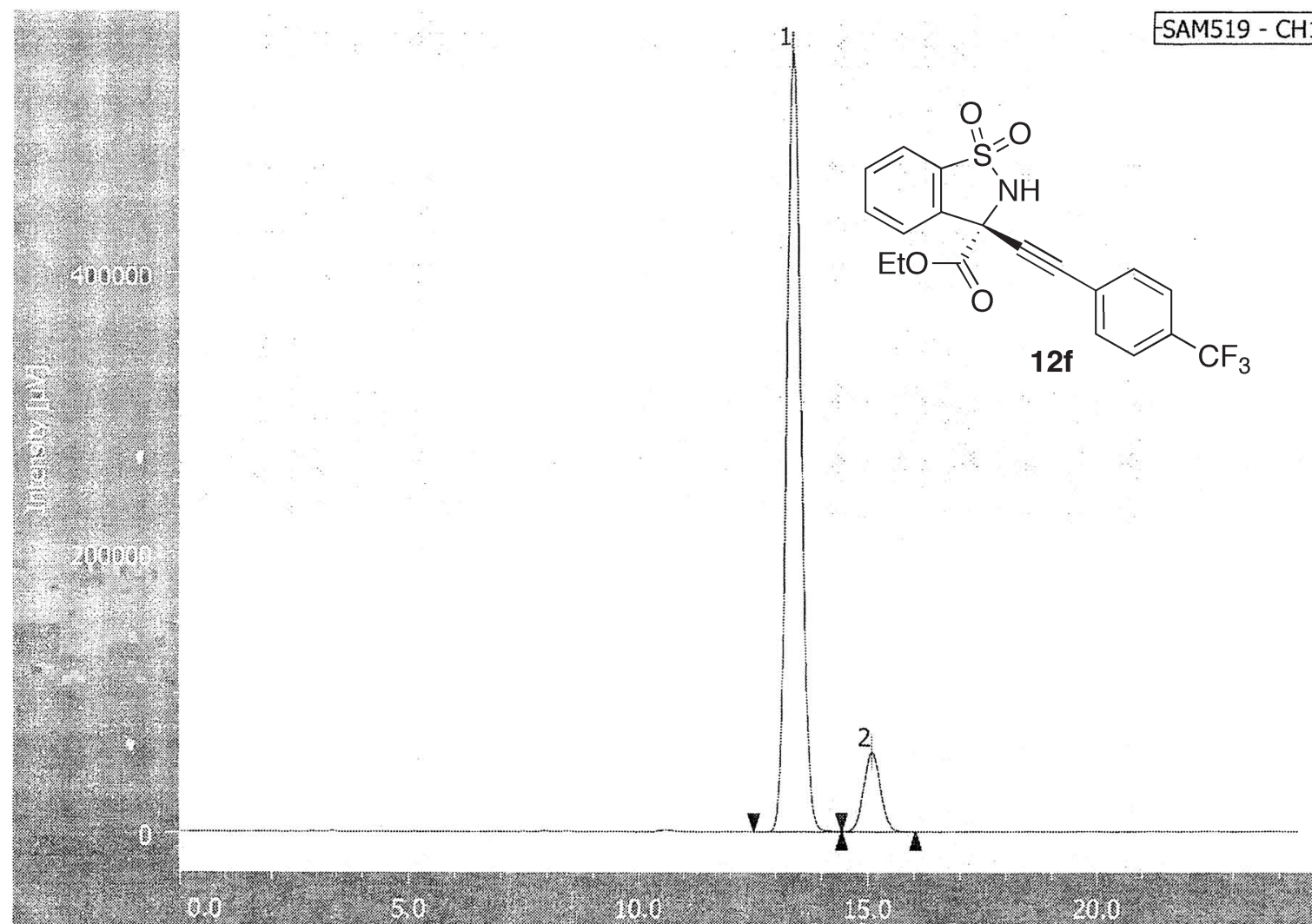

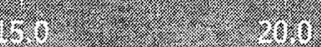

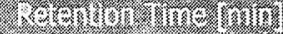

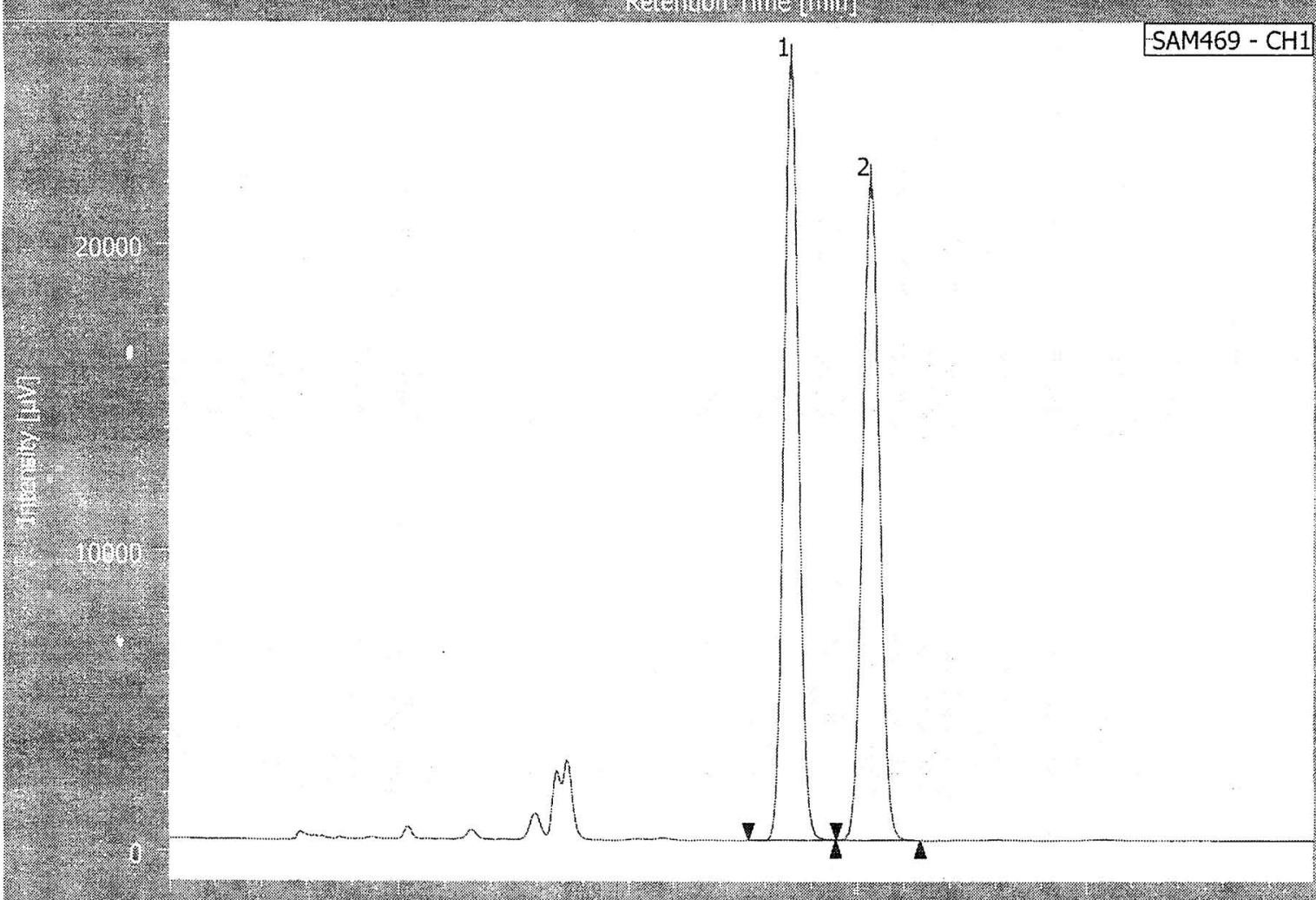

3.

.

28.6

チャンネル情報+ピーク情報

クロマトグラム名

SAM519-CH1

サンプル名

チャンネル名

$\mathrm{CH} 1$

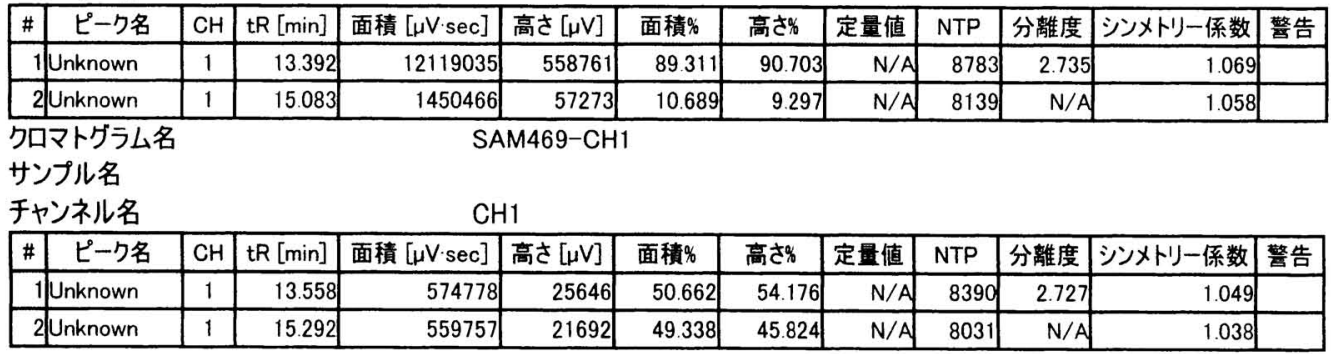




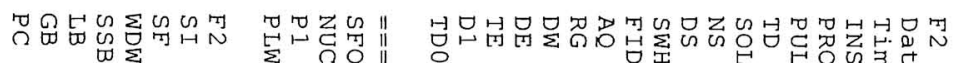
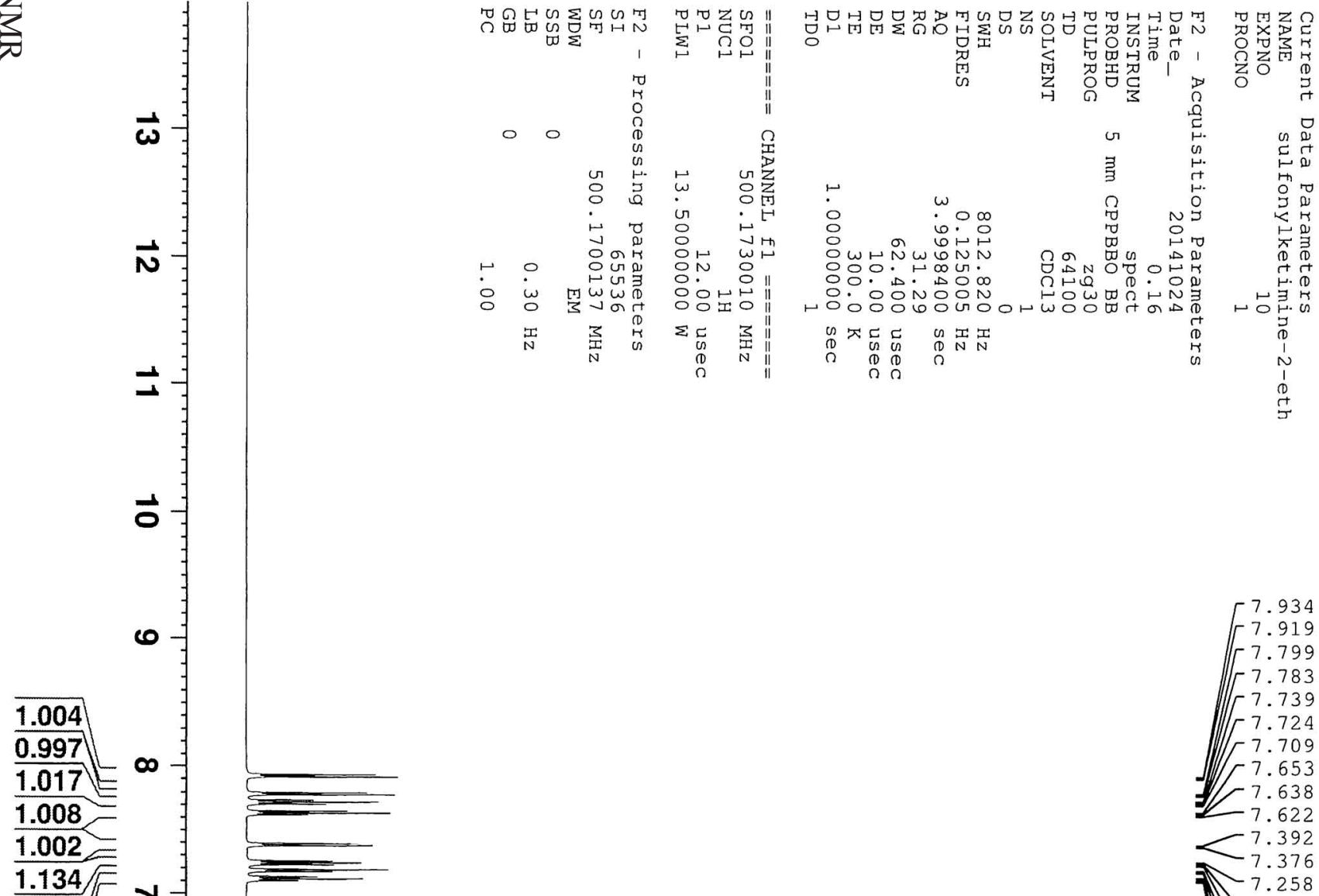

$\omega$

궁

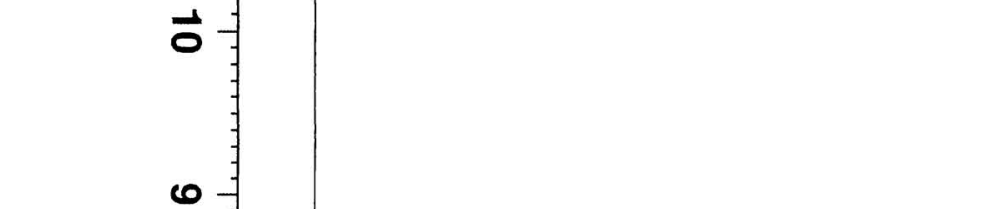

7.934

7.919

7.799

7.783

7.739

7.724

7.709

7.653

$\begin{array}{r}7.638 \\ 7.622 \\ \hline\end{array}$

$-7.392$

$-7.376$

$-7.258$

$-7.245$

$-7.230$

7.191

7.176

7.133

7.118

7.103

5.904

4.452

4.438

4.430

$\Gamma^{4.423}$

4.416

4.409

4.401

4.387
-4.379

$-4.379$

$-4.372$

4.365

4.358

4.351

4.337

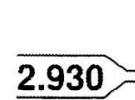

$\boldsymbol{\omega}$

$\omega$

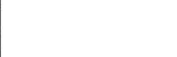

$-2.394$
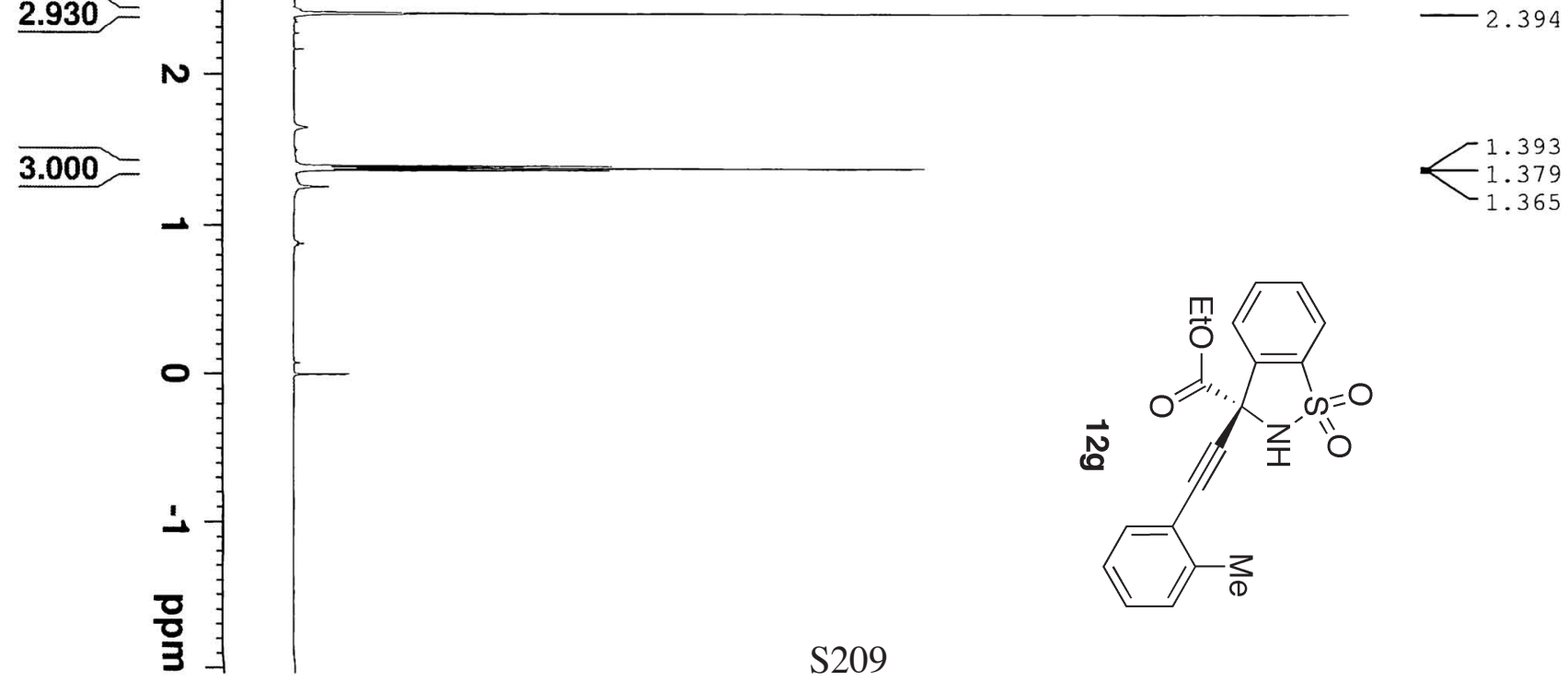
$\overbrace{0}^{\bar{\omega}}$
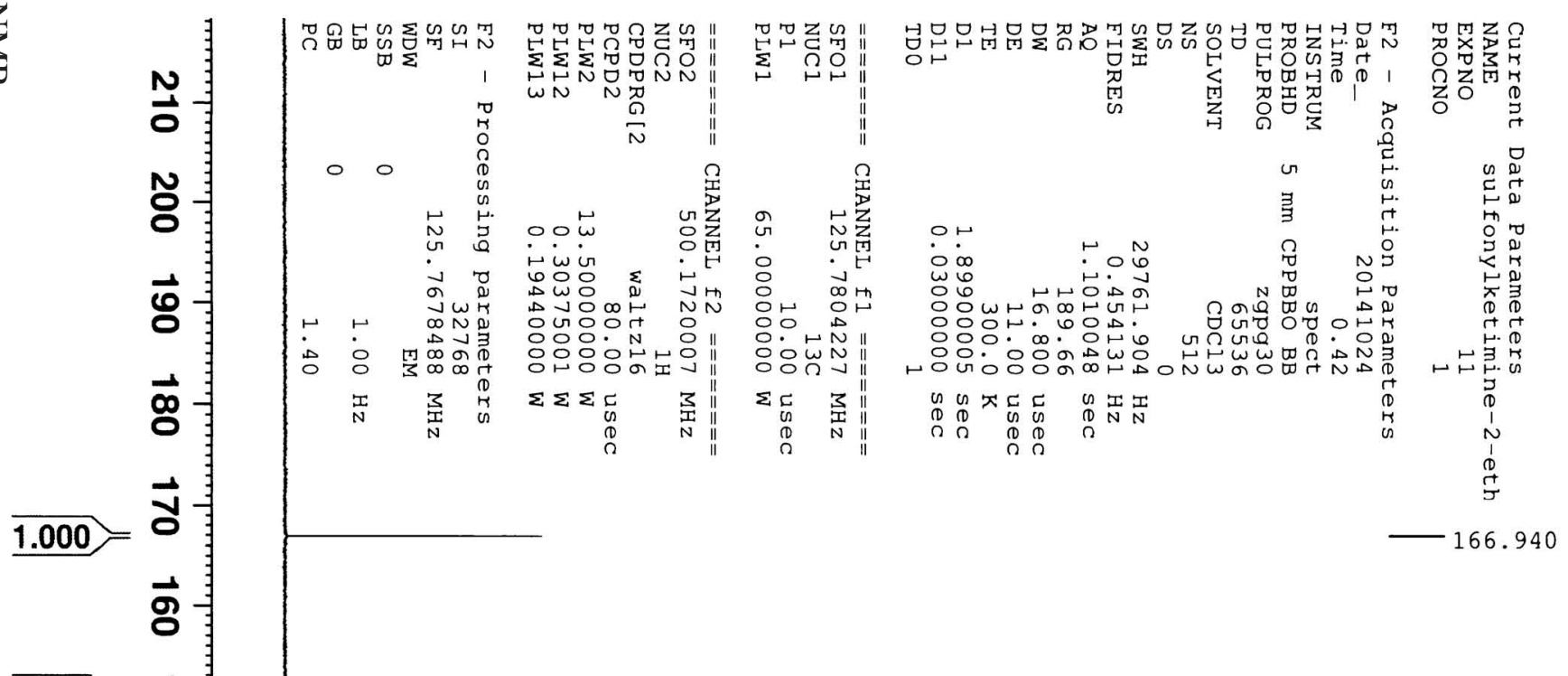

$\frac{1.153}{1.065}$ 응

$\frac{1.073}{2.834}=\vec{F}$

$2.753=$

2.830 虫

$2.909 / \Gamma$

$2.763=$

2.670

2.802

0.930

홍

$\overline{0.904}=8$

$0.952=$

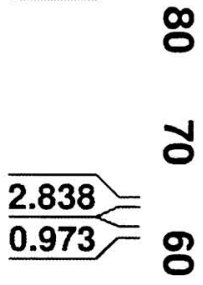

o

w

$\underline{2.199}=\mathrm{N}$

$2.964=$

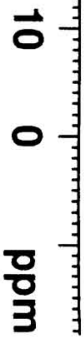

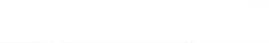

140.941

136.141

134.296

133.878

$-132.19$

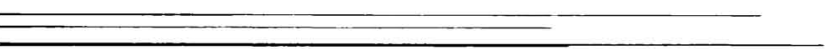

$-131.052$

$-129.532$

129.391

$-126.020$

125.614

$-121.342$

120.836

青

8

青

웍

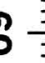

61.929

春

ㅇ.
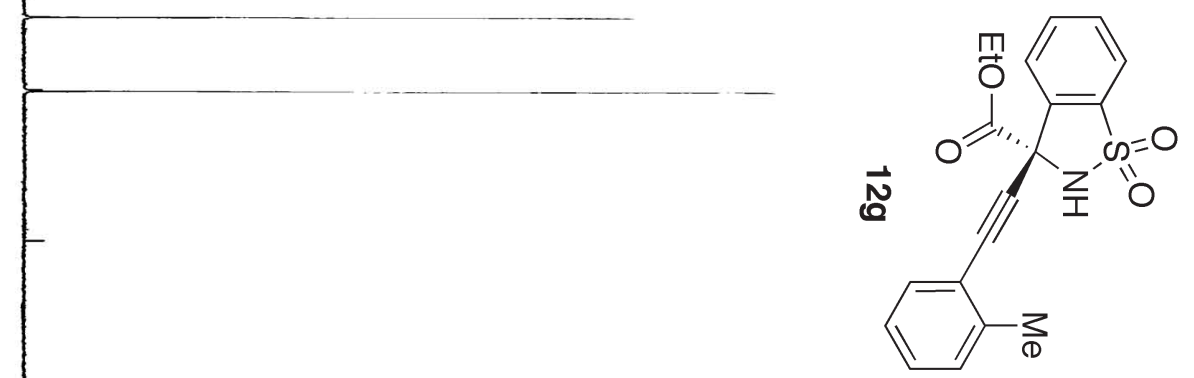

88.101

84.749

77.306

77.052

76.798 


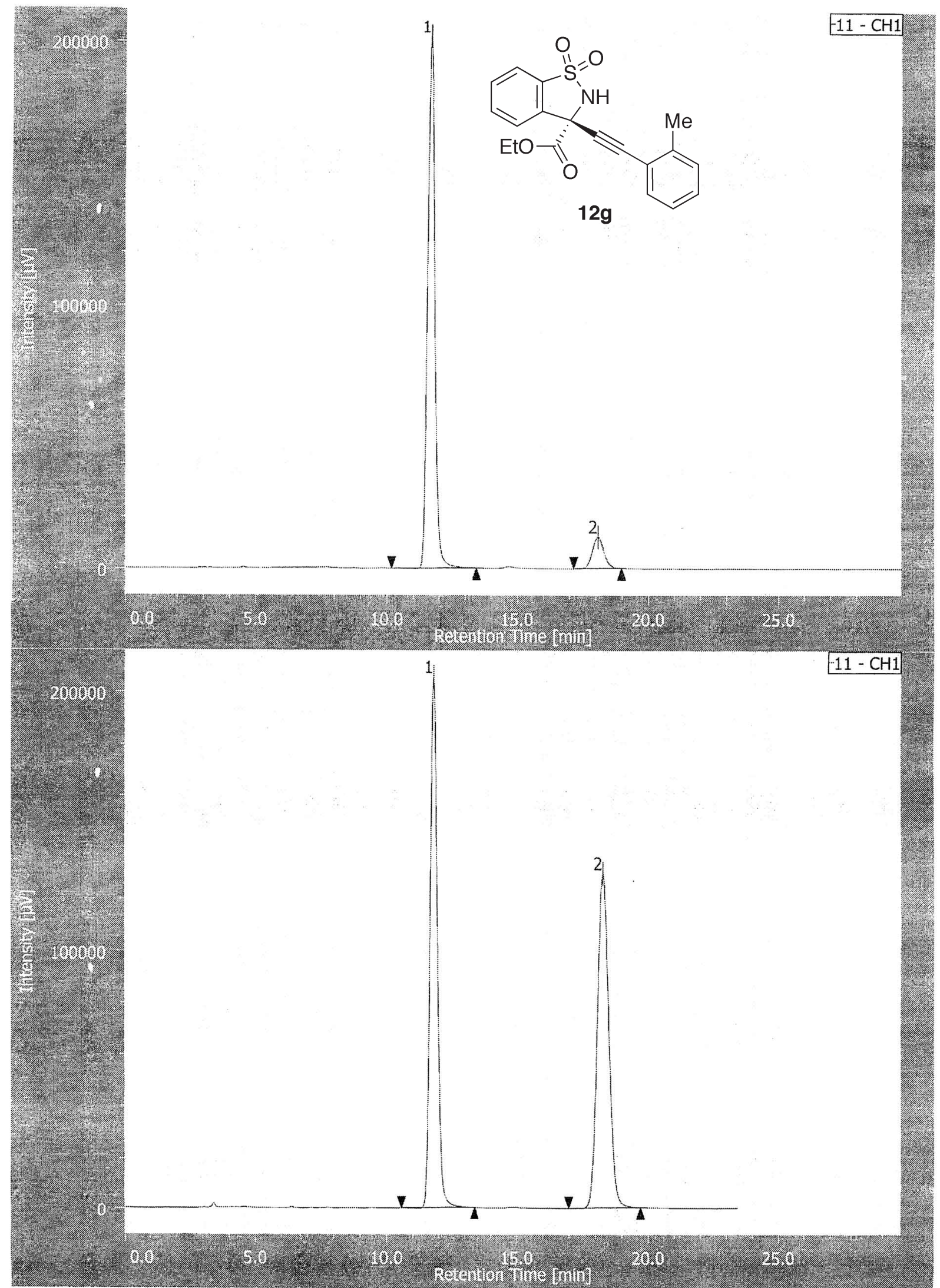

チャンネル情報+ピーク情報

クロマトグラム名

$11-\mathrm{CH} 1$

サンプル名

チャンネル名

$\mathrm{CH} 1$

\begin{tabular}{|c|c|c|c|c|c|c|c|c|c|c|c|c|}
\hline$\#$ & ピーク名 & $\mathrm{CH}$ & $\mathrm{tR}[\mathrm{min}]$ & 面積 $[u \vee \cdot s e c]$ & 高さ $[\mu \mathrm{V}]$ & 面積\% & 高さ\% & 定量値 & NTP & 分離度 & シンメトリリー係数 & 警告 \\
\hline & Jnknown & 1 & 11.667 & 3858003 & 202142 & 91.274 & 94.355 & N/A & 9117 & 9.979 & 1.125 & \\
\hline & Unknown & 1 & 18.075 & 368811 & 12094 & 8.726 & 5.645 & N/A & 8187 & N/A & 1.098 & \\
\hline & $\begin{array}{l}\text { マトグラム } \\
\text { プル名 }\end{array}$ & & & & -0 & & & & & & & \\
\hline & ンネル名 & & & $\mathrm{CH}$ & & & & & & & & \\
\hline$\#$ & ピーク名 & $\mathrm{CH}$ & $\operatorname{tR}[\mathrm{min}]$ & 面皘 $[\mu \mathrm{V} \cdot \mathrm{sec}]$ & 高さ [ $\mu \mathrm{V}]$ & 面積\% & 高さ\% & 定量値 & NTP & 分離度 & シンメトリー係数 & 警告 \\
\hline & Unknown & 1 & 11.792 & 3953498 & 205605 & 49.942 & 61.311 & N/A & 9068 & 10.035 & 1.136 & \\
\hline & Unknown & 1 & 18.275 & 3962678 & 129742 & 50.058 & 38.689 & N/A & 8343 & N/A & 1.084 & \\
\hline
\end{tabular}


$\underset{z}{z}$

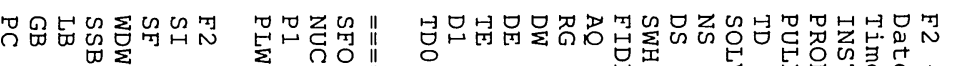

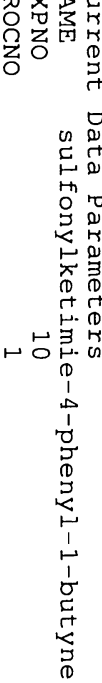

7.741

$-7.704$

7.689

7.671

7.668

7.656

7.654

7.641

7.638

7.619

7.617

7.604
7.602
7.590

$-7.590$

7.587

$-7.284$

$-7.281$

7.271

7.258

7.256

7.228

7.226

7.218

$-7.214$

7.199

7.181

7.178

7.164

$-5.699$

4.328

4.314

4.300

4.286

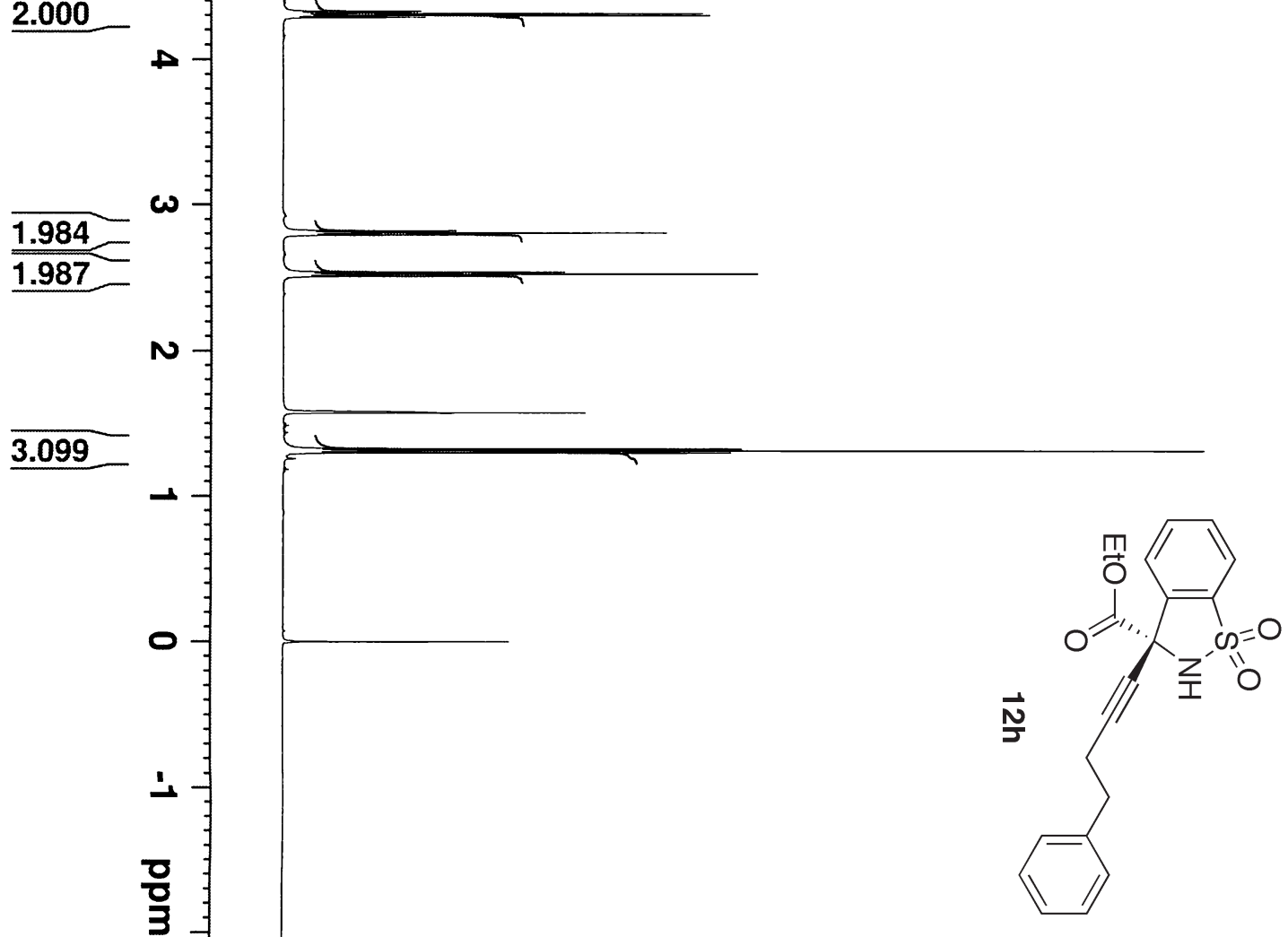

2.822
2.807
2.792
2.538
2.524
2.509 


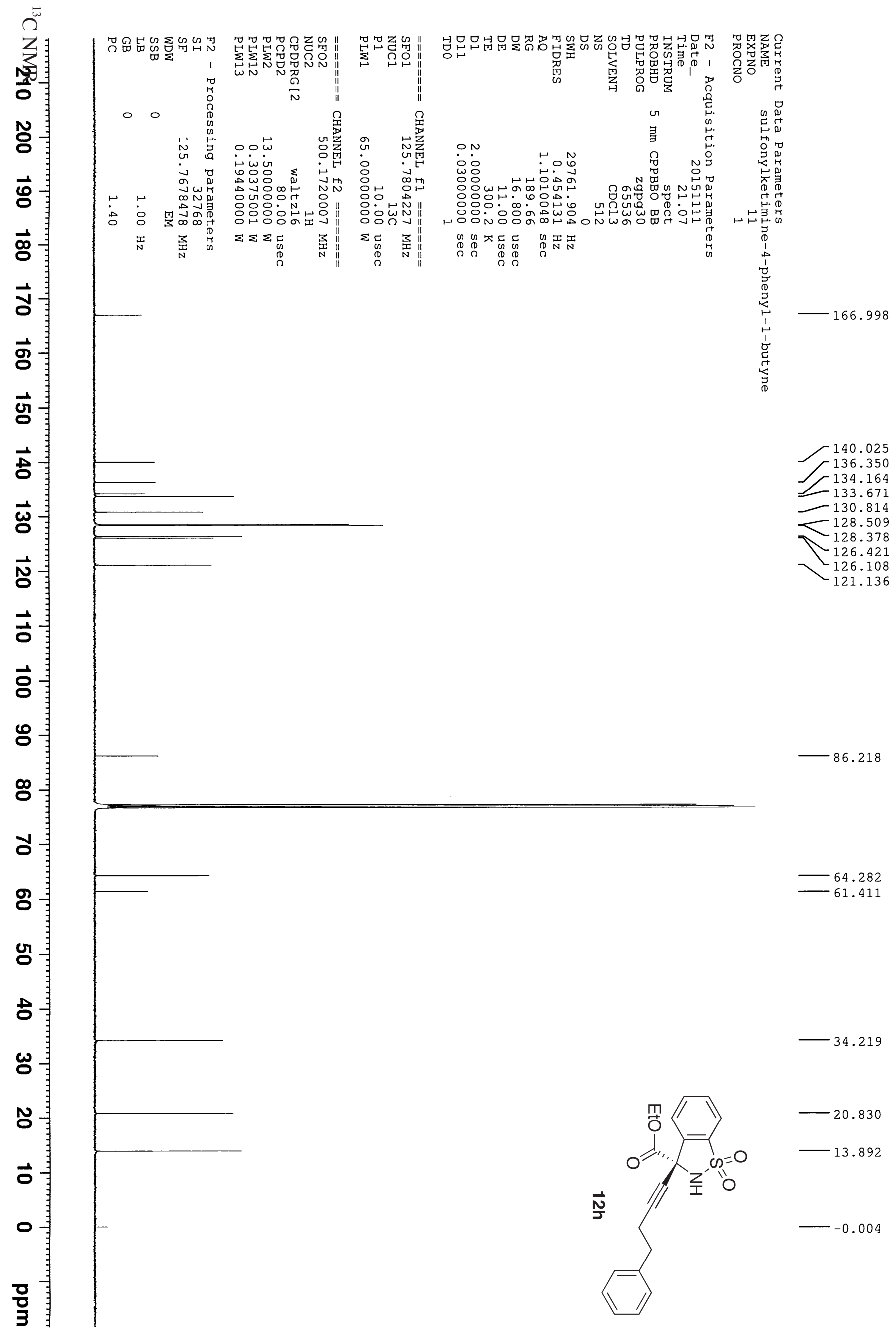




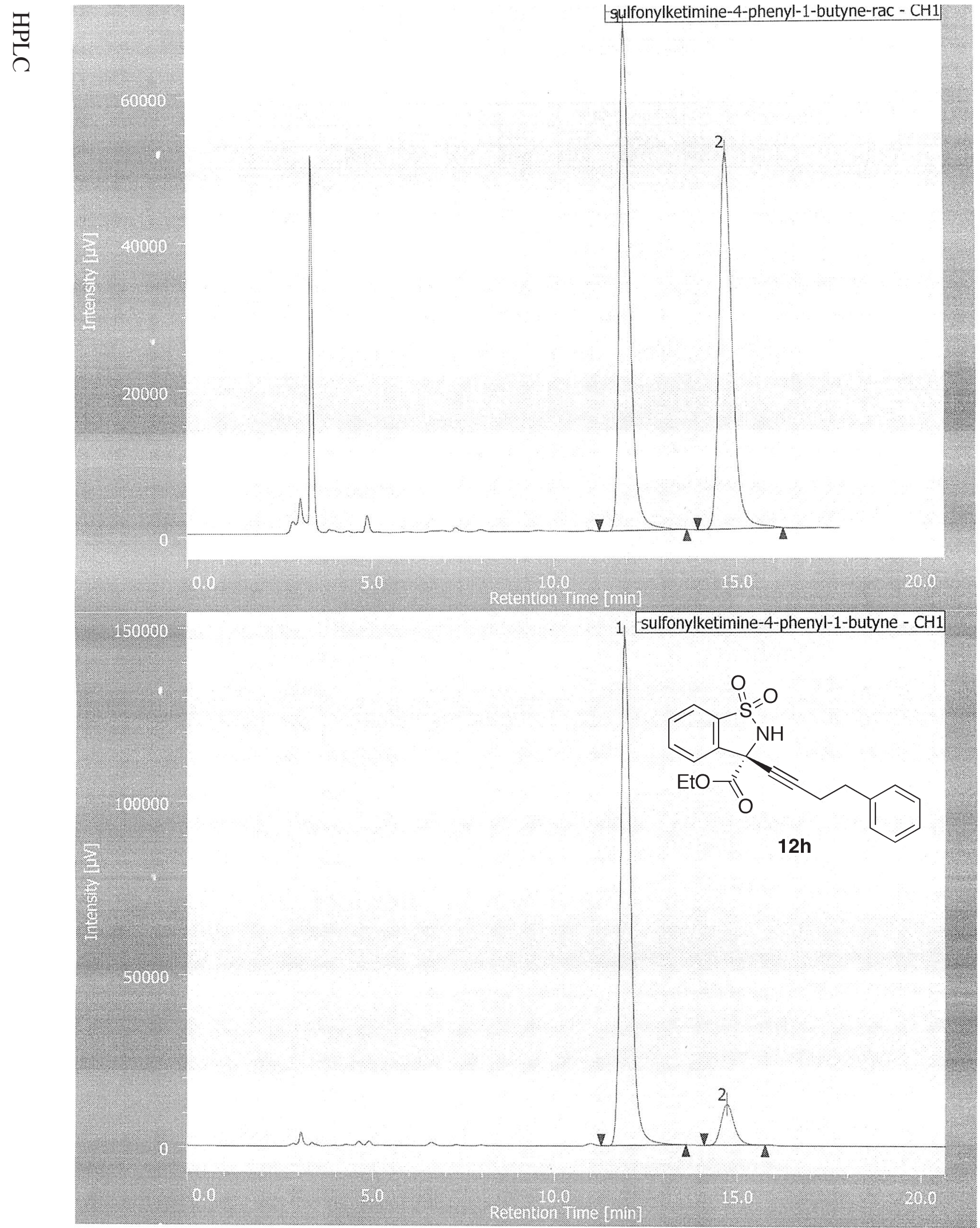

チャンネル情報+ピーク情報

クロマトグラム名

sulfonylketimine-4-phenyl-1-butyne-rac- $\mathrm{CH} 1$

サンプル名

チャンネル名

$\mathrm{CH} 1$

\begin{tabular}{|l|c|c|r|r|r|r|r|r|r|r|r|r|}
\hline$\#$ & ピーク名 & $\mathrm{CH}$ & $\mathrm{tR}[\mathrm{min}]$ & 面積 $[\mu \mathrm{V} \cdot \mathrm{sec}]$ & 高さ $[\mu \mathrm{V}]$ & \multicolumn{1}{|c|}{ 面積\% } & 高さ\% & 定量値 & NTP & 分離度 & シンメトリー係数 & 警告 \\
\hline 1 Unknown & 1 & 11.958 & 1354480 & 68710 & 51.910 & 57.316 & $\mathrm{~N} / \mathrm{A}$ & 9348 & 4.941 & 1.286 & \\
\hline 2 Unknown & 1 & 14.708 & 1254810 & 51169 & 48.090 & 42.684 & $\mathrm{~N} / \mathrm{A}$ & 8966 & $\mathrm{~N} / \mathrm{A}$ & 1.221 & \\
\hline
\end{tabular}

クロマトグラム名 sulfonylketimine-4-phenyl-1-butyne-CH1

サンプル名

チャンネル名

$\mathrm{CH} 1$

\begin{tabular}{|c|c|c|c|c|c|c|c|c|c|c|c|}
\hline ピーク名 & $\mathrm{CH}$ & $t R[\min ]$ & 面積 [ $\mu \mathrm{V} \cdot \mathrm{sec}]$ & 高さ $[\mu \mathrm{V}]$ & 面積\% & 高さ\% & 定量値 & NTP & 分離度 & シンメトリー係数 & 警告 \\
\hline 1 Unknown & 1 & 11.942 & 2866911 & 146250 & 90.828 & 92.584 & N/A & 9363 & 4.946 & 1.257 & \\
\hline 2 Unknown & 1 & 14.717 & 289511 & 11715 & 9.172 & 7.416 & $N / A$ & 8692 & $\mathrm{~N} / \mathrm{A}$ & 1.237 & \\
\hline
\end{tabular}



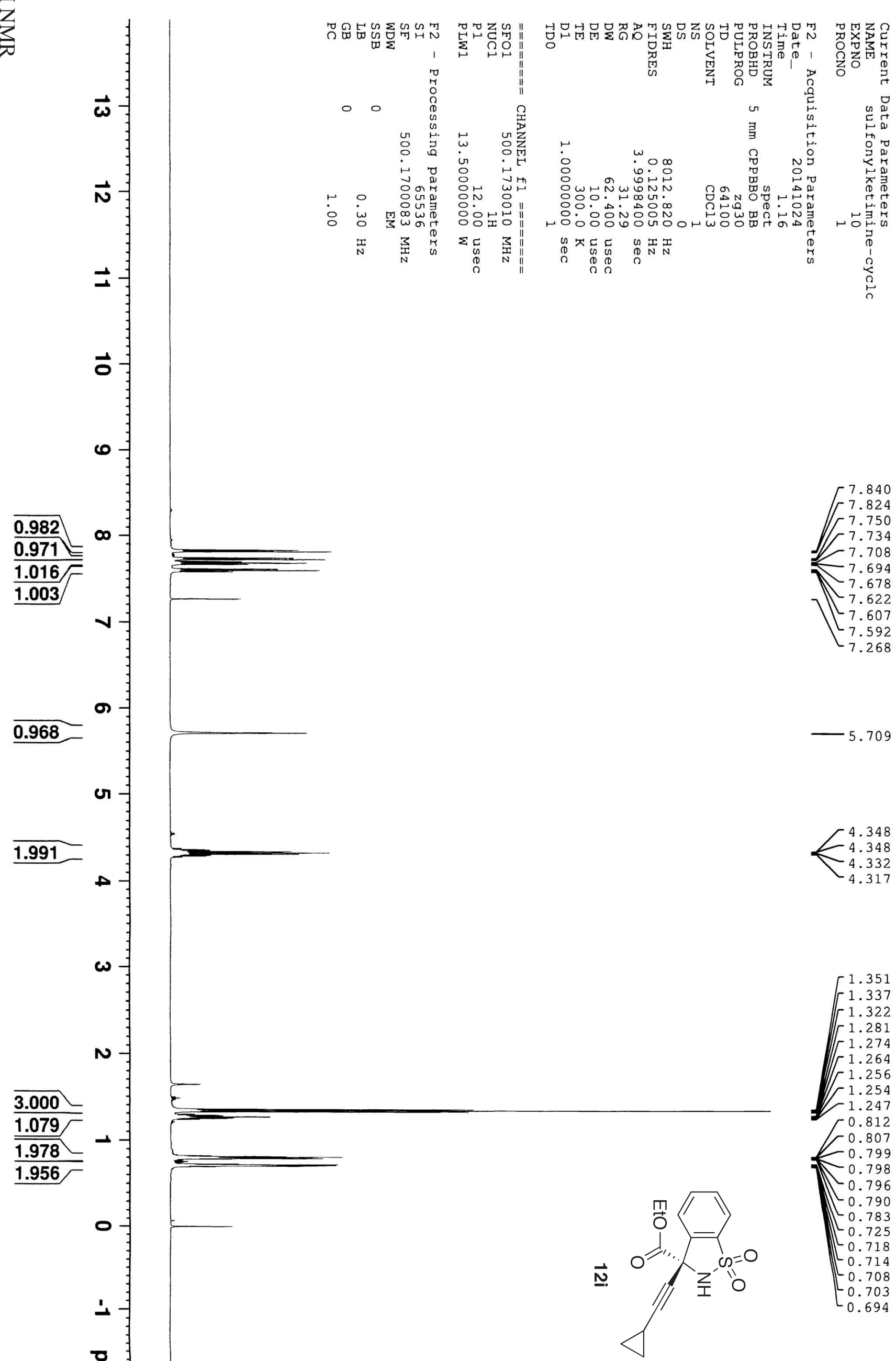

1.351

1.337

1.322

1.281

F 1.264

1.256

1.254

1.247

0.812

0.807

$-0.799$

0.798

$-0.796$

0.796
-0.790
0.783

0.783

0.725

0.718

0.714

0.708

0.703

0.694

밈 


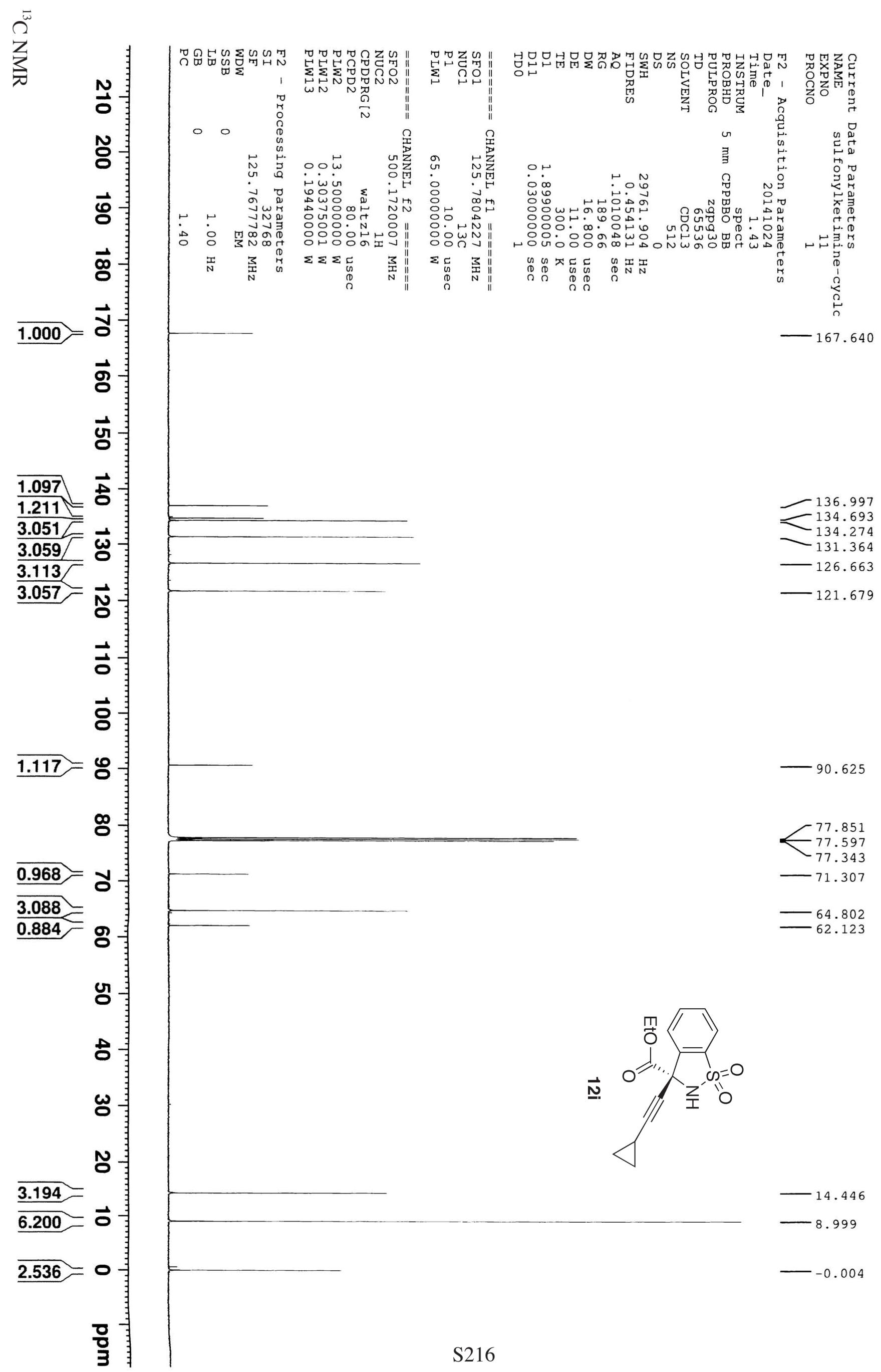




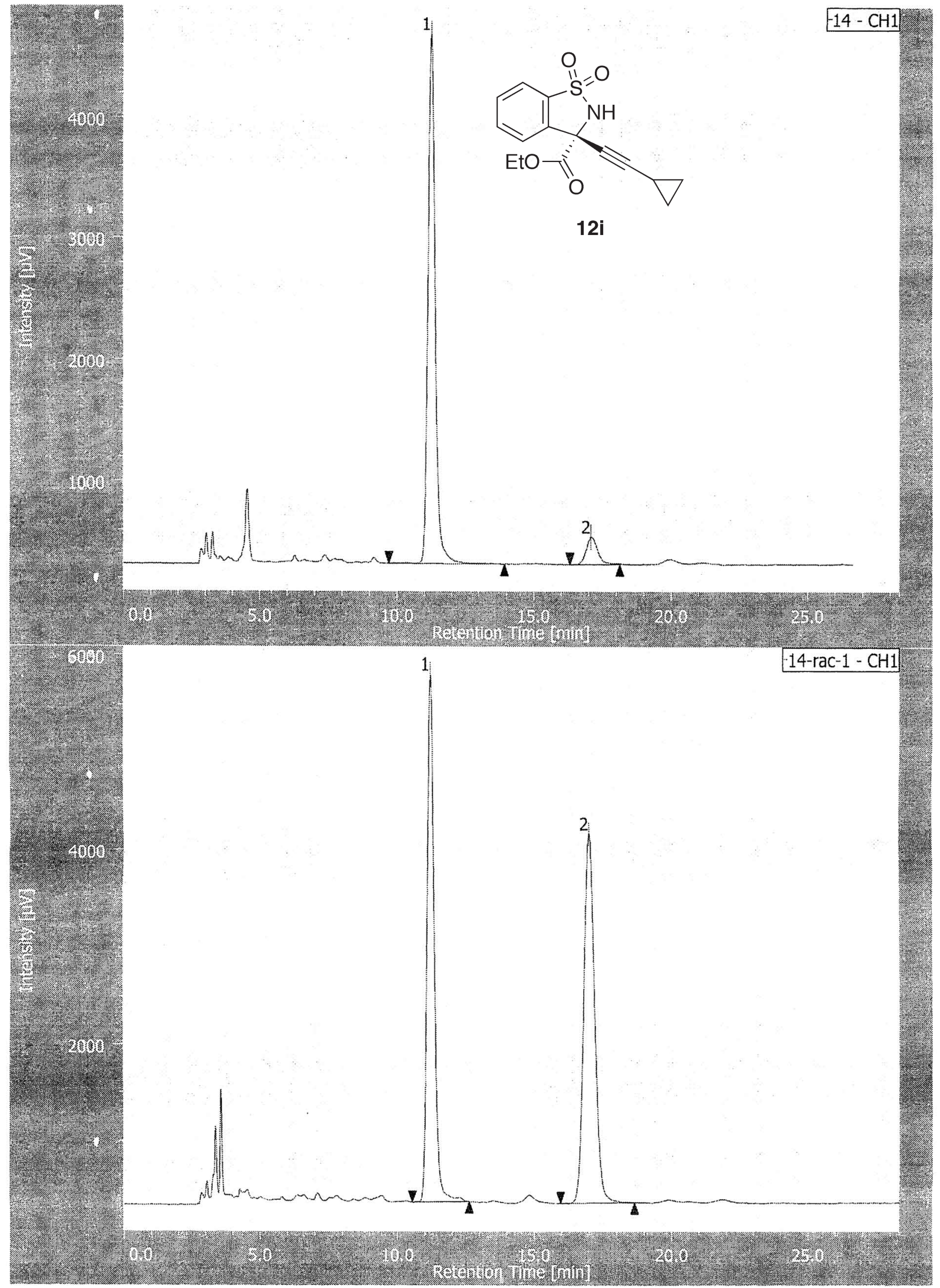

チャンネル情報 サピーク情報

クロマトグラム名

14- $\mathrm{CH} 1$

サンプル名

チャンネル名

$\mathrm{CH} 1$

\begin{tabular}{|c|c|c|c|c|c|c|c|c|c|c|c|}
\hline ピーク名 & $\mathrm{CH}$ & $t R[\mathrm{~min}]$ & 面積 [ $[\mu \mathrm{V} \cdot \mathrm{sec}]$ & 高さ $[\mu \mathrm{V}]$ & 面積\% & 高さ\% & 定量値 & NTP & 分離度 & シンメトリー係数 & 警告 \\
\hline 1 Unknown & 1 & 11.233 & 80098 & 4365 & 92.311 & 95.050 & N/A & 9522 & 9.800 & 1.191 & \\
\hline 2 Unknown & 1 & 17.075 & 6672 & 227 & 7.689 & 4.950 & N/A & 8638 & $N / A$ & 1.554 & \\
\hline
\end{tabular}

クロマトグラム名

14-rac-1-CH1

サンプル名

チャンネル名

$\mathrm{CH} 1$

\begin{tabular}{|c|c|c|c|c|c|c|c|c|c|c|c|}
\hline ピーク名 & $\mathrm{CH}$ & $t R$ [min] & 面積 $[\mu \mathrm{V} \cdot \mathrm{sec}]$ & 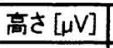 & 面積\% & 高さ\% & 定量值 & NTP & 分離度 & シンメトリー係数 & 警告 \\
\hline Unknown & 1 & 11.200 & 98792 & 5454 & 48.177 & 58.712 & $N / A$ & 9659 & 9.878 & 1.175 & \\
\hline 2 Unknown & 1 & 17.000 & 106270 & 3836 & 51.823 & 41.288 & N/A & 8880 & N/A & 1.094 & \\
\hline
\end{tabular}


望

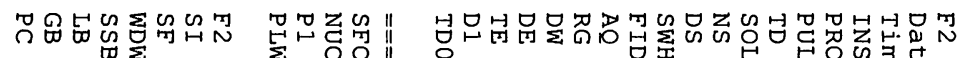

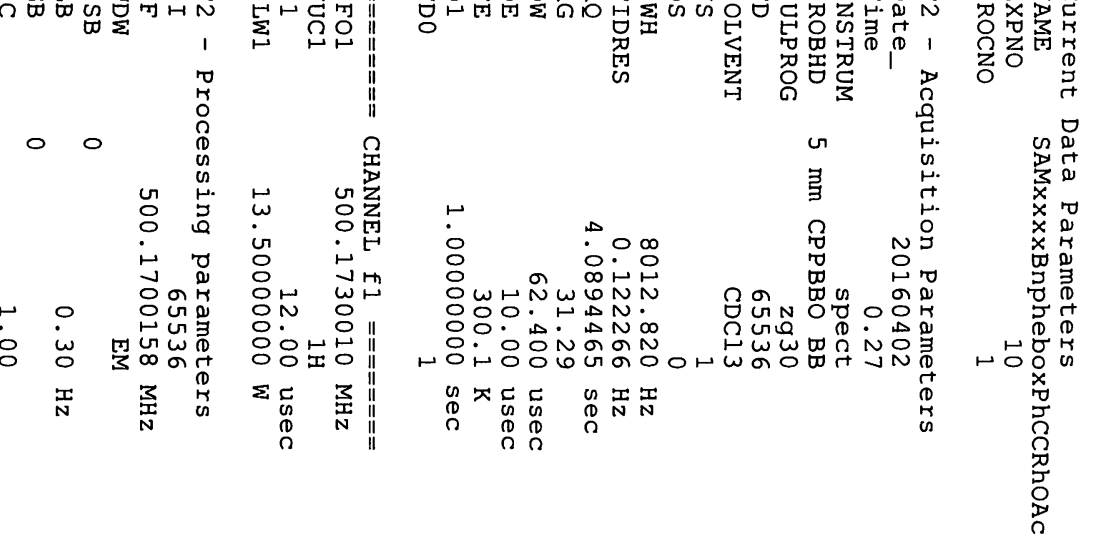

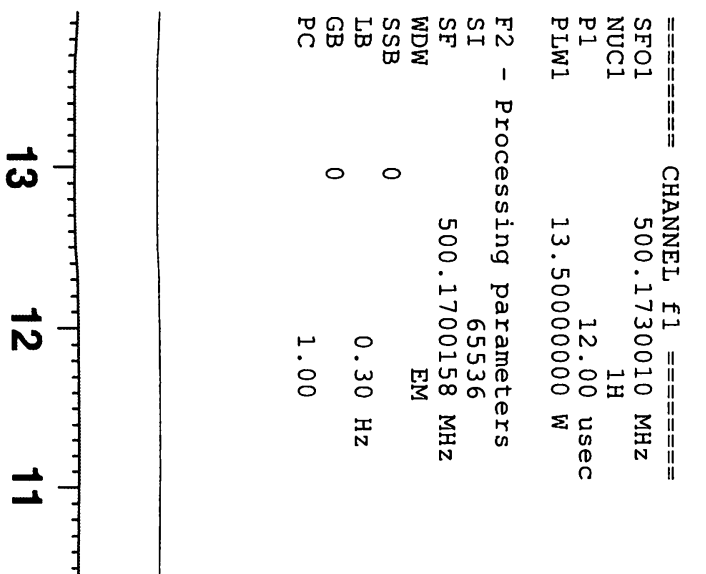

\section{$\frac{3.951 £}{1.072 \digamma}$}

$\boldsymbol{\omega}$

$\infty$

or

\subsection{8}

2.000

0.994

1.039

1.079

1.280

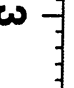

a

or

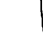
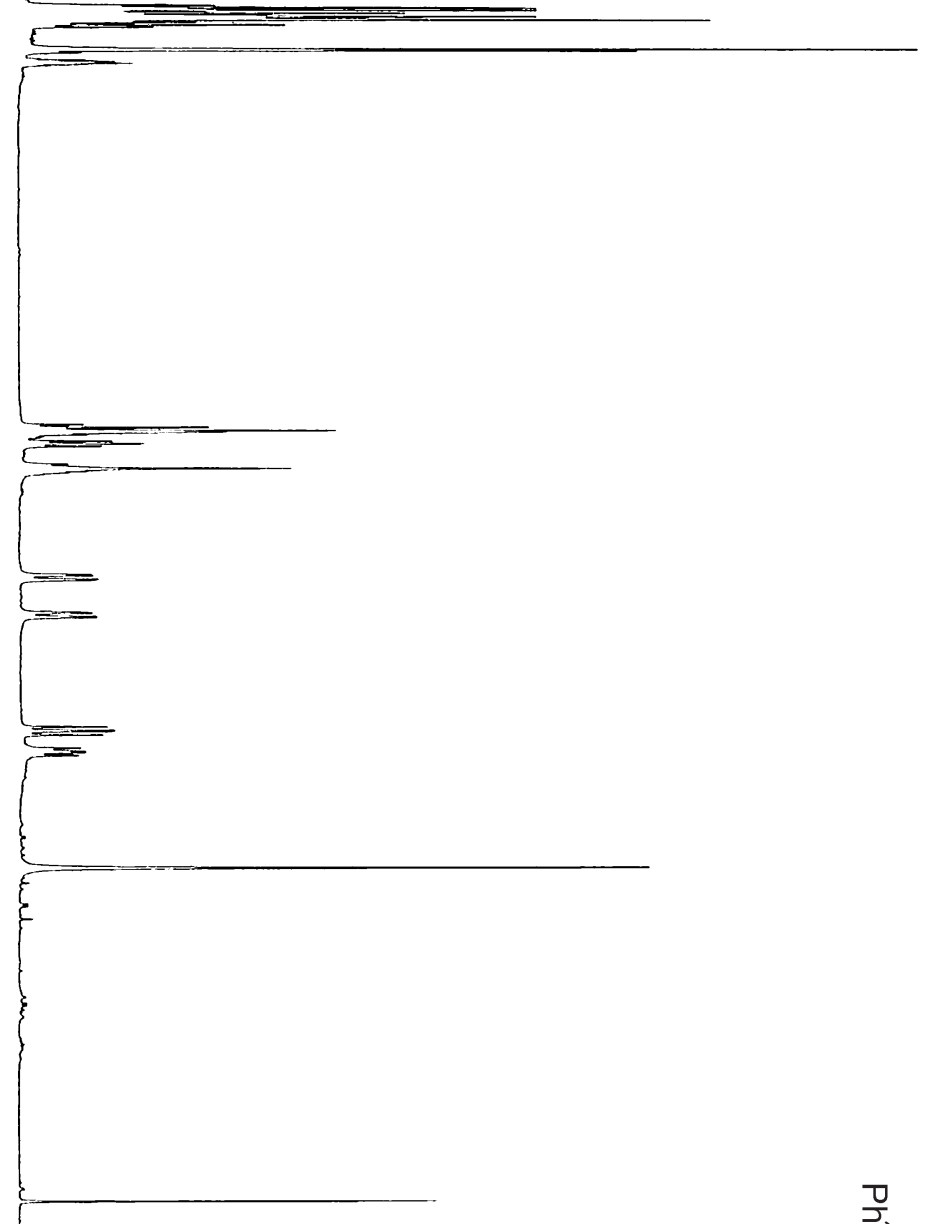

$\stackrel{\square}{s}$<smiles>C[C@H]1C=COC1</smiles>

$\vec{\omega}$

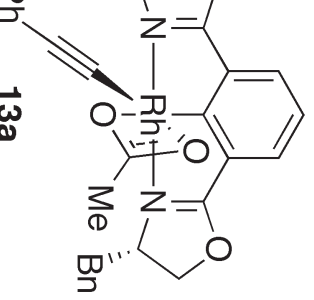

$\left[\begin{array}{r}7.551 \\ -7.536\end{array}\right.$

$-7.342$

$-7.327$

7.313

7.295

7.274

7.253

7.236

$-7.221$

7.206

7.073

7.063

7.051
-7.046

$-7.001$

6.994
-6.991
6.984

6.984

6.977

4.755

4.736

4.729
4.710

4.710

4.699

4.691

4.670

4.656

4.638

4.528

4.502
4.485

4.476

4.464

3.855

3.849

3.827

3.820

3.622

-3.600

3.594

2.921

2.900

2.893

2.871

2.790

$-2.770$

2.763
2.744

2.047

o

$\stackrel{\square}{g}$ 


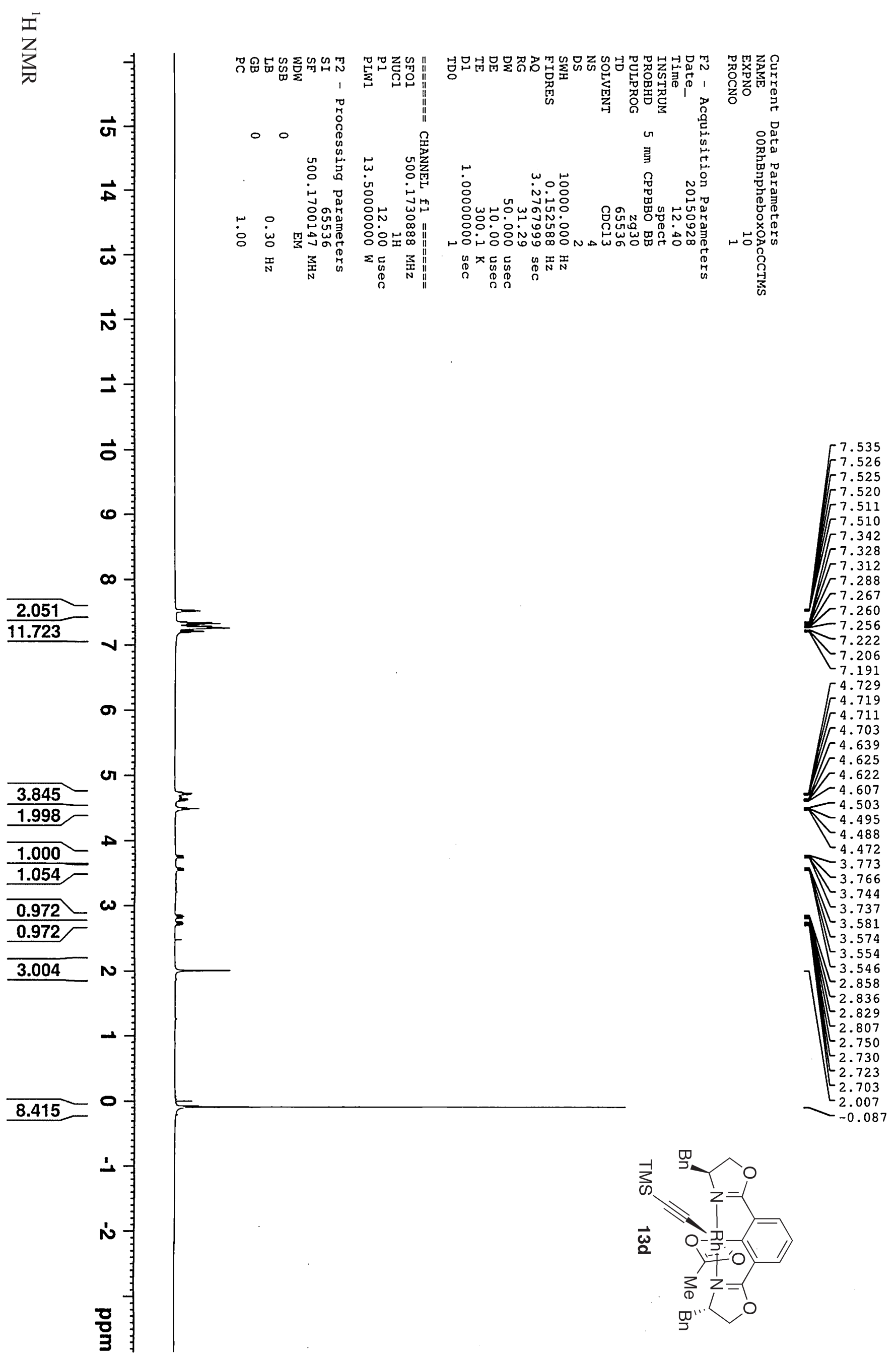




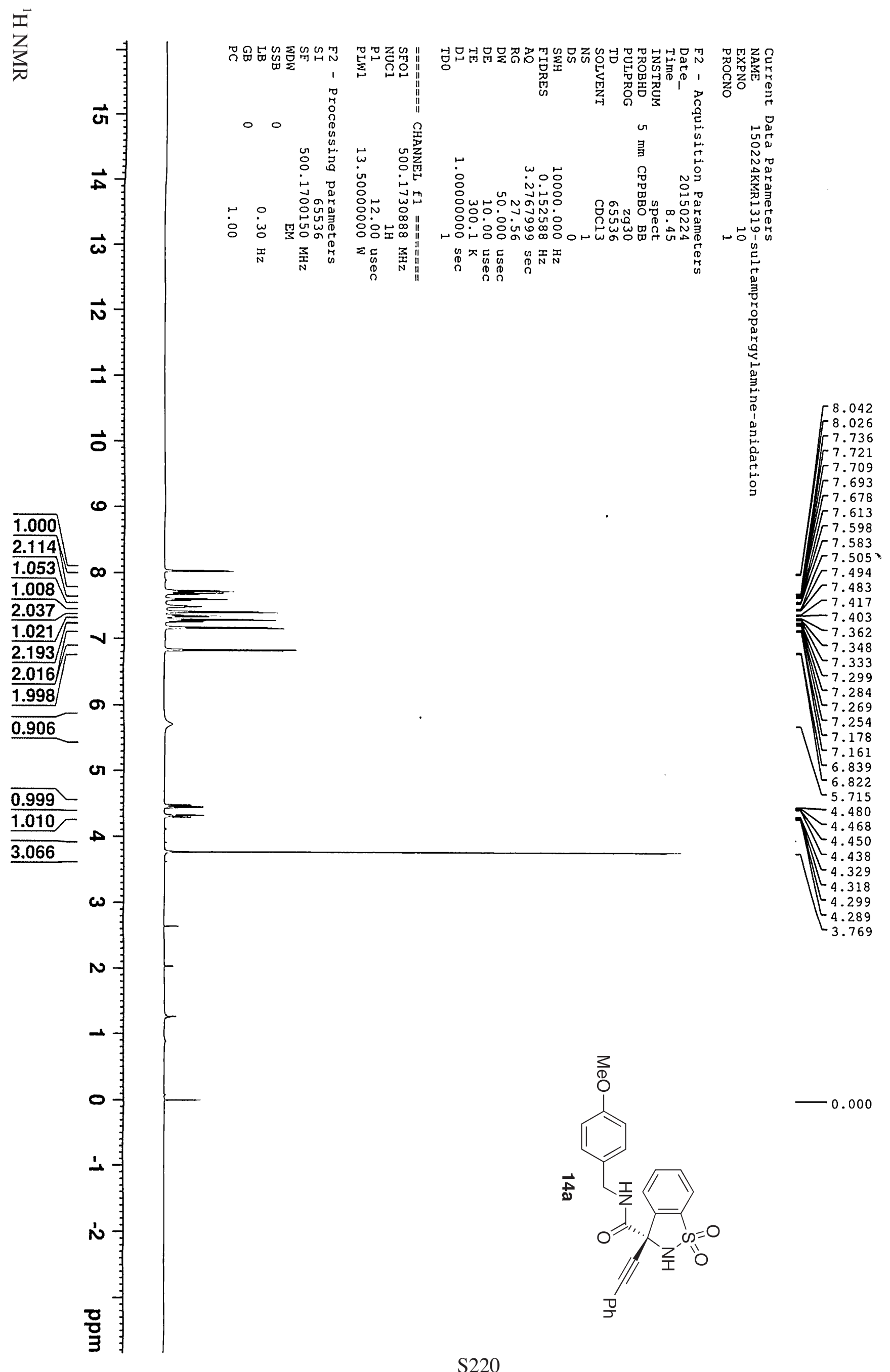




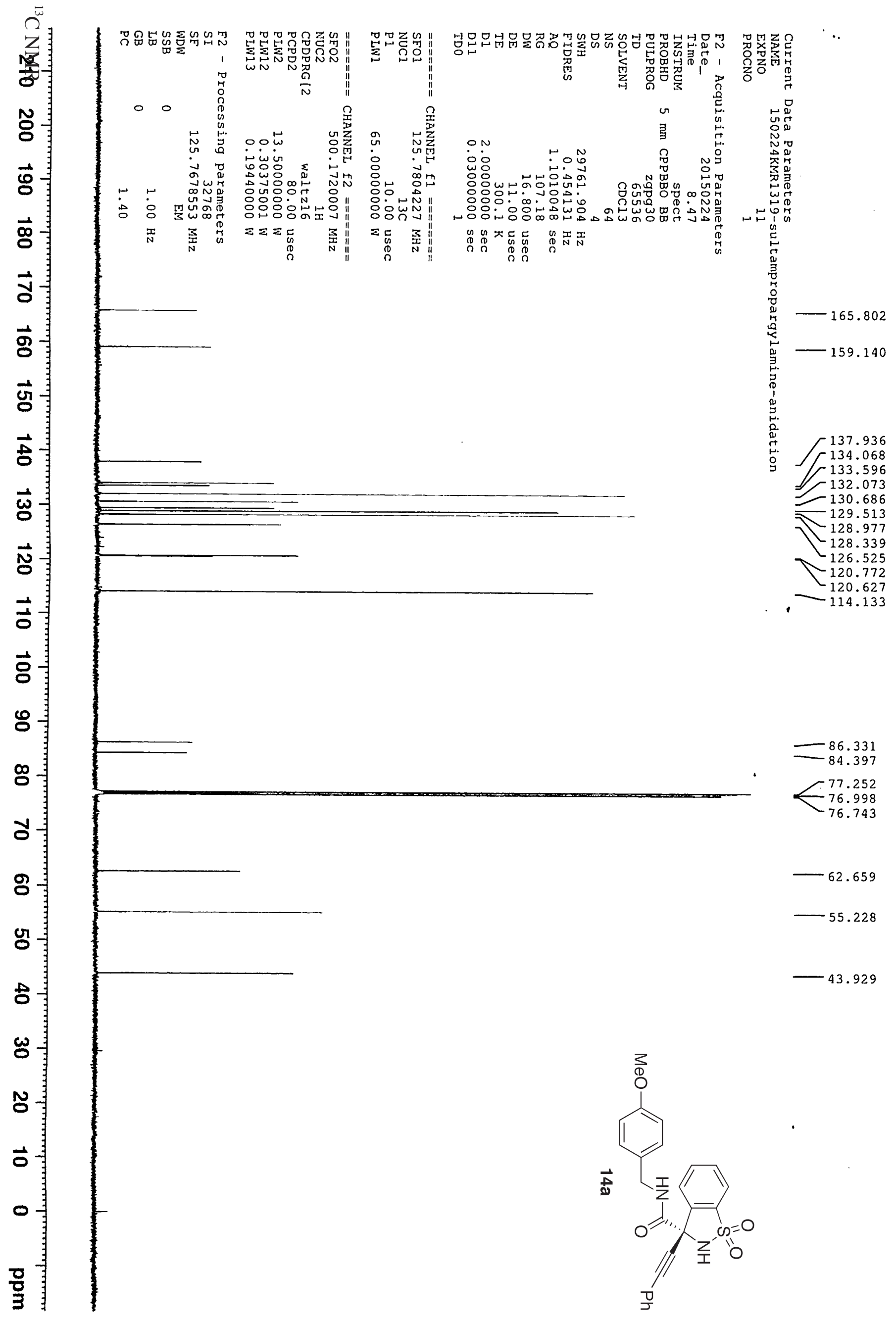




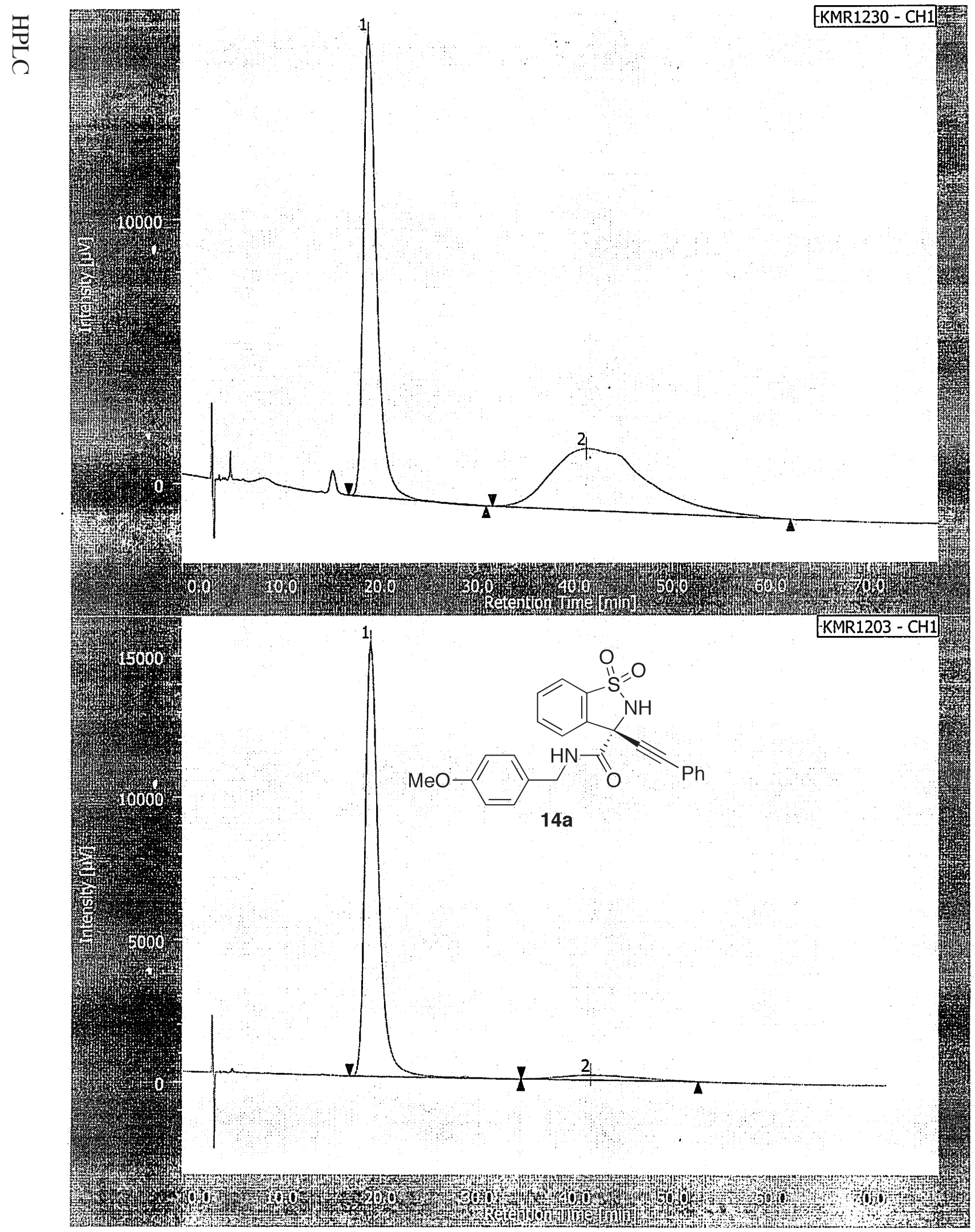

チャンネル情報+ピーク憎報

クロマトグラム名

KMR1230-CH1

サンプル名

チャンネル名

$\mathrm{CHI}$

\begin{tabular}{|c|c|c|c|c|c|c|c|c|c|c|c|}
\hline ピーク名 & $\mathrm{CH}$ & $t R$ [min] & 面皘 $[\mu \mathrm{V} \cdot \mathrm{sec}]$ & 高さ [ $[\mu V]$ & 面栍\% & 高さ\% & 定量值 & NTP & 分離度 & シンメトリー係数 & 鳘告 \\
\hline 1 Unknown & 1 & 18.967 & 1644847 & 17632 & 50.270 & 88.232 & N/A & 1066 & 2.220 & 1.385 & \\
\hline 2. Unknown & 1 & 41.258 & 1627185 & 2352 & 49.730 & 11.768 & $N / A$ & 86 & $\mathrm{~N} / \mathrm{A}$ & 1.559 & \\
\hline
\end{tabular}

クロマトグラム名 KMR1203-CH1

サンプル名

チャンネル名

$\mathrm{CH} 1$

\begin{tabular}{|c|c|c|c|c|c|c|c|c|c|c|c|}
\hline ピーク名 & $\mathrm{CH}$ & $t R[\mathrm{~min}]$ & 面積 $[\mu \mathrm{V} \cdot \mathrm{sec}]$ & 高さ [ [ V V] & 面積X & 高さో & 定晴值 & NTP & 分離度 & シンメトリー係数 & 敕告 \\
\hline Unknown & 1 & 19.025 & 1456316 & 15281 & 93.683 & 98.883 & $N / A$ & 1051 & 2.433 & 1.382 & \\
\hline 2) Unknown & 1 & 41.592 & 98201 & 173 & 6.317 & 1.117 & N/A & 105 & N/A & 1.272 & \\
\hline
\end{tabular}

